\title{
Decomposition for Compositional Verification
}

Genehmigte Dissertation

zur Erlangung des Grades eines

Doktors der Naturwissenschaften

der Fakultät für Elektrotechnik, Informatik und Mathematik

der Universität Paderborn

vorgelegt von

Dipl.-Inform. Björn Metzler

Paderborn, im Mai 2010 
Mitglieder der Promotionskommission:

- Prof. Dr. Heike Wehrheim (Vorsitzende, Gutachterin)

- Prof. Dr. Steve Schneider (Gutachter)

- Prof. Dr. Gitta Domik

- Prof. Dr. Wilhelm Schäfer

- Dr. Matthias Tichy

Die Dissertation wurde am 15. Januar 2010 bei der Fakultät für Elektrotechnik, Informtik und Mathematik der Universität Paderborn eingereicht und am 27. April 2010 vor der Promotionskommission verteidigt und durch die Fakultät angenommen. 


\section{Abstract}

Within the domain of safety-critical systems, software engineering becomes a major challenge, as failures of a system may have life-threatening ramifications. In order to ensure the reliability of software, its correctness is essential. For the correctness proof of a model, integrated formalisms with an underlying formal semantics can be used.

Several obstacles complicate a successful application of model checking software models. The main challenge is to cope with the state explosion problem, that is, the exponential growth of the system's state space in the size of the model. Several approaches deal with this well-known problem. One of them is compositional verification.

The basic idea of compositional verification is that the check of correctness of a complex system can be divided into smaller verification tasks. The technique avoids to build up the entire state space of the model, as it solely needs to deal with the individual state spaces of the single components of a system.

In order to facilitate an application of this technique, two problems need to be addressed: the model itself must be assembled from several components which is, in general, not the case. Furthermore, an application of compositional reasoning must provide an efficiency advantage over monolithic model checking.

Within this thesis, we develop a technique on how to decompose software models specified in the integrated formalism CSP-OZ. Such a decomposition results in two components suitable for the application of compositional reasoning.

A first challenge is posed by a proof of correctness, showing the equivalence of the original specification and a decomposition in our semantic domain. In order to achieve this, we carry out a dependence analysis by means of a specification's dependence graph. The analysis leads to a set of correctness criteria, based on which the graph is fragmented into two parts. The fragmentation then results in the decomposition of the specification. In addition, we introduce several techniques and algorithms to restore the specification's original control flow and its data flow.

As a second challenge, we address the practicability of compositional reasoning: we identify heuristics for measuring the quality of a valid decomposition. Here, we neglect inefficient decompositions. This allows us to consider only those, which most likely result in an effective compositional verification.

Overall, our approach facilitates a general application of compositional reasoning, as it does not rely on systems composed of several components. Moreover, valid decompositions, which are assessed as good by our heuristics, are beneficial for a compositional verification.

The whole approach is tool-supported due to an integration into a graphical modelling environment, allowing for the modelling, analysis, decomposition and (compositional) verification of integrated specifications. Model checking itself is performed within an assume-guarantee-based verification framework. Here, we use two proof rules, which are shown to be valid in our semantic domain. Along with this, we provide several case studies and experimental results. 



\section{Zusammenfassung}

Die Softwareentwicklung im Bereich von sicherheitskritischen Systemen stellt eine große Herausforderung dar, da Systemfehler lebensgefährliche Konsequenzen haben können. Die Korrektheit von Software ist essentiell, um ihre Verlässlichkeit zu garantieren. Für den Korrektheitsbeweis eines Softwaremodells eignen sich integrierte Formalismen, welchen eine formale Semantik zu Grunde liegt.

Das Model Checking von Softwaremodellen wird durch verschiedene Hindernisse erschwert. Die größte Herausforderung ist die Bewältigung der Zustandsexplosion, des exponentiellen Wachstums des Zustandsraums mit der Größe des betrachteten Systems. Eine Reihe von Techniken beschäftigt sich mit diesem populären Problem, unter anderem die kompositionelle Verifikation.

Die grundlegende Idee bei der kompositionellen Verifikation ist die Zerlegung des Korrektheitsbeweises in Teilaufgaben. Diese Methodik vermeidet die Konstruktion des Zustandsraums des gesamten Systems, stattdessen werden die Zustandsräume der einzelnen Systemkomponenten betrachtet.

Die Anwendbarkeit dieser Technik ist an zwei Voraussetzungen gebunden. Zum einen muss das Softwaremodell aus mehreren Einzelkomponenten zusammengesetzt sein, was im Allgemeinen nicht der Fall ist. Des Weiteren muss die Anwendung der kompositionellen Verifikation einen Effizienzvorteil gegenüber dem direkten Model Checking erbringen.

Diese Arbeit beschäftigt sich mit der Dekomposition von Softwaremodellen, spezifiziert in dem integrierten Formalismus CSP-OZ. Eine solche Zerlegung definiert zwei Komponenten, welche sich für die kompositionelle Verifikation eignen.

Eine erste Herausforderung dieser Arbeit stellt ein Korrektheitsbeweis dar, welcher die Äquivalenz der ursprünglichen Spezifikation und einer Dekomposition in der zugrunde liegenden semantischen Domäne zeigt. Dazu wird eine Abhängigkeitsanalyse durchgeführt, die auf dem Abhängigkeitsgraphen einer Spezifikation basiert. Diese Analyse führt zu einer Menge von Korrektheitsbedingungen, auf deren Basis der Graph in zwei Teile zerlegt wird. Daraus ergibt sich die Dekomposition der Spezifikation. Zusätzlich werden Techniken und Algorithmen zur Wiederherstellung des Kontroll- und Datenflusses der ursprünglichen Spezifikation vorgestellt.

Eine zweite Schwierigkeit betrifft die Praktikabilität der kompositionellen Verifikation. Dazu werden in dieser Arbeit Heuristiken zur Messung der Qualität einer validen Dekomposition ermittelt, wobei ineffiziente Dekompositionen vernachlässigt werden. Dies erlaubt es, ausschließlich solche Zerlegungen zu betrachten, die eine effektive kompositionelle Verifikation in Aussicht stellen.

Insgesamt ermöglicht die beschriebene Technik die Anwendung von kompositioneller Verifikation, da sich der Ansatz nicht nur auf zusammengesetzte Systeme beschränkt. Außerdem sind durch die Heuristiken favorisierte valide Dekompositionen vorteilhaft für die Anwendung der kompositionellen Verifikation.

Für den gesamten Ansatz existiert eine Werkzeugunterstützung. Diese basiert auf einer Integration in eine grafische Modellierungsumgebung, welche die Modellierung, 
Analyse, Dekomposition und (kompositionelle) Verifikation von integrierten Spezifikationen erlaubt. Das Model Checking wird im Rahmen eines Frameworks im Kontext des Assume-Guarantee Beweisverfahrens durchgeführt. Dabei werden zwei Beweisregeln verwendet, deren Korrektheit gezeigt wird. Schließlich werden einige Fallstudien sowie experimentelle Ergebnisse präsentiert. 


\section{Acknowledgments}

I am grateful to a number of persons for their support, guidance and patience over the last couple of years.

First of all, I would like to express my profound gratitude to Professor Dr. Heike Wehrheim for the supervision, the everlasting support and the opportunity to write this $\mathrm{PhD}$ thesis. She constantly pointed the right direction and helped in a dedicated manner, which is anything but naturally.

I also would like to thank the members of my PhD committee, Professor Dr. Steve Schneider, Professor Dr. Wilhelm Schäfer, Professor Dr. Gitta Domik and Dr. Matthias Tichy for their assistance and invaluable advice.

For the proof reading of this thesis and the English review, sincere thanks to Isabela Anciutti, Dr. Uwe Bubeck, Christian Estler, Dr. Dorina Ghindici, Dr. Andreas Goebels, Nils Timm, Simon Titz and Daniel Wonisch.

In our research group, I enjoyed a very warm and collaborative atmosphere. Here, special acknowledgements to Thomas Ruhroth, who always lent a helping hand and never backed down from assisting on all kinds of problems.

There are several students to whom I am greatly thankful for doing most of the implementation of the thesis' approach within Syspect: Klaus Herbold for implementing the decomposition, Meik Piepmeyer for developing the heuristics-based mass validation, Sebastian Micus for integrating the counterexample analysis and, last but not least, Daniel Wonisch for doing an excellent job in writing a compiler for the translation of Syspect export into $\mathrm{CSP}_{\mathrm{M}}$, integrating FDR2 into Syspect and developing the learning-based CSPLChecker.

I am also grateful to the members of the research group "Correct System Design" supervised by Professor Dr. Ernst-Rüdiger Olderog. In particular, I would like to thank Johannes Faber, who provided assistance to our extension of Syspect in many aspects, always coming up with helpful ideas or additional features. Furthermore, I am in debt to Ingo Brückner and Sven Linker who theoretically and technically provided the basis for this work by developing the slicing approach. It was a great pleasure to work with Ingo on several papers and discuss our related topics.

Most of all, there are three persons to whom my gratefulness is never-ending: my parents, Peter and Inge Metzler, for taking care of me in so many different aspects of life and for their limitless encouragement and patience. Finally, my heartfelt gratitude to my beloved girlfriend Celina: you are an inspiration to my life and the most warm-hearted and affectionate person I ever met. No words can describe the love and emotions I have for you. Your perpetual care, support and love mean the world to me. 



\section{Contents}

1 Introduction 1

1.1 A Vision of Correct Software . . . . . . . . . . . . . . . . . . . 1

1.2 Formal Methods and their Combination . . . . . . . . . . 2

1.3 Compositional Verification . . . . . . . . . . . . . . . . 3

1.4 Contributions ..................... 5

1.5 Thesis Structure . . . . . . . . . . . . . . . . . . 6

2 Background: Integrated Formal Methods 9

2.1 A Survey of (Integrated) Formal Methods . . . . . . . . . . . . . . . . 9

2.2 The Integrated Formalism CSP-OZ . . . . . . . . . . . . . . . . . . . 11

2.2.1 Case Study: Candy Machine . . . . . . . . . . . . . . . . . 11

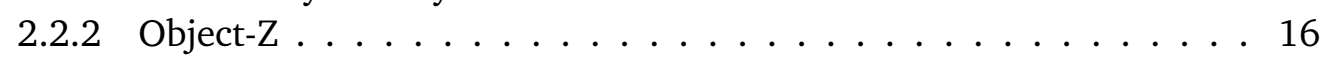

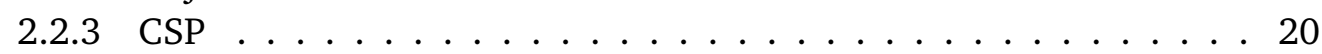

2.2 .4 Semantics of CSP-OZ . . . . . . . . . . . . . . . . 24

2.3 Dependence Analysis . . . . . . . . . . . . . . . . . . . . . 27

2.3.1 Dependence Analysis for CSP-OZ: Motivation . . . . . . . . . 27

2.3.2 Definition of the Control Flow Graph . . . . . . . . . . . . . . . 29

2.3.3 Definition of the Data Dependence Graph . . . . . . . . . . . . 32

2.3.4 Definition of the Dependence Graph . . . . . . . . . . . . 36

3 Background: Compositional Reasoning 41

3.1 Approaches to the State Space Explosion . . . . . . . . . . . . . . . . . . 42

3.2 Compositional Reasoning . . . . . . . . . . . . . . . . . . 43

3.2.1 Assume Guarantee Proof Rules . . . . . . . . . . . . . . . . 43

3.2.2 Obstacles to the Application of Assume Guarantee Reasoning . . 45

3.2.3 Learning for Compositional Verification . . . . . . . . . . . . . . . . 45

3.3 Assume-Guarantee Reasoning for CSP . . . . . . . . . . . . . . . . . 47

3.3.1 Application Example: Elevator System . . . . . . . . . . . . . . . . . 49

3.3.2 Soundness of Assume-Guarantee Proof Rules . . . . . . . . . . . 50

3.4 Related Work . . . . . . . . . . . . . . . . . . . . . 53

4 Decomposition of a Specification $\quad \mathbf{5 5}$

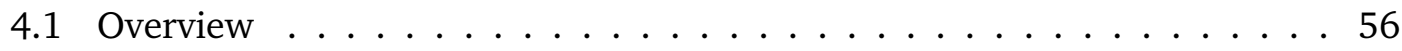

4.2 Cut of a Dependence Graph . . . . . . . . . . . . . . . 58

4.2.1 Fragmentation of the Control Flow Graph . . . . . . . . . . 58

4.2.2 Correctness Criteria for the Fragmentation . . . . . . . . . . . . 61

4.2 .3 Definition of a Cut . . . . . . . . . . . . . 666

4.2.4 Candy Machine Revisited: Cut of the Dependence Graph . . . . . 70 
4.3 Decomposing CSP-OZ Specifications _ . . . . . . . . . . . . . 72

4.3.1 Intermediate Definition of the Decomposition . . . . . . . . . 75

4.3.2 Preservation of the Data Dependences . . . . . . . . . . . . 81

4.3.3 Preservation of the Control Flow . . . . . . . . . . . . . 86

4.3.4 Renaming for the Decomposition . . . . . . . . . . . . 98

4.3.5 Definition of the Decomposition . . . . . . . . . . . . . . . 100

4.3.6 Candy Machine Revisited: Decomposition . . . . . . . . . . . . . 101

4.3.7 Improvement of the Decomposition . . . . . . . . . . . . . . 103

4.4 Decomposition for the General Case: Number Swapper . . . . . . . . . . 106

4.5 Related Work . . . . . . . . . . . . . . . . . . . . . . . . . . 109

5 Correctness of the Decomposition $\quad 111$

5.1 Ensuring Correct Synchronisation . . . . . . . . . . . . . . . . . 113

5.2 Correctness for the CSP Part . . . . . . . . . . . . . . . . . . . . . . 119

5.2.1 Properties of the Decomposition: CSP Part . . . . . . . . . . . . . 119

5.2.2 Correctness of the Decomposition: CSP part . . . . . . . . . . . . 132

5.3 Correctness for the Object-Z Part . . . . . . . . . . . . . . . 138

5.3.1 Properties of the Decomposition: Object-Z Part . . . . . . . . . . 140

5.3.2 Correctness of the Decomposition: Object-Z part . . . . . . . . . 146

5.4 Correctness of the Renaming for the Decomposition . . . . . . . . . . . . 159

5.5 CSP Laws for Parallel Composition . . . . . . . . . . . . . . . . . . . . 164

5.6 Proof of the Main Theorem . . . . . . . . . . . . . . . 166

6 Finding Reasonable Decompositions 167

6.1 Decomposition Heuristics ． . . . . . . . . . . . . . . . . . . . . . 168

6.1 .1 First Heuristic: Cut Size . . . . . . . . . . . . . . . . . . . 169

6.1.2 Second Heuristic: Even Distribution . . . . . . . . . . . . . 170

6.1.3 Third Heuristic: Few Transmission . . . . . . . . . . . . . . 170

6.1 .4 Fourth Heuristic: Few Addressing . . . . . . . . . . . . . . . . . 172

6.2 Evaluation of Decomposition Heuristics . . . . . . . . . . . . . . . . 172

6.3 Candy Machine Revisited: Evaluation of Cuts . . . . . . . . . . . . . 174

6.4 Case Study: Two Phase Commit Protocol . . . . . . . . . . . . . . . . . 175

6.5 Discussion . . . . . . . . . . . . . . . . . . . . . . 180

6.6 Related Work . . . . . . . . . . . . . . . . . . . 181

7 Implementation and Experimental Results 183

7.1 Syspect . . . . . . . . . . . . . . . . . . . . . . . . . . 184

7.1 .1 Class Diagrams . . . . . . . . . . . . . . . . 184

7.1 .2 State Machines . . . . . . . . . . . . . . . 186

7.1 .3 Component Diagrams . . . . . . . . . . . . . . 187

7.1 .4 Export to CSP-OZ . . . . . . . . . . . . . . . . . . 187

7.2 Decomposition Framework for Syspect ～. . . . . . . . . . . . . . . . 188

7.2.1 Decomposition Plug-In . . . . . . . . . . . . . . . . . 189

7.2 .2 Mass Validation . . . . . . . . . . . . . . . . . . . . . 191 
7.2.3 Model Checking with FDR2 and the CSPLChecker . . . . . . . . . . 192

7.2 .4 Counterexample Analysis . . . . . . . . . . . . . . 196

7.2 .5 Overall Workflow . . . . . . . . . . . . . . . . . . . 198

7.3 Experimental Results . . . . . . . . . . . . . . . . . . . 200

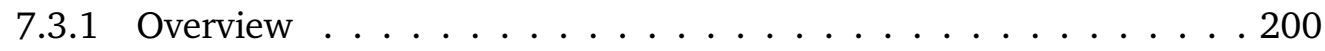

7.3.2 Verification Results for the Candy Machine . . . . . . . . . . . . . 201

7.3.3 Verification Results for the Two Phase Commit Protocol . . . . . . . 204

7.3.4 Verification Results for the Number Swapper . . . . . . . . . . . . 207

7.3 .5 Discussion . . . . . . . . . . . . . . . 207

8 Conclusion $\quad 217$

8.1 Summary . . . . . . . . . . . . . . . . . . 217

8.2 Future Work . . . . . . . . . . . . . . . . . . . . 219

$\begin{array}{ll}\text { Glossary of Symbols } & 223\end{array}$

$\begin{array}{lr}\text { Bibliography } & 229\end{array}$

$\begin{array}{lr}\text { List of Figures } & 239\end{array}$

$\begin{array}{ll}\text { List of Tables } & 243\end{array}$

$\begin{array}{ll}\text { Index } & 245\end{array}$ 



\section{Introduction}

\section{Contents}

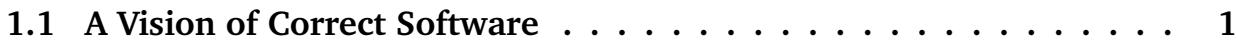

1.2 Formal Methods and their Combination $\ldots \ldots \ldots \ldots \ldots$

1.3 Compositional Verification . . . . . . . . . . . . 3

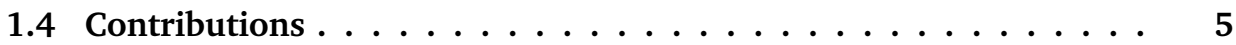

1.5 Thesis Structure $\ldots \ldots \ldots \ldots \ldots \ldots \ldots$

\subsection{A Vision of Correct Software}

Over the last decades, research in Computer Science underwent a major focus change: as hardware and software systems influence our daily lives in many critical and even life-threatening situations, systematic approaches to ensure their quality in terms of correct functionality are essential. Trustworthiness and safety-critical hardware and software are required in many areas such as aerospace manufacturing, the automotive industry and medical care, to mention only a few. The more we depend on these systems, the more confidence we need to have in their reliability.

Software quality assurance (SQA) [Gal04] is an approach to observe the engineering process regarding to the quality of the resulting software. Since weaknesses and errors can be introduced at any given point in the process of software development, they need to be excluded at an early stage of the design process.

One SQA methodology is the model-driven development (MDD) [MDA]: software systems are described as models in some (domain specific) language. For modelling object-oriented systems, the Unified Modelling Language (UML) [BJR99] is the current de facto standard.

In order to ensure software quality, techniques for early model analysis have been developed, which makes MDD highly useful. One specific analysis technique is software testing [Xie96], aiming at the detection of errors in the model. Automated testing can be of great benefit if hidden faults can be determined and corrected early in the development process. However, correctness of a program can never be achieved by testing:

Program testing can be a very effective way to show the presence of bugs, but it is hopelessly inadequate for showing their absence.

[Dij72]

Since malfunctions are in many cases unacceptable, errors in critical parts of the system have to be ruled out completely. Limited computing resources make the verification of 
large models practically impossible. Therefore, a possible strategy is to verify vital parts of a system complemented by testing the system's functionality and non-critical aspects.

For a system's verification, the model needs to be specified in some mathematical formalism incorporating a well-defined semantics. One particular kind of mathematicalbased techniques are formal specification languages (formal methods [CW96]). Based on their precise semantics, they allow for the application of verification techniques. In order to guarantee a reliable system, the developer needs to adhere to the specification, which has to be proven correct.

\subsection{Formal Methods and their Combination}

An informal description of a software model - such as by using an intuitive description based on natural language - is not sufficient for mathematical-based proof techniques due to its missing formal semantics. Owed to its expressive power, the UML does not have a common mathematical-based representation and is often referred to as a semi formal modelling language. This lack of a precise underlying semantics makes the verification of UML models generally impossible.

Formal methods [CW96] are widely-used as a mathematical-based approach of designing software and hardware systems and they receive considerable attention from the research community. The existence of a well-defined underlying semantics, making a precise analysis of the system technically feasible, is common to all formal languages. There are a lot of different notations and techniques, with all of them holding their specific advantages and disadvantages. In general, formal methods can be classified into several categories for describing different aspects of a system, among them are state-based techniques using set theory and predicate logic such as Z [ISO00] and the B-method [Abr96]. In contrast, process algebras like CSP [Hoa85] and CCS [Mil89], for instance, specify the system's behavioural aspects.

Individual formalisms do not cover all relevant aspects to describe a complex system as a whole. Instead of redefining existing methods moving away from the original intention for a specific method, recent research has shifted to the domain of integrated formal methods. Focusing on more than one specific facet, they combine different languages to model different viewpoints of a system within one, well-defined formalism. By defining a common and consistent semantics, these notations incorporate the advantages of each individual formalism. Some examples are the combination of the process algebra CSP with the state-based formalism B into CSP||B [ST02], the formalism Event-B [AH06], a combination of the B method [Abr96] with events, and the method we are focusing on in this thesis, CSP-OZ [Fis97], a combination of CSP with the object-oriented extension of Z, Object-Z [Smi00].

In terms of the overall goal (that is, the verification of a system model), the system has to be specified in an (integrated) formalism and needs to be proven correct against certain requirements of the system. This act is called formal verification. 


\subsection{Compositional Verification}

Besides theorem proving, model checking [CGP99] is the most widespread formal verification method. Given a specification $S$, specified as a finite state-transition system, and a requirement $P$, formulated in some logical formalism, model checking fully automatically proves or disproves that the system meets the requirement. This is in general denoted by $S \models P$.

As the complexity of software and hardware systems increases, so does the complexity of its models. The most common and major problem for the applicability of model checking is the state explosion: the size of the software model, represented by a state transition system, exponentially grows with the size and number of its components and data domains. In particular, model checking for integrated specifications needs to deal with the state explosion problem: for instance, the behavioural part of the specification can incorporate concurrency, leading to an exponential blow-up of its branching structure. In addition, its state space can be large or even infinite due to its possibly infinite data types.

In general, building up the full state space of a model is infeasible. In order to allow model checking to scale to complex systems, several techniques to tackle this problem were proposed. To mention some of them, symbolic model checking aims at an efficient representation of the model's state space whereas partial order reduction and data abstraction techniques try to reduce the state space of a model by exploring its concurrency structure and by abstracting from concrete data values, respectively. These techniques complement each other and can be combined.

Amongst these techniques, compositional verification $\left[\mathrm{dRHH}^{+} 01\right]$ is one promising approach: instead of verifying a software model as a whole, the components of the model are analysed separately. The verification results can then be combined into one global result. For an application of this divide-and-conquer approach, the system needs to be structured into several (parallel) components. That being the case, different strategies can be applied in order to incrementally prove a system correct without ever building up its full state space.

The main technique of compositional verification is assume-guarantee reasoning [FP78, Jon83, MC81], applied to a system usually structured into two components. For a given property $P$ on the overall system composed of $S_{1}$ and $S_{2}$, both components can be verified separately without building the global state space. In order to do so, an environment assumption $A$ needs to be identified, describing the connection and interdependences between the components. The application of an appropriate proof rule, employing $A$, yields the correctness of the system with respect to $P$.

Assume-guarantee reasoning has been researched for more than three decades. Recently, a new strategy to fully automatically generate the assumption [CGP03, BGP03] gave a new impulse to this area of research. The strategy is based on automatic learning, thereby freeing the user from a manual computation of the assumptions used in assume-guarantee reasoning.

However, the technique relies on a given structuring of the system into parallel components. Moreover, the efficiency of this approach depends on several factors: if the 
generated assumption is too large or the size of the components is not well-balanced, applying the approach can again lead to large state spaces and even worse verification run-times compared to monolithic (direct) verification. It is essential to think about good decompositions to ensure applicability and scalability of the approach [CAC06].

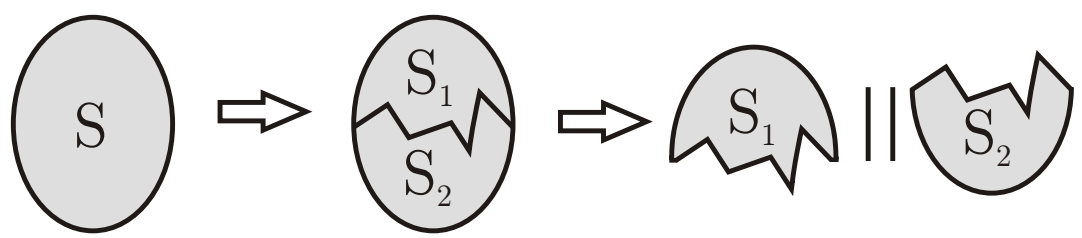

Figure 1.1: Decomposition of a specification $S$ into $S_{1}$ and $S_{2}$

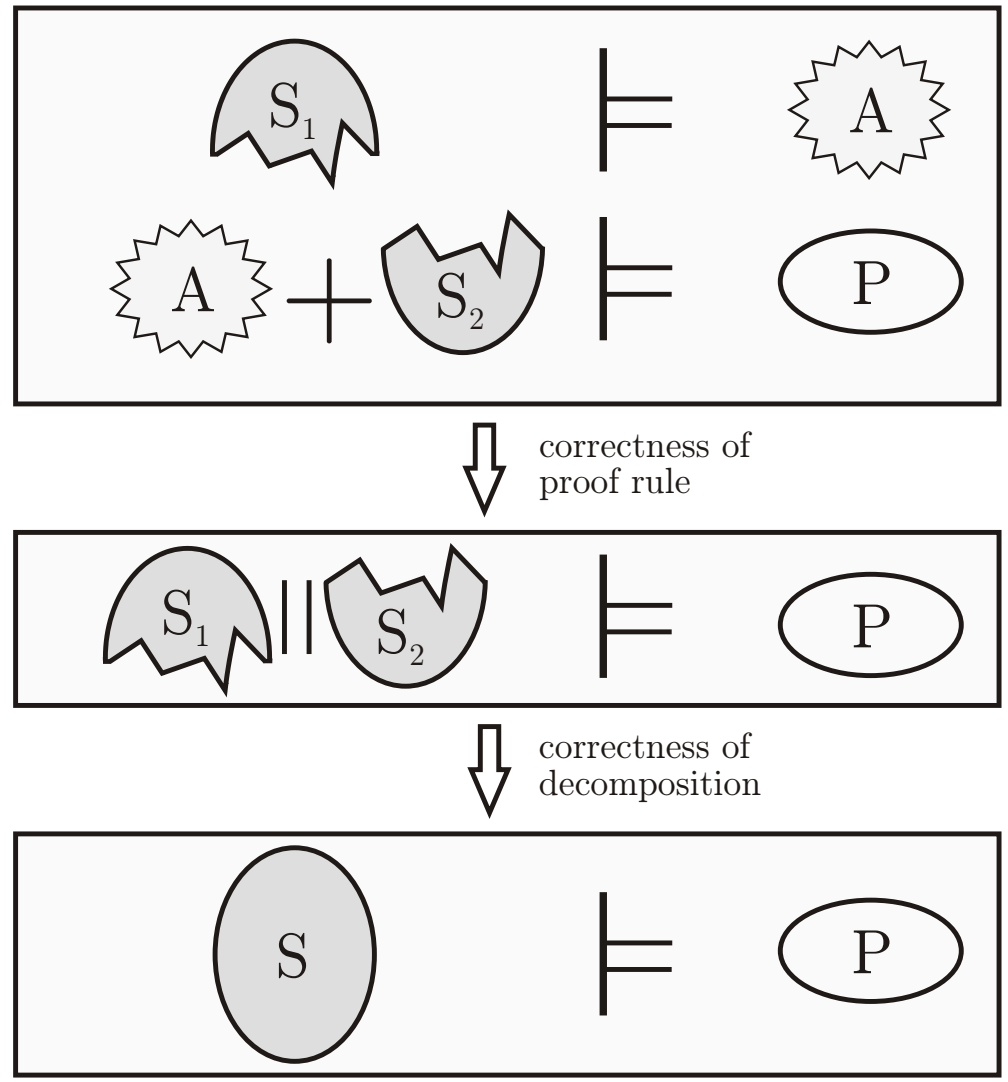

Figure 1.2: Illustration of the overall approach of this thesis

In this thesis, we construct and evaluate decompositions of integrated specifications. The starting point is a specification $S$ for which we want to show a specific property $P$. We 
define a set of correctness criteria, serving as the basis for the decomposition of $S$. Figure 1.1 illustrates the overall idea. The decomposition results in two specification parts, $S_{1}$ and $S_{2}$. These two parts represent the two parallel components of the decomposed system. An appropriate synchronisation between $S_{1}$ and $S_{2}$ ensures that the decomposition and the original system are behaviourally equivalent which is subsequently shown in the correctness proof.

$S_{1}$ and $S_{2}$ then serve as the input for assume-guarantee-based proof rules. The proof rule, as illustrated in Figure 1.2, states the following: if $S_{1}$ satisfies an assumption $A$ (described by the symbol ' $=$ ') and if $S_{2}$ satisfies $P$ under the assumption $A$, then the overall system composed of $S_{1}$ and $S_{2}$ satisfies $P$. Correctness of the decomposition yields that $S$ satisfies $P$, if, and only if, the conclusion of the proof rule can be inferred.

The approach is based on several context-specific heuristics pointing the direction for reasonable decompositions. The technique thus allows for an efficient application of assume-guarantee reasoning. Within our implemented framework for CSP-OZ, we translate the obtained components to the input language of a model checker and ultimately apply the learning-based approach. We are able to evaluate different decompositions by comparing verification run-times with those for monolithic verification.

\subsection{Contributions}

Compositional verification for integrated formal methods has been researched in [ST04, But09]. These works perform the decomposition of a system by hand and rely on the fact that it can be carried out effectively.

Learning for compositional verification, especially to automate the verification process, was introduced in [CGP03] and further developed in $\left[\mathrm{PGB}^{+} 08\right]$. The techniques are, however, not applied in the context of formal methods and rely on systems which are already composed of several components.

Alur and Nam [NA06, Nam07] use assume-guarantee-based reasoning in the context of symbolic model checking. They apply the learning framework to automatically generate assumptions and decompose a given system. In addition, they propose heuristics to improve the decomposition process. In their semantic domain of symbolic transition modules solely based on boolean variables, they do not deal with the aspects of integrated formalisms such as data flow, control flow and synchronisation. Furthermore, the developed heuristics only focus on aspects of the learning framework and they do not consider the (dependence structure of the) original system.

The key contribution of this thesis is an approach on how to combine all of these strategies, that is, how to effectively apply compositional verification for integrated formal methods: based on several correctness criteria and certain heuristics, we explicitly decompose the given system. The result of the decomposition serves as the input for the learning-based automated verification process.

Overall, the thesis' contributions are given as follows. We define an approach to decompose specifications written in CSP-OZ. The approach does not rely on systems which are already composed of several processes but, instead, leads to self-defined decompositions. 


\begin{tabular}{|c|c|}
\hline CATEgory & CONTRIBUTIONS \\
\hline Decomposition & $\begin{array}{ll}\checkmark & \text { Decomposition for integrated specifications. } \\
\checkmark & \text { Exploitation of specification's dependence structure. } \\
\checkmark & \text { Heuristics-based approach to detect reasonable decomposi- } \\
& \text { tions. }\end{array}$ \\
\hline Soundness Proof & $\begin{array}{ll}\checkmark & \text { Equivalence between original and decomposed system. } \\
\checkmark & \text { Correctness in context of assume-guarantee framework. }\end{array}$ \\
\hline Implementation & $\begin{array}{ll}\checkmark & \text { Integration into graphical modelling framework. } \\
\checkmark & \text { Integration into assume-guarantee-based framework. } \\
\checkmark & \text { Evaluation-based on case studies. }\end{array}$ \\
\hline
\end{tabular}

Table 1.1: Contributions of this thesis

In order to achieve reasonable decompositions, we investigate heuristics, exploiting the dependence structure of the specification as well as algorithms for the assumption identification. We present a correctness proof, showing that our decomposition preserves the observable behaviour of the specification. Since the decomposition mandatorily modifies the specification's internal behaviour, the proof incorporates several techniques to link the original system to its decomposition. We integrate the approach along with the learning strategy into a graphical modelling framework for CSP-OZ [Sys06]. An evaluation of the approach is performed based on several case studies and two different learning strategies according to [CGP03] and [BGP03].

\subsection{Thesis Structure}

This thesis is structured as follows.

Chapter 2 provides an overview of (integrated) formal methods and introduces the employed formalism CSP-OZ [Fis97], a combination of the process algebra CSP [Hoa85], and the state-based formalism Object-Z [Smi00]. The semantics of CSP-OZ and necessary definitions are given. For an illustration of CSP-OZ, we present the running case study of this thesis. Along with this, we provide background on the dependence analysis for CSP$\mathrm{OZ}$, which serves as the basis for the decomposition approach. The dependence structure of a specification is defined by means of a dependence graph developed in [Brü08], reflecting the control flow of a specification's CSP part as well as data dependences with 
respect to the Object-Z part. We present the definition and slightly modify it for our purpose.

Chapter 3 introduces compositional reasoning and starts with an overview on relevant techniques to cope with the state explosion problem in model checking. We survey compositional verification, particularly in the context of integrated formal methods. Afterwards, we present the specific method we deal with in this thesis: the assumeguarantee paradigm. The learning strategy to automatically generate assumptions is introduced next. In order to integrate assume-guarantee reasoning into our setting, we show the correctness of two compositional proof rules in the semantic domain of CSP-OZ. The chapter concludes with a discussion of related work.

Chapters 4-6 are the core chapters of this thesis. In Chapter 4, we introduce our definition for the decomposition of a CSP-OZ specification. We start by defining correctness criteria for a fragmentation of a specification's dependence graph into two parts. Subsequently, we define the decomposition of the specification itself resulting in two specification parts. These parts represent the two parallel components for the employed compositional proof rules. We motivate and describe the employed techniques to guarantee a semantics-preserving decomposition and illustrate the individual steps on the running case study. Finally, we discuss works closely related to our approach.

Chapter 5 presents the correctness proof of our approach. Ultimately, we show that the decomposition does not change the overall semantics of the specification. Several properties, relating the decomposed specification to the original system, are proven. We show that the original specification and the decomposed system, that is, the composition of the two parallel components, are behaviourally equivalent in our semantic domain. Achieving this is done through employing the compositional semantics of CSP-OZ along with the criteria on a correct decomposition.

Chapter 6 describes techniques and heuristics for finding reasonable decompositions. These are the ones for which model-checking-based on our approach will presumably outperform monolithic model checking. We motivate and discuss some context-specific heuristics for good decompositions. Furthermore, we introduce a second, bigger case study, on which we illustrate the application of the heuristics.

Chapter 7 introduces our implementation framework and experimental results. The graphical modelling framework Syspect [Sys06] for modelling CSP-OZ specifications serves as the platform. We describe our integration of the decomposition approach and the integration of the learning framework along with the heuristics-based identification for reasonable decompositions. Additionally, we evaluate our approach on three case studies and discuss the results.

Chapter 8 summarises this thesis, discusses the main results and points out possible topics for future work. 



\title{
Background: Integrated Formal Meth- ods
}

\author{
Contents \\ 2.1 A Survey of (Integrated) Formal Methods . . . . . . . . . . . 9 \\ 2.2 The Integrated Formalism CSP-OZ . . . . . . . . . . . . . . 11 \\ 2.2.1 Case Study: Candy Machine . . . . . . . . . . . . . . . 11

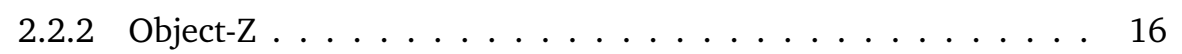

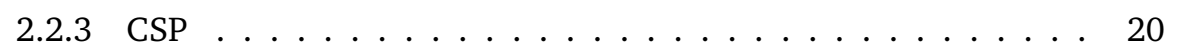 \\ 2.2 .4 Semantics of CSP-OZ . . . . . . . . . . . . . . . . . . 24 \\ 2.3 Dependence Analysis $\ldots \ldots \ldots \ldots$. . . . . . . . . . 27 \\ 2.3.1 Dependence Analysis for CSP-OZ: Motivation . . . . . . . 27 \\ 2.3.2 Definition of the Control Flow Graph . . . . . . . . . . . . 29 \\ 2.3.3 Definition of the Data Dependence Graph . . . . . . . . . 32 \\ 2.3.4 Definition of the Dependence Graph $\ldots \ldots \ldots$
}

The introduction gave a brief overview on the subject area and goals of this thesis. The following two chapters provide the necessary background for the main part of this work. In this chapter, (integrated) formal methods, and in particular CSP-OZ, will be introduced in Sections 2.1 and 2.2. The dependence analysis for CSP-OZ, which serves as the basis for the decomposition, is presented in Section 2.3.

\subsection{A Survey of (Integrated) Formal Methods}

Model-driven software development aims at the abstract description of a system by specifying a software model in some domain specific language. A model needs to precisely reflect the relevant aspects of the software product to be developed. After an accurate analysis, tools are used to automatically generate code from the model.

The Unified Modelling Language (UML) [BJR99] is undeniable the notation to model object-oriented systems in a graphical and intuitive way. The acceptance of the UML as a standard, not only in the academic but also in the industrial field, was not an overnight process. Over many years, researchers defined and evaluated different notations to finally end up with the UML 1.0 proposed in 1997.

Due to the lack of a common precise formal semantics, the UML is not adequate for a rigorous formal analysis. Even though there exist several tools supporting the automated verification of $\mathrm{UML}$ diagrams $\left[\mathrm{BGH}^{+} 05\right.$, DWQQ01, $\left.\mathrm{BBK}^{+} 04\right]$, they are all restricted to part of the language.

In the perspective to define mathematically-based languages suitable for formal specification and verification, researchers all over the world investigate different techniques 
and formalisms. Over the last three decades, a huge amount of formal methods has been developed. In [CW96], Clarke and Wing surveyed the current state of the art. More recently, Bowen [Bow09] set up a Wiki [Wik06] used by the formal methods community which gives a detailed overview over many individual formalisms and shows the broad spectrum of research in this area.

Formal methods can be classified into different categories. Mainly, these are behaviour oriented techniques concentrating on the dynamic aspects of a system such as communication, concurrence and control flow, state-based formalisms for the specification of the data and functional aspects and languages to describe hybrid systems which incorporate both, discrete and continuous behaviour.

Behaviour Oriented Formalisms: Among the formalisms to describe behavioural aspects of a system, Petri Nets [Rei85] are a graphical notation to illustrate distributed systems. Process algebras such as CCS [Mil89], CSP [Hoa85] and LOTOS [ISO89] describe concurrent systems by using an algebraic language. Milner also developed the strongly CCS related $\pi$-calculus [Mil99]. Another widely used formalism, particularly in the context of the UML, are State Charts [Har87].

State Based Formalisms: The most popular techniques concentrating on the data aspects of a system, that is, describing a system's state space, are Z [Spi92, ISO00], a set theory and first-order-predicate-logic-based formalism, and the $\mathrm{Z}$ related $\mathrm{B}$ method [Abr96], where B is slightly more low-level and focused on automatic code generation with great success in industrial application [Abr06]. Object-Z [Smi00] is an extension of $\mathrm{Z}$ to additionally integrate object-oriented concepts into Z. Event-B [AH06] extends the B method with guarded events. Abstract State Machines [BS03] describe a system's state space and its modifications by using transformation rules and functions.

Formalisms for Hybrid Systems: For the specification of hybrid systems, hybrid automata [ACHH92] combine the description of discrete and continuous behaviour of a system. For the description of continuous real time aspects, in 1994, Alur and Dill developed a real time extension for finite state automata, called timed automata [AD94].

Naturally, different description languages specify different viewpoints of a system. The analysis of large systems thus requires more than one dedicated formalism to reason about different aspects. Many researchers advocating formal methods agree on the statement that there exists no single notation covering all aspects of complex software systems. For this reason, they aim at combining existing, well researched languages, into one consistent new formalism, an integrated formal method.

These combinations range from the integration of two or more viewpoints into a single formalism. Combinations of a process calculus with a state-based technique are, for instance, CCS-Z [TA97] combining CCS and Z, the combination of CSP and Z into CSP-Z [MS98], along with CSP||B [TS99], a combination of CSP with the B-method. Fischer [Fis97] integrated CSP and Object-Z into the formalism CSP-OZ. 
The integration of time aspects into existing formalisms is, for instance, researched in the context of Timed CSP [Sch99], an integration of real time into CSP. E-LOTOS [ISO01] supplements LOTOS to support time and incorporates a functional-language-based data typing part. In [Hoe06], Hoenicke extended CSP-OZ with the real time interval logic Duration Calculus [ZH04] into CSP-OZ-DC.

The differences between these combinations can also be found in how the new semantics is defined. As an example, Circus [WC02], a combination of CSP, Z and a refinement calculus [SWC02], introduces a new semantics from scratch, that is, the semantics of CSP and $\mathrm{Z}$ are redefined into a new model, using Hoare's approach of Unifying Theories of Programs [HJ98]. Other formalisms, such as CSP||B, keep the original semantics and are thus able to use existing tools.

The following section stepwise introduces the applied formalism, CSP-OZ, illustrated by an example. In order to familiarise this formalism for the core chapters, we introduce the syntax and semantics of CSP-OZ along with necessary definitions and characteristics.

\subsection{The Integrated Formalism CSP-OZ}

Ever since its introduction in 1978 by Sir Anthony Hoare [Hoa78], the process algebra Communicating Sequential Processes (CSP) draws a lot of attention and is widely used for the specification of concurrent systems. The basic underlying concept is a description of a system by events and processes: a process defines the communication and interaction aspects by using an underlying alphabet, its set of events.

The state-based $\mathrm{Z}$ notation was developed by Jean-Raymond Abrial and others in the late 1970s. By using the concept of operation schemas, a Z specification describes the state space and its modifications based on mathematical theory [Spi92]. Smith [Smi00] defined an object-oriented extension of Z, Object-Z.

The integrated formalism we will concentrate on in this thesis is CSP-OZ, a combination of CSP with the object-oriented specification language Object-Z, introduced in [Fis97] and further elaborated on in [Fis00]. In his $\mathrm{PhD}$ thesis, Fischer developed the formalism by preserving the original semantics of both, CSP and Object-Z, with the objective to reuse existing theories and tools for both, CSP and Object-Z. In comparison to [Smi00], he introduced a slightly modified notation for Object-Z to which we will refer in this thesis.

We introduce CSP-OZ by means of an example serving as the running case study for this thesis. Afterwards, we give an overview on the syntax and semantics along with required definitions for the incorporated formalisms CSP, Object-Z and CSP-OZ itself.

\subsubsection{Case Study: Candy Machine}

The following example of a CSP-OZ specification describes a candy machine allowing for the payment and collection of several goodies. At first, we define some basic types needed for the specification and start with a free type Candies denoting the set of possible candies a customer may order. These are either a chocolate, a cookie or crisps: 


\section{Candies $::=$ CHOC $\mid$ COOKIE $\mid$ CRISPS}

For simplification, the candy machine only accepts coins with value 1 or 2 :

$$
\text { Coins }==\{1,2\}
$$

We define a constant identifying the maximal value of all inserted coins, which we set to 5:

$$
\operatorname{Max}==5
$$

Next, we give an axiomatic definition for a function determining the price of each of the candies:

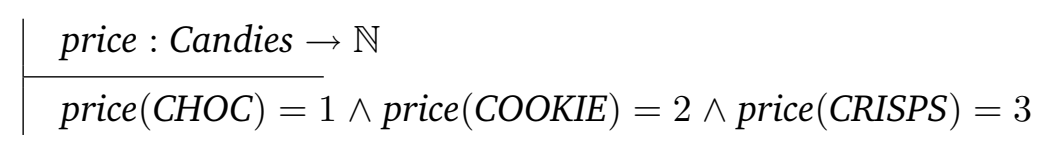

In general, a CSP-OZ specification consists of a set of a classes which can then be combined to define the overall system. In Chapter 4, we will consider a specification consisting of several classes. As of now, in our running example, we will sufficiently deal with a specification comprising one class only.

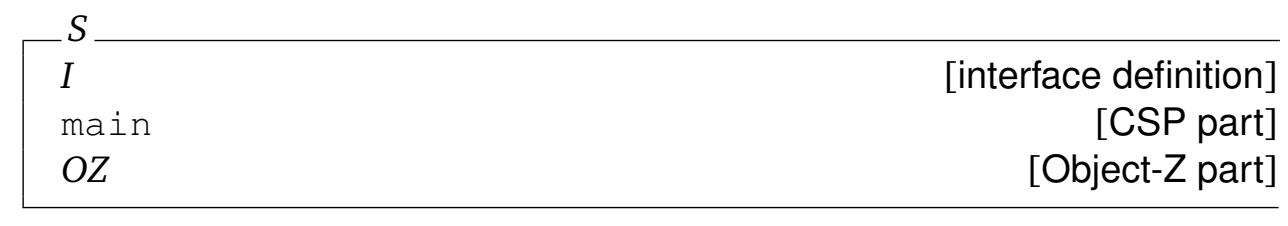

Figure 2.1: Structure of a CSP-OZ specification

The general structure of a CSP-OZ class named $S$ is depicted in Figure 2.1. A class consists of three parts, namely its interface, its CSP part and its Object-Z part. The Object-Z

\begin{tabular}{|c|c|}
\hline State & [state schema] \\
\hline Init & [initial state schema] \\
\hline enable_op & [enable-schemas] \\
\hline effect_op & [effect-schemas] \\
\hline
\end{tabular}
part is again divided into its state schema, initial state schema and its set of operation schemas as shown in Figure 2.2.

Figure 2.2: Structure of the Object-Z part of a CSP-OZ specification

The fundamental concept of CSP-OZ is the connection between CSP part and Object-Z part by using the interface $I$ as the common alphabet for both viewpoints of the system: one operation schema of the Object-Z part corresponds to a set of events of the CSP part. 
For achieving this correspondence, the interface defines a set of typed channels. A channel declaration has the form

$$
\text { chan name }\left[p_{1}: t_{1} ; \ldots p_{n}: t_{n}\right]
$$

where name identifies the name of the channel and $p_{i}$ is a decorated parameter of type $t_{i}$. We distinguish between three different parameter categories:

Input: Input parameters are decorated with '?' and controlled by the environment of the class. Neither the CSP part nor the Object-Z part can control input parameters. However, the guard of an operation can refer to input parameters, thus allowing the operation to be blocked for a subset of the values of the parameter's type.

Output: Output parameters are decorated with '!' and controlled by the class itself. Predicates of an operation schema can restrict output values. If the operation is executed, the value is determined non-deterministically.

Simple: In contrast to input and output parameters known from CSP and Object-Z, simple parameters are an extension in CSP-OZ and they are in general used for indexing purposes. Simple parameters are undecorated and controlled by the class and its environment. They can be restricted by both, the Object-Z part and the CSP part.

Figure 2.3 shows the actual CSP-OZ specification of the candy machine. Here, the interface comprises eight channels. For instance, channel pay has one input parameter of type Coin modelling the customer's payment. In contrast, channel deliver has one output parameter of type Candies modelling a goody the machine dispenses. Note that all parameters are inputs to the CSP part since neither of them is a simple parameter. Some channels such as abort do not use any parameters.

As already mentioned, the CSP part of the specification describes the dynamic behaviour of a system by means of the possible sequences of events and their orderings. This is achieved by a set of process equations. As a convention, the initial process of a class' CSP part is named main. The remaining set of process equations comprises four process names: Payout describes the behaviour of the system if the customer chooses to abort the procedure and collects his money. Select models the selection of an item and Order its actual ordering. Finally, Deliver describes the delivery of the ordered items.

The Object-Z part starts with the class' state schema, containing the set of state variables for the description of the class' state space and its modifications. These are two variables, sum and credits, of type $\mathbb{N}$ to denote the current sum of money paid by the customer and the remaining credits, respectively. A sequence of coins paid models the inserted coins, and a second sequence items the previously ordered candies before the actual delivery. Finally, the variable selected of type Candies describes the current item, selected by the customer.

The initial state schema of the class defines the set of valid initial configurations by using predicates, restricting the values of the state variables. In our example, both 


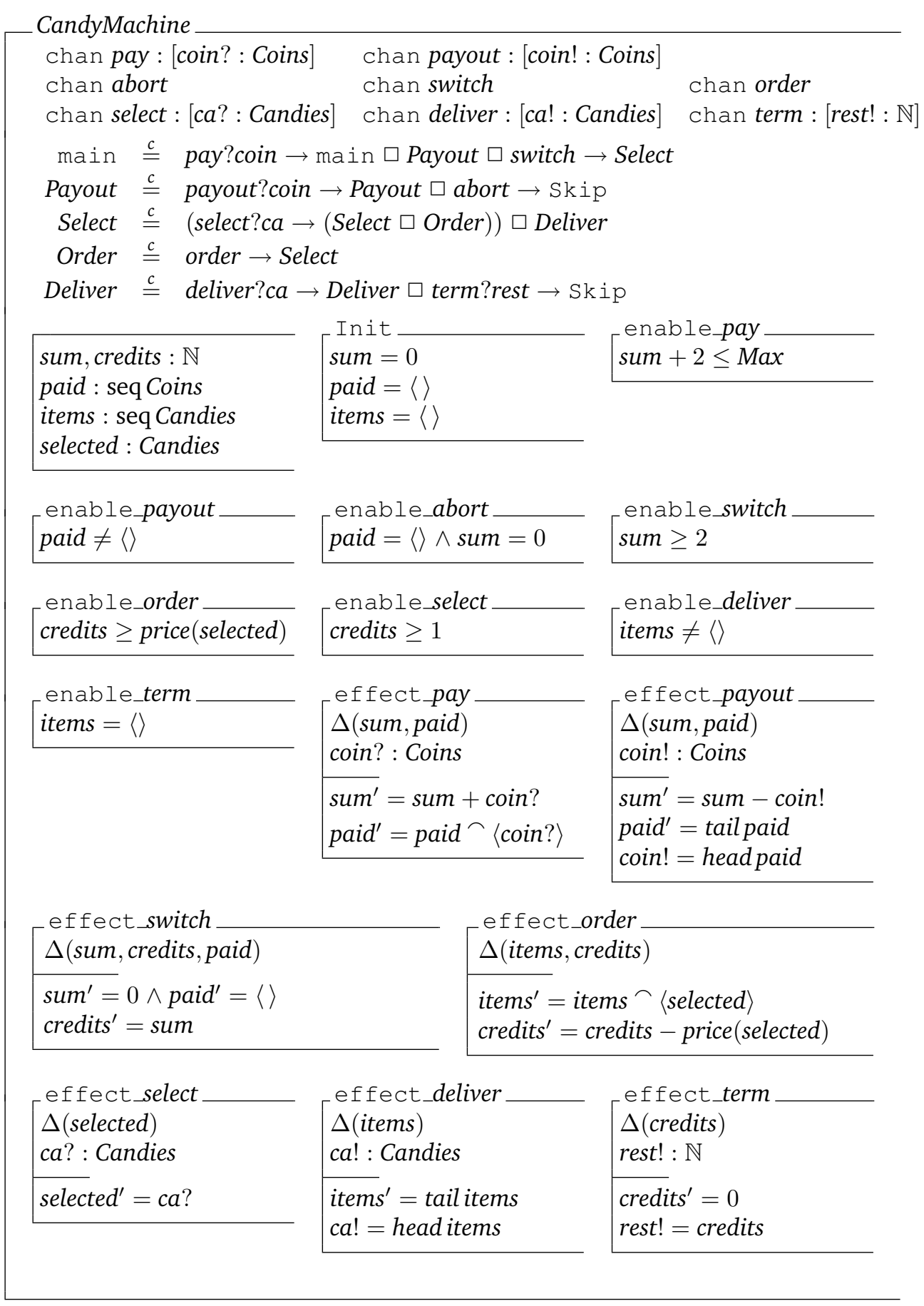

Figure 2.3: Candy machine specification 
sequences need to be initially empty, and the initial sum of money is equal to zero. The remaining state variables are not restricted by the initial state schema.

The static behaviour of the class is described in terms of a set of enable- and effect schemas, conjointly defining the behaviour of an operation schema. An enable schema defines the precondition of an operation by again using predicates referring to state variables of the class. An operation can only be executed if its respective precondition is satisfied. Otherwise, the operation is blocked. For instance, operation deliver is blocked if there are no items to deliver, that is, items $=\langle\rangle$ holds. Besides, order can only be executed if there are enough credits left to pay the price of the selected items.

An effect schema defines an operation and how its execution modifies the state space. It starts with its $\Delta$-list, comprising the set of state variables, modified by the operation. All variables not appearing in this list remain unchanged. As an example, the effect schema of payout modifies the variables sum and paid. Next, the schema can contain a set of parameter declarations, corresponding to the parameters in the operation's interface declaration. Finally, the predicate part of the schema defines the actual modifications of the state variables. For that purpose, a predicate can refer to the possible values of a state variable after execution of the operation; these post-state values are depicted in primed form. For instance, payout restricts the post value of sum, sum', to sum - coin!. Thus, the operation ensures that the only possible value of sum after execution of payout is exactly the original amount of money, reduced by the value of the dispensed coin. Note that the operation abort possesses an empty ef fect schema which leaves all variable values unchanged. In this thesis, we will leave out empty schemas.

We will now describe the dynamic behaviour of the class and its state space modifications by clarifying and illustrating its workflow. Figure 2.4 illustrates the CSP part of the specification as a state transition graph, according to the operational semantics of CSP as given in [Ros98].

A customer has three initial options, modelled by the CSP operator $\square$ for external choice by the environment: first, if the amount of already inserted money increased by two is smaller than Max, a user can insert a coin into the machine (pay) followed by (using the prefix operator $\rightarrow$ ) a call of the initial process main. The coin and its value are stored in the variables paid and sum, respectively. Second, the customer can chose to cancel buying candies as described in the process Payout, where he repeatedly collects his coins (payout) by emptying the paid sequence. After a possibly empty sequence of payouts, the process is finally aborted and terminates (denoted by Skip, the basic CSP process for termination). As a last option, if the user inserted at least coins of an overall value of 2 , he can request to process to the ordering of candies (switch), for which the process Select is called. The customer may now select an item which she wants to order. If enough credits are left, the item is ordered by storing it in the sequence items and reducing the credits by the respective amount. Otherwise, the customer needs to reselect another item. If he ordered at least one item, he can proceed to get his candies delivered. In this case, the machine dispenses the items one by one in the correct order. The process terminates after the potential order and delivery of candies. Remaining spare money is returned.

Next, we clarify some syntactical aspects of Object-Z, CSP and CSP-OZ along with 


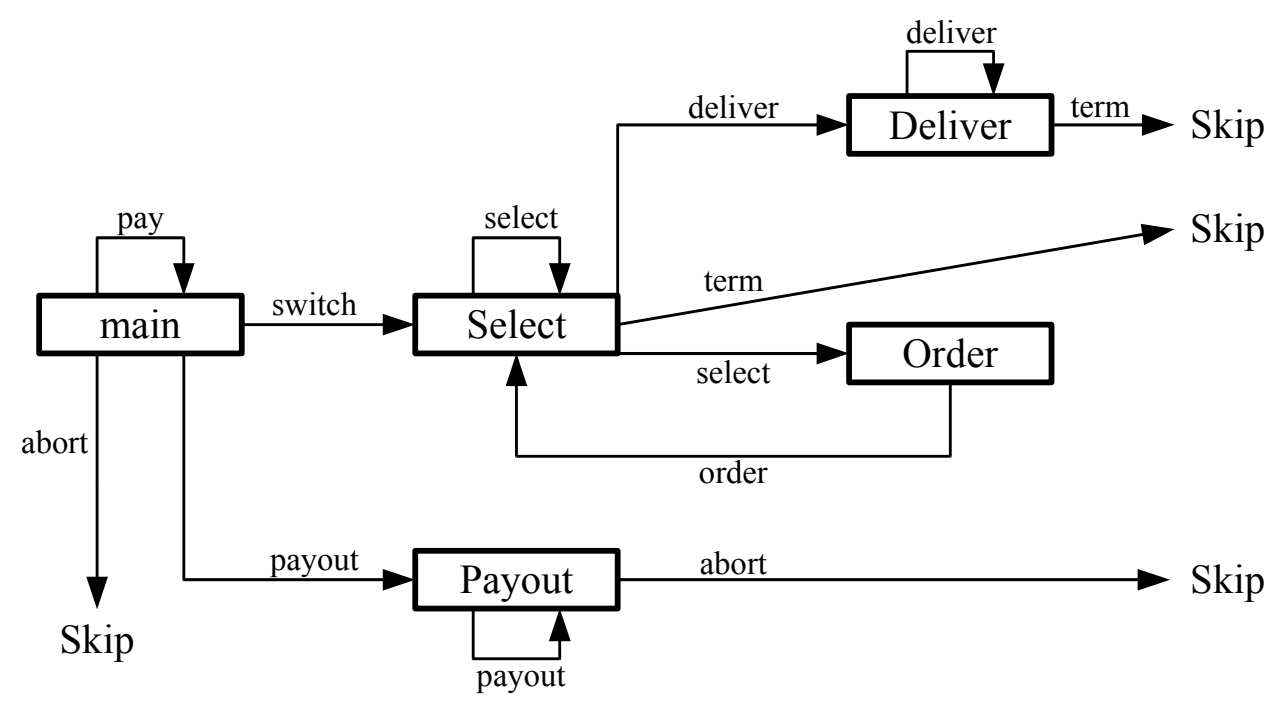

Figure 2.4: Illustration of the CSP part of the candy machine specification

defining their semantics. For more details, we refer to [Smi00, Spi92, ISO00], [Ros98, Sch99] and [Fis00] for comprehensive documentations on (Object-)Z, CSP and CSP-OZ, respectively.

\subsubsection{Object-Z}

As already explained, we use a sightly adapted version of the Object-Z language as introduced in [Fis00]. Therefore, we will continue to refer to the Object-Z part of a CSP-OZ class specification, denoted by $O Z$, instead of pure Object-Z class specifications.

$O Z$ generally consists of a state schema, an initial state schema and a set of operation schemas, where elements of the latter comprise an enable schema and ef fect schema, as depicted in Figure 2.2. The keywords State and Init denote the state schema and initial state schema of a class, respectively. Thus, $O Z$ can be denoted by a tuple:

$$
O Z=\left(\text { State, } \text { Init },(\text { enable_op })_{o p \in O p},(\text { effect_op })_{o p \in O p}\right)
$$

In the remainder of this thesis, we denote the sets of all values for input parameters, output parameters and simple parameters of an operation schema op by $\operatorname{In}(o p)$, Out(op) and Simple(op), respectively. Elements of these sets are tuples adhering to the types of the operation parameters. The set Events is defined as the set of operation names of $O Z$, completed by values for their parameters:

$$
\text { Events }=\{\text { op.in.sim.out } \mid \text { op } \in \text { Op }, \text { in } \in \operatorname{In}(o p), \text { sim } \in \text { Simple }(o p), \text { out } \in \text { Out }(o p)\}
$$

The state schema State defines the state space of $O Z$ and comprises the set of state variables the class uses along with their types. Additionally, the state schema contains a 
(possibly empty) set of state invariants - a set of predicates, which have to be satisfied initially as well as for any reachable state of the class. The set of state variables will be referred to as $V$. A state of $O Z$ is defined as a valuation of all state variables: for $V=\left\{x_{1}, \ldots, x_{n}\right\}$, a state $s$ is denoted as the tuple $s=\left(v_{1}, \ldots, v_{n}\right)$ where $v_{i}$ are values of $x_{i}$ within the variable's domain. We write State $(s)$ or, equivalently, $s \in$ State, to refer to states of the OZ state space, and we use s. $x_{i}$ to denote the value of $x_{i}$ in the state $s$.

For the definition of our decomposition, we need to project a state $s \in$ State on a subset of the state variables $V^{\prime} \subseteq V$ :

Definition 2.2.1. (State Projection)

Let $V=\left\{x_{1}, \ldots, x_{n}\right\}$, and let $s=\left(v_{1}, \ldots, v_{n}\right)$ with $s \in$ State. We use

$$
\left(v_{1}, \ldots, v_{n}\right)=\left(\left(v_{1}, \ldots, v_{n-1}\right), v_{n}\right) .
$$

The projection of $s$ on the set of state variables $V^{\prime} \subseteq V$, denoted by $s \uparrow V^{\prime}$, is inductively defined as

$$
\left(\left(\ldots\left(v_{1}\right), \ldots v_{n-1}\right), v_{n}\right)\left\lceil V^{\prime}:= \begin{cases}\left(\ldots\left(v_{1}\right), \ldots v_{n-1}\right) \uparrow V^{\prime}, & x_{n} \notin V^{\prime} \\ \left(\left(\ldots\left(v_{1}\right), \ldots v_{n-1}\right) \uparrow V^{\prime}, v_{n}\right), & x_{n} \in V^{\prime}\end{cases}\right.
$$

The initial state schema restricts the initial valuation of the state variables. The enable schema defines an operation's guard. It consists of a declaration part for possible inputand simple parameters (enable-schemas must not declare output variables) and a predicate part, containing predicates solely referring to unprimed state variables, that is, to the state before the operation took place. If the conjunction of these predicates is not satisfied, the operation is blocked. enable_op can be interpreted as a predicate, denoted by enable_op $(s$, in, sim) with $s \in$ State, in $\in \operatorname{In}(o p)$ and $\operatorname{sim} \in \operatorname{Simple}(o p)$.

An operation's ef fect schema declares the possible post states after the operation took place. It consists of a $\Delta$-list, comprising all variables which are modified by the operation. The subsequent declaration part contains the schema's parameters and its predicate part defines the restriction on the post-state. For this, variables denoted in primed form refer to post state values. For any variable $x$ not contained in the $\Delta$-list, $x^{\prime}=x$ implicitly holds. An effect schema can be denoted as the predicate effect_op $\left(s\right.$, in, sim, out, $\left.s^{\prime}\right)$ with $s \in$ State, in $\in \operatorname{In}(o p)$, sim $\in$ Simple $(o p)$, out $\in$ Out $(o p)$ and $s^{\prime} \in$ State'.

In the remainder of this thesis, we let ref(op) denote the set of referenced variables of an operation (those occurring in unprimed form), whereas $\bmod (o p)$ denotes its set of modified variables (those occurring in its $\Delta$-list). In addition, we set $\operatorname{all}(o p):=\operatorname{ref}(o p) \cup \bmod (o p)$.

The precondition of an effect-schema can be defined as

$$
\begin{aligned}
& \text { pre effect_op }(s, \text { in }, \text { sim }):= \\
& \exists \text { out } \in \text { Out }(\text { op }), s^{\prime} \in \text { State }^{\prime} \bullet \text { effect_op }\left(s, \text { in }, \text { sim, out }, s^{\prime}\right)
\end{aligned}
$$

In this thesis, we assume that enable_op $(s$, in, $\operatorname{sim}) \Rightarrow$ pre effect_op $(s$, in, sim $)$ holds. This corresponds to the blocking view of operations as described in [Fis00]: an operation can only be executed if its precondition is satisfied, otherwise it is blocked. 
As an enable-schema can always be strengthened such that enable $\wedge \neg$ pre effect is impossible, this is not a restriction. For instance, consider the following operation op:

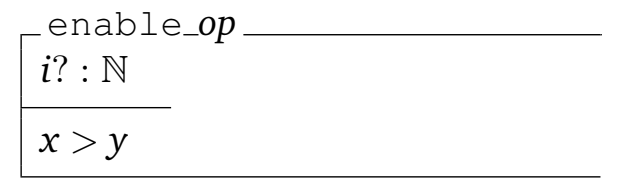

$\left[\begin{array}{l}\text { effect_op } \\ \Delta(x) \\ i ?: \mathbb{N} \\ \hline x^{\prime}=i ? \wedge x^{\prime}>y^{\prime}\end{array}\right.$

For any value of $i$ ? such that $i ? \leq y$ holds, pre effect_op is not satisfied. Adding $i$ ? $>y$ to enable_op schema ensures the previous implication.

When referring to an operation op, comprising enable_op and effect_op, we denote its entire predicate part by op.pred whereas the declaration part will be denoted by op.dec. In case we need to refer to the delta list of an operation, we write op.delta.

\section{Semantics of Object-Z}

As we are interested in the sequences of events of a specification, our approach is based on an operational semantics for Object-Z and ultimately for CSP-OZ.

For the Object-Z part of a specification, we need to reason about events and states. The decomposition approach analyses a specification's dependence structure. A description of paths solely referring to events is insufficient, since we need to incorporate the state space and its modifications as well.

In order to be precise, execution of an event op.in.sim.out within the Object-Z part, changing the before state $s$ into the after state $s^{\prime}$, refers to an operation's enable- and effect-schema:

$$
s \stackrel{\text { op.in.sim.out }}{\longrightarrow} s^{\prime} \Leftrightarrow\left(\text { enable_op }(s, \text { in }, \text { sim }) \wedge \text { effect_op }\left(s, \text { in }, \text { sim }, \text { out }, s^{\prime}\right)\right)
$$

The notation we are using is closely related to the Object-Z semantics of [Brü08] which itself is based on the history semantics of Object-Z [Smi95]: sequences of state valuations and operation calls describe the possible behaviours.

As a semantic model, we use labelled transitions systems (LTS). In order to reason about states of the Object-Z part, a path of a labelled transition system is an alternating sequence of states and events.

Definition 2.2.2. (Labelled Transition System)

Let $E$ be an alphabet of events. A labelled transition system (LTS) $M=\left(S, S_{0}, \rightarrow\right)$ over $E$ consists of

- a set of states $S$,

- a set of initial states $S_{0} \subseteq S$ and

- a transition relation $\rightarrow \subseteq S \times E \times S$.

A path of an LTS is a finite or infinite sequence $\left\langle s_{0}, e_{0}, s_{1}, e_{1}, \ldots\right\rangle$ alternating between states and events such that $\left(s_{i}, e_{i}, s_{i+1}\right) \in \rightarrow$ holds for all $i \geq 0$. 
Note that paths of LTS can be infinite but do not need to be infinite. Next, we define the operational semantics of $O Z$ in terms of labelled transitions systems.

Definition 2.2.3. (Labelled Transition System for Object-Z)

Let $O Z$ be the Object-Z part of a CSP-OZ class specification. The LTS semantics of OZ is defined as the labelled transition system $M^{O Z}=\left(S, S_{0}, \rightarrow O Z\right)$, defined over $E:=$ Events, with

- $S=\{s \mid s \in$ State $\}$,

- $S_{0}=\{s \in S \mid \operatorname{Init}(s)\}$,

- $\rightarrow O Z=\left\{\left(s\right.\right.$, op.in.sim.out,$\left.s^{\prime}\right) \mid$ enable_op $(s$, in, sim $) \wedge$ effect_op $\left(s\right.$, in, sim, out,$\left.\left.s^{\prime}\right)\right\}$.

The set of all paths of $M^{O Z}$ is defined as Traces(OZ). Moreover, let

$$
\operatorname{traces}(\mathrm{OZ}):=\{\pi\lceil\text { Events } \mid \pi \in \operatorname{Traces}(\mathrm{OZ})\}
$$

and for $\operatorname{tr} \in \operatorname{traces}(\mathrm{OZ})$,

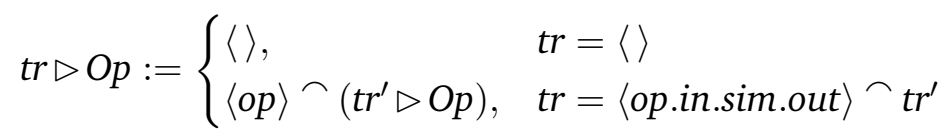

Finally, for $\pi \in \operatorname{Traces}(\mathrm{OZ})$, let $\pi[i]$ denote the $i$-th state and $\pi . i$ the $i$-th event of $\pi$.

For clarification, $\pi$ denotes a trace within Traces(OZ) distinguishing it from $\operatorname{tr} \in$ traces $(\mathrm{OZ})$ not comprising states. We exemplify the definition with our case study:

Example 2.2.4. The following trace, named $\pi$, is a valid path of the LTS of the Object-Z part of the candy machine:

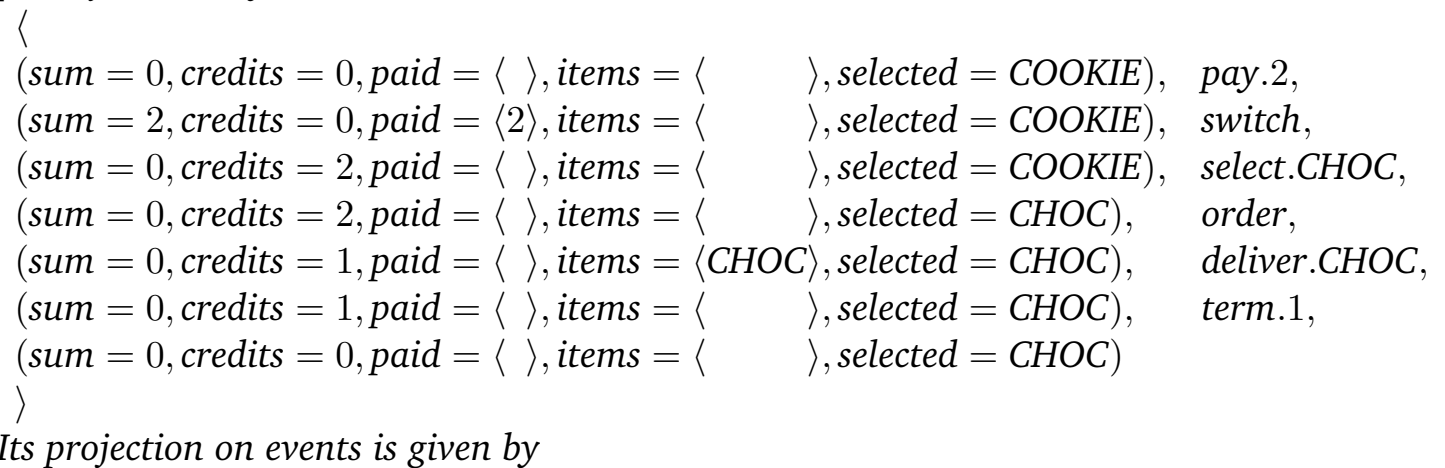

$$
\operatorname{tr}=\pi\lceil\text { Events }=\langle\text { pay.2, switch, select.CHOC, order, deliver.CHOC, term.1 }\rangle .
$$

The projection of tr on its set of operation names yields

$$
\operatorname{tr} \triangleright O p=\langle\text { pay, switch, select, order, deliver, term }\rangle .
$$




\begin{tabular}{c|lc}
$P:=$ & Skip & (Termination) \\
Stop & (Deadlock) \\
& $a \rightarrow P_{1}$ & (Prefixing) \\
& $P_{1} \square P_{2}$ & (External Choice) \\
& $P_{1} \sqcap P_{2}$ & (Internal Choice) \\
& $P_{1}{ }_{9} P_{2}$ & (Sequential Composition) \\
& $P_{1} \|_{A} P_{2}$ & (Interface Parallel) \\
$\mid$ & $P_{1} A_{1} \|_{A_{2}} P_{2}$ & (Alphabetised Parallel) \\
& $P_{1} \| P_{2}$ & (Interleaving) \\
$P_{1} \backslash A$ & (Hiding) \\
$X$ & (Process Call) \\
& $P \llbracket \mathrm{R} \rrbracket$ & (Renaming)
\end{tabular}

Figure 2.5: Simplified grammar of CSP

\subsubsection{CSP}

In general, a CSP process $P$ is defined over a set of communication events which the process can perform: its alphabet. For this, we need the notion of channels. A channel consists of a name and a finite, possibly empty, sequence of data types $T_{1} \times \cdots \times T_{k}$, the type of the channel. An event is then composed of the channel name and possible data values, corresponding to the channel's type.

In our example specification of a candy machine, the channel payout is of type Coins. Thus, payout.1 denotes a possible event, communicated by the CandyMachine which is composed of the channel name payout and the value 1 according to its type.

In this thesis, we will, in general, refer to an alphabet Events, denoting a global set of all events which corresponds to the set of events for the Object- $Z$ part. These are comprised of the operation names and values for their parameters. If we want to refer to the distinguished alphabet of a process $P$, we use the notation $\alpha P$. Accordingly, we let Op denote the set of channel names, corresponding to the set of operation names for the Object-Z part. We use the terms operation and channel synonymously throughout this thesis.

The inductive definition of a CSP process, which we will refer to in this thesis, is summarised in the grammar, given in Figure 2.5. Here, $a \in$ Events denotes an event and $A, A_{1}, A_{2} \subseteq$ Events sets of events.

Skip and Stop are basic CSP processes for termination and deadlock, respectively. Stop does not communicate at all whereas Skip solely communicates the reserved event $\checkmark$ to indicate successful termination. The prefix process $a \rightarrow P_{1}$ communicates the event $a$ and subsequently behaves as $P_{1} . P_{1} \square P_{2}$ describes the external choice (resolved by the environment) between both processes $P_{1}$ and $P_{2}$ whereas $P_{1} \sqcap P_{2}$ denotes the internal choice (resolved internally). $P_{1}{ }_{9}^{\circ} P_{2}$ describes sequential composition meaning that first, $P_{1}$ is executed and, if $P_{1}$ successfully terminates, $P_{2}$ is allowed to occur. $P_{1} \|_{A} P_{2}$ defines the interface parallel composition of two processes, which need to synchronise on all events in $A$. Similarly, the alphabetised parallel composition $P_{1 A_{1}} \|_{A_{2}} P_{2}$ needs to synchronously perform any events within $A_{1} \cap A_{2}$. We will sometimes leave out the 
synchronisation alphabet(s) and denote the parallel composition of two processes by $P_{1} \| P_{2}$, if the alphabet is not considered. The interleave process $P_{1} \| P_{2}$ is a special case of parallel composition where the synchronisation alphabet is empty. $P_{1} \backslash A$ behaves similar to $P_{1}$, except that events from $A$ are hidden, that is, invisible to the environment. All events within $A$ are renamed to a distinguished, internal event $\tau$. Since CSP processes are defined via process equations, $X$ denotes the body, that is, the right hand side, of a process equation. Finally, $P \llbracket \mathrm{R} \rrbracket$ depicts the process where all events $e$ occurring in $P$ are renamed to $R(e)$, according to a relation $R:$ Events $\rightarrow \mathbb{P}($ Events $)$.

From now on, we let $L^{C S P}$ denote the set of all CSP terms. We introduce some additional generalisations and abbreviations, which we will use in the remainder of this thesis. First, binary operators can be indexed over some finite indexing set $I$. As an example, the indexed external choice, denoted by

$$
\square_{i \in I} P_{i}
$$

defines the external choice over all processes $P_{i}$ with $i \in I$. Similarly, $N$-way indexed parallel composition can be denoted by

$$
\|_{i=1}^{N} P_{i}
$$

Based on associativity laws, $N$-way indexed parallel composition can be transformed into a chain of binary parallel compositions. The same holds for the remaining binary operators. Therefore, in the following definitions and proofs, we do not need to deal separately with indexed operators.

The prefix choice process $a: A \rightarrow P_{1}$ initially offers any event of $A$ and subsequently behaves as $P_{1}$. Prefix choice can be seen as generalisation of prefixing. For finite $A$, according to [Ros98], prefix choice can equivalently be transformed into indexed external choice based on the equivalence

$$
a: A \rightarrow P_{1} \Leftrightarrow \square_{a: A} a \rightarrow P_{1}
$$

The process Run $_{A}$ defined as

$$
\operatorname{Run}_{A} \stackrel{c}{=} a: A \rightarrow \operatorname{Run}_{A}
$$

can always communicate any member of $A$. If no alphabet is specified, we assume $A=$ Events and set Run $:=$ Run $_{\text {Events }}$.

Sometimes, it is convenient to refer to the set of events extending a set of channel names with all possible parameter values. This motivates the following definition from [Ros98]:

Definition 2.2.5. (Extension of channels)

Let $c$ be a channel of type $T_{1} \times \cdots \times T_{k}$. The extension set of $c$ is defined as

$$
\{|c|\}:=\left\{c . v_{1} \ldots v_{k} \mid v_{i} \in T_{i}\right\}
$$


The definition allows us to refer to a set of channel names as the synchronisation alphabet, meaning that the extension sets of their operations are synchronised.

A channel includes an ordering on its data types. Partially defined events fix a (possibly empty) subset of its type while the remaining data values (possibly none) are undetermined. Achieving this is done through using the underscore- (don't care-) symbol "-" in order to refer to positions within a channel's type and define:

Definition 2.2.6. ((Extensions of) partial events)

Let $c$ be a channel of type $T_{1} \times \cdots \times T_{k}$. c. $v_{1} \ldots v_{k}$ is a partial event if $v_{j} \in T_{j} \cup\left\{{ }_{-}\right\}$. Its extension set is defined as

$$
\left\{\left|c . v_{1} \ldots v_{k}\right|\right\}:=\left\{c . v_{1}^{\prime} \ldots . v_{k}^{\prime} \mid\left\{\begin{array}{ll}
v_{j}^{\prime}=v_{j}, & v_{j} \neq{ }_{-} \\
v_{j}^{\prime} \in T_{j}, & \text { otherwise }
\end{array}\right\}\right\} .
$$

Note that by definition, the set of partial events includes the set of (complete) events. We give an example for Definition 2.2.6:

Example 2.2.7. Let $c$ be a channel of type $\mathbb{N} \times \mathbb{B}$. Then,

$$
\begin{aligned}
& \{\mid \text { c._. true } \mid\}=\left\{\text { c. } v_{1} . \text { true } \mid v_{1} \in \mathbb{N}\right\} \text { and } \\
& \{\mid \text { c.3.- } \mid\}=\{\text { c.3.true, } \text { c. } 3 . \text { false }\} .
\end{aligned}
$$

\section{Semantics of CSP}

In order to analyse specifications and, in particular, CSP processes, we need to consider the formalism's semantics. The standard semantic model of CSP is the failures-divergences model. In addition, the less discriminating stable failures model and the least restrictive traces model can be chosen.

Traces of a CSP process describe its observable behaviour by means of sequences of events. The prefix closed set of all finite traces of a CSP process $P$ is denoted by $\operatorname{traces}(P) \subseteq \mathbb{P}\left(\right.$ Events $\left.^{*}\right)$. Elements are described as sequences $\left\langle e_{1}, e_{2}, \ldots, e_{n}\right\rangle$ with $e_{i} \in$ Events. Internal events ( $\tau$-events) do not appear in the traces of a process. For instance, $\langle$ pay.2, switch, select.CRISPS, order, deliver.COOKIE, term.2〉 describes a valid trace of the candy machine's CSP part. ${ }^{1}$

A failure of a CSP process $P$ is expressed as a tuple $(\operatorname{tr}, A) \in$ Events $^{*} \times \mathbb{P}($ Events $)$ where tr denotes a trace and $A$ a set of events which $P$ is unable to accept after $t r$ has been performed. For instance, ( $\langle$ switch $\rangle,\{$ pay $\})$ is a failure of the CSP part of the candy machine.

Divergence within a CSP process $P$ describes the ability of $P$ to perform an infinite sequence of internal events. The set of divergences of a process $P$ contains the set of traces after which $P$ can diverge.

Our decomposition approach focusses on the verification of safety properties. As explained in [Weh00] and [OW05], this allows us to move to the semantic domain of the CSP traces model.

\footnotetext{
${ }^{1}$ Note that this is not a valid trace if we additionally consider the Object-Z part.
} 
Next, we introduce some notations adopted from [Sch99] and [Ros98] which we will use in this thesis, and we start with the projection of a trace on a set of events:

Definition 2.2.8. (Trace Projection)

The projection of $\operatorname{tr} \in \operatorname{traces}(P)$ with respect to a set of events $A$ is denoted by $\operatorname{tr}\lceil A$ and defined as:

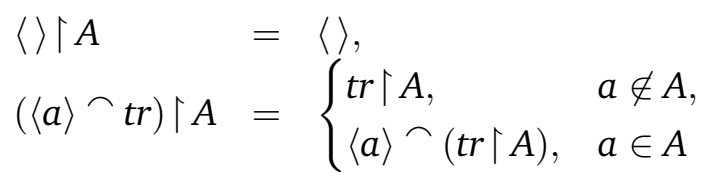

As an example:

$\langle$ pay.2, switch, select.CRISPS, order, deliver.COOKIE, term. 2$\rangle \uparrow\{$ pay, term $\}=$ $\langle$ pay.2, term. 2$\rangle$.

The set of initial events, a process is able to perform, is defined as follows:

Definition 2.2.9. (Initials)

Let $P$ be a CSP process. Then,

$$
\text { initials }(P):=\{a \mid\langle a\rangle \in \operatorname{traces}(P)\}
$$

For instance, initials(main) $=\{$ pay, switch,payout $\}$.

In order to describe that a certain CSP process satisfies a given property, also described as a process, we need to be able to effectively compare processes. The general concept behind this is to show refinement of one process by another. If a specification $Q$ refines another specification $P$, then $Q$ is more restrictive and preserves the behaviour of $P$. In our semantic domain of traces, preservation means that $Q$ offers fewer traces than $P$ thus not allowing more behaviour. This gives rise to the following definition:

Definition 2.2.10. (Trace Refinement)

Let $P, Q$ be CSP processes. $Q$ is a trace refinement of $P$, if traces $(Q) \subseteq \operatorname{traces}(P)$. We write $P \sqsubseteq_{T} Q$. $P$ is trace equivalent to $Q, P=_{T} Q$, if, and only if, $P \sqsubseteq_{T} Q$ and $Q \sqsubseteq_{T} P$.

The traces of a CSP process can be obtained by defining its transition graph. A labelled transition system for a process can be deduced from the operational semantics of CSP. LTSs are the standard way for describing CSP processes in terms of transition graphs. For more details on the operational semantics of CSP, we refer to [Ros98].

Definition 2.2.11. (Labelled Transition System for CSP)

The LTS semantics of a CSP process main over a set of events $A$ is defined as the labelled transition system $M^{C S P}=\left(S, S_{0}, \rightarrow C S P\right)$ with

- $S=L^{C S P}$ the set of all CSP terms,

- $S_{0}=\{\operatorname{main}\}$

- $\rightarrow$ CSP according to the operational semantics of CSP.

The labelled transition system definitions for Object-Z and CSP will be used to define the operational semantics of CSP-OZ. 


\section{Case Study Revisited}

One particular property of the candy machine specification can informally be described as follows:

"The amount of money, paid by the customer, must be equal to the sum of the values of all delivered candies plus the potential spare money. "

For specifying properties of a specification, we will use CSP as the modelling language. This is reasonable since the semantics of CSP-OZ specifications can jointly be given in terms of CSP alone as we will see in Section 2.2.4.

Figure 2.6 defines a CSP process Prop, exactly describing the previously introduced property. Here, all three comprised processes have a parameter of type $\mathbb{N}$, counting the current amount of inserted money and credits, respectively. This yields three sets of families of process equations. Paying $(i)$ monitors the sum of inserted money whereas Collecting $(i)$ decreases the sum by the specific costs of the delivered candies. In order to identify the respective candy delivered by the machine, we explicitly denote the parameter for the event deliver. Finally, Terminate $(i)$ calls the event term with the remaining money of value $i$.

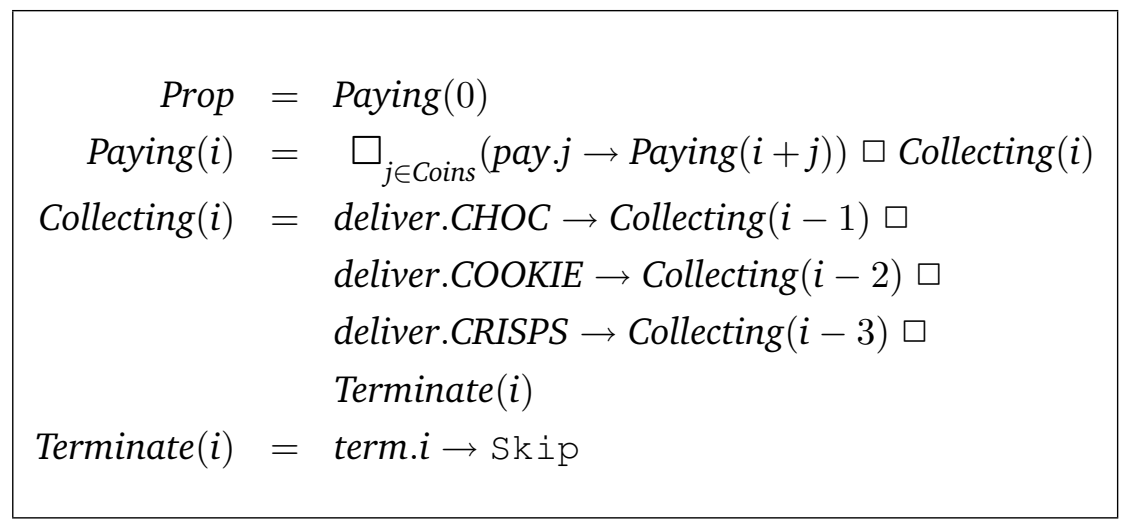

Figure 2.6: Correctness requirement for the candy machine specification

In order to show that Prop is valid for the specification of a candy machine, we need to prove

$$
\text { Prop } \sqsubseteq_{T} \text { CandyMachine } \backslash\{\mid \text { payout, abort, startOrder, select, order } \mid\} \text {. }
$$

As we are only interested in the behaviour reflected by Prop, all events not occurring in Prop are hidden.

\subsubsection{Semantics of CSP-OZ}

For the definition of the semantics of CSP-OZ, Fischer [Fis00] uses an extension of CSP, which he calls $\mathrm{CSP}_{Z}$, and ultimately defines a $\mathrm{CSP}_{Z}$ process capturing the semantics 
of a CSP-OZ class. Figure 2.7 shows a simplified version of his definition: a function Semantics inputs a CSP-OZ class and translates it into a CSP process. Here, the operator $\&$ represents guarding of an event defined as

$b \& P: \Leftrightarrow($ if $b$ then $P$ else $S t o p)$.

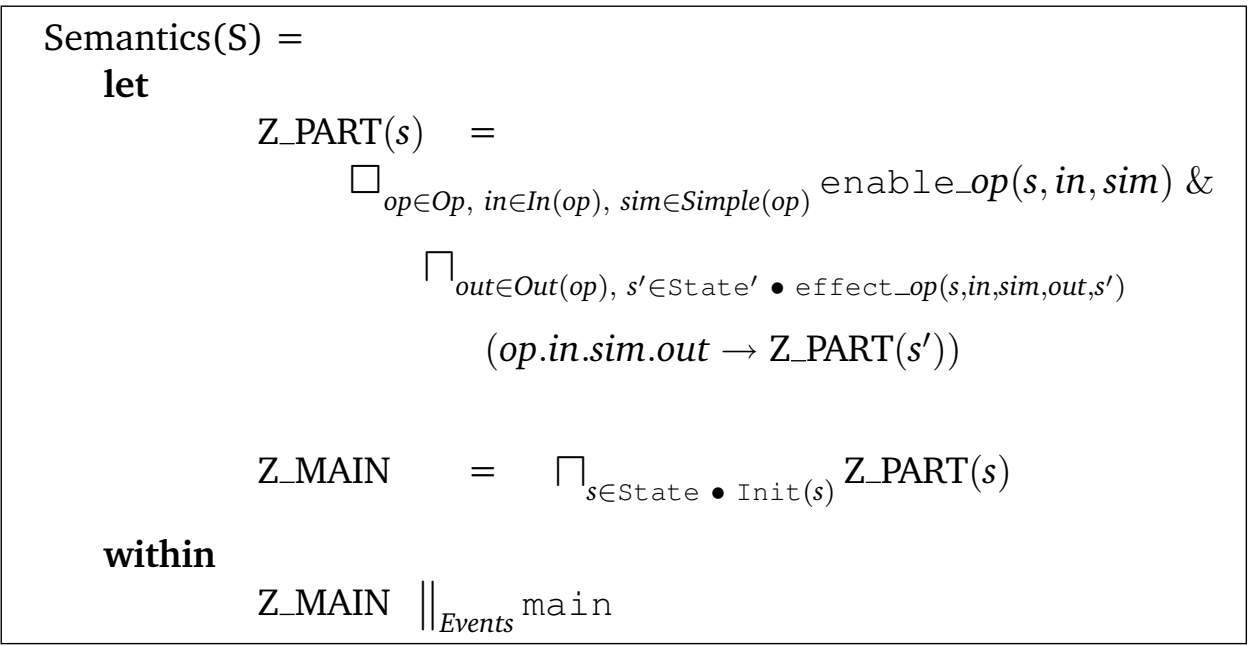

Figure 2.7: Translation of a CSP-OZ specification into a CSP process

The basic underlying idea for this definition is to define a CSP process Z MAIN modelling the Object-Z part of the specification and putting it in parallel with the specification's original CSP part main. Both processes need to synchronise on Events. Z MAIN non-deterministically chooses a valid initial state $s$ and subsequently calls Z_PART $(s)$. Z_PART $(s)$ recursively executes operations of the Object-Z part in an arbitrary order as long as the operation's enable-schema is satisfied. Input parameters are deterministically chosen (using $\square$ ) whereas output parameters and post states are determined non-deterministically (using $\sqcap$ ). This is motivated by the idea that output parameters and post states are internally chosen by a specification.

We aim at using the model checker FDR2 [For05] for verifying CSP-OZ specifications against certain requirements. For this, we need to translate a CSP-OZ specification to the input language of $\mathrm{FDR} 2, \mathrm{CSP}_{\mathrm{M}}$, without changing its semantics. A tool-supported translation of CSP-Z to CSP $\mathrm{M}_{\mathrm{M}}$ has been accomplished in [MS01], [FMS01]. Bolton and Davies compare data refinement in Object-Z with failures-refinement in CSP based on the Object-Z semantics as given in [Smi95] and use a translation of Object-Z to $\mathrm{CSP}_{\mathrm{M}}$. In the context of refinement, Schneider [Sch05] introduced a more general translation from abstract data types (ADTs) [LZ74] to CSP, which can be applied for (part of) Object-Z as well.

In our context of CSP-OZ, Fischer [Fis97] derives a failures-divergences semantics for CSP-OZ based on the definition from Figure 2.7. This allows us to generally use a CSP model checker based on this transformation of CSP-OZ specifications. A transformation function using the above translation and resulting in a process defined in $\operatorname{CSP}_{M}$ is 
introduced in [FW99]. In Chapter 7, we will apply this transformation to use FDR2 for checking trace refinements. We give more details on FDR2 and the tools we are using there.

Our aim is to give an operational semantics for CSP-OZ by using the definition from Fischer. In this thesis, we are interested in the paths a specification might execute. In this particular case, we do not need to deal separately with external choice and internal choice: based on the operational semantics of CSP, the trace semantics does not distinguish between external and internal choice. More precisely, for two CSP processes $P_{1}$ and $P_{2}$ :

$$
P_{1} \square P_{2}={ }_{T} P_{1} \sqcap P_{2} .
$$

Next, we define the operational semantics of CSP-OZ by putting the labelled transition systems for the specification's CSP part and Object-Z part in parallel. The parallel composition of two labelled transition systems is defined as follows:

Definition 2.2.12. (Parallel composition of labelled transition systems)

Let $M^{1}=\left(S^{1}, S^{1}{ }_{0}, \rightarrow_{1}\right)$ and $M^{2}=\left(S^{2}, S^{2}{ }_{0}, \rightarrow_{2}\right)$ be two labelled transition systems over the same set of events $E$. The parallel composition of $M^{1}$ and $M^{2}$ is defined as $M^{1} \|_{E} M^{2}=\left(S, S_{0}, \rightarrow\right)$ with

- $S=S^{1} \times S^{2}, S_{0}=S_{0}^{1} \times S_{0}^{2}$,

- $\left(s_{1}, s_{2}\right) \stackrel{e}{\rightarrow}\left(s_{1}^{\prime}, s_{2}^{\prime}\right)$ if one of the three conditions

a) $s_{1} \stackrel{e}{\rightarrow}_{1} s_{1}^{\prime} \wedge s_{2} \stackrel{e}{\rightarrow}_{2} s_{2}^{\prime}$,

b) $s_{1} \stackrel{\tau}{\rightarrow}_{1} s_{1}^{\prime} \wedge s_{2}^{\prime}=s_{2}$,

c) $s_{1}^{\prime}=s_{1} \wedge s_{2} \stackrel{\tau}{\rightarrow}_{2} s_{2}^{\prime}$.

holds.

The operational semantics of CSP-OZ is then defined as the parallel composition of $M^{C S P}$ and $M^{O Z}$, synchronising on Events. Note that we assume the alphabets of operations of the CSP part and the Object-Z part to be equal. This is not a restriction, as any operation solely represented in the CSP part of a class can be added to its Object-Z part by using an empty predicate part not modifying the state space of the class. Conversely, operations exclusively appearing in the Object-Z part can be integrated into the CSP process by globally offering them based on an additional interleaving. Besides, based on the operational semantics of CSP, $M^{C S P}$ can indeed perform $\tau$-events whereas $M^{O Z}$ cannot.

Table 2.1 gives an overview on the two semantics of CSP-OZ which we introduced in this section. When showing correctness of our approach, we will refer to the LTS semantics of CSP-OZ, incorporating state valuations and events in their paths. The more discriminating $\mathrm{CSP}_{Z}$ semantics maps a CSP-OZ class specification on a CSP process, preserving the original behaviour within the failures-divergences model.

Even though the translation of CSP-OZ to $\mathrm{CSP}_{\mathrm{M}}$ uses the $\mathrm{CSP}_{Z}$ semantics and is thus semantics-preserving for any of the three models of CSP, our approach solely focusses on the traces model. 


\begin{tabular}{|c|l|l|}
\hline & LTS SEMANTICS & CSP $_{Z}$ SEMANTICS \\
\hline Semantic model & traces model of CSP & failures-divergences model of CSP \\
\hline Alphabet for paths & $($ State $\times$ Events $)$ & Events \\
\hline Application in thesis & correctness proof & model checking (trace refinement) \\
\hline
\end{tabular}

Table 2.1: Comparison between the different semantics for CSP-OZ

\subsection{Dependence Analysis}

The main aspects of this thesis are the construction and evaluation of decompositions for software models, specified in CSP-OZ. We require correctness of our approach, meaning that the decomposition must preserve the observable behaviour of the specification. This is achieved by a correctness proof, requiring a representation of the model on which the decomposition and the general proof can be carried out. This representation must reflect the structure of the specification as well as the interdependences between its elements.

In his $\mathrm{PhD}$ thesis [Brü08], Brückner introduced a dependence analysis for CSP-OZ based on the definition of a (program) dependence graph. In the context of program slicing [Wei81], he uses it to show correctness of his approach. Since a dependence graph precisely reflects all the specification's interdependences, we can take advantage of this construction and use his graph in a slightly modified version.

This section introduces the dependence analysis for CSP-OZ specifications mainly according to [Brü08]. We start with a small motivation, stepwise present the dependence graph and illustrate its definition by means of our case study. Instead of repeating all the details of the dependence analysis, we concentrate on the main aspects and an illustration of the concept. Along with that, we describe our context-specific modifications and introduce some necessary properties of the dependence graph.

\subsubsection{Dependence Analysis for CSP-OZ: Motivation}

The introduction already gave an overview on the overall goals of this thesis. In particular, Figure 1.1 illustrated the approach for decomposing a given specification $S$ into two components $S_{1}$ and $S_{2}$, yielding a system $S_{1} \| S_{2}$.

Decomposing a CSP-OZ specification $S$ means that $S$ is split-up into two smaller CSP-OZ specifications $S_{1}$ and $S_{2}$. For that purpose, the specification's elements, such as operation schemas and state variables, are distributed over $S_{1}$ and $S_{2}$. In order to define correct decompositions, we cannot simply assign these constituents to $S_{1}$ and $S_{2}$ at random: the specification's elements might depend on each other. The distribution of dependent elements over both components is not beneficial but generally possible. A definition of $S_{1}$ and $S_{2}$ needs to conform to the structure of the original model such that the 


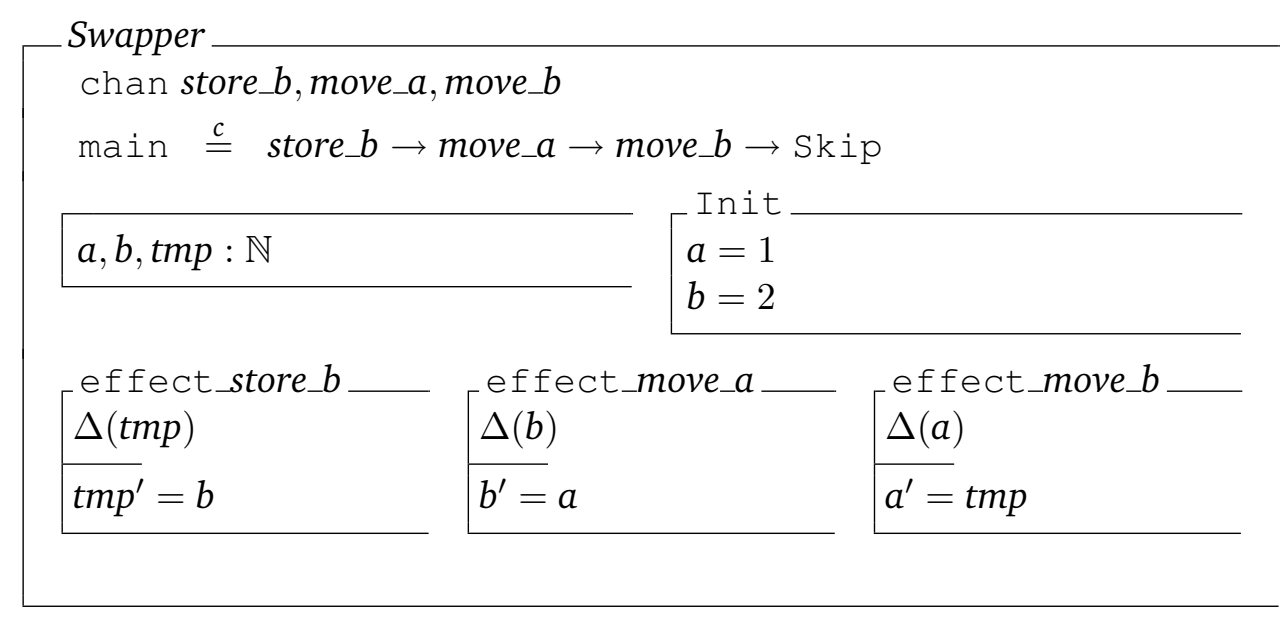

Figure 2.8: Simple CSP-OZ class specification for swapping two numbers

(observational) equivalence of $S$ and $S_{1} \| S_{2}$ can be deduced.

We illustrate the need for a precise specification's analysis with a small example. Consider the simple CSP-OZ specification Swapper as given in Figure 2.8. The specification swaps two natural numbers $a$ and $b$ with respective values 1 and 2 by using a temporary variable tmp.

A decomposition could, for instance, yield two specifications Swapper ${ }_{1}$ and Swapper 2 such that store $\_b$ and move $b$ are distributed over different components. This definition bears some problems: first, the parallel composition of the resulting CSP parts needs to preserve the original ordering of events $\left\langle\right.$ store $\_b$, move $a$, move $\left.\_b\right\rangle$ according to Swapper.main. In the parallel composition Swapper ${ }_{1} \|$ Swapper $_{2}$, the operation move $b$ must not be performed prior to any other event. The dependence graph must therefore comprise edges reflecting the control flow of a specification's CSP part.

Second, consider the Object-Z part of the resulting specification part Swapper 2 : the variable tmp is modified within store_b. We need to ensure that move_b refers to the correct value of tmp. The modified value somehow needs to be restored within Swapper $_{2}$. This interconnection needs to be reflected in the dependence graph as well. Here, we use edges representing the specification's data dependences.

In general, we need to preserve the dependence structure of both, a specification's CSP part and Object-Z part. Our dependence analysis for CSP-OZ specifications addresses this issue by using two graphs:

a) a control flow graph $(C F G)$, which represents the workflow of the specification's CSP part and

b) a data dependence graph ( $D D G$ ), representing the interdependences between state variables and parameters of the specification's Object-Z part.

The overall dependence structure is subsequently defined in the specification's (program) dependence graph (DG) combining the CFG and DDG. Our definition of the DG 
mainly corresponds to the one by Brückner [Brü08]. However, in this thesis and contrary to Brueckner's definitions, the DG is defined with respect to operation nodes. We do not separately consider an operation's enable- and effect-schema and its contained predicates.

A decomposition defines a split-up of the dependence graph which then leads to the decomposition of the underlying specification. Preservation of the observable behaviour is defined in terms of correctness criteria on this fragmentation in Chapter 4.

\subsubsection{Definition of the Control Flow Graph}

In order to analyse a program in respect of its execution paths, control flow analysis [All70] is a standard practice. A control flow graph is a graph theoretical representation of a program.

The definition of [Brü08] yields a control flow graph representing a CSP process. Nodes of this graph mainly correspond to CSP events and CSP operators. We start with the general definition of the control flow graph. Ultimately, we are interested in a dependence graph for a CSP-OZ specification $S$. To this end, the following definitions refer to the CSP part main of a CSP-OZ specification and to the set of operation schemas $O p$ of $S$.

Definition 2.3.1. (Control Flow Graph (CFG) of $S$ )

The control flow graph $(\mathrm{CFG}) \mathrm{CFG}_{\mathrm{S}}=(N, \longrightarrow)$ of a CSP-OZ specification $S$ is defined over a set of nodes $N=\operatorname{cf}(N) \cup \mathrm{op}(N)$ and a set of edges $\longrightarrow \subseteq N \times N$.

Nodes of the CFG either correspond to a CSP operator or to an operation schema of the underlying specification. Table 2.2 denotes all nodes along with the corresponding CSP operators, if existent.

We use a unique node start, representing the start of main. The set $N$ comprises the set of nodes op $(N)$ which is defined as

$$
\mathrm{op}(N)=\left\{\mathrm{op}^{i} \mid \mathrm{op} \in O p\right\} \cup\{\text { init }\} .
$$

Here, a special node init represents the initial state schema of a class, comprising all initial predicates. For the definition of the $\mathrm{CFG}$, the init-node is conjoined with the start-node of the class. As an operation schema op may occur more than once in main and thus in its CFG, we denote the $i$-th occurrence of the respective operation node by op ${ }^{i}$.

$\operatorname{cf}(N)$ is the set of CSP operator nodes plus a set of additional nodes representing entry and leaving of a process. The whole set complies with the elements of the CSP grammar as given in Figure 2.5. Some of these operators, namely the ones corresponding to external choice, internal choice, both parallel operators (which are not separately dealt with in the CFG) and interleaving, introduce branching into the CFG. Here, we introduce split nodes and corresponding join nodes, which are denoted by cfop and uncfop for cfop $\in$ \{extch, intch, par, interleave $\}$, respectively. According to operation nodes, the same notation for the $i$-th occurrence of a CSP operator node applies.

Note that we do not separately consider parallel composition of classes since, on graph level, parallel composition of classes and processes is equally dealt with [Brü08]. Thus, we equally treat specifications consisting of one and several classes. 


\begin{tabular}{|l|c|l|}
\hline Node & CSP operator & Name \\
\hline start & - & (Start of main) \\
\hline \hline op $^{i}$ & - & (Operation Node for op $\in O p$ ) \\
\hline \hline skip $^{i}$ & Skip & (Termination) \\
stop $^{i}$ & Stop & (Deadlock) \\
extch $^{i}$ & $\square$ & (Split External Choice) \\
unextch $^{i}$ & - & (Join External Choice) \\
intch $^{i}$ & $\sqcap$ & (Split Internal Choice) \\
unintch $^{i}$ & - & (Join Internal Choice) \\
seq $^{i}$ & 9 & (Sequential Composition) \\
par $^{i}$ & $\|$ & (Split Parallel) \\
unpar $^{i}$ & - & (Join Parallel) \\
interleave $^{i}$ & $\|$ & (Split Interleave) \\
uninterleave $^{i}$ & - & (Join Interleave) \\
\hline \hline start.X & - & (Process Entry) \\
term.X & - & (Process Termination) \\
call.X & - & (Process Call) \\
ret.X $^{\mid}$ & - & (Process Return) \\
\hline
\end{tabular}

Table 2.2: Table of nodes of the control flow graph

The remaining four nodes are used for structuring of CSP process definitions: start of a process $X$, termination of $X$, call of $X$ and returning from $X$. As an example, executing switch in the candy machine and subsequently calling the process Select corresponds to a CFG path $\langle\ldots$, switch, call.Select, start.Select, ... $\rangle$.

In general, a CFG node $\mathrm{n} \in N$ must always have zero, one or two successor nodes. We denote a single successor node by succ(n), in case of two successor nodes we separately denote each one with succ_one(n) and succ_two(n), respectively.

Paths of the CFG precisely reflect the control flow of main. For the correctness proof, we make one important observation: according to the definition of the CFG, the sole possibility of cycles within the CFG are process calls within the CSP part. This is reflected in [Brü08] where the definition of $G_{C F G}$ introduces cycles into the CFG solely for the case of call-nodes.

Figure 2.9 shows a slightly simplified version of the CFG for the candy machine specification. We omit unextch nodes, term nodes and ret nodes to avoid a blow up in the illustration. Operation nodes are highlighted in grey.

The following notations for paths of the CFG are mainly corresponding to [Brü08]:

Definition 2.3.2. (Paths of the Control Flow Graph)

Let $\mathrm{CFG}_{\mathrm{S}}=(N, \longrightarrow)$ be the $C F G$ of $\mathrm{S}$, and let $\mathrm{n}, \mathrm{n}^{\prime} \in N$. We use the following notations for paths of the CFG, that is, sequences of nodes, visited, when walking along the edges of the graph:

- path $_{\mathrm{CFG}}$ denotes the set of all paths of the CFG whereas path $_{\mathrm{CFG}}\left(\mathrm{n}, \mathrm{n}^{\prime}\right)$ denotes the set of paths starting in $\mathrm{n}$ and terminating in $\mathrm{n}$. 


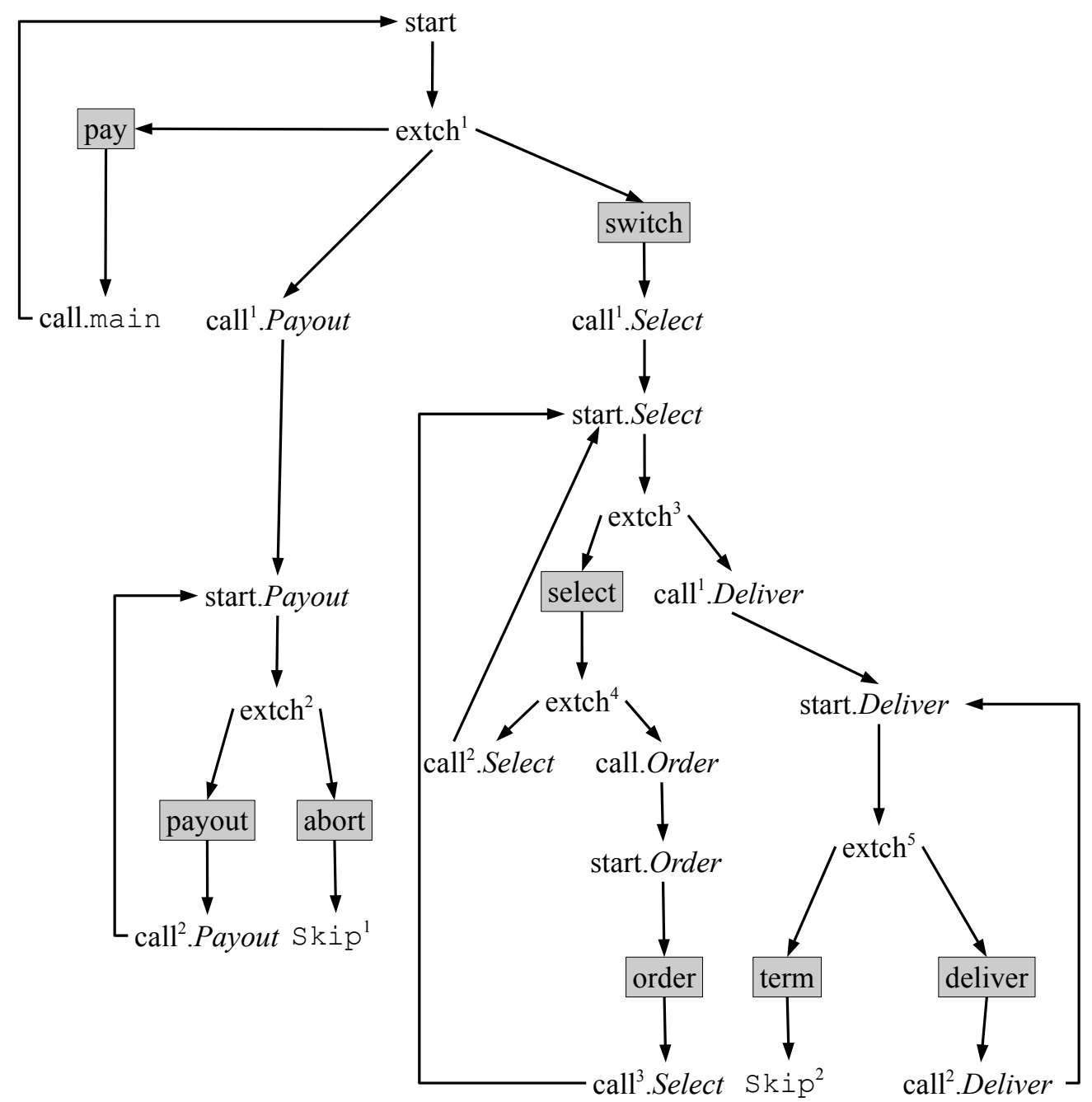

Figure 2.9: Control flow graph (CFG) for the candy machine specification

- $\mathrm{n} \stackrel{\pi}{\longrightarrow} \mathrm{n}^{\prime}$ denotes that $\pi \in$ path $_{\mathrm{CFG}}\left(\mathrm{n}, \mathrm{n}^{\prime}\right)$ whereas $\mathrm{n} \stackrel{*}{\longrightarrow} \mathrm{n}^{\prime}$ denotes that there exists some path from $\mathrm{n}$ to $\mathrm{n}^{\prime}$, that is, $\operatorname{path}_{\mathrm{CFG}}\left(\mathrm{n}, \mathrm{n}^{\prime}\right) \neq \varnothing$.

- For $\mathrm{n}, \mathrm{n}^{\prime} \in \mathrm{op}(N)$, we write

$$
\mathrm{n} \stackrel{\bullet}{\longrightarrow} \mathrm{n}^{\prime} \text { if, and only if, }\left(\exists \pi \in \operatorname{path}_{\mathrm{CFG}}\left(\mathrm{n}, \mathrm{n}^{\prime}\right) \bullet \pi \cap \mathrm{op}(N)=\left\{\mathrm{n}, \mathrm{n}^{\prime}\right\}\right) .
$$

We will sometimes need to refer to paths connecting two operation nodes n, n' without additional operation nodes in between. For this, we use the last definition. For $\pi \in$ $\operatorname{path}_{\mathrm{CFG}}\left(\mathrm{n}, \mathrm{n}^{\prime}\right)$, we let $\mathrm{x} \in \pi$ denote that $\mathrm{x} \in N$ is an arbitrary node on the path $\pi$, including $n$ and $n$ ' themselves. 
We give a small illustrating example for the previous definition:

Example 2.3.3. For the CFG of the candy machine from Figure 2.9, we get:

- $\left\langle\right.$ start, $\operatorname{extch}^{1}$, switch, call ${ }^{1}$.Select, start.Select, extch $^{3}$, select $\rangle \in$ path $_{\mathrm{CFG}}$,

- $\left\langle\right.$ switch, call ${ }^{1}$.Select, start.Select, extch $^{3}$, select $\rangle \in$ path $_{\mathrm{CFG}}$ (switch, select),

- start.Deliver $\longrightarrow$ extch $^{5}$,

- switch $\stackrel{*}{\longrightarrow}$ order and

- select $\stackrel{\bullet}{\longrightarrow}$ order.

Next, we define a mapping between the set of operation nodes of the CFG, op $(N)$, and the set of operations $O p$ of a specification:

Definition 2.3.4. (Labelling of CFG nodes)

Let $\mathrm{CFG}_{\mathrm{S}}=(N, \longrightarrow)$ be the $C F G$ of $\mathrm{S}$, and let $O p$ be the set of all operation schemas of $S$. The labelling function $l: o \mathrm{p}(N) \rightarrow O p$ maps an operation node of the $C F G$ on its corresponding schema name: $l\left(\mathrm{op}^{i}\right):=$ op. For $O \subseteq O p$, we define

$$
l^{-1}[O]:=\{\mathrm{n} \in \mathrm{op}(N) \mid l(\mathrm{n}) \in O\} .
$$

As multiple occurrences of an Object-Z operation within the CSP part of a specification's class are possible, the cardinality of $\mathrm{op}(N)$ is greater or equal than the cardinality of $O p$ : for all op $\in O p$, there exists at least - but in many cases more than - one occurrence $o p^{i}$ within the DG. Thus, the mapping $l$ is surjective but in general not injective. If $l^{-1}[\{o p\}]$ only contains one element, we denote it by op, leaving out the index.

For a more precise definition and description of the CFG, we refer to [Brü08].

\subsubsection{Definition of the Data Dependence Graph}

The control flow of a program can be represented in a graph theoretical way, and the same applies to its data flow. Data flow analysis and data dependence graphs [Den74] aim at an evaluation and description of dependent program statements, incorporating data values. A data dependence is, for instance, given if one statement modifies a certain program variable, while another statement refers to it, and the variable is not overwritten in between.

The data dependence graph, which we consider, is solely defined over the set of nodes $\mathrm{op}(N)$, that is, the set of operation schemas of a specification plus its initial state schema. It supplements the CFG in the sense that its edges are related to paths of the CFG and that it is mainly derived from the Object-Z part of a specification.

As already mentioned, enable- and effect-schemas are comprised into one operation node. Dealing with operation nodes instead of its constituents is reasonable, since, as we will see in Chapter 4, our decomposition approach does not further decompose an operation but rather keeps operations as atoms. 
Besides, we refer to a normalisation of the Object-Z part of $S$ according to [Brü08]: as a state invariant needs to hold before and after execution of each method, it can safely be copied to the effect-schema of each method and eliminated from the state schema, without changing the behaviour of the specification.

The definition of the data dependence graph is as follows:

Definition 2.3.5. (Data Dependence Graph (DDG) of $S$ )

The data dependence graph (DDG) $\mathrm{DDG}_{\mathrm{S}}=(\mathrm{op}(N),--\rightarrow)$ of a specification $S$ is defined over a set of nodes $\mathrm{op}(N)$ and a set of edges $\rightarrow-\rightarrow \subseteq \mathrm{op}(N) \times \mathrm{op}(N)$.

Edges of the DDG incorporate several dependences with all of them being introduced in [Brü08]. Table 2.3 denotes all comprised edges. Note that we do not consider control dependences, as we will explain in the next section.

\begin{tabular}{|c|l|}
\hline Edge & Name \\
\hline$-d d$ & (Direct Data Dependence) \\
idd \\
$---\rightarrow$ & (Initial Data Dependence) \\
ifdd \\
$---\rightarrow$ & (Interference Data Dependence) \\
sd \\
$\leftrightarrow---\rightarrow$ & (Synchronisation Dependence) \\
sdd \\
$---\rightarrow$ & (Synchronisation Data Dependence) \\
\hline
\end{tabular}

Table 2.3: Table of edges of the data dependence graph

The simplest example is a (direct) data dependence: assume a certain state variable $v \in V$ being modified in some operation schema $o p_{1}$ and referenced in some other operation schema $o p_{2}$, that is, $v \in\left(\bmod \left(o p_{1}\right) \cap \operatorname{ref}\left(o p_{2}\right)\right)$. For all operation nodes $\mathrm{n} \in l^{-1}\left(o p_{1}\right)$, $\mathrm{n}^{\prime} \in l^{-1}\left(o p_{2}\right)$, such that there exists a CFG path from the first to the latter node and $v$ is not further modified on this path, the DDG contains a data dependence edge $n \stackrel{d d}{-\rightarrow} \rightarrow n$.

Initial data dependences are a special case of direct data dependences. Since the initial state schema poses restrictions on the set of state variables, an initial data dependence connects the representation of the initial state schema with an operation if some variable $v$ is restricted in Init and referenced in op, without being overwritten in between. As initial data dependences will frequently be used in the following chapters, we introduce a separate notation: init $\stackrel{\text { idd }}{-\rightarrow}$ n', if, and only if, init $-\mathrm{dd}_{-\rightarrow} \mathrm{n}^{\prime}$ for $\mathrm{n}^{\prime} \in l^{-1}(o p)$.

An interference data dependence exists from one node to another if both nodes are located in different branches of an interleaving or parallel composition and, again, the source node modifies a variable that the target node references. Note that, in general, there is no CFG path connecting both nodes.

Synchronisation dependences model the fact that synchronised events within a parallel composition have a mutual dependence on each other. These edges can more likely be seen as a representation of the control flow. However, we integrate them in the DDG, since we want to keep the original definition of the CFG. Note that synchronisation dependences are always symmetric. 
Finally, synchronisation data dependences complement synchronisation dependences. They connect two operation nodes if they are connected by a synchronisation dependence, and one of the corresponding operations declares an output variable which the other corresponding operation uses as an input.

Since we will need to refer to the precise conditions for some of these edges later on, we give their definitions next.

Definition 2.3.6. ((Direct-, Interference-) Data Dependence, Synchronisation Dependence)

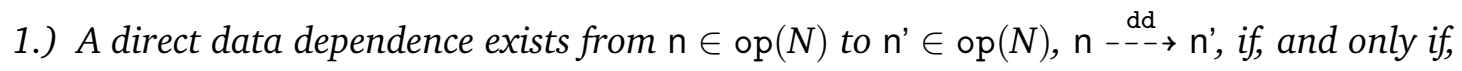
$\exists o p_{1}, o p_{2} \in O p \bullet \mathrm{n} \in l^{-1}\left(o p_{1}\right), \mathrm{n}^{\prime} \in l^{-1}\left(o p_{2}\right) \wedge$
(nodes corresp. to two operations)
$\exists v \in\left(\bmod \left(o p_{1}\right) \cap \operatorname{ref}\left(o p_{2}\right)\right) \wedge$
$\left(v\right.$ modified in $o p_{1}$, referenced in $o p_{2}$ )
$\exists \pi \in \operatorname{path}_{\mathrm{CFG}}\left(\mathrm{n}, \mathrm{n}^{\prime}\right) \bullet$
(nodes are connected by $C F G$ path)
$\forall \mathrm{m} \in \pi \bullet v \in \bmod (l(\mathrm{~m})) \Rightarrow(\mathrm{m}=\mathrm{n}) \vee\left(\mathrm{m}=\mathrm{n}^{\prime}\right)$
(no further modification of $v$ )

2.) An interference data dependence exists from $\mathrm{n} \in \mathrm{op}(N)$ to $\mathrm{n}^{\prime} \in \mathrm{op}(N), \mathrm{n} \stackrel{\text { ifdd }}{---\rightarrow} \mathrm{n}^{\prime}$, if, and only if,

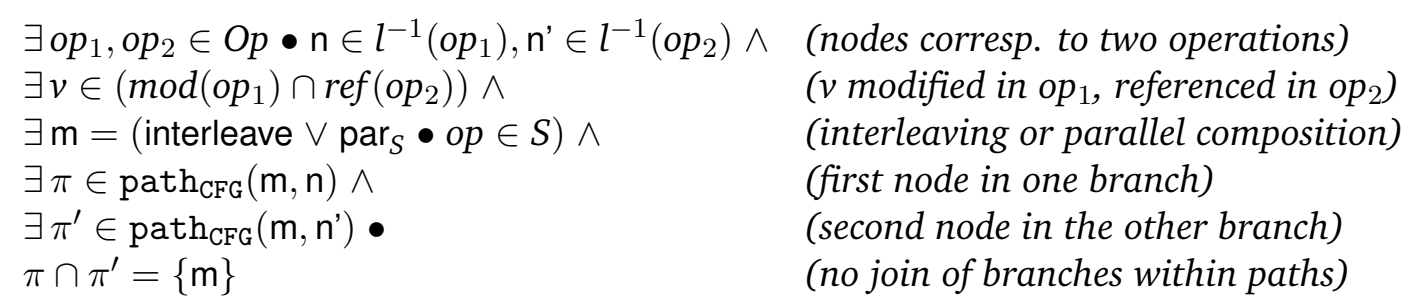

3.) A synchronisation dependence exists between $\mathrm{n}, \mathrm{n}^{\prime} \in \mathrm{op}(N), \mathrm{n} \stackrel{\mathrm{sd}}{\rightarrow--} \mathrm{n}^{\prime}$, if, and only if,
$\exists o p \in O p \bullet \mathrm{n}, \mathrm{n}^{\prime} \in l^{-1}(o p) \wedge$
$\exists \mathrm{m}=\operatorname{par}_{S} \bullet o p \in S \wedge$
(two nodes corresponding to same operation)
$\exists \pi \in \operatorname{path}_{\mathrm{CFG}}(\mathrm{m}, \mathrm{n}) \wedge$
(parallel composition with operation synchronised)
$\exists \pi^{\prime} \in \operatorname{path}_{\mathrm{CFG}}\left(\mathrm{m}, \mathrm{n}^{\prime}\right) \bullet$
(first node in one branch of parallel composition)
$\pi \cap \pi^{\prime}=\{\mathrm{m}\}$
(second node in the other branch of par. composition)
(no join of branches within paths)

Sometimes, we explicitly need to refer to the state variable responsible for a data dependence. This leads to the following notation:

Definition 2.3.7. ((Direct-, Interference-) Data Dependence by Reason) Let $\mathrm{n} \stackrel{\mathrm{dd}}{\mathrm{d}} \mathrm{n}$, and let the state variable $v$ satisfy the criteria from Definition 2.3.6, 1.). In

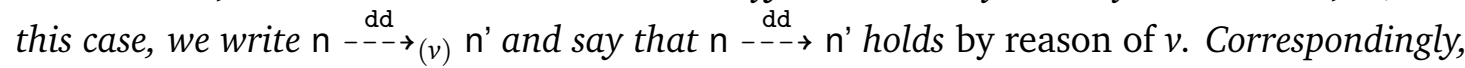
we define $\mathrm{n} \stackrel{\text { ifdd }}{-\rightarrow-\rightarrow(v)} \mathrm{n}$ '.

Note that $\mathrm{n} \stackrel{\mathrm{dd}}{--\rightarrow} \mathrm{n}$ ' and $\mathrm{n} \stackrel{\text { ifdd }}{--\rightarrow} \mathrm{n}$ ' can hold by reason of more than one variable. The definitions for all kinds of dependences can be found in [Brü08]. We immediately deduce a small lemma which we will frequently use in the following chapters:

Lemma 2.3.8. (Direct data dependence requires $C F G$ path)

Let $\mathrm{n}, \mathrm{n}^{\prime} \in \mathrm{op}(N)$ such that $\mathrm{n}-\stackrel{\mathrm{dd}}{\rightarrow} \mathrm{n}^{\prime}$. Then, $\mathrm{n} \stackrel{*}{\longrightarrow} \mathrm{n}$. 
Proof. Immediately follows from Definition 2.3.6, 1.).

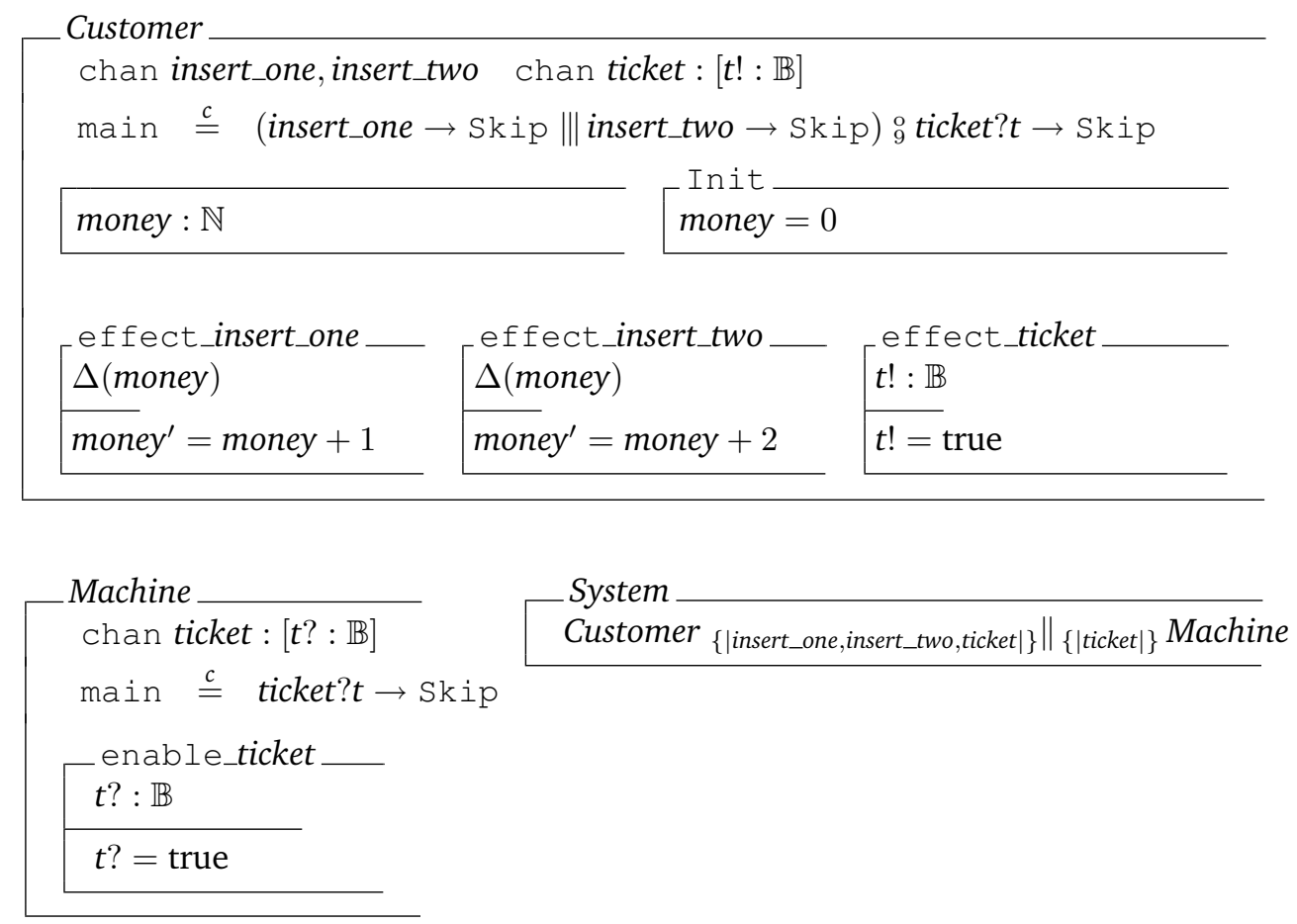

Figure 2.10: Simple CSP-OZ class specification for a ticket machine

Since our main case study does not incorporate all kinds of data dependences, we give a small example to illustrate them. Figure 2.10 shows a ticket machine specification consisting of two classes Customer and Machine. The overall system is defined as the parallel composition of both classes, synchronising on the set $\{\mid$ ticket $\mid\}$. The customer can insert coins of value 1 and 2 in an arbitrary order and afterwards, the machine dispenses the ticket. The full DDG of this small specification is given in Figure 2.11. Edges are labelled according to Table 2.3.

The specification incorporates the following dependences:

Initial Data Dependence: Since the state variable money is restricted in the initial state schema of Customer, referenced within insert_one, insert_two and possibly not overwritten in between, there exist two initial data dependences (1), (2) from Init to the respective operations.

Synchronisation Dependence: The operation schema ticket is synchronised between both classes thus yielding a synchronisation dependence (3) between Customer.ticket and Machine.ticket. 

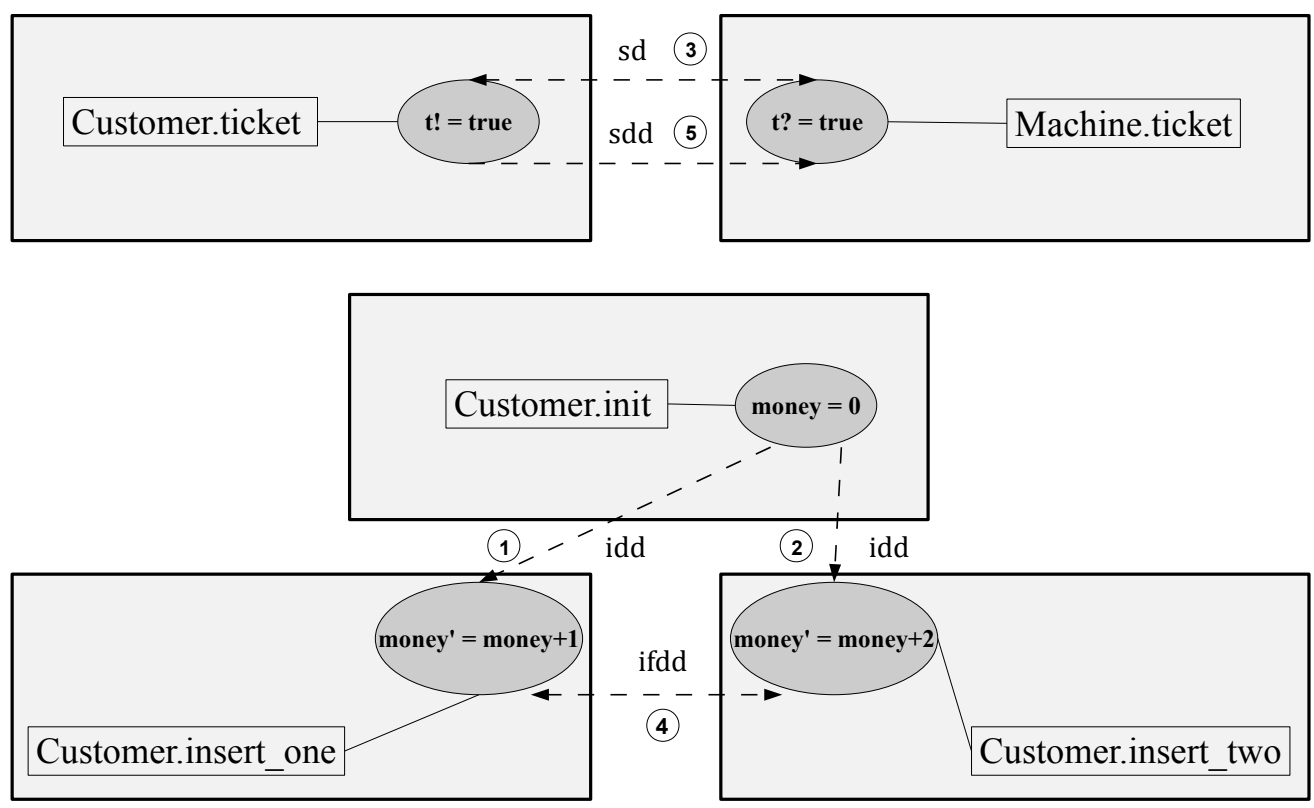

Figure 2.11: Data dependence graph (DDG) for the ticket machine specification

Interference Data Dependence: Based on money $\in(\bmod ($ insert_one $) \cap$ ref(insert_two $))$ and vice versa money $\in(\bmod ($ insert_two $) \cap$ ref(insert_one $))$, both nodes are connected via a (symmetric) interference data dependence (4)).

Synchronisation Data Dependence: As Customer.ticket sends the value of the parameter $t$ to Machine.ticket, a synchronisation data dependence (5), with Customer.ticket as the source node and Machine.ticket as the target node, connects both operation nodes.

The sole remaining data dependence, which we did not yet exemplify, is the direct data dependence. In the candy machine specification, one such edge is the link from switch to select due to credits $\in(\bmod ($ switch $) \cap$ ref $($ select $))$.

Figure 2.12 gives an extract of the DDG for the candy machine specification which solely comprises direct data dependences and initial data dependences. All edges of the DDG will be given in the next section as part of the specification's dependence graph.

\subsubsection{Definition of the Dependence Graph}

The idea of the definition of a (program) dependence graph (PDG), as introduced in [FOW87], is the unification of all the dependences of a program into one determined graph which can then serve as the sole basis for the analysis of a program. 


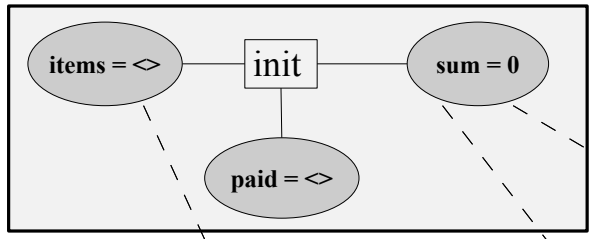

idd

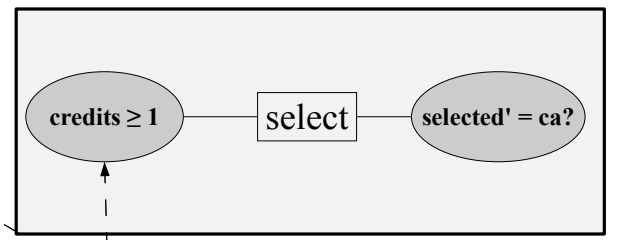

idd

idd

dd
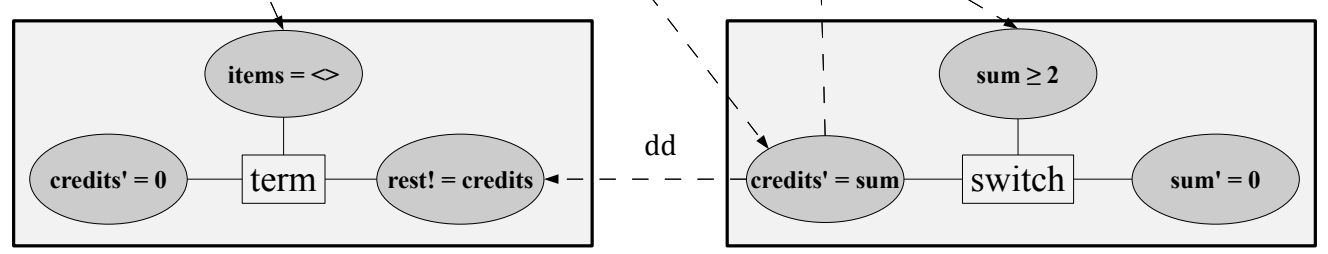

Figure 2.12: Extract of DDG for the candy machine specification

According to the structure of CSP-OZ specifications, the analysis of their dependence structure is two-folded: the construction of the overall dependence graph of the specification comprises the control flow graph for representing the control flow of a specification and the data dependence graph as a representation of its data flow. We will now consolidate both graphs into one. Again, we start with the general definition:

Definition 2.3.9. (Dependence Graph (DG) of $S$ )

The dependence graph $(\mathrm{DG}) \mathrm{DG}_{\mathrm{S}}=(N, \longrightarrow \mathrm{DG})$ of a CSP-OZ specification $S$ is defined over a set of nodes $N$ and a set of edges $\longrightarrow \mathrm{DG} \subseteq N \times N$, where

- $N=\mathrm{cf}(N) \cup \mathrm{op}(N)$ and

- $\longrightarrow{ }_{\mathrm{DG}}=(\longrightarrow \cup--\rightarrow)$,

according to Definition 2.3.1 and Definition 2.3.5 for the CFG and DDG, respectively.

The dependence graph is defined over the same set of nodes $N=\operatorname{cf}(N) \cup \operatorname{op}(N)$ as the CFG and comprises both, edges of the CFG and the DDG. Recall that edges of the DDG always connect operation nodes.

Our definition of the DG differs from the one defined in [Brü08] in several points:

Definition based on Operation Nodes: Our set of DG nodes comprises operation nodes instead of predicate nodes. Data dependences thus connect the respective operation nodes which contain the responsible predicates. This definition corresponds to Brückner's simplified graph representation, using super nodes. However, we additionally consolidate enable- and effect schemas of an operation into one node. The coarsening is motivated by the idea that in our decomposition, we will 
keep operations atomic, that is, we will either assign all or none of the original predicates of an operation to the generated components.

Inclusion of the CFG: In our context, a decomposition completely needs to adhere to the $\mathrm{CFG}$, since we must not destroy the overall dependence structure of a specification. Therefore, in contrast to Brückner, we integrate the full CFG into our dependence graph.

Neglect of Control Dependences: Based on the previous explanations, neither direct nor indirect control dependences as defined in [Brü08] are relevant in our context.

Neglect of Symmetric Data Dependences: Symmetric data dependences model sharing of modified variables between two predicates. These edges are only used for connecting two predicates within the same operation. Analogous to the previous explanations, we can safely omit them.

Paths of the DG are defined according to paths of the CFG, except that we use the notation path $h_{D G}$. Finally, we present the dependence graph for our case study in Figure 2.13. We do not explicitly distinguish between the several types of edges of the DDG here. The Init schema of the specification, attached with outgoing initial data dependences, is linked to the start-node of the graph. 


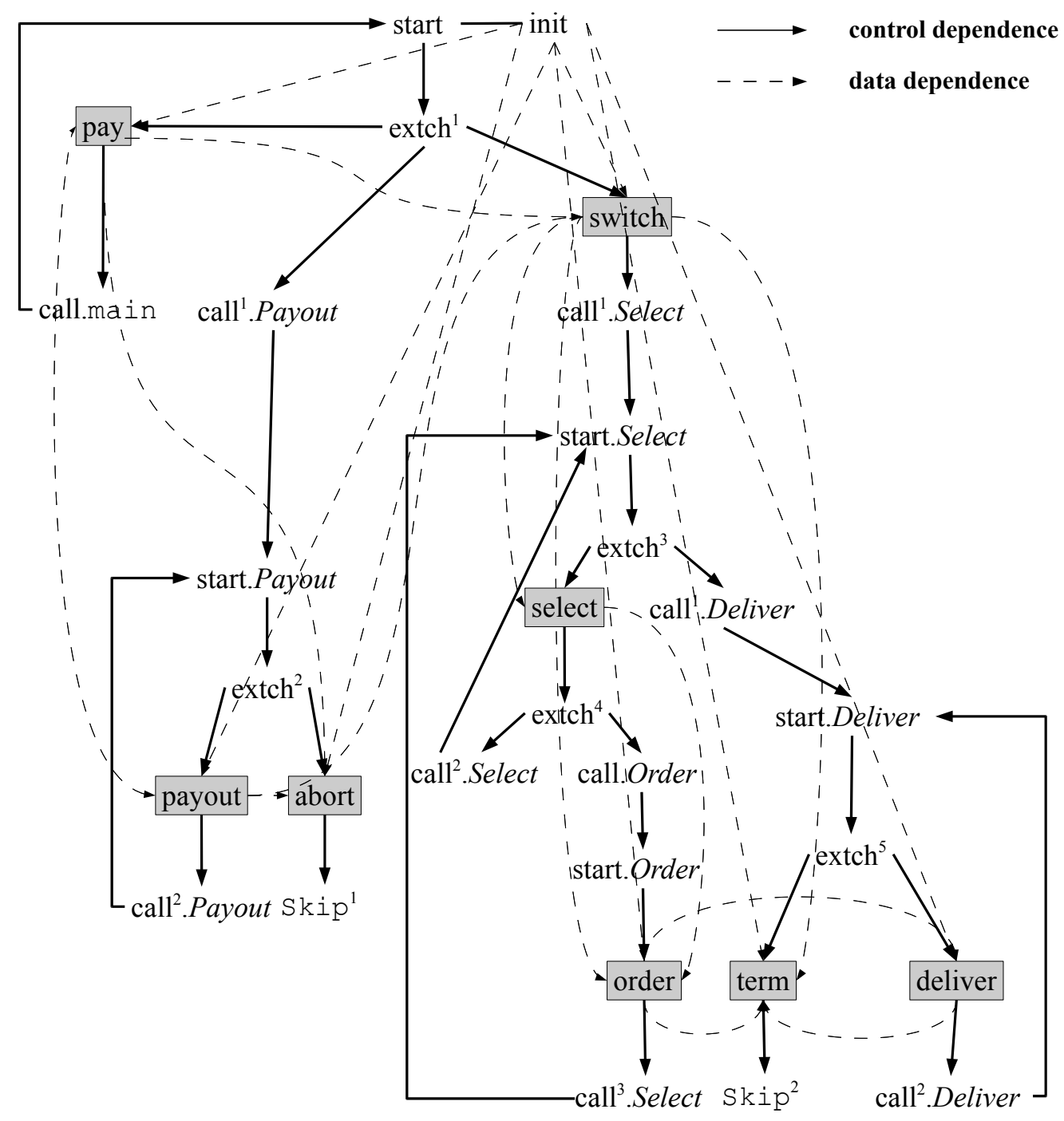

Figure 2.13: Dependence graph (DG) for the candy machine specification 



\section{Background: Compositional Reasoning}

\section{Contents}

3.1 Approaches to the State Space Explosion . . . . . . . . . 42

3.2 Compositional Reasoning . . . . . . . . . . . . . 43

3.2.1 Assume Guarantee Proof Rules . . . . . . . . . . . . . . 43

3.2.2 Obstacles to the Application of Assume Guarantee Reasoning 45

3.2.3 Learning for Compositional Verification . . . . . . . . . 45

3.3 Assume-Guarantee Reasoning for CSP . . . . . . . . . . . 47

3.3.1 Application Example: Elevator System . . . . . . . . . . . . . 49

3.3.2 Soundness of Assume-Guarantee Proof Rules . . . . . . . . 50

3.4 Related Work . . . . . . . . . . . . . . . . . . . . 53

In the introduction, we discussed strategies to ensure the reliability of a software system. Our approach concentrates on the verification of a system model with respect to certain requirements. This is achieved by specifying the system in the integrated formalism CSP-OZ, as introduced in the previous chapter, and employ model checking.

Model checking [CGP99] is a technique to automatically verify a system model, represented as a finite state machine, against desired properties of the system, described in some logical formalism. It either shows the validity of the desired properties or produces counterexamples, giving some insight on why the model is invalid. The methodology is introduced in [EC80, CES86], and extensive research has been devoted to it over the last years.

Even though model checking algorithms generally have a linear or at worst polynomial complexity in the size of their underlying models [Sch02], they all need to compute the state space of the system, which exponentially grows in the number of its components. Therefore, the main focus of attention is to cope with this decisive task, know as the state explosion problem.

This chapter provides the necessary background on model checking techniques and particularly on compositional verification as our object of research. Section 3.1 elaborates on the most relevant techniques to tackle the state explosion problem. Subsequently, Section 3.2 gives an overview on compositional verification and introduces our employed proof method, assume-guarantee reasoning. Along with this, we describe a methodology on learning assumptions for an automation of assume-guarantee-based verification. Section 3.3 puts assume-guarantee reasoning into our semantic context. Finally, Section 3.4 discusses related work on compositional verification. 


\subsection{Approaches to the State Space Explosion}

Verification of program correctness incorporates the analysis of any possible program execution and any reachable state. In order to achieve this, mathematical-based techniques aim to build a model, representing all possible program configurations. This structure is in general referred to as a program's state space. Model checking verifies the system model against certain requirements by analysing its state space.

Due to limited computing resources, automated verification of a software model can only construct models up to a certain extent. Thus, if the state space of a model becomes larger and larger, model checking becomes infeasible.

Model checking of specifications written in an integrated formal method are highly afflicted from the state explosion problem: as the data-oriented description of a system may cause an enormous state space due to large or even infinite data types, so does the behaviour-oriented description, owed to its concurrency. If two diverse formalisms are combined into one, automated formal verification suffers from both of these problems at the same time.

There are many strategies to tackle state explosion, with most of them having their specific advantages in certain domains. The most important techniques are described in the following.

Partial order reduction [KP88, God96] concentrates on the analysis of the concurrency of a system. More precisely, it aims at identifying independent and thus commutative transition paths in asynchronous systems. As a result, different orderings on these transitions can be conjoined. This technique clearly has its key benefits if applied to behaviour-oriented formalisms, incorporating asynchronous concurrency.

In order to apply model checking for infinite state systems, abstraction techniques need to be employed. In general, these techniques aim at either removing or simplifying parts of the system model.

One such technique is data abstraction [CGL94], which aims at handling large data domains. It is based on the idea of abstract interpretations [CC77]. Instead of evaluating a property with respect to all possible data values, an abstraction mapping identifies a set of concrete values for one abstract value. If the mapping satisfies certain correctness criteria, properties of the abstract system also hold for the concrete system. Data abstraction techniques for CSP-OZ were introduced in [Weh00].

However, too coarse abstractions can lead to wrong verification results. Counterexample guided abstraction-refinement $\left[\mathrm{CGJ}^{+} 03\right]$ iteratively refines an initially minimal abstraction and is guided by the model checker's counterexamples. In case of a spurious counterexample, based on an over-approximation of the system, the model is refined, and the verification process is repeated. In the context of CSP and $\mathrm{Z}$, this technique has been applied in [DW07].

Symmetry reduction [CJEF96] aims at finding behavioural symmetries to subsequently reduce the model. A similar approach is followed in [RW94] in the context of sequential composition.

Another abstraction technique is cone-of-influence reduction [Kur94]. Based on a certain property under interest, this technique aims at eliminating all specification elements 
which do not influence the verification property. For that purpose, the dependence structure of the specification is computed and analysed. A similar technique is program slicing [Wei81] which was successfully applied in the context of CSP-OZ [Brü08], as already mentioned in Section 2.3.

Symbolic model checking [JEK ${ }^{+}$90, McM93] aims at representing the state space in a canonical and more efficient form by means of a boolean encoding (ordered binary decision diagrams, [Bry86]). Many existing model checkers work on a symbolic representation of the original state machine and by using symbolic model checking algorithms.

Bounded model checking,[BCCZ99] as one branch of symbolic model checking [CBRZ01], incrementally tries to find finite prefixes of counterexamples by examining paths up to a certain bound $k$. If no counterexample is found, the bound is incremented, and the algorithm continues. The bounded model checking problem can efficiently be reduced to the propositional satisfiability problem (SAT) [DP60].

\subsection{Compositional Reasoning}

The strategy to cope with the state explosion problem we focus on is compositional verification. Many systems are not defined as one single large component but more likely composed of smaller parts. Compositional verification $\left[\mathrm{dRHH}^{+} 01\right]$ uses this property by means of a "divide and conquer" approach: instead of verifying the system as a whole, the verification task is split up into smaller subtasks. The components of the system are verified independently, and the verification results are combined.

The benefits are evident: instead of computing the global state space of the overall system, compositional verification merely needs to deal with the individual state spaces of the system components and thus avoids the state explosion problem up to a certain extent.

There are a lot of different compositional proof strategies [BCC98] but the most popular ones are based on the assume-guarantee paradigm [FP78, Jon83, MC81]: since, in general, a system component $S$ depends on its environment, it cannot be verified in isolation. However, if a certain environment assumption $A$ is assumed for $S$, a guarantee condition $G$ of $S$ can be inferred. Typically, this is expressed by a logical triple $\langle A\rangle S\langle G\rangle$, stating that if $S$ is part of an overall system satisfying $A$, then the system must guarantee $G$.

Assume guarantee reasoning uses the previously described paradigm in terms of inference rules. In our context and in the context of [BGP03], $A, S$ and $G$ represent labelled transition systems. Thus, we may let $\mathcal{L}(A)$ denote the language of the assumption $A$, that is, its set of traces over $\Sigma^{*}$ on the underlying LTS, where $\Sigma$ denotes the trace alphabet of $A$. Furthermore, let $\mathcal{L}(A)^{C}$ denote the complement of this language, that is, $\mathcal{L}(A)^{C}=\Sigma^{*} \backslash \mathcal{L}(A)$.

Next, we present the different proof rules, which we will deal with in this thesis.

\subsubsection{Assume Guarantee Proof Rules}

Proof rules adhering to the assume-guarantee paradigm can be classified into different categories. Suppose an overall system $S$ to be composed of two components $S_{1}$ and 
$S_{2}$ running in parallel: $S=S_{1} \| S_{2}$. The simplest assume-guarantee proof rule can be described as follows: if component $S_{1}$ guarantees (satisfies) an assumption $A$, and if component $S_{2}$ satisfies a property Prop under the assumption $A$, then the overall system $S_{1} \| S_{2}$ satisfies Prop. This can be denoted as an inference rule as given in Figure 3.1.

$$
\begin{gathered}
\langle\text { true }\rangle S_{1}\langle A\rangle \\
\langle A\rangle S_{2}\langle\text { Prop }\rangle \\
\hline\langle\text { true }\rangle S_{1} \| S_{2}\langle\text { Prop }\rangle
\end{gathered}
$$

Figure 3.1: Basic assume-guarantee proof rule (B-AGR)

$$
\begin{gathered}
\left\langle A_{1}\right\rangle S_{1}\langle\text { Prop }\rangle \\
\left\langle A_{2}\right\rangle S_{2}\langle\text { Prop }\rangle \\
\mathcal{L}\left(A_{1}\right)^{C} \cap \mathcal{L}\left(A_{2}\right)^{C}=\varnothing \\
\hline\langle\text { true }\rangle S_{1} \| S_{2}\langle\text { Prop }\rangle
\end{gathered}
$$

Figure 3.2: Parallel assume-guarantee proof rule (P-AGR)

From now on, this rule will be denoted by (B-AGR) and it will be called the basic assume-guarantee proof rule. It can be classified as being sequential in the sense that the first premise, $\langle$ true $\rangle S_{1}\langle A\rangle$, needs to be evaluated before the second premise, $\langle A\rangle S_{2}\langle$ Prop $\rangle$, can be considered $-A$ must already be determined before it can serve as an assumption for $S_{2}$.

Another proof rule is motivated by the need for a symmetric computation of assumptions for both components. One particular symmetric proof rule is given in [BGP03] and depicted in Figure 3.2. In contrast to the basic proof rule, we call this rule the parallel proof rule and refer to it as (P-AGR).

The main difference to rule (B-AGR) is the usage of one additional premise and assumption. Moreover, the rule allows for a parallel computation of the first and second premise, since both assumptions do not appear on the right hand side of both logical triples.

The first premise states that under the assumption $A_{1}$, component $S_{1}$ satisfies Prop. The second premise states the corresponding for $A_{2}$ and $S_{2}$. In order to show that $S_{1} \| S_{2}$ satisfies Prop, we need a third premise: the intersection of the complements of both assumption languages needs to be empty.

In [BGP03], the authors show that the third premise, which is equivalent to $\mathcal{L}\left(A_{1}\right) \cup$ $\mathcal{L}\left(A_{2}\right)=\Sigma^{*}$, is indeed necessary. The intuitive reason can roughly be described as follows: $A_{1}$ restricts $S_{1}$ to Prop and $A_{2}$ restricts $S_{2}$ to Prop, whereas the conclusion states that $S_{1} \| S_{2}$ satisfies Prop without any restriction. Thus, the unification of the languages of both assumptions must contain all possible words. This ensures that no possible behaviour is ruled out by both assumptions at the same time.

A third class of assume-guarantee proof rules are referred to as circular proof rules. These rules either involve circularity on the assumptions or, as in our case, on the components: one circular proof rule as introduced originally in [GL91] is depicted in Figure 3.3 which we will refer to as rule (C-AGR). Here, two premises coincide on their component. In general, in comparison to non-circular ones, proving soundness and completeness of circular proof rules is rather difficult [Mai03].

In this thesis, we will focus on non-circular rules and on the the first two proof rules, 


$$
\begin{gathered}
\langle\text { true }\rangle S_{1}\left\langle A_{1}\right\rangle \\
\left\langle A_{1}\right\rangle S_{2}\left\langle A_{2}\right\rangle \\
\left\langle A_{2}\right\rangle S_{1}\langle\text { Prop }\rangle \\
\hline\langle\text { true }\rangle S_{1} \| S_{2}\langle\text { Prop }\rangle
\end{gathered}
$$

Figure 3.3: Circular assume-guarantee rule (C-AGR)

rules (B-AGR) and (P-AGR). For an application of these proof rules, one needs to identify appropriate assumptions.

\subsubsection{Obstacles to the Application of Assume Guarantee Reasoning}

Several issues complicate the application of assume-guarantee reasoning. First, the system needs to be composed of several components. If this is not the case, assume-guarantee reasoning is not applicable at all.

Furthermore, the identification of environment assumptions had to be done manually by the user. By the use of a new technique based on a learning approach and proposed in [CGP03], this process can now fully be automated. We will introduce the approach in the next section.

Even though that automated learning of an assumption removes one of the obstacles assume-guarantee reasoning has to deal with, its usefulness in comparison to monolithic verification is still questionable: the major aim of this technique is to explore smaller state spaces. However, an unadvantageous decomposition can still lead to large assumptions and thus large state spaces. In [CAC06], the authors investigated the effectiveness of assume-guarantee reasoning based on exploring different decompositions of a given system and comparing memory usage. The results show that only in very few cases, assume-guarantee reasoning indeed outperforms non-compositional verification. Even worse, in most cases, the explored state spaces are actually larger in compositional verification.

In particular in the context of compositional verification for formal methods, these considerations motivate the need for a technique to define decompositions which are advantageous for an application of assume-guarantee-based techniques. We address this problem in Chapter 6.

\subsubsection{Learning for Compositional Verification}

In order to apply an assume-guarantee-based proof rule, environment assumptions need to be identified. Consider the basic proof rule (B-AGR). Unfortunately, it is a non-trivial process to find an assumption which, on the one hand, abstracts from $S_{1}$ by over-approximating it and which is, on the other hand, strong enough for $S_{2}$, such that Prop can be deduced. This applies to any of these proof rules. 
Over many years, the development of an assumption had to be done manually by the user, not allowing assume-guarantee reasoning to be performed in an automatic manner. Recently, a new technique to fully automatically generate assumptions [CGP03] based on a learning algorithm [Ang87] has been developed. The core idea for this technique is to use a model checker to learn the assumption. This technique can be applied with respect to several assume-guarantee proof rules $\left[\mathrm{PGB}^{+} 08\right]$ and in a framework, freeing the user from manual interference.

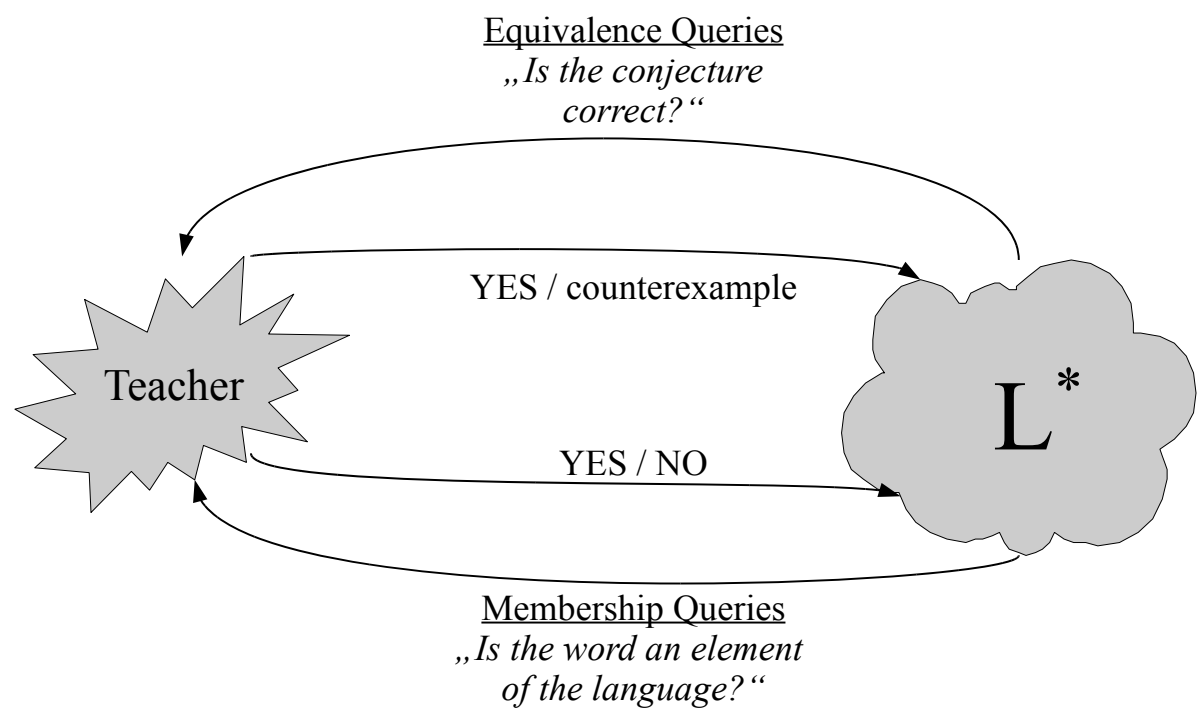

Figure 3.4: Illustration of the $\mathrm{L}^{*}$ algorithm

The basis for this approach is an algorithm which learns an unknown regular language (in our case: the language of the assumption) and returns a deterministic finite automaton (DFA) accepting this language. The algorithm is called $\mathrm{L}^{*}$, and it was introduced in [Ang87]. We describe the basic idea of the algorithm: suppose that $U$ is an unknown regular language over some alphabet $\Sigma$. For an effective learning of $U$, the algorithm requires an oracle which correctly answers two different questions:

Question (Membership Query):

Given a word $w$ over the alphabet $\Sigma$, is $w$ an element of $U$ ?

Answer:

Yes, if $w$ is an element of $U$, no otherwise.

Question (Equivalence Query):

Does the DFA $D$ accept the language $U$ ?

Answer:

Yes, if $\mathcal{L}(D)=U$ holds, a counterexample $w \in(\mathcal{L}(D) \backslash U) \cup(U \backslash \mathcal{L}(D))$ otherwise. 
If the oracle (or teacher, as it is called in the context of $\mathrm{L}^{*}$ ) correctly answers this question, the algorithm always terminates and outputs a DFA $D_{U}$, such that $\mathcal{L}\left(D_{U}\right)=U$ holds.

Figure 3.4 illustrates this concept. The approach presented in [CGP03] incorporates the $\mathrm{L}^{*}$ algorithm into an assume-guarantee-based framework for the automatic computation of the required assumptions. The technique can be applied to all three previously introduced proof rules, as shown in $\left[\mathrm{PGB}^{+} 08\right]$. Here, a model checker serves as the teacher. The idea is to incrementally compute the assumption.

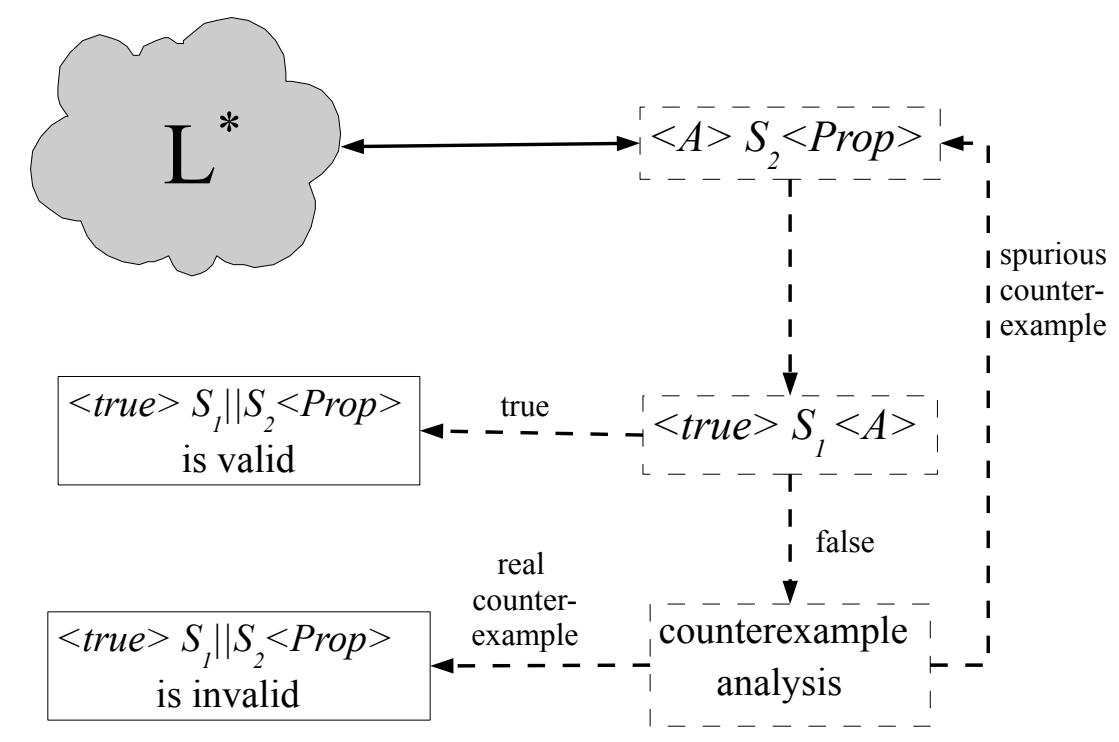

Figure 3.5: Illustration of the $\mathrm{L}^{*}$ based learning framework

As an example, for the basic proof rule (B-AGR), the framework starts by making use of $\mathrm{L}^{*}$ to compute an assumption $A$ such that $\langle A\rangle S_{2}\langle$ Prop $\rangle$ holds. Afterwards, $\langle$ true $\rangle S_{1}\langle A\rangle$ is checked. ${ }^{1}$ If the result is true, correctness of the proof rule yields that $\langle$ true $\rangle S_{1} \| S_{2}\langle$ Prop $\rangle$ holds. Otherwise, the counterexample is analysed. A spurious counterexample leads to a refinement of the verification process, a valid counterexample to the refutation of $\langle$ true $\rangle S_{1} \| S_{2}\langle$ Prop $\rangle$. This is illustrated in Figure 3.5.

Next, we put assume-guarantee reasoning into our context by translating both rules, (B-AGR) and (P-AGR), into the semantic domain of CSP-OZ. Subsequently, we show their soundness.

\subsection{Assume-Guarantee Reasoning for CSP}

Since our application of assume-guarantee reasoning lies in the domain of CSP-OZ specifications, we need to translate the previously identified proof rules into our context and show their correctness. Fortunately, as already explained in Chapter 2, CSP-OZ

\footnotetext{
${ }^{1}$ In terms of an LTS, true corresponds to the empty language.
} 
specifications can be translated into semantic equivalent CSP processes. Therefore, it is sufficient to consider the semantic domain of CSP.

Verification properties can mainly be classified into two categories [OL82]: safety and liveness properties. Safety properties follow the principle of

\section{"Nothing bad will ever happen!"}

meaning that a violation of a safety property is given by a finite counterexample. In contrast, liveness properties can be described by

\section{"Something good will eventually happen!"}

describing that at some point, the property will be satisfied, not allowing to contradict a liveness property by a finite counterexample.

Our decomposition approach focuses on safety properties. This allows us to move to the domain of the CSP trace semantics instead of the more discriminating failuresdivergences semantics: as explained in [Weh00] and [OW05], in contrast to liveness properties dealing with deadlock or livelock freedom, when dealing with safety properties, the CSP traces model is sufficient. An approach for verifying liveness properties in the context of compositional reasoning is, for instance, given in [CGK97]. According to this, the learning-based approach, as explained in the previous section, is also considering safety properties.

By translating assume-guarantee proof rules into the CSP traces model, a logical triple $\langle A\rangle S\langle$ Prop $\rangle$ becomes a trace refinement condition Prop $\sqsubseteq_{T} A \| S$ which is by definition equivalent to traces $(A \| S) \subseteq \operatorname{traces}(\operatorname{Prop})$.

We need to be more precise and consider the respective alphabets of $A, S$ and Prop. Here, the alphabet of the assumption depends on the particular proof rule: for the basic rule, (B-AGR), $\alpha A=\left(X_{2} \cup Y\right) \cap X_{1}$ whereas for the parallel rule, (P-AGR), $\alpha A=\left(X_{1} \cap X_{2}\right) \cup Y$. Setting $\alpha S=X$, $\alpha$ Prop $=Y$ and $\alpha A=\Sigma$, the condition becomes

$$
\text { Prop } \sqsubseteq_{T}\left(A_{\Sigma} \|_{X} S\right) \backslash(\text { Events } \backslash Y) \text {, }
$$

where the right hand side processes need to be restricted to the alphabets of the left hand side processes by using hiding.

Figures 3.6 and 3.7 specify rules (B-AGR) and (P-AGR), rephrased in terms of CSP trace refinement, where we additionally set $\alpha S_{1}=X_{1}$ and $\alpha S_{2}=X_{2}$.

We take a closer look at the third premise of rule (P-AGR): in comparison to the work [CGP03], the authors move from the domain of labelled transitions system (LTS) to finite state machines (FSM) [BGP03] and construct the complement $c o-M$ of a FSM $M$ to denote the third premise of rule (P-AGR) by

$$
\mathcal{L}\left(\operatorname{co} \_A_{1} \| \operatorname{co} \_A_{2}\right)=\varnothing
$$

However, it is impossible to construct a CSP process co_P for some process $P$, accepting the complement of its language. This is based on the fact that the set of traces of a CSP process is always prefix-closed whereas its complement is not. Thus, co $P$ does not exist 


$$
\begin{gathered}
A \sqsubseteq_{T} S_{1} \backslash(\text { Events } \backslash \Sigma) \\
\text { Prop } \sqsubseteq_{T}\left(A_{\Sigma} \|_{X_{2}} S_{2}\right) \backslash(\text { Events } \backslash Y) \\
\text { Prop } \sqsubseteq_{T}\left(S_{1 X_{1}} \|_{X_{2}} S_{2}\right) \backslash(\text { Events } \backslash Y)
\end{gathered}
$$

Figure 3.6: Rule (B-AGR) rephrased in terms of CSP trace refinement

$$
\begin{array}{r}
\text { Prop } \sqsubseteq_{T}\left(A_{1} \Sigma \|_{X_{1}} S_{1}\right) \backslash(\text { Events } \backslash Y) \\
\text { Prop } \sqsubseteq_{T}\left(A_{2} \Sigma \|_{X_{2}} S_{2}\right) \backslash(\text { Events } \backslash Y) \\
\quad\left(A_{1} \square A_{2}\right) \sqsubseteq_{T} \text { Run }_{\Sigma} \\
\hline \text { Prop } \sqsubseteq_{T}\left(S_{1 X_{1}} \|_{X_{2}} S_{2}\right) \backslash(\text { Events } \backslash Y)
\end{array}
$$

Figure 3.7: Rule (P-AGR) rephrased in terms of CSP trace refinement

and we use the equivalent ${ }^{2}$ condition $\mathcal{L}\left(A_{1}\right)^{C} \cap \mathcal{L}\left(A_{2}\right)^{C}=\varnothing$. In our semantic domain of the CSP traces model, this means traces $\left(A_{1}\right)^{C} \cap \operatorname{traces}\left(A_{2}\right)^{C}=\varnothing$. We will now show that $\left(A_{1} \square A_{2}\right) \sqsubseteq_{T}$ Run $_{\Sigma}$ and traces $\left(A_{1}\right)^{C} \cap \operatorname{traces}\left(A_{2}\right)^{C}=\varnothing$ are equivalent, implying that rule (P-AGR) corresponds to rule 1 from [BGP03].

Lemma 3.3.1. (Correspondence between rule (P-AGR) and rule 1 from [BGP03]) Let $A_{1}$ and $A_{2}$ be two CSP processes over the alphabet $\Sigma$. Then,

$$
\left(A_{1} \square A_{2}\right) \sqsubseteq_{T} \operatorname{Run}_{\Sigma}
$$

holds, if, and only if,

$$
\operatorname{traces}\left(A_{1}\right)^{C} \cap \operatorname{traces}\left(A_{2}\right)^{C}=\varnothing .
$$

Proof.

$$
\begin{array}{rll} 
& \left(A_{1} \square A_{2}\right) \sqsubseteq_{T} \operatorname{Run}_{\Sigma} & \\
\Leftrightarrow \quad \operatorname{traces}\left(A_{1} \square A_{2}\right)=\operatorname{traces}\left(\operatorname{Run}_{\Sigma}\right) & \text { (Definition of } \left.\operatorname{Run}_{\Sigma}\right) \\
\Leftrightarrow \quad \operatorname{traces}\left(A_{1} \square A_{2}\right)=\Sigma^{*} & \text { (Definition of } \left.\operatorname{traces}\left(\operatorname{Run}_{\Sigma}\right)\right) \\
\Leftrightarrow \quad \operatorname{traces}\left(A_{1} \square A_{2}\right)^{C}=\varnothing & \\
\Leftrightarrow \quad\left(\operatorname{traces}\left(A_{1}\right) \cup \operatorname{traces}\left(A_{2}\right)\right)^{C}=\varnothing & \text { (Definition of traces for external choice) } \\
\Leftrightarrow \quad \operatorname{traces}\left(A_{1}\right)^{C} \cap \operatorname{traces}\left(A_{2}\right)^{C}=\varnothing &
\end{array}
$$

Next, we give a small example illustrating the application of rule (B-AGR).

\subsubsection{Application Example: Elevator System}

Figure 3.8 defines a CSP specification of a simple elevator system. It consists of two processes Elevator and User. The overall system is defined as the parallel composition of both processes, synchronising on the intersection of their alphabets

$$
X_{1}:=\{\text { req_floor }, \text { req_close, move, stop, req_open }\}
$$

and

$$
X_{2}:=\{\text { req_floor, enter, req_close, req_open, leave }\} .
$$




$$
\begin{aligned}
\text { Elevator } \stackrel{c}{=} & \text { req_floor } \rightarrow \text { req_close } \rightarrow \text { move } \rightarrow \text { stop } \rightarrow \text { req_open } \rightarrow \text { Elevator } \\
\text { User } \stackrel{c}{=} & \text { req_floor } \rightarrow \text { enter } \rightarrow \text { User } \square \\
& \text { req_close } \rightarrow \text { User } \square \\
& \text { req_open } \rightarrow \text { leave } \rightarrow \text { User } \\
\text { System } \stackrel{c}{=} & \text { Elevator } X_{X_{1}} \|_{X_{2}} \text { User }
\end{aligned}
$$

Figure 3.8: CSP specification of a simple elevator system

The property, which we want to verify, is given as follows: a user entering the elevator (enter) will always lead to him leaving (leave) the elevator. As a CSP process, we write: Prop $\stackrel{c}{=}$ enter $\rightarrow$ leave $\rightarrow$ Prop. Let $Y:=\{$ enter, leave $\}$ denote the alphabet of the property. Based on the definition of [CGP03], we get

$$
\Sigma=\left(X_{2} \cup Y\right) \cap X_{1}=\{\text { req_floor, req_close, req_open }\} .
$$

In order to show that

$$
\text { Prop } \sqsubseteq_{T}\left(\text { Elevator } X_{X_{1}} \|_{X_{2}} \text { User }\right) \backslash(\text { Events } \backslash Y)
$$

holds, we can apply rule (B-AGR) by defining

$$
\begin{aligned}
& A \stackrel{c}{=} \text { req_close } \rightarrow A \square \text { req_floor } \rightarrow A^{\prime} \\
& A^{\prime} \stackrel{c}{=} \text { req_close } \rightarrow A^{\prime} \square \text { req_open } \rightarrow A
\end{aligned}
$$

Then, both premises of the rule are satisfied, that is, traces(Elevator) $\uparrow \Sigma \subseteq \operatorname{traces}(A)$ and traces $\left(A_{\Sigma} \|_{X_{2}}\right.$ User $)\lceil Y \subseteq \operatorname{traces}($ Prop $)$ hold.

\subsubsection{Soundness of Assume-Guarantee Proof Rules}

After translating both rules, (B-AGR) and (P-AGR), into our setting of CSP, we need to show their soundness. In his bachelor's thesis, Wonisch [Won08] integrated the approach of [CGP03] into a framework for compositional reasoning about CSP processes, which he implemented by using the CSP model checker FDR2 as the teacher. For that purpose, he showed the following soundness theorem for rule (B-AGR): ${ }^{3}$

Theorem 3.3.2. (Soundness of basic proof rule)

Let $S_{1}, S_{2}$ and Prop be CSP processes. Let $X_{1}, X_{2}, Y$ be alphabets, and let $A$ be a CSP process

\footnotetext{
${ }^{2}$ This is based on $\mathcal{L}(A)^{C}=\mathcal{L}\left(\operatorname{co\_ } A\right)$ and $\mathcal{L}(A \| B)=\mathcal{L}(A) \cap \mathcal{L}(B)$.

${ }^{3}$ We omit dealing with the technical aspect of $\checkmark$-freedom.
} 
defined over the alphabet $\Sigma=\left(X_{2} \cup Y\right) \cap X_{1}$. Then, the following proof rule is sound:

$$
\begin{gathered}
A \sqsubseteq_{T} S_{1} \backslash(\text { Events } \backslash \Sigma) \\
\text { Prop } \sqsubseteq_{T}\left(A_{\Sigma} \|_{X_{2}} S_{2}\right) \backslash(\text { Events } \backslash Y) \\
\text { Prop } \sqsubseteq_{T}\left(S_{1 X_{1}} \|_{X_{2}} S_{2}\right) \backslash(\text { Events } \backslash Y)
\end{gathered}
$$

Proof. See [Won08], Theorem 1.

We will now correspondingly show soundness of the parallel proof rule (P-AGR).

Theorem 3.3.3. (Soundness of parallel proof rule)

Let $S_{1}, S_{2}$ and Prop be CSP processes. Let $X_{1}, X_{2}, Y$ be alphabets such that $Y \subseteq X_{1} \cup X_{2}$, and let $A_{1}, A_{2}$ be CSP processes defined over the alphabet $\Sigma=\left(X_{1} \cap X_{2}\right) \cup Y$. Then, the following proof rule is sound:

$$
\begin{gathered}
\text { Prop } \sqsubseteq_{T}\left(A_{1} \Sigma \|_{X_{1}} S_{1}\right) \backslash(\text { Events } \backslash Y) \\
\text { Prop } \sqsubseteq_{T}\left(A_{2} \|_{X_{2}} S_{2}\right) \backslash(\text { Events } \backslash Y) \\
\quad\left(A_{1} \square A_{2}\right) \sqsubseteq_{T} \operatorname{Run}_{\Sigma} \\
\left.\hline \text { Prop } \sqsubseteq_{T}\left(S_{1 X_{1}} \|_{X_{2}} S_{2}\right) \backslash \text { (Events } \backslash Y\right)
\end{gathered}
$$

Proof. Let

$$
t \in \operatorname{traces}\left(\left(S_{1 X_{1}} \|_{X_{2}} S_{2}\right) \backslash(\text { Events } \backslash Y)\right) .
$$

We need to show $t \in \operatorname{traces}(\operatorname{Prop}$ ). The definition of traces for hiding ([Ros98]) yields the existence of

$$
s \in \operatorname{traces}\left(S_{1 X_{1}} \|_{X_{2}} S_{2}\right),
$$

such that $t=s\left\lceil Y\right.$. Moreover, since $s$ is defined over $X_{1} \cup X_{2}$ and by applying the definition of traces $\left(P_{X} \|_{Y} Q\right)$ ([Ros98]), for $u_{1}:=s\left\lceil X_{1}\right.$ and $u_{2}:=s\left\lceil X_{2}\right.$, we get $u_{i} \in \operatorname{traces}\left(S_{i}\right)$. Mainly corresponding to the correctness proof of the basic assume-guarantee rule, [Won08], we will now show:

(i) $s \uparrow\left(\Sigma \cup X_{1}\right) \in \operatorname{traces}\left(A_{1} \Sigma \|_{X_{1}} S_{1}\right)$ or $s \uparrow\left(\Sigma \cup X_{2}\right) \in \operatorname{traces}\left(A_{2} \Sigma \|_{X_{2}} S_{2}\right)$,

(ii) $(*) \Rightarrow t_{1}^{\prime}:=s_{1}^{\prime} \uparrow Y \in \operatorname{traces}\left(\right.$ Prop) and $t_{1}^{\prime}=t$,

(iii) $\left(^{* *}\right) \Rightarrow t_{2}^{\prime}:=s_{2}^{\prime}\left\lceil Y \in \operatorname{traces}(\operatorname{Prop})\right.$ and $t_{2}^{\prime}=t$,

where both, $(*)$ and $(* *)$ lead to the conclusion $t \in \operatorname{traces}($ Prop).

For property (i), let $s_{i}^{\prime}:=s \uparrow\left(\Sigma \cup X_{i}\right)$. We first deduce

$$
\begin{aligned}
& s_{1}^{\prime}\left\lceil\Sigma=\left(s \uparrow\left(\Sigma \cup X_{1}\right)\right) \uparrow \Sigma=s \uparrow \Sigma\right. \text { and } \\
& s_{2}^{\prime} \uparrow \Sigma=\left(s\left\lceil\left(\Sigma \cup X_{2}\right)\right) \uparrow \Sigma=s\lceil\Sigma \text {. }\right.
\end{aligned}
$$


Based on the third premise and the fact that $s\left\lceil\Sigma \in \operatorname{traces}\left(\operatorname{Run}_{\Sigma}\right)\right.$ by definition of Run $\Sigma$, we have $s\left\lceil\Sigma \in \operatorname{traces}\left(A_{1} \square A_{2}\right)\right.$. Thus, either $s\left\lceil\Sigma \in \operatorname{traces}\left(A_{1}\right)\right.$ or $s\left\lceil\Sigma \in \operatorname{traces}\left(A_{2}\right)\right.$ holds. Second, we get

$$
\begin{aligned}
& s_{1}^{\prime}\left\lceil X_{1}=\left(s \uparrow\left(\Sigma \cup X_{1}\right)\right) \uparrow X_{1}=s\left\lceil X_{1}=u_{1}\right. \text { and }\right. \\
& s_{2}^{\prime}\left\lceil X_{2}=\left(s \lceil ( \Sigma \cup X _ { 2 } ) ) \left\lceilX_{2}=s\left\lceil X_{2}=u_{2},\right.\right.\right.\right.
\end{aligned}
$$

with $u_{1} \in \operatorname{traces}\left(S_{1}\right)$. Both combined: if $s \mid \Sigma=s_{1}^{\prime}\left\lceil\Sigma \in \operatorname{traces}\left(A_{1}\right)\right.$, we use $s_{1}^{\prime} \mid X_{1} \in \operatorname{traces}\left(S_{1}\right)$ to deduce $s_{1}^{\prime} \in \operatorname{traces}\left(A_{1} \Sigma \|_{X_{1}} S_{1}\right)$. Otherwise, we get $s_{2}^{\prime} \in \operatorname{traces}\left(A_{2} \Sigma \|_{X_{1}} S_{2}\right)$. This concludes the proof of (i).

Next, we show (ii), (iii) is analogous. If (*) holds, $t_{1}^{\prime}:=s_{1}^{\prime} \uparrow Y \in \operatorname{traces}($ Prop) follows immediately from the first premise. We are left to show $t_{1}^{\prime}=t$ :

$$
\begin{aligned}
& t_{1}^{\prime} & & \\
= & s_{1}^{\prime} \uparrow Y & & \text { (Definition of } \left.t_{1}^{\prime}\right) \\
= & \left(s \uparrow\left(\Sigma \cup X_{1}\right)\right) \uparrow Y & & \text { (Definition of } \left.s_{1}^{\prime}\right) \\
= & s \uparrow\left(\left(\Sigma \cup X_{1}\right) \cap Y\right) & & \text { (Definition of trace projection) } \\
= & s \uparrow\left(\left(\left(X_{1} \cap X_{2}\right) \cup Y \cup X_{1}\right) \cap Y\right) & & \text { (Definition of } \Sigma \text { ) } \\
= & s \uparrow Y & & \\
= & t & & \text { (Definition of } t \text { ) }
\end{aligned}
$$

This concludes the proof.

The following example [Sch09] shows that the restriction $\Sigma=\left(X_{1} \cap X_{2}\right) \cup Y$ is indeed required.

Example 3.3.4. Let $S_{1}=a \rightarrow a \rightarrow$ Stop, $S_{2}=b \rightarrow b \rightarrow$ Stop and

$$
\text { Prop }=(a \rightarrow a \rightarrow \text { Stop }) \square(b \rightarrow b \rightarrow \text { Stop }) .
$$

Thus, we get $X_{1}=\{a\}, X_{2}=\{b\}$ and $Y=\{a, b\}$. Now assume $\Sigma=\varnothing$ and $A_{1}=A_{2}=$ Stop. Then, all three premises of the parallel rule are satisfied:

- We get (Stop $\left.\varnothing \|_{\{a\}} S_{1}\right)=a \rightarrow a \rightarrow$ Stop and thus Prop $\bigsqcup_{T} a \rightarrow a \rightarrow$ Stop.

- Also, (Stop $\left.\varnothing \|_{\{b\}} S_{2}\right)=b \rightarrow b \rightarrow$ Stop and therefore Prop $\bigsqcup_{T} b \rightarrow b \rightarrow$ Stop.

- Finally, Run $\varnothing=_{T}$ Stop, hence (Stop $\square$ Stop) $\sqsubseteq_{T} \operatorname{Run}_{\varnothing}$.

However, Prop $\sqsubseteq_{T}\left(a \rightarrow a \rightarrow\right.$ Stop $\{a\} \|_{\{b\}} b \rightarrow b \rightarrow$ Stop) does not hold as Prop does not allow the trace $\langle a, b, a, b\rangle$ which $\left(a \rightarrow a \rightarrow \operatorname{Stop}\{a\} \|_{\{b\}} b \rightarrow b \rightarrow\right.$ Stop) is able to conduct. In case of $\Sigma=\left(X_{1} \cap X_{2}\right) \cup Y=\{a, b\}$, the third premise becomes

$$
\text { (Stop } \square \text { Stop) } \sqsubseteq_{T} \operatorname{Run}_{\{a, b\}} \text {, }
$$

which is clearly not satisfied.

Summing up, we have shown the applicability of the proof rules (B-AGR) and (P-AGR) in the semantic domain of CSP. This allows us to apply compositional reasoning, based on the following decomposition approach in our context. Moreover, we can evaluate the efficiency of different decompositions by using the CSP model checker FDR2 [For05]. 


\subsection{Related Work}

Model checking and (automated) compositional verification of specifications, written in (integrated) formal methods, is extensively researched. We give a brief overview on recent works, mainly in the context of our employed methods.

Model Checking for Formal Methods: In order to allow model checking of a software system, it needs to be specified in some formal language.

Leuschel examines LTL model checking [LMC01] for CSP by using the model checker EDR2 [For05]. In [SW05], Smith and Wildman consider model checking of $\mathrm{Z}$ specifications by translating $\mathrm{Z}$ into the input language of the model checker SAL $\left[\mathrm{BGL}^{+}\right.$00]. Derrick et al. also investigate $\mathrm{Z}$ model checking by using SAL in [DNS08]. Smith deals with model checking Object-Z specifications with respect to temporal logic formulae in [KS01].

CSP-Z model checking is researched in [MS01]. Model checking CSP-OZ specifications by again using FDR2 is described in [FW99]. We use this approach in our implementation framework.

Compositional Verification for Formal Methods: Compositional verification has its early application within the scope of model checking in [EDK89] and later in [GL91, CGP99]. Proof rules for verifying real time system have been developed in [CMP94]. In the context of UML, compositional verification (and model checking) of embedded real time systems is, for instance, investigated in [SGT $\left.{ }^{+} 03\right]$. By defining a formal semantics for a domain specific subset of the UML, the authors allow themselves to reason about individual software components instead of the complete system.

In our context, Winter and Smith [WS03] deal with compositional verification for Object-Z. They analyse the class structure of an Object-Z specification and argument about restricted environments, allowing for the definition of a compositional proof rule. Modular reasoning of Object-Z is also investigated by Griffiths [Gri97, Gri98].

In [MG07], Moffat and Goldsmith examine compositional reasoning for CSP by identifying and showing several proof rules with respect to some CSP operators and certain structures of the overall system. Compositional reasoning for CSP is also analysed in [Moo90].

Compositional verification for integrated formal methods has extensively be researched in the context of CSP||B [ST02]. Amongst other works [ST04, ST05], Evans, Schneider and Treharne investigated how to decompose specifications into so-called chunks [STE05]. For Event-B, Butler [But09] described how to decompose specifications for independent refinement checks.

Assume-Guarantee Reasoning: Assume-guarantee reasoning was first introduced in [FP78, Jon83] and further developed in [Pnu84]. Several variants being applied in different domains, such as assumption-commitment for synchronous message 
passing [MC81] and rely-guarantee for shared-variable concurrency [Jon83], exist. All of them can be subsumed under the roof of the assume-guarantee paradigm.

The book $\left[\mathrm{dRHH}^{+} 01\right]$ gives a profound overview on compositional reasoning and the assume-guarantee paradigm in particular.

Automated Compositional Reasoning: Ever since the introduction of compositional reasoning, one of the major goals is to fully automate this verification process. The idea to automatically generate assumptions in the context of assume-guarantee reasoning was first proposed in [GPB02].

Learning assumptions for compositional reasoning was introduced in [CGP03] and initially with respect to the basic proof rule (B-AGR). The following paper [BGP03] extended the idea to symmetric proof rules, such as rule (P-AGR). Apart from these authors, several other articles investigate this particular field of research: [GP08] contains a selection of articles on learning techniques for automated assumeguarantee reasoning. Nam and Alur [AMN05, NA06, Nam07] investigate L*-based learning of assumption in the context of symbolic model checking. In the same context, the article [APR $\left.{ }^{+} 01\right]$ presents a SAT-based technique for lazy learning of assumptions. Several articles concentrate on the optimisation of the $\mathrm{L}^{*}$ algorithm to more effectively compute the assumptions [GGP07, GMF07, CS07, CS08].

Besides the application of learning in the area of model checking, the $\mathrm{L}^{*}$ algorithm is used in several other software verification domains. For instance, in [CCST05], the authors use assumption learning in the context of simulations. Alur et al. [AČMN05] tackle synthesis of interface specifications based on learning. In the context of black box checking, that is, verifying a software system without a model, $\mathrm{L}^{*}$ is used to learn an unknown system [GPY02]. 


\title{
Decomposition of a Specification
}

\author{
Contents

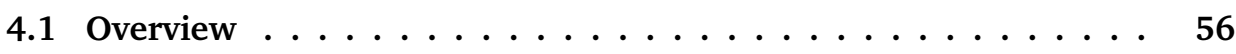 \\ 4.2 Cut of a Dependence Graph $\ldots \ldots \ldots \ldots \ldots$ \\ 4.2.1 Fragmentation of the Control Flow Graph . . . . . . . . 58 \\ 4.2.2 Correctness Criteria for the Fragmentation . . . . . . . . . 61

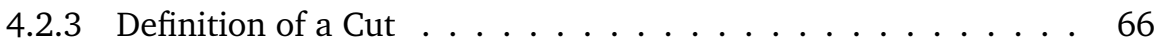 \\ 4.2.4 Candy Machine Revisited: Cut of the Dependence Graph . . . . 70 \\ 4.3 Decomposing CSP-OZ Specifications . . . . . . . . . . . . 72 \\ 4.3.1 Intermediate Definition of the Decomposition . . . . . . . 75 \\ 4.3.2 Preservation of the Data Dependences . . . . . . . . . . . 81 \\ 4.3.3 Preservation of the Control Flow . . . . . . . . . . . . 86 \\ 4.3.4 Renaming for the Decomposition . . . . . . . . . . . . 98 \\ 4.3.5 Definition of the Decomposition . . . . . . . . . . . . 100 \\ 4.3.6 Candy Machine Revisited: Decomposition . . . . . . . . . . 101 \\ 4.3.7 Improvement of the Decomposition . . . . . . . . . . . . . . . 103
}

4.4 Decomposition for the General Case: Number Swapper . . . . . 106

4.5 Related Work . . . . . . . . . . . . . . . . . . . . . . . . 109

As previously stated, we focus on decomposing specifications, allowing for an efficient application of compositional reasoning. To this end, we analyse a specification's dependence structure by means of its dependence graph, as defined in Chapter 2.

The following core chapter presents the correctness criteria and the definitions for the decomposition of CSP-OZ specifications. Before going into the technical details, we start by outlining our approach in Section 4.1. Section 4.2 defines and illustrates the fragmentation of a dependence graph, denoted as cut. The fragmentation is based on certain correctness criteria, resulting in the decomposition of the specification itself, as introduced in Section 4.3. A special case of the definition will be illustrated by means of the case study from Chapter 2. Additionally, Section 4.4 introduces a second, smaller case study, exemplifying the general case of a decomposition. In the final section, we discuss related work.

In order to facilitate an illustrative and fluent description of the approach, we postpone most of the correctness proofs to the next chapter. 


\subsection{Overview}

Compositional verification follows a "divide and conquer" approach: to cope with the state explosion problem, a local verification with respect to the components of a software model is applied.

However, as already stated in Section 3.2.2, two major obstacles complicate the application of compositional verification and particularly assume-guarantee reasoning.

First, the technique is only applicable if the overall model is composed of at least two components. If this is not the case, the model needs to be decomposed, without changing its observable behaviour.

Less evident, second, a decomposition itself does not always lead to an effective application of compositional verification. Disadvantageous decompositions may still cause large state spaces during model checking. We will deal with the aspect of classifying decompositions in Chapter 6.

In this chapter, we construct decompositions of specifications written in CSP-OZ, preserving the specification's semantics in the domain of the CSP traces model. As the dependence graph comprises the complete dependence structure of a specification $S$, our strategy primarily targets the distribution of the DG. Henceforward, $S$ itself is decomposed such that the resulting specification parts $S_{1}$ and $S_{2}$ correspond to the generated segments of the DG.

A distribution of the DG is accomplished on the level of its operation nodes. Correctness criteria refer to the control flow and thus to CSP operator nodes as well. In order to fragment the DG into two subgraphs, we define a set $\mathbf{C} \subseteq$ op $(N)$, which serves as the link between them. We will call this set a cut motivated by the intuition that it identifies the line(s) of intersection of the graph. The set of cut nodes is common to both subgraphs and, consecutively, to both specification parts. From the specification point of view, the cut serves as the interface, that is, the synchronisation alphabet, between the specification parts $S_{1}$ and $S_{2}$.

Figure 4.1 illustrates the individual steps of our approach.

Computation of the DG, (1): Given a specification $S$, we first compute its dependence graph $\mathrm{DG}_{\mathrm{S}}=\left(N, \longrightarrow_{\mathrm{DG}}\right)$, as introduced in Chapter 2 . We mainly focus our considerations on its set of operation nodes.

Identification of the Cut, (2): Next, we identify a cut of the dependence graph: a set of operation nodes, yielding a correct fragmentation of the DG (represented by grey nodes in the figure). In Section 4.2, we present the definition of a cut along with the correctness criteria for the segmentation.

Fragmentation of the DG, 3): Determining the set of cut nodes and distributing the set of operation nodes results in two subgraphs. The cut itself is represented in both subgraphs.

Decomposition of the Specification, (4): The fragmentation of the DG leads to the definition of the two specification parts of $S, S_{1}$ and $S_{2}$. Section 4.3 precisely defines 

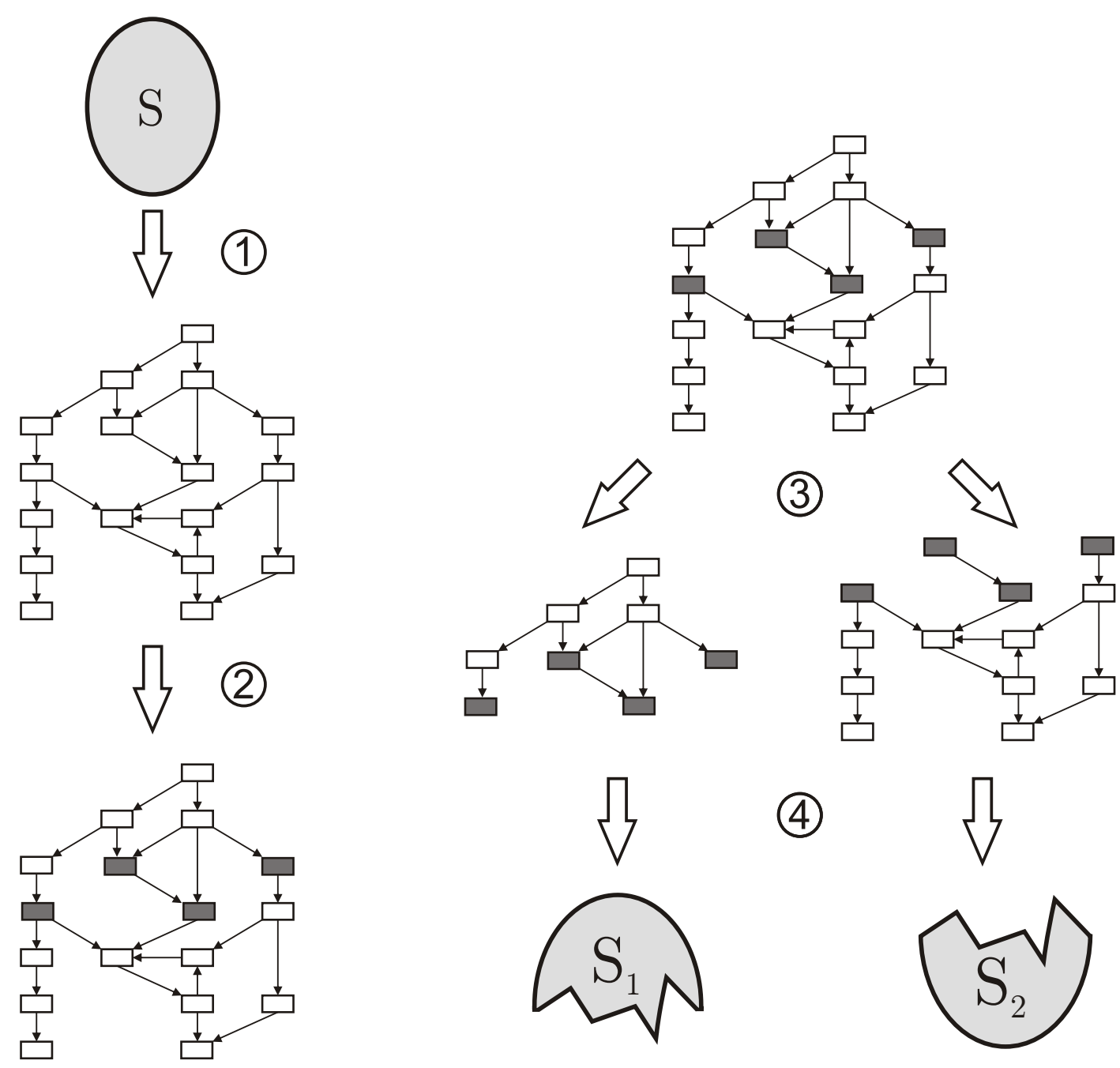

Figure 4.1: Cut identification, fragmentation of the dependence graph and decomposition of the specification

the decomposition and introduces the additional constructs required to ensure the (trace) equivalence of $S$ and $S_{1} \| S_{2}$.

Next, we introduce our definition of a cut along with the criteria which need to be satisfied such that the observable behaviour of the specification is preserved. We illustrate the definitions and criteria on several small examples and especially on our case study. 


\subsection{Cut of a Dependence Graph}

Before we introduce the definition of a valid cut of a dependence graph, we start with identifying its fragmentation with respect to two sets of operation nodes. Correctness criteria on the fragmentation consecutively lead to the definition of the cut. Since most of our definitions are not restricted to the dependence graph, we introduce them for arbitrary graphs and subsequently apply them in our specific context.

\subsubsection{Fragmentation of the Control Flow Graph}

We are interested in identifying two different subgraphs of the DG. In particular, these subgraphs should not arbitrarily intersect. Thus, we need to define different segments of the graph which are disjoint.

The control flow graph comprises all nodes of the dependence graph and defines the workflow and the dynamic behaviour of a specification. Therefore, we will define a fragmentation of the control flow graph alone instead of considering the dependence graph. Subsequently, the data flow needs to be evaluated to verify that a corresponding fragmentation of the DG is correct.

In general, the technique needs to deal with all different kinds of nodes and edges. However, the subsequent distribution of nodes refers to operation nodes, which is sufficient in our context: we do not distribute the set of CSP operator nodes. This will be achieved in Section 4.3, where we define a projection of a CSP process with respect to a set of events.

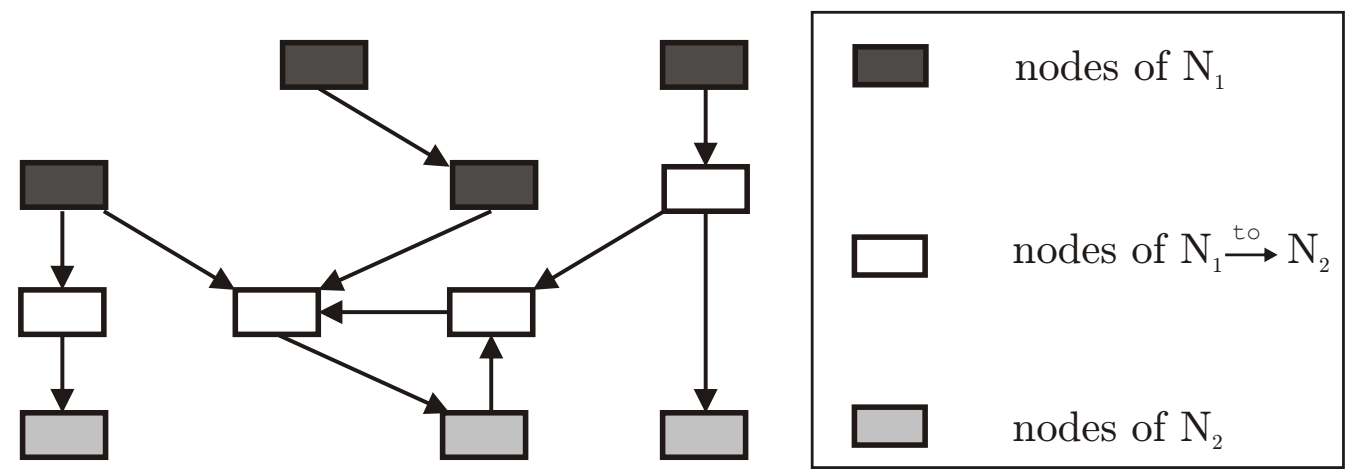

Figure 4.2: Illustration of Definition 4.2.1

First, the following definition determines all nodes reachable from one set of nodes $N_{1}$ not intersecting with another set of nodes $N_{2}$.

Definition 4.2.1. (Interval from $N_{1}$ to $N_{2}$ )

Let $\mathrm{G}=(N, \longrightarrow)$ be a graph, and let $N_{1}, N_{2} \subseteq N$. Then,

$$
N_{1} \stackrel{\text { to }}{\longrightarrow} N_{2}:=\left\{\mathrm{n}^{\prime} \in N \mid \exists \mathrm{n} \in N_{1}, \pi \in \operatorname{path}_{\mathrm{G}}\left(\mathrm{n}, \mathrm{n}^{\prime}\right) \bullet \pi \cap N_{2}=\varnothing\right\} \backslash N_{1} .
$$


The interval excludes both, $N_{1}$ and $N_{2}$, as illustrated in Figure 4.2. Intuitively, it can be regarded as the set of nodes "between" $N_{1}$ and $N_{2}$. Note that both, $N_{1}$ and $N_{2}$, are allowed to be empty.

The previous definition allows us to divide the set of nodes of a graph into several subsets (or phases, as we call them). Next, we introduce the fragmentation of the CFG, which is defined with respect to two sets of operation nodes, $\mathbf{C}_{1}$ and $\mathbf{C}_{2}$ :

Definition 4.2.2. (Fragmentation of the control flow graph)

Let $\mathrm{CFG}_{\mathrm{S}}=(N, \longrightarrow)$ be the control flow graph of a specification $S$, and let $\mathbf{C}_{1}, \mathbf{C}_{2} \subseteq \mathrm{op}(N)$. Moreover, let

$$
\text { StartNodes : }=\left\{\text { start. } P \mid P \in L^{C S P}\right\}
$$

and

$$
\text { start }_{1}:=\left(\{\text { start }\} \stackrel{\text { to }}{\longrightarrow} \mathbf{C}_{1}\right) \cap \text { StartNodes. }
$$

A fragmentation of (the set of operation nodes of) $\mathrm{CFG}_{S}$ with respect to a tuple $\left(\mathbf{C}_{1}, \mathbf{C}_{2}\right)$ is a set of three phases $\mathbf{P} \mathbf{h}_{1}, \mathbf{P h}_{2}$ and $\mathbf{P h}$ defined as

1.) $\mathbf{P h}_{1}:=\left(\left(\{\right.\right.$ start $\left.\left.\} \stackrel{\text { to }}{\longrightarrow} \mathbf{C}_{1}\right) \cap \mathrm{op}(N)\right) \cup\{$ init $\}$, (Phase 1)

2.) $\mathbf{P h}_{2}:=\left(\mathbf{C}_{1} \stackrel{\text { to }}{\longrightarrow} \mathbf{C}_{2}\right) \cap \circ \mathrm{p}(N)$, (Phase 2)

3.) $\mathbf{P h}_{3}:=\left(\mathbf{C}_{2} \stackrel{\text { to }}{\longrightarrow} \operatorname{start}_{1}\right) \cap \mathrm{op}(N)$. (Phase 3)

$\mathbf{C}_{1}$ and $\mathbf{C}_{2}$ serve as the two lines of intersection for the graph. The first phase $\mathbf{P h}_{1}$ contains all operation nodes before the first line of intersection. We add the unique init-node of the specification to $\mathbf{P h}_{1}$, comprising the set of initial predicates.

The second phase includes the set of operation nodes between both lines of intersection. Finally, the third phase comprises the set of operation nodes behind the second line of intersection. A first correctness criterion will exclude that any two of the five sets have a common element.

Intuitively, one would expect $\mathbf{P h}_{3}:=\left(\mathbf{C}_{2} \stackrel{\text { to }}{\longrightarrow} \varnothing\right) \cap \mathrm{op}(N)$. However, we need to "stop" adding nodes to $\mathbf{P h}_{3}$ after reaching a recursive call back to $\mathbf{P h}_{1}$. Otherwise, our subsequently defined correctness criteria on a fragmentation would rule out allowed recursive calls. Therefore, we define a set start ${ }_{1}$, comprising all nodes start. $X$ occurring before the first line of intersection. This specific point will become clearer in the next section.

In the general case of a cut, as introduced in Section 4.2.3, we use the previous definition as follows: we determine two sets of operation nodes, namely $\mathbf{C}_{1}$ and $\mathbf{C}_{2}$, which will from now be called the first cut and the second cut. The definition results in five disjoint sets of operation nodes $\mathbf{P h}_{1}, \mathbf{C}_{1}, \mathbf{P h}_{2}, \mathbf{C}_{2}$ and $\mathbf{P h}_{3}$. Henceforth, we will refer to $\mathbf{P h}_{1}, \mathbf{P h}_{2}$ and $\mathbf{P} \mathbf{h}_{3}$ as the phases of a fragmentation, whereas $\mathbf{C}_{1}$ and $\mathbf{C}_{2}$ will be referred to as its cut sets.

The following lemma states that a fragmentation of the CFG is always complete in the sense that no nodes are left out: 
Lemma 4.2.3. (Completeness of Fragmentation)

Let $\mathrm{CFG}_{\mathrm{S}}=(N, \longrightarrow)$ be the control flow graph of a specification $S$, and let $\left(\mathbf{C}_{1}, \mathbf{C}_{2}\right)$ be a fragmentation. Then,

$$
\mathbf{P h}_{1} \cup \mathbf{C}_{1} \cup \mathbf{P h}_{2} \cup \mathbf{C}_{2} \cup \mathbf{P h}_{3}=o p(N) .
$$

Proof. The left-to-right inclusion is obvious. For the opposite inclusion, let $n \in o p(N)$. Based on the definition, the special init-node is an element of $\mathbf{P h} \mathbf{h}_{1}$. Moreover, as any CFG node is reachable from the unique start-node, there exists $\pi \in \operatorname{path}_{\mathrm{CFG}}(\operatorname{start}, \mathrm{n})$. Without loss of generality let $\pi=\left\langle\right.$ start, $\left.\mathrm{n}_{1}, \ldots, \mathrm{n}_{k}\right\rangle$ and $\mathrm{n}_{k}=\mathrm{n}$.

If $\mathrm{n} \in \mathbf{P h}_{1}$, we immediately deduce the right-to-left inclusion. Otherwise, $\pi \cap \mathbf{C}_{1} \neq \varnothing$ holds. $\mathrm{n} \in \mathbf{C}_{1}$ would again conclude the proof. If $\mathrm{n} \notin \mathbf{C}_{1}$, there exists an index $1 \leq l_{1}<k$ such that $\mathrm{n}_{l_{1}} \in \mathbf{C}_{1}$. Since $\mathrm{n}$ is reachable from $\mathrm{n}_{l_{1}}$, either $\mathrm{n} \in \mathbf{P h}_{2}$ or, otherwise, there exists $\mathrm{n}_{l_{2}} \in \mathbf{C}_{2}$ for some $l_{1}<l_{2} \leq k$. If $l_{2}=k$, we have shown $\mathrm{n} \in \mathbf{C}_{2}$. In the opposite case, we deduce that $\mathrm{n}$ is reachable from $\mathbf{C}_{2}$ which either leads to $\mathrm{n} \in \mathbf{P h}_{3}$ or to $\pi \cap \operatorname{start}_{1} \neq \varnothing$ based on the definition of $\mathbf{P h}_{3}$. In this case, we infer that there exists some $l_{3}>l_{2}$ and $\mathrm{n}_{l_{3}} \in$ start $_{1}$. Here, $l_{3} \neq l_{2}$ since $l_{2} \in \mathrm{op}(N)$ and $l_{3} \in$ StartNodes. Hence, the path $\left\langle\right.$ start, $\left.\ldots, \mathrm{n}_{l_{3}}\right\rangle$ contains at least three different nodes $\mathrm{n}_{l_{1}}, \mathrm{n}_{l_{2}}$ and $\mathrm{n}_{l_{3}}$.

Reapplication of the previous ideas now starting in $n_{l_{3}}$ yields a sequence of nodes which continuously traverses the CFG through its five fragments. As the length of the sequence increases with every cycle, but never leaves the set $\mathbf{P h}_{1} \cup \mathbf{C}_{1} \cup \mathbf{P h}_{2} \cup \mathbf{C}_{2} \cup \mathbf{P h}_{3}$, it eventually reaches $n$, yielding the right-to-left inclusion.
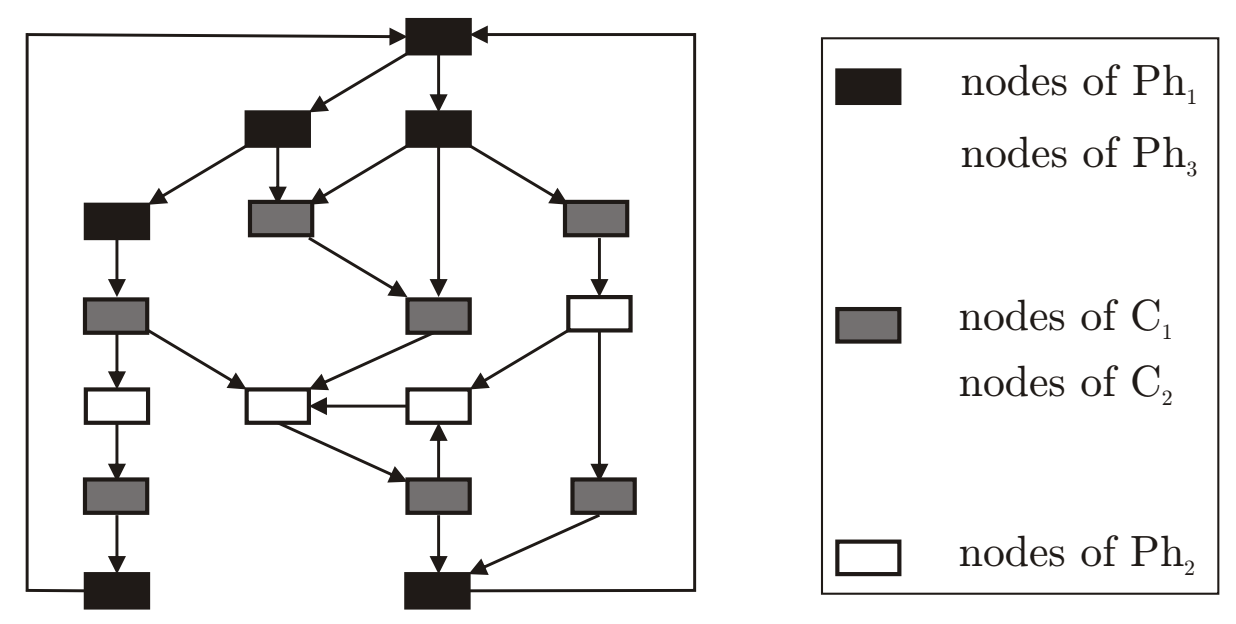

Figure 4.3: Fragmentation of the DG

Figure 4.3 illustrates the fragmentation. As already mentioned, we do not deal with nodes of $\operatorname{cf}(N)$ here. Thus, all boxes denote operation nodes. Besides, nodes of $\mathbf{P h} \mathbf{h}_{1}$ and $\mathbf{P h}_{3}$ have the same colour, since both segments will be assigned to the same component in Section 4.3. Hence, we will mostly not distinguish between $\mathbf{P h} \mathbf{h}_{1}$ and $\mathbf{P} \mathbf{h}_{3}$. 
Since the definition of the fragmentation cannot be arbitrary, we need to specify additional correctness constraints. These criteria coarsely describe the following aspects:

Criterion 1 - disjointness: All fragments are disjoint.

Criterion 2 - no crossing: The lines of intersection (cut sets) are not circumvented by data dependence edges.

Criterion 3 - no reaching back: Paths of the CFG have to comply to the ordering of the fragments.

Criterion 4 - all-or-none: The set of operation nodes corresponding to the same schema must not be distributed over different fragments.

We give a detailed definition of the correctness criteria next.

\subsubsection{Correctness Criteria for the Fragmentation}

In order to define a correct fragmentation of the DG and ultimately a correct decomposition of the specification, several correctness criteria need to be satisfied. If possible, a criterion will again be defined for arbitrary graphs.

Most of the criteria will rule out specific edges of the DG with respect to the fragmentation. We illustrate these edges by means of a recurrent figure. Recall that nodes of the cut sets and phases are always operation nodes, that is, elements of op $(N)$.

\section{Criterion 1: disjointness}

As a first and straightforward correctness criterion, we require that all segments resulting from the graph fragmentation are pairwise disjoint. Intuitively, this is motivated by the fact that we aim at a partitioning of the dependence graph. We recall the set theoretical definition for disjointness:

\section{Definition 4.2.4. (disjointness)}

Let $\mathrm{G}=(N, \longrightarrow)$ be a graph, and let $N_{1}, N_{2} \subseteq N$. Then, $N_{1}$ and $N_{2}$ satisfy disjointness, if, and only if, $N_{1}$ and $N_{2}$ are disjoint, that is, $N_{1} \cap N_{2}=\varnothing$.

The definition of a cut will comprise the condition that $\mathbf{P h}_{1}, \mathbf{C}_{1}, \mathbf{P h}_{2}, \mathbf{C}_{2}$ and $\mathbf{P} \mathbf{h}_{3}$ are pairwise disjoint. Based on the construction of the different phases, this constraint is particularly related to CFG paths, as it excludes several edges between the different segments. For instance, as $\mathbf{P} \mathbf{h}_{1}$ and $\mathbf{P} \mathbf{h}_{2}$ have to be disjoint, a direct edge from a node of $\mathbf{P h}$ to a node of $\mathbf{P h}$ is impossible: the definition of $\mathbf{P h}$ 解 yields that the target node would be an element of $\left(\mathbf{P h}_{1} \cap \mathbf{P \mathbf { h } _ { 2 }}\right)$.

Figure 4.4 illustrates that CFG edges with the source node in $\mathbf{P h}_{3}\left(\mathbf{P h}_{2}\right)$ and the target node in $\mathbf{P h}_{2}\left(\mathbf{P h}_{1}\right)$ are not allowed. Note that edges in the opposite direction are already ruled out by definition of the fragmentation. Further note that we intentionally allow edges connecting nodes of $\mathbf{P h}_{3}$ and $\mathbf{P h}$. This substantiates the definition of $\mathbf{P} \mathbf{h}_{3}$. 


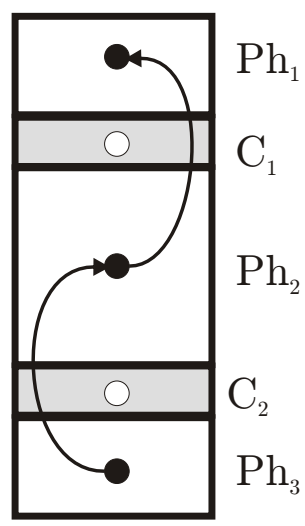

Figure 4.4: Disallowed control flow edges based on disjointness

\section{Criterion 2: no crossing}

The second correctness criterion tackles the previously described aspect of a cut identifying the lines of intersection of the dependence graph. Since it is generally impossible to decompose the graph into two completely independent (that is, unconnected) subgraphs, the cut needs to serve as the link between them. Intuitively, this link should not be evaded when switching from one subgraph to the other. Therefore, paths of the DG

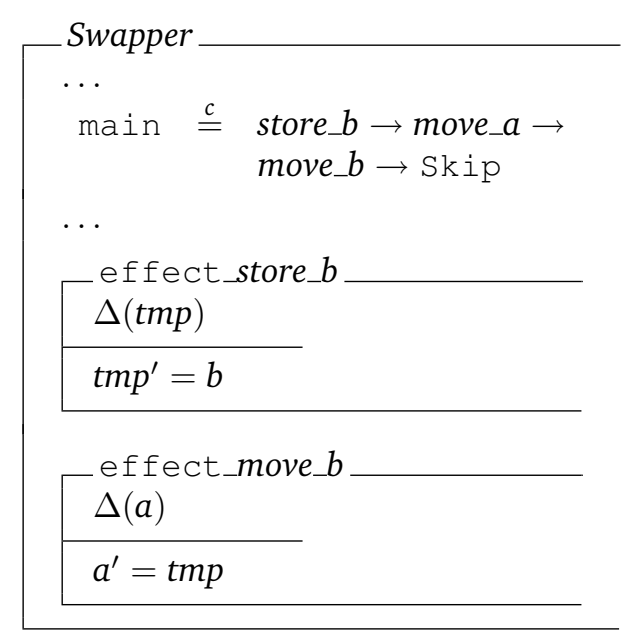

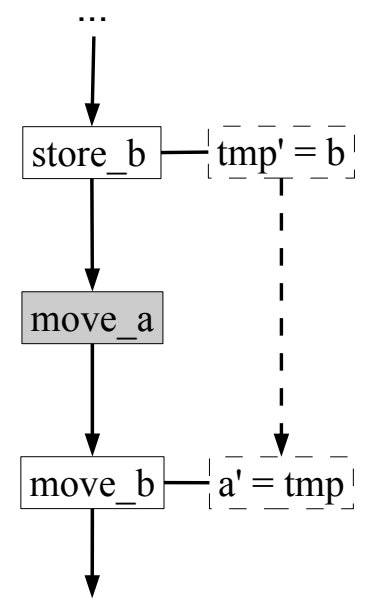

$\cdots$

Figure 4.5: Motivation for the correctness criterion no crossing

must not circumvent the cut. Based on our fragmentation of the CFG and the criterion disjointness, we implicitly ensure this for control flow edges. However, we also need to guarantee that data dependence edges do not evade the cut as well: on the level of 
the underlying specification, the set of operation schemas of the cut defines the interface between both resulting specification parts. If the behaviour of the specification parts depends on each other, these shared operations are responsible for preserving the mutual influence. This will be achieved by using them as transmitters for the correct values of modified state variables. If a data dependence circumvents the cut, it would be impossible to transmit the influence of one component on the other.

As an example, recall the small specification for a number swapper from Chapter 2 , Figure 2.8. The modification of $t m p$ within $s t o r e \_b$ and the reference to tmp within move_b yields a direct data dependence from the first to the latter operation node. Choosing the set $\{$ move_a \} as the set of cut nodes is not reasonable: the modified value of tmp cannot be transmitted. In this case, the data dependence edge circumvents the cut as illustrated in Figure 4.5.

In general, we have to disallow data dependence edges connecting the different fragments of the dependence graph if neither of the involved nodes is an element of the cut. These edges cross the cut in the sense that there exists a direct link between different sides of the cut. This motivates the following definition of a predicate called no crossing, which we will subsequently use with respect to $\left(\mathbf{P h}_{1} \cup \mathbf{P h}_{3}\right)$ and $\mathbf{P h}$ :

\section{Definition 4.2.5. (no crossing)}

Let $\mathrm{G}=(N, \longrightarrow)$ be a graph, and let $N_{1}, N_{2} \subseteq N$. Then, $\operatorname{noCr}\left(N_{1}, N_{2}, \mathrm{G}\right)$, if, and only if,

$$
\nexists \mathrm{n}_{1} \in N_{1} \nexists \mathrm{n}_{2} \in N_{2} \bullet \mathrm{n}_{1} \longrightarrow \mathrm{n}_{2} \vee \mathrm{n}_{2} \longrightarrow \mathrm{n}_{1}
$$

This condition will be called no crossing between $N_{1}$ and $N_{2}$.

For the definition of the cut, we require noCr $\left(\left(\mathbf{P h}_{1} \cup \mathbf{P h}_{3}\right), \mathbf{P h}_{2}, \mathrm{DDG}_{\mathrm{S}}\right)$. The disallowed data dependence edges are illustrated in Figure 4.6.

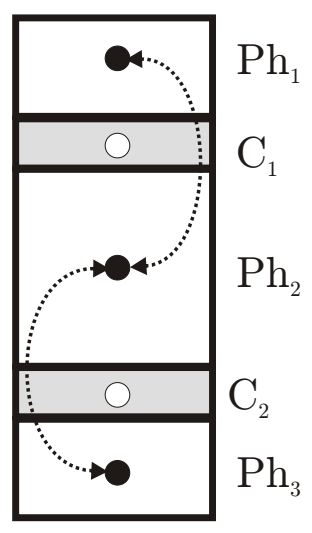

Figure 4.6: Disallowed data dependences based on no crossing 


\section{Criterion 3: no reaching back}

The next constraint needs to be defined with respect to the DG of a specification since here, we explicitly need to refer to operation nodes and CSP operator nodes.

First, we consider the control flow graph and its fragmentation: the two lines of intersection, namely $\mathbf{C}_{1}$ and $\mathbf{C}_{2}$, dissect the graph into several fragments. We require that paths of the control flow graph need to comply to the ordering of the segments as follows: any path of the CFG starts in start and either remains in $\mathbf{P h} \mathbf{h}_{1}$ or subsequently reaches $\mathbf{C}_{1}$. Consecutively, the path remains in the respective segment or advances to either $\mathbf{P h}$ or directly to $\mathbf{C}_{2}$. Next, the path may reach $\mathbf{P h}_{3} \cup \mathbf{P h}_{1}$ or immediately $\mathbf{C}_{1}$. Following up on this, all paths need to comply with the ordering $\mathbf{P h}_{1}, \mathbf{C}_{1}, \mathbf{P h}_{2}, \mathbf{C}_{2}, \mathbf{P h}_{3}$, possibly repeated. Phases are potentially skipped in between.

Thus, we generally allow the control flow to advance with respect to the ordering of the segments or to remain in a segment. However, a path must not directly return to a previous fragment.
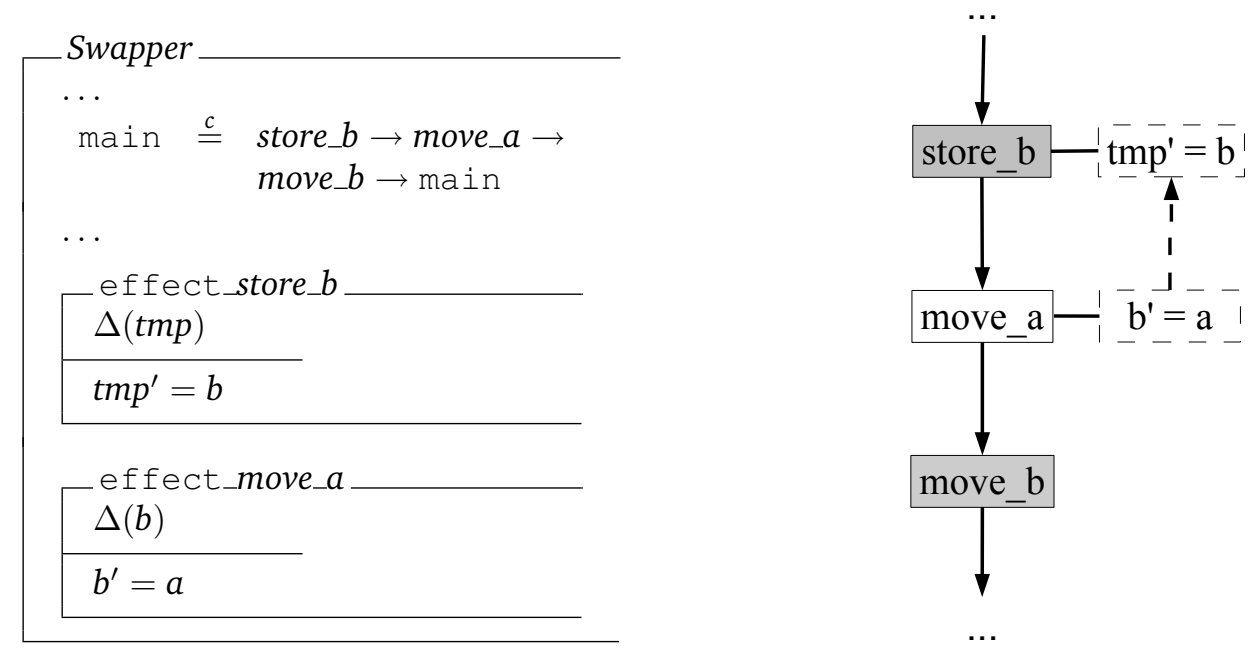

Figure 4.7: Motivation for the correctness criterion no reaching back

The application of the following criterion is two-folded: besides the fact that paths of the control flow graph should comply with the ordering of its segments, we also consider data dependences. For them, the motivation for this constraint is similar to no crossing, which already excludes a skipping of the cut sets. In addition, we need to exclude data dependences, returning to a previous segment.

Recall the example from Figure 2.8 with a small modification: we replace Skip with a recursive call of main. The modification of $b$ within move_a and the reference to $b$ within store $b$ yields a direct data dependence from the first to the latter operation node. Choosing the sets $\{$ store $b\}$ and $\left\{\right.$ move $\left.\_b\right\}$ as the sets of cut nodes is not reasonable: in this case, the data dependence edge reaches back to the first cut as illustrated in Figure 
4.7. The modified value of $b$ cannot be transmitted in between.

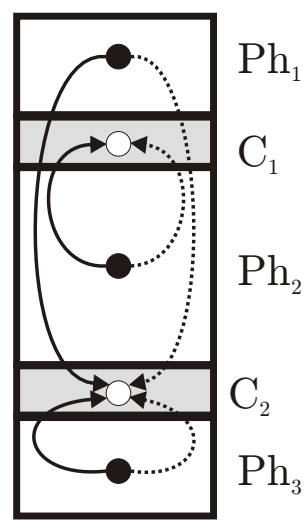

Figure 4.8: Disallowed edges based on no reaching back

It is sufficient to disallow edges reaching back to a cut segment: control flow edges reaching back from the cut to the previous phase are already excluded by definition of the fragmentation and the criterion disjointness. Moreover, corresponding data dependences do not need to be excluded. Figure 4.8 shows the additionally disallowed edges of the DG.

In order to formally express that a CFG path or a data dependence edge must not return to a previous segment, we define a predicate no reaching back which inputs two sets of operation nodes: the first set denotes the source nodes, the second set the target nodes. Data dependence edges must not connect the first to the latter set of nodes, the same needs to hold for control flow edges. As CFG paths from one operation node to another possibly comprise CSP operator nodes in between, we need to rule out those paths from the first to the latter set of nodes without operation nodes in between. Recall that

$$
\mathrm{n} \stackrel{\bullet}{\longrightarrow} \mathrm{n}^{\prime} \text {, if, and only if, }\left(\exists \pi \in \text { path }_{\mathrm{CFG}}\left(\mathrm{n}, \mathrm{n}^{\prime}\right) \bullet \pi \cap \mathrm{op}(N)=\left\{\mathrm{n}, \mathrm{n}^{\prime}\right\}\right) \text {. }
$$

\section{Definition 4.2.6. (no reaching back)}

Let $\mathrm{DG}_{\mathrm{S}}=\left(N, \longrightarrow_{\mathrm{DG}}\right)$ be the dependence graph of a specification $S$, and let $N_{1}, N_{2} \subseteq \mathrm{op}(N)$. Then, $\operatorname{noRB}\left(N_{1}, N_{2}, \mathrm{DG}_{\mathrm{S}}\right)$, if, and only if,

$$
\forall \mathrm{n}_{1} \in N_{1} \bullet\left(\nexists \mathrm{n}_{2} \in N_{2} \bullet \mathrm{n}_{1} \stackrel{\bullet}{\longrightarrow} \mathrm{n}_{2} \wedge \nexists \mathrm{n}_{2}^{\prime} \in N_{2} \bullet \mathrm{n}_{1---\rightarrow} \mathrm{n}_{2}^{\prime}\right)
$$

This condition will be called no reaching back from $N_{1}$ to $N_{2}$.

The definition will be instantiated as

$$
\operatorname{noRB}\left(\mathbf{P h}_{2}, \mathbf{C}_{1}, \mathrm{DG}_{\mathrm{S}}\right) \wedge \operatorname{noRB}\left(\left(\mathbf{P h}_{1} \cup \mathbf{P h}_{3}\right), \mathbf{C}_{2}, \mathrm{DG}_{\mathrm{S}}\right) .
$$




\section{Criterion 4: all-or-none}

The last correctness criterion restricts the distribution of the set of operation nodes of the DG. Definition 2.3.4 introduced a labelling function $l$, mapping an operation node on its schema name. In our decomposition, we have to require that for any operation schema $o p \in O p$, all corresponding nodes $\mathrm{op}^{i}$ are assigned to the same graph fragment.

Intuitively, this condition is necessary, since schemas corresponding to operation nodes occurring in the cut are generally modified. For the different cut sets $\mathbf{C}_{1}$ and $\mathbf{C}_{2}$, this modification can differ. Moreover, schemas occurring outside of the cut remain unchanged. A distribution of $\left\{\mathrm{op}^{i} \in \mathrm{op}(N) \mid l\left(\mathrm{op}^{i}\right)=o p\right\}$ over at least two different segments would require a duplication of the schema which is undesirable and technically infeasible.

The following predicate defines this all-or-none law - it will subsequently be used with respect to the cut sets $\mathbf{C}_{1}, \mathbf{C}_{2}$ and the complement of $\mathbf{C}_{1} \cup \mathbf{C}_{2}$ :

\section{Definition 4.2.7. (all-or-none)}

Let $\mathrm{G}=(N, \longrightarrow)$ be a graph, and let $N_{1}, N_{2} \subseteq N$. Then, $\mathrm{AoN}\left(N_{1}, N_{2}, \mathrm{G}\right)$, if, and only if,

$$
N_{1} \subseteq N_{2} \vee N_{1} \subseteq\left(N \backslash N_{2}\right)
$$

This condition will be called all-or-none law for $N_{1}$ relative to $N_{2}$.

This completes the definition of the correctness criteria. They will consecutively be used to define a cut, that is, a correct fragmentation of the DG, and subsequently the decomposition of a specification.

\subsubsection{Definition of a Cut}

The previously introduced correctness criteria along with Definition 4.2.2 immediately lead to the first of two core definitions of this thesis, the definition of a cut: ${ }^{1}$

Definition 4.2.8. ([General] Cut of the $D G$ )

Let $\mathrm{DG}_{\mathrm{S}}=\left(N, \longrightarrow{ }_{\mathrm{DG}}\right)$ be the dependence graph and $\mathrm{CFG}_{\mathrm{S}}=(N, \longrightarrow)$ the control flow graph of a specification $S$, respectively. A fragmentation $\mathbf{C}=\left(\mathbf{C}_{1}, \mathbf{C}_{2}\right)$ of the $C F G$ according to Definition 4.2.2 is called a (valid) cut of the DG, if, and only if, the following correctness criteria are satisfied:

Criterion 1 (disjointness): The following five sets are pairwise disjoint:

- $\mathbf{P h}_{1}, \mathbf{P h}_{2}, \mathbf{P h}_{3}$,

(phases)

- $\mathrm{C}_{1}, \mathrm{C}_{2}$.

(cut sets)

\section{Criterion 2 (no crossing):}

$$
\operatorname{noCr}\left(\left(\mathbf{P h}_{1} \cup \mathbf{P h}_{3}\right), \mathbf{P h}_{2}, \mathrm{DDG} \text { ), } \quad\right. \text { (no crossing between different components) }
$$

\footnotetext{
${ }^{1}$ In the following definition, allowing a cut set to be empty does not pose a problem: if $\mathbf{C}_{1}=\varnothing$, the fragmentation either yields a trivial decomposition or a contradiction to the criterion disjointness. $\mathbf{C}_{2}=\varnothing$ will subsequently be identified as a special case of the cut definition.
} 
Criterion 3 (no reaching back):

- $\operatorname{noRB}\left(\mathbf{P h}_{2}, \mathbf{C}_{1}, \mathrm{DG}_{\mathrm{S}}\right)$ and

- $\operatorname{noRB}\left(\left(\mathbf{P h}_{1} \cup \mathbf{P h}_{3}\right), \mathbf{C}_{2}, \mathrm{DG}_{\mathrm{S}}\right)$, (no reaching back to first cut set) (no reaching back to second cut set)

Criterion 4 (all-or-none): For all operation nodes op $\in$ Op:

- $\operatorname{AoN}\left(l^{-1}[\{o p\}], \mathbf{C}_{1}, \mathrm{DG}_{\mathrm{S}}\right)$ and

- $\operatorname{AoN}\left(l^{-1}[\{o p\}], \mathrm{C}_{2}, \mathrm{DG}_{\mathrm{S}}\right)$. (no cut-distribution of nodes associated to one operation)

We ultimately aim at the definition of two specification parts $S_{1}$ and $S_{2}$, resulting from the decomposition of the dependence graph of $S$. The previous definition of a cut identifies a fragmentation of the set of operation nodes of the dependence graph in the following way: the unification of $\mathbf{C}_{1}$ and $\mathbf{C}_{2}$ together with $\mathbf{P} \mathbf{h}_{3}$ and $\mathbf{P h} \mathbf{h}_{1}$ yields the set of operations of the first component $S_{1}$. Accordingly, $\mathbf{C}_{1}$ and $\mathbf{C}_{2}$ together with $\mathbf{P h}_{2}$ constitute the second component $S_{2}$. This is illustrated in Figure 4.9. Operations corresponding to the first cut set identify the link from $S_{1}$ to $S_{2}$, whereas the second cut set determines the opposite link. The precise definition of $S_{1}$ and $S_{2}$ will be given in Section 4.3.

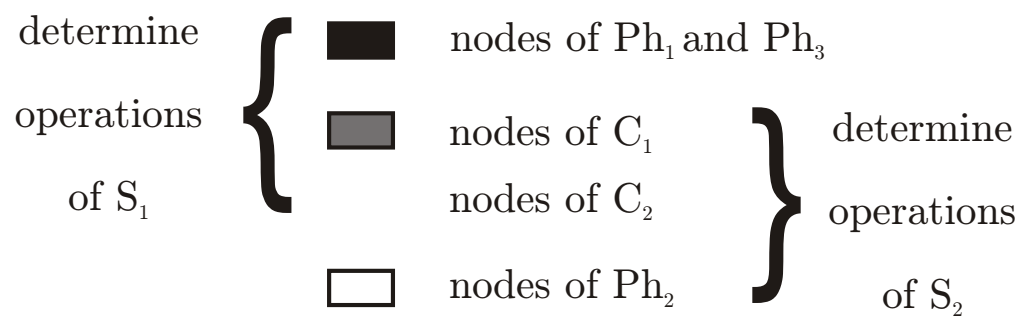

Figure 4.9: Fragmentation of the set of operation nodes in general case

In order to establish a well-defined fragmentation of the original dependence graph and thus well-defined specification components, CSP operator nodes need to be considered as well. In Section 4.3, we will determine the CSP parts of the components $S_{1}$ and $S_{2}$, resulting from a projection of the CSP part of $S$ onto the specific sets of operation schemas. This definition will provide a correct distribution of the CSP operators and thus, operator nodes of the DG.

Figure 4.10 shows all allowed edges of the DG. Dotted edges depict data dependences, whereas solid edges represent a unification of both, control flow edges and data dependences.

As we introduced $\mathbf{C}_{1}$ as the first line of intersection and $\mathbf{C}_{2}$ as the second, we need to substantiate that $\mathbf{C}_{2}$ is located behind $\mathbf{C}_{1}$. The following lemma shows that our definition indeed matches with the intuition. It states that there are no direct CFG paths from the second cut to the first cut - any such path needs to proceed over $\mathbf{P h}_{1}$ via a recursive call. Recall that

$$
\text { start }_{1}:=\left(\{\text { start }\} \stackrel{\text { to }}{\longrightarrow} \mathbf{C}_{1}\right) \cap \text { StartNodes. }
$$




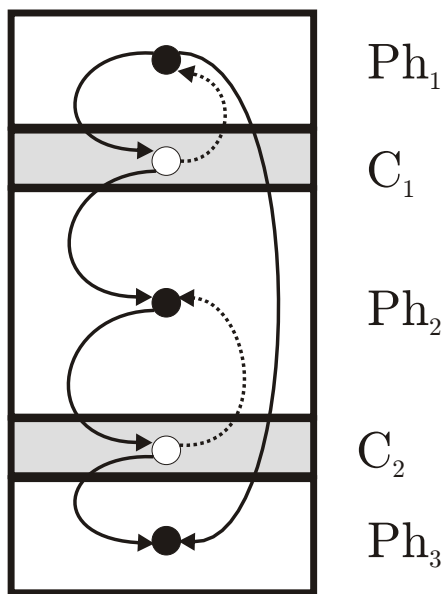

Figure 4.10: Assignment of DG edges to the subgraphs

Lemma 4.2.9. (No direct CFG paths from second to first cut)

Let $\mathrm{DG}_{\mathrm{S}}=\left(N, \longrightarrow_{\mathrm{DG}}\right)$ be the $D G$ of a specification $S$ and let $\left(\mathbf{C}_{1}, \mathbf{C}_{2}\right)$ be a cut of the DG. Then, the following holds:

$$
\forall c_{1} \in \mathbf{C}_{1}, c_{2} \in \mathbf{C}_{2} \bullet\left(\pi \in \operatorname{path}_{\mathrm{CFG}}\left(c_{2}, c_{1}\right) \Rightarrow \pi \cap \operatorname{start}_{1} \neq \varnothing\right) .
$$

Proof. Assume the opposite: let $\pi \in \operatorname{path}_{\mathrm{CFG}}\left(c_{2}, c_{1}\right)$ with $c_{2} \stackrel{\pi}{\longrightarrow} c_{1}$ and $\pi \cap \operatorname{start}_{1}=\varnothing$. In this case, by definition of $\mathbf{P h}_{3}$, the node $c_{1} \in\left(\mathbf{P h}_{3} \cap \mathbf{C}_{1}\right)$ violates Definition 4.2.8, correctness criterion disjointness.

Since the CFG of a specification may include recursive calls, yielding paths from $\mathbf{P h}_{2}$ back to $\mathbf{P h}_{1}$, we generally need to identify two lines of intersection. The first subgraph thus contains nodes located before the first cut $\left(\mathbf{P h}_{1}\right)$ as well as nodes located behind the second cut $\left(\mathbf{P h}_{3}\right)$. We will now additionally consider a special case of the segmentation, which corresponds to the definitions of [MWW08].

Assume that the dependence graph of a specification can be fragmented in such a way that there are no paths from $\mathbf{P h}_{2}$ back to $\mathbf{P h}_{1}$. Intuitively, this means that recursion can only occur within the same phase. In particular, such a DG does not incorporate "outer" recursive calls in the sense that a path reaching $\mathbf{P h}_{2}$ never returns to the start-node.

In this case, the dependence graph can reasonably be segmented into two subgraphs without the need for a second line of intersection: the first subgraph contains the nodes before the sole line of intersection and the second subgraph the nodes behind it, whereas both subgraphs include the cut set.

In this specific case, we call the dependence graph sequential based on the possibility to fragment it without outer recursion. The now simplified fragmentation is illustrated in Figure 4.11. This leads to the following definition:

Definition 4.2.10. (Single Cut)

Let $\mathbf{C}=\left(\mathbf{C}_{1}, \mathbf{C}_{2}\right)$ be a cut. We call $\mathbf{C}$ a single cut, if, and only if, $\mathbf{C}_{2}=\varnothing$. 


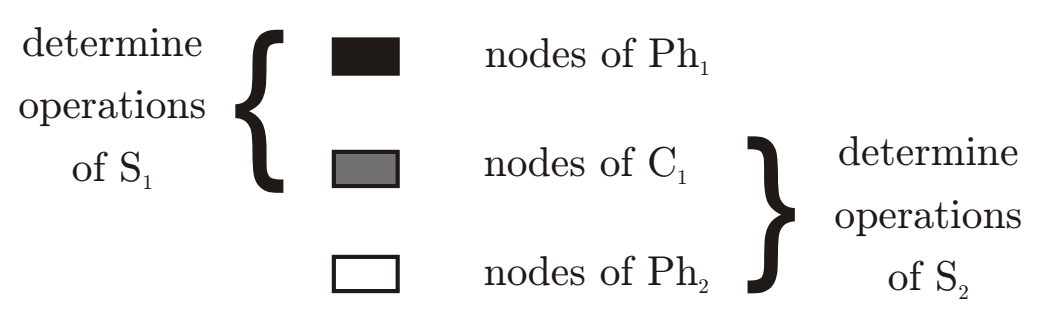

Figure 4.11: Fragmentation of the set of operation nodes in the special case

In the case of a single cut, we synonymously write $\mathbf{C}$ and $\mathbf{C}_{1}$. The restriction $\mathbf{C}_{2}=\varnothing$ incorporates several repercussions. First of all, the fragmentation yields $\mathbf{P h}_{2}=\left(\mathbf{C}_{1} \stackrel{\text { to }}{\rightarrow}\right.$ $\varnothing) \cap \operatorname{op}(N)$ from which we can deduce that no CFG paths from $\mathbf{P h}_{2}$ back to $\mathbf{C}_{1}$ are allowed at all. Moreover, no paths from $\mathbf{P h}$ to $\mathbf{P h}$ can exist. Finally, $\mathbf{P h}_{3}=\varnothing$ holds. We will summarise and proof these claims in the following lemma:

Lemma 4.2.11. (Properties of single cut)

Let $\mathbf{C}$ be a single cut. Then, the following holds:

1. $\mathbf{P h}_{2}=\left(\mathbf{C}_{1} \stackrel{\text { to }}{\longrightarrow} \varnothing\right) \cap \mathrm{op}(N)$,

2. $\forall \mathrm{n} \in \mathbf{P h}_{2}, \mathrm{n}^{\prime} \in \mathbf{C}_{1} \bullet \operatorname{path}_{\mathrm{CFG}}\left(\mathrm{n}, \mathrm{n}^{\prime}\right)=\varnothing$,

3. $\forall \mathrm{n} \in \mathbf{P h}_{2}, \mathrm{n}^{\prime} \in \mathbf{P h}_{1} \bullet \operatorname{path}_{\mathrm{CFG}}\left(\mathrm{n}, \mathrm{n}^{\prime}\right)=\varnothing$,

4. $\mathrm{Ph}_{3}=\varnothing$,

Proof:

1. Obvious. $\checkmark$

2. Assume that there exist $\mathrm{n} \in \mathbf{P h}_{2}, \mathrm{n}^{\prime} \in \mathbf{C}_{1}$ such that $\pi \in \operatorname{path}_{\mathrm{CFG}}\left(\mathrm{n}, \mathrm{n}^{\prime}\right)$. We distinguish two cases for $\pi$ : if $\pi \cap \mathbf{P h}_{1}=\varnothing$, there exist some nodes $I \in \mathbf{P h}_{2}, \mathbf{m} \in \mathbf{C}_{1}$ of $\pi$ such that $\mathrm{I} \stackrel{\bullet}{\longrightarrow} \mathrm{m}$. This yields a contradiction to the correctness criterion no reaching back. Otherwise, let $\mathrm{m}$ be the first node of $\pi$ which is an element of $\mathbf{P h}_{1}$. Then, $\pi$ either reaches $\mathrm{m}$ via some direct edge from $\mathbf{P h}_{2}$, violating the correctness criterion disjointness ( $\mathrm{m} \in \mathbf{P h}_{2} \cap \mathbf{P h _ { 1 }}$ ). Otherwise, there is an indirect connection via $\mathbf{C}_{1}$, which again violates no reaching back at some point within $\pi$. $\checkmark$

3. Now assume there exist some $n \in \mathbf{P h}_{2}, \mathrm{n}^{\prime} \in \mathbf{P h}_{1}$ such that $\pi \in \operatorname{path}_{\mathrm{CFG}}\left(\mathrm{n}, \mathrm{n}^{\prime}\right)$. According to the previous case, second part, this path violates one of the correctness criteria disjointness and no reaching back.

4. Since, in particular, $\varnothing \stackrel{\text { to }}{\rightarrow} M=\varnothing$ for any set $M$, we immediately deduce the equation. $\checkmark$

Table 4.1 summarises the differences between a general cut and a single cut. Based on our case study from Chapter 2, we consecutively illustrate the definition for the special case of a single cut. 


\begin{tabular}{|l|l|l|}
\hline & General Cut & Single Cut \\
\hline \hline Number of Cut Sets & two & one \\
\hline disjointness & $\begin{array}{l}\mathbf{P h}_{1}, \mathbf{C}_{1}, \mathbf{P h}_{2}, \mathbf{C}_{2}, \mathbf{P h}_{3} \\
\text { are pairwise disjoint }\end{array}$ & $\begin{array}{l}\mathbf{P h}_{1}, \mathbf{C}_{1}, \mathbf{P h}_{2} \\
\text { are pairwise disjoint }\end{array}$ \\
\hline First Subgraph & $\begin{array}{l}\text { comprises } \\
\mathbf{P h}_{1}, \mathbf{C}_{1}, \mathbf{C}_{2} \text { and } \mathbf{P h}\end{array}$ & $\begin{array}{l}\text { comprises } \\
\mathbf{P h}_{1} \text { and } \mathbf{C}_{1}\end{array}$ \\
\hline Second Subgraph & $\begin{array}{l}\text { comprises } \\
\mathbf{C}_{1}, \mathbf{P} \mathbf{h}_{2} \text { and } \mathbf{C}_{2}\end{array}$ & $\begin{array}{l}\text { comprises } \\
\mathbf{C}_{1} \text { and } \mathbf{P h}\end{array}$ \\
\hline Allowed Recursion & $\begin{array}{l}\text { within one segment, } \\
\text { between } \mathbf{P h}_{3} \text { and } \mathbf{P h}\end{array}$ & within one segment \\
\hline
\end{tabular}

Table 4.1: Comparison between the general cut and the single cut

\subsubsection{Candy Machine Revisited: Cut of the Dependence Graph}

Chapter 2 introduced the specification CandyMachine. We illustrate the previous definitions of a fragmentation and a cut by means of this particular example. The example complies to the general restrictions for a single cut and thus allows a demonstration of the special case. Section 4.4 additionally illustrates the general case.

Here, we will neglect three specific data dependences, namely the three initial data dependences originating from the Init predicate items $=\langle\rangle$ to the respective operation nodes order, term and deliver. The reason why we are allowed to do this will precisely be given in Section 4.3.7, where we will deal with the neglect of specific initial data dependences. Intuitively, these dependences originate from a predicate restricting a variable which is never modified or referenced in any of the schemas pay, payout, abort and switch. We will show that the source of this dependence can safely be moved to the second subgraph.

We start the illustration of the cut definition with the fragmentation of the CFG, according to Definition 4.2.2. Figure 2.9 from Section 2.3.2 depicts the control flow graph of the candy machine. Let $\mathbf{C}_{1}:=\{$ switch $\}$ and, according to the definition of a single cut, $\mathrm{C}_{2}:=\varnothing$. This leads to the following fragmentation:

$$
\begin{aligned}
& \mathbf{P h}_{1}=\{\text { pay, payout, abort }\} \\
& \mathbf{C}_{1}=\{\text { switch }\} \text { and } \\
& \mathbf{P h}_{2}=\{\text { select, order, term, deliver }\}
\end{aligned}
$$

For showing that this fragmentation satisfies the constraints of Definition 4.2.8, recall 
the DG of the candy machine specification as given in Figure 2.13, and consider the four correctness criteria for the decomposition:

Criterion $\mathbf{1}$ (disjointness): $\mathbf{P h} \mathbf{h}_{1}, \mathbf{C}_{1}$ and $\mathbf{P h}_{2}$ are disjoint. In particular, this is due to the non-existent recursive calls from $\mathbf{P h}_{2}$ to $\mathbf{P h}_{1}$.

Criterion 2 (no crossing): In case we neglect the previously identified initial data dependences, noCr $\left(\mathbf{P h}_{1}, \mathbf{P h}_{2}, \mathrm{DDG}_{\mathrm{S}}\right)$ holds. No data dependences connect a node of $\mathbf{P h}_{1}$ and $\mathbf{P h}_{2}$.

Criterion 3 (no reaching back): $\operatorname{noRB}\left(\mathbf{P h}_{2}, \mathbf{C}_{1}, \mathrm{DG}_{\mathrm{S}}\right)$ holds as well. There are no CFG paths or data dependences originating from $\mathbf{P h}_{2}$ targeting $\{$ switch $\}$.

Criterion 4 (all-or-none): Obvious, since there are no multiple occurrences of an operation within the CSP part of CandyMachine.

The fragmentation based on $\mathbf{C}_{1}=$ \{switch $\}$ thus yields a valid (single) cut. This is illustrated in Figure 4.12. The left hand side depicts the first subgraph, and the right hand side displays the second subgraph. Note that for the reduced parts of the graphs, we applied a simplification on the sets of CSP operators and control flow edges. The precise definition of the modified CSP part is given in the next section.
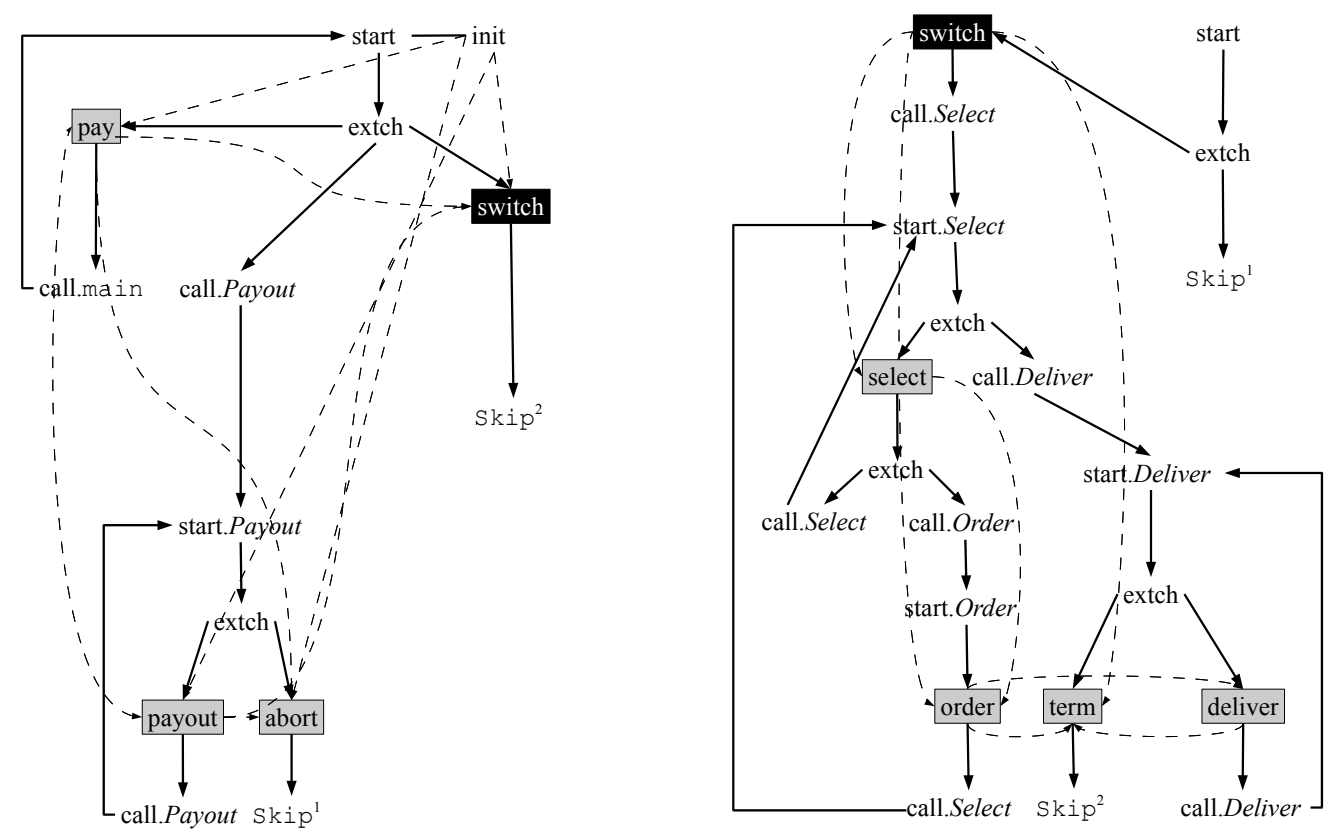

Figure 4.12: Cut of the dependence graph for the candy machine

This concludes the illustration of a (single) cut. So far, we considered the dependence graph of a specification which represents its dependence structure. We defined the cut of 
the DG, separating it into two parts. Next, we need to transfer the fragmentation of the graph $\mathrm{DG}_{\mathrm{S}}$ to the decomposition of the specification $S$.

\subsection{Decomposing CSP-OZ Specifications}

A cut of the dependence graph of a specification $S$ as defined in the previous section determines a fragmentation of the DG, resulting in several clusters of nodes. This segmentation serves as the cornerstone for the identification of two specifications $S_{1}$ and $S_{2}$, representing a correspondent decomposition of $S$.

\begin{tabular}{|lr}
$S$ & \\
$I$ & \\
main & [interface definition] \\
State & [CSP part] \\
Init & [Object-Z part: state schema] \\
enable_op & [Object-Z part: initial state schema] \\
effect_op & [Object-Z part: enable-schemas] \\
\end{tabular}

Figure 4.13: Constituents of a CSP-OZ class specification

In this section, we transfer the previous definitions from the graph level to the specification level. Again, we do not distinguish between specifications consisting of one and several classes. The decomposition of a specification is defined with respect to the fragmentation of the DG and is thus independent of the class structure. Therefore, throughout this thesis, we will synonymously refer to class and specification.

Recall the structure of a CSP-OZ class specification as given in Figure 4.13. At first, we have to identify the different constituents of $S_{1}$ and $S_{2}$, namely its interface definition, its CSP part and its Object-Z part. Subsequently, we assemble both specifications by identifying a synchronisation alphabet $A$, employed for the definition of $S_{1} \|_{A} S_{2}$. The construction has to make sure that $S$ and $S_{1} \|_{A} S_{2}$ have the same observable behaviour.

A first fingerpost for the definition of $S_{1}$ and $S_{2}$ is directly given by the fragmentation of the DG: the sets of operation nodes corresponding to $\mathbf{C}_{1}$ and $\mathbf{C}_{2}$ take the role of connecting the different specification parts where $\mathbf{C}_{1}$ is responsible for preserving the influence of $S_{1}$ on $S_{2}$ whereas $\mathbf{C}_{2}$ identifies the opposite link. Additionally, the cut is the basis for the definition of the synchronisation alphabet $A$. Moreover, nodes of $\mathbf{P h} \mathbf{h}_{1}, \mathbf{P h}_{2}$ and $\mathbf{P h}_{3}$ represent the operations local to $S_{1}\left(\mathbf{P h}_{1}, \mathbf{P h}_{3}\right)$ and $S_{2}\left(\mathbf{P h}_{2}\right)$. This is illustrated in Figure 4.14.

In order to construct two well-defined specifications $S_{i}, i \in\{1,2\}$, we start with a first, intermediate definition of a decomposition in Section 4.3.1, where we need to deal with the following subtasks:

Definition of the Interfaces of $S_{i}$ : Identifying the set of operations of a component, the 

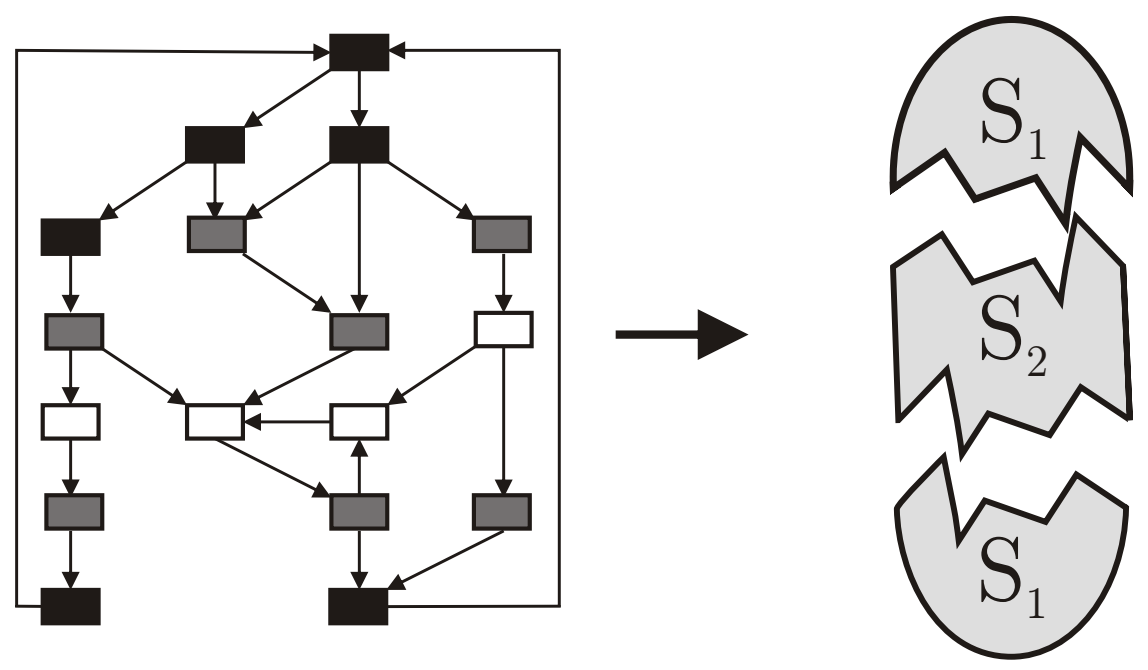

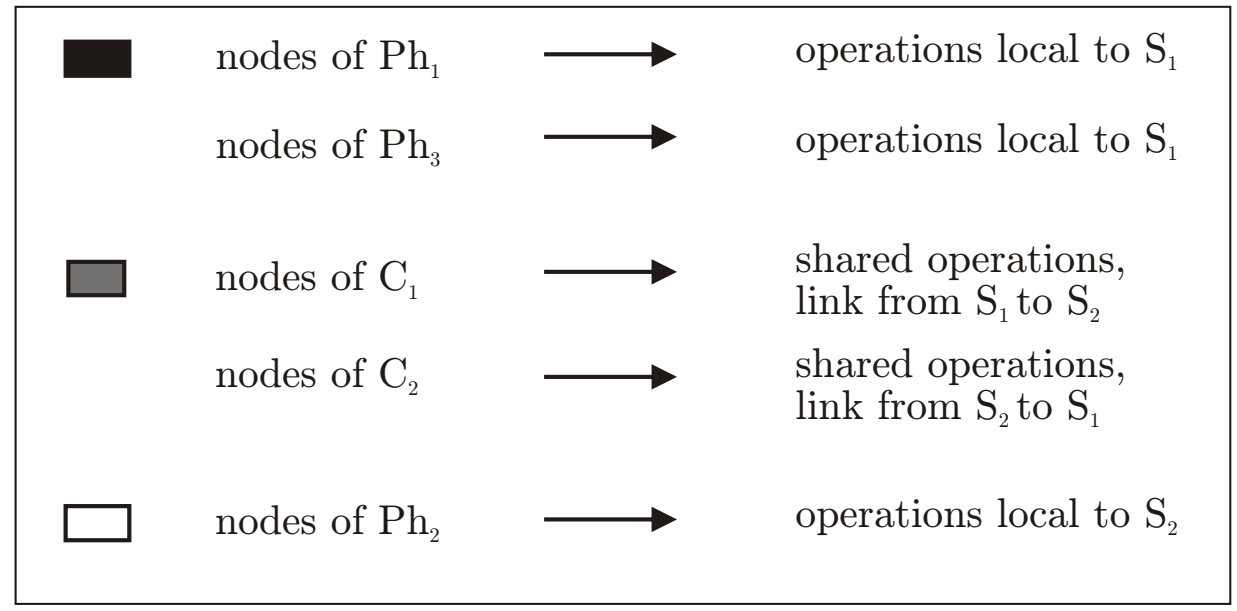

Figure 4.14: Correspondence between graph nodes and specification operations

fragmentation of the dependence graph immediately yields the set of channel declarations of $S_{i}$.

Definition of the CSP Parts $S_{i}$-main: According to its interface, the CSP part of $S_{i}$ needs to be restricted to the component's set of channels. For that purpose, we define a projection of the original CSP part on the remaining operations of a component, according to [Brü08].

Definition of the State Schemas of $S_{i}$ : One of the decisive aspects for an effective application of compositional reasoning is the size of the state space of the involved components. As the set $V$ of state variables of a specification's Object-Z part determines the size of the Object-Z state space, the sets $S_{1} . V$ and $S_{2} . V$ necessarily need 
to be smaller than S.V. Hence, we need to identify two subsets of $S . V$, forming the sets of required state variables for the specification parts. Additionally, we need to deal with the state invariants of the state schema.

Definition of the Initial State Schemas $S_{i}$. Init: Following up on the restriction of the sets of state variables, we accordingly need to restrict the original initial state schema. Moreover, an optimisation for this definition, as already indicated in the last section, will be given in Section 4.3.7.

Definition of the Operation Schemas for $S_{i}$ : According to the definition of the set of channels, we use the fragmentation of the dependence graph in order to identify the sets of operation schemas of a component. The determination of their respective declaration parts and predicate parts is straightforward.

Definition of the Synchronisation Alphabet: The definition of both specification parts leads to the overall system $S_{1} \|_{A} S_{2}$. The assembly requires a definition of the synchronisation alphabet $A$.

Carrying out the previous considerations will result in two well-defined specifications $S_{1}$ and $S_{2}$ and an assembly of $S_{1}$ and $S_{2}$ into $S_{1} \| S_{2}$. However, the pure definition of two specification parts, resulting from a cut, is insufficient. Additionally, we need to preserve the behaviour of the specification. To this end, we have to modify part of the generated components, mainly by adding parameters to some operations:

Preservation of Data Dependences: Even though we do not allow data dependences to circumvent the cut based on the correctness criterion no crossing, we still have to transmit the allowed influence of one on the other specification part. Data dependences may indeed target the set of cut operation nodes as well as originate from them. From a specification level, this means that modifications of state variables within one component influence state variables of the other component. In order to preserve these dependences, we introduce additional transmission parameters, passing the relevant state variable modifications of one to the other component. Section 4.3.2 deals with this aspect.

Preservation of CSP Part: The definition of the CSP parts for $S_{1}$ and $S_{2}$ based on a projection does not automatically yield an equivalence of the original CSP part and the CSP part of $S_{1} \| S_{2}$. In particular, the synchronisation of both CSP processes may introduce additional sequences of events which are infeasible for the original specification. For ensuring the equivalence of both, the CSP parts of $S$ and $S_{1} \| S_{2}$, we introduce additional address parameters, ensuring a correct synchronisation of both resulting CSP processes in Section 4.3.3.

Renaming of Events: Based on the introduction of additional parameters to some of the specification's channels, $S$ and $S_{1} \| S_{2}$ are solely equivalent modulo different channel types. In Section 4.3.4, we introduce an event renaming relation, linking the modified to the original channels. 
These are the crucial aspects which we will deal with in the upcoming sections. We proceed in two steps: first, in Section 4.3.1, we introduce a decomposition of $S$ with respect to a cut into two well-defined specification parts $S_{1}$ and $S_{2}$. Subsequently, we modify the decomposition to achieve a thorough decomposition by modifying part of the components elements. The complete definition of the thorough decomposition of $S$ into $S_{1}$ and $S_{2}$ will be given in Section 4.3.5, incorporating all the definitions and considerations of the previous sections. After illustrating the approach on our candy machine specification in Section 4.3.6, Section 4.3.7 gives an improvement for the decomposition by pointing out an optimisation for dealing with initial state predicates.

\subsubsection{Intermediate Definition of the Decomposition}

The current section stepwise introduces the different constituents of two specifications $S_{1}$ and $S_{2}$, resulting from a valid cut of $\mathrm{DG}_{\mathrm{S}}$. As of now, we are interested in developing a welldefined decomposition. Some of the subsequent definitions are marked as intermediate, as the corresponding specification elements will later by modified to ensure a semanticspreserving decomposition.

We start the definition of the components $S_{1}$ and $S_{2}$ by identifying their respective interfaces and CSP parts. In order to bridge the gap between the set of operation nodes, resulting from a cut and the corresponding set of operations, we use Definition 2.3.4:

Definition 4.3.1. (Sets of operations of components)

Let $\mathrm{DG}_{\mathrm{S}}=\left(N, \longrightarrow_{\mathrm{DG}}\right)$ be the dependence graph of a specification $S$, and let $\mathbf{C}=\left(\mathbf{C}_{1}, \mathbf{C}_{2}\right)$ be a cut. The sets of operation schemas for the decomposition of $S$ are defined as

- $O p_{1}:=l\left[\left(\mathbf{P h}_{1} \cup \mathbf{P h}_{3}\right) \backslash\{\right.$ init $\left.\}\right]$,

- $O p_{2}:=l\left[\mathbf{P h}_{2}\right]$,

- $O p_{\mathbf{C}_{1}}:=l\left[\mathbf{C}_{1}\right]$ and

- $O p_{\mathbf{C}_{2}}:=l\left[\mathbf{C}_{2}\right]$.

We let $O p_{\mathrm{C}}:=O p_{\mathrm{C}_{1}} \cup O p_{\mathrm{C}_{2}}$.

We exclude init from the definition since we will separately deal with the initial state schema. It is important to note that in general, $O p_{1}$ and $O p_{2}$ are not disjoint, as a multiple occurrence of an operation may lead to one occurrence being assigned to $\mathbf{P h}_{1} \cup \mathbf{P h}_{3}$ and another to $\mathbf{P h}_{2}$. However, the three sets $O p_{\mathbf{C}_{1}}, O p_{\mathbf{C}_{2}}$ and $O p_{1} \cup O p_{2}$ are indeed disjoint based on the correctness criterion all-or-none.

Next, we deduce the interfaces of the components $S_{1}$ and $S_{2}$ from the previous definition, where $\left.I\right|_{O}$ denotes the restriction of the interface $I$ on the operations of $O$ :

Definition 4.3.2. (Interfaces of components, intermediate definition)

Let $\mathrm{DG}_{\mathrm{S}}=\left(N, \longrightarrow_{\mathrm{DG}}\right)$ be the dependence graph of a specification $S$, and let $\mathbf{C}=\left(\mathbf{C}_{1}, \mathbf{C}_{2}\right)$ be a cut. The interfaces for the decomposition of $S$ into $S_{1}$ and $S_{2}$ are defined as

- $S_{1} I:=\left.I\right|_{\left(O p_{1} \cup O p_{\mathrm{C}}\right)}$ and

(Interface for $S_{1}$ ) 
- $S_{2} . I:=\left.I\right|_{\left(O p_{2} \cup O p_{\mathrm{C}}\right)}$.

(Interface for $S_{2}$ )

This definition will slightly be adapted in Section 4.3.3, based on the introduction of additional parameters to the channels.

For determining the CSP parts of $S_{i}$, the process $S$.main is restricted on the sets of events corresponding to the component's sets of operations. To this end, we define the projection of a CSP process on a subset of its events according to [Brü08]. The definition also applies, if the specification is composed of several classes:

Definition 4.3.3. (Projection of CSP processes, [Brü08])

Let $P$ be the right-hand side of a CSP process definition and $E \subseteq$ Events. The projection of $P$ on $E$, denoted by $\left.P\right|_{E}$, is inductively defined:

1. Skip $\left.\right|_{E}:=\operatorname{Skip}$ and Stop $\left.\right|_{E}:=$ Stop,

2. $\left.(e \rightarrow P)\right|_{E}:= \begin{cases}\left.P\right|_{E}, & e \notin E \\ \left.e \rightarrow P\right|_{E}, & \text { otherwise, }\end{cases}$

3. $\left.(P \circ Q)\right|_{E}:=\left(\left.P\right|_{E}\right) \circ\left(\left.Q\right|_{E}\right)$ for $\circ \in\{;,|| \mid, \square, \sqcap\}$,

4. $\left.\left(P \|_{A} Q\right)\right|_{E}:=\left(\left.P\right|_{E}\right) \|_{A \cap E}\left(\left.Q\right|_{E}\right)$.

According to [Brü08], we can apply several simplifications to the resulting CSP processes. Such a modification is, for instance, given by replacing a process equation $P \stackrel{c}{=} P$ by $P \stackrel{c}{=}$ Stop or $P \stackrel{c}{=}(P \circ Q)$ with $P \stackrel{c}{=} Q$ for $\circ \in\{\square, \sqcap\}$. Note that an equation $P \stackrel{c}{=} P$ introduces divergence [Ros98] into the overall process, that is, an infinite loop without an execution of an external event. In the semantic model of traces, replacing it with $P \stackrel{c}{=}$ St op does not influence the behaviour of the process. For more details, see [Brü08].

This definition of the projection allows us to inductively define the processes $S_{1}$.main and $S_{2}$.main. As the definition is applied with respect to a set of events, we use the extension sets of the respective sets of operations.

Definition 4.3.4. (CSP parts of components, intermediate definition) Let $\mathrm{DG}_{\mathrm{S}}=\left(N, \longrightarrow_{\mathrm{DG}}\right)$ be the dependence graph of a specification $S$, and let $\mathbf{C}=\left(\mathbf{C}_{1}, \mathbf{C}_{2}\right)$ be a cut. The CSP parts for the decomposition of $S$ into $S_{1}$ and $S_{2}$ are defined as

- $S_{1} \cdot \operatorname{main}:=\left.S \cdot \operatorname{main}\right|_{\left\{\left|O p_{1}\right|\right\} \cup\left\{\left|O p_{\mathrm{C}}\right|\right\}}$ and

(CSP part for $S_{1}$ )

- $S_{2} \cdot \operatorname{main}:=\left.S \cdot \operatorname{main}\right|_{\left\{\left|O p_{2}\right|\right\} \cup\left\{\left|O p_{\mathbf{C}}\right|\right\}}$.

(CSP part for $S_{2}$ )

Again, due to the additional parameters, the CSP parts of the components will slightly be modified in Section 4.3.4 by introducing a renaming function.

Next, we define the Object-Z parts of $S_{1}$ and $S_{2}$. We have to identify their state schemas, initial state schemas and operation schemas.

The state schema of $S$ comprises a set of state variables $S . V$ with their respective types, along with a possibly empty set of state invariants. In order to define the state schemas of $S_{1}$ and $S_{2}$, we first identify two subsets of S.V. By setting 
- $S_{1} \cdot V:=\operatorname{all}\left(O p_{1} \cup O p_{\mathbf{C}_{1}}\right)$ and

- $S_{2} \cdot V:=\operatorname{all}\left(O p_{2} \cup O p_{\mathrm{C}_{2}}\right)$,

we restrict both state schemas to those variables which are referenced or modified in at least one of the component's local operations or operations of one specific cut set. Not adding $O p_{\mathrm{C}_{1}}$ and $O p_{\mathrm{C}_{2}}$ to both sets will become clearer when we define the predicate parts of the operations and when we deal with transmitting the state space modification between the components in Section 4.3.2. Note that we do not additionally refer to variables occurring in $S$.Init.

As a consequence of invariants influencing the execution of any operation, according to the previous definition, variables occurring in some invariant need to be represented in both, $S_{1} . V$ and $S_{2} . V$. This is implicitly guaranteed by the normalisation as introduced in Section 2.3.3, attaching all state invariants to any ef fect-schema.

For the complete definition of $S_{i}$.State, we will use Definition 2.2.1:

Definition 4.3.5. (State schemas of components)

Let $\mathrm{DG}_{\mathrm{S}}=\left(N, \longrightarrow_{\mathrm{DG}}\right)$ be the dependence graph of a specification $S$, and let $\mathbf{C}=\left(\mathbf{C}_{1}, \mathbf{C}_{2}\right)$ be a cut. The state schemas for the decomposition of $S$ into $S_{1}$ and $S_{2}$ are defined over

- $S_{1} \cdot V:=\operatorname{all}\left(O p_{1} \cup O p_{\mathrm{C}_{1}}\right)$ and

- $S_{2} . V:=\operatorname{all}\left(O p_{2} \cup O p_{\mathrm{C}_{2}}\right)$,

as

- $S_{1}$. State $:=\left\{s \uparrow\left(S_{1} . V\right) \mid s \in\right.$ S.State $\}$ and

- $S_{2}$. State $:=\left\{s \uparrow\left(S_{2} . V\right) \mid s \in\right.$ S.State $\}$.

Next, we are concerned with the initial state schema of a class, that is, the decomposition of $S$.Init into $S_{1}$. Init and $S_{2}$. Init. The question arises of how to deal with predicates referring to elements of both, $S_{1} . V$ and $S_{2} . V$.

Consider some initial state predicate $p(x, y)$ with $x$ being assigned to $S_{1} . V \backslash S_{2} . V$ and $y$ being assigned to $S_{2} . V \backslash S_{1} . V$. The predicate can neither be assigned to $S_{1}$. Init nor to $S_{2}$.Init, since one of the specific variables is not an element of the respective component. However, an elimination of the predicate is infeasible, since the relation between $x$ and $y$ would get lost.

Therefore, a simple restriction of $S$. Init onto predicates dealing with $S_{i} . V$ is insufficient. The general definition of the initial state schemas of $S_{1}$ and $S_{2}$ will refer to $S$. Init and use an existential quantification for a subset of S.V. This leads to the following definition:

Definition 4.3.6. (Init schemas of components)

Let $\mathrm{DG}_{\mathrm{S}}=\left(N, \longrightarrow_{\mathrm{DG}}\right)$ be the dependence graph of a specification $S$, and let $\mathbf{C}=\left(\mathbf{C}_{1}, \mathbf{C}_{2}\right)$ be a cut. Furthermore, let $\left(S . V \backslash S_{1} . V\right)=\left\{v_{1}, \ldots, v_{n}\right\}$ and let $S_{1} . V=\left\{w_{1}, \ldots, w_{m}\right\}$. The initial state schemas for the decomposition of $S$ into $S_{1}$ and $S_{2}$ are defined as 
- $S_{1}$.Init $:=\exists v_{1}, \ldots, v_{n} \bullet$ S.Init and

- $S_{2}$.Init $:=\exists w_{1}, \ldots, w_{n} \bullet$ S.Init.

Both Init-predicates are well-defined, that is, all free variables occurring in $S_{i}$. Init are elements of its respective sets of state variables $S_{i} . V$. Note that for the initial state schema of $S_{2}$, shared variables, that is, elements of $S_{1} \cdot V \cap S_{2} \cdot V$, are also quantified: these variables are already restricted in the first specification part.

We use the following abbreviation: variables not occurring in the initial state schema will not be quantified. Precisely, if $p$ is a predicate referring to variables $x_{1}, \ldots, x_{k}$,

$$
\exists y_{1}, \ldots, y_{m} \bullet p\left(x_{1}, \ldots, x_{k}\right)
$$

is abbreviated by

$$
\exists z_{1}, \ldots, z_{n} \bullet p\left(x_{1}, \ldots, x_{k}\right),
$$

where $\left\{z_{1}, \ldots, z_{n}\right\}=\left\{x_{1}, \ldots, x_{k}\right\} \cap\left\{y_{1}, \ldots, y_{m}\right\}$. Moreover, we omit trivially satisfied predicates as, for instance, $\exists v \bullet v=n$ with $n \in t_{v}$.

Recall the abstract example from before: the initial state predicate $p(x, y)$ will be changed to $\exists y \bullet p(x, y)$ for $S_{1}$. Init and to $\exists x \bullet p(x, y)$ for $S_{2}$.Init. A proof of the adequateness of this definition will be given in Chapter 5. In addition, Section 4.3.7 indicates that a subset of a specification's initial data dependences does not need to be considered when it comes to validating the correctness of a cut.

We remain to define the declaration parts and predicate parts of the component's operations. For local operations to $S_{i}$, we simply keep the original definition as-is. For the set of cut operations, we solely keep the predicate parts in one of the specifications parts. In order to ensure corresponding types, we always need to preserve the original declaration parts. Precisely:

Definition 4.3.7. (Operation schemas of components, intermediate definition) Let $\mathrm{DG}_{\mathrm{S}}=\left(N, \longrightarrow_{\mathrm{DG}}\right)$ be the dependence graph of a specification $S$, and let $\mathbf{C}=\left(\mathbf{C}_{1}, \mathbf{C}_{2}\right)$ be a cut. The operation schemas for the decomposition of $S$ into $S_{1}$ and $S_{2}$ are defined as

$$
\begin{aligned}
S_{1} . o p & := \begin{cases}\text { S.op, } & o p \in\left(O p_{1} \cup O p_{\mathbf{C}_{1}}\right), \\
{[\text { S.op.dec } \mid \text { true }],} & o p \in O p_{\mathbf{C}_{2}} .\end{cases} \\
S_{2} . o p & := \begin{cases}\text { S.op, } & o p \in\left(O p_{2} \cup O p_{\mathbf{C}_{2}}\right), \\
{[\text { S.op.dec } \mid \text { true }],} & \text { op } \in O p_{\mathbf{C}_{1}} .\end{cases}
\end{aligned}
$$

Again, this definition needs to be modified, when we are dealing with data dependences between both components. Finally, we unify all the previous definitions into one, the intermediate decomposition of $S$ into two components $S_{1}$ and $S_{2}$ : 
Definition 4.3.8. (Decomposition with respect to a cut, intermediate definition) Let $\mathrm{DG}_{\mathrm{S}}=\left(N, \longrightarrow_{\mathrm{DG}}\right)$ be the dependence graph of a specification $S$, and let $\mathbf{C}=\left(\mathbf{C}_{1}, \mathbf{C}_{2}\right)$ be a cut. Let

$$
O p_{1}, O p_{2}, O p_{\mathbf{C}_{1}}, O p_{\mathbf{C}_{2}}, O p_{\mathbf{C}}
$$

be defined according to Definition 4.3.1. The (intermediate) decomposition of $S$ with respect to $\left(\mathbf{C}_{1}, \mathbf{C}_{2}\right)$ into $S_{1}$ and $S_{2}$ is defined as

\begin{tabular}{|ll}
$S_{1} . I$ & [according to Definition 4.3.2] \\
$S_{1} \cdot$ main & [according to Definition 4.3.4] \\
$S_{1}$. State & [according to Definition 4.3.5] \\
$S_{1}$. Init & [according to Definition 4.3.6] \\
$S_{1} . \mathrm{op}$ & [according to Definition 4.3.7] \\
\hline & \\
$S_{2}$ & \\
$S_{2} . I$ & [according to Definition 4.3.2] \\
$S_{2} \cdot$ main & [according to Definition 4.3.4] \\
$S_{2}$. State & [according to Definition 4.3.5] \\
$S_{2}$. Init & [according to Definition 4.3.6] \\
$S_{2} . \mathrm{op}$ & [according to Definition 4.3.7] \\
\hline
\end{tabular}

The system generated from the components is defined as the parallel composition of both classes, synchronising on the set of cut events, that is,

$$
S_{1} \|_{\left\{\left|O p_{\mathbf{C}}\right|\right\}} S_{2} .
$$

In Section 4.3.6, we carry out the decomposition for the candy machine. For a stepwise illustration of the decomposition on a simpler example, we consider a trivial CSP-OZ specification for subsequently increasing three natural numbers $l, m$ and $n$ as given in Figure 4.15. The set $\mathbf{C}=\left\{\right.$ change $\left.\_\mathrm{m}\right\}$ defines a valid single cut. We get

- $O p_{1}:=\{$ change_l $\}$,

- $O p_{2}:=\left\{\right.$ change $\left.\_n\right\}$ and

- $O p_{\mathrm{C}}:=\{$ change_m $\}$.

The intermediate definition of the components Increaser ${ }_{1}$ and Increaser 2 is given in Figure 4.16. The overall system is defined as

$$
\text { Increaser }_{1} \|_{\{\mid \text {change_m } \mid\}} \text { Increaser }_{2} \text {. }
$$

Note that currently, Increaser 2 .change $m$ is empty. Moreover, the generated initial state predicates can be simplified: 
Increaser

chan change_l $:[x !: \mathbb{N}]$ chan change_m $:[y !: \mathbb{N}]$ chan change_n $:[z !: \mathbb{N}]$

main $\stackrel{c}{=}$ change_l? $x \rightarrow$ change_m? $y \rightarrow$ change_n?z $\rightarrow$ Skip

$l, m, n: \mathbb{N}$

$\stackrel{\operatorname{Init}}{l>n}$

effect_change_l
$\Delta(l)$
$x !: \mathbb{N}$
$l^{\prime}=l+1 \wedge x !=l^{\prime}$

effect_change_m _

$\Delta(m)$
$y !: \mathbb{N}$
$m^{\prime}=l+1 \wedge y !=m^{\prime}$

_effect_change_n_

$\Delta(n)$

$z !: \mathbb{N}$

$\overline{n^{\prime}}=m+1 \wedge z !=n^{\prime}$

Figure 4.15: Simple CSP-OZ specification for increasing two natural numbers
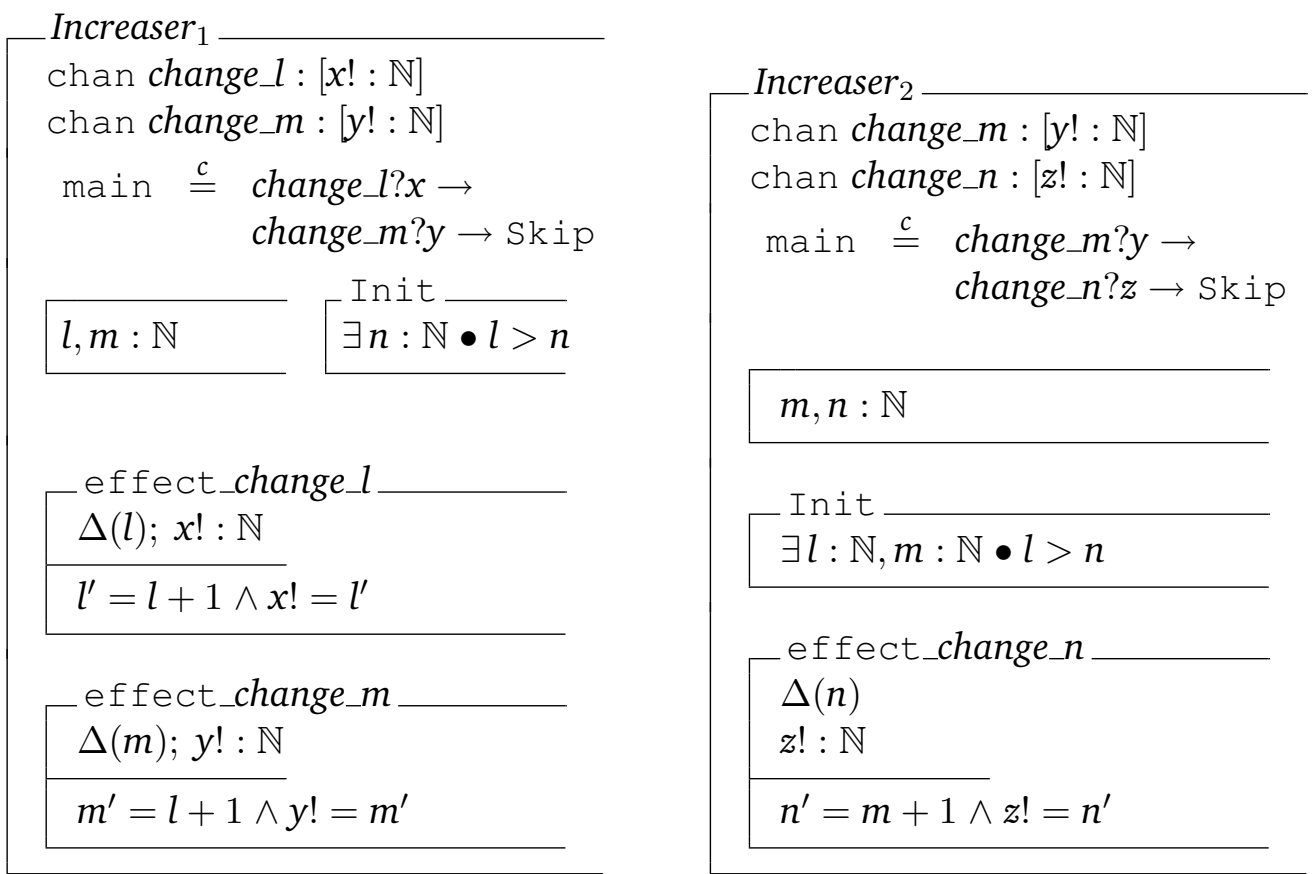

Figure 4.16: Intermediate decomposition of Increaser

- $\exists n: \mathbb{N} \bullet l>n \Leftrightarrow l>0$ and

- $\exists l: \mathbb{N}, m: \mathbb{N} \bullet l>n \equiv$ true, respectively.

This completes the intermediate definition of the different constituents of the components, resulting in a well-defined decomposition of $S$. In an optimum way, the de- 
composition results in two completely independent specification parts $S_{1}$ and $S_{2}$. In this case, the previously given intermediate decomposition is final in the sense that no further modification is required. In the context of assume-guarantee reasoning, this is preferable, as no supplemental constructs need to be added, ensuring that the size of the components remains rather small.

However, two completely independent specification parts are far from realistic. This would, for instance, require the cut to split a graph into two unrelated pieces, not sharing any ingoing and outgoing data dependences. Along with that, any branching within the control flow graph would have to be assigned to one component.

In order to ensure a universally valid decomposition in our context, the introduction of additional parameters and an event renaming is required. These extensions are given next, yielding a modification of the previously as intermediate marked definitions.

\subsubsection{Preservation of the Data Dependences}

As a first step, we are interested in preserving the original data flow, that is, the state space modifications. In particular, both components sharing the same state variables requires that a modification within one component is visible to the other component. Even though it is impossible that data dependences circumvent the set of cut operations based on the criterion no crossing, they can indeed target the cut and originate from it, thus causing mutual influence between both components, based on the data flow. Figure 4.17 again illustrates the fragmentation of a specification's dependence graph.

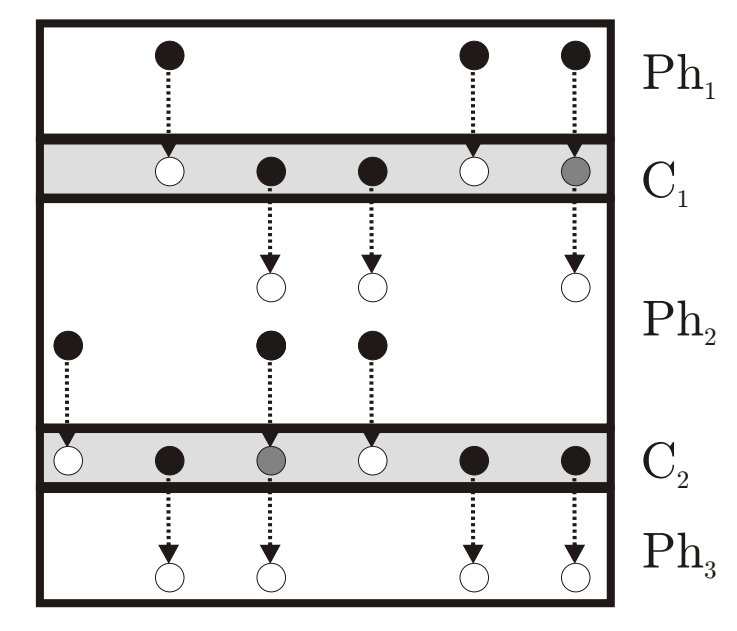

- modification of a variable

- modification of / reference to variable

reference to a variable

Figure 4.17: Possible data dependences targeting the cut and originating from the cut 
Dotted edges denote data dependences between two operation nodes, where the schema corresponding to the source node modifies a certain state variable, and the target schema references a variable. Nodes highlighted in grey depict schemas which modify one and reference another state variable.

The crucial edges are the ones originating from a cut operation and targeting an operation in the subsequent phase or the other cut: they represent variables modified in one specification part (within a cut schema and possibly before as well) and referenced in the other. These modifications must be preserved to not refer to inconsistent values.

In the example Increaser, a particular sequence of two data dependences conforms to this specific problem: the schema change_l modifies the variable $l$, the schema change_m references $l$ and modifies $m$ and change_ $n$ references $m$. This sequence of state modifications is not reflected in the decomposition of Increaser as given in the last section. For an illustration, assume that initially, $l=3, m=2$ and $n=1$ holds. Table 4.2 denotes the state valuations of Increaser during the processing of the event trace $\langle$ change_l.4, change_m.5, change_n.6〉. Additionally, assuming the same initial state, the corresponding traces of the components are given.

\begin{tabular}{|c|c|c|}
\hline Trace of Increaser & ${\text { Trace of } \text { Increaser }_{1}}$ & ${\text { Trace of } \text { Increaser }_{2}}$ \\
\hline \hline$\langle(l=3, m=2, n=1)$, & $\langle(l=3, m=2)$, & \\
change_l. 4, & change_l.4, & \\
$(l=4, m=2, n=1)$, & $(l=4, m=2)$, & $\langle(m=2, n=1)$, \\
change_m.5, & change_m.5, & change_m.2, \\
$(l=4, m=5, n=1)$, & $(l=4, m=5)\rangle$, & $(\mathbf{m}=\mathbf{2}, n=1)$, \\
change_n.6, & & change_n.3, \\
$(l=4, m=5, n=6)\rangle$ & & $(\mathbf{m}=\mathbf{2}, \mathbf{n}=\mathbf{3})\rangle$ \\
\hline
\end{tabular}

Table 4.2: Comparison of two traces for Increaser and its components

As the modification of $m$ depends on $l$ and is no longer represented in Increaser $_{2}$, the value of $m$ is inconsistent after the operation change $m$ took place. This inconsistency is in particular visible to the outside, as the parameter value of the event change $m$ has changed from 5 to 2 . Even worse, this inconsistency is propagated to the value of $n$ as well. The inconsistency changes the behaviour of the original specification as the trace $\langle$ change_l.4, change_m.5, change_n.6〉 cannot be restored within Increaser $_{1} \|_{\{\mid \text {change_m } \mid\}}$ Increaser $_{2}$. Since we are interested in the equivalence of traces of events the specification and its decomposition may perform, this inconsistency must be prohibited.

The set of cut operations serves as the (sole) link between both specification parts, and any influence of one component on the other must be transmitted via the cut. A correspondence of the values of shared variables between both components is achieved by the introduction of additional parameters. The type of a cut operation is possibly extended based on this set of transmission parameters, each representing one specific shared state variable modified in one and referenced in the other specification part. 
Precisely, these parameters are outputs to the modifying specification part and inputs to the referencing component, while transmitting the values of the respective state variables.

First, we have to clarify which variables actually need to be transmitted, that is, which variables exert influence from one on the other component. The following definition identifies two sets of state variables, namely the ones which need to be transmitted via the first cut set and the second cut set:

Definition 4.3.9. (Cut variables)

Let $\mathrm{DG}_{\mathrm{S}}=\left(N, \longrightarrow_{\mathrm{DG}}\right)$ be the dependence graph of a specification $S$, and let $\mathbf{C}=\left(\mathbf{C}_{1}, \mathbf{C}_{2}\right)$ be a cut. The modifications of $\mathrm{n} \in \mathrm{op}(N)$ influencing $X \subseteq \mathrm{op}(N)$ are defined as

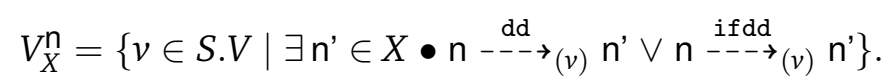

The sets of cut variables for the decomposition of $S$ into $S_{1}$ and $S_{2}$ are given by

- $C V\left(\mathbf{C}_{1}\right):=\bigcup_{\mathbf{n} \in \mathbf{C}_{1}} V_{\left(\mathbf{P h}_{2} \cup \mathbf{C}_{2}\right)}^{\mathrm{n}}$ and

- $C V\left(\mathbf{C}_{2}\right):=\bigcup_{\mathbf{n} \in \mathbf{C}_{2}} V_{\left(\mathbf{P h}_{1} \cup \mathbf{P h}_{3} \cup \mathbf{C}_{1}\right)}^{\mathrm{n}}$.

$v \in C V\left(\mathbf{C}_{1}\right)$ holds, if there exists a (direct- or interference-) data dependence by reason of $v$ originating from the first set of cut operations and targeting an operation from $\mathbf{P h}_{2}$ or $\mathbf{C}_{2} . C V\left(\mathbf{C}_{2}\right)$ is analogously defined. The definition is complete in the sense that all variables exerting influence from one on the other component are included: the correctness criterion no crossing ensures that data dependences must not circumvent the set of cut nodes.

As we need to refer to operation schemas instead of operation nodes when adding transmission parameters to an operation, we set

$$
V_{X}^{o p}=\bigcup_{\mathrm{n} \in l^{-1}(o p)} V_{X}^{\mathrm{n}}
$$

and let

$$
C V_{1}:=V_{\left(\mathbf{P h}_{2} \cup \mathbf{C}_{2}\right)}^{o p} \text { and } C V_{2}:=V_{\left(\mathbf{P h}_{1} \cup \mathbf{P h}_{3} \cup \mathbf{C}_{1}\right)}^{o p} .
$$

Even though we might have different sets of cut variables for $\mathrm{n}, \mathrm{n}^{\prime} \in l^{-1}(o p)$, the definition is reasonable: the correctness criterion all-or-none ensures that two different operation nodes corresponding to one operation schema must not be distributed over a cut set and its complement.

The previous considerations lead to the following, final definition for the operation schemas of $S_{1}$ and $S_{2}$ :

Definition 4.3.10. (Operation schemas of components, final definition)

Let $\mathrm{DG}_{\mathrm{S}}=\left(N, \longrightarrow_{\mathrm{DG}}\right)$ be the dependence graph of a specification $S$, and let $\mathbf{C}=\left(\mathbf{C}_{1}, \mathbf{C}_{2}\right)$ be a cut. For $C V_{1}=\left\{v_{1}, \ldots, v_{n}\right\}$ and $C V_{2}=\left\{w_{1}, \ldots, w_{m}\right\}$, let

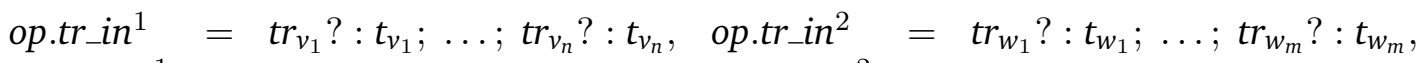

$$
\begin{aligned}
& \text { op.tr_out }{ }^{1}=t r_{v_{1}} !: t_{v_{1}} ; \ldots ; t r_{v_{n}} \text { !: } t_{v_{n}}, \quad \text { op.tr_out }{ }^{2}=t r_{w_{1}} !: t_{w_{1}} ; \ldots ; t r_{w_{m}} !: t_{w_{m}} \text {. }
\end{aligned}
$$


The operation schemas for the decomposition of $S$ into $S_{1}$ and $S_{2}$ are defined as $S_{1}$. op $:=$ $\begin{cases}\text { S.op, } & \text { op } \in O p_{1}, \\ {\left[\text { S.op.delta; S.op.dec; op.tr_out }{ }^{1} \mid \text { op.pred } \wedge \wedge_{v \in C V_{1}} t_{v} !=v^{\prime}\right],} & o p \in O p_{\mathbf{C}_{1}}, \\ {\left[\Delta\left(w_{1}, \ldots, w_{m}\right) ; \text { S.op.dec; op.tr_in }{ }^{2} \mid \bigwedge_{w \in C V_{2}} w^{\prime}=t r_{w} ?\right],} & o p \in O p_{\mathbf{C}_{2}} .\end{cases}$

$S_{2} . o p:=$

$\begin{cases}\text { S.op, } & \text { op } \in O p_{2}, \\ {\left[\Delta\left(v_{1}, \ldots, v_{n}\right) ; \text { S.op.dec; op.tr_in }{ }^{1} \mid \bigwedge_{v \in C V_{1}} v^{\prime}=t r_{v} ?\right],} & o p \in O p_{\mathbf{C}_{1}}, \\ {\left[\text { S.op.delta; S.op.dec; op.tr_out }{ }^{2} \mid \text { op.pred } \wedge \bigwedge_{w \in C V_{2}} t r_{w} !=w^{\prime}\right],} & o p \in O p_{C_{2}} .\end{cases}$

The declaration parts of all cut operations are extended by additional transmission parameters. For the influence of $S_{1}$ on $S_{2}$, we add predicates $t r_{v} !=v^{\prime}$ for each cut variable $v \in C V_{1}$ to the first cut set and corresponding predicates $v^{\prime}=t r_{v}$ ? to the second. We proceed accordingly for variables of $S_{2}$ influencing $S_{1}$. The delta lists of the receiving operations need to comprise all modified cut variables. Figure 4.18 illustrates the concept of these parameters. In Chapter 5 , we will show that this technique is sufficient to restore the data flow of a specification in its decomposition.

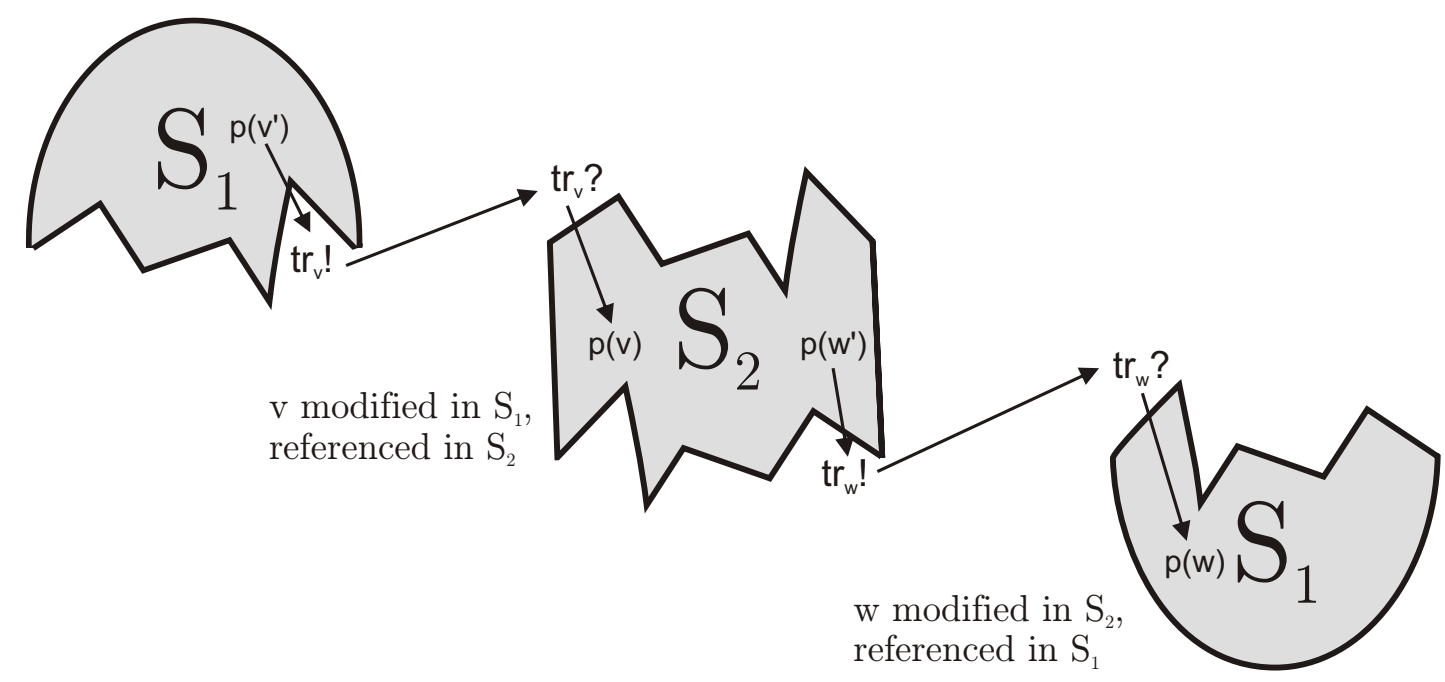

Figure 4.18: Illustration of the transmission parameters

In our example, due to the data dependence change $\_m \stackrel{d d}{--\rightarrow_{(m)}}$ change $\_n$, the state variable $m$ is a cut variable of change_m. Thus, we add one transmission parameter $t r_{m}$ to change $m$, serving as an output to Increaser $_{1}$.change $m$ and an input to 

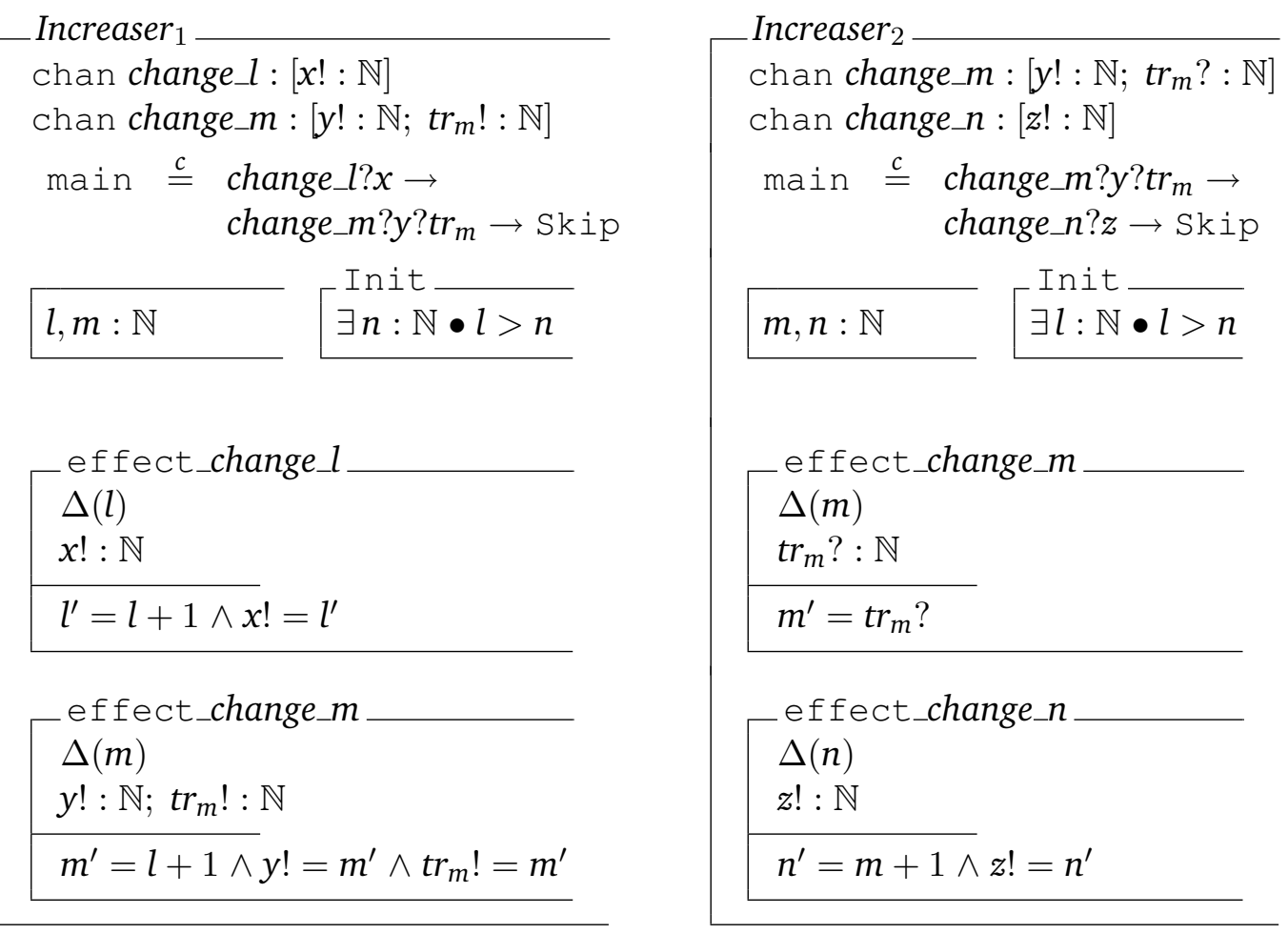

Figure 4.19: Decomposition of Increaser, modified according to Definition 4.3.10

Increaser $_{2}$.change $m$. The modified decomposition is shown in Figure 4.19. Note that we have to modify the specification's interfaces and CSP parts as well. We deal with this aspect in Section 4.3.4.

This modification fixes the previously identified inconsistency as shown in Table 4.3. Next, we deal with the reconstitution of the control flow of the original specification within its decomposition. The underlying concept similarly uses additional parameters.

\begin{tabular}{|c|c|c|}
\hline Trace of Increaser & Trace of Increaser 1 & Trace of Increaser $_{2}$ \\
\hline $\begin{array}{c}\langle(l=3, m=2, n=1), \\
\quad \text { change } l .4, \\
(l=4, m=2, n=1), \\
\\
\quad \text { change_m. } 5, \\
(l=4, m=5, n=1), \\
\quad \text { change } n .6, \\
(l=4, m=5, n=6)\rangle\end{array}$ & $\begin{array}{c}\langle(l=3, m=2), \\
\text { change_l. } 4, \\
(l=4, m=2), \\
\text { change } m .5, \\
(l=4, m=5),\rangle\end{array}$ & $\begin{array}{c}\langle(m=2, n=1), \\
\text { change } \_ \text {.5 }, \\
(\mathbf{m}=\mathbf{5}, n=1), \\
\text { change } n \cdot \mathbf{6}, \\
(\mathbf{m}=\mathbf{5}, \mathbf{n}=\mathbf{6})\rangle\end{array}$ \\
\hline
\end{tabular}

Table 4.3: Comparison of two traces of Increaser and its components after modification 


\subsubsection{Preservation of the Control Flow}

The fact that one specification part influences the other one due to its data flow is quite intuitive. Additional to that and less obvious, the intermediate decomposition and reassembly can also cause a modification of the original control flow of a specification. For instance, it is possible that the CSP part of $S_{1} \| S_{2}$ allows for additional traces, thus causing a violation of the trace equivalence between $S$ and $S_{1} \| S_{2}$.

As the problem of preserving the control flow of a specification is solely related to the CSP part of a specification, we entirely omit dealing with the Object-Z part in this section.

\section{Restoring the Original Synchronisation}

First, we will deal with ensuring a correct synchronisation between $S_{1}$ and $S_{2}$. In order to illustrate the general problem, we give a small example.

Example 4.3.11. Let $S$ be a specification over a set of events $\{a, b, c, d, e\}$, and let

$$
\text { S.main }:=(a \rightarrow c \rightarrow d \rightarrow \text { Skip }) \square(b \rightarrow c \rightarrow e \rightarrow \text { Skip }) .
$$

Let $\mathbf{C}=\{c\}$ be a valid single cut yielding

- $S_{1} \cdot \operatorname{main}:=(a \rightarrow c \rightarrow$ Skip $) \square(b \rightarrow c \rightarrow$ Skip $)$ and

- $S_{2}$ main $:=(c \rightarrow d \rightarrow$ Skip $) \square(c \rightarrow e \rightarrow$ Skip $)$.

Let $\operatorname{tr}:=\langle a, c, e\rangle$. Then, $\operatorname{tr} \in \operatorname{traces}\left(S_{1} \cdot\right.$ main $\|_{\{c\}} S_{2}$.main) but $\operatorname{tr} \notin \operatorname{traces}($ S.main).

The example points out the following: Definition 4.2 .8 allows the cut sets to contain several nodes with the same operation name - for $\mathrm{n}_{1}, \mathrm{n}_{2} \in \mathrm{C}_{i}$, the equation op $=l\left(\mathrm{n}_{1}\right)=$ $l\left(\mathrm{n}_{2}\right)$ is possible. Let us denote two different occurrences of op within the CSP part of a specification by $o p^{1}$ and $o p^{2}$.

In the decomposition of the specification, $o p^{1}$ and $o p^{2}$ occur in both parts, $S_{1}$ and $S_{2}$. Obviously, a synchronisation of op must be restricted to originally corresponding occurrences of $o p$, that is, $S_{1} . o p^{1}$ should be synchronised with $S_{2} . o p^{1}$ and, accordingly, $S_{1} . o p^{2}$ with $S_{2} . o p^{2}$.

However, the synchronisation alphabet can no longer distinguish between these different occurrences. Therefore, non-corresponding instances of operations can be synchronised as well. This can particularly lead to additional traces for the CSP part of $S_{1} \| S_{2}$.

In our example, the event $c$ occurs twice within $S$.main and thus twice in $S_{1}$.main and $S_{2}$.main. A synchronisation of $c$ within $S_{1}$.main $\| S_{2}$.main can either result in the joint execution of corresponding occurrences, namely $S_{1} \cdot c^{1}$ synchronising with $S_{2} \cdot c^{1}$ and $S_{1} \cdot c^{2}$ synchronising with $S_{2} \cdot c^{2}$, as shown on the left hand side of Figure 4.20, or to an invalid synchronisation of $S_{1} \cdot c^{1}$ with $S_{2} \cdot c^{2}$ and $S_{1} \cdot c^{2}$ with $S_{2} \cdot c^{1}$, as shown on the right hand side of the same figure. The latter synchronisation triggers the previously identified path $\langle a, c, e\rangle$, which is invalid for S.main.

In Section 2.2.1, we introduced simple parameters [Fis00, Fis97] which can be restricted by both, the CSP part and the Object-Z part of a specification. This specific type 

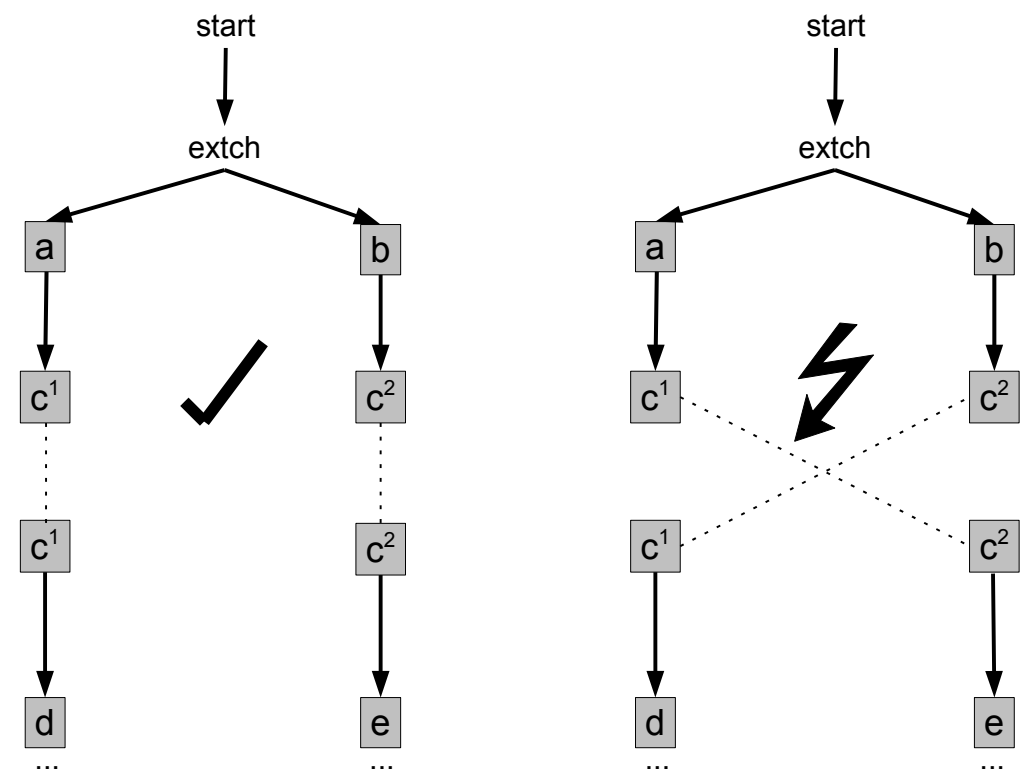

Figure 4.20: Synchronisation of events for external choice

of parameters will be used to define a set of additional address parameters to operations with a multiple occurrence in the cut. In our case, they will solely be restricted by the CSP part, and they do not occur in the Object-Z part of a component. We will modify the CSP parts of $S_{1}$ and $S_{2}$ by fixing the values for some of these parameters. As a synchronisation of two instances of an operation is only possible if their extension sets are not disjoint, differently fixed parameters can prevent a false synchronisation.

We illustrate the outcome of this extension on the previous example. For the event $c$, we will use one address parameter $p_{1}$ of type $\{1,2\}$ and redefine

- $S_{1}$.main $:=(a \rightarrow c .1 \rightarrow$ Skip $) \square(b \rightarrow c .2 \rightarrow$ Skip $)$ and

- $S_{2}$ main $:=(c .1 \rightarrow d \rightarrow$ Skip $) \square(c .2 \rightarrow e \rightarrow$ Skip $)$.

A synchronisation of $c^{1}$ with $c^{2}$ over different components is now impossible.

In general, if no parallel composition is involved in a process, one additional address parameter is sufficient to separate two different occurrences of an operation from each other. However, when dealing with parallel composition, synchronising the operation under interest, one parameter is no longer adequate, since it would exclude part of the originally allowed synchronisation.

Recall Example 4.3.11 after replacing the external choice operator with $\|_{\{c\}}$. We get

$$
\begin{array}{lll}
S_{1} \text { main } & := & (a \rightarrow c \rightarrow \text { Skip }) \\
& \|_{\{c\}} & (b \rightarrow c \rightarrow \text { Skip }), \\
S_{2} \text { main } & := & (c \rightarrow d \rightarrow \text { Skip }) \\
& \|_{\{c\}} & (c \rightarrow e \rightarrow \text { Skip }) .
\end{array}
$$


In this case, a joint synchronisation of the event $c$ within $S_{1}$.main $\|_{\{c\}} S_{2}$.main is allowed. This requires us to add two fresh parameters, not affecting each other, with one of them subsequently restricted for one branch of the parallel composition and the other one restricted for the other branch.

Summarising, we need to preserve and neither extend nor restrict the original synchronisation structure of $S$ within $S_{1} \| S_{2}$. The following definitions especially need to ensure that only corresponding instances of operations can be synchronised between $S_{1}$ and $S_{2}$.

In order to find a general solution for this problem by identifying a correct addressing extension for any process, including nesting of different types of branching, we recursively traverse its CFG with respect to any operation schema with multiple occurrence in the cut. An algorithm yielding a correct synchronisation is given in Section 5.1. To this end, we outline the general strategy. In addition, we define and show the required conditions on a correct addressing, which are realised by the algorithm. The algorithm proceeds as follows:

Traversing the CFG: Starting with the unique start-node, we recursively traverse the $\mathrm{CFG}$ of the process $S$.main. Let $o p \in O p$ be the current operation under interest.

Initial parameter: Initially, we use one address parameter $p_{1}$ of type $\{1\}$. The type of any parameter can be extended throughout the traversal.

Active Parameters: Any branch of the CFG has one dedicated, active address parameter. The underlying idea is that this parameter possibly needs to be assigned with a specific value to prevent a false synchronisation within the associated branching. Initially, $p_{1}$ is declared active for the sole initial branch and assigned with the value 1. All assigned values are possibly modified during an execution of the algorithm. Besides, one parameter can be active for more than one branch.

No Branching: In case we proceed over a CFG operator which does not introduce branching, no changes to the parameters are committed.

Branching for cfop $\in\left\{\right.$ extch, intch, interleave, $\left.\operatorname{par}_{X}\right\}$ and $o p \notin X$ : Branching without parallel composition of the operation under interest can lead to two occurrences of op within the cut, which need to be separated. In this case, the currently active parameter is declared active for both, the left and right branch. For the left branch, we keep the originally assigned value whereas for the right branch, we increase it by one, and we add the new value to the parameter's type. This ensures that an operation occurring in both branches cannot wrongly be synchronised.

Branching for $\operatorname{par}_{X}$ and $o p \in X$ : Branching with a synchronisation of $o p$ possibly leads to two occurrences of $o p$, which still need to be able to be synchronised. In this case, the active parameter $p_{i}$, which belongs to the branch entering the parallel composition, can no longer be used: it may already be used to prevent a wrong synchronisation within a previous branching. The algorithm introduces two additional, fresh parameters $p_{i+1}$ and $p_{i+2}$. The first parameter is declared active for the left branch, the second parameter is declared active for the right branch. As we solely 
restrict each parameter on one side, a synchronisation of occurrences within the left branch and the right branch is always possible, independent of further restrictions of $p_{i+1}$ and $p_{i+2}$.

Figure 4.21 illustrates the two different cases for branching.

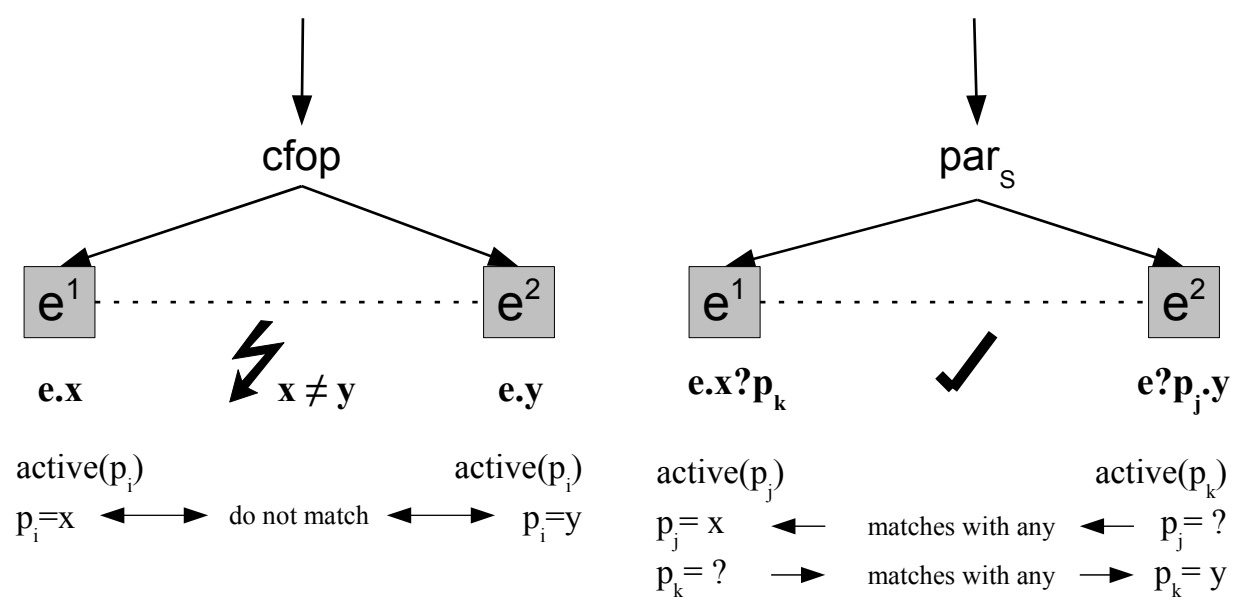

Figure 4.21: Addressing extension for CFG branching

In order to exemplify the necessity for introducing additional parameters in the case of a parallel composition and to clarify the general idea, we give an example. Figure 4.22 shows an extract of a possible control flow graph, for which we consider one operation $b$, element of a valid cut. The CFG proceeds over an external choice, followed by a parallel composition with $b$ being synchronised and, finally, a two-sided external choice. As $b$ occurs multiple times in the cut, an addressing extension is required. Based on our strategy, we introduce three additional parameters:

- $p_{1}$ is responsible for ensuring that no false synchronisation with respect to the outer external choice is possible, that is, $b^{1}$ must not be synchronised with any element of $\left\{b^{2}, b^{3}, b^{4}, b^{5}\right\}$. This is achieved by fixing $p_{1}$ to the value of 1 for the left branch and to 2 for the right branch.

- $p_{2}$ is responsible for excluding a wrong synchronisation within the left inner external choice, that is, between $b^{2}$ and $b^{3}$.

- $p_{3}$ forbids a synchronisation within the right inner external choice, that is, between $b^{4}$ and $b^{5}$.

- Finally, $p_{2}$ and $p_{3}$ are indeed necessary to ensure that any two elements of $\left\{b^{2}, b^{3}\right\}$ and $\left\{b^{4}, b^{5}\right\}$ can still be synchronised. 


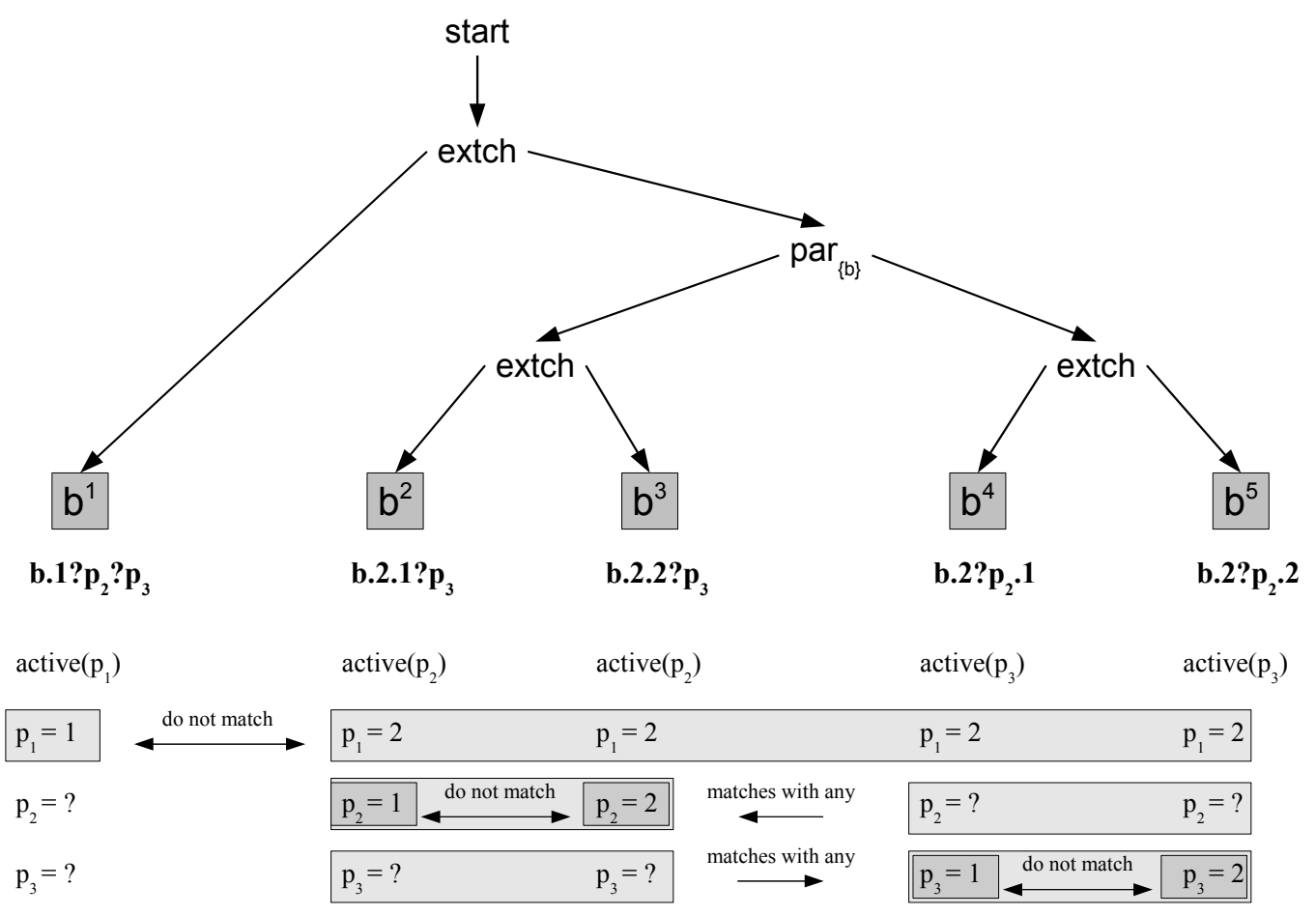

Figure 4.22: Addressing extension for nested branching

Having illustrated and exemplified our general strategy, we now give the details on the addressing extension. Based on the criterion all-or-none, all occurrences of an operation have to be assigned to one cut set, which we denote by $\mathrm{C}_{i}$.

We define two conditions on a parameter extension and subsequently show that they are sufficient to preserve the synchronisation structure of $S$ within $S_{1} \| S_{2}$. Here, we omit dealing with the original parameters of an operation $o p$, since they are irrelevant for the subsequent proof. Both conditions correspond to the previously identified different cases for branching with and without a synchronisation of op.

In Definition 2.2.6, we introduced partial events. As the CSP part of a specification may restrict the set of simple parameters of an operation, any occurrence of an operation within the CSP part is a partial event. Subsequently, $o p_{p}$ denotes an arbitrary partial event for the channel op.

Definition 4.3.12. (Conditions for correct addressing extension) Let $\mathrm{CFG}_{\mathrm{S}}=(N, \longrightarrow)$ be the control flow graph of a specification $S$, and let $\mathbf{C}=\left(\mathbf{C}_{1}, \mathbf{C}_{2}\right)$ be a cut. Furthermore, let op $\in O p_{\mathbf{C}}$ such that op occurs at least twice in either $\mathbf{C}_{1}$ or $\mathbf{C}_{2}$. Let op ${ }^{k}$ denote an arbitrary occurrence of the operation op within $\mathrm{CFG}_{\mathrm{S}}$ and $o p_{p}^{k}$ its corresponding occurrence within S.main. The address requirements for a correct synchronisation are given by the following two conditions, which need to hold for any $i \neq j$ : 
Branching without Synchronisation: If op $^{i}$ and op $^{j}$ are located inside different branches of either an external choice operator, internal choice operator, interleaving operator or a parallel composition operator par $_{X}$ with op $\notin X$, op $p_{p}$ needs to comprise one parameter $p_{1}$ such that its type includes $x, y \in \mathbb{N}$ with $x \neq y$. This parameter is fixed to $x$ for op $p_{p}^{i}$ and to $y$ for opp in both, $S_{1}$.main and $S_{2}$.main :

$$
o p_{p}^{i} \text { becomes } o p_{p}^{i} \cdot x \text { and } o p_{p}^{j} \text { becomes } o p_{p}^{j} \cdot y \text {. }
$$

This corresponds to the left hand side of Figure 4.21.

Branching with Synchronisation: If $\mathrm{op}^{i} \stackrel{\mathrm{sd}}{\leftrightarrow--\rightarrow} \mathrm{op}^{j}$, the (partial) event op needs to comprise two parameters $p_{1}$ and $p_{2}$, such that the type of $p_{1}$ includes $x \in \mathbb{N}$ and the type of $p_{2}$ includes $y \in \mathbb{N}$ for arbitrary $x, y$. The first parameter is fixed to $x$ for $o p_{p}^{i}$ whereas the second parameter is fixed to $y$ for $o p_{p}^{j}$ in both, $S_{1}$.main and $S_{2}$.main :

$$
o p_{p}^{i} \text { becomes } o p_{p}^{i} \cdot x ? p_{2} \text { and } o p_{p}^{j} \text { becomes } o p_{p}^{j} ? p_{1} \cdot y \text {. }
$$

This corresponds to the right hand side of Figure 4.21.

We give an intuitive description of these conditions. Example 4.3.11 illustrated that two different occurrences of an operation can spuriously be synchronised over different branches of an external choice operator. This problem can correspondingly occur for any CSP operator, which introduces branching into the CFG. In order to prevent this from happening, the first condition uses a parameter $p_{1}$ for the respective operation, which is differently fixed in both branches. Thus, a wrong synchronisation is no longer possible, as the extension sets of the partial events are now disjoint. If any two occurrences of the same operation were not allowed to synchronise beforehand, our addressing extension ensures an empty intersection of their extensions, not allowing for a synchronisation afterwards.

Additionally, we have to ensure that a previous synchronisation is not excluded due to our extension. Here, the second condition requires that for any parallel composition including op, two additional parameters are introduced, which can subsequently be restricted for their corresponding branch without influencing a synchronisation over both branches.

In order to restore the original synchronisation structure of a specification by the introduction of additional address parameters, we need to precisely state when two occurrences of the same operation were previously allowed to synchronise. Beforehand, we define a condition, stating that a certain synchronisation dependence can be realised by means of the underlying CSP process: there indeed exist traces, leading to the joint execution of both events. From now on, we let foot(tr) denote the last element of the CSP trace $t r$ according to [Sch99].

Definition 4.3.13. (Realisation of synchronisation dependence) Let op $\in$ Op such that op $p_{p}^{i}$ and $o p_{p}^{j}, i \neq j$, are two different occurrences of op within the CSP part of $S$. Let $\mathrm{op}^{i}$ and $\mathrm{op}^{j}$ denote their corresponding nodes of $\mathrm{CFG}_{\mathrm{S}}$ such that $\mathrm{op}^{i} \stackrel{\mathrm{sd}}{---\mathrm{op}^{j}}$. 
For the CFG node par $_{X}$ being responsible for $\mathrm{op}^{i} \stackrel{\mathrm{sd}}{\leftrightarrow} \rightarrow$ op $\mathrm{p}^{j}$, let $P_{1}$ and $P_{2}$ denote the CSP processes corresponding to the first branch and the second branch of $\operatorname{par}_{X}$ within $\mathrm{CFG}_{S}$, respectively. If

$$
\begin{aligned}
& \exists \operatorname{tr}_{1} \in \operatorname{traces}\left(P_{1}\right), \operatorname{tr}_{2} \in \operatorname{traces}\left(P_{2}\right) \bullet \\
& \left(\operatorname { t r } _ { 1 } \left\lceilX=\operatorname{tr}_{2}\lceil X) \wedge\left(\text { foot }\left(\operatorname{tr}_{1}\right)=o p_{p}^{i}\right) \wedge\left(\text { foot }\left(\operatorname{tr}_{2}\right)=o p_{p}^{j}\right),\right.\right.
\end{aligned}
$$

we say that the synchronisation dependence connecting $o p_{p}^{i}$ and $o p_{p}^{j}$ can be realised.

For two events to allow for synchronisation, their corresponding operation nodes of the CFG must be connected via a synchronisation dependence which can be realised. In addition, the intersection of the extension sets of the partial events corresponding to these nodes is non-empty. All conditions combined ensure that op ${ }^{i}$ and $\mathrm{op}^{j}$ can indeed synchronously be executed.

Definition 4.3.14. (Allowed synchronisation)

Let op $\in$ Op such that op $p_{p}^{i}$ and $o p_{p}^{j}, i \neq j$, are two different occurrences of op within the CSP part of $S$. Let $\mathrm{op}^{i}$ and $\mathrm{op}^{j}$ denote their corresponding nodes of the CFG. We say that $\mathrm{p}_{p}^{i}$ and $o p_{p}^{j}$ allow for synchronisation within $S$, if, and only if,

a) $\mathrm{op}^{i} \stackrel{\mathrm{sd}}{\leftrightarrow--\rightarrow} \mathrm{op}^{j}$ within the $C F G$ of $S$,

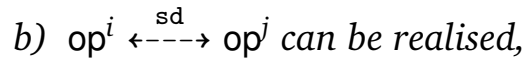

c) $\left\{\left|o p_{p}^{i}\right|\right\} \cap\left\{\left|o p_{p}^{j}\right|\right\} \neq \varnothing$.

Before proving the correctness of the conditions of the previous definition, we show the following property: if two nodes $x, y$ of are not located in different CFG branches attached to the same node, they have to be connected by a CFG path.

Lemma 4.3.15. (Non-opposite branching requires CFG path)

Let $\mathrm{CFG}_{\mathrm{S}}=(N, \longrightarrow)$ be the $C F G$ of a specification $S$. For any node cfop $\in$ \{extch, intch, par, interleave\}, let fst(cfop) denote one branch and snd(cfop) the other branch of cfop, before reaching the join-node uncfop. For any n, $n^{\prime} \in \mathrm{cf}(N)$, if

$\nexists c f o p \in\{$ extch, intch, par, interleave $\} \bullet$

$\left(\mathrm{n} \in\right.$ fst $($ cfop $) \wedge \mathrm{n}^{\prime} \in$ snd(cfop) $) \vee\left(\mathrm{n} \in\right.$ snd(cfop) $\wedge \mathrm{n}^{\prime} \in$ fst(cfop) $)$,

either $\mathrm{n} \stackrel{*}{\longrightarrow} \mathrm{n}^{\prime}$ or $\mathrm{n}^{\prime} \stackrel{*}{\longrightarrow} \mathrm{n}$.

Proof. Let cfop ${ }^{1}$, cfop ${ }^{2}$ denote the innermost operators with $\mathrm{n}, \mathrm{n}^{\prime}$ being located inside one of their respective branches. In case that a node is not located inside of any branching, cfop $^{1}=$ cfop $^{2}=$ start. Thus, we do not separately need to deal with start.

Case 1: cfop $^{1}=$ cfop $^{2}$ Based on the assumption, $n$ and $n^{\prime}$ have to be located in the same branch of the operator. As we chose cfop $^{i}$ to be the innermost branching, $n$ and $n^{\prime}$ are both located on the sole path from cfop ${ }^{i}$ to $n$ or from cfop ${ }^{i}$ to $\mathrm{n}^{\prime}$, dependent on which node is visited first. 
Case 2: cfop $^{1} \neq$ cfop $^{2}$ Based on the assumption, there is not outer operator cfop ${ }^{0}$ with $n$ and $\mathrm{n}^{\prime}$ being located in different branches of cfop $^{o}$. Therefore, for $i \neq j$, either cfop ${ }^{i}$ terminates before cfop ${ }^{j}$, yielding a path from one node to the other one via uncfop ${ }^{i}$. Otherwise, cfop $^{i}$ is located inside of cfop ${ }^{j}$, also yielding a CFG path from one node to the other one.

The following theorem shows that the previously identified conditions on an addressing extension are sufficient.

Theorem 4.3.16. (Definition 4.3.12 ensures correct synchronisation of $S_{1}$ and $S_{2}$ )

Let $\mathrm{CFG}_{\mathrm{S}}=(N, \longrightarrow)$ be the control flow graph of a specification $S$, and let $\mathbf{C}=\left(\mathbf{C}_{1}, \mathbf{C}_{2}\right)$ be a cut. For any op $\in O p_{\mathrm{C}}$ with multiple occurrence within the CFG and CSP part of S, let both conditions of Definition 4.3 .12 be satisfied. Then, the original horizontal synchronisation structure of $S$ is preserved within $S_{1} \| S_{2}$, whereas no additional synchronisation is introduced. Precisely, for $o p_{p}^{i} \neq o p_{p}^{j}$ :

(1) Possible synchronisation for duplicated nodes:

$S_{1} . o p_{p}^{i}$ and $S_{2} . o p_{p}^{i}$ allow for synchronisation in $S_{1} \| S_{2}$.

(2) Original synchronisation is preserved within $S_{1}$ and $S_{2}$ :

If $S . o p_{p}^{i}$ and $S . o p_{p}^{j}$ allow for synchronisation in $S$, then $S_{1} . o p_{p}^{i}\left[S_{2} . o p_{p}^{i}\right]$ and $S_{1} . o p_{p}^{j}$ $\left[S_{2} . o p_{p}^{j}\right]$ allow for synchronisation in $S_{1}\left[S_{2}\right]$.

(3) Original synchronisation is preserved within $S_{1} \| S_{2}$ :

If $S . o p_{p}^{i}$ and $S . o p_{p}^{j}$ allow for synchronisation in $S$, then $S_{1} . o p_{p}^{i}\left[S_{1} . o p_{p}^{j}\right]$ and $S_{2} . o p_{p}^{j}$ $\left[S_{2} . o p_{p}^{i}\right]$ allow for synchronisation in $S_{1} \| S_{2}$.

(4) No additional synchronisation within $S_{1}$ :

If $S . o p_{p}^{i}$ and $S . o p_{p}^{j}$ do not allow for synchronisation in $S$, then $S_{1} . o p_{p}^{i}$ and $S_{1} . o p_{p}^{j}$ do not allow for synchronisation in $S_{1}{ }^{2}$

(5) No additional synchronisation within $S_{1} \| S_{2}$ :

If $S . o p_{p}^{i}$ and $S . o p_{p}^{j}$ do not allow for synchronisation in $S$, then $S_{1} . o p_{p}^{i}\left[S_{2} . o p_{p}^{i}\right]$ and $S_{2} . o p_{p}^{j}\left[S_{1} . o p_{p}^{j}\right]$ do not allow for synchronisation in $S_{1} \| S_{2}$.

Proof. Assume that both conditions of Definition 4.3.12 hold. In case we refer to $o p_{p}^{i}, o p_{p}^{j}$, we implicitly assume $i \neq j$. We show the respective properties by applying the conditions from the definition:

(1) $S_{1} . o p_{p}^{i}$ and $S_{2} . o p_{p}^{i}$ result from a duplication of $S . o p_{p}^{i}$. Let us denote the corresponding CFG nodes within the CFG of $S_{1} \| S_{2}$ by op $p_{1}^{i}$ and $\mathrm{op}_{2}^{i}$. We have to show all three conditions of Definition 4.3.14.

\footnotetext{
${ }^{2}$ Note that both events might indeed allow for synchronisation within $S_{2}$. This does not pose a problem as in this case, a synchronisation over the cut would have to involve all four events $S_{1} . o p_{p}^{i}, S_{1} . o p_{p}^{j}, S_{2} . o p_{p}^{i}$ and $S_{2} . o p_{p}^{j}$ which is impossible if $S_{1} . o p_{p}^{i}$ and $S_{1} . o p_{p}^{j}$ cannot be synchronised.
} 
a) Starting from par $_{O p_{\mathrm{C}}}$, there exist paths $\operatorname{par}_{O p_{\mathrm{C}}} \stackrel{\pi}{\longrightarrow} \mathrm{op}_{1}^{i}$ and $\operatorname{par}_{O p_{\mathrm{C}}} \stackrel{\pi^{\prime}}{\longrightarrow} \mathrm{op}_{2}^{i}$. $\pi$ and $\pi^{\prime}$ do not share any additional nodes, as par $_{O p_{\mathrm{c}}}$ is the outermost operator of the CFG for $S_{1} \| S_{2}$. This ensures the conditions on a synchronisation dependence, as given in Definition 2.3.6. $\checkmark$

b) This dependence can be realised: for the traces $t r$ and $t r^{\prime}$ corresponding to the paths $\pi$ and $\pi^{\prime}$, the equation $\operatorname{tr}\left\lceil O p_{\mathrm{C}}=t r^{\prime}\left\lceil O p_{\mathrm{C}}\right.\right.$ holds. Traces restricted on the set of cut operations are preserved by the projection of the CSP process $S$.main on $S_{i}$.ma in, as cut events occur in both, $S_{1}$ and $S_{2}$, and as Definition 4.3.3 does not modify the structure of a process. $\checkmark$

c) Additionally, since we refer to two nodes corresponding to the same node of $\mathrm{CFG}_{S}$, and since the addressing extension is identical for both, $S_{1}$.ma in and $S_{2}$.ma in, the inequality $\left\{\mid S_{1} . o p_{p}^{i}\right.$.add $\left.\mid\right\} \cap\left\{\mid S_{2} . o p_{p}^{i}\right.$. add $\left.\mid\right\} \neq \varnothing$ trivially holds for any possible addressing extension add. $\checkmark$

(2) Assume that $o p_{p}^{i}$ and $o p_{p}^{j}$ allow for synchronisation in $S$. Again, we show all three conditions for allowed synchronisation of $S_{i} . o p_{p}^{i}$ and $S_{i} . o p_{p}^{j}$.

a) By assumption, $\mathrm{op}^{i}$ and $\mathrm{op}^{j}$ are connected via a synchronisation dependence in $S$, and both nodes are elements of $\mathbf{C}_{i}$. Corresponding to the previous case, they are still connected via a synchronisation dependence in $S_{1}$ and in $S_{2}$, as Definition 4.3.3 does not modify the branching structure of a process. $\checkmark$

b) Let $\operatorname{par}_{X}$ be responsible for the synchronisation dependence between $o p_{p}^{i}$ and $o p_{p}^{j}$, and let $t r$ and $t r^{\prime}$ be the traces realising the dependence. Then, $\operatorname{tr}\left\lceil X=t r^{\prime}\lceil X\right.$. As both paths are correspondingly projected within $S_{i}$.main, we get

$$
\left(\operatorname{tr} \uparrow\left(O p_{i} \cup O p_{\mathbf{C}}\right)\right)\left\lceil X=\left(\operatorname{tr}^{\prime} \uparrow\left(O p_{i} \cup O p_{\mathbf{C}}\right)\right)\lceil X .\right.
$$

Thus, the synchronisation dependence can be realised. $\checkmark$

c) Finally, the second condition on correct addressing results in $o p_{p}^{i}$ being replaced by $o p_{p}^{i} \cdot x ? p_{2}$ and $o p_{p}^{j}$ being replaced by $o p_{p}^{j} ? p_{1} . y$ in both, $S_{1}$ and $S_{2}$, for some $x, y \in \mathbb{N}$. This implies

$$
\left\{\left|o p_{p}^{i} \cdot x \cdot-\right|\right\} \cap\left\{\left|o p_{p \cdot-\cdot}^{j} \cdot y\right|\right\} \neq \varnothing
$$

based on the assumption

$$
\left\{\left|o p_{p}^{i}\right|\right\} \cap\left\{\left|o p_{p}^{j}\right|\right\} \neq \varnothing . \checkmark
$$

(3) Assume that $o p_{p}^{i}$ and $o p_{p}^{j}$ allow for synchronisation in $S$.

a) According to (1), we get two paths $\pi$ and $\pi^{\prime}$ in the CFG of $S_{1} \| S_{2}$, which start in $\operatorname{par}_{O p_{\mathrm{C}}}$ and reach the respective occurrences of op ${ }^{i}$ and op ${ }^{j}$ without additional shared nodes, thus yielding a synchronisation dependence.

b) This dependence can be realised: the projection of $S$.main on $O p_{C}$ yields the same traces within $S_{1}$.main and $S_{2}$ main. $\checkmark$ 
c) Finally, in correspondence to the previous case,

$$
\left\{\left|o p_{p}^{i} \cdot x_{-}\right|\right\} \cap\left\{\left|o p_{p \cdot-\cdot}^{j} \cdot y\right|\right\} \neq \varnothing . \checkmark
$$

(4) Assume that $\mathrm{op}^{i}$ and $\mathrm{op}^{j}$ do not allow for synchronisation in $S$ due to a violation of conditions a), b) or c):

a): In either case, a missing synchronisation dependence cannot be introduced due to the projection. $\checkmark$

b): Let $\operatorname{par}_{X}$ be responsible for the synchronisation dependence between $o p_{p}^{i}$ and $o p_{p}^{j}$. Let $P_{1}$ and $P_{2}$ denote the CSP processes corresponding, to the first and second branch of $\operatorname{par}_{X}$. Then, there are no traces $\operatorname{tr}_{1} \in \operatorname{traces}\left(P_{1}\right)$ and $\operatorname{tr}_{2} \in \operatorname{traces}\left(P_{2}\right)$, such that $\operatorname{tr}_{1}\left\lceil X=t r_{2}\left\lceil X\right.\right.$. As op $\in O p_{\mathrm{C}}$, the projection of the CSP process $S$.main on $S_{1}$.main preserves the original traces with respect to $X$ : no events of $O p_{2}$ can be involved, thus ensuring that the synchronisation dependence cannot be realised within $S_{1}$.main. $\checkmark$

c): $\left\{\left|o p_{p}^{i}\right|\right\} \cap\left\{\left|o p_{p}^{j}\right|\right\}=\varnothing$ is preserved by the addressing extension.

(5) Again assume that one of three conditions for allowed synchronisation is violated:

a): We distinguish between two cases:

Case 1: Both nodes are located inside different branches of either an external choice-, internal choice- or interleaving operator or a parallel composition operator $\operatorname{par}_{X}$ with $o p \notin X$. The first condition on correct addressing results in $o p_{p}^{i}$ being replaced by $o p_{p}^{i} \cdot x$ and $o p_{p}^{j}$ being replaced by $o p_{p}^{i} \cdot y, x \neq y$, within $S_{1}$.main and $S_{2}$.main. Thus, even though both nodes are possibly connected via a newly added synchronisation dependence within the CFG of $S_{1} \| S_{2}$, the events $S_{1} . o p_{p}^{i} . x$ and $S_{2} . o p_{p}^{j} . y$ do not allow for synchronisation according to Definition 4.3.14, since $\left\{\left|o p_{p}^{i} \cdot x\right|\right\} \cap\left\{\left|o p_{p}^{j} \cdot y\right|\right\}=\varnothing$ holds. The same holds for $S_{1}$ and $S_{2}$ switched.

Case 2: The premise of case 1 does not hold. A parallel composition with op being synchronised is impossible, as there is no synchronisation dependence connecting both nodes. Thus, branching is ruled out. Based on Lemma 4.3.15, there exists a CFG path $\pi$ starting in op ${ }^{i}$ and reaching op ${ }^{j}$ (opposite direction accordingly). This path does not include any operation nodes outside of $\mathbf{C}_{i}$ since otherwise, the cut would be left and re-entered, causing a violation of the correctness criterion no reaching back. In particular, for any two paths par $_{O p_{\mathrm{C}}} \stackrel{\pi_{1}}{\longrightarrow} \mathrm{op}^{i}$ and par $_{O p_{\mathrm{C}}} \stackrel{\pi_{2}}{\longrightarrow} \mathrm{op}^{j}$, the traces $t r, \mathrm{tr}_{1}$ and $\operatorname{tr}_{2}$ corresponding to the paths $\pi, \pi_{1}$ and $\pi_{2}$ yield $t r_{1}\left\lceil O p_{\mathbf{C}} \neq t r_{2}\left\lceil O p_{\mathbf{C}}\right.\right.$, as $o p_{p}^{j}$ is an element of the latter but not the first trace. This violates that the synchronisation dependence can be realised. The same holds for $S_{1}$ and $S_{2}$ switched. $\checkmark$

b): Again, let $\operatorname{par}_{X}$ be responsible for the synchronisation dependence between $o p_{p}^{i}$ and $o p_{p}^{j}$. A violation of the possible realisation of a synchronisation dependence 
yields that there are no $\operatorname{tr}_{1} \in \operatorname{traces}\left(P_{1}\right)$ and $\operatorname{tr}_{2} \in \operatorname{traces}\left(P_{2}\right)$ such that $\operatorname{tr}_{1}\lceil X=$ $t r_{2}\left\lceil X\right.$. In particular, as op $\in\left(O p_{\mathrm{C}} \cap X\right)$, a synchronisation of op within $S_{1} \| S_{2}$ would have to involve all four occurrences of op. However, according to $(4, \mathrm{~b}$.), the projection of $S$.main preserves the traces with respect to $X$ up to reaching the cut within $S_{1}$.main. Thus, $o p_{p}^{i}$ and $o p_{p}^{j}$ are not allowed to synchronise within $S_{1}$. A diagonal synchronisation between $o p_{p}^{i}$ and $o p_{p}^{j}$ is impossible as well, as the synchronisation dependence cannot be realised due to $S_{1}$. $\checkmark$

c): A violation of Condition c) is trivially preserved within $S_{1} \| S_{2} . \checkmark$

Figure 4.23 illustrates the allowed and forbidden synchronisations due to the three conditions of the lemma. A solid line depicts an allowed synchronisation, whereas a dotted lines depicts the opposite.

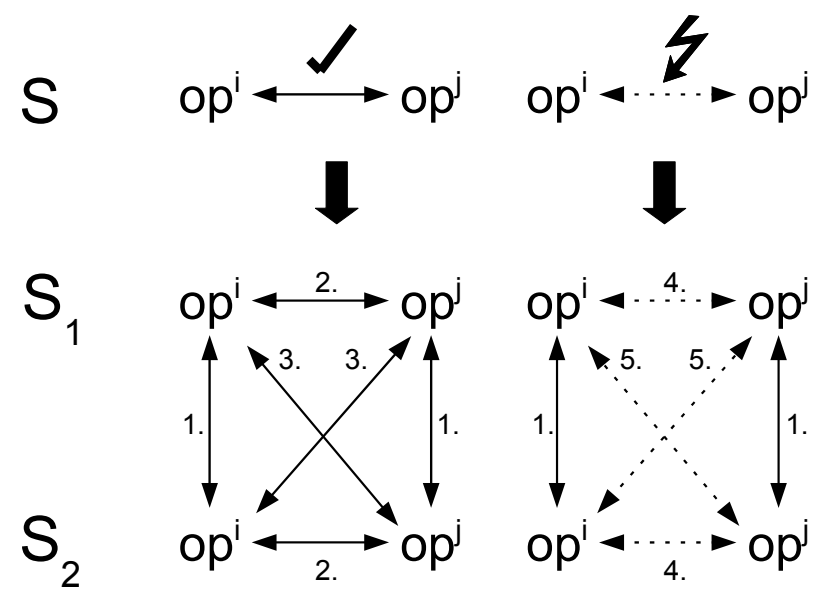

Figure 4.23: Illustration of Theorem 4.3.16

\section{Separating Operations Shared between $O p_{1}$ and $O p_{2}$}

Another aspect which we have to deal with tackles the fact that in general, $O p_{1}$ and $O p_{2}$ are not disjoint. This can lead to one operation being assigned to both, $S_{1}$ and $S_{2}$. We need to ensure that the projection of a CSP process correctly eliminates the subset of occurrences of an operation which are no longer part of the respective component. A projection, keeping the set of all occurrences, is generally insufficient:

Example 4.3.17. Let S.main $:=a \rightarrow b \rightarrow a \rightarrow$ Skip and $\mathbf{C}=\{b\}$ be a valid (single) cut. Then, the first occurrence of a should be an element of $S_{1}$.main whereas the second one should be an element of $S_{2}$.main. A projection of $S$.main on $\{a, b\}$ would result in $S_{1}$.main $=S_{2}$.main $=S$.main and is therefore infeasible. 
As $O p_{1}$ and $O p_{2}$ are not disjoint, we need to separate the occurrences of an operation $o p \in O p_{1} \cap O p_{2}$ within $S_{1}$ from the ones within $S_{2}$. Corresponding to the previous section, we will use one additional address parameter $p_{1}:\{1,2\}$ for any operation with its occurrences distributed over $O p_{1}$ and $O p_{2}$. The parameter is fixed to 1 for occurrences within $S_{1}$ and accordingly fixed to 2 for occurrences within $S_{2}$.

For the event $a$ of Example 4.3.17, we get

- $S_{1}$.main $:=a .1 \rightarrow b \rightarrow$ Skip and

- $S_{2} \cdot \operatorname{main}:=b \rightarrow a .2 \rightarrow$ Skip.

\section{Defining the Sets of Events for $S_{1}$ and $S_{2}$}

Based on the additional parameters and their restrictions, the overall system definition needs to be adapted. First, we observe the following:

- For any op $\notin O p_{\mathrm{C}}$, there exist one (if op $\in\left(O p_{1} \cap O p_{2}\right)$ ) or zero additional address parameters. For simplification, we will denote this possible additional parameter by $p_{1}$. $[v]$ denotes that the value of the parameter $p_{1}$ is set to $v$, if the parameter exists.

- For $o p \in O p_{\mathrm{C}}$, any number of parameters is possible. However, for the set of cut operations, all possible extensions of operations need to be represented in the synchronisation alphabet. This is due to the correctness criterion all-or-none and the fact that the respective addressing is identical for both, $S_{1}$ and $S_{2}$.

Both observations allow for the following definition:

Definition 4.3.18. (Event sets of components)

Let $\mathrm{DG}_{\mathrm{S}}=\left(N, \longrightarrow_{\mathrm{DG}}\right)$ be the control flow graph of a specification $S$, and let $\mathbf{C}=\left(\mathbf{C}_{1}, \mathbf{C}_{2}\right)$ be a cut, yielding the four sets $O p_{1}, O p_{2}, O p_{\mathrm{C}_{1}}$ and $O p_{\mathrm{C}_{2}}$, now possibly comprising additional address parameters. The event sets for the decomposition of $S$ into $S_{1}$ and $S_{2}$ are given by

$$
\begin{array}{lll}
E_{1}:=\bigcup_{o p \in O p_{1}}\{\mid o p \cdot[.[1] \mid\}, & E_{2}:=\bigcup_{o p \in O p_{2}}\{|o p .-[.2]|\}, \\
E_{\mathbf{C}_{1}}:=\left\{\left|O p_{\mathbf{C}_{1}}\right|\right\}, & E_{\mathbf{C}_{2}}:=\left\{\left|O p_{\mathbf{C}_{2}}\right|\right\}
\end{array}
$$

where "_" denotes the original parameters of the channel. Let $E_{\mathbf{C}}:=E_{\mathbf{C}_{1}} \cup E_{\mathbf{C}_{2}}, E_{S_{1}}:=E_{1} \cup E_{\mathbf{C}}$ and $E_{S_{2}}:=E_{2} \cup E_{\mathbf{C}}$.

The following lemma describes that all events shared between $S_{1}$ and $S_{2}$ are elements of $E_{\mathrm{C}}$ :

Lemma 4.3.19. (Common events of $S_{1}$ and $S_{2}$ solely occur in the cut)

Let $E_{1}, E_{2}$ be defined according to Definition 4.3.18. Then:

$$
E_{1} \cap E_{2}=\varnothing .
$$

Proof. Assume that there exists $e \in\left(E_{1} \cap E_{2}\right)$. Then, $e \in\{\mid$ op $\mid\}$ holds for some $o p \in\left(O p_{1} \cap O p_{2}\right)$. Based on the addressing extension for shared operations, op is thus extended by one address parameter of type $\{1,2\}$. Either this value is set to 1 implying $e \notin E_{2}$ or to 2 implying $e \notin E_{1}$, contradiction. 


\subsubsection{Renaming for the Decomposition}

The previous section introduced additional parameters to operations of $S_{1}$ and $S_{2}$, required to ensure an equivalent data flow and control flow between $S$ and $S_{1} \| S_{2}$. These parameters modify the original types of the channels of $S$. In our correctness proof, which is given in Chapter 5, we thus show that $S$ and $S_{1} \| S_{2}$ are equivalent modulo different channel types. As we need to refer to the precise sets of events of a specification, we will from now on write $E_{S}$ to denote the set of events of a specification $S$.

For describing the difference between $E_{S}$ and $E_{S_{i}}$, we introduce

- a function $f$, mapping a channel of $S$ on the corresponding channel within $S_{i}$, now comprising additional parameters and

- two event renaming relations $\mathrm{R}_{1}^{\mathrm{C}}: E_{S} \rightarrow E_{S_{1}}$ and $\mathrm{R}_{2}^{\mathrm{C}}: E_{S} \rightarrow E_{S_{2}}$, applied on the process $S$.main, in order to determine $S_{1}$.main and $S_{2}$.main. ${ }^{3}$

We start with the function $f$ mapping channels of $S$ on channels of $S_{i}$. It implicitly defines a corresponding extension of the declaration parts of the Object- $Z$ schemas, now additionally containing transmission parameters. ${ }^{4}$ According to the notation for transmission parameters, let

$$
\text { op.add }=a d d_{1}: r_{1} ; \ldots ; a d d_{k}: r_{k}
$$

denote the set of address parameters of an operation $o p$, and let

$$
\text { op.orig }=p_{1} d_{1}: s_{1} ; \ldots ; p_{l} d_{l}: s_{l}
$$

with $d_{i} \in\{?, !, \epsilon\}$ (where $\epsilon$ denotes the empty decoration used for simple parameters) the set of original parameters of $o p$, as defined within the interface of $S$ :

Definition 4.3.20. (Renaming of channels)

Let $S$ be a specification, and let $\mathbf{C}=\left(\mathbf{C}_{1}, \mathbf{C}_{2}\right)$. The channel renaming for the decomposition of $S$ into $S_{1}$ and $S_{2}$ is given by

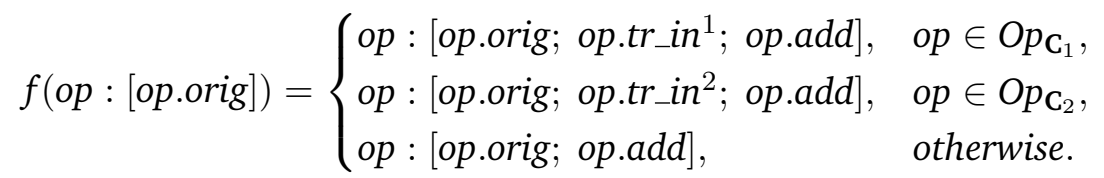

Note that, in the last case, op.add comprises zero or one address parameter whereas in the other cases, the amount is indefinite. Further note that we never leave out any original parameters, as the types of the shared operations have to coincide.

\footnotetext{
${ }^{3}$ Note that in CSP-OZ, according to [Fis00], and in contrast to pure Z, renaming of CSP processes is not restricted to functions - relations can be used as well.

${ }^{4}$ As address parameters are not restricted by the Object-Z part, we omit them in the declaration parts of Object-Z schemas.
} 
Next, we introduce two renaming relations, determining two processes, which are subsequently used for the definition of $S_{1}$.main and $S_{2}$.main. For an operation $o p \in O p$, we let

$$
\text { op.tr_in }=t r_{1} ?: t_{1} ; \ldots ; t r_{n} ?: t_{n}
$$

denote the additional transmission parameters of an arbitrary operation. Moreover, let $a_{i}$ denote the possibly fixed value of the address parameter $a d d_{i}$ according to the restriction of address parameters. The following event renaming is relational, as it maps an event on a set of events. We simply write $o p ? p$ to denote the set $\left\{o p \cdot x \mid x: t_{p}\right\}$. This notation is motivated by the equivalence between $o p ? p \rightarrow P$ and $\square_{x: t_{p}}$ op. $x \rightarrow P$.

Definition 4.3.21. (Renaming of events)

Let $S$ be a specification, and let $\mathbf{C}=\left(\mathbf{C}_{1}, \mathbf{C}_{2}\right)$. The (relational) event renaming for the decomposition of $S$ into $S_{1}$ and $S_{2}$ is given by

$$
\mathrm{R}_{1}^{\mathrm{C}}: E_{S} \rightarrow E_{S_{1}} \text { and } \mathrm{R}_{2}^{\mathrm{C}}: E_{S} \rightarrow E_{S_{2}},
$$

defined as

$$
\mathrm{R}_{1}^{\mathrm{C}}(\text { op.x }):= \begin{cases}\text { op.x.1, } & o p \in\left(O p_{1} \cap O p_{2}\right) \backslash\left(O p_{\mathbf{C}_{1}} \cup O p_{\mathbf{C}_{2}}\right), \\ o p \cdot x ? \operatorname{tr}_{1} \ldots ? t t_{n} \cdot a_{1} \ldots a_{k}, & o p \in O p_{\mathbf{C}} \wedge\left|l^{-1}(o p)\right|>1 \\ o p \cdot x ? \operatorname{tr}_{1} \ldots ? t t_{n}, & o p \in O p_{\mathbf{C}} \wedge\left|l^{-1}(o p)\right|=1 \\ o p \cdot x, & \text { otherwise }\end{cases}
$$

and

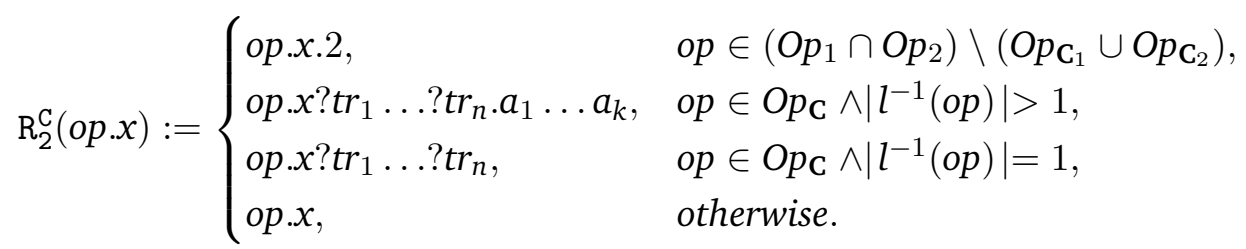

Graphically explained, the renaming introduces additional transmission and address parameters to the original events, if required. For operations not represented in the cut, no transmission parameters are introduced. Shared operations of $O p_{1}$ and $O p_{2}$ receive one address parameter fixed to 1 and 2, respectively, whereas local operations to one specification do not receive any additional parameters. For the cut, we introduce a possibly empty set of transmission parameters. For the address parameters, we separate operations with multiple occurrence in the cut from the ones with single occurrence: the first operations receive additional address parameters, whereas the latter ones do not.

The previous definitions allow us to give the final definitions for the interfaces and CSP parts of $S_{1}$ and $S_{2}$. We start with a modification of Definition 4.3.2, which now takes the channel renaming $f$ into account:

Definition 4.3.22. (Interfaces of components, final definition)

Let $\mathrm{DG}_{\mathrm{S}}=\left(N, \longrightarrow_{\mathrm{DG}}\right)$ be the dependence graph of a specification $S$, and let $\mathbf{C}=\left(\mathbf{C}_{1}, \mathbf{C}_{2}\right)$ be a cut. Let $f$ be the channel renaming function according to Definition 4.3.20. The interfaces for the decomposition of $S$ into $S_{1}$ and $S_{2}$ are defined as 
- $S_{1} . I:=\left.f(I)\right|_{\left(O p_{1} \cup O p_{\mathrm{C}}\right)}$ and

(Interface for $S_{1}$ )

- $S_{2} . I:=\left.f(I)\right|_{\left(O p_{2} \cup O p_{\mathrm{C}}\right)}$.

(Interface for $S_{2}$ )

In order to modify the CSP parts of the components according to Definition 4.3.4, we apply the event renaming on main. Note that the following holds for any renaming relation $R$ ([Sch09]):

$$
(e \rightarrow P) \llbracket \mathrm{R} \rrbracket=e^{\prime}: \mathrm{R}(e) \rightarrow P \llbracket \mathrm{R} \rrbracket .
$$

Definition 4.3.23. (CSP parts of components, final definition)

Let $\mathrm{DG}_{\mathrm{S}}=\left(N, \longrightarrow_{\mathrm{DG}}\right)$ be the dependence graph of a specification $S$, and let $\mathbf{C}=\left(\mathbf{C}_{1}, \mathbf{C}_{2}\right)$ be a cut. Let $\mathrm{R}_{1}^{\mathrm{C}}$ and $\mathrm{R}_{2}^{\mathrm{C}}$ be the event renaming relations according to Definition 4.3.21. The CSP parts for the decomposition of $S$ into $S_{1}$ and $S_{2}$ are defined as

- $S_{1} \cdot \operatorname{main}:=\left.\left(S \cdot \operatorname{main} \llbracket \mathrm{R}_{1}^{\mathrm{C}} \rrbracket\right)\right|_{E_{S_{1}}}$ and

(CSP part for $\left.S_{1}\right)$

- $S_{2} \cdot \operatorname{main}:=\left.\left(S \cdot \operatorname{main} \llbracket \mathrm{R}_{2}^{\mathrm{C}} \rrbracket\right)\right|_{E_{S_{2}}}$.

(CSP part for $\left.S_{2}\right)$

In Figure 4.19, we implicitly modified the channel change_m of Increaser after the introduction of one transmission parameter. As address parameters are not required for the decomposition, the specification's decomposition is final.

Summarising the previous definition, we are now able to give the final definition for the thorough decomposition of $S$ into $S_{1}$ and $S_{2}$.

\subsubsection{Definition of the Decomposition}

After ensuring a correct data flow within $S_{1} \| S_{2}$ based on the introduction of additional transmission parameters and ensuring a correct control flow based on additional address parameters, we finally give the definition of the thorough decomposition of $S$ into components $S_{1}$ and $S_{2}$ by modifying Definition 4.3.8:

Definition 4.3.24. (Decomposition with respect to a cut, final definition)

Let $\mathrm{DG}_{\mathrm{S}}=\left(N, \longrightarrow{ }_{\mathrm{DG}}\right)$ be the dependence graph of a specification $S$, and let $\mathbf{C}=\left(\mathbf{C}_{1}, \mathbf{C}_{2}\right)$ be a cut. Let

$$
O p_{1}, O p_{2}, O p_{\mathbf{C}_{1}}, O p_{\mathrm{C}_{2}}, O p_{\mathbf{C}}
$$

be defined according to Definition 4.3.1. The decomposition of $S$ with respect to $\left(\mathbf{C}_{1}, \mathbf{C}_{2}\right)$ into $S_{1}$ and $S_{2}$ is defined as

\begin{tabular}{|lr}
$S_{1}$ & \\
$S_{1} . I$ & [according to Definition 4.3.22] \\
$S_{1} . \mathrm{main}$ & [according to Definition 4.3.23] \\
$S_{1}$. State & [according to Definition 4.3.5] \\
$S_{1}$. Init & [according to Definition 4.3.6] \\
$S_{1} . \mathrm{op}$ & [according to Definition 4.3.10] \\
\hline
\end{tabular}




\begin{tabular}{|lr}
$S_{2}$ & \\
$S_{2} . I$ & [according to Definition 4.3.22] \\
$S_{2} . \mathrm{main}$ & [according to Definition 4.3.23] \\
$S_{2}$. State & [according to Definition 4.3.5] \\
$S_{2}$. Init & [according to Definition 4.3.6] \\
$S_{2} . \mathrm{op}$ & [according to Definition 4.3.10] \\
\hline
\end{tabular}

The system, generated from the components, is defined according to Definition 4.3.8 as the parallel composition of both classes, synchronising on the set of cut events:

$$
S_{1} \|_{E_{\mathrm{C}}} S_{2} \text {. }
$$

For the remainder of this thesis, we let $E_{S^{\prime}}:=E_{S_{1}} \cup E_{S_{2}}$ and $O p^{\prime}:=O p_{1} \cup O p_{2} \cup O p_{\mathrm{C}}$. The following theorem states the main result of this thesis. The correctness proof will be shifted to the next chapter.

Theorem 4.3.25. (Correctness of the decomposition)

Let $S$ be a specification, and let $\mathbf{C}=\left(\mathbf{C}_{1}, \mathbf{C}_{2}\right)$ be a cut, yielding a decomposition into $S_{1}$ and $S_{2}$ according to Definition 4.3.24. Then, the following holds:

$$
S={ }_{T}\left(S_{1} \|\left.\right|_{E_{\mathrm{C}}} S_{2}\right) \llbracket \mathrm{R}^{\prime} \rrbracket,
$$

where $\mathrm{R}^{\prime}: E_{S^{\prime}} \rightarrow E_{S}$ is defined as

$$
\mathrm{R}^{\prime}\left(\text { op.x.t. } t_{1} \ldots t_{n} \cdot a_{1} \ldots a_{k}\right):=o p \cdot x
$$

with $t_{i}$ denoting the values for the possible transmission parameters of op and $a_{i}$ the values for its possible address parameters.

Based on several lemmas and some additional prearrangements, the proof is given in Chapter 5, Section 5.6. The next section illustrates the decomposition on our case study of a candy machine. It is based on the single cut $\mathbf{C}:=\{$ switch $\}$.

\subsubsection{Candy Machine Revisited: Decomposition}

Recall the main case study of this thesis, the specification of a candy machine, as given in Figure 2.3. We already identified the set $\mathbf{C}:=\{$ switch $\}$ to be a valid single cut in Section 4.2.4.

First, the definition for $\mathbf{P h}_{1}, \mathbf{C}_{1}$ and $\mathbf{P h}$ yields

- $O p_{1}=\{$ pay, payout, abort $\}$,

- $O p_{\mathrm{C}}=\{$ switch $\}$ and

- $O p_{2}=\{$ select, order, term, deliver $\}$.

The projections of S.main on the remaining sets of events, 
- $S_{1} \cdot \operatorname{main}:=\left.S \cdot \operatorname{main}\right|_{\{\mid \text {pay,payout,abort,switch } \mid\}}$ and

- $S_{2}$.ma in $:=S . m a$ in $\left.\right|_{\{\mid \text {switch,select,order,term,deliver } \mid\}}$,

lead to

CandyMachine ${ }_{1}$

$[\ldots]$

main $\stackrel{c}{=}$ pay?coin $\rightarrow$ main $\square$ Payout $\square$ switch $\rightarrow$ Skip

Payout $\stackrel{c}{=}$ payout?coin $\rightarrow$ Payout $\square$ abort $\rightarrow$ Skip

$[\ldots]$

CandyMachine 2

$[\ldots]$

main $\stackrel{c}{=}$ Skip $\square$ switch $\rightarrow$ Select

Select $\stackrel{c}{=}($ select?ca $\rightarrow($ Select $\square$ Order $)) \square$ Deliver

Order $\stackrel{c}{=}$ order $\rightarrow$ Select

Deliver $\stackrel{c}{=}$ deliver?ca $\rightarrow$ Deliver $\square$ term?rest $\rightarrow \mathrm{Skip}$

$[\ldots]$

after applying several simplifications. For the sets of state variables of CandyMachine ${ }_{1}$ and CandyMachine 2 , we get

- $S_{1} \cdot V=\{$ sum, paid, credits $\}$ and

- $S_{2} . V=\{$ credits, items, selected $\}$.

$S_{1} . V$ and $S_{2} . V$ determine the respective state schemas. The initial state schemas are given by

$$
\begin{aligned}
S_{1} \cdot \text { Init }= & \exists \text { selected }: \text { Candies, } \text { items }: \text { seq Candies } \bullet \\
& (\text { sum }=0 \wedge \text { paid }=\langle\rangle \wedge \text { items }=\langle\rangle) \\
\equiv & (\text { sum }=0 \wedge \text { paid }=\langle\rangle)
\end{aligned}
$$

and

$$
\begin{aligned}
S_{2} . \text { Init }= & \exists \text { sum }: \mathbb{N} \text {, paid }: \text { seq Coins, credits }: \mathbb{N} \\
& (\text { sum }=0 \wedge \text { paid }=\langle\rangle \wedge \text { items }=\langle\rangle) \\
\equiv & \text { items }=\langle\rangle .
\end{aligned}
$$

In order to determine the operation schemas of the components, we first need to compute the set of cut variables with respect to $\mathrm{C}_{1}=\{$ switch $\}$. The operation schema switch modifies three different variables, namely sum, credits and paid. However, only one of them is subsequently referenced: credits. Based on the three data dependences by reason of credits, 
- switch $\stackrel{\mathrm{dd}}{--\rightarrow(\text { credits })}$ select,

- switch $\stackrel{\mathrm{dd}}{-\rightarrow}$ (credits) order and

- switch $\stackrel{\mathrm{dd}}{-\rightarrow}$ (credits) term,

we get $C V_{1}=\{$ credits $\}$. Therefore, switch needs to be extended by one additional transmission parameter $\operatorname{tr}_{c}: \mathbb{N}$. We are now able to define CandyMachine ${ }_{1}$.switch and CandyMachine $_{2}$. switch:
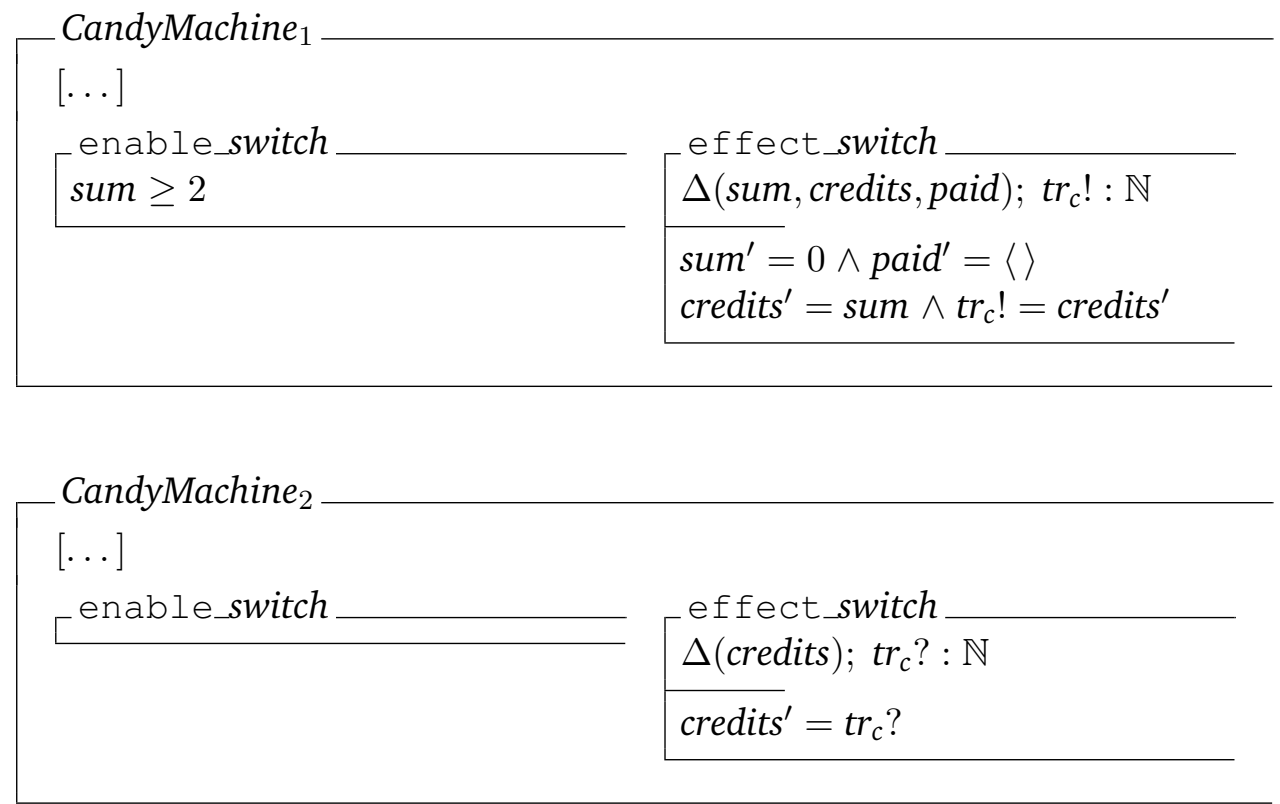

As the sole cut operation switch only occurs once in the specification, no address parameters are required. We remain to apply the renaming of the channel switch and all of its occurrences within S.main, according to the introduction of the sole transmission parameter. The final decomposition is depicted in Figures 4.24 and 4.25.

When dealing with the identification of reasonable decompositions, Chapter 6 introduces a bigger case study, consisting of several classes and requiring address parameters as well as transmission parameters.

\subsubsection{Improvement of the Decomposition}

Up to now, we defined a valid decomposition of a specification, based on a fragmentation of its dependence graph. The given correctness criteria exclude invalid decompositions, thus restricting the set of possible decompositions.

In Section 4.3.1, we defined a restriction of the initial state schema of $S$ on the possible initial valuations of the generated components $S_{1}$ and $S_{2}$. The implementation of our decomposition approach is based on this specific definition and needs to take any initial 


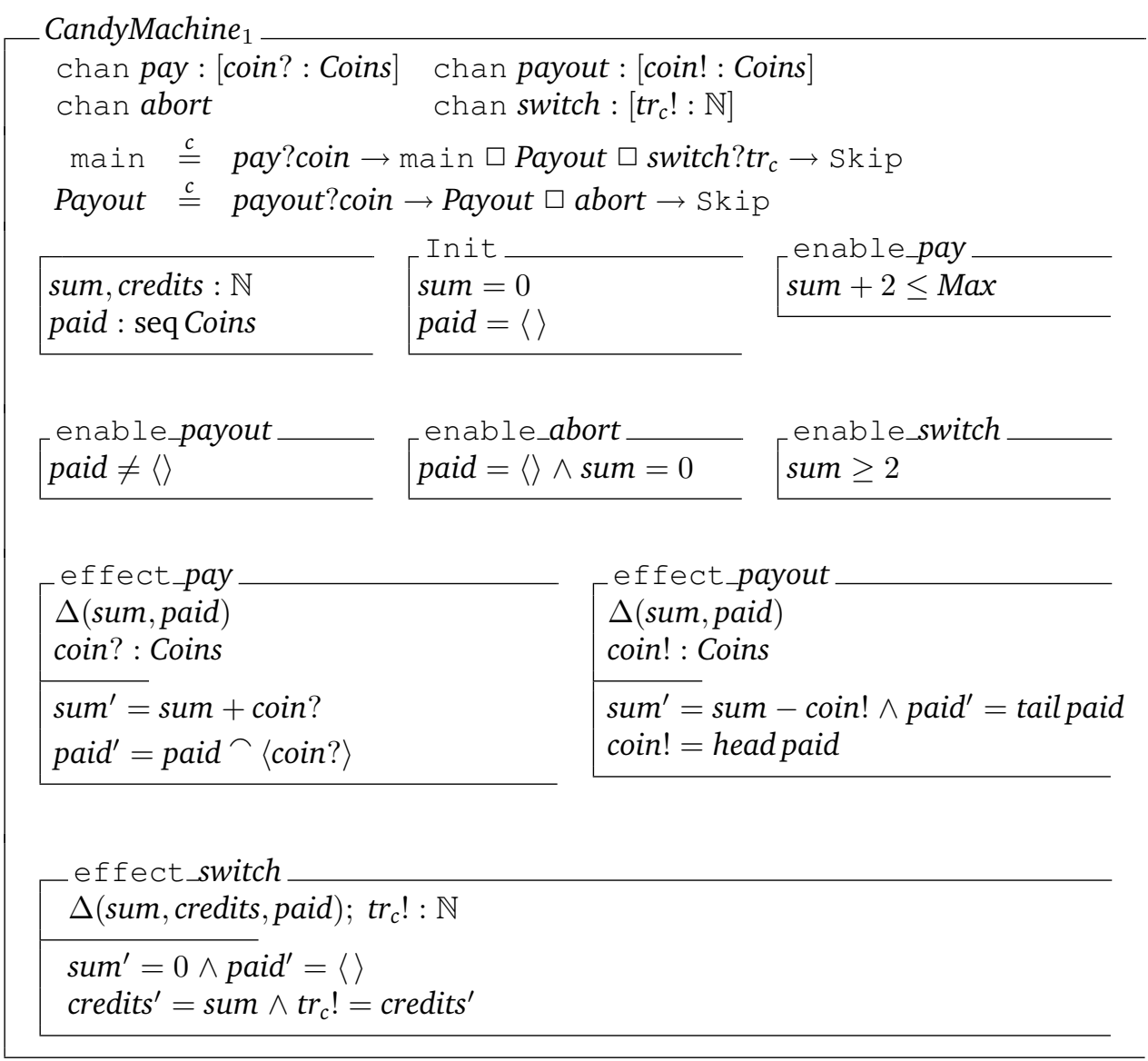

Figure 4.24: Decomposition of the candy machine, first component

data dependence into account. The definition can, however, slightly by altered and improved.

The specification CandyMachine comprises an initial state predicate items $=\langle\rangle$, which forms the source of three initial data dependences:

- init $\stackrel{\text { idd }}{--{ }_{(\text {items })}}$ term, based on enable_term $=[$ items $=\langle\rangle]$,

- init $\stackrel{\text { idd }}{--\rightarrow_{(i t e m s)}}$ deliver, based on (amongst others) enable_term $=[$ items $\neq\langle\rangle]$ and

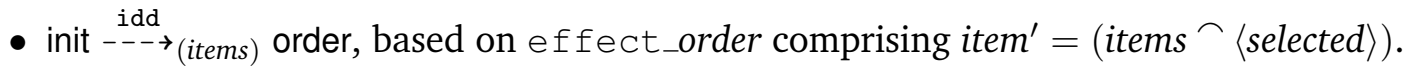

We identified \{switch\} as a valid single cut in Sections 4.2.4 and 4.3.6 due to the fact that all of these three initial data dependences do not violate the correctness criterion no crossing. The reason is as follows: the variable items is never modified or referenced in any operation schema of $O p_{1} \cup O p_{\mathrm{C}}$. In particular, items $\notin S_{1} . V$. Therefore, items 


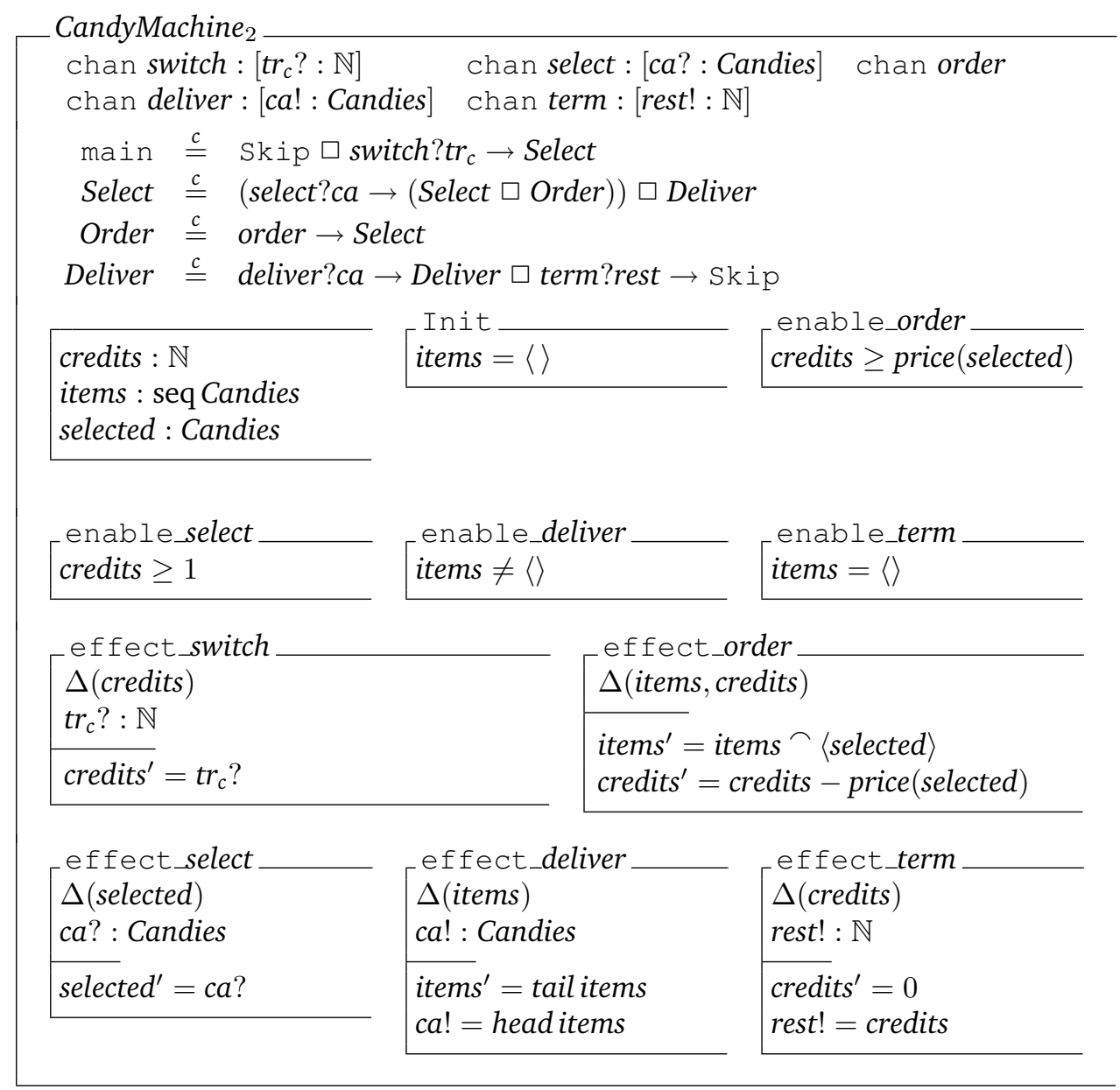

Figure 4.25: Decomposition of the candy machine, second component

does not influence the behaviour of $S_{1}$ at all. In this case, the respective initial state predicate can completely be eliminated from $S_{1}$. Init and the corresponding initial data dependence can safely be neglected.

Indeed, this elimination of an initial data dependence is only possible for corresponding predicates not being related to the variables of $S_{1}$ at all. These observations serve as the basis for the following definitions.

First, when explicitly dealing with predicates within a CSP-OZ specification, we do not refer to the single top-level predicate of an operation but rather to its atomic sub-predicates. This is according to [Brü08]. For op $\in$ Op, the set Atoms(op) depicts the set of all atomic predicates such that the conjunction of all these predicates yields the predicate part of $o p$. We use the same notation for the initial state schema:

$$
\bigwedge_{p \in \text { Atoms(op.pred) }} p=\text { op.pred and } \bigwedge_{p \in \text { Atoms(Init) }} p=\text { Init. }
$$


Next, we define an equivalence relation on $S . V$ and a closure set of a state variable with respect to this relation. Let $\operatorname{vars}(p)$ denote the set of state variables, occurring in the predicate $p$ :

Definition 4.3.26. (Initial closure of state variables) Let $S$ be a specification. We define an equivalence relation $\mathcal{R}$ over $(S . V \times S . V) b^{5}$

$$
\mathcal{R}:=\{(x, y) \mid \exists a \in \operatorname{Atoms}(\text { Init }) \bullet x, y \in \operatorname{vars}(a)\} \cup \operatorname{ld}_{S . V} .
$$

For any $x \in S . V$, the initial closure of $x$ is inductively defined as the set $\operatorname{InitClos}(x)$, satisfying the following two conditions:

- $x \in \operatorname{InitClos}(x)$,

- $y_{1} \in \operatorname{InitClos}(x) \wedge\left(y_{1}, y_{2}\right) \in \mathcal{R} \Rightarrow y_{2} \in \operatorname{InitClos}(x)$.

$\mathcal{R}$ relates any two state variables such that there exists an atomic predicate within Init containing both variables. The initial closure of a state variable $x$ is the set of all state variables, directly or indirectly influencing $x$ within the initial state schema.

Example 4.3.27. Let $S$ be a specification, $S . V=\{x, y, z\}$ with all elements of type $\mathbb{N}$, and let $S$. Init $=(x>2) \wedge(x<y) \wedge(z>5)$. Then, $\mathcal{R}=\{(x, y),(y, x)\} \cup \operatorname{ld}_{\{x, y, z\}}$. This yields $\operatorname{InitClos}(z)=\{z\}$ and $\operatorname{InitClos}(x)=\operatorname{InitClos}(y)=\{x, y\}$.

Now let $S$. Init $=(x=y) \wedge(y=z)$. Then, $\mathcal{R}=\{(x, y),(y, x),(y, z),(z, y)\} \cup \operatorname{ld}_{\{x, y, z\}}$. This yields $\operatorname{InitClos}(x)=\operatorname{InitClos}(y)=\operatorname{InitClos}(z)=\{x, y, z\}$.

These considerations do not influence Definition 4.3.6. The correctness proof in Chapter 5 shows the following: we can safely neglect all initial data dependences originating from an atomic predicate $a$, such that $\operatorname{InitClos}(x) \subseteq\left(S_{2} . V \backslash S_{1} . V\right)$ for all $x \in \operatorname{vars}(a)$.

In our specific case, $\operatorname{InitClos}($ items $)=\{$ items $\}$ and $\{$ items $\} \subseteq\left(S_{2} . V \backslash S_{1} . V\right)$ holds. Thus, the three previously identified initial data dependences originating from items $=\langle\rangle$ can indeed be neglected, justifying the correctness of the cut $\{$ switch $\}$. In particular, the predicate items $=\langle\rangle$ is already removed from CandyMachine $e_{2}$. Init by applying our definition for a decomposition and further simplifications on CandyMachine ${ }_{2}$. Init.

We pointed out an optimisation for the decomposition in the following sense: some data dependences do not need to be considered when the correctness criterion no crossing is validated. Thus, a larger set of valid decompositions is possible.

\subsection{Decomposition for the General Case: Number Swapper}

We recall the small case study of a number swapper from Chapter 2 and slightly adapt it as displayed in Figure 4.26: the specification swaps two natural numbers $a$ and $b$ with $a$ initially possessing the value 1 and $b$ continuously receiving a new value as an input. The protocol starts by inputting the new value for $b$, subsequently swaps both numbers and outputs the new value of $b$. As Swapper.ma in restarts, the specification does not allow for the definition of a single cut.

\footnotetext{
${ }^{5}$ For any set $X$, we let $\operatorname{ld}_{X}$ denote the identity on $X$, that is, $\operatorname{ld}_{X}:=\{(x, x) \mid x \in X\}$.
} 


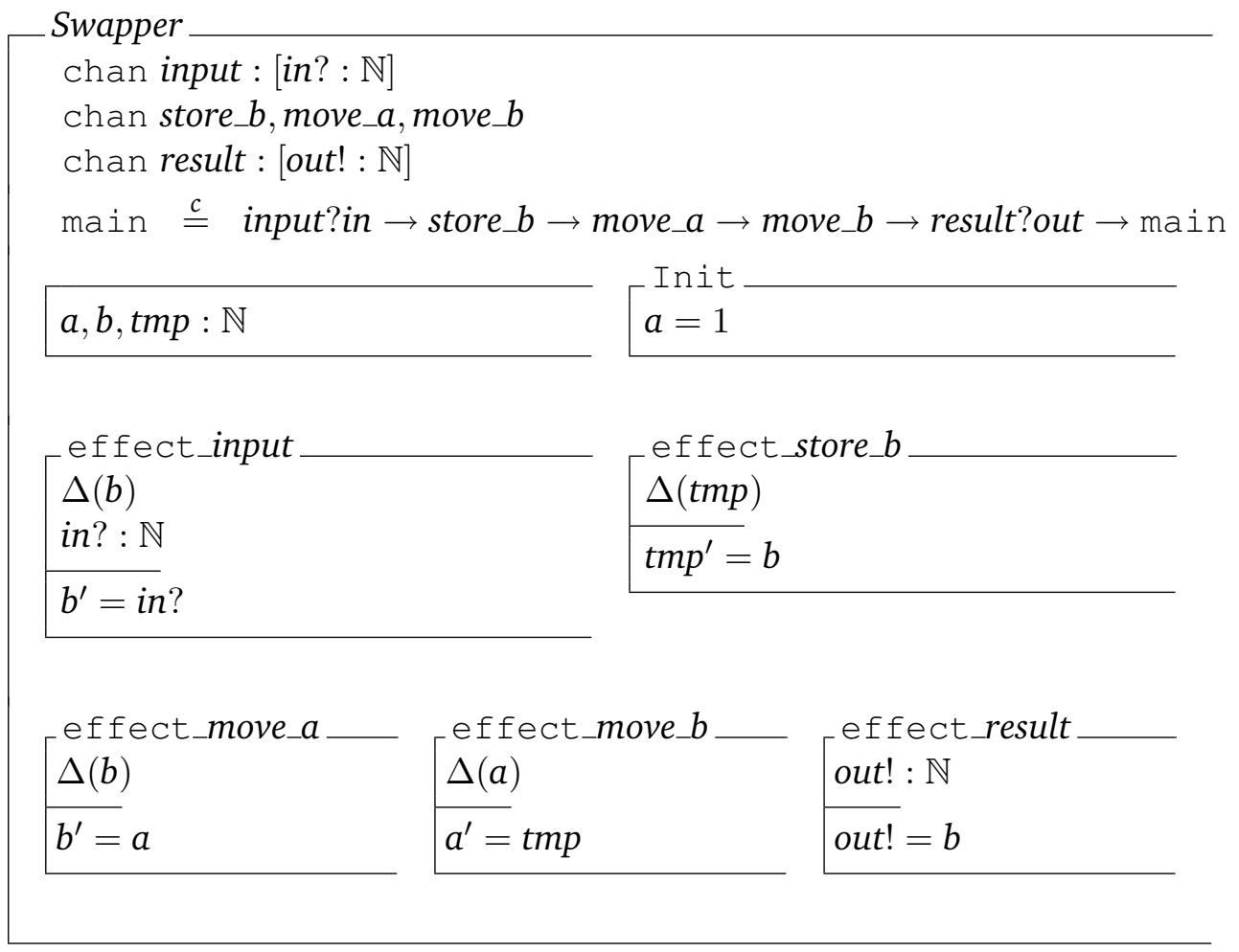

Figure 4.26: CSP-OZ specification for swapping two numbers, extended

A valid (general) cut for this specification is given by $\left(\mathbf{C}_{1}, \mathbf{C}_{2}\right)$ with $\mathbf{C}_{1}=\{$ store_b $\}$ and $\mathbf{C}_{2}=\{$ result $\}$. The definition yields

- $O p_{1}=\{$ input $\}$,

- $O p_{\mathbf{C}_{1}}=\left\{\right.$ store $\left.\_b\right\}$,

- $O p_{2}=\{$ move_a, move_b $\}$ and

- $O p_{\mathrm{C}_{2}}=\{$ result $\}$.

For the sets of state variables, we get

- $S_{1} . V=\{b, t m p\}$ and

- $S_{2} \cdot V=\{a, b, t m p\}$.

The initial state schemas are given by

$$
\begin{array}{ll}
S_{1} \text {.Init }=\exists a: \mathbb{N} \bullet(a=1) & \equiv \text { true, } \\
S_{2} \text {.Init }=\exists b: \mathbb{N}, \operatorname{tmp}: \mathbb{N} \bullet(a=1) & \equiv a=1 .
\end{array}
$$




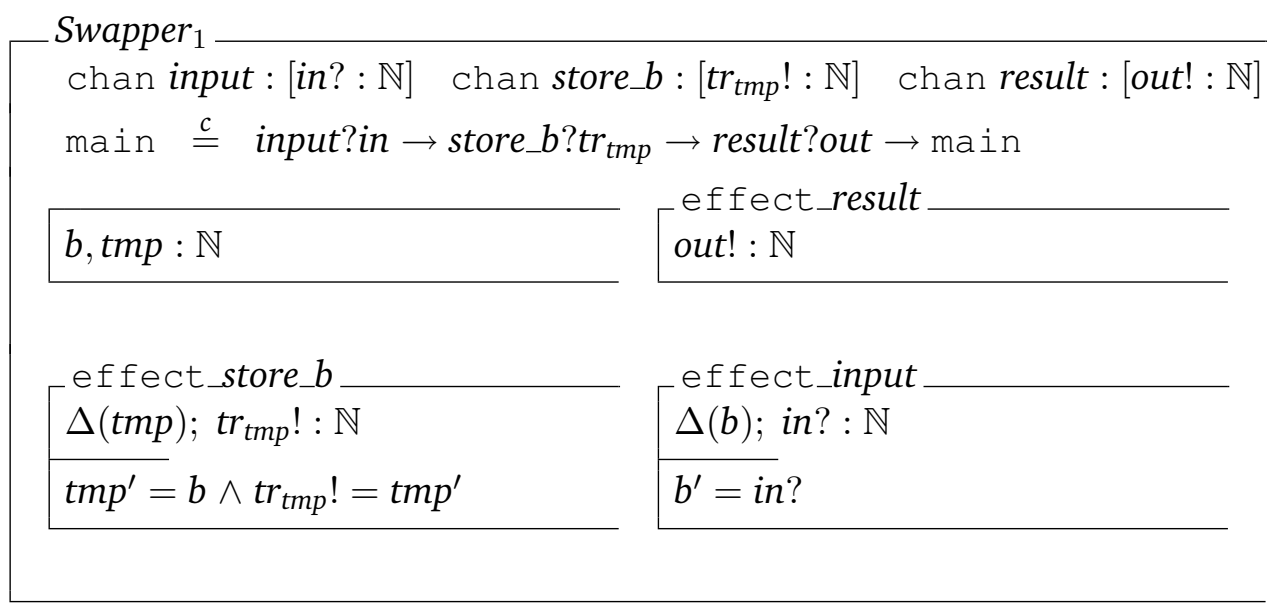

Figure 4.27: Decomposition of the number swapper, first component

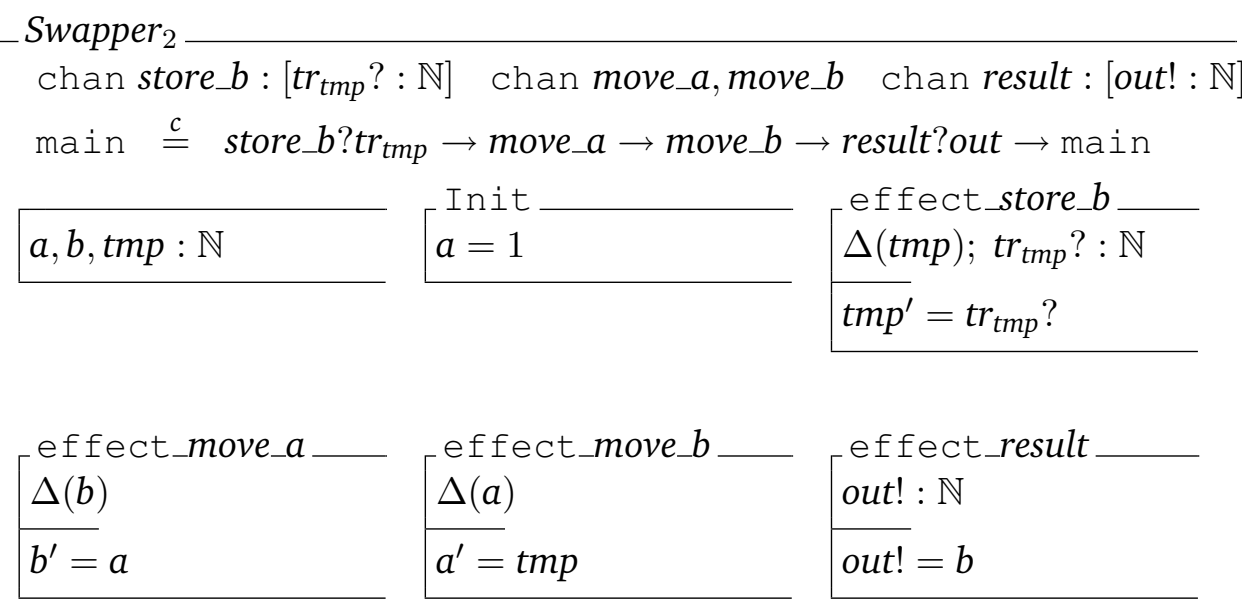

Figure 4.28: Decomposition of the number swapper, second component 
As InitClos $(a)=\{a\}$ and $\{a\} \subseteq\left(S_{2} . V \backslash S_{1} . V\right)$, the initial data dependence init idd $_{-\rightarrow-\rightarrow}(a)$ move $\_$a is not cut-crossing. Based on the data dependence store $\_b-{ }_{--\rightarrow} \rightarrow(t m p)$ move $\_$, we get $C V_{1}=\{t m p\}$, necessitating one transmission parameter $t_{\text {tmp }}$. As the operation result does not modify any state variable, $C V_{2}=\varnothing$ holds. No addressing extension is required, thus leading to the final decomposition as given in Figures 4.27 and 4.28.

The correctness property on the specification as described in Figure 4.29 models that the value received by input corresponds to the output value of result in the next iteration of the protocol. Model checking this property with FDR2 yields its validity for Swapper as well as Swapper $1 \|_{\{\mid \text {store_b,result } \mid\}}$ Swapper $_{2}$.

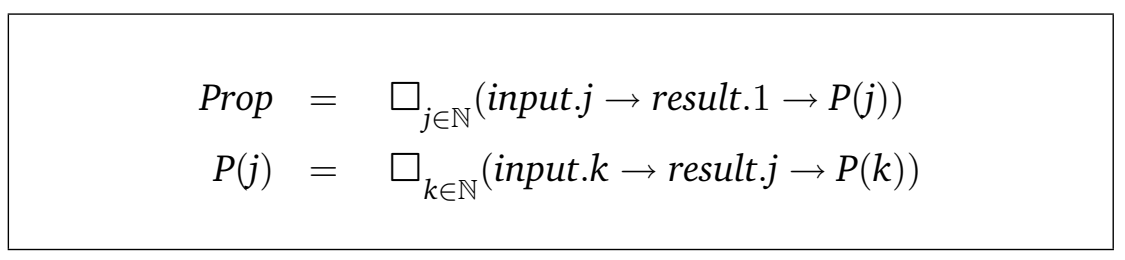

Figure 4.29: Correctness requirement for Swapper

\subsection{Related Work}

The technique proposed in this chapter targets the manual decomposition of a given specification into two components. These subsystems are used in a compositional verification framework which is based on two assume-guarantee proof rules. The approach is closely related to several works, with some of them described next.

The dependence analysis, as given in Section 2.3, is based upon the methodology by Brückner [Brü08] for slicing CSP-OZ specifications. Besides applying a similar analysis of a specification, slicing does not decompose a given specification but rather eliminates irrelevant parts from it. These irrelevant specification elements depend on a certain property under interest, the slicing criterion. A correct decomposition in our context is independent of the verification properties. The decomposition approach is more closely related to program chopping [RR95]: chopping is likewise based on the analysis of a (program) dependence graph and tries to identify program points affecting a certain target node based on a specific source node.

Several works in the context of formal specifications present techniques for decomposing a given system into several components. Recently, Butler [But09] sketched a technique for composing Event-B models and decomposing them into sub-models. Here, events can be split, without allowing common variables to different machines. Similar to our approach dealing with transmission parameters, shared parameters are used to pass the influence of one to another machine. The technique is not applied in the context of compositional verification, but rather in the scope of model refinements. In the context of CSP ||B and for separate checking of divergence freedom of a model, Evans, Schneider and Treharne [STE05] developed a methodology to decompose CSP||B specifications 
into smaller subsystems, called chunks. They can consist of a set of CSP processes or contain $\mathrm{B}$ machines as well. The decomposition is conducted by examining the existing subsystems and parallel components of a CSP||B specification.

The technique closest to ours is the one by Alur and Nam [NA06, AMN05, Nam07] dealing with assume-guarantee-based reasoning in the context of symbolic model checking. Using symbolic transition systems (STS) as the semantic model, the authors fully automatically decompose and verify a system. A decomposition of a STS yields a set of symbolic modules, now comprising additional boolean input- and output variables which are similar to our transmission parameters. The choice of the decomposition is carried out by an automatic partitioning of the set of boolean variables of the STS and it is based on an equal distribution of the set of variables along with a minimisation of required inputs and outputs. The approach is also based on the $\mathrm{L}^{*}$ algorithm, using a generalised version of rule (B-AGR) in the validation process. In their semantic domain solely dealing with boolean variables, the authors do not incorporate a dependence analysis based on data flow and control flow, and they do not tackle communication and synchronisation aspects of a specification. The decomposition is based on one particular heuristic which does not take the alphabet size of the assumption into account. As the decomposition is performed automatically, it is impossible to lead the framework to a superior decomposition by hand which does not satisfy the constraints for an equal distribution and minimisation.

Another related work discusses the usefulness of assume-guarantee reasoning. The authors investigate the possible decompositions of a program specified as a labelled transition system, based on several case studies and model checkers [CAC06]. The results show that assume-guarantee reasoning outperforms monolithic verification in only a few cases. Two conclusions can be drawn from this work: assume-guarantee reasoning is not in general more effective than direct model checking. Moreover, its effectiveness highly depends on the choice of the decomposition. The authors state that analysts need some guidance to identify those decompositions which are indeed less time- and memory consuming. Chapter 6 will provide some theory on how this can be achieved.

Beforehand, the next chapter will show correctness of our approach by particularly proving Theorem 4.3.25. 


\section{Correctness of the Decomposition}

\section{Contents}

5.1 Ensuring Correct Synchronisation . . . . . . . . . . . . . 113

5.2 Correctness for the CSP Part . . . . . . . . . . . . . . . 119

5.2.1 Properties of the Decomposition: CSP Part . . . . . . . . . . . 119

5.2.2 Correctness of the Decomposition: CSP part . . . . . . . . . . 132

5.3 Correctness for the Object-Z Part _ . . . . . . . . . . . 138

5.3.1 Properties of the Decomposition: Object-Z Part . . . . . . . 140

5.3.2 Correctness of the Decomposition: Object-Z part . . . . . . . 146

5.4 Correctness of the Renaming for the Decomposition . . . . . . . 159

5.5 CSP Laws for Parallel Composition . . . . . . . . . . . . . . 164

5.6 Proof of the Main Theorem . . . . . . . . . . . . . . . . 166

The previous chapter introduced a technique on how to decompose a given CSP-OZ specification into two components, based on an analysis of the specification's dependence graph. Theorem 4.3.25 states the main result of this thesis: in our semantic domain of the CSP traces model, the original specification and its decomposition are trace-equivalent. The result is essential and ensures the following: for a property $P$, specified as a CSP process,

$$
\left(P \sqsubseteq_{T} S\right) \Leftrightarrow\left(P^{\prime} \sqsubseteq_{T}\left(S_{1} \|_{E_{\mathrm{C}}} S_{2}\right)\right) .
$$

Here, we need to refer to a process $P^{\prime}$, resulting from the process $P$ after a renaming with respect to the set of all additional parameters. Recall that $S_{1}$ and $S_{2}$ already comprise transmission parameters and address parameters according to Section 4.3.4. $P^{\prime} \sqsubseteq_{T}\left(S_{1} \| S_{2}\right)$ can be deduced from $A \sqsubseteq_{T} S_{1}$ and $P^{\prime} \sqsubseteq_{T}\left(A \| S_{2}\right)$ within the compositional learning framework, introduced in Chapter 3. Thus, correctness of the compositional proof rules (B-AGR) and (P-AGR) along with Theorem 4.3.25 yield the overall correctness of our approach.

Correctness of (B-AGR) and (P-AGR) were already shown in Chapter 3. The verification of Theorem 4.3.25 will be carried out in the present chapter. The main strategy for the proof uses the compositional semantics of CSP-OZ specifications in terms of $\mathrm{CSP}_{Z}$ according to Figure 2.7: the traces of a CSP-OZ specification $S$ are given by

$$
\text { traces(S.main } \left.\|_{E_{S}} S . O Z\right) \text {. }
$$

Figure 5.1 illustrates the individual proof steps. Precisely, we show: 


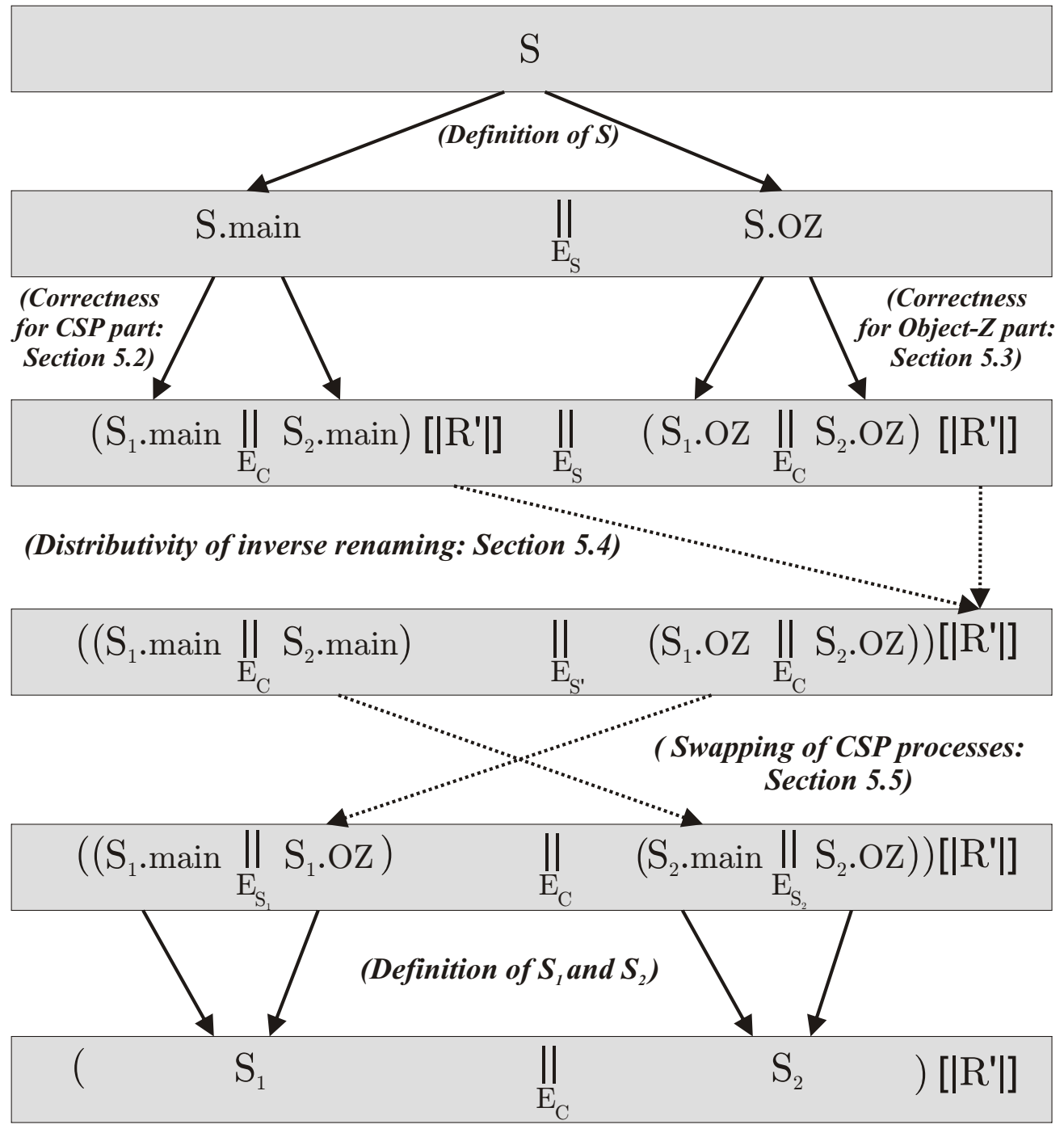

Figure 5.1: Illustration of the steps of the correctness proof

Correctness for the CSP Part, Section 5.2: Based on the compositional semantics of CSP-OZ, $S=_{T}$ (S.main $\|_{E_{S}} S . O Z$ ) holds. In order to refer to the individual parts of the components $S_{i}$, we first need to decompose the CSP part and show that the original CSP part is trace equivalent to its decomposition modulo the (inverse) renaming relation, that is,

$$
\text { S.main }=_{T}\left(S_{1} \cdot \text { main } \|_{E_{\mathrm{C}}} S_{2} \cdot \operatorname{main}\right) \llbracket \mathrm{R}^{\prime} \rrbracket \text {. }
$$

Correctness for the OZ Part, Section 5.3: Accordingly, we have to show correctness for 
the decomposition of the Object-Z part. However,

$$
S . O Z={ }_{T}\left(S_{1} . O Z \|_{E_{\mathrm{C}}} S_{2} . O Z\right) \llbracket \mathrm{R}^{\prime} \rrbracket
$$

does not hold in general: traces of the Object-Z part alone do not adhere to the CSP part. We need to take the orderings of events with respect to the CSP part into account and show $S . O Z=_{T}\left(S_{1} . O Z \|_{E_{\mathrm{C}}} S_{2} . \mathrm{OZ}\right) \llbracket \mathrm{R}^{\prime} \rrbracket$ for the set of traces conforming to the CSP part.

Distributivity of Inverse Renaming, Section 5.4: After showing the individual correctness of both decompositions, we have to distribute the inverse renaming relation $\mathrm{R}^{\prime}$ over the parallel composition $E_{S}$. Thus, we show

$$
\begin{aligned}
& \left.\left(S_{1} \cdot \text { main } \|_{E_{\mathrm{C}}} S_{2} \cdot \mathrm{main}\right) \llbracket \mathrm{R}^{\prime} \rrbracket\right] \|_{E_{S}}\left(S_{1} \cdot \mathrm{OZ} \|_{E_{\mathrm{C}}} S_{2} \cdot \mathrm{OZ}\right) \llbracket \mathrm{R}^{\prime} \rrbracket={ }_{T} \\
& \left(\left(S_{1} \cdot \mathrm{main} \|_{E_{\mathrm{C}}} S_{2} \cdot \mathrm{main}\right) \|_{E_{S^{\prime}}}\left(S_{1} \cdot O Z \|_{E_{\mathrm{C}}} S_{2} \cdot O Z\right)\right) \llbracket \mathrm{R}^{\prime} \rrbracket .
\end{aligned}
$$

Redistribution of CSP Processes, Section 5.5: Now being able to refer to $S_{i}$.main and $S_{i} . O Z$ without the need for considering the renaming, we have to swap $S_{2}$.main and $S_{1} . O Z$ to step from the parallel composition of the CSP parts and Object-Z parts to the parallel composition of the components $S_{1}$ and $S_{2}$. We show

$$
\begin{aligned}
& \left(S_{1} \text {.main } \|_{E_{\mathrm{C}}} S_{2} \text {.main }\right) \|_{E_{S^{\prime}}}\left(S_{1} \cdot \mathrm{OZ} \|_{E_{\mathrm{C}}} S_{2} . \mathrm{OZ}\right)={ }_{T} \\
& \left(S_{1} \text {.main } \|_{E_{S_{1}}} S_{1} \cdot \mathrm{OZ}\right) \|_{E_{\mathrm{C}}}\left(S_{2} \cdot \mathrm{main} \|_{E_{S_{2}}} S_{2} . \mathrm{OZ}\right),
\end{aligned}
$$

which subsequently leads to the overall conclusion, as $S_{i}={ }_{T}\left(S_{i}\right.$.main $\|_{E_{S_{i}}} S_{i}$.OZ $)$ holds.

Before getting under way with the individual proof steps, Section 5.1 presents an algorithm, which satisfies the requirements for correct addressing. Afterwards, we show correctness of the decomposition of the CSP part and the Object-Z part in Sections 5.2 and 5.3 , respectively. The correctness for the distributivity of the inverse renaming is given in Section 5.4, followed by a lemma, stating the possible redistribution of CSP processes within a context-specific parallel composition in Section 5.5. The chapter concludes with the proof of Theorem 4.3.25, now joining together all the individual proof steps.

\subsection{Ensuring Correct Synchronisation}

In order to ensure an equivalent control flow of the original specification and its decomposition, Section 4.3.3 introduced the concept of address parameters. In particular, Definition 4.3.12 presented two conditions on these additional parameters and Theorem 4.3.16 showed that they are sufficient to preserve the original control flow.

In this section, we define an algorithm, realising both conditions of Definition 4.3.12. The algorithm was successfully implemented in Java as part of a diploma thesis [Her09] focusing on the integration of the decomposition approach into Syspect [Sys06], a graphical modelling environment for CSP-OZ. In this thesis, the algorithm will be presented in pseudo code. 
The root procedure inputs the control flow graph of a specification $S$ along with the set of operations $O p^{\prime}:=O p_{1} \cup O p_{2} \cup O p_{C}$. It computes the modified interfaces $S_{i} . I$ and CSP processes $S_{i}$.main, according to Definition 4.3.22 and Definition 4.3.23, respectively.

For $\mathrm{CFG}_{\mathrm{S}}=(N, \longrightarrow)$ being the $\mathrm{CFG}$ of the specification under interest, let $\mathrm{n}$ denote an arbitrary node of the $\mathrm{CFG}$ and, in case that $\mathrm{n}$ introduces branching, unn its corresponding join node. For any $o p \in O p$, we do not denote the original type, but solely refer to the additional address parameters.

$$
\begin{aligned}
& \text { procedure ADDRESSMAIN }\left(\mathrm{CFG}_{\mathrm{S}}, O p^{\prime}\right) \\
& \text { for each }\left(o p \in\left(O p_{1} \cap O p_{2}\right) \backslash O p_{\mathbf{C}}\right) \\
& \qquad \mathbf{d o}\left\{\begin{array}{l}
o p \leftarrow o p:\left[p_{1}:\{1,2\}\right] \text { for the definition of } S_{i} . I \\
\text { if }\left(o p^{i} \in\left(\mathbf{P h}_{1} \cup \mathbf{P h}_{3}\right)\right) \text { do } \\
o p_{p}^{i} \leftarrow o p_{p}^{i} .1 \text { for the definition of } S_{1} \text {.main } \\
\text { if }\left(o p^{j} \in \mathbf{P h}_{2}\right) \text { do } \\
o p_{p}^{j} \leftarrow o p_{p}^{j} .2 \text { for the definition of } S_{2} \text {.main }
\end{array}\right. \\
& \text { for each }\left(o p \in O p_{\mathbf{C}_{1}} \text { such that } l^{-1}(o p)>1\right) \text { do ADDRESCUT }\left(o p, \mathbf{C}_{1}\right) \\
& \text { for each }\left(o p \in O p_{\mathbf{C}_{2}} \text { such that } l^{-1}(o p)>1\right) \text { do AdDRESCUT }\left(o p, \mathbf{C}_{2}\right)
\end{aligned}
$$

Figure 5.2: Algorithm for the address extension: procedure ADDRESSMAIN

The algorithm comprises four different procedures. The root procedure ADDRESSMAIN is given in Figure 5.2. It first processes over all shared operations of $S_{1}$ and $S_{2}$, which are not located in a cut set. Their corresponding occurrences need to be separated, and they are addressed by one parameter, according to Section 4.3.3.

$$
\begin{aligned}
& \text { procedure AdDRESSCUT }\left(o p, \mathbf{C}_{i}\right) \\
& \text { global } \operatorname{Decl}(o p) \leftarrow\left\{p_{1}:\{1\}\right\} \\
& \text { global } \operatorname{Val}(o p) \leftarrow \varnothing \\
& \text { comment: ADD modifies } \operatorname{Decl}(o p) \text { and } \operatorname{Val}(o p) \\
& \text { ADD }\left(\text { start, op, }\left\langle p_{1}=1\right\rangle, \mathbf{C}_{i}, \text { false }\right) \\
& \text { ModifyCuT }(o p, \operatorname{Decl}(o p), \operatorname{Val}(o p))
\end{aligned}
$$

Figure 5.3: Algorithm for the address extension: procedure ADDRESSCUT

The procedure ADDRESSCUT, depicted in Figure 5.3, is successively called for all operations op with multiple occurrence in either $\mathbf{C}_{1}$ or $\mathbf{C}_{2}$. For each operation, ADDRESSCUT holds two global lists:

- $\operatorname{Decl}(o p)$ comprises the set of additional address parameters of op with their corresponding types. Initially, $\operatorname{Decl}(o p)$ holds one parameter of type $\{1\}$. The set is used 
for the definition of the interfaces $S_{i} . I$.

- $\operatorname{Val}(o p)$ contains a set of tuples ( $o p_{p}^{j}$, values), where values is a sequence of valuations $p_{i}=v_{i}$ with $v_{i}$, denoting the restriction of the address parameter of $p_{i}$ for the specific occurrence $o p_{p}^{j}$ of $o p$ within S.main. Initially, the set is empty. $\operatorname{Val}(o p)$ is used to define the renaming of $S_{i}$.main.

ADDRESSCUT calls the core procedure ADD, which recursively traverses the CFG and modifies the global variables $\operatorname{Decl}(o p)$ and $\operatorname{Val}(o p)$. This procedure will be explained below.

$$
\begin{aligned}
& \text { procedure ModifyCuT }(o p, \operatorname{Decl}(o p), \operatorname{Val}(o p)) \\
& \text { let }\left\{p_{1}: t_{1}, \ldots, p_{k}: t_{k}\right\}=\operatorname{Decl}(o p) \text { in } \\
& o p \leftarrow o p:\left[p_{1}: t_{1} ; \ldots ; p_{k}: t_{k}\right] \text { for the definition of } S_{1} . I \text { and } S_{2} . I \\
& \text { for each }\left(o p_{p}^{j}, \text { values }\right) \in \operatorname{Val}(o p) \text { do } \\
& \text { let }\left(\text { if }\left(\left\langle p_{i}=w_{i}\right\rangle \text { in } v a l u e s\right) \text { then } v_{i}=w_{i} \text { else } v_{i}=? p_{i}\right) \text { in } \\
& o p_{p}^{j} \leftarrow o p_{p}^{j} \cdot v_{1} \ldots v_{k} \text { for the definition of } S_{1} \text {.main and } S_{2} . \text { main }
\end{aligned}
$$

Figure 5.4: Algorithm for the address extension: procedure ModIFYCuT

After the procedure ADD terminated, ADDREssCut calls a procedure ModifyCuT (Figure 5.4) which inputs the respective operation and both sets, $\operatorname{Decl}(o p)$ and $\operatorname{Val}(o p)$. ModifyCuT carries out the actual modification of S.I and the renaming of S.main, according to the results of ADD. In particular, the interfaces $S_{i} . I$ are modified based on the parameter declarations within $\operatorname{Decl}(o p)$. Each occurrence $o p_{p}^{j}$ of op within $S$.main is modified with respect to the tuple (op $p_{p}^{j}$,values). Here, address parameters $p_{i}$ are either restricted by $p_{i}=w_{i}$ or remain unrestricted, if values does not comprise a restriction on $p_{i}$.

Finally, the core procedure ADD, as shown in Figure 5.5, proceeds as follows. It traverses the CFG and inputs five parameters.

- The first parameter $\mathrm{n}$ denotes the current node visited by the procedure. For the initial call of ADD, this node is the unique start-node of the CFG.

- The second parameter op denotes the operation under interest.

- As ADD keeps track of all parameter valuations a subsequent occurrence of $o p$ needs to adhere to, the third parameter comprises the current restrictions for the address parameters. Initially, according to the singleton of initial parameters, values $=\left\langle p_{1}=1\right\rangle$. Corresponding to the explanations of Section 4.3.3, the last element of values denotes the currently active parameter and its actual restriction.

- Parameter four identifies the cut set, corresponding to the occurrence of op. 
procedure $\operatorname{ADD}(\mathrm{n}$, op, values, $\mathrm{CS}$, cutVisited $)$

case $\left(\mathrm{n}=\operatorname{term}^{i} X \wedge \operatorname{succ}\left(\operatorname{term}^{i} X\right)=\varnothing\right) \vee \mathrm{n}=\operatorname{stop}^{i}$ then exit

case $\mathrm{n} \in\left\{\right.$ skip $^{i}$, seq $^{i}$, call $\left.^{i} . X, \operatorname{ret} . X\right\} \vee\left(\mathrm{n}=\operatorname{term}^{i} . X \wedge \operatorname{succ}\left(\operatorname{term}^{i} X\right) \neq \varnothing\right)$

then $\operatorname{ADD}(\operatorname{succ}(\mathrm{n})$, op, values, $\mathrm{CS}$, cutVisited $)$

case $\mathrm{n}=$ start $P$ then

if (start. $P$ already visited) then exit

else $\operatorname{ADD}(\operatorname{succ}(\mathrm{n})$, op, values, CS, cutVisited)

case $\mathrm{n} \in \mathrm{op}(N)$

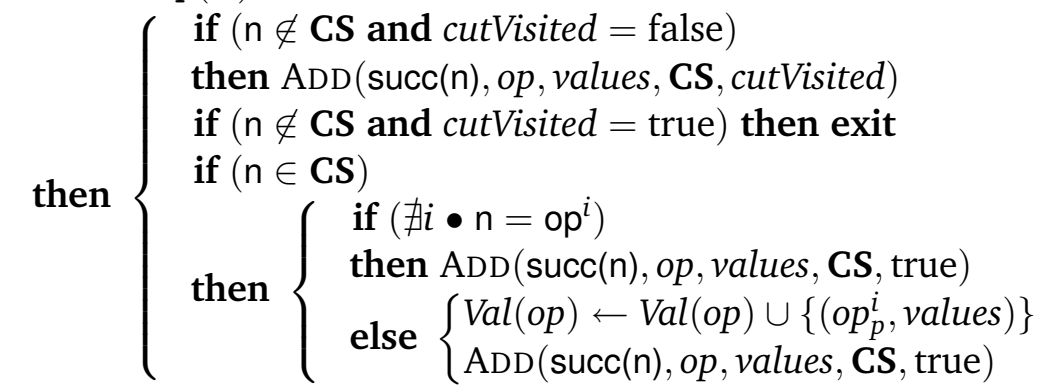

case $\left(\left(\mathrm{n}=\operatorname{par}_{X}^{i} \wedge o p \notin X\right) \vee\left(\mathrm{n} \in\left\{\right.\right.\right.$ extch $^{i}$, intch $^{i}$, interleave $\left.\left.\left.{ }^{i}\right\}\right)\right)$

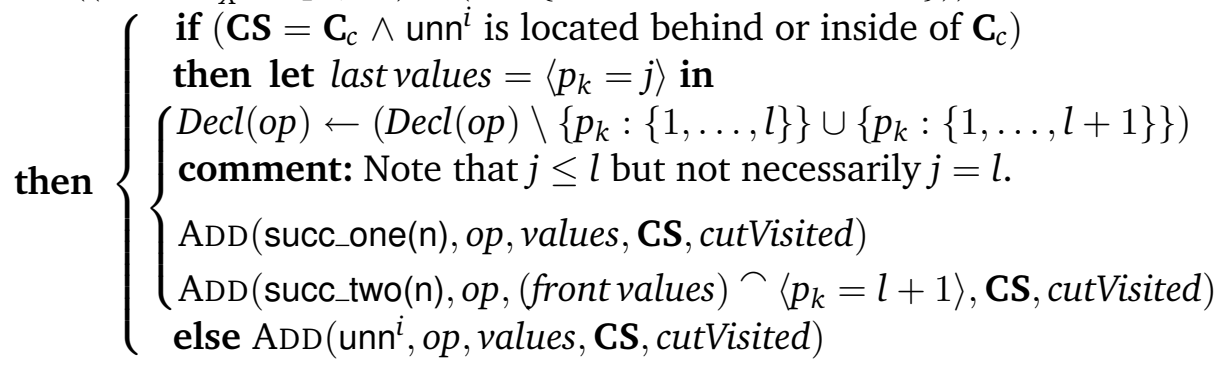

case $\left(\mathrm{n}=\operatorname{par}_{X}^{i} \wedge o p \in X\right)$

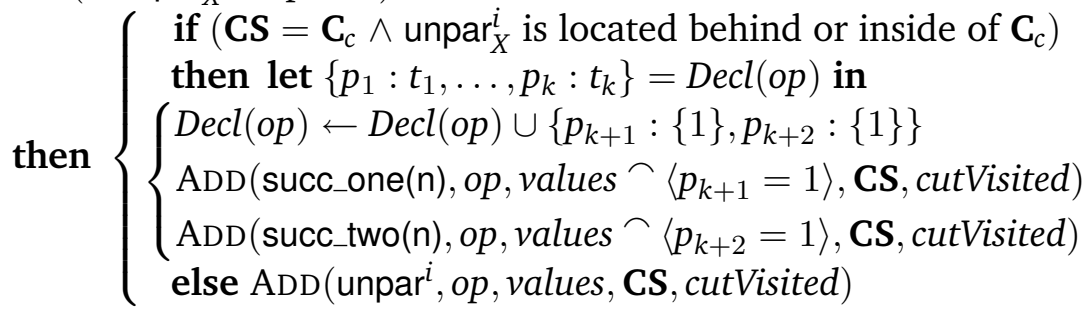

case $\left(\mathrm{n}=\right.$ uncfop $\left.^{i}\right)$

then $\left\{\begin{array}{l}\text { if }\left(\text { uncfop }^{i} \text { already visited }\right) \text { then exit } \\ \text { else } \operatorname{ADD}(\operatorname{succ}(\mathrm{n}), \text { op, values, CS, cutVisited })\end{array}\right.$

Figure 5.5: Algorithm for the address extension: procedure ADD 
- Finally, a fifth parameter, initially assigned to false, specifies if ADD has already reached the respective cut set.

The general idea of ADD is to carry over and realise the requirements on a correct addressing from Definition 4.3.12. The procedure recursively traverses the CFG. As already explained, ADD has side effects on the global variables $\operatorname{Decl}(o p)$ and $\operatorname{Val}(o p)$ : it continuously adds address parameters (in case of parallel composition with op being synchronised) and modifies their values (in case of any other branching).

Precisely, in case of $\mathrm{n}$ not having any successor node, the procedure stops. If $\mathrm{n}$ is an element of $\left\{\right.$ skip $^{i}$, seq $^{i}$, call $^{i} . X$, ret. $X$, term $\left.^{i} . X\right\}$ with the latter node being followed by a ret-node, the procedure is recursively called for the sole successor node.

Termination of the algorithm is achieved by the fact that $\mathrm{n}=$ start. $P$ only leads to a recursive call if start. $P$ was not already visited before. Otherwise, the respective call of ADD terminates. Note that for simplification, our pseudo code-algorithm does not explicitly keep track of the already visited nodes. This can obviously be achieved by adding a global variable.

Next, if $\mathrm{n}$ is an operation node of the CFG, a case differentiation is required: if $\mathrm{n}$ does not correspond to an operation of the cut set, the procedure either continues (if the traversal did not reach the cut set yet) or terminates (in the opposite case, as this signalises that the cut set is left). Accordingly, if $\mathrm{n}$ does not represent an occurrence of the operation under interest, the procedure is called for its successor node. In the final case of $\mathrm{n}=\mathrm{op}^{i}$ for some $i$, the current tuple (op $p_{p}^{i}$,values) is stored in $\operatorname{Val}(\mathrm{op})$. This assignment signalises the modification of $o p_{p}^{i}$ within the procedure ModifyCuT.

The first core case of the procedure handles the case of branching without synchronisation of op. Here, the type of the currently active parameter $p_{k}$ is modified according to the first case of Definition 4.3.12. Precisely, it is extended by one additional value within $\operatorname{Decl}(o p)$. Additionally, the restriction of $p_{k}$ within one branch is preserved, whereas it is assigned with the new value within the other branch. An additional if-clause ensures that the branching indeed reaches the cut set under interest and does not terminate beforehand. Otherwise, the procedure simply steps over the branching.

In the second core case, the algorithm deals with parallel composition with op being synchronised. According to the second case of Definition 4.3.12, we introduce two additional address parameters, with one of them restricted for the first and the other one for the second branch. This is carried out in this specific case of the procedure: $\operatorname{Decl}(o p)$ is extended by two additional parameters of initial type $\{1\}$, whereas $\operatorname{Val}(o p)$ is extended by two additional tuples, denoting the initial restrictions for the first and second branch, respectively. The procedure is recursively called for both branches. Again we use an if-clause to prevent proceeding of branching, terminating before the cut set.

The final case deals with joining of branching. Here, we again simply proceed with the node's successor. However, as a join node has two incoming edges, we need to ensure that we only proceed once with the node's sole successor.

We will now substantiate the termination and correctness of the algorithm.

Proposition 5.1.1. (Termination of ADDRESSMAIN)

The algorithm ADDRESSMAIN terminates for any control flow graph. 
Proof (Sketch). Obviously, we solely need to show termination of ADD. Let $\pi$ be a path of the CFG of an arbitrary specification. We distinguish two cases:

1. $\pi$ is a finite path. In accordance to the definition of the CFG, the final node of the path is either term ${ }^{i} X$ or stop ${ }^{i}$. However, in both cases, ADD terminates, according to the first case.

2. $\pi$ is an infinite path. Thus, the path must contain a cycle,, since the CFG's set of nodes is finite. According to the definition of the CFG and our explanations from Section 2.3.2, the sole possibility for cycles are combinations of call. $P^{i}$ and start. $P$ for some process $P$. However, in case the algorithm visits start. $P$ for the second time, it again terminates.

Proposition 5.1.2. (Correctness of ADDRESSMAIN)

The algorithm ADDRESSMAIN satisfies both conditions of Definition 4.3.12.

Proof (Sketch). We recall both conditions from Definition 4.3.12.

Branching without Synchronisation: If op $^{i}$ and op $^{j}$ are located inside different branches of either an external choice operator, internal choice operator, interleaving operator or a parallel composition operator par $_{X}$ with op $\notin X$, op $p_{p}$ needs to comprise one parameter $p_{1}$, such that its type includes $x, y \in \mathbb{N}$ with $x \neq y$. This parameter is fixed to $x$ for op $i$ and to $y$ for op $p_{p}^{j}$ in both, $S_{1}$.main and $S_{2}$.main :

$o p_{p}^{i}$ becomes $o p_{p}^{i} . x$ and $o p_{p}^{j}$ becomes $o p_{p}^{j} \cdot y$.

Let $\mathrm{op}^{i}$ and $\mathrm{op}^{j}$ be two according nodes. Consider the first of the two core cases of ADD: it applies for $o p^{i}$ and $o p^{j}$ and thus, $o p_{p}^{i}$ and $o p_{p}^{j}$ will be addressed according to this case. The addressing sets the value of the parameter $p_{k}$ to the value $l$ in one and to the value $l+1$ in the other branch. Any further branching preserves the inequality of both values. Thus, $p_{k}$ satisfies the first condition of the definition.

Branching with Synchronisation: If $\mathrm{op}^{i} \stackrel{\mathrm{sd}}{\rightarrow} \mathrm{op}^{j}$, the (partial) event op $p_{p}$ needs to comprise two parameters $p_{1}$ and $p_{2}$, such that the type of $p_{1}$ includes $x \in \mathbb{N}$ and the type of $p_{2}$ includes $y \in \mathbb{N}$ for arbitrary $x, y$. The first parameter is fixed to $x$ for $o p_{p}^{i}$, whereas the second parameter is fixed to y for op $p_{p}^{j}$ in both, $S_{1}$.main and $S_{2}$.main :

$o p_{p}^{i}$ becomes $o p_{p}^{i} \cdot x ? p_{2}$ and $o p_{p}^{j}$ becomes $o p_{p}^{j} ? p_{1} \cdot y$.

Let $\mathrm{op}^{i}$ and $\mathrm{op}^{j}$ be two according nodes. Consider the second of the two core cases of ADD: $o p_{p}^{i}$ and $o p_{p}^{j}$ will be addressed according to this case as both nodes are located in different branches of a parallel composition $\operatorname{par}_{X}^{i}$ with op $\in X$. Two additional parameters $p_{k+1}, p_{k+2}$ are introduced, with one of them fixed for $o p_{p}^{i}$ and the other one fixed for $o p_{p}^{j}$ (initially by 1 and possibly modified later on). Therefore, the second condition of the definition is satisfied as well.

This completes the definition of the algorithm and the motivation for its termination and correctness. The following sections carry out the individual steps of the proof of Theorem 4.3.25. 


\subsection{Correctness for the CSP Part}

The operational semantics of CSP-OZ allows for a compositional proof of Theorem 4.3.25 in the following sense: we show that the individual decompositions of the CSP part $S$.main and the Object-Z part S.OZ are semantics-preserving in the domain of the CSP traces model. Subsequently, and by using some additional properties, we combine both results to deduce the overall correctness of the decomposition.

For both, the CSP part and the Object-Z part, we will show semantic equivalence modulo renaming. This means, that we relate the original events from $E_{S}$ to events from $E_{S^{\prime}}$, now possibly comprising transmission parameters and address parameters.

In this section, the correctness proof of the CSP part is conducted. We have to show

$$
\text { S.main }=_{T}\left(S_{1} \cdot \text { main } \|_{E_{\mathrm{C}}} S_{2} \cdot \operatorname{main}\right) \llbracket \mathrm{R}^{\prime} \rrbracket
$$

that is, the proof will show the equivalence of both CSP processes, factoring out the different parameter extensions. ${ }^{1}$ At first sight, this particular proof step seems to be rather easy, as the CSP process S.main is one-to-one reflected in the CFG of a specification.

However, as a first obstacle, the set of traces of $S$.main does not correspond to the set of paths of the CFG: in general, the first set is strictly larger due to possible interleaving. This complicates the proof, as reasoning with respect to the specification's CFG becomes impractical.

Another difficulty arises from the projection of CSP processes and traces according to Definitions 4.3.3 and 2.2.8, respectively. Unfortunately, their definitions do not satisfy the law

$$
\operatorname{traces}\left(\left.P\right|_{X}\right)=\operatorname{traces}(P)\lceil X
$$

when we are dealing with parallel composition of processes. As we need to bridge the gap between both definitions, this particularly complicates dealing with this individual operator.

Before carrying out the actual correctness proof of the decomposition of the CSP part, we start with some related properties.

\subsubsection{Properties of the Decomposition: CSP Part}

Showing correctness of the decomposition of the CSP part requires several properties, which the actual correctness proof uses. They are given next.

\section{Disallowed Distribution of Initial Branching Events}

A first property describes that in case of branching within the CFG, the initial events (see Definition 2.2.9) of both branches are never distributed over $E_{1}$ and $E_{2}$, that is, over the sets of local events for the components:

\footnotetext{
${ }^{1}$ We explicitly deal with the renaming relation in Section 5.4.
} 
Lemma 5.2.1. (No distribution of initial events)

For $\circ \in\left\{\square, \sqcap,\left\|_{S},\right\| \|\right\}$, let $P=Q_{1} \circ Q_{2}$ be a process, occurring within S.main. Then, for any valid decomposition of $S$ according to Definition 4.3.24:

$$
\left(\text { initials }\left(Q_{1}\right) \cap E_{i}\right) \neq \varnothing \Rightarrow\left(\text { initials }\left(Q_{2}\right) \cap E_{j}\right)=\varnothing,
$$

for $i \neq j$, and vice versa.

Proof. Without loss of generality, assume that $e_{1} \in\left(\right.$ initials $\left.\left(Q_{1}\right) \cap E_{1}\right)$ and that there exists some $e_{2} \in$ (initials $\left(Q_{2}\right) \cap E_{2}$ ), yielding $\mathbf{e}_{2} \in \mathbf{P h}_{2}$ for the corresponding DG node. Based on the definition for $\mathbf{P h}_{1}$ and $e_{1} \in E_{1}$, there exists a path $\pi$, such that

$$
\text { start } \stackrel{\pi}{\longrightarrow} \mathrm{e}_{1}
$$

and $\pi \cap \mathbf{C}_{1}=\varnothing$. Obviously, any prefix of this path does not intersect with $\mathbf{C}_{1}$ as well. Let $\pi^{\prime}$ denote the prefix, leading from start to the binary operator $\circ$. As $e_{2} \in$ initials $\left(Q_{2}\right)$, the path $\pi^{\prime}$ can be extended to a path $\pi_{2}$ not comprising any additional nodes from op $(N)$ :

$$
\text { start } \stackrel{\pi_{2}}{\longrightarrow} \mathrm{e}_{2} \text { corresponding to start } \stackrel{\pi^{\prime}}{\longrightarrow} \text { cfop } \stackrel{\bullet}{\longrightarrow} \mathrm{e}_{2},
$$

and $\pi_{2} \cap \mathbf{C}_{1}=\varnothing$. We deduce that $\mathrm{e}_{2} \in\left(\mathbf{P h}_{1} \cap \mathbf{P h}_{2}\right)$, contradicting the correctness criterion disjointness for a valid cut.

The lemma basically states that a branching introduced within a certain phase yields that all initial events of both branches are represented in this specific phase or the subsequent cut set. Figure 5.6 shows one instance of a disallowed distribution of initial events. Here, $\circ=$ extch, and the violation occurs with respect to the first cut set.

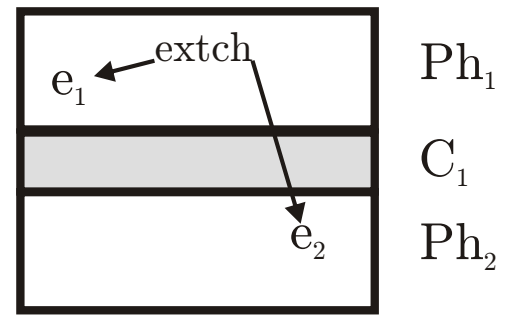

Figure 5.6: Illustration of a violation of Lemma 5.2.1

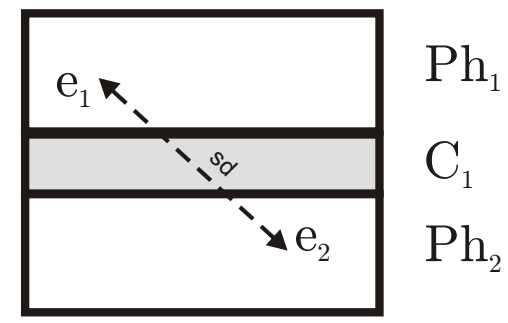

Figure 5.7: Illustration of a violation of Lemma 5.2.2

\section{Disallowed Split of Synchronisation}

A rather obvious property is the following: in case that two operation nodes are connected by a synchronisation dependence, they must not be distributed over different elements of $\left\{\left(\mathbf{P h}_{1} \cup \mathbf{P h}_{3}\right), \mathbf{C}_{1}, \mathbf{P h}_{2}, \mathbf{C}_{2}\right\}$ : 
Lemma 5.2.2. (No split of synchronisation)

Let $\mathrm{CFG}_{\mathrm{S}}=(N, \longrightarrow)$ be the control flow graph of a specification $S$, and let $\left(\mathbf{C}_{1}, \mathbf{C}_{2}\right)$ be a cut. Let $\mathcal{D}=\left\{\left(\mathbf{P h}_{1} \cup \mathbf{P h}_{3}\right), \mathbf{C}_{1}, \mathbf{P h}_{2}, \mathbf{C}_{2}\right\}$. Then:

$$
\mathrm{n} \stackrel{\mathrm{sd}}{\leftrightarrow--\rightarrow} \mathrm{n}^{\prime} \Rightarrow\left(\forall M \in \mathcal{D} \bullet \mathrm{n} \in M \Leftrightarrow \mathrm{n}^{\prime} \in M\right)
$$

Proof. As $\mathrm{n}$ and $\mathrm{n}$ ' are connected by a synchronisation dependence, both nodes have the same operation name: $l^{-1}(\mathrm{n})=l^{-1}(\mathrm{n})$ holds. The correctness criterion all-or-none rules out that both nodes are either distributed over both cut sets or over one cut set and one phase. We remain to show that both nodes must not be distributed over $\left(\mathbf{P h}_{1} \cup \mathbf{P h}_{3}\right)$ and $\mathbf{P h}_{2}$. However, if this was the case, the connecting synchronisation dependence would violate no crossing.

A possible violation of the lemma, with two synchronised nodes distributed over $\mathbf{P} \mathbf{h}_{1}$ and $\mathbf{P h}_{2}$, is illustrated in Figure 5.7.

\section{Redistribution of Processes for Binary Operators}

Next, we show a property which we will use throughout the actual proof. The property states that we can redistribute the component processes with respect to the parallel composition $\|_{E_{\mathrm{C}}}$ and all binary operators, that is, $\circ \in\left\{\square, \sqcap, \stackrel{\circ}{9},\left\|_{S},\right\|\right\}$. Precisely, for $P=(T \circ U), T_{i}=\left.T\right|_{E_{S_{i}}}$ and $U_{i}=\left.U\right|_{E_{S_{i}}}$, Figure 5.8 illustrates our proof strategy for $S$.main being composed of two processes. The top-down-equivalence will be shown in the following lemma.

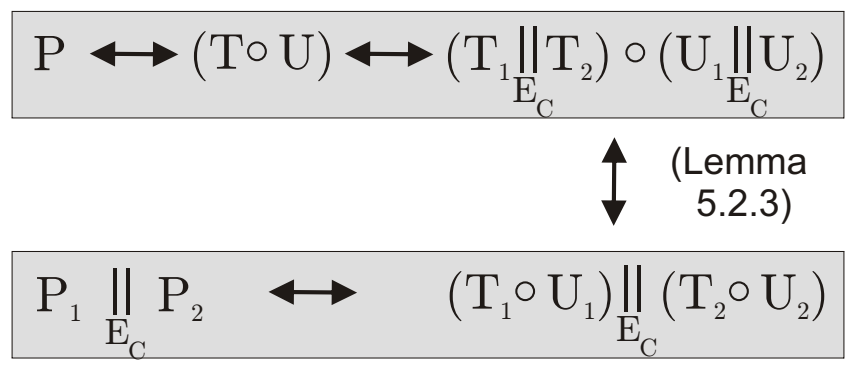

Figure 5.8: Illustration of the CSP correctness proof of binary operators

The subsequent lemma uses the LTS semantics of CSP according to Definition 2.2.11. Here, we will refer to the firing rules or CSP [Ros98], which determine the set of possible transitions and thus the labelled transition system of a process. In addition to the set of possible events a process may perform, we need to deal with $\tau$-transitions, symbolising invisible events.

In our semantic domain of the CSP traces model, we apply several simplifications: first of all and according to [Brü08], we do not distinguish between the processes Stop and Div. This is justified by the fact that both processes are incapable of performing any event, and they are thus trace equivalent. Furthermore, we do not consider the special 
event $\checkmark$, signalising termination of a process. As a consequence, we do not separate the processes Skip and Stop. The LTS semantics makes use of an additional symbol $\Omega$, denoting the end state of the transition system. Here, we do not separately deal with this symbol and rather refer to the corresponding process Stop. These considerations allow us to simplify and restrict some of the CSP firing rules.

Lemma 5.2.3. (Redistribution of CSP processes within the decomposition)

Let $P=(T \circ U)$ for $\circ \in\left\{\square, \sqcap, \stackrel{\circ}{9},\|\|, \|_{S}\right\}$ be a reachable state of the LTS of S.main. Then,

$$
\left(T_{1} \|_{E_{\mathbf{C}}} T_{2}\right) \circ\left(U_{1} \|_{E_{\mathbf{C}}} U_{2}\right)=_{T}\left(T_{1} \circ U_{1}\right) \|_{E_{\mathbf{C}}}\left(T_{2} \circ U_{2}\right)
$$

for $\circ \neq \|_{S}$ and

$$
\left(T_{1} \|_{E_{\mathbf{C}}} T_{2}\right)\left\|_{S}\left(U_{1} \|_{E_{\mathbf{C}}} U_{2}\right)=_{T}\left(T_{1} \|_{S \cap E_{S_{1}}} U_{1}\right)\right\|_{E_{\mathbf{C}}}\left(T_{2} \|_{S \cap E_{S_{2}}} U_{2}\right),
$$

where $T_{i}=\left.T\right|_{E_{S_{i}}}$ and $U_{i}=\left.U\right|_{E_{S_{i}}}, i \in\{1,2\}$.

Proof. As we are interested in trace equivalence, external choice and internal choice can equally be treated. Moreover, being a special case of parallel composition with an empty synchronisation alphabet, we do not explicitly need to deal with interleaving.

The method of proof, which we choose here, is (weak) bisimilarity [Mil89]: if we can show that the labelled transition systems of the left hand side and the right hand side of the equation are bisimilar, we can deduce their trace equivalence [Pnu85]. In the following, let op.x.t.a indicate an event of $E_{S^{\prime}}$ with the valuations for

- the original parameters according to $x$,

- the transmission parameters according to $t$ and

- the address parameters according to $a$.

Subject to the individual operator we refer to, we define a weak bisimulation

$$
\mathcal{R}=\left\{(A, B) \mid A=\left(C_{1} \|_{E_{\mathrm{C}}} C_{2}\right) \circ\left(D_{1} \|_{E_{\mathrm{C}}} D_{2}\right), B=\left(C_{1} \circ D_{1}\right) \|_{E_{\mathrm{C}}}\left(C_{2} \circ D_{2}\right)\right\} \cup \mathcal{R}^{\prime},
$$

and we show that $\left(T_{1} \|_{E_{\mathrm{C}}} T_{2}\right) \circ\left(U_{1} \|_{E_{\mathrm{C}}} U_{2}\right)$ and $\left(T_{1} \circ U_{1}\right) \|_{E_{\mathrm{C}}}\left(T_{2} \circ U_{2}\right)$ are the initial states of $\mathcal{R}$. Here, $C_{i} \in L^{C S P}\left(D_{i} \in L^{C S P}\right)$ denotes any reachable state within the labelled transition system of $T_{i}\left(U_{i}\right)$, and $\mathcal{R}^{\prime}$ denotes a case-specific extension of $\mathcal{R}$.

Based on the definition of bisimulation, we have to show two directions:

(1) If $(A, B) \in \mathcal{R}$ and $A \stackrel{e}{\longrightarrow} A^{\prime}$ for $e \in E_{S^{\prime}} \cup\{\tau\}$, then there exists some $B^{\prime}$ such that $B \stackrel{\widehat{e}}{\longrightarrow} B^{\prime}$ and $\left(A^{\prime}, B^{\prime}\right) \in \mathcal{R}$.

(2) If $(A, B) \in \mathcal{R}$ and $B \stackrel{e}{\longrightarrow} B^{\prime}$ for $e \in E_{S^{\prime}} \cup\{\tau\}$, then there exists some $A^{\prime}$ such that $A \stackrel{\widehat{e}}{\longrightarrow} A^{\prime}$ and $\left(A^{\prime}, B^{\prime}\right) \in \mathcal{R}$. 
Here, we let $P \stackrel{\widehat{e}}{\longrightarrow} P^{\prime}$ stand for

$$
P \stackrel{\tau}{\longrightarrow} \ldots \stackrel{\tau}{\longrightarrow} P_{k} \stackrel{e}{\longrightarrow} P_{k+1} \stackrel{\tau}{\longrightarrow} \ldots \stackrel{\tau}{\longrightarrow} P^{\prime},
$$

that is, $P$ transits into $P^{\prime}$ by $e$, possibly surrounded by additional $\tau$-transitions. We show the property for $\circ \in\left\{\square,{ }_{9}^{\circ}, \|_{S}\right\}$ and we construct the bisimulation relations $\mathcal{R}$ with respect to the individual binary operator, instantiating $\circ$. In any case, we separate between both required conditions (1) and (2). Within the individual proofs, we additionally distinguish between $(A, B) \in \mathcal{R}^{\prime}$ and $(A, B) \notin \mathcal{R}^{\prime}$, and we need to consider $\tau$-transitions.

External Choice: For the case of external choice, we extend the relation $\mathcal{R}$ by defining

$$
\begin{aligned}
\mathcal{R}^{\prime}:=\operatorname{Id}_{L^{C S P}} \cup\{(A, B) \mid A & \left.=\left(C_{1} \|_{E_{\mathrm{C}}} C_{2}\right), B \in X_{1}\right\} \cup \\
\{(A, B) \mid A & \left.=\left(D_{1} \|_{E_{\mathrm{C}}} D_{2}\right), B \in X_{2}\right\},
\end{aligned}
$$

for

$$
\begin{aligned}
X_{1}:= & \left\{\left(C_{1} \|_{E_{\mathbf{C}}}\left(C_{2} \square D_{2}\right)\right) \mid \text { initials }\left(D_{2}\right) \subseteq E_{\mathbf{C}}\right\} \cup \\
& \left\{\left(\left(C_{1} \square D_{1}\right) \|_{E_{\mathbf{C}}} C_{2}\right) \mid \text { initials }\left(D_{1}\right) \subseteq E_{\mathbf{C}}\right\},
\end{aligned}
$$

and

$$
\begin{aligned}
X_{2}:= & \left\{\left(D_{1} \|_{E_{\mathrm{C}}}\left(C_{2} \square D_{2}\right)\right) \mid \text { initials }\left(C_{2}\right) \subseteq E_{\mathrm{C}}\right\} \cup \\
& \left\{\left(\left(C_{1} \square D_{1}\right) \|_{E_{\mathrm{C}}} D_{2}\right) \mid \text { initials }\left(C_{1}\right) \subseteq E_{\mathbf{C}}\right\},
\end{aligned}
$$

where again, $C_{i} \in L^{C S P}\left(D_{i} \in L^{C S P}\right)$ denotes any reachable state within the labelled transition system of $T_{i}\left(U_{i}\right)$. Here, $\operatorname{ld}_{L C S P}:=\left\{(P, P) \mid P \in L^{C S P}\right\}$, depicting the identity on $L^{C S P}$. We do not explicitly deal with $(A, B) \in \mathrm{Id}_{L} C S P$, as in this case, the bisimulation diagram can trivially be completed.

(1) $(A, B) \in \mathcal{R}^{\prime}$ and $A \stackrel{e}{\rightarrow} A^{\prime}$.

$\tau$-case: Let $A \stackrel{\tau}{\longrightarrow} A^{\prime}$. We start with the case of $(A, B) \in \mathcal{R}^{\prime}$. If

$$
\left(C_{1} \|_{E_{\mathrm{C}}} C_{2}\right) \stackrel{\tau}{\longrightarrow}\left(C_{1}^{\prime} \|_{E_{\mathrm{C}}} C_{2}^{\prime}\right),
$$

according to the firing rules for parallel composition, the transition is either performed by $C_{1}$ or $C_{2}$. We consider the first case, the other case is analogous. Then, $C_{2}^{\prime}=C_{2}$. From $C_{1} \stackrel{\tau}{\longrightarrow} C_{1}^{\prime}$, we get

$$
C_{1}\left\|_{E_{\mathbf{C}}}\left(C_{2} \square D_{2}\right) \stackrel{\tau}{\longrightarrow} C_{1}^{\prime}\right\|_{E_{\mathbf{C}}}\left(C_{2} \square D_{2}\right)
$$

as well as

$$
\left(C_{1} \square D_{1}\right)\left\|_{E_{\mathrm{C}}} C_{2} \stackrel{\tau}{\longrightarrow}\left(C_{1}^{\prime} \square D_{1}\right)\right\|_{E_{\mathrm{C}}} C_{2}
$$

The following bisimulation diagram illustrates this case. $\checkmark$ 


\begin{tabular}{|cccc|c|}
\hline$A=\left(C_{1} \|_{E_{\mathrm{C}}} C_{2}\right)$ & $\stackrel{\tau}{\longrightarrow}$ & $\left(C_{1}^{\prime} \|_{E_{\mathrm{C}}} C_{2}^{\prime}\right)$ & $=A^{\prime}$ \\
$\mid$ & & $\vdots$ & \\
$\mathcal{R}$ & & $\mathcal{R}$ & \\
$\mid$ & & $\vdots$ & \\
$B=\left(C_{1} \|_{E_{\mathrm{C}}}\left(C_{2} \square D_{2}\right)\right)$ & $\stackrel{\tau}{\longrightarrow}$ & $\left(C_{1}^{\prime} \|_{E_{\mathrm{C}}}\left(C_{2}^{\prime} \square D_{2}\right)\right)$ & $=B^{\prime}$ \\
\hline
\end{tabular}

Next, we consider $A \stackrel{\tau}{\longrightarrow} A^{\prime}$ and $(A, B) \notin \mathcal{R}^{\prime} . \tau$-transitions do not resolve an external choice. For the case of $C_{1}$ performing $\tau$, based on the firing rules for external choice and parallel composition, the bisimulation diagram can be completed as follows. The other cases are similar. $\checkmark$

\begin{tabular}{|ccccc||}
\hline$A=\left(\left(C_{1} \|_{E_{\mathrm{C}}} C_{2}\right) \square\left(D_{1} \|_{E_{\mathrm{C}}} D_{2}\right)\right)$ & $\stackrel{\tau}{\longrightarrow}\left(\left(C_{1}^{\prime} \|_{E_{\mathrm{C}}} C_{2}\right) \square\left(D_{1} \|_{E_{\mathrm{C}}} D_{2}\right)\right)$ & $=A^{\prime}$ \\
$\mid$ & & $\vdots$ & & \\
$\mathcal{R}$ & & $\mathcal{R}$ & & \\
$\mid$ & & $\vdots$ & \\
$B=\left(\left(C_{1} \square D_{1}\right) \|_{E_{\mathrm{C}}}\left(C_{2} \square D_{2}\right)\right)$ & $\stackrel{\tau}{\longrightarrow}\left(\left(C_{1}^{\prime} \square D_{1}\right) \|_{E_{\mathrm{C}}}\left(C_{2} \square D_{2}\right)\right)$ & $=B^{\prime}$ \\
\hline
\end{tabular}

op-case: Next, let $A \stackrel{\text { op.x.t.a }}{\longrightarrow} A^{\prime}$. For the case of $(A, B) \in \mathcal{R}^{\prime}$, let $A=C_{1} \|_{E_{\mathrm{C}}} C_{2}$ and $A \stackrel{\text { op.x.t.a }}{\rightarrow} A^{\prime}$. Obviously, any of the processes from $X_{1}$ can simulate op.x.t.a, resulting in two $\mathcal{R}^{\prime}$-related processes, since the comprised external choice solely extends the set of possible steps, independent of any restriction on initials $\left(D_{i}\right)$. The case $A=D_{1} \|_{E_{\mathrm{C}}} D_{2}$ and $X_{2}$ is analogous. $\checkmark$

\begin{tabular}{|cccc||}
\hline$A=\left(C_{1} \|_{E_{\mathrm{C}}} C_{2}\right)$ & $\stackrel{\text { op.x.t.a }}{\longrightarrow}$ & $\left(C_{1}^{\prime} \|_{E_{\mathrm{C}}} C_{2}^{\prime}\right)$ & $=A^{\prime}$ \\
$\mid$ & & $\vdots$ & \\
$\mathcal{R}$ & & $\mathcal{R}$ & \\
$\mid$ & & $\vdots$ & \\
$B=\left(C_{1} \|_{E_{\mathrm{C}}}\left(C_{2} \square D_{2}\right)\right) \stackrel{\text { op.x.t.a }}{\longrightarrow}\left(C_{1}^{\prime} \|_{E_{\mathrm{C}}}\left(C_{2}^{\prime} \square D_{2}\right)\right)$ & $=B^{\prime}$ \\
\hline
\end{tabular}

Now consider the case $(A, B) \notin \mathcal{R}^{\prime}$, that is,

$$
A=\left(C_{1} \|_{E_{\mathrm{C}}} C_{2}\right) \square\left(D_{1} \|_{E_{\mathrm{C}}} D_{2}\right) .
$$

Then, either $C_{1} \|_{E_{\mathrm{C}}} C_{2} \stackrel{\text { op.x.t.a }}{\rightarrow} A^{\prime}$ or $D_{1} \|_{E_{\mathrm{C}}} D_{2} \stackrel{\text { op.x.t.a }}{\rightarrow} A^{\prime}$. Without loss of generality, we assume the first. Two separate cases have to be considered: op.x.t.a $\in E_{\mathrm{C}}$ : Then, $C_{1}$ and $C_{2}$ have to synchronise on the execution of op.x.t.a. Thus,

$$
C_{1} \stackrel{\text { op.x.t.a }}{\rightarrow} A_{1}^{\prime} \text { and } C_{2} \stackrel{\text { op.x.t.a }}{\rightarrow} A_{2}^{\prime}
$$

for some $A^{\prime}=A_{1}^{\prime} \|_{E_{\mathrm{C}}} A_{2}^{\prime}$, again based on the firing rules of the operational semantics of CSP. This yields that

$$
\left(C_{1} \square D_{1}\right) \stackrel{\text { op.x.t.a }}{\rightarrow} A_{1}^{\prime} \text { and }\left(C_{2} \square D_{2}\right) \stackrel{\text { op.x.t.a }}{\rightarrow} A_{2}^{\prime}
$$


and therefore,

$$
\left(\left(C_{1} \square D_{1}\right) \|_{E_{\mathbf{C}}}\left(C_{2} \square D_{2}\right)\right) \stackrel{\text { op.x.t.a }}{\rightarrow}\left(A_{1}^{\prime} \|_{E_{\mathbf{C}}} A_{2}^{\prime}\right) .
$$

As both successor states are identical, they are related by $\mathcal{R}^{\prime}$. The bisimulation diagram for this case is given next. $\checkmark$

\begin{tabular}{|ccccc|}
\hline$A=\left(\left(C_{1} \|_{E_{\mathrm{C}}} C_{2}\right) \square\left(D_{1} \|_{E_{\mathrm{C}}} D_{2}\right)\right)$ & $\stackrel{\text { op.x.t.a }}{\longrightarrow}$ & $\left(A_{1}^{\prime} \|_{E_{\mathrm{C}}} A_{2}^{\prime}\right)$ & $=A^{\prime}$ \\
$\mid$ & $\vdots$ & & \\
$\mathcal{R}$ & & $\mathcal{R}$ & \\
$\mid$ & $\vdots$ & \\
$B=\left(\left(C_{1} \square D_{1}\right) \|_{E_{\mathrm{C}}}\left(C_{2} \square D_{2}\right)\right)$ & $\stackrel{\text { op.x.t.a }}{\longrightarrow}\left(A_{1}^{\prime} \|_{E_{\mathrm{C}}} A_{2}^{\prime}\right)$ & $=B^{\prime}$ \\
\hline
\end{tabular}

op.x.t.a $\notin E_{\mathrm{C}}$ : Then, either, but exactly one of the four components can execute op.x.t.a. Without loss of generality, let this component be $C_{1}$.

$$
\left(\left(C_{1} \|_{E_{\mathrm{C}}} C_{2}\right) \square\left(D_{1} \|_{E_{\mathrm{C}}} D_{2}\right)\right) \stackrel{\text { op.x.t.a }}{\longrightarrow} A^{\prime}
$$

yields

$$
\left(C_{1} \|_{E_{\mathrm{C}}} C_{2}\right) \stackrel{\text { op.x.t.a }}{\longrightarrow} A^{\prime} .
$$

We get $C_{1} \stackrel{\text { op.x.t.a }}{\longrightarrow} C_{1}^{\prime}$ for some process $C_{1}^{\prime}$, such that $A^{\prime}=\left(C_{1}^{\prime} \|_{E_{\mathrm{C}}} C_{2}\right)$. Furthermore,

$$
\left(C_{1} \square D_{1}\right) \stackrel{\text { op.x.t.a }}{\longrightarrow} C_{1}^{\prime}
$$

and thus,

$$
\left(\left(C_{1} \square D_{1}\right) \|_{E_{\mathbf{C}}}\left(C_{2} \square D_{2}\right)\right) \stackrel{\text { op.x.t.a }}{\longrightarrow}\left(C_{1}^{\prime} \|_{E_{\mathbf{C}}}\left(C_{2} \square D_{2}\right)\right) .
$$

Finally, initials $\left(D_{2}\right) \subseteq E_{\mathrm{C}}$ holds: as op.x.t.a $\in$ initials $\left(C_{1} \|_{E_{\mathrm{C}}} C_{2}\right)$, the set of initial events of $D_{1} \|_{E_{\mathrm{C}}} D_{2}$ and therefore the one of $D_{2}$ needs to be a subset of $E_{S_{1}}$ due to Lemma 5.2.1. $D_{2}$ being a reachable state of $\left.U\right|_{E_{S_{2}}}$ yields initials $\left(D_{2}\right) \subseteq E_{\mathbf{C}}$, as no events from $E_{1}$ are possible. Thus,

$$
\left(\left(C_{1}^{\prime} \|_{E_{\mathbf{C}}} C_{2}\right),\left(C_{1}^{\prime} \|_{E_{\mathbf{C}}}\left(C_{2} \square D_{2}\right)\right)\right) \in \mathcal{R}^{\prime},
$$

which concludes this case. $\checkmark$

\begin{tabular}{||cccc||}
\hline$A=\left(\left(C_{1} \|_{E_{\mathrm{C}}} C_{2}\right) \square\left(D_{1} \|_{E_{\mathrm{C}}} D_{2}\right)\right)$ & $\stackrel{\text { op.x.t.a }}{\longrightarrow}$ & $\left(C_{1}^{\prime} \|_{E_{\mathrm{C}}} C_{2}\right)$ & $=A^{\prime}$ \\
$\mid$ & & $\vdots$ & \\
$\mathcal{R}$ & & $\mathcal{R}$ & \\
$\mid$ & & $\vdots$ & \\
$B=\left(\left(C_{1} \square D_{1}\right) \|_{E_{\mathrm{C}}}\left(C_{2} \square D_{2}\right)\right)$ & $\stackrel{\text { op.x.t.a }}{\longrightarrow}$ & $\left.\left(C_{1}^{\prime} \|_{E_{\mathrm{C}}}\left(C_{2} \square D_{2}\right)\right)\right)$ & $=B^{\prime}$ \\
\hline
\end{tabular}


(2) For the reverse direction, let $(A, B) \in \mathcal{R}$ and $B \stackrel{e}{\rightarrow} B^{\prime}$.

$\tau$-case: Again, we start with $B \stackrel{\tau}{\rightarrow} B^{\prime}$. For the case of $\mathcal{R}^{\prime}$, consider

$$
\left(C_{1} \|_{E_{\mathbf{C}}}\left(C_{2} \square D_{2}\right)\right) \stackrel{\tau}{\longrightarrow}\left(C_{1} \|_{E_{\mathbf{C}}}\left(C_{2} \square D_{2}^{\prime}\right)\right),
$$

that is, $D_{2} \stackrel{\tau}{\longrightarrow} D_{2}^{\prime}$. First, as initials $\left(D_{2}\right) \subseteq E_{\mathrm{C}}$, the process $D_{2}$ can solely perform synchronised events with $C_{1}$. However, a synchronisation between $C_{1}$ and $D_{2}$ is impossible due to Theorem 4.3.16, (5). Therefore, $D_{2}$ is incapable of performing any event within $C_{1} \|_{E_{\mathbf{C}}}\left(C_{2} \square D_{2}\right)$. This allows us to simulate the $\tau$-transition by $\left(C_{1} \|_{E_{\mathrm{C}}} C_{2}\right) \stackrel{\tau}{\longrightarrow}\left(C_{1} \|_{E_{\mathrm{C}}} C_{2}\right)$. In any other case, including $(A, B) \notin \mathcal{R}^{\prime}$, we apply the exact same rules and ideas of the forward direction. $\checkmark$.

op-case: In the case of $(A, B) \in \mathcal{R}^{\prime}$, we solely consider $B=\left(C_{1} \|_{E_{\mathrm{C}}}\left(C_{2} \square D_{2}\right)\right)$ - the other three cases are accordingly shown. Let $B \stackrel{\text { op.x.t.a }}{\rightarrow} B^{\prime}$. Again, it is impossible that $D_{2}$ performs any event within $C_{1} \|_{E_{\mathbf{C}}}\left(C_{2} \square D_{2}\right)$. Furthermore, any (local or synchronised) step of $C_{i}$ can be simulated by $C_{1} \|_{E_{\mathrm{C}}} C_{2} \cdot \checkmark$

$$
\begin{array}{|ccccc||}
\hline B=\left(C_{1} \|_{E_{\mathrm{C}}}\left(C_{2} \square D_{2}\right)\right) & \stackrel{\text { op.x.t.a }}{\longrightarrow} & \left(C_{1}^{\prime} \|_{E_{\mathrm{C}}}\left(C_{2}^{\prime} \square D_{2}\right)\right) & =B^{\prime} \\
\mid & & \vdots & \\
\mathcal{R} & & \mathcal{R} & \\
\mid & & \vdots & \\
A=\quad\left(C_{1} \|_{E_{\mathrm{C}}} C_{2}\right) & \stackrel{\text { op.x.t.a }}{\longrightarrow} & \left(C_{1}^{\prime} \|_{E_{\mathrm{C}}} C_{2}^{\prime}\right) & =A^{\prime} \\
\hline
\end{array}
$$

Now let $B=\left(\left(C_{1} \square D_{1}\right) \|_{E_{\mathrm{C}}}\left(C_{2} \square D_{2}\right)\right)$. Here, both sides need to synchronise on op.x.t.a. Again, two cases need to be considered:

op.x.t.a $\in E_{\mathrm{C}}$ : Then, there exist $B_{1}^{\prime}, B_{2}^{\prime}$ such that

$$
\left(C_{1} \square D_{1}\right) \stackrel{\text { op.x.t.a }}{\rightarrow} B_{1}^{\prime} \text { and }\left(C_{2} \square D_{2}\right) \stackrel{\text { op.x.t.a }}{\rightarrow} B_{2}^{\prime}
$$

for some $B_{1}^{\prime}, B_{2}^{\prime}$ and $B^{\prime}=\left(B_{1}^{\prime} \|_{E_{\mathrm{C}}} B_{2}^{\prime}\right)$. Based on Theorem 4.3.16, (5), a synchronisation between $C_{1}$ and $D_{2}$ or $C_{2}$ and $D_{1}$ is impossible, as $C$ and $D$ were unable to synchronise before the decomposition. Therefore, without loss of generality, we deduce $C_{1} \stackrel{\text { op.x.t.a }}{\rightarrow} B_{1}^{\prime}$ and $C_{2} \stackrel{\text { op.x.t.a }}{\rightarrow} B_{2}^{\prime}$. Following up,

$$
\left(C_{1} \|_{E_{\mathrm{C}}} C_{2}\right) \stackrel{\text { op.x.t.a }}{\rightarrow}\left(B_{1}^{\prime} \|_{E_{\mathbf{C}}} B_{2}^{\prime}\right)
$$

and thus,

$$
\left(\left(C_{1} \|_{E_{\mathrm{C}}} C_{2}\right) \square\left(D_{1} \|_{E_{\mathrm{C}}} D_{2}\right)\right) \stackrel{\text { op.x.t.a }}{\rightarrow}\left(B_{1}^{\prime} \|_{E_{\mathrm{C}}} B_{2}^{\prime}\right) .
$$

As both successor states are identical, they are $\mathcal{R}$-related. $\checkmark$ 


\begin{tabular}{|ccccc|}
\hline$B=\left(\left(C_{1} \square D_{1}\right) \|_{E_{\mathrm{C}}}\left(C_{2} \square D_{2}\right)\right)$ & $\stackrel{\text { op.x.t.a }}{\longrightarrow}$ & $\left(B_{1}^{\prime} \|_{E_{\mathrm{C}}} B_{2}^{\prime}\right)$ & $=B^{\prime}$ \\
$\mid$ & & $\vdots$ & \\
$\mathcal{R}$ & $\mathcal{R}$ & \\
$\mid$ & $\vdots$ & \\
$A=\left(\left(C_{1} \|_{E_{\mathrm{C}}} C_{2}\right) \square\left(D_{1} \|_{E_{\mathrm{C}}} D_{2}\right)\right)$ & $\stackrel{\text { op.x.t.a }}{\longrightarrow}$ & $\left(B_{1}^{\prime} \|_{E_{\mathrm{C}}} B_{2}^{\prime}\right)$ & $=A^{\prime}$ \\
\hline
\end{tabular}

op.x.t.a $\notin E_{\mathrm{C}}$ : Again, exactly one of the four components executes op.x.t.a, which we assume to be $C_{1}$. From

$$
\left(\left(C_{1} \square D_{1}\right) \|_{E_{\mathbf{C}}}\left(C_{2} \square D_{2}\right)\right) \stackrel{\text { op.x.t.a }}{\longrightarrow} B^{\prime}
$$

we get

$$
\left(C_{1} \square D_{1}\right) \stackrel{\text { op.x.t.a }}{\longrightarrow} C_{1}^{\prime}
$$

for some process $C_{1}^{\prime}$ such that $B^{\prime}=\left(C_{1}^{\prime} \|_{E_{\mathrm{C}}}\left(C_{2} \square D_{2}\right)\right)$. From this, we get $C_{1} \stackrel{\text { op.x.t.a }}{\longrightarrow} C_{1}^{\prime}$ and thus,

$$
\left(C_{1} \|_{E_{\mathrm{C}}} C_{2}\right) \stackrel{\text { op.x.t.a }}{\longrightarrow}\left(C_{1}^{\prime} \|_{E_{\mathrm{C}}} C_{2}\right) \text {. }
$$

This yields

$$
\left(\left(C_{1} \|_{E_{\mathbf{C}}} C_{2}\right) \square\left(D_{1} \|_{E_{\mathbf{C}}} D_{2}\right)\right) \stackrel{\text { op.x.t.a }}{\longrightarrow}\left(C_{1}^{\prime} \|_{E_{\mathbf{C}}} C_{2}\right) .
$$

Finally, again based on Lemma 5.2.1, we get initials $\left(D_{2}\right) \subseteq E_{\mathrm{C}}$ and

$$
\begin{aligned}
& \left(\left(C_{1}^{\prime} \|_{E_{\mathbf{C}}} C_{2}\right),\left(C_{1}^{\prime} \|_{E_{\mathbf{C}}}\left(C_{2} \square D_{2}\right)\right)\right) \in \mathcal{R}^{\prime} \cdot \checkmark \\
& \begin{array}{|cccc|}
\hline \hline B=\left(\left(C_{1} \square D_{1}\right) \|_{E_{\mathrm{C}}}\left(C_{2} \square D_{2}\right)\right) & \stackrel{\text { op.x.t.a }}{\longrightarrow}\left(C_{1}^{\prime} \|_{E_{\mathrm{C}}}\left(C_{2} \square D_{2}\right)\right) & =B^{\prime} \\
\mid & & \vdots & \\
\mathcal{R} & & \vdots & \\
\mid & & \vdots \\
A=\left(\left(C_{1} \|_{E_{\mathrm{C}}} C_{2}\right) \square\left(D_{1} \|_{E_{\mathrm{C}}} D_{2}\right)\right) & \stackrel{\text { op.x.t.a }}{\longrightarrow} & \left(C_{1}^{\prime} \|_{E_{\mathrm{C}}} C_{2}\right) & =A^{\prime} \\
\hline
\end{array}
\end{aligned}
$$

Sequential Composition: Here, $\mathcal{R}^{\prime}=\mathrm{ld}_{L^{C S P}}$.

(1) Let $(A, B) \in \mathcal{R}$ and $A \stackrel{e}{\rightarrow} A^{\prime}$ for

$$
A=\left(\left(C_{1} \|_{E_{\mathrm{C}}} C_{2}\right)_{9}^{\circ}\left(D_{1} \|_{E_{\mathrm{C}}} D_{2}\right)\right) \text {. }
$$

$\tau$-case: $A \stackrel{\tau}{\rightarrow} A^{\prime}$ yields that $C_{1}=$ Skip and $C_{2}=$ Skip, based on the firing rule for sequential composition and thus, $A^{\prime}=\left(D_{1} \|_{E_{\mathrm{C}}} D_{2}\right)$. Therefore,

$$
C_{1} \stackrel{\circ}{9} D_{1} \stackrel{\tau}{\longrightarrow} D_{1} \text { and } C_{2} \stackrel{\circ}{{ }_{9}} D_{2} \stackrel{\tau}{\longrightarrow} D_{2},
$$

yielding $\left(\left(C_{1} \stackrel{\circ}{9} D_{1}\right) \|_{E_{\mathrm{C}}}\left(C_{2} \stackrel{\circ}{9} D_{2}\right)\right) \stackrel{\tau^{2}}{\longrightarrow}\left(D_{1} \|_{E_{\mathrm{C}}} D_{2}\right)$. Both successor states are related by $\operatorname{ld}_{L C S P}$, that is, $\mathcal{R}^{\prime} . \checkmark$ 


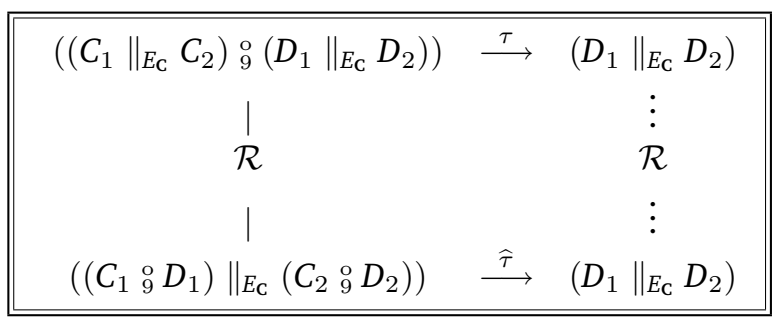

op-case: Let $A \stackrel{\text { op.x.t.a }}{\rightarrow} A^{\prime}$. Two cases need to be considered:

op.x.t.a $\in E_{\mathbf{C}}:$ If $\left(C_{1} \|_{E_{\mathbf{C}}} C_{2}\right) \stackrel{\text { op.x.t.a }}{\rightarrow} A^{\prime}$, we have

$$
A^{\prime}=\left(\left(C_{1}^{\prime} \|_{E_{\mathrm{C}}} C_{2}^{\prime}\right)_{9}^{\circ}\left(D_{1} \|_{E_{\mathrm{C}}} D_{2}\right)\right)
$$

for some $C_{i}^{\prime} \in L^{C S P}$ and thus,

$$
C_{1} \stackrel{\text { op.x.t.a }}{\rightarrow} C_{1}^{\prime} \text { and } C_{2} \stackrel{\text { op.x.t.a }}{\rightarrow} C_{2}^{\prime},
$$

from which can stepwise deduce

$$
\left(\left(C_{1} \stackrel{\circ}{9} D_{1}\right) \|_{E_{\mathbf{C}}}\left(C_{2} \stackrel{\circ}{9} D_{2}\right)\right) \stackrel{\text { op.x.t.a }}{\rightarrow}\left(\left(C_{1}^{\prime} \stackrel{\circ}{9} D_{1}\right) \|_{E_{\mathbf{C}}}\left(C_{2}^{\prime} \stackrel{\circ}{9} D_{2}\right)\right) .
$$

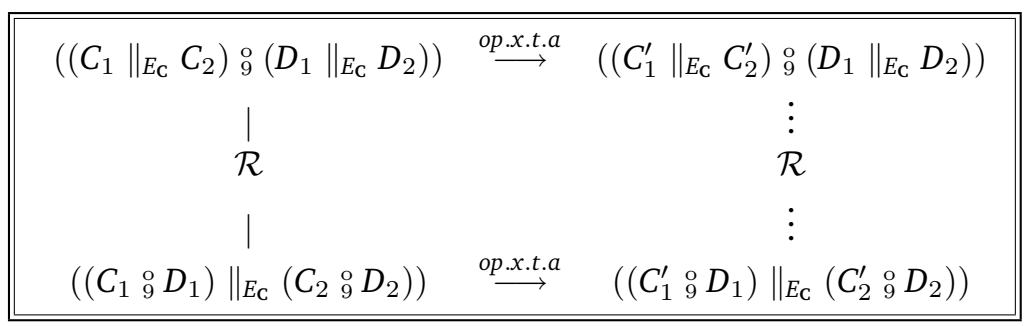

If $\left(D_{1} \|_{E_{\mathrm{C}}} D_{2}\right) \stackrel{\text { op.x.t.a }}{\rightarrow} A^{\prime}$, both, $C_{1}$ and $C_{2}$, need to have terminated, thus requiring $C_{1}=$ Skip, $C_{2}=$ Skip, and we proceed analogously. $\checkmark$

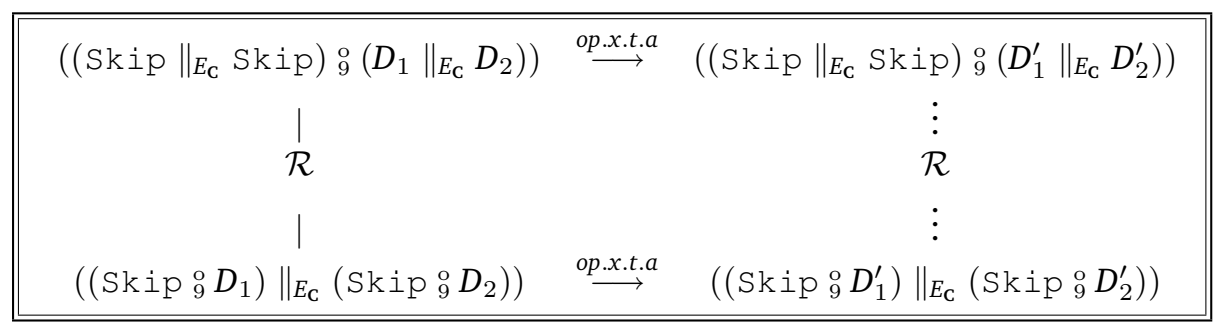

op.x.t.a $\notin E_{\mathrm{C}}$ : In this case, again, either $C_{1} \|_{E_{\mathrm{C}}} C_{2}$ or $D_{1} \|_{E_{\mathrm{C}}} D_{2}$ perform op.x.t.a, where the latter requires $\left(C_{1} \|_{E_{\mathrm{C}}} C_{2}\right)=\mathrm{Skip}$. The proof is straightforward and according to the previous proof steps. The bisimulation diagram for the first case, where we assume that $C_{1}$ performs op.x.t.a, is given next. $\checkmark$ 


$$
\begin{array}{|ccc|}
\hline\left(\left(C_{1} \|_{E_{\mathrm{C}}} C_{2}\right){ }_{9}^{\circ}\left(D_{1} \|_{E_{\mathrm{C}}} D_{2}\right)\right) & \stackrel{\text { op.x.t.a }}{\longrightarrow} & \left(\left(C_{1}^{\prime} \|_{E_{\mathrm{C}}} C_{2}\right) \stackrel{\circ}{9}\left(D_{1} \|_{E_{\mathrm{C}}} D_{2}\right)\right) \\
\mid & & \vdots \\
\mathcal{R} & & \vdots \\
\mid & \stackrel{\mathcal{R}}{\longrightarrow} & \left(\left(C_{1}^{\prime}{ }_{9}^{\circ} D_{1}\right) \|_{E_{\mathrm{C}}}\left(C_{2}{ }_{9}^{\circ} D_{2}\right)\right) \\
\left(\left(C_{1}{ }_{9}^{\circ} D_{1}\right) \|_{E_{\mathrm{C}}}\left(C_{2}{ }_{9}^{\circ} D_{2}\right)\right) & \stackrel{\text { op.x.t.a }}{\longrightarrow} \\
\hline
\end{array}
$$

(2) Let $(A, B) \in \mathcal{R}$ and $B \stackrel{e}{\rightarrow} B^{\prime}$ for $B=\left(\left(C_{1} \stackrel{\circ}{9} D_{1}\right) \|_{E_{\mathrm{C}}}\left(C_{2} \stackrel{\circ}{9} D_{2}\right)\right)$.

$\tau$-case: Let $B \stackrel{\tau}{\rightarrow} B^{\prime}$. Then, without loss of generality, $\left(C_{1} \stackrel{\circ}{9} D_{1}\right) \stackrel{\tau}{\longrightarrow} D_{1}$, based on the firing rule for sequential composition. We deduce that $\left(C_{1} \|_{E_{\mathrm{C}}} C_{2}\right) \stackrel{\tau}{\longrightarrow}\left(\mathrm{Skip} \|_{E_{\mathrm{C}}} C_{2}\right)$ holds. The bisimulation diagram is given next. $\checkmark$

\begin{tabular}{|ccc|}
\hline$\left(\left(C_{1}{ }_{9}^{\circ} D_{1}\right) \|_{E_{\mathrm{C}}}\left(C_{2}{ }_{9}^{\circ} D_{2}\right)\right)$ & $\stackrel{\tau}{\longrightarrow}$ & $\left(\left(\operatorname{Skip}{ }_{9}^{\circ} D_{1}\right) \|_{E_{\mathrm{C}}}\left(C_{2}{ }_{9}^{\circ} D_{2}\right)\right)$ \\
$\mid$ & & $\vdots$ \\
$\mathcal{R}$ & & $\mathcal{R}$ \\
$\mid$ & $\vdots$ \\
$\left(\left(C_{1} \|_{E_{\mathrm{C}}} C_{2}\right) \stackrel{\circ}{9}\left(D_{1} \|_{E_{\mathrm{C}}} D_{2}\right)\right)$ & $\stackrel{\tau}{\longrightarrow}$ & $\left(\left(\operatorname{Skip} \|_{E_{\mathrm{C}}} C_{2}\right) \stackrel{\circ}{9}\left(D_{1} \|_{E_{\mathrm{C}}} D_{2}\right)\right)$ \\
\hline
\end{tabular}

op-case: Let $B \stackrel{\text { op.x.t.a }}{\rightarrow} B^{\prime}$. Again, there are two separate cases:

op.x.t.a $\in E_{\mathrm{C}}$ : Theorem 4.3.16, (5), ensures that a synchronisation within $\left(C_{1}{ }_{9}^{\circ} D_{1}\right) \|_{E_{\mathrm{C}}}\left(C_{2}{ }_{9}^{\circ} D_{2}\right)$ can only occur between $C_{1}$ and $C_{2}$ or between $D_{1}$ and $D_{2}$. In case that $D_{1}$ and $D_{2}$ synchronise on op.x.t.a, $C_{1}$ and $C_{2}$ are equivalent to $\mathrm{Skip}$. The remainder of this particular proof step is straightforward. We give the bisimulation diagram for the $C$-case next. $\checkmark$

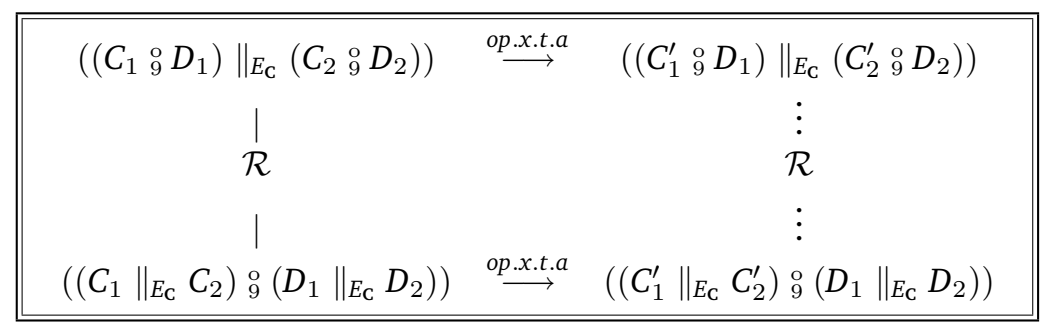

op.x.t.a $\notin E_{\mathrm{C}}$ : From the structure of the process $\left(C_{1}{ }_{9}^{\circ} D_{1}\right) \|_{E_{\mathrm{C}}}\left(C_{2}{ }_{9}^{\circ} D_{2}\right)$, we know that $C_{i}$ terminates before $D_{i}$. In addition, we have to show that $C_{2}$ terminates before $D_{1}$, which implies that $C_{1}$ terminates before $D_{2}$. Based on that, in order to complete this case, we may safely use $C_{1}=\mathrm{Skip}$ and $C_{2}=\mathrm{Skip}$, in case that $D_{i}$ performs op.x.t.a $\notin$ $E_{\mathrm{C}}$. Assume that $D_{1}$ performs op.x.t.a $\notin E_{\mathrm{C}}$. Then, by definition, either op.t.x. $a \in l\left[\mathbf{P h}_{1}\right]$ or op.t.x.a $\in l\left[\mathbf{P h}_{3}\right]$. In the first case, as $C$ terminates before $D$ and based on the definition of $\mathbf{P h}_{1}$, the process $C$ is completely assigned to $\mathbf{P h}_{1}$. Thus, due to $\alpha C_{2} \subseteq E_{S_{2}}, C_{2}$ can only perform synchronised events with $C_{1}$, which need to happen prior to 
op.x.t.a. In the latter case, the set of events for $C_{2}$ are executed before any event of $l\left[\mathbf{P h}_{3}\right]$, again yielding the termination of $C_{2}$ prior to $D_{1}$. The bisimulation diagram for a sole step of $D_{1}$ is given next.

\begin{tabular}{|ccc|}
\hline$\left(\left(\right.\right.$ Skip $\left.{ }_{9}^{\circ} D_{1}\right) \|_{E_{\mathrm{C}}}\left(\right.$ Skip $\left.\left.{ }_{9} D_{2}\right)\right)$ & $\stackrel{\text { op.x.t.a }}{\longrightarrow}$ & $\left(\left(\right.\right.$ Skip $\left.{ }_{9}^{\prime} D_{1}^{\prime}\right) \|_{E_{\mathrm{C}}}\left(\right.$ Skip $\left.\left.{ }_{9}^{\circ} D_{2}\right)\right)$ \\
$\mid$ & & $\vdots$ \\
$\mathcal{R}$ & & $\vdots$ \\
$\mid$ & $\stackrel{\mathcal{R}}{\longrightarrow}$ & $\left(\left(\right.\right.$ Skip $\|_{E_{\mathrm{C}}}$ Skip $\left.){ }_{9}^{\circ}\left(D_{1}^{\prime} \|_{E_{\mathrm{C}}} D_{2}\right)\right)$ \\
\hline
\end{tabular}

Parallel Composition: Again, $\mathcal{R}^{\prime}=\varnothing$. For the case of parallel composition, weak bisimilarity of the processes

$$
\begin{aligned}
& A=\left(C_{1} \|_{E_{\mathrm{C}}} C_{2}\right) \|_{S}\left(D_{1} \|_{E_{\mathrm{C}}} D_{2}\right) \text { and } \\
& B=\left(C_{1} \|_{S \cap E_{S_{1}}} D_{1}\right) \|_{E_{\mathrm{C}}}\left(C_{2} \|_{S \cap E_{S_{2}}} D_{2}\right)
\end{aligned}
$$

has to be shown. First, we consider the case of a $\tau$-transition for both directions.

(1) Let $(A, B) \in \mathcal{R}$ and $A \stackrel{\tau}{\longrightarrow} A^{\prime}$. This case is immediate based on the rules for promoting $\tau$-transitions within a parallel composition.

(2) For $B \stackrel{\tau}{\longrightarrow} B^{\prime}$, we proceed analogously.

For either $A \stackrel{\text { op.x.t.a }}{\longrightarrow} A^{\prime}$ or $B \stackrel{\text { op.x.t.a }}{\longrightarrow} B^{\prime}$, several cases need to be separated, making a case differentiation over all cases rather tedious. As most of these cases refer to the transition laws for CSP, corresponding to the applications for the external choice and sequential composition, we precisely deal with the decisive cases and only sketch the straightforward cases.

Figure 5.9 shows the different cases, which need to be considered for $A$ or $B$ performing op.x.t.a. These are:

(a) op.x.t.a $\in\left(S \cap E_{\mathbf{C}}\right)$,

(b) op.x.t.a $\in\left(S \cap E_{1}\right)$,

(c) op.x.t.a $\in\left(S \cap E_{2}\right)$,

(d) op.x.t.a $\in\left(E_{\mathbf{C}} \backslash S\right)$,

(e) op.x.t.a $\in\left(E_{1} \backslash S\right)$ and

(f) op.x.t.a $\in\left(E_{2} \backslash S\right)$.

For the bisimulation proof, there is one decisive case for both directions. We give the intuitive ideas first:

- $A=\left(C_{1} \|_{E_{\mathrm{C}}} C_{2}\right) \|_{S}\left(D_{1} \|_{E_{\mathrm{C}}} D_{2}\right)$ performing an event from $S \backslash E_{\mathrm{C}}$ might cause a wrong synchronisation between $C_{2}$ and $D_{1}$ or $C_{1}$ and $D_{2}$. However, as the event is either an element of $E_{1}$ or $E_{2}$ but never an element of both sets, this is impossible. 


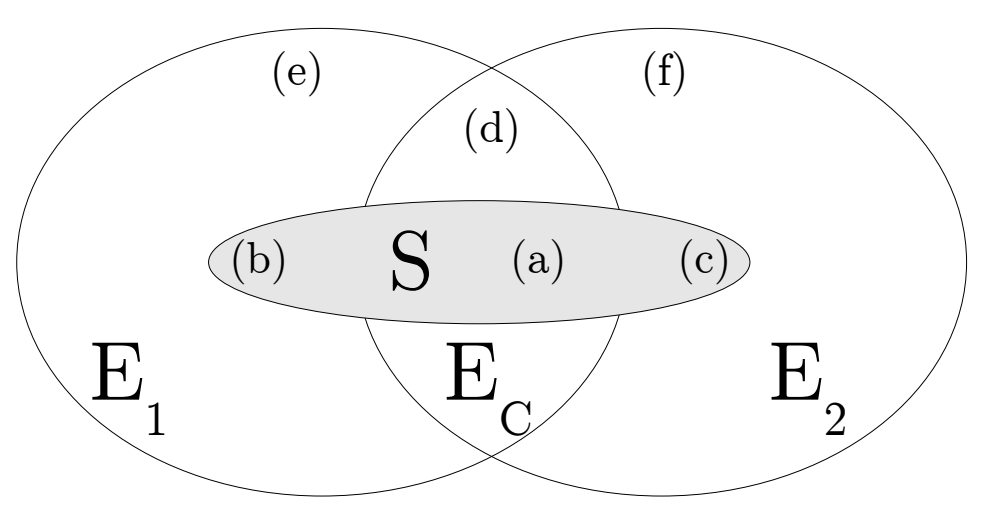

Figure 5.9: Case differentiation for Lemma 5.2.3, parallel composition

- $B=\left(C_{1} \|_{S \cap E_{S_{1}}} D_{1}\right) \|_{E_{\mathbf{C}}}\left(C_{2} \|_{S \cap E_{S_{2}}} D_{2}\right)$ performing an event from $E_{\mathbf{C}} \backslash S$ might cause a wrong synchronisation between $C_{2}$ and $D_{1}$ or $C_{1}$ and $D_{2}$ as well. Here, Theorem 4.3.16 shows that the addressing extension prevents this from happening.

Next, we show the bisimilarity conditions for all six cases:

op.x.t.a $\in\left(S \cap E_{\mathbf{C}}\right)$ : Independent of Condition (1) or (2), all four processes $C_{1}$, $C_{2}, D_{1}$ and $D_{2}$ have to synchronise on op.x.t.a. Showing both conditions is immediate, based on applying the firing rules for CSP.

op.x.t.a $\in\left(S \cap E_{1}\right)$ : For implication (1), assume that

$$
\left(\left(C_{1} \|_{E_{\mathrm{C}}} C_{2}\right) \|_{S}\left(D_{1} \|_{E_{\mathrm{C}}} D_{2}\right)\right) \stackrel{\text { op.x.t.a }}{\longrightarrow}\left(\left(C_{1}^{\prime} \|_{E_{\mathrm{C}}} C_{2}^{\prime}\right) \|_{S}\left(D_{1}^{\prime} \|_{E_{\mathrm{C}}} D_{2}^{\prime}\right)\right) .
$$

Based on op.x.t.a $\in E_{1}$, the synchronisation must be performed by $C_{1}$ and $D_{1}$. Thus, $C_{2}^{\prime}=C_{2}, D_{2}^{\prime}=D_{2}$ and $\left(C_{1} \|_{S \cap E_{S_{1}}} D_{1}\right) \stackrel{\text { op.x.t.a }}{\longrightarrow}\left(C_{1}^{\prime} \|_{S \cap E_{S_{1}}} D_{1}^{\prime}\right)$. This yields $\left(\left(C_{1} \|_{S \cap E_{S_{1}}} D_{1}\right) \|_{E_{\mathbf{C}}}\left(C_{2} \|_{S \cap E_{S_{2}}} D_{2}\right)\right) \stackrel{\text { op.x.t.a }}{\longrightarrow}\left(\left(C_{1}^{\prime} \|_{S \cap E_{S_{1}}} D_{1}^{\prime}\right) \|_{E_{\mathbf{C}}}\left(C_{2} \|_{S \cap E_{S_{2}}} D_{2}\right)\right)$, as op.x.t.a $\notin E_{\mathrm{C}}$.

\begin{tabular}{|ccc|}
\hline$\left(\left(C_{1} \|_{E_{\mathrm{C}}} C_{2}\right) \|_{S}\left(D_{1} \|_{E_{\mathrm{C}}} D_{2}\right)\right)$ & $\stackrel{\text { op.x.t.a }}{\longrightarrow}$ & $\left(\left(C_{1}^{\prime} \|_{E_{\mathrm{C}}} C_{2}\right) \|_{S}\left(D_{1}^{\prime} \|_{E_{\mathrm{C}}} D_{2}\right)\right)$ \\
$\mid$ & & $\vdots$ \\
$\mathcal{R}$ & & $\mathcal{R}$ \\
$\mid$ & $\stackrel{\text { op.x.t.a }}{\longrightarrow}$ & $\left(\left(C_{1}^{\prime} \|_{S \cap E_{S_{1}}} D_{1}^{\prime}\right) \|_{E_{\mathrm{C}}}\left(C_{2} \|_{S \cap E_{S_{2}}} D_{2}\right)\right)$ \\
$\left(\left(C_{1} \|_{S \cap E_{S_{1}}} D_{1}\right) \|_{E_{\mathrm{C}}}\left(C_{2} \|_{S \cap E_{S_{2}}} D_{2}\right)\right)$ & $\stackrel{2}{\longrightarrow}$ \\
\hline
\end{tabular}

Implication (2) is straightforward. $\checkmark$ 
op.x.t.a $\in\left(S \cap E_{2}\right)$ : Analogous to the previous case. $\checkmark$

op.x.t.a $\in\left(E_{\mathbf{C}} \backslash S\right)$ : Here, implication (1) is straightforward. For implication (2), let

$$
\left(\left(C_{1} \|_{S \cap E_{S_{1}}} D_{1}\right) \|_{E_{\mathbf{C}}}\left(C_{2} \|_{S \cap E_{S_{2}}} D_{2}\right)\right) \stackrel{\text { op.x.t.a }}{\longrightarrow}\left(\left(C_{1}^{\prime} \|_{S \cap E_{S_{1}}} D_{1}^{\prime}\right) \|_{E_{\mathbf{C}}}\left(C_{2}^{\prime} \|_{S \cap E_{S_{2}}} D_{2}^{\prime}\right)\right) \text {. }
$$

Theorem 4.3.16 yields that only $C_{1}$ and $C_{2}$ or $D_{1}$ and $D_{2}$ are able to synchronise. We assume the first, thus yielding $D_{1}^{\prime}=D_{1}$ and $D_{2}^{\prime}=D_{2}$. We get $\left(C_{1} \|_{E_{\mathrm{C}}}\right.$ $\left.C_{2}\right) \stackrel{\text { op.x.t.a }}{\longrightarrow}\left(C_{1}^{\prime} \|_{E_{\mathrm{C}}} C_{2}^{\prime}\right)$ and finally

$$
\left(\left(C_{1} \|_{E_{\mathrm{C}}} C_{2}\right) \|_{S}\left(D_{1} \|_{E_{\mathrm{C}}} D_{2}\right)\right) \stackrel{\text { op.x.t.a }}{\longrightarrow}\left(\left(C_{1}^{\prime} \|_{E_{\mathrm{C}}} C_{2}^{\prime}\right) \|_{S}\left(D_{1} \|_{E_{\mathrm{C}}} D_{2}\right)\right) \cdot \checkmark
$$

\begin{tabular}{|ccc|}
\hline$\left(\left(C_{1} \|_{S \cap E_{S_{1}}} D_{1}\right) \|_{E_{\mathrm{C}}}\left(C_{2} \|_{S \cap E_{S_{2}}} D_{2}\right)\right)$ & $\stackrel{\text { op.x.t.a }}{\longrightarrow}$ & $\left(\left(C_{1}^{\prime} \|_{S \cap E_{S_{1}}} D_{1}\right) \|_{E_{\mathrm{C}}}\left(C_{2}^{\prime} \|_{S \cap E_{S_{2}}} D_{2}\right)\right)$ \\
$\mid$ & & $\vdots$ \\
$\mathcal{R}$ & & $\vdots$ \\
$\mid$ & $\stackrel{\mathcal{R}}{\longrightarrow}$ & $\left(\left(C_{1}^{\prime} \|_{E_{\mathrm{C}}} C_{2}^{\prime}\right) \|_{S}\left(D_{1} \|_{E_{\mathrm{C}}} D_{2}\right)\right)$ \\
$\left(\left(C_{1} \|_{E_{\mathrm{C}}} C_{2}\right) \|_{S}\left(D_{1} \|_{E_{\mathrm{C}}} D_{2}\right)\right)$ & $\stackrel{\text { op.x.t.a }}{\longrightarrow}$ \\
\hline
\end{tabular}

op.x.t.a $\in\left(E_{1} \backslash S\right)$ : Independent of Condition (1) or (2), exactly one of the four processes needs to perform op.x.t.a, which is straightforward.

op.x.t.a $\in\left(E_{2} \backslash S\right)$ : Analogous to the previous case. $\checkmark$

\subsubsection{Correctness of the Decomposition: CSP part}

Finally, we show correctness for the decomposition of $S$.main by using the results from the previous sections. Again, we use weak bisimulation as the method of proof: we construct a weak bisimulation relation, comprising tuples

$$
\left(P,\left.P \llbracket \mathrm{R}_{1}^{\mathrm{C}} \rrbracket\right|_{E_{S_{1}}} \|_{E_{\mathrm{C}}} P \llbracket \mathrm{R}_{2}^{\mathrm{C}} \rrbracket \mid E_{S_{2}}\right),
$$

where $P$ denotes any reachable state of the LTS of $S$.main. As we show trace equivalence modulo the renaming by transmission parameters and address parameters, we accordingly show bisimilarity not explicitly denoting the renaming. ${ }^{2}$ For simplification, we let $P_{\mathrm{R}}$ denote $P \llbracket \mathrm{R}^{\mathrm{C}} \rrbracket$.

In the theorem, we will use a lemma from [Brü08], namely Lemma 6.1.2. It relates the possible transitions of a CSP process to transitions of a projection of this process. Next, we state the main theorem of this section:

Theorem 5.2.4. (Correctness of the decomposition: CSP part)

Let $S$ be a specification, and let $\mathbf{C}=\left(\mathbf{C}_{1}, \mathbf{C}_{2}\right)$ be a cut, yielding a decomposition into $S_{1}$ and $S_{2}$, according to Definition 4.3.24. Then, the following holds:

$$
\text { S.main }=_{T}\left(S_{1} \cdot \text { main } \|_{E_{\mathrm{C}}} S_{2} \cdot \text { main }\right) \llbracket \mathrm{R}^{\prime} \rrbracket .
$$

\footnotetext{
${ }^{2}$ As a matter of course, the renaming is solely syntactically neglected. We still have to use the properties of the additional parameters, as they ultimately ensure the correctness of the decomposition.
} 
Proof: We show that $S$.main and $S_{1}$.main $\|_{E_{\mathrm{C}}} S_{2}$.main are the initial states of a weak bisimulation

$$
\mathcal{R}:=\left\{\left(P,\left.P_{\mathrm{R}}\right|_{E_{S_{1}}} \|\left._{E_{\mathrm{C}}} P_{\mathrm{R}}\right|_{E_{S_{2}}}\right) \mid P \in L^{C S P} \text { reachable state of LTS of } S \text {.main, } P_{\mathrm{R}}=P \llbracket \mathrm{R}^{\mathrm{C}} \rrbracket\right\} .
$$

Again, we need to show two implications:

(1) If $\left(P,\left.P_{\mathrm{R}}\right|_{E_{S_{1}}} \|\left._{E_{\mathrm{C}}} P_{\mathrm{R}}\right|_{E_{S_{2}}}\right) \in \mathcal{R}$ and $P \stackrel{o p . x}{\longrightarrow} P^{\prime}$ for op.x $\in E_{S}\left[P \stackrel{\tau}{\longrightarrow} P^{\prime}\right]$, then there exists some $Q^{\prime}$, such that

$$
\left(\left.P_{\mathrm{R}}\right|_{E_{S_{1}}} \|\left.\left.\right|_{E_{\mathrm{C}}} P_{\mathrm{R}}\right|_{E_{S_{2}}}\right) \stackrel{\text { op.x.t.a }}{\longrightarrow} Q^{\prime}\left[\left(\left.P_{\mathrm{R}}\right|_{E_{S_{1}}} \|\left._{E_{\mathrm{C}}} P_{\mathrm{R}}\right|_{E_{S_{2}}}\right) \stackrel{\widehat{\tau}}{\longrightarrow} Q^{\prime}\right]
$$

for some op.x.t.a $\in E_{S^{\prime}}$ and $\left(P^{\prime}, Q^{\prime}\right) \in \mathcal{R}$.

(2) If $\left(P,\left.P_{\mathrm{R}}\right|_{E_{S_{1}}} \|\left. E_{E_{\mathrm{C}}} P_{\mathrm{R}}\right|_{E_{S_{2}}}\right) \in \mathcal{R}$ and

$$
\left.\left(\left.P_{\mathrm{R}}\right|_{E_{S_{1}}} \|\left.\left.\right|_{E_{\mathrm{C}}} P_{\mathrm{R}}\right|_{E_{S_{2}}}\right) \stackrel{\text { op.x.t.a }}{\longrightarrow} Q^{\prime}\left[\left.P_{\mathrm{R}}\right|_{E_{S_{1}}} \|\left._{E_{\mathrm{C}}} P_{\mathrm{R}}\right|_{E_{S_{2}}}\right) \stackrel{\tau}{\longrightarrow} Q^{\prime}\right]
$$

then there exists some $P^{\prime}$, such that $P \stackrel{\widehat{o p . x}}{\longrightarrow} P^{\prime}$ for op. $x \in E_{S}\left[P \stackrel{\widehat{\tau}}{\longrightarrow} P^{\prime}\right]$ and $\left(P^{\prime}, Q^{\prime}\right) \in \mathcal{R}$.

For the first implication, it is sufficient to show that op.x.t.a $\in E_{S^{\prime}}$ exists.

(1): First, let $P \stackrel{\tau}{\longrightarrow} P^{\prime}$. Based on the firing laws for CSP, a $\tau$-transition is preserved by a renaming as well as hiding. Thus, we immediately get

$$
\left(\left.P_{\mathrm{R}}\right|_{E_{S_{1}}} \|\left._{E_{\mathrm{C}}} P_{\mathrm{R}}\right|_{E_{S_{2}}}\right) \stackrel{\tau}{\longrightarrow}\left(\left.P_{\mathrm{R}}^{\prime}\right|_{E_{S_{1}}} \|\left._{E_{\mathrm{C}}} P_{\mathrm{R}}^{\prime}\right|_{E_{S_{2}}}\right) .
$$

Now let $P \stackrel{o p . x}{\longrightarrow} P^{\prime}$ for op. $x \in E_{S}$. First, assume that $P$ is composed of two parallel processes, with op being synchronised. Based on Theorem 4.3.16, (2) and (3), if $P$ performs a synchronised step, the process $P_{\mathrm{R}}$ can accordingly perform this step, as the renaming preserves the synchronisation structure. Thus, we do not separately need to deal with this particular structure of $P$. Next, we have to distinguish between three cases for op.x:

op. $x \in E_{\mathrm{C}}:$ In this case, we apply the first property of Lemma 6.1.2, [Brü08]. For $e \in E$, it states that performing $e$ for $P$ and $\left.P\right|_{E}$ leads to corresponding successor states $Q$ and $\left.Q\right|_{E}$ :

$$
\left.\left.(P \stackrel{e}{\longrightarrow} Q \wedge e \in E) \Rightarrow P\right|_{E} \stackrel{e}{\longrightarrow} Q\right|_{E} .
$$

In our context and based upon the previous observation, the property yields

$$
\left.\left.P_{\mathrm{R}}\right|_{E_{S_{1}}} \stackrel{\text { op.x.t. } t_{1} \cdot a_{1}}{\longrightarrow} P_{\mathrm{R}}^{\prime}\right|_{E_{S_{1}}} \text { and }\left.\left.P_{\mathrm{R}}\right|_{E_{S_{2}}} \stackrel{\text { op.x.t.t. }}{\longrightarrow} P_{\mathrm{R}_{2}}^{\prime}\right|_{E_{S_{2}}}
$$

for some op.x.t. $t_{i} \cdot a_{i} \in E_{S^{\prime}}$. In order to deduce that $\left.P_{\mathrm{R}}\right|_{E_{S_{1}}}$ and $\left.P_{\mathrm{R}}\right|_{E_{S_{2}}}$ can do a synchronous step, there needs to exist some op.t.x.a $\in E_{S^{\prime}}$, which both processes can perform. This is the case: the transmission parameters are not 
restricted by the CSP part at all. Thus, any values are possible for $t_{1}$ and $t_{2}$. For the address parameters, Theorem 4.3.16, (1), showed that their values are identical for both, $S_{1}$.main and $S_{2}$.main. We deduce that there exists op.t.x. $a \in E_{S^{\prime}}$, such that

$$
\left(\left.P_{\mathrm{R}}\right|_{E_{S_{1}}} \|\left._{E_{\mathrm{C}}} P_{\mathrm{R}}\right|_{E_{S_{2}}}\right) \stackrel{\text { op.x.t.a }}{\longrightarrow}\left(\left.P_{\mathrm{R}}^{\prime}\right|_{E_{S_{1}}} \|\left._{E_{\mathrm{C}}} P_{\mathrm{R}}^{\prime}\right|_{E_{S_{2}}}\right)
$$

and $\left(P^{\prime},\left.P_{\mathrm{R}}^{\prime}\right|_{E_{S_{1}}} \|\left._{E_{\mathrm{C}}} P_{\mathrm{R}}^{\prime}\right|_{E_{S_{2}}}\right) \in \mathcal{R} . \checkmark$

op. $x \in E_{1}$ : Again, we deduce

$$
\left.\left.P_{\mathrm{R}}\right|_{E_{S_{1}}} \stackrel{\text { op.x.t.t. }}{\longrightarrow} P_{\mathrm{R}}^{\prime}\right|_{E_{S_{1}}} .
$$

Based on op.x $\notin E_{\mathrm{C}}$ and the operational semantics of CSP, we get

$$
\left(\left.P_{\mathrm{R}}\right|_{E_{S_{1}}} \|\left._{E_{\mathrm{C}}} P_{\mathrm{R}}\right|_{E_{S_{2}}}\right) \stackrel{\text { op.x.t. } t_{1} \cdot a_{1}}{\longrightarrow}\left(\left.P_{\mathrm{R}}^{\prime}\right|_{E_{S_{1}}} \|\left.\left.\right|_{E_{\mathrm{C}}} P_{\mathrm{R}}\right|_{E_{S_{2}}}\right) .
$$

By using the firing rule for CSP hiding and op. $x \notin E_{2}$, we deduce that

$$
\left.\left.P_{\mathrm{R}}\right|_{E_{S_{2}}} \stackrel{\tau}{\longrightarrow} P_{\mathrm{R}}^{\prime}\right|_{E_{S_{2}}}
$$

and thus,

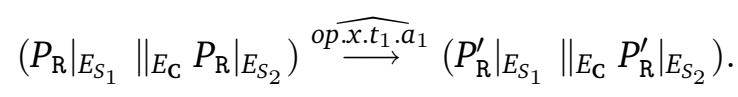

Again, $\left(P^{\prime},\left.P_{\mathrm{R}}^{\prime}\right|_{E_{S_{1}}} \|\left._{E_{\mathrm{C}}} P_{\mathrm{R}}^{\prime}\right|_{E_{S_{2}}}\right) \in \mathcal{R}$.

op. $x \in E_{2}$ : Analogous to the second case. $\checkmark$

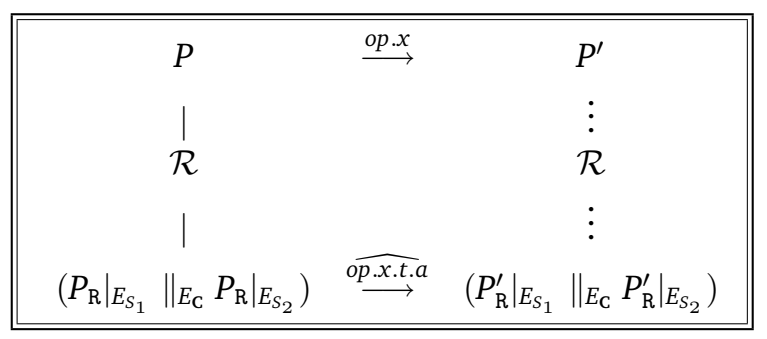

(2): Let

$$
\left(\left.P_{\mathrm{R}}\right|_{E_{S_{1}}} \|\left._{E_{\mathrm{C}}} P_{\mathrm{R}}\right|_{E_{S_{2}}}\right) \stackrel{\text { op.x.t.a }}{\longrightarrow} Q^{\prime}
$$

for some op.x.t.a $\in E_{S^{\prime}}$. We show that there exists $P^{\prime} \in L^{C S P}$, such that $P \stackrel{\text { op.x }}{\longrightarrow} P^{\prime}$ and

$$
Q^{\prime}=\left(\left.P_{\mathrm{R}}^{\prime}\right|_{E_{S_{1}}} \|\left._{E_{\mathrm{C}}} P_{\mathrm{R}}^{\prime}\right|_{E_{S_{2}}}\right),
$$

by induction on the structure of $P$. 


\begin{tabular}{||ccc||}
\hline \hline$\left(\left.P_{\mathrm{R}}\right|_{E_{S_{1}}} \|\left._{E_{\mathrm{C}}} P_{\mathrm{R}}\right|_{E_{S_{2}}}\right) \stackrel{\text { op.x.t.a }}{\longrightarrow}$ & $\left(\left.P_{\mathrm{R}}^{\prime}\right|_{E_{S_{1}}} \|\left._{E_{\mathrm{C}}} P_{\mathrm{R}}^{\prime}\right|_{E_{S_{2}}}\right)$ & $=Q^{\prime}$ \\
$\mid$ & & $\vdots$ \\
$\mathcal{R}$ & & $\mathcal{R}$ \\
$\mid$ & $\stackrel{\widehat{o p . x}}{\longrightarrow}$ & $P^{\prime}$ \\
$P$ & &
\end{tabular}

Induction Basis: Let $P=e \rightarrow Q$ for some $e \in E_{S}$ and $Q \in L^{C S P}$. Based on Definition 4.3.3, we have

$$
\left.(e \rightarrow Q)\right|_{E}:= \begin{cases}\left.Q\right|_{E}, & e \notin E, \\ \left.e \rightarrow Q\right|_{E}, & \text { otherwise }\end{cases}
$$

We have to distinguish between three cases: ${ }^{3}$

$\mathrm{R}^{\mathrm{C}}(e) \subseteq E_{\mathrm{C}}:$ Based on $(e \rightarrow P) \llbracket \mathrm{R} \rrbracket=e^{\prime}: \mathrm{R}(e) \rightarrow P \llbracket \mathrm{R} \rrbracket([$ Sch09] $)$,

$$
\left(\left.P_{\mathrm{R}}\right|_{E_{S_{1}}} \|\left._{E_{\mathrm{C}}} P_{\mathrm{R}}\right|_{E_{S_{2}}}\right)=\left(\left(e^{\prime}:\left.\mathrm{R}_{1}^{\mathrm{C}}(e) \rightarrow Q_{\mathrm{R}}\right|_{E_{S_{1}}}\right) \|_{E_{\mathrm{C}}}\left(e^{\prime \prime}:\left.\mathrm{R}_{2}^{\mathrm{C}}(e) \rightarrow Q_{\mathrm{R}}\right|_{E_{S_{2}}}\right)\right) .
$$

As $\left(\left.P_{\mathrm{R}}\right|_{E_{S_{1}}} \|\left._{E_{\mathrm{C}}} P_{\mathrm{R}}\right|_{E_{S_{2}}}\right) \stackrel{\text { op.x.t.a }}{\longrightarrow} Q^{\prime}$, we get op.x.t.a $\in\left(\mathrm{R}_{1}^{\mathrm{C}}(e) \cap \mathrm{R}_{2}^{\mathrm{C}}(e)\right)$. Thus, $e=$ op.x. Moreover,

$$
\left(\left.P_{\mathrm{R}}\right|_{E_{S_{1}}} \|\left. E_{E_{\mathrm{C}}} P_{\mathrm{R}}\right|_{E_{S_{2}}}\right) \stackrel{\text { op.x.t.a }}{\longrightarrow}\left(\left.Q_{\mathrm{R}}\right|_{E_{S_{1}}} \|\left._{E_{\mathbf{C}}} Q_{\mathrm{R}}\right|_{E_{S_{2}}}\right),
$$

as both processes have to synchronise on op.x.t.a $\in E_{\mathbf{C}}$. Obviously, $P \stackrel{e}{\longrightarrow} Q$ holds, and finally, $\left(Q,\left.Q_{\mathrm{R}}\right|_{E_{S_{1}}} \|\left._{E_{\mathrm{C}}} Q_{\mathrm{R}}\right|_{E_{S_{2}}}\right) \in \mathcal{R}$.

$\mathrm{R}^{\mathrm{C}}(e) \in E_{1}$ : Let $e^{\prime}=\mathrm{R}^{\mathrm{C}}(e)$. In this case,

$$
\left(\left.P_{\mathrm{R}}\right|_{E_{S_{1}}} \|\left._{E_{\mathrm{C}}} P_{\mathrm{R}}\right|_{E_{S_{2}}}\right)=\left(\left(\left.e^{\prime} \rightarrow Q_{\mathrm{R}}\right|_{E_{S_{1}}}\right) \|\left._{E_{\mathrm{C}}} Q_{\mathrm{R}}\right|_{E_{S_{2}}}\right)=: X,
$$

as the projection eliminates $e^{\prime}$ for the right hand side of the parallel composition. In case that op.t.x. $a=e^{\prime}$ holds, the process $X$ switches to $\left.Q_{\mathrm{R}}\right|_{E_{S_{1}}} \|\left._{E_{\mathrm{C}}} Q_{\mathrm{R}}\right|_{E_{S_{2}}}$, and we reside in the first case. However, we need to show that $X$ must not be able to perform any other event than $e^{\prime}$, that is, $\left.Q_{\mathrm{R}}\right|_{E_{S_{2}}}$ is incapable of performing a non-synchronised step. But this is the case: from Lemma 5.2.1 and based on $e^{\prime} \in E_{1}$, we know that the set of initial events of $\left.Q_{\mathrm{R}}\right|_{E_{S_{2}}}$ is a subset of $E_{S_{1}}$ and thus $E_{\mathrm{C}}$. Therefore, $\left.Q_{\mathrm{R}}\right|_{E_{S_{2}}}$ can initially only do a synchronous step which is impossible as the sole initial event for the parallel composition is an event from $E_{1} \cdot \checkmark$

$\mathrm{R}^{\mathrm{C}}(e) \in E_{2}$ : According to the previous case.

Induction Hypothesis: Assume that the property is shown for $T$ and $U$.

\footnotetext{
${ }^{3}$ Here, we need to refer to the renaming $\mathrm{R}^{\mathrm{C}}$ as we have to distinguish between the different sets of events
} which $e$ is assigned to in the decomposition. 
Induction Step: The induction step needs to distinguish between $P=T \circ U$ with $\circ \in\left\{\square, \sqcap, \stackrel{\circ}{9},\left\|_{S},\right\| \mid\right\}$. Both choice operators have the same interpretation in the CSP traces model. Moreover, interleaving is a special case of parallel composition with an empty synchronisation alphabet. That leaves a case differentiation for $\circ \in\left\{\square, \stackrel{\circ}{9}, \|_{S}\right\}$. In any of the three cases, we apply Lemma 5.2.3. As the lemma already dealt with $\tau$-transitions, we do not need to consider them again.

$P=T \square U:$ Let

$$
\left(\left.(T \square U)_{\mathrm{R}}\right|_{E_{S_{1}}} \|\left._{E_{\mathbf{C}}}(T \square U)_{\mathrm{R}}\right|_{E_{S_{2}}}\right) \stackrel{\text { op.x.t.a }}{\longrightarrow} Q^{\prime} .
$$

Definition 4.3.3 yields

$$
\left(\left(\left.\left.T_{\mathrm{R}}\right|_{E_{S_{1}}} \square U_{\mathrm{R}}\right|_{E_{S_{1}}}\right) \|_{E_{\mathbf{C}}}\left(\left.\left.T_{\mathrm{R}}\right|_{E_{S_{2}}} \square U_{\mathrm{R}}\right|_{E_{S_{2}}}\right)\right) \stackrel{\text { op.x.t.a }}{\longrightarrow} Q^{\prime} .
$$

Next, we apply Lemma 5.2.3 for the case of external choice and deduce

$$
\left(\left(\left.T_{\mathrm{R}}\right|_{E_{S_{1}}} \|\left._{E_{\mathrm{C}}} T_{\mathrm{R}}\right|_{E_{S_{2}}}\right) \square\left(\left.U_{\mathrm{R}}\right|_{E_{S_{1}}} \|\left._{E_{\mathrm{C}}} U_{\mathrm{R}}\right|_{E_{S_{2}}}\right)\right) \stackrel{\text { op.x.t.a}}{\longrightarrow} Q^{\prime} .
$$

Without loss of generality, the left hand side performs a (synchronous or asynchronous) step. From the induction hypothesis, we deduce the existence of $T^{\prime}$, such that $T \stackrel{\widehat{o p . x}}{\longrightarrow} T^{\prime}$ and $Q^{\prime}=\left(\left.T_{\mathrm{R}}^{\prime}\right|_{E_{S_{1}}} \|\left._{E_{\mathrm{C}}} T_{\mathrm{R}}^{\prime}\right|_{E_{S_{2}}}\right)$. The operational semantics of CSP yields $(T \square U) \stackrel{\widehat{o p . x}}{\longrightarrow} T^{\prime} . \checkmark$

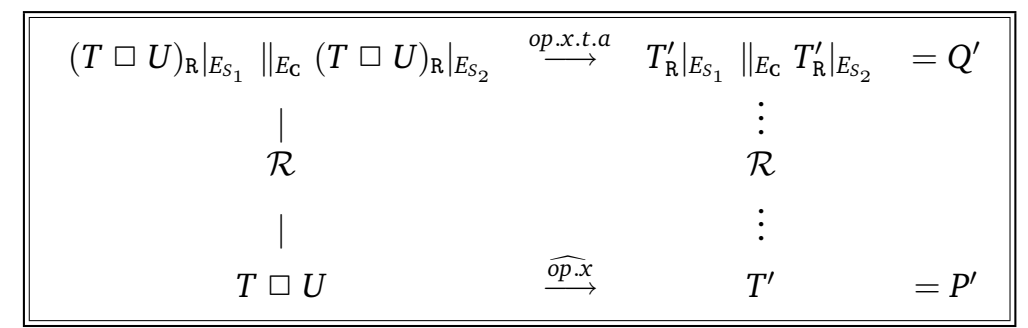

$P=T{ }_{9}^{\circ} U:$ Let

$$
\left(\left.\left(T{ }_{9}^{\circ} U\right)_{\mathrm{R}}\right|_{E_{S_{1}}} \|\left._{E_{\mathbf{C}}}(T \stackrel{\circ}{\circ} U)_{\mathrm{R}}\right|_{E_{S_{2}}} \stackrel{\text { op.x.t.a }}{\longrightarrow} Q^{\prime} .\right.
$$

Definition 4.3 .3 yields

$$
\left(\left(\left.\left.T_{\mathrm{R}}\right|_{E_{S_{1}}} \stackrel{\circ}{9} U_{\mathrm{R}}\right|_{E_{S_{1}}}\right) \|_{E_{\mathrm{C}}}\left(\left.\left.T_{\mathrm{R}}\right|_{E_{S_{2}}} \stackrel{\circ}{9} U_{\mathrm{R}}\right|_{E_{S_{2}}}\right)\right) \stackrel{\text { op.x.t.a }}{\longrightarrow} Q^{\prime}
$$

and the application of Lemma 5.2.3 for the case of sequential composition

$$
\left(\left(\left.T_{\mathrm{R}}\right|_{E_{S_{1}}} \|\left._{E_{\mathrm{C}}} T_{\mathrm{R}}\right|_{E_{S_{2}}}\right)_{9}^{\circ}\left(\left.U_{\mathrm{R}}\right|_{E_{S_{1}}} \|\left._{E_{\mathrm{C}}} U_{\mathrm{R}}\right|_{E_{S_{2}}}\right)\right) \stackrel{\text { op.x.t.a}}{\longrightarrow} Q^{\prime} .
$$

First, let

$$
\left(\left(\left.\left.T_{\mathrm{R}}\right|_{E_{S_{1}}}\|\|_{E_{\mathrm{C}}} T_{\mathrm{R}}\right|_{E_{S_{2}}}\right)_{9}^{\circ}\left(\left.\left.U_{\mathrm{R}}\right|_{E_{S_{1}}}\|\|_{E_{\mathbf{C}}} U_{\mathrm{R}}\right|_{E_{S_{2}}}\right)\right) \stackrel{\text { op.x.t.a }}{\longrightarrow}\left(Q_{1}^{\prime} \stackrel{\circ}{9}\left(\left.U_{\mathrm{R}}\right|_{E_{S_{1}}} \|\left._{E_{\mathrm{C}}} U_{\mathrm{R}}\right|_{E_{S_{2}}}\right)\right) .
$$


From the induction hypothesis, we deduce the existence of $T^{\prime}$, such that $T \stackrel{\widehat{o p . x}}{\longrightarrow} T^{\prime}$ and

$$
Q_{1}^{\prime}=\left(\left.T_{\mathrm{R}}^{\prime}\right|_{E_{S_{1}}} \|\left.\left.\right|_{E_{\mathrm{C}}} T_{\mathrm{R}}^{\prime}\right|_{E_{S_{2}}}\right) \text {. }
$$

The operational semantics of CSP yields $\left(T{ }_{9}^{\circ} U\right) \stackrel{\widehat{o p . x}}{\longrightarrow}\left(T^{\prime}{ }_{9}^{\circ} U\right)$. Finally,

$$
Q^{\prime}=\left(\left.T_{\mathrm{R}}^{\prime}\right|_{E_{S_{1}}} \|\left._{E_{\mathrm{C}}} T_{\mathrm{R}}^{\prime}\right|_{E_{S_{2}}}\right) \stackrel{\circ}{9}\left(\left.U_{\mathrm{R}}\right|_{E_{S_{1}}} \|\left._{E_{\mathbf{C}}} U_{\mathrm{R}}\right|_{E_{S_{2}}}\right),
$$

which is equivalent to

$$
\left(\left.\left.T_{\mathrm{R}}^{\prime}\right|_{E_{S_{1}}} \stackrel{\circ}{9} U_{\mathrm{R}}\right|_{E_{S_{1}}}\right) \|_{E_{\mathrm{C}}}\left(\left.\left.T_{\mathrm{R}}^{\prime}\right|_{E_{S_{2}} \stackrel{\circ}{9}} U_{\mathrm{R}}\right|_{E_{S_{2}}}\right),
$$

according to Lemma 5.2.3. The latter process is equal to

$$
\left.\left(T^{\prime}{ }_{9}^{\circ} U\right)_{\mathrm{R}}\right|_{E_{S_{1}}} \|\left._{E_{\mathrm{C}}}\left(T^{\prime}{ }_{9}^{\circ} U\right)_{\mathrm{R}}\right|_{E_{S_{2}}},
$$

based on Definition 4.3.3. We conclude that the successor states are $\mathcal{R}$ related. A step for the process $\left.U_{\mathrm{R}}\right|_{E_{S_{1}}} \|\left.\left.\right|_{E_{\mathrm{C}}} U_{\mathrm{R}}\right|_{E_{S_{2}}}$ is analogous, based on the fact that in this case, $\left.T_{\mathrm{R}}\right|_{E_{S_{1}}}=\mathrm{Skip}$ and $\left.T_{\mathrm{R}}\right|_{E_{S_{2}}}=\mathrm{Skip}$ needs to hold.

\begin{tabular}{|ccc|}
\hline \hline$\left(\left.\left(T{ }_{9}^{\circ} U\right)_{\mathrm{R}}\right|_{E_{S_{1}}} \|\left._{E_{\mathrm{C}}}\left(T{ }_{9}^{\circ} U\right)_{\mathrm{R}}\right|_{E_{S_{2}}}\right)$ & $\stackrel{\text { op.x.t.a }}{\longrightarrow}$ & $\left(\left.\left(T_{9}^{\prime}{ }_{9}^{\circ} U\right)_{\mathrm{R}}\right|_{E_{S_{1}}} \|\left._{E_{\mathrm{C}}}\left(T^{\prime}{ }_{9}^{\circ} U\right)_{\mathrm{R}}\right|_{E_{S_{2}}}\right)$ \\
$\mid$ & & $\vdots$ \\
$\mathcal{R}$ & & $\mathcal{R}$ \\
$\mid$ & $\stackrel{\widehat{o p . x}}{\longrightarrow}$ & $\vdots$ \\
$(T \stackrel{\circ}{\circ} U)$ & $\left(T_{9}^{\prime}{ }_{9}^{\circ} U\right)$ \\
\hline
\end{tabular}

$P=T \|_{S} U:$ We assume

$$
\left(\left.\left(T \|_{S} U\right)_{\mathrm{R}}\right|_{E_{S_{1}}} \|\left._{E_{\mathrm{C}}}\left(T \|_{S} U\right)_{\mathrm{R}}\right|_{E_{S_{2}}} \stackrel{\text { op.x.t.a }}{\longrightarrow} Q^{\prime} .\right.
$$

Applying 4.3.3 and subsequently Lemma 5.2.3 for the case of parallel composition yields

$$
\left(\left(\left.T_{\mathrm{R}}\right|_{E_{S_{1}}} \|\left._{E_{\mathrm{C}}} T_{\mathrm{R}}\right|_{E_{S_{2}}}\right) \|_{S}\left(\left.U_{\mathrm{R}}\right|_{E_{S_{1}}} \|\left._{E_{\mathrm{C}}} U_{\mathrm{R}}\right|_{E_{S_{2}}}\right)\right) \stackrel{\text { op.x.t.a }}{\longrightarrow}\left(Q_{1}^{\prime} \|_{E_{\mathrm{C}}} Q_{2}^{\prime}\right) .
$$

Two cases for op.x.t.a have to be considered:

op.x.t.a $\in S$ : The induction hypothesis yields the existence of two processes $T^{\prime}$ and $U^{\prime}$, such that $T \stackrel{\widehat{o p . x}}{\longrightarrow} T^{\prime}$ and $U \stackrel{\widehat{\text { p.x }}}{\longrightarrow} U^{\prime}$ holds for

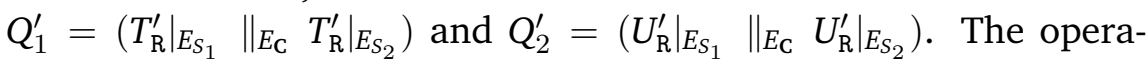
tional semantics of CSP yields $\left(T \|_{S} U\right) \stackrel{\widehat{o p . x}}{\longrightarrow}\left(T^{\prime} \|_{S} U^{\prime}\right)$. Finally,

$$
\begin{aligned}
Q^{\prime} & =Q_{1}^{\prime} \|_{E_{\mathrm{C}}} Q_{2}^{\prime} \\
& =\left(\left.T_{\mathrm{R}}^{\prime}\right|_{E_{S_{1}}} \|\left._{E_{\mathrm{C}}} T_{\mathrm{R}}^{\prime}\right|_{E_{S_{2}}}\right) \|_{S}\left(\left.U_{\mathrm{R}}^{\prime}\right|_{E_{S_{1}}} \|\left._{E_{\mathrm{C}}} U_{\mathrm{R}}^{\prime}\right|_{E_{S_{2}}}\right),
\end{aligned}
$$


with the latter process being equivalent to

$$
\left(\left.\left.T_{\mathrm{R}}^{\prime}\right|_{E_{S_{1}}}\|\|_{S \cap E_{S_{1}}} U_{\mathrm{R}}^{\prime}\right|_{E_{S_{1}}}\right) \|_{E_{\mathrm{C}}}\left(\left.T_{\mathrm{R}}^{\prime}\right|_{E_{S_{2}}} \|\left. S \cap E_{S_{2}} U_{\mathrm{R}}^{\prime}\right|_{E_{S_{2}}}\right),
$$

according to Lemma 5.2.3 and, consecutively, equivalent to

$$
\left.\left(T^{\prime} \|_{S} U^{\prime}\right)_{\mathrm{R}}\right|_{E_{S_{1}}} \|\left._{E_{\mathrm{C}}}\left(T^{\prime} \|_{S} U^{\prime}\right)_{\mathrm{R}}\right|_{E_{S_{2}}}
$$

by Definition 4.3.3. We conclude that the successor states are $\mathcal{R}$ related.

\begin{tabular}{|ccc|}
\hline \hline$\left(\left.\left(T \|_{S} U\right)_{\mathrm{R}}\right|_{E_{S_{1}}} \|\left._{E_{\mathrm{C}}}\left(T \|_{S} U\right)_{\mathrm{R}}\right|_{E_{S_{2}}}\right)$ & $\stackrel{\text { op.x.t.a }}{\longrightarrow}$ & $\left(\left.\left(T^{\prime} \|_{S} U^{\prime}\right)_{\mathrm{R}}\right|_{E_{S_{1}}} \|\left._{E_{\mathrm{C}}}\left(T^{\prime} \|_{S} U^{\prime}\right)_{\mathrm{R}}\right|_{E_{S_{2}}}\right)$ \\
$\mid$ & & $\vdots$ \\
$\mathcal{R}$ & & $\mathcal{R}$ \\
$\mid$ & $\widehat{\text { op.x }}$ & $\vdots$ \\
$\left(T \|_{S} U\right)$ & $\left(T^{\prime} \|_{S} U^{\prime}\right)$ \\
\hline
\end{tabular}

op.x.t.a $\notin S$ : Analogous to the previous case, except for applying the induction hypothesis only once for either $T$ or $U . \checkmark$

This completes the proof of the correct decomposition of the CSP part. Next, we accordingly show correctness for the decomposition of the Object-Z part of a specification.

\subsection{Correctness for the Object-Z Part}

In the introduction of this chapter, we pointed out the general strategy for the correctness proof. In particular, for showing correctness of the decomposition of a specification's Object-Z part, we need to take the traces of its CSP part into account:

$$
S . O Z=_{T}\left(S_{1} . O Z \|_{E_{\mathrm{C}}} S_{2} . \mathrm{OZ}\right) \llbracket \mathrm{R}^{\prime} \rrbracket
$$

is only satisfied, if the ordering of events for the Object-Z part adheres to the sequences of the CSP part. We illustrate this with a small example:

Example 5.3.1. Consider the following specification Simple. Its CSP part subsequently performs three operations. The operation first assigns the value 1 to the sole state variable $x$. Next, second assigns 2 to $x$ in case that the precondition $x=1$ is satisfied. Finally, third outputs the value of $x$ :

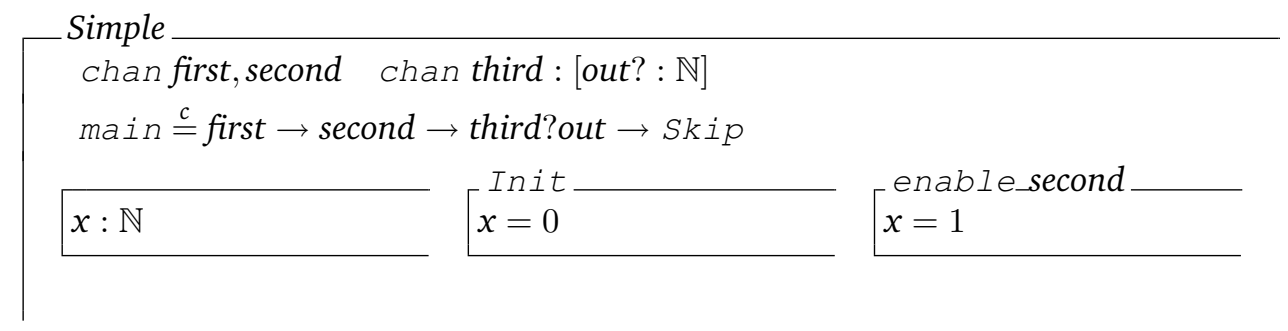




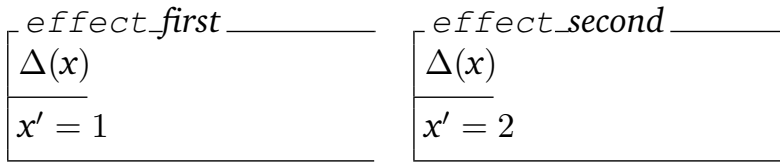

effect_third out! : $\mathbb{N}$

out! $=x$

The possible (single) cut $E_{\mathrm{C}}=\{\mid$ second $\mid\}$ leads to the following decomposition, requiring an additional transmission parameter:
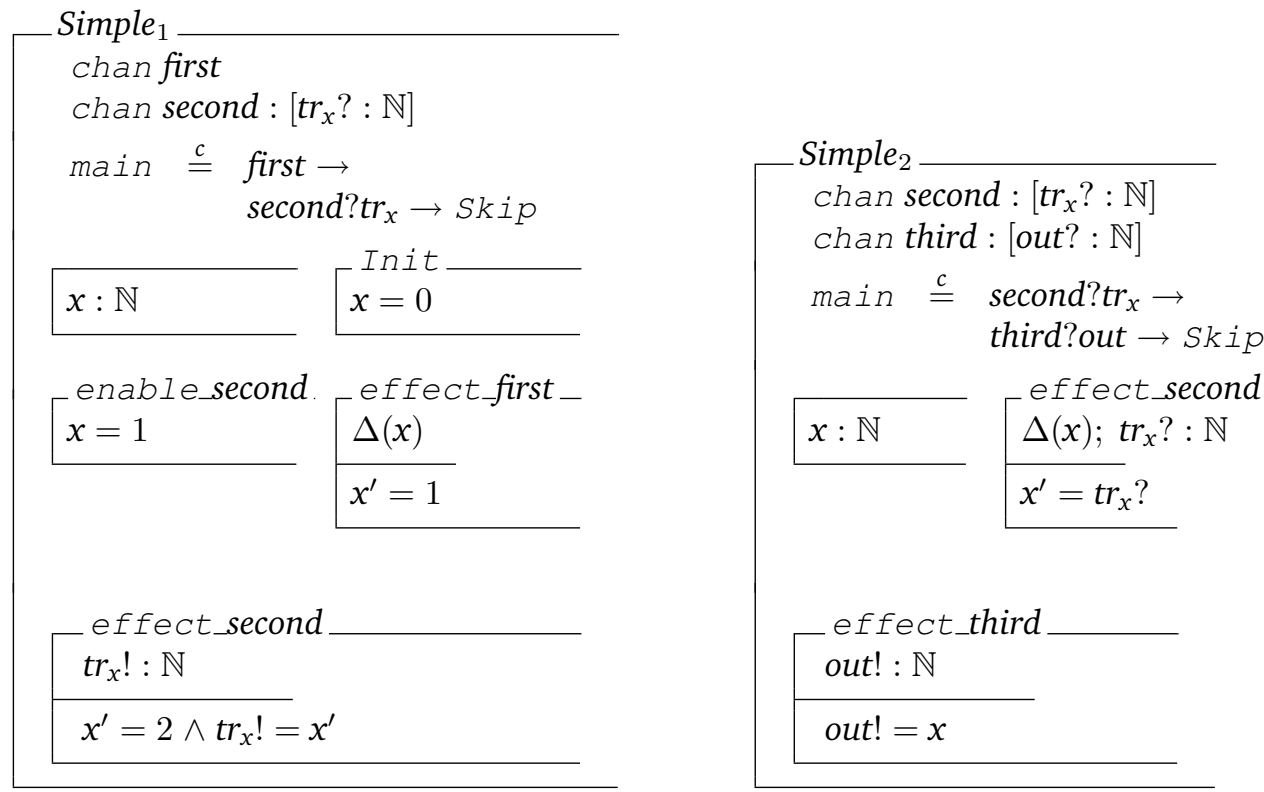

Based on the CSP part of Simple, there is only one possible sequence of operations, namely $\langle$ first, second, third $\rangle$. However, in sole regard to the specification's Object-Z part, the ordering 〈first, third, second $\rangle$ is possible. As the introduction of transmission parameters refers to the CFG and thus the CSP part of a specification, correct values for the state variables cannot be ensured. In particular, the event trace 〈first, third.1, second.2〉 is an element of traces(Simple ${ }_{1} . \mathrm{OZ} \|_{E_{\mathrm{C}}}$ Simple $_{2} . \mathrm{OZ}$ ), whereas (first, third.1, second.2〉 is not an element of traces(Simple.OZ): in the decomposition, the value of $x$ needs to be transmitted before third takes place, which is not the case, if the execution of the final two events is switched.

In the following correctness proof, we refer to the LTS semantics of Object-Z, as introduced in Definition 2.2.3. The proof itself requires us to reason about the intermediate states of $S . O Z$, that is, its state valuations, as we are now explicitly dealing with data dependences. However, the semantic equivalence we aim at, is trace equivalence within the CSP traces model, disregarding states of the Object- $Z$ part. This allows that the valuations of the state variables within $S . O Z$ and $S_{1} . O Z \|_{E_{\mathrm{C}}} S_{2} . \mathrm{OZ}$ are possibly inconsistent if their values do not influence the observable behaviour of the class, that is, the traces of the CSP part. 
In Section 5.3.2, we clarify what inconsistency between state valuations means. Moreover, we define the assumption that traces of the Object-Z part need to adhere to the CSP part.

Beforehand, we start by showing some properties related to the decomposition of the Object-Z part, which the actual correctness proof uses.

\subsubsection{Properties of the Decomposition: Object-Z Part}

Corresponding to the previous section for the CSP part, we introduce and prove some properties of the decomposition of the Object-Z part: the first section will summarise some characteristics of the DG, which the definition for a valid cut necessitates. Afterwards, we show correctness of the restriction of the initial state schema and its optimisation.

\section{No Dependences between Different Segments}

A valid cut rules out several dependence edges between different segments of the DG. In particular, edges must not reach back to a cut set or circumvent the cut.

As we continuously need to refer to the correctness criteria no reaching back and no crossing with respect to the phases and cut sets of the fragmented DG, we will now give a lemma, summarising possible violations of these conditions. Here, we consider edges with the target node being at an earlier stage than the source node:

Definition 5.3.2. (Earlier stage)

Let $\mathrm{DG}_{\mathrm{S}}=\left(N, \longrightarrow_{\mathrm{DG}}\right)$ be the $D G$ of a specification $S$ and let $\left(\mathbf{C}_{1}, \mathbf{C}_{2}\right)$ be a cut. We say that $\mathrm{n} \in \mathrm{op}(N)$ is at an earlier stage than $\mathrm{n}^{\prime} \in \mathrm{op}(N)$, if and only if one of the four conditions

1) $\mathrm{n} \in \mathbf{P h}_{1}$ and $\mathrm{n}^{\prime} \in \mathbf{P h}_{2}$,

2) $\mathrm{n} \in \mathbf{C}_{1}$ and $\mathrm{n}^{\prime} \in \mathbf{P h}_{2}$,

3) $\mathrm{n} \in \mathbf{P h}_{2}$ and $\mathrm{n}^{\prime} \in \mathbf{P h}_{3}$,

4) $\mathrm{n} \in \mathbf{C}_{2}$ and $\mathrm{n}^{\prime} \in \mathbf{P h}_{3}$

holds.

The definition is motivated by a property, which we subsequently show: if a DG node is at an earlier stage with respect to another one, a control flow edge or data dependence from the latter to the first node causes a violation of one of the correctness criteria disjointness, no reaching back or no crossing:

Lemma 5.3.3. (No data dependences to an earlier stage)

Let $\mathrm{DG}_{\mathrm{S}}=\left(N, \longrightarrow_{\mathrm{DG}}\right)$ be the $D G$ of a specification $S$ and let $\left(\mathbf{C}_{1}, \mathbf{C}_{2}\right)$ be a cut. If $\mathrm{n} \in \mathrm{op}(N)$ is at an earlier stage than $\mathrm{n}^{\prime} \in \mathrm{op}(N)$, there must not be a data dependence from n' to $\mathrm{n}$ : $\mathrm{n}{ }^{\prime}--\rightarrow \mathrm{n}$ is impossible. 
Proof. For the first and the third case of Definition 5.3.2, a data dependence $n--\rightarrow n$ violates the correctness criterion no crossing. For the other cases, no reaching back is violated.

A corresponding lemma considering control flow edges is given next.

Lemma 5.3.4. (No control flow edges to an earlier stage)

Let $\mathrm{DG}_{\mathrm{S}}=\left(N, \longrightarrow_{\mathrm{DG}}\right)$ be the $D G$ of a specification $S$ and let $\left(\mathbf{C}_{1}, \mathbf{C}_{2}\right)$ be a cut. If $\mathrm{n} \in \mathrm{op}(N)$ is at an earlier stage than $\mathrm{n}^{\prime} \in \mathrm{op}(N)$, there must not be a control flow edge from n' to $\mathrm{n}$ : $\mathrm{n}^{\prime} \longrightarrow \mathrm{n}$ is impossible.

Proof. Consider the first case of Definition 5.3.2: $\mathrm{n} \in \mathbf{P h}_{1}$ and $\mathrm{n}^{\prime} \in \mathbf{P h}_{2}$. Assume that n' $\longrightarrow \mathrm{n}$. But then, $\mathrm{n} \in\left(\mathbf{P h}_{1} \cap \mathbf{P h}_{2}\right)$, contradicting disjointness. The third case is analogous. For the other cases, no reaching back is violated, according to Lemma 5.3.3.

Figure 5.10 illustrates the definition and both lemmas, where edges denote disallowed control flow edges and data dependences.

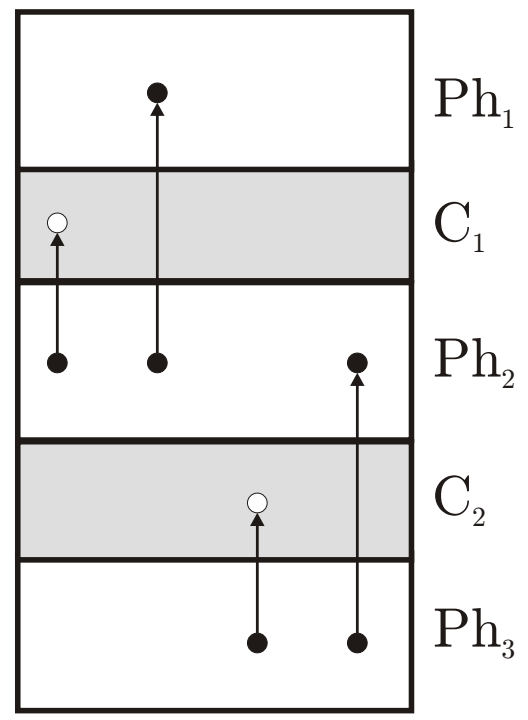

Figure 5.10: Illustration of Definition 5.3.2 and Lemmas 5.3.3, 5.3.4

We aim at lifting Lemma 5.3.4 to paths of the CFG, that is, we want to state that the CFG must not comprise paths connecting a node with another one from an earlier stage. However, this is not always the case, as recursive calls from the third phase back to the first one are possible. More generally, our correctness proof will need to distinguish between paths returning to an earlier stage with recursion (which is possible) and without recursion (which is impossible). This motivates the following definition:

Definition 5.3.5. (Recursion-free $C F G$ path)

Let $\mathrm{CFG}_{\mathrm{S}}=(N, \longrightarrow)$ be the CFG of a specification $S$, and let $\pi \in$ path $_{\mathrm{CFG}}$. We say that 
$\pi$ is (outer-) recursion-free, if, and only if, $\pi$ does not comprise two subsequent nodes call. $X \in \mathbf{P h}_{3}$ and start. $X \in \mathbf{P h}_{1}$ for any $X \in L^{C S P}$.

The following lemma bridges the gap between paths of the CFG of $S$ and traces of the CSP part by characterising traces of $S$.main without corresponding CFG paths:

Lemma 5.3.6. (CSP trace to an earlier stage requires interleaving or recursion) Let $\mathrm{DG}_{\mathrm{S}}=\left(N, \longrightarrow_{\mathrm{DG}}\right)$ be the $D G$ of a specification $S$, and let $\left(\mathbf{C}_{1}, \mathbf{C}_{2}\right)$ be a cut. Let $\mathrm{n} \in \mathrm{op}(N)$ be at an earlier stage than $\mathrm{n}^{\prime} \in \mathrm{op}(N)$, and let $n$ and $n^{\prime}$ denote their corresponding occurrences within S.main. If there exists $t r \in \operatorname{traces}$ (S.main) and indices $i<j$, such that $t r . i=n^{\prime}$ and tr. $j=n$, then one of the following two cases applies:

1) there exists a CFG path from n' to $\mathrm{n}$, which is not recursion-free, or

2) $\mathrm{n}$ and $\mathrm{n}$ ' are located in different branches of the $C F G$, attached to the same interleaving node or parallel composition node.

Proof. We show the following: if the opposite of 1 ) holds, that is, if no recursion-free CFG path from n' to n exists, both nodes must not be connected by a CFG path at all, based on Lemma 5.3.4. From this, we deduce that the second case needs to apply.

Assume that there exists a CFG path $\pi$ from n' to $\mathrm{n}$, which is recursion-free. Let $\mathrm{n}^{\prime} \in \mathbf{P h}_{2}$ and $\mathrm{n} \in \mathbf{P h}_{1}$. According to Lemma 5.3.4, it is impossible that $\pi$ proceeds from $\mathbf{P h}_{2}$ over $\mathbf{C}_{1}$ to $\mathbf{P h} \mathbf{h}_{1}$ or directly from $\mathbf{P h} \mathbf{h}_{2}$ to $\mathbf{P h}$. Therefore, $\pi$ has to proceed over $\mathbf{C}_{2}$ and $\mathbf{P h} \mathbf{h}_{3}$ back to $\mathbf{P h}_{1}$, which requires a recursion within $\pi$, contradiction. The other cases are similar.

Therefore, a CFG path from n' to $n$ does not exist. We conclude the proof by applying Lemma 6.1.4 from [Brü08]: two events with a subsequent execution within S.main require a CFG path from the first to the latter node or, if such a path does not exist, they have to be located in different branches of the CFG, attached to the same interleaving node or parallel composition node.

\section{Correctness of Init-restriction}

Chapter 4, Definition 4.3.6, introduced the restriction of $S$. Init to determine the initial state schemas of $S_{1}$ and $S_{2}$. In addition, Section 4.3.7 introduced an optimisation for the decomposition of a specification, allowing us to neglect certain initial data dependences when checking the correctness criterion no crossing.

In this section, we show that both, the definition and the optimisation, are correct in the following sense, where we let $V:=S . V, V_{1}:=S_{1} . V, V_{2}:=S_{2} . V$ and $i \in\{1,2\}$ :

1.) for any state $s \in S$.State such that $S$.Init $(s)$ holds, the restriction $s\left\lceil V_{i} \in S_{i}\right.$. State satisfies $S_{i}$.Init and

2.) two states $s^{i} \in S_{i}$. State, for which $S_{i}$. Init $\left(s^{i}\right)$ holds, can appropriately be combined to a state $s \in S$. State such that $S$.Init $(s)$ holds.

Before proving these particular properties, we introduce some notations. First, recall that the set Atoms(S.Init) denotes the set of all atomic predicates for the initial state schema:

$$
\bigwedge_{a \in \operatorname{Atoms}(\text { S.Init) }} a=\text { S.Init. }
$$


For any such predicate $a$, let $a[x / v]$ depict the predicate, resulting from replacing any free occurrence of the variable $x$ in $a$ with the value $v$ of type $t_{x}$. Henceforth, if $a$ is defined over a set of state variables $\left\{x_{1}, \ldots, x_{n}\right\}$ and $v_{i}: t_{x_{i}}$, we write

$$
\left(v_{1}, \ldots, v_{n}\right) \models a \Leftrightarrow a\left[x_{i} / v_{i}\right]=\text { true } .
$$

For instance, $(7,3) \models x>y$. Here, we assume an ordering on the state variables, such that a unique mapping $x_{i} \rightarrow v_{i}$ is indeed possible. Moreover, we write $s=S$. Init instead of $S$.Init(s). Finally, let Init $\lceil V$ denote the Init-schema of a specification, restricting only variables from $V$.

The next lemma states the first of the two properties specified above:

Lemma 5.3.7. (Correctness of Init-restriction, first part)

Let $\mathrm{DG}_{\mathrm{S}}=\left(N, \longrightarrow_{\mathrm{DG}}\right)$ be the $D G$ of a specification $S$, and let $\left(\mathbf{C}_{1}, \mathbf{C}_{2}\right)$ be a cut, separating the set $V$ into $V_{1}$ and $V_{2}$, according to Definition 4.3.5. Then, for all states $s \in S$.State:

$$
s \models S \text {.Init } \Rightarrow\left(s \left\lceilV_{1} \models S_{1} \text {. Init } \wedge s\left\lceil V_{2} \models S_{2} \text {. Init }\right) .\right.\right.
$$

Proof. Assume $s \models S$.Init for some $s \in$ S.State. Let $\left(V \backslash V_{1}\right)=\left\{v_{1}, \ldots, v_{n}\right\}$, and let $V_{1}=\left\{w_{1}, \ldots, w_{m}\right\}$. We have to show

$$
s\left\lceil V_{1} \models \exists v_{1}, \ldots, v_{n} \bullet S\right. \text {.Init }
$$

and

$$
s \uparrow V_{2} \models \exists w_{1}, \ldots, w_{m} \bullet S \text {.Init. }
$$

Recall that, in general, $V_{1} \cap V_{2} \neq \varnothing$, and thus, $\left(V \backslash V_{1}\right) \neq V_{2}$. Since

$$
\text { S.Init }=\bigwedge_{a \in A t o m s(S . I n i t)} a,
$$

$s \models S$.Init is equivalent to $\forall a \in \operatorname{Atoms}(S$.Init) $\bullet s \models a$. Let Free(a) be the set of free state variables within $a$. Without loss of generality, let

$$
\operatorname{Free}(a)=\left\{x_{1}, \ldots, x_{n}, y_{1}, \ldots, y_{m}\right\}
$$

for $x_{i} \in V_{1}$ and $y_{j} \in\left(V \backslash V_{1}\right)$. Then,

$$
S_{1} \text {.Init. } a=\exists y_{1}, \ldots, y_{m} \bullet a\left(x_{1}, \ldots, x_{n}, y_{1}, \ldots, y_{m}\right)
$$

and

$$
S_{2} \text {.Init. } a=\exists x_{1}, \ldots, x_{n} \bullet a\left(x_{1}, \ldots, x_{n}, y_{1}, \ldots, y_{m}\right) .
$$

We deduce

$$
\begin{aligned}
s \models a & \Leftrightarrow a\left[x_{i} / s . x_{i}\right]\left[y_{j} / s . y_{j}\right]=\text { true } \\
& \Leftrightarrow s\left\lceilV _ { 1 } \models a [ y _ { j } / s . y _ { j } ] \text { and } s \left\lceil V_{2} \models a\left[x_{i} / s . x_{i}\right]\right.\right. \\
& \Rightarrow s\left\lceilV _ { 1 } \models \exists y _ { 1 } , \ldots , y _ { m } \bullet a \text { and } s \left\lceil V_{2} \models \exists x_{1}, \ldots, x_{n} \bullet a\right.\right. \\
& \Leftrightarrow s \uparrow V_{1} \models S_{1} \text {. Init. } a \text { and } s\left\lceil V_{2} \models S_{2} .\right. \text { Init.a. }
\end{aligned}
$$


For the second property, we have to consider the optimisation from Section 4.3.7: as already mentioned, we aim at neglecting several initial data dependences. These are the ones originating from an atomic predicate $a$ solely referring to variables $x$, such that $\operatorname{InitClos}(x) \subseteq\left(V_{2} \backslash V_{1}\right)$ holds. It is reasonable to neglect those dependences, because all of these predicates remain unchanged within $S_{2}$.Init, which we show next. As additionally, state variables from $V_{2} \backslash V_{1}$ are not modified within $S_{1}$ at all, the initial data dependences within $\mathrm{DG}_{\mathrm{S}}=\left(N, \longrightarrow_{\mathrm{DG}}\right)$, originating from these predicates, no longer cause a violation of no crossing. Note that all remaining initial data dependences must not be neglected.

Lemma 5.3.8. (Correctness of optimisation) Let $\mathrm{DG}_{\mathrm{S}}=\left(N, \longrightarrow_{\mathrm{DG}}\right)$ be the $D G$ of a specification $S$, and let $\left(\mathbf{C}_{1}, \mathbf{C}_{2}\right)$ be a cut, separating the set $V$ into $V_{1}$ and $V_{2}$, according to Definition 4.3.5. In addition, let

$$
\left\{y_{1}, \ldots, y_{m}\right\}=\left\{x \mid \operatorname{InitClos}(x) \subseteq\left(V_{2} \backslash V_{1}\right)\right\} .
$$

Then, the initial state predicate of $S$ restricted to $\left\{y_{1}, \ldots, y_{m}\right\}$ is equal to the initial state predicate of $S_{2}$ restricted to the same set, that is

$$
\text { S.Init }\left\{\left\{y_{1}, \ldots, y_{m}\right\}=S_{2} \text {. Init } \uparrow\left\{y_{1}, \ldots, y_{m}\right\}\right. \text {. }
$$

Proof. Let $\operatorname{InitClos}(x) \subseteq\left(V_{2} \backslash V_{1}\right)$. Then, for any atomic predicate $a \in A$ toms(S. Init), we get $\operatorname{vars}(a) \subseteq\left(V_{2} \backslash V_{1}\right)$. Based on

$$
S_{2} \text {.Init }=\exists w_{1}, \ldots, w_{m} \bullet \text { S.Init }
$$

for $V_{1}=\left\{w_{1}, \ldots, w_{m}\right\}$, any of these atoms is preserved within $S_{2}$.Init.

Next, we complement Lemma 5.3.7 by proving the second of the two properties, specified above: from two states $s^{i} \in S_{i}$. State satisfying $S_{i}$. Init, we can construct $s \in$ State satisfying $S$.Init. For finally showing correctness of the optimisation, this construction necessarily needs to take the previously described subset of $\left(V_{2} \backslash V_{1}\right)$ into account.

Lemma 5.3.9. (Correctness of Init-restriction, second part) Let $\mathrm{DG}_{\mathrm{S}}=\left(N, \longrightarrow_{\mathrm{DG}}\right)$ be the $D G$ of a specification $S$, and let $\left(\mathbf{C}_{1}, \mathbf{C}_{2}\right)$ be a cut, separating the set $V$ into $V_{1}$ and $V_{2}$, according to Definition 4.3.5. Let $V_{1}=\left\{x_{1}, \ldots, x_{n}\right\}$,

$$
\begin{aligned}
& \left\{y_{1}, \ldots, y_{m}\right\}=\left\{x \mid \operatorname{InitClos}(x) \subseteq\left(V_{2} \backslash V_{1}\right)\right\} \text { and } \\
& \left\{z_{1}, \ldots, z_{l}\right\}=\left(V_{2} \backslash V_{1}\right) \backslash\left\{y_{1}, \ldots, y_{m}\right\} .
\end{aligned}
$$

For all states of $S$, we use the variable ordering $\left(x_{1}, \ldots, x_{n}, y_{1}, \ldots, y_{m}, z_{1}, \ldots, z_{l}\right)$. Let $s^{i} \in S_{i}$.State, such that $s^{i} \mid=S_{i}$. Init. Then, there exist $c_{i}: t_{z_{i}}, i \in\{1, \ldots, l\}$, such that for

$$
s:=\left(s^{1} \cdot x_{1}, \ldots, s^{1} \cdot x_{n}, s^{2} \cdot y_{1}, \ldots, s^{2} \cdot y_{m}, c_{1}, \ldots, c_{l}\right),
$$

$s \models S$.Init. 
Proof. Again, let $\left(V \backslash V_{1}\right)=\left\{v_{1}, \ldots, v_{n}\right\}$, and let $V_{1}=\left\{w_{1}, \ldots, w_{m}\right\}$. Based on the definition of $S_{i}$. Init, we have

1) $\left(s^{1} \cdot x_{1}, \ldots, s^{1} \cdot x_{n}\right)=\exists v_{1}, \ldots, v_{n} \bullet$ S.Init and

2) $\left(s^{2} \cdot y_{1}, \ldots, s^{2} \cdot y_{m}, s^{2} \cdot z_{1}, \ldots, s^{2} \cdot z_{l}\right) \mid=\exists w_{1}, \ldots, w_{m} \bullet$ Init.

We need to show that there indeed exist $c_{i}$ of type $t_{z_{i}}$, such that

$$
S . \operatorname{Init}\left[x_{1} / s^{1} . x_{1}\right] \ldots\left[x_{n} / s^{1} \cdot x_{n}\right]\left[y_{1} / s^{2} \cdot y_{1}\right] \ldots\left[y_{m} / s^{2} \cdot y_{m}\right]\left[z_{1} / c_{1}\right] \ldots\left[z_{l} / c_{l}\right]
$$

evaluates to true. First, for all $a \in \operatorname{Atoms}(S$.Init):

$$
\operatorname{vars}(a) \cap\left\{x_{1}, \ldots, x_{n}, z_{1}, \ldots, z_{l}\right\} \cap\left\{y_{1}, \ldots, y_{m}\right\}=\varnothing .
$$

This is based on Definition 4.3.26: assume the opposite, then there exists an atomic predicate from $S$. Init, containing a variable $y \in\left(V_{2} \backslash V_{1}\right)$, such that $\operatorname{InitClos}(y) \subseteq\left(V_{2} \backslash V_{1}\right)$ holds. In addition, the predicate either refers to a variable $x \in V_{1}$ or to a variable $z \in\left(V_{2} \backslash V_{1}\right)$, for which $\operatorname{InitClos}(z) \nsubseteq\left(V_{2} \backslash V_{1}\right)$. In both cases, we get a contradiction, as either $x$ itself or some $z^{\prime} \in \operatorname{InitClos}(z)$ is an element of $\left(V_{1} \cap \operatorname{InitClos}(y)\right)$. Therefore, any atomic predicate within $S$. Init is either defined over a subset of $\left\{x_{1}, \ldots, x_{n}, z_{1}, \ldots, z_{l}\right\}$ or a subset of $\left\{y_{1}, \ldots, y_{m}\right\} . s$ indeed satisfies $S$.Init: let $a \in A t o m s$ (S.Init) be defined over $\left\{x_{1}, \ldots, x_{n}, z_{1}, \ldots, z_{l}\right\}$. As

$$
\left(s^{1} . x_{1}, \ldots, s^{1} \cdot x_{n}\right)=\exists v_{1}, \ldots, v_{n} \bullet \text { S.Init, }
$$

in particular,

$$
\left(s^{1} \cdot x_{1}, \ldots, s^{1} \cdot x_{n}\right) \models \exists c_{1}, \ldots, c_{n} \bullet a
$$

holds. Now assume that $a \in \operatorname{Atoms}\left(S\right.$. Init) is defined over $\left\{y_{1}, \ldots, y_{m}\right\}$.

$$
\left(s^{2} \cdot y_{1}, \ldots, s^{2} \cdot y_{m}, s^{2} \cdot z_{1}, \ldots, s^{2} . z_{l}\right) \models \exists w_{1}, \ldots, w_{m} \bullet \text { Init }
$$

particularly implies that $\left(s^{2} \cdot y_{1}, \ldots, s^{2} \cdot y_{m}\right) \models a$. As

$$
\operatorname{vars}(a) \cap\left\{x_{1}, \ldots, x_{n}, z_{1}, \ldots, z_{l}\right\} \cap\left\{y_{1}, \ldots, y_{m}\right\}=\varnothing,
$$

we conclude that $s=S$.Init.

Example 5.3.10. Recall the initial state schema of the candy machine from Figure 2.3:

$$
\text { CandyMachine. Init }=(\text { sum }=0) \wedge(\text { paid }=\langle\rangle) \wedge(\text { items }=\langle\rangle) .
$$

For the valid single cut $\mathbf{C}=\{$ switch $\}$, we get sum, paid $\in V_{1}$ and items $\in\left(V_{2} \backslash V_{1}\right)$. Obviously, InitClos $($ items $) \subseteq\left(V_{2} \backslash V_{1}\right)$. Thus,

$$
\text { S.Init } \uparrow\{\text { items }\}=S_{2} \text {. Init } \uparrow\{\text { items }\}=(\text { items }=\langle\rangle) .
$$

In addition, any state $s=\left(s^{1}\right.$.sum, $s^{1}$.paid, $s^{2}$.items $)$. such that $s^{i} \models S_{i}$. Init, yields $s \mid=S$.Init. 
Note, that the previous lemmas showed the correctness of the optimisation from Section 4.3.7 but not ultimately of the Init-restriction. It remains to be shown that the existential quantification does not violate the initial correlation between several state variables. For instance, one could assume that a split-up of a predicate $x \geq y$ into two predicates $\exists x \bullet x \geq y$ and $\exists y \bullet x \geq y$ may cause an observable difference between $S$ and $S_{1} \|_{E_{\mathrm{C}}} S_{2}$. The correctness proof of the decomposition of the Object-Z part in Section 5.3.2 will show that this is not the case, mainly by using the correctness criterion no crossing with respect to initial data dependences.

\subsubsection{Correctness of the Decomposition: Object-Z part}

Next, we show correctness for the decomposition of S.OZ by using the previous results of this section. We start by clarifying the restriction that traces of the Object-Z part need to adhere to the ones of the CSP part. Instead of showing that

$$
S . O Z=_{T}\left(S_{1} . \mathrm{OZ} \|_{E_{\mathrm{C}}} S_{2} . \mathrm{OZ}\right) \llbracket \mathrm{R}^{\prime} \rrbracket
$$

holds, we show the weaker property

$$
\begin{gathered}
\forall \operatorname{tr} \text { such that }(\operatorname{tr} \triangleright O p) \in \operatorname{traces}(S . m a \text { in }) \triangleright O p \bullet \\
\operatorname{tr} \in \operatorname{traces}(S . O Z) \Leftrightarrow \operatorname{tr} \in \operatorname{traces}\left(\left(S_{1} . O Z \|_{E_{\mathrm{C}}} S_{2} . O Z\right) \llbracket \mathrm{R}^{\prime} \rrbracket\right),
\end{gathered}
$$

It describes that any trace, for which we assume the ordering of operations to be determined by the CSP part, is an element of traces (S.OZ), if, and only if, it is contained in traces $\left(\left(S_{1} . O Z \|_{E_{\mathrm{C}}} S_{2} . O Z\right) \llbracket \mathrm{R}^{\prime} \rrbracket\right)$.

The crucial point considering the renaming is as follows: addressing parameters are not restricted by the Object-Z part, and we can entirely omit dealing with them. However, we have to consider the set of transmission parameters, as they are necessary to restore the original data flow within the decomposition. In correspondence to the correctness proof of the CSP part and for simplification, we omit denoting the additional parameters, which are introduced by the renaming relation.

We sketch the main strategy for showing Equation 5.1: for any trace of S.OZ, we define a trace of $S_{1} . O Z \|_{E_{\mathrm{C}}} S_{2} . O Z$ and vice versa, such that both traces are equivalent. Recall Section 2.2.2 and the definition of Traces(S.OZ): according to the LTS semantics from Definition 2.2.3, an Object-Z trace consists of an alternating sequence of states and events. As we ultimately aim at showing trace equivalence with respect to the CSP traces model, we solely have to require trace equivalence over the set $\operatorname{traces}(\mathrm{OZ})$, the set of Object-Z traces projected on events. In particular, we will observe that the trace equivalence with respect to Traces (S.OZ) cannot be shown: some state variables do not need to have corresponding values within $\pi \in \operatorname{Traces}(S . O Z)$ and its analogon $\pi_{i}$ within $\operatorname{Traces}\left(S_{1} . O Z \|_{\left\{E_{\mathrm{C}}\right\}} S_{2} . O Z\right)$.

In general, the decomposition of a specification eliminates a subset of the original set of operations. Therefore, a trace of $\pi_{i} \in S_{i}$.OZ is a projection of a trace of $\pi \in S$.OZ. In order to simplify reasoning about their correspondence and the usage of indices, we assume an event noev, depicting stuttering [CGP99] in $\pi_{i}$ : it substitutes for any event $e$ of $\pi$, not 
occurring in $\pi_{i}$. Thus, both traces have a corresponding length. Furthermore, for any noev-step in a trace, the succeeding state is identical to the one before noev, that is

$$
s \stackrel{\text { noev }}{\longrightarrow} s^{\prime} \Rightarrow s=s^{\prime}
$$

When dealing with traces $\pi \in \operatorname{Traces}(S . O Z)$, we use $s_{i}:=\pi[i]$ to denote the ith state of the trace $\pi$ and $e_{i}:=\pi . i$ to denote its $i$ th event. Furthermore, we let $e_{i}=o p_{i}$. in $_{i} \cdot \operatorname{sim}_{i}$. out $_{i}$. We proceed accordingly for $\pi_{i} \in \operatorname{Traces}\left(S_{i} . \mathrm{OZ}\right)$, where we use an additional top index. Based on the usage of noev, we can always refer to corresponding positions within $\pi$ and $\pi_{i}$. Summarising, traces are referred to as

$$
\begin{aligned}
\pi & =\left\langle s_{0}, e_{0}, s_{1}, e_{1}, \ldots\right\rangle \\
\pi_{1} & =\left\langle s_{0}^{1}, e_{0}^{1}, s_{1}^{1}, e_{1}^{1}, \ldots\right\rangle \text { and } \\
\pi_{2} & =\left\langle s_{0}^{2}, e_{0}^{2}, s_{1}^{2}, e_{1}^{2}, \ldots\right\rangle .
\end{aligned}
$$

Before carrying out the actual proof, we illustrate our general strategy and the possible inconsistencies in the state space valuations by an example.

Example 5.3.11. Consider the extended number swapper from Figure 4.26 and its decomposition from Figures 4.27 and 4.28. The following table compares three valid traces

\begin{tabular}{|c|c|c|}
\hline$\pi$ & $\pi_{1}$ & $\pi_{2}$ \\
\hline 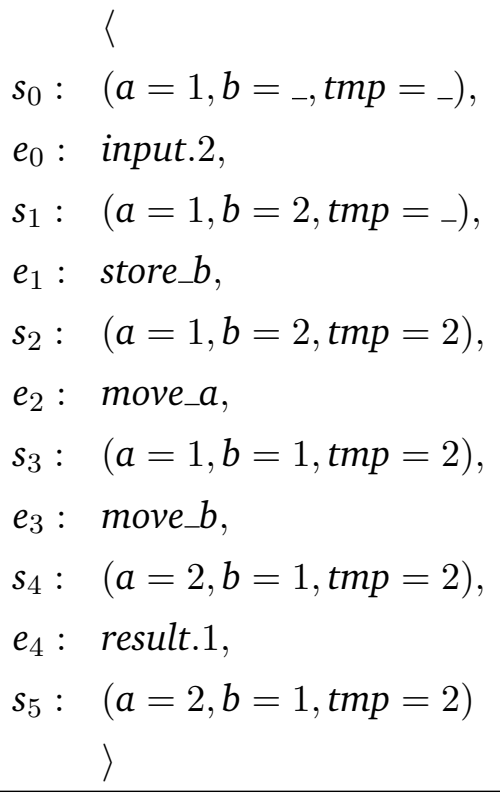 & $\begin{aligned} & \langle \\
s_{0}^{1}: & \left(b={ }_{-}, t m p={ }_{-}\right), \\
e_{0}^{1}: & \text { input.2, } \\
s_{1}^{1}: & \left(b=2, t m p={ }_{-}\right), \\
e_{1}^{1}: & \text { store_b.2, } \\
s_{2}^{1}: & (b=2, t m p=2), \\
e_{2}^{1}: & \text { noev, } \\
s_{3}^{1}: & (\mathbf{b}=\mathbf{2}, \text { tmp }=2), \\
e_{3}^{1}: & \text { noev, } \\
s_{4}^{1}: & (\mathbf{b}=\mathbf{2}, t m p=2), \\
e_{4}^{1}: & \text { result.., } \\
s_{5}^{1}: & (\mathbf{b}=\mathbf{2}, t m p=2) \\
& \rangle\end{aligned}$ & $\begin{array}{ll} & \langle \\
s_{0}^{2}: & \left(a=1, b==_{-}, t m p={ }_{-}\right), \\
e_{0}^{2}: & \text { noev }, \\
s_{1}^{2}: & \left(a=1, \mathbf{b}={ }_{-}, t m p={ }_{-}\right), \\
e_{1}^{2}: & \text { store_b.2, } \\
s_{2}^{2}: & \left(a=1, \mathbf{b}={ }_{-}, t m p=2\right), \\
e_{2}^{2}: & \text { move_a }, \\
s_{3}^{2}: & (a=1, b=1, t m p=2) \\
e_{3}^{2}: & \text { move_b }, \\
s_{4}^{2}: & (a=2, b=1, t m p=2) \\
e_{4}^{2}: & \text { result.1, } \\
s_{5}^{2}: & (a=2, b=1, t m p=2) \\
& \rangle\end{array}$ \\
\hline
\end{tabular}
$\pi \in \operatorname{Traces}\left(\right.$ Swapper.OZ), $\pi_{1} \in$ Traces(Swapper ${ }_{1} . \mathrm{OZ}$ ) and $\pi_{2} \in$ Traces(Swapper $_{2} . \mathrm{OZ}$ ). Here, "_" denotes an arbitrary value. We choose the value 2 for the input parameter of the operation input.

As move $a$ and move $b$ do not occur in $S_{1}$ and as input is not represented in $S_{2}$, these events are replaced by noev within $\pi_{1}$ and $\pi_{2}$, respectively. 
Within the decomposition, the parameter value for result is solely determined by $S_{2}$.result. Therefore, the event has an arbitrary parameter value within $\pi_{1}$, the synchronisation ensures that exclusively the value from $\pi_{2}$ is possible.

Five cells are highlighted in gray: for these states, the value for $b$ is inconsistent between either $\pi$ and $\pi_{1}$ or between $\pi$ and $\pi_{2}$. However, these inconsistent values do not influence the equivalence between $\pi\left\lceil E_{S}\right.$ and the joint execution of $\pi_{1}\left\lceil E_{S^{\prime}}\right.$ and $\pi_{2}\left\lceil E_{S^{\prime}}\right.$ : in both cases, we get

$\langle$ input.2, store_b[.2], move_a, move_b, result.1 $\rangle$,

where store_b receives an additional transmission parameter within the parallel composition of $\pi_{1}\left\lceil E_{S^{\prime}}\right.$ and $\pi_{2}\left\lceil E_{S^{\prime}}\right.$.

As we will show in this section, inconsistent values for some state variables never affect the trace equivalence in the CSP traces model.

The decomposition of $S$ leads to a partitioning of $V$ into $V_{1}$ and $V_{2}$. In the remainder of this proof, we need to distinguish between four different sets of state variables:

$V_{1} \backslash V_{2}$ : the set of state variables solely represented in $S_{1}$,

$V_{2} \backslash V_{1}$ : the set of state variables solely represented in $S_{2}$,

$C V:=C V_{1} \cup C V_{2}$ : the set of cut variables, according to Definition 4.3.9,

$\overline{C V}:=\left(V_{1} \cap V_{2}\right) \backslash C V$ : the set of remaining shared state variables.

The latter set can be characterised as the set of state variables occurring in both, $S_{1}$ and $S_{2}$, which are either not modified within $\mathbf{C}_{1}\left(\mathbf{C}_{2}\right)$ or which do not influence $\mathbf{P h}_{2}$ $\left(\mathbf{P h}_{3} \cup \mathbf{P h}_{1}\right)$. As we will see later, this is the sole set of state variables, for which values in $\pi$ and $\pi_{i}$ are possibly inconsistent.

We start the proof with the forward direction of Equation 5.1.

\section{Left-to-Right Implication}

Let $\operatorname{tr} \in \operatorname{traces}(S . O Z)$, such that $(\operatorname{tr} \triangleright O p) \in \operatorname{traces}(S$ main $) \triangleright O p$. We have to show

$$
\operatorname{tr} \in \operatorname{traces}\left(\left(S_{1} . O Z \|_{E_{\mathrm{C}}} S_{2} . O Z\right) \llbracket \mathrm{R}^{\prime} \rrbracket\right) .
$$

Let $\pi=\left\langle s_{0}, e_{0}, s_{1}, e_{1}, \ldots\right\rangle \in \operatorname{Traces}(S . O Z)$, such that $\pi\left\lceil E_{S}=\operatorname{tr}\right.$ holds. Recall that $e_{i}=o p_{i} \cdot i_{i} \cdot \operatorname{sim}_{i} \cdot$ out $_{i}$. We proceed in three steps: based upon $\pi$, we define two traces

$$
\pi_{i}=\left\langle s_{0}^{i}, e_{0}^{i}, s_{1}^{i}, e_{1}^{i}, \ldots\right\rangle(\operatorname{Step} \mathbf{1}),
$$

with possibly $e_{j}^{i}=$ noev for some events. Next, we inductively show

$$
\pi_{i} \in \operatorname{Traces}\left(S_{i} . \mathrm{OZ}\right) \text { (Step 2). }
$$

Finally, we deduce

$$
\operatorname{tr}=\pi\left\lceilE _ { S } \in \left(\pi _ { 1 } \left\lceil E_{S^{\prime}} \|_{E_{\mathrm{C}}} \pi_{2}\left\lceil E_{S^{\prime}}\right) \llbracket \mathrm{R}^{\prime} \rrbracket\right.\right.\right. \text { (Step 3). }
$$


Here, we refer to the definition of [Ros98] for the parallel composition of two traces.

Step 1: For the definition of $\pi_{i} \in \operatorname{Traces}\left(S_{i} . \mathrm{OZ}\right)$, we need to consider events and states. Obviously, as we aim at showing equivalence within the CSP traces model, the definition for $\pi_{i}$ on events needs to match with the trace $t r$, except for events replaced by noev. This gives rise to the following definition: ${ }^{4}$

$$
\begin{aligned}
e_{j}^{1} & := \begin{cases}e_{j} . t, & \mathrm{op}_{j} \notin \mathbf{P h}_{2}, \\
\text { noev, } & \text { otherwise, }\end{cases} \\
e_{j}^{2} & := \begin{cases}e_{j} . t, & \mathrm{op}_{j} \notin\left(\mathbf{P h}_{1} \cup \mathbf{P h}_{3}\right), \\
\text { noev, } & \text { otherwise, }\end{cases}
\end{aligned}
$$

where $t$ denotes the values for the additional transmission parameters. Note that these are uniquely defined, based on Definition 4.3.10.

The definition of the states is more complicated. The initial states of $\pi_{1}$ and $\pi_{2}$ are simply defined as the restrictions of $s_{0}$ on $V_{1}$ and $V_{2}$, respectively:

$$
s_{0}^{1}:=s_{0} \uparrow V_{1} \text { and } s_{0}^{2}:=s_{0} \uparrow V_{2} .
$$

Next, we define $s_{k}^{i}$ for $k \geq 1$. The states for $\pi_{i}$ mostly correspond to the states of $\pi$, restricted to the remaining set of state variables. Therefore, in most cases, we simply set $s_{k}^{i}:=s_{k}\left\lceil V_{i}\right.$. However, in some cases, the state valuations do not match. This is the case, if some modification of a state variable within $\pi$ is not represented in $\pi_{i}$, thus causing an inconsistency between the values of the respective state variable, which is possibly preserved afterwards.

Precisely, there are three different cases, for which the value for a state variable $x$ within $\pi_{i}$ must not be modified, that is $s_{k}^{i}:=s_{k-1}^{i}$, instead of $s_{k}^{i}:=s_{k}$.

(1) In Example 5.3.11, consider the transition $s_{2} \stackrel{\text { move } a}{\longrightarrow} s_{3}$ within $\pi$. The event is replaced by noev within $\pi_{1}$. As the modification for $b$ to the value 1 gets lost within the trace for $S_{1} . O Z$, setting $s_{3}^{1} . b$ to $s_{3} . b$ would contradict $\pi_{1} \in \operatorname{Traces}\left(S_{1} . \mathrm{OZ}\right)$ and is therefore unreasonable. Instead, we have to define $s_{3}^{1} \cdot b:=s_{2}^{1} \cdot b$ - the original value is preserved in case that an event is replaced by noev within $\pi_{i}$.

(2) Now consider the transition $s_{4} \stackrel{\text { result.1 }}{\longrightarrow} s_{5}$. As the value for $b$ is not modified within result, the equation $s_{5} . b=s_{4} \cdot b=1$ holds. The corresponding call of result within $\pi_{1}$ does not change $b$ as well. Therefore, we again need to preserve the (inconsistent) value $s_{4}^{1} \cdot b=2$.

(3) For the final case, assume that in the example, the predicate part of the operation store $\_b$ additionally comprises a modification of $b$. As store $b \in O p_{\mathbf{C}_{1}}$ and as $b$ is not a cut variable, this modification is solely conducted in $S_{1}$. The old value for $b$ needs to be preserved within $\pi_{2}$.

\footnotetext{
${ }^{4}$ Here, we refer to the specific occurrence of the DG node $\mathrm{op}_{j}$ corresponding to the uniquely related occurrence $o p_{j}$ of the operation $o p$.
} 
The previous considerations motivate the following definition. Let $i, j \in\{1,2\}$. For any $k \geq 1$ and $x \in V_{i}$, we define the value of $s_{k}^{i} \cdot x$ within $\pi_{i}$ as:

$$
s_{k}^{i} \cdot x:= \begin{cases}s_{k-1}^{i} \cdot x, & e_{k-1}^{i}=\text { noev or } \\ & \left(x \in\left(V_{1} \cap V_{2}\right) \text { and } x \notin \operatorname{ref}\left(o_{k-1}\right) \text { and } x \notin \bmod \left(\operatorname{op}_{k-1}\right)\right) \text { or } \\ & \left(x \notin C V_{j} \text { and } x \in \bmod \left(o p_{k-1}\right) \text { and } e_{k-1}^{i} \in E_{\mathbf{C}_{j}}, \text { for } j \neq i\right) \\ s_{k} \cdot x, & \text { otherwise. }\end{cases}
$$

The three cases for $s_{k-1}^{i} x$ correspond to the previous explanations. The definition can be summarised as follows: any modification of $x$ within $\pi_{i}$ results in corresponding values $s_{k} \cdot x$ and $s_{k}^{i} \cdot x$. In any other case, the pre-state value for the variables is kept.

Step 2: In a next step, we have to show $\pi_{i} \in \operatorname{Traces}\left(S_{i} . \mathrm{OZ}\right)$. Based on the previous definition, the values for the state variables within $\pi$ and $\pi_{i}$ are possibly diverse. However, the following, crucial lemma shows that if some state variable $x$ is referenced by the operation $o p_{k}^{i}$, the pre-state value of $x$ is identical in $\pi$ and $\pi_{i}$, that is, $s_{k} \cdot x=s_{k}^{i} \cdot x$ holds:

Lemma 5.3.12. (Equality of values for referenced state variables, left-to-right)

Let $\pi \in \operatorname{Traces}(S . O Z)$, and let $\pi_{i}$ be defined according to Equations 5.2, 5.3 and 5.4. Then:

$$
\forall n \geq 0, \forall x \in V_{i}, \forall o p^{i} \in S_{i} \cdot O p \bullet x \in \operatorname{ref}\left(o p_{n}^{i}\right) \Rightarrow s_{n} \cdot x=s_{n}^{i} \cdot x .
$$

Proof. The property obviously holds for $n=0$, as $s_{0}^{i}=s_{0}\left\lceil V_{i}\right.$. Let $n>0, x \in \operatorname{ref}\left(o p_{n}^{1}\right)$ and thus, $x \in \operatorname{ref}\left(o p_{n}\right)$, for some $x \in S$. State. Assume that $s_{n} \cdot x \neq s_{n}^{1} \cdot x$. The case $i=2$ is analogous. Initially, $s_{0} \cdot x=s_{0}^{1} \cdot x$ holds. Therefore, there exist some $0 \leq k<n$ and $o p_{k}$.par $r_{k}$, such that $x \in \bmod \left(o p_{k}\right)$, but $x \notin \bmod \left(o p_{k}^{1}\right)$. This is due to Equation 5.4: if some state variable is modified within $\pi_{1}$, the modification is identical for $\pi$ and $\pi_{1}$. Assume that $k$ is the latest such position, that is, there is no further modification of $x$ between $s_{k+1}$ and $s_{n}$. For the transition sequence $\left\langle e_{k}, \ldots, e_{n}\right\rangle$, we apply Lemma 6.1.4 from [Brü08]: either, there exists a CFG path, connecting both corresponding DG-nodes $e_{k}$ and $e_{n}$ or they are located in different CFG branches, attached to the same interleaving node or parallel composition node. As $x$ is not modified in between, we deduce that there either exists a direct data dependence (in the first case) or an interference data dependence (in the latter case) from $e_{k}$ to $e_{n}$.

This particular dependence will now be used to deduce a contradiction. Here, several different cases have to be considered. Figure 5.11 illustrates the current situation, where the DG nodes corresponding to $e_{k}$ and $e_{n}$ are connected by a data dependence.

As $x \in \bmod \left(o p_{k}\right)$ and $s_{k+1} x \neq s_{k+1}^{1} x$, either $e_{k}^{1}=$ noev or $e_{k}^{1} \in E_{\mathrm{C}_{2}}$ according to Equation 5.4.

$e_{k}^{1}=$ noev: In this case, the corresponding DG node $\mathbf{e}_{k}$ is an element of $\mathbf{P h}_{2}$, as only operations corresponding to nodes from $\mathbf{P h}_{2}$ are eliminated from $S_{1}$. We need to consider four cases for $\mathrm{e}_{n}$ : 


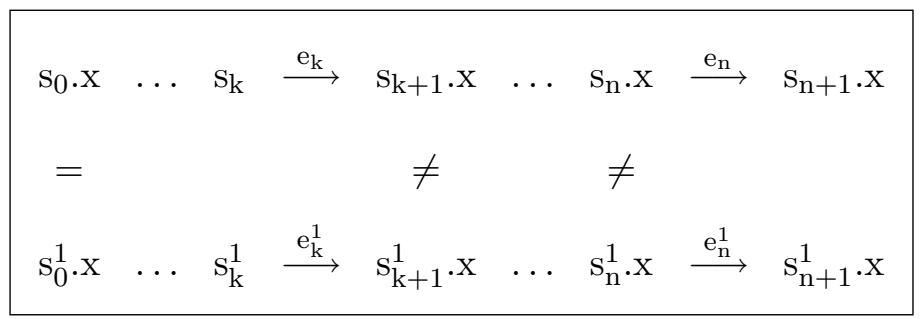

Figure 5.11: Illustration of Lemma 5.3.12

$\mathrm{e}_{n} \in\left(\mathbf{P h}_{1} \cup \mathbf{P h}_{3}\right)$ : The data dependence from from $\mathrm{e}_{k}$ to $\mathrm{e}_{n}$ violates condition no crossing.

$\mathrm{e}_{n} \in \mathbf{C}_{1}$ : The data dependence from $\mathrm{e}_{k}$ to $\mathrm{e}_{n}$ violates Lemma 5.3.3 (and particularly condition no reaching back). $\checkmark$

$\mathrm{e}_{n} \in \mathbf{C}_{2}$ : In this case, the original predicate part of $o p_{n}$ is eliminated for the definition of $S_{1} . o p_{n}$ and solely replaced by the transmission parameter predicates. Thus, $x \in \operatorname{ref}\left(o p_{n}^{1}\right)$ is impossible.

$\mathrm{e}_{n} \in \mathbf{P h}_{2}: o p_{n}$ is eliminated from $S_{1}$ in its entirety and again, $x \in \operatorname{ref}\left(o p_{n}^{1}\right)$ is impossible.

$e_{k}^{1} \in E_{\mathbf{C}_{2}}$ : In particular, $x \notin C V_{2}$ needs to hold, according to the third case of Equation 5.4 .

$\mathrm{e}_{n} \in\left(\mathbf{P h}_{1} \cup \mathbf{P h}_{3}\right):$ The data dependence $\mathrm{e}_{k}$ to $\mathrm{e}_{n}$ causes $x$ to be contained in the set of cut variables of $E_{\mathrm{C}_{2}}$, contradicting $x \notin C V_{2}$.

$\mathrm{e}_{n} \in \mathbf{C}_{1}$ : According to the previous case, as the referencing of $x \in \mathbf{C}_{1}$ still causes $x$ to be a cut variable. $\checkmark$

$\mathrm{e}_{n} \in \mathrm{C}_{2}$ : Analogous to the corresponding case for $e_{k}^{1}=$ noev. $\checkmark$

$\mathrm{e}_{n} \in \mathbf{P h}_{2}$ : Analogous to the corresponding case for $e_{k}^{1}=$ noev. $\checkmark$

Next, we show a corresponding lemma for the values of local state variables:

Lemma 5.3.13. (Equality of values for local state variables, left-to-right)

Let $\pi \in$ Traces(S.OZ), and let $\pi_{i}$ be defined according to Equations 5.2, 5.3 and 5.4. Let $j \neq i$. Then:

$\forall n \geq 0, \forall x \in V_{i}, \forall o p^{i} \in S_{i} \cdot O p \bullet x \in\left(V_{i} \backslash V_{j}\right) \Rightarrow s_{n} \cdot x=s_{n}^{i} \cdot x$.

Proof. Again, the property holds for $n=0$. As $x \in\left(V_{i} \backslash V_{j}\right)$, none of the first three cases from Equation 5.4 ever applies: an event replaced by noev within $\pi_{i}$ is solely represented in $S_{j}$ and never refers to local variables from $S_{i}$. The remaining two cases only apply for $x \in\left(V_{1} \cap V_{2}\right)$. Therefore, $s_{n} \cdot x=s_{n}^{i} \cdot x$ always holds.

The previous lemmas will be used throughout the following theorem, which shows that $\pi_{i}$ is indeed an element of Traces $\left(S_{i} . \mathrm{OZ}\right)$ : 
Theorem 5.3.14. (Correctness of the decomposition: Object- $Z$ part, first part) Let $\pi_{i}$ be defined according to Equations 5.2, 5.3 and 5.4. Then, $\pi_{i} \in \operatorname{Traces}\left(S_{i} . \mathrm{OZ}\right)$.

Proof. The proof is conducted by induction on the length of $\pi_{i}$. In the induction base, we show $s_{0}^{i}=S_{i}$. Init. In the induction step, based on the assumption that $\left\langle s_{0}^{i}, e_{0}^{i}, \ldots, s_{k}^{i}\right\rangle$ is an element of Traces $\left(S_{i} . \mathrm{OZ}\right)$, we show

$$
s_{k}^{i} \stackrel{e_{k}^{i}}{\longrightarrow} s_{k+1}^{i}
$$

where $s_{k+1}^{i}$ complies to the conditions of Equation 5.4.

Induction Base:

$s_{0}^{i}=S_{i}$. Init directly follows from Equation 5.3 and Lemma 5.3.7.

\section{Induction Step:}

We start by considering the guard of $o p_{k}^{i}$, which needs to be satisfied. Furthermore, the operation must be executable with parameter values corresponding to $e_{k}$. Finally, performing $o p_{k}^{i}$ needs to allow for the successor state $s_{k+1}^{i}$ to comply to the conditions of Equation 5.4 .

In the following proof, we use the predicate interpretation of an enable- and ef fectschema in terms of $Z$ :

$$
s \stackrel{\text { op.in.sim.out }}{\longrightarrow} s^{\prime} \Leftrightarrow\left(\text { enable_op }(s, \text { in }, \text { sim }) \wedge \text { effect_op }\left(s, \text { in }, \text { sim }, \text { out }, s^{\prime}\right)\right)
$$

Furthermore, we write $s \oplus t$ to denote that the state $t$ overrides the state $s$. Precisely, for $s$ defined over $V$ and $t$ defined over a subset $V^{\prime}$, let $s \oplus t$ be defined over $V$ as follows:

$$
(s \oplus t) . x:= \begin{cases}t . x, & x \in V^{\prime}, \\ s . x, & \text { otherwise }\end{cases}
$$

Let $e_{k}=o p_{k} \cdot$ in $_{k} \cdot \operatorname{sim}_{k} \cdot$ out $_{k}$. By assumption,

$$
\text { enable_op } k\left(s_{k}, \operatorname{in}_{k}, \operatorname{sim}_{k}\right) \wedge \text { effect_op }\left(s_{k}, \operatorname{in}_{k}, \operatorname{sim}_{k}, \text { out }_{k}, s_{k+1}\right)
$$

holds. Besides, by using Lemma 5.3.12, we know that $s_{k} \cdot x=s_{k}^{i} \cdot x$ for all referenced variables within $o p_{k}^{i}$.

Guard is Satisfied: We need to show enable_op $p_{k}^{i}\left(s_{k}^{i}, i n_{k}, \operatorname{sim}_{k}\right)$. For $o p_{k}^{i}=$ noev, there is obviously nothing to show. Moreover, if $o p_{k}^{i} \in O p_{\mathbf{C}_{j}}$ for $j \neq i$, then enable_op $p_{k}^{i}=$ true. In any other case, enable_op $p_{k}^{i}=e$ nable_op $p_{k}$ holds, according to Definition 4.3.10. We deduce: 


$$
\begin{array}{ll} 
& \text { enable_op } k\left(s_{k}, i n_{k}, \operatorname{sim}_{k}\right) \\
\stackrel{(1)}{\Rightarrow} & \text { enable_op }\left(s_{k}\left\lceil V_{i}, i n_{k}, \operatorname{sim}_{k}\right)\right. \\
\stackrel{(2)}{\Rightarrow} & \text { enable_op } p_{k}^{i}\left(\left(s_{k}\left\lceil V_{i}\right) \oplus\left(s_{k}^{i}\left\lceil r e f\left(o p_{k}\right)\right), i n_{k}, \operatorname{sim}_{k}\right)\right.\right. \\
\stackrel{(3)}{\Rightarrow} & \text { enable_op }{ }_{k}^{i}\left(s_{k}^{i}, i n_{k}, \operatorname{sim}_{k}\right) .
\end{array}
$$

Implication (1) is due to enable_op $p_{k}^{i}=e$ nable_op $p_{k}$ and the fact that any operation from $S_{i}$ solely refers to variables from $V_{i}$. Implication (2) is due to $s_{k}=s_{k}^{i}$ on $r e f\left(o p_{k}\right)$ (induction hypothesis and Lemma 5.3.12). The last implication, (3), follows by the fact that non-referenced variables within enable_op $k$ do not affect the truth-value of the associated predicate.

Operation is Executable with Compatible Successor State: We will now show

$$
\text { effect_op } p_{k}^{i}\left(s_{k}^{i}, \text { in }_{k}, \operatorname{sim}_{k}, \text { out }_{k}, s_{k+1}^{i}\right)
$$

by distinguishing the three cases

1. $o p_{k}^{i}=$ noev,

2. $o p_{k}^{i} \in O p_{\mathrm{C}_{j}}$ for $i \neq j$ and

3. all remaining possibilities.

$o p_{k}^{i}=$ noev: Again, noev does not pose a problem, as in this case, $s_{k+1}^{i}=s_{k+1}$, corresponding to Equation 5.4.

$o p_{k}^{i} \in O p_{\mathbf{C}_{j}}$ for $i \neq j$ : In this case, the predicate part of $o p_{k}$ is replaced by $\bigwedge_{w \in C V_{j}} w^{\prime}=t r_{w}$ ? within $o p_{k}^{i}$. We deduce:

effect_op ${ }_{k}\left(s_{k}\right.$, in $_{k}, \operatorname{sim}_{k}$, out $\left.{ }_{k}, s_{k+1}\right)$

$\stackrel{(1)}{\Rightarrow} \operatorname{effect}_{\text {opp }}^{i}\left(s_{k}\left\lceil V_{i}\right.\right.$, in $_{k}, \operatorname{sim}_{k}$, out $_{k},\left(s_{k}\left\lceil V_{i}\right) \oplus\left(s_{k+1}\left\lceil C V_{j}\right)\right)\right.$

$\stackrel{(2)}{\Rightarrow}$ effect_op ${ }_{k}^{i}\left(\left(s_{k}\left\lceil V_{i}\right) \oplus s^{\prime}\right.\right.$, in $_{k}, \operatorname{sim}_{k}$, out $_{k},\left(\left(s_{k}\left\lceil V_{i}\right) \oplus s^{\prime}\right) \oplus\left(s_{k+1}\left\lceil C V_{j}\right)\right)\right.$

$\stackrel{(3)}{\Rightarrow}$ effect_op ${ }_{k}^{i}\left(s_{k}^{i}\right.$, in $_{k}, \operatorname{sim}_{k}$, out $_{k}, s_{k}^{i} \oplus\left(s_{k+1}\left\lceil C V_{j}\right)\right)$,

for

$$
s^{\prime}:=\left(s_{k}^{i} \uparrow\left(r e f\left(o p_{k}\right) \cup V_{i} \backslash V_{j}\right)\right) .
$$

The first implication is based on the fact that solely cut variables are modified by $o p_{k}^{i}$, that is, the pre-state value needs to be kept for all remaining variables. Moreover, as the value for the output parameters are not restricted within $o p_{k}^{i}$, the output value $o u t_{k}$ can indeed be used. Implication (2) follows by $s_{k} \cdot x=s_{k}^{i} \cdot x$ on $r e f\left(o p_{k}\right)$ and all local variables (induction hypothesis, Lemma 5.3.12 and Lemma 5.3.13). As all other variables do not affect the execution of the operation, implication (3) is immediate.

The state $s_{k+1}^{i}=s_{k}^{i} \oplus\left(s_{k+1}\left\lceil C V_{j}\right)\right.$ satisfies the conditions of Equation 5.4 for any $x \in V$ : 
$x \notin\left(V_{1} \cap V_{2}\right)$ : Impossible, as for $o p_{k}^{i} \in O p_{\mathbf{C}_{j}}$, all variables occurring within $o p_{k}^{i}$ are shared variables. $\checkmark$

$x \in C V_{j}$ : Here, $s_{k+1}^{i}=s_{k+1}$, according to Equation 5.4. $\checkmark$

$x \in\left(V_{1} \cap V_{2}\right) \backslash C V_{j}:$ For $x \in \bmod \left(o_{k}\right)$, we get $s_{k+1}^{i}=s_{k}^{i}$, according to Equation 5.4 , third case. Otherwise, the variable is not modified within $o p_{k}$. If it is referenced, $s_{k}=s_{k}^{i}$, which is preserved by the operation and thus, $s_{k+1}^{i}=$ $s_{k+1}$, again matching with Equation 5.4. Otherwise, $x \notin\left(\bmod \left(\operatorname{op}_{k}\right) \cup\right.$ $\left.r e f\left(o p_{k}\right)\right)$, and $s_{k+1}^{i}=s_{k}^{i}$ matches with Equation 5.4, second case.

All remaining cases: Now, effect_op $p_{k}^{i}=$ effect_op $p_{k}$ holds according to Definition 4.3.10, and we get:

$$
\begin{aligned}
& \text { effect_op }\left(s_{k}, \text { in }_{k}, \operatorname{sim}_{k}, \text { out }_{k}, s_{k+1}\right) \\
& \stackrel{(1)}{\Rightarrow} \text { effect_op }{ }_{k}^{i}\left(s _ { k } \left\lceilV_{i}, \text { in }_{k}, \operatorname{sim}_{k}, \text { out }_{k}, s_{k+1}\left\lceil V_{i}\right)\right.\right. \\
& \stackrel{(2)}{\Rightarrow} \text { effect_op } p_{k}^{i}\left(\left(s_{k}\left\lceil V_{i}\right) \oplus s^{\prime}, \text { in }_{k}, \operatorname{sim}_{k}, \text { out }_{k}, s_{k+1}\left\lceil V_{i}\right)\right.\right. \\
& \stackrel{(3)}{\Rightarrow} \text { effect_op }{ }_{k}^{i}\left(s_{k}^{i}, \text { in }_{k}, \operatorname{sim}_{k}, \text { out }_{k},\left(s_{k+1}\left\lceil V_{i}\right) \oplus\left(s_{k}^{i}\lceil X)\right)\right. \text {, }\right.
\end{aligned}
$$

for $i \neq j$,

$$
s^{\prime}:=\left(s_{k}^{i} \uparrow\left(r e f\left(o p_{k}\right) \cup V_{i} \backslash V_{j}\right)\right),
$$

and

$$
X:=\left(V_{1} \cap V_{2}\right) \backslash\left(\bmod \left(o p_{k}\right) \cup \operatorname{ref}\left(o p_{k}\right)\right) .
$$

For implication (1), we use effect_op $p_{k}^{i}=$ effect_op $p_{k}$. Implication (2) follows by $s_{k} \cdot x=s_{k}^{i} \cdot x$ on $r e f\left(o p_{k}\right)$ and all local variables (induction hypothesis, Lemma 5.3.12 and Lemma 5.3.13).

For the final implication, (3), we use effect_op $p_{k}^{i}=$ effect_op $p_{k}$ and $s_{k} \cdot x=$ $s_{k}^{i} . x$, yielding that any modification of a variable within $o p_{k}$ is correspondingly possible within $o p_{k}^{i}$. We are left to deal with non-modified variables: for these, as all local state variables and referenced state variables have consistent values in the pre-state, they have consistent values in the post state as well. The remaining state variables are exactly those described by the set $X$ : variables neither modified nor referenced within $o p_{k}$, which are not local to $S_{i}$. For these, the pre-state value must be preserved within the post state.

Finally, the state $\left(s_{k+1}\left\lceil V_{i}\right) \oplus\left(s_{k}^{i}\lceil X)\right.\right.$ indeed satisfies the conditions of Equation 5.4 (where only the second and fourth case can apply), as for any state variable, the pre-state value is kept for $\left(V_{1} \cap V_{2}\right) \backslash\left(\operatorname{ref}\left(o p_{k}\right) \cup \bmod \left(o p_{k}\right)\right)$ and otherwise, the value from $s_{k+1}$ is used.

Step 3: So far, we constructed two traces $\pi_{i}$ out of $\pi \in \operatorname{Traces}(S . O Z)$ for which we showed $\pi_{i} \in \operatorname{Traces}\left(S_{i} . \mathrm{OZ}\right)$. It remains to be shown that $\operatorname{tr}=\pi\left\lceil E_{S} \in\left(\pi_{1}\left\lceil E_{S^{\prime}} \|_{E_{\mathbf{C}}} \pi_{2}\left\lceil E_{S^{\prime}}\right) \llbracket \mathrm{R}^{\prime} \rrbracket\right.\right.\right.$ 
holds. This is an immediate deduction due to $\pi_{i}\left\lceil E_{S_{i}}=\operatorname{tr}\left\lceil E_{S_{i}}\right.\right.$ modulo renaming and the definition for the parallel composition of two traces ([Ros98]). This completes the proof of the left-to-right implication.

\section{Right-to-Left Implication}

Let $\operatorname{tr} \in \operatorname{traces}\left(\left(S_{1} . \mathrm{OZ} \|_{E_{\mathrm{C}}} S_{2} . \mathrm{OZ}\right) \llbracket \mathrm{R}^{\prime} \rrbracket\right)$, such that $(\operatorname{tr} \triangleright O p) \in \operatorname{traces}(S$.main $) \triangleright O p .{ }^{5} \mathrm{We}$ have to show $\operatorname{tr} \in \operatorname{traces}(S . O Z)$. Based on $\operatorname{tr} \in \operatorname{traces}\left(\left(S_{1} . \mathrm{OZ} \|_{E_{\mathrm{C}}} S_{2} . \mathrm{OZ}\right) \llbracket \mathrm{R}^{\prime} \rrbracket\right)$, there exist $\pi_{i} \in \operatorname{Traces}\left(S_{i} . \mathrm{OZ}\right)$, such that $\operatorname{tr} \in\left(\pi_{1}\left\lceil E_{S^{\prime}} \|_{E_{\mathrm{C}}} \pi_{2}\left\lceil E_{S^{\prime}}\right) \llbracket \mathrm{R}^{\prime} \rrbracket\right.\right.$.

Again, we proceed in three steps: we define a trace

$$
\pi=\left\langle s_{0}, e_{0}, s_{1}, e_{1}, \ldots\right\rangle
$$

out of $\pi_{i}=\left\langle s_{0}^{i}, e_{0}^{i}, s_{1}^{i}, e_{1}^{i}, \ldots\right\rangle$ (Step 1). In (Step 2), we inductively show $\pi \in \operatorname{Traces}($ S.OZ). Finally, we deduce $t r=\pi\left\lceil E_{S} \in \operatorname{traces}(S . O Z)\right.$ (Step 3).

Step 1: For the definition of $\pi$ on events, we obviously choose the events from tr:

$$
e_{j}:=t r . j
$$

For the definition of the states of $t r$, we start by defining $s_{0}$. Here, we use the result from Lemma 5.3.9: in the following, let $V_{1}=\left\{x_{1}, \ldots, x_{n}\right\}$,

$$
V_{Y}:=\left\{y_{1}, \ldots, y_{m}\right\}=\left\{x \mid \operatorname{InitClos}(x) \subseteq\left(V_{2} \backslash V_{1}\right)\right\}
$$

and

$$
V_{Z}:=\left\{z_{1}, \ldots, z_{l}\right\}=\left(V_{2} \backslash V_{1}\right) \backslash\left\{y_{1}, \ldots, y_{m}\right\} .
$$

Furthermore, let $c_{1}, \ldots, c_{l}$, with $c_{i}: t_{z_{i}}$, such that

$$
\text { S.Init }\left[x_{1} / s_{0}^{1} \cdot x_{1}\right] \ldots\left[x_{n} / s_{0}^{1} \cdot x_{n}\right]\left[y_{1} / s_{0}^{2} \cdot y_{1}\right] \ldots\left[y_{m} / s_{0}^{2} \cdot y_{m}\right]\left[z_{1} / c_{1}\right] \ldots\left[z_{l} / c_{l}\right]
$$

holds. Then:

$$
s_{0}:=\left(s_{0}^{1} \cdot x_{1}, \ldots, s_{0}^{1} \cdot x_{n}, s_{0}^{2} \cdot y_{1}, \ldots, s_{0}^{2} \cdot y_{m}, c_{1}, \ldots, c_{l}\right) .
$$

Note that we can freely choose any values $c_{i}$ for $z_{i}$, as long as they extend $s_{0}^{1} \cdot x_{j}$ and $s_{0}^{2} \cdot y_{k}$ to a valid initial valuation. Lemma 5.3.9 showed that such values for $z_{i}$ indeed exist. Intuitively, the freedom of choice is substantiated by the fact that for the set $V_{Z}$, the initial values within $S_{2}$. Init are irrelevant for the specification $S_{2}$ : in case that any such variable is referenced, it must have been modified before, as otherwise, an initial data dependence would violate the condition no crossing. Thus, these values never become relevant within $S_{2}$, and we can safely refrain from using them within $s_{0}$.

\footnotetext{
${ }^{5}$ Based on the correctness for the CSP part from Section 5.2, tr can equally refer to both, traces (S.main) or $\operatorname{traces}\left(\left(S_{1}\right.\right.$.main $\|_{E_{\mathrm{C}}} S_{2}$.main $\left.) \llbracket \mathrm{R}^{\prime} \rrbracket\right)$.
} 
The definition for $s_{k}, k \geq 1$, is given next:

$$
s_{k} \cdot x:= \begin{cases}s_{k}^{1} \cdot x, & x \in\left(V_{1} \backslash V_{2}\right), \\ s_{k}^{2} \cdot x, & x \in\left(V_{2} \backslash V_{1}\right), \\ s_{k}^{1} \cdot x, & x \in\left(V_{1} \cap V_{2}\right), x \in\left(\bmod \left(o p_{k-1}^{1}\right) \cap \bmod \left(o p_{k-1}^{2}\right)\right), \\ s_{k}^{1} \cdot x, & x \in\left(V_{1} \cap V_{2}\right), x \in\left(\bmod \left(o p_{k-1}^{1}\right) \backslash \bmod \left(o p_{k-1}^{2}\right)\right), \\ s_{k}^{2} \cdot x, & x \in\left(V_{1} \cap V_{2}\right), x \in\left(\bmod \left(o p_{k-1}^{2}\right) \backslash \bmod \left(o p_{k-1}^{1}\right)\right), \\ s_{k-1} \cdot x, & x \in\left(V_{1} \cap V_{2}\right), x \notin\left(\bmod \left(o p_{k-1}^{1}\right) \cup \bmod \left(o p_{k-1}^{2}\right)\right) .\end{cases}
$$

Summarising, for state variables local to $S_{i}$, we choose the value of $s_{k}^{i}$. For shared variables, we adopt modifications from the respective traces and keep the pre-state value, if no modification is conducted. If a variable is modified in both traces, the modification must be corresponding. This is based on the usage of transmission parameters, ensuring that shared state variables must not distinctly be modified by the same operation. Thus, for the third case, we could equally define $s_{k} \cdot x:=s_{k}^{2} \cdot x$.

Step 2: In accordance with the left-to-right implication, we show a property describing that state variables referenced by an operation $o p_{k}^{i}$ always have identical values within $\pi$ and $\pi_{i}$ :

Lemma 5.3.15. (Equality of values for referenced state variables, right-to-left)

Let $\pi_{i} \in \operatorname{Traces}\left(S_{i} . O Z\right)$, and let $\pi$ be defined according to Equations 5.5, 5.6 and 5.7. Then:

$$
\forall n \geq 0, \forall x \in V_{i}, \forall o p^{i} \in S_{i} . O p \bullet x \in \operatorname{ref}\left(o p_{n}^{i}\right) \Rightarrow s_{n} \cdot x=s_{n}^{i} . x .
$$

Proof. We first show that the property holds for $n=0$. For the sets $V_{1}$ and $V_{Y}$, the states $s_{0}$ and $s_{0}^{i}$ are identically defined. This is not the case for the set $V_{Z}$. However, $z \in \operatorname{ref}\left(o p_{0}^{i}\right)$ would yield that $e_{0}^{i} \in E_{2}$, as the set $V_{Z}$ solely comprises variables local to $S_{2}$. This is impossible: any event, which can initially be executed within a trace of $S$.main, is an element of $E_{S_{1}}$. Otherwise, the corresponding DG-node $e_{0}^{i}$ would violate the correctness criterion disjointness, based on $\mathrm{e}_{0}^{i} \in \mathbf{P h}_{2} \cap\left(\mathbf{P h}_{1} \cup \mathbf{C}_{1}\right)$.

Let $n>0, x \in \operatorname{ref}\left(o p_{n}^{i}\right)$ and thus, $x \in \operatorname{ref}\left(o p_{n}\right)$ for some $x \in$ S. St ate. Based on Equation 5.7, any modification conducted within $\pi_{i}$ is identical within $\pi$. This allows us to apply the same ideas from Lemma 5.3.12 for $o p_{n}^{1}$. In particular, if $x \in\left(\bmod \left(o p_{k}\right) \backslash \bmod \left(o p_{k}^{1}\right)\right)$ for some $o p_{k}$, either $o p_{k}^{1}=$ noev or it is an element of $E_{\mathbf{C}_{2}}$, resulting in the exact same case differentiation as in Lemma 5.3.12.

For $o p_{n}^{2}$, we have to consider one additional case: for $x \in V_{Z}$, we cannot assume $s_{0} \cdot x=s_{0}^{2} x$. If $x$ is modified somewhere in $\pi_{2}$, the modification is identical to the one in $\pi$, and we reside in the previous case. Now assume that $x \in \operatorname{ref}\left(o p_{n}\right)$, and $x$ is never modified in $\pi_{2}$. In this case, there exists an initial data dependence from $S$. Init to the corresponding DG node $\mathrm{e}_{n}$. Since $o p_{n}^{2}$ references $x \in V_{Z}, o p_{n}$ is an element of $O p_{2}$ and thus, $e_{n}$ is an element of $\mathbf{P h}$. This yields a contradiction, as the connecting initial data dependence violates no crossing.

Figure 5.12 illustrates the proof idea of the lemma. Here, $(*)$ denotes that $\mathrm{s}_{0} \cdot \mathrm{x}=\mathrm{s}_{0}^{2} \cdot \mathrm{x}$ only holds for $V \backslash V_{Z}$. 


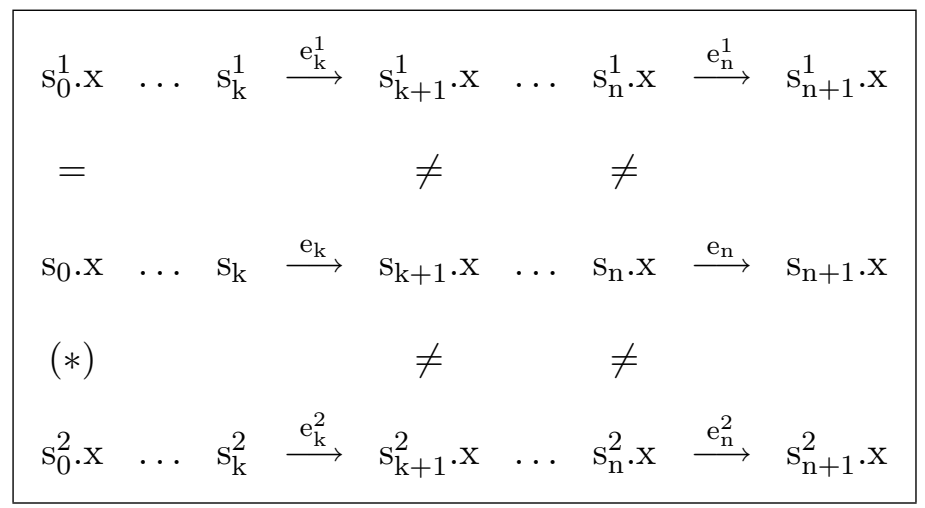

Figure 5.12: Illustration of Lemma 5.3.15

The corresponding lemma for local state variables is immediate:

Lemma 5.3.16. (Equality of values for local state variables, right-to-left)

Let $\pi_{i} \in \operatorname{Traces}\left(S_{i} . \mathrm{OZ}\right)$, and let $\pi$ be defined according to Equations 5.5, 5.6 and 5.7. Let $j \neq i$. Then:

$$
\forall n \geq 1, \forall x \in V_{i}, \forall o p^{i} \in S_{i} . O p \bullet x \in\left(V_{i} \backslash V_{j}\right) \Rightarrow s_{n} \cdot x=s_{n}^{i} \cdot x .
$$

Proof. The property holds based on Equation 5.7.

Note that the previous property does not hold for $n=0$, as the initial states do not correspond on the set $\left\{z_{1}, \ldots, z_{l}\right\}$. Next, we show that $\pi$ is an element of Traces(S.OZ):

Theorem 5.3.17. (Correctness of the decomposition: Object-Z part, second part) Let $\pi$ be defined according to Equations 5.5, 5.6 and 5.7. Then, $\pi \in \operatorname{Traces}(S . O Z)$.

Proof. Again, the proof is conducted by induction on the length of $\pi$.

Induction Base:

$s_{0}=S$.Init directly follows by Equation 5.6 and Lemma 5.3.9.

Induction Step:

Again, let $e_{k}=o p_{k} \cdot \operatorname{in}_{k} \cdot \operatorname{sim}_{k} \cdot$ out $_{k}$. By assumption,

$$
\text { enable_op } p_{k}^{i}\left(s_{k}^{i}, i n_{k}^{i}, \operatorname{sim}_{k}^{i}\right) \wedge \text { effect_op } p_{k}^{i}\left(s_{k}^{i}, \operatorname{in}_{k}^{i}, \operatorname{sim}_{k}^{i}, o u t_{k}^{i}, s_{k+1}^{i}\right)
$$

holds. By using Lemma 5.3.15, we know that $s_{k} \cdot x=s_{k}^{i} \cdot x$ holds for all referenced variables within $o p_{k}^{i}$.

Guard is Satisfied: In order to show enable_op $\left(s_{k}, i n_{k}^{i}, \operatorname{sim}_{k}^{i}\right)$, we start with $o p_{k} \in O p_{i}$, that is, $o p_{k}$ is a non-cut operation: enable_op $p_{k}=$ enable_op $p_{k}^{i}$ holds according to Definition 4.3.10. We deduce: 


$$
\begin{aligned}
& \text { enable_op } p_{k}^{i}\left(s_{k}^{i}, i n_{k}^{i}, \operatorname{sim}_{k}^{i}\right) \\
\stackrel{(1)}{\Rightarrow} & \text { enable_op } k\left(s_{k} \oplus s_{k}^{i}, i n_{k}^{i}, \operatorname{sim}_{k}^{i}\right) \\
\stackrel{(2)}{\Rightarrow} & \text { enable_op } k\left(s_{k} \oplus\left(s_{k}\left\lceil V_{i}\right), i n_{k}^{i}, \operatorname{sim}_{k}^{i}\right)\right. \\
\stackrel{(3)}{\Rightarrow} & \text { enable_op }\left(s_{k}, i n_{k}^{i}, \operatorname{sim}_{k}^{i}\right) .
\end{aligned}
$$

Implication (1) is due to enable_op $p_{k}^{i}=e_{\text {nable }} o p_{k}$ and the fact that only variables of $V_{i}$ are referenced in enable_op $p_{k}^{i}$. The second implication follows by the induction hypothesis and Lemma 5.3.15, again using that non-referenced variables within enable_op $k$ do not affect the truth-value of the associated predicate. The last implication is immediate.

If we assume $o p_{k} \in O p_{C_{i}}$, the equation enable_op $p_{k}=e n a b l e \_o p_{k}^{i}$ holds as well, and we proceed accordingly.

Operation is Executable with Compatible Successor State: Next, we show

$$
\text { effect_op }\left(s_{k}, \operatorname{in}_{k}^{i}, \operatorname{sim}_{k}^{i}, \text { out }_{k}^{i}, s_{k+1}\right),
$$

by distinguishing the two cases

1. $o p_{k} \in O p_{i}$ and

2. $o p_{k} \in O p_{\mathrm{C}_{i}}$.

$o p_{k} \in O p_{i}$ : Here, effect_op $p_{k}=$ effect_op $p_{k}^{i}$ and $o p_{k}^{j}=$ noev, $j \neq i$, according to Definition 4.3.10. We get:

$$
\begin{aligned}
& \text { effect_op } p_{k}^{i}\left(s_{k}^{i}, \operatorname{in}_{k}^{i}, \operatorname{sim}_{k}^{i}, o u t_{k}^{i}, s_{k+1}^{i}\right) \\
& \stackrel{(1)}{\Rightarrow} \text { effect_op }\left(s_{k} \oplus s_{k}^{i}, \text { in }_{k}^{i}, \operatorname{sim}_{k}^{i}, \text { out }_{k}^{i}, s_{k} \oplus s_{k+1}^{i}\right) \\
& \stackrel{(2)}{\Rightarrow} \text { effect_op } p_{k}\left(s_{k} \oplus\left(s_{k}\left\lceil V_{i}\right), \text { in }_{k}^{i}, \operatorname{sim}_{k}^{i}, \text { out }_{k}^{i}, s_{k} \oplus s^{\prime}\right)\right. \\
& \stackrel{(3)}{\Rightarrow} \text { effect_op }{ }_{k}\left(s_{k}, i n_{k}^{i}, \operatorname{sim}_{k}^{i}, o u t_{k}^{i}, s_{k} \oplus s^{\prime}\right) \text {, }
\end{aligned}
$$

for

$$
s^{\prime}:=\left(s_{k+1}^{i}\left\lceil\left(\operatorname{ref}\left(o p_{k}\right) \cup \bmod \left(o p_{k}\right)\right)\right) .\right.
$$

The first implication is analogous to the considerations for the enable-schema, and the last implication is obvious. For implication (2), the value $s_{k+1}^{i} x$ can solely be used in case that either $x$ is correspondingly modified by $o p_{k}$ and $o p_{k}^{i}$ or the identical pre-state values is kept. For the remaining variables, the value $s_{k} \cdot x$ needs to be used.

The state $s_{k+1}=s_{k} \oplus s^{\prime}$ satisfies the conditions of Equation 5.7 for any $x \in V$ : the sole case of $s_{k+1}$ and Equation 5.7 differing is $x \in\left(\operatorname{ref}\left(o p_{k}^{i}\right) \backslash \bmod \left(o p_{k}^{i}\right)\right)$. But then, $s_{k} \cdot x=s_{k}^{i} \cdot x=s_{k+1}^{i}$. 
$o p_{k} \in O p_{\mathrm{C}_{i}}$ : Again, effect_op $p_{k}=$ effect_op $p_{k}^{i}$, according to Definition 4.3.10. In addition, effect_op $p_{k}^{j}$ for $j \neq i$ solely comprises $\bigwedge_{w \in C V_{j}} w^{\prime}=t r_{w}$ ?. The proof is corresponding to the previous case, except for the fact that now, $x \in \bmod \left(o p_{k}^{i}\right) \cap \bmod \left(o p_{k}^{j}\right)$ is possible. However, in this case, the modification is identical based on Definition 4.3.10, which corresponds to Equation 5.7, where we choose the modification from $o p_{k}^{1}$.

Step 3: As $\pi \in \operatorname{Traces}(S . O Z)$ and $\pi \mid E_{S}=\operatorname{tr}$ hold, we immediately deduce $t r \in \operatorname{traces}(S . O Z)$. This completes the proof of the right-to-left implication and thus, the correctness proof of the decomposition of the Object-Z part.

\subsection{Correctness of the Renaming for the Decomposition}

The previous sections showed correctness for the decomposition of both, the CSP part and the Object-Z part of $S$. Preservation of control flow and data flow can only be achieved by the introduction of additional parameters. One drawback of these parameters is the required modification of the types of operations from $S$ : equivalence of $S$ and $S_{1} \| S_{2}$ can only be shown modulo a renaming of events.

According to Section 4.3.4, the addition of parameters requires a channel renaming $f$. As the interface of a specification declares the types of operations, the set of additional parameters is identical for the CSP part and the Object-Z part. However, according to Section 4.3, transmission parameters are solely restricted by the Object-Z part, whereas the restriction of address parameters is limited to the CSP part.

For the definition of the CSP parts of $S_{1}$ and $S_{2}$, we already introduced two renaming relations

$$
\mathrm{R}_{1}^{\mathrm{C}}: E_{S} \rightarrow E_{S_{1}} \text { and } \mathrm{R}_{2}^{\mathrm{C}}: E_{S} \rightarrow E_{S_{2}} .
$$

These relations determine the possible events the CSP parts of $S_{i}$ can communicate, and they fix the values for the address parameters, whereas transmission parameters remain unrestricted. Subsequently, in case that no restriction on either the transmission parameters or address parameters is conducted, we write ?tr and ?add, respectively. If the number of additional parameters is irrelevant, we write op.x.t.a to denote an event of $E_{S^{\prime}}$, according to Section 5.2. Note that none of these parameters have to exist. We recall the definitions of $\mathrm{R}_{1}^{\mathrm{C}}$ and $\mathrm{R}_{2}^{\mathrm{C}}$ :

$$
\mathrm{R}_{\mathrm{i}}^{\mathrm{C}}(\text { op.x }):= \begin{cases}\text { op.x.i, } & o p \in\left(O p_{1} \cap O p_{2}\right) \backslash\left(O p_{\mathbf{C}_{1}} \cup O p_{\mathbf{C}_{2}}\right), \\ \text { op.x?tr.a, } & o p \in O p_{\mathbf{C}} \wedge\left|l^{-1}(o p)\right|>1, \\ o p \cdot x ? t r, & o p \in O p_{\mathbf{C}} \wedge\left|l^{-1}(o p)\right|=1, \\ o p \cdot x, & \text { otherwise. }\end{cases}
$$

The definition of $S_{i} . \mathrm{OZ}$ implicitly defines two renaming relations for the Object-Z parts as well. The roles for restricting the different types of added parameters are switched: for an event op.x $\in E_{S}$, the Object-Z part of $S_{i}$ is able to communicate any event op.x.t?add, 
as address parameters are unrestricted for the Object-Z part. Precisely, we get a renaming relation for the Object-Z part, given as:

$$
\mathrm{R}_{\mathrm{i}}^{0}(\text { op.x }):= \begin{cases}\text { op.x.add, } & o p \in\left(O p_{1} \cap O p_{2}\right) \backslash\left(O p_{\mathbf{C}_{1}} \cup O p_{\mathbf{C}_{2}}\right), \\ \text { op.x.t?add, } & o p \in O p_{\mathbf{C}} \wedge\left|l^{-1}(o p)\right|>1, \\ \text { op.x.t, } & o p \in O p_{\mathbf{C}} \wedge\left|l^{-1}(o p)\right|=1, \\ \text { op.x, } & \text { otherwise. }\end{cases}
$$

The renaming needs to be considered, when it comes to showing trace equivalence between the original system and the decomposition. We use several notations for the combinations of the four renaming relations $R_{1}^{C}, R_{2}^{C}, R_{1}^{0}$ and $R_{2}^{0}$ :

- $R_{i}$ denotes the union of $R_{i}^{C}$ and $R_{i}^{0}$.

- $\mathrm{R}^{\mathrm{C}}$ denotes the union of $\mathrm{R}_{1}^{\mathrm{C}}$ and $\mathrm{R}_{2}^{\mathrm{C}}$ and, accordingly, $\mathrm{R}^{0}$ the union of $\mathrm{R}_{1}^{0}$ and $\mathrm{R}_{2}^{0}$.

- Finally, $\mathrm{R}$ denotes the union of all renaming relations, that is, the union of $\mathrm{R}^{\mathrm{C}}$ and $\mathrm{R}^{0}$ or, accordingly, $\mathrm{R}_{1}$ and $\mathrm{R}_{2}$.

For achieving a comparison between both, the original system and its decomposition, we consider the - in regard of $\mathrm{R}$ - inverse relation $\mathrm{R}^{\prime}$, which removes the additional transmission parameters and address parameters. More precisely, $\mathrm{R}^{\prime}: E_{S^{\prime}} \rightarrow E_{S}$ and

$$
\mathrm{R}^{\prime}(\text { op.x.t.a) }:=\text { op.x. }
$$

We are now able to relate $S$ to $\left(S_{1} \|_{E_{\mathrm{C}}} S_{2}\right)$ by means of $\mathrm{R}^{\prime}$. Correctness for the decomposition of the Object-Z part and the CSP part was carried out modulo $\mathrm{R}^{\prime}$, and we showed the equivalences

- $S$.main $=_{T}\left(S_{1} \cdot\right.$ main $\left.\|_{E_{\mathrm{C}}} S_{2} \cdot \operatorname{main}\right) \llbracket \mathrm{R}^{\prime} \rrbracket$ and

- $S . O Z=_{T}\left(S_{1} . O Z \|_{E_{\mathrm{C}}} S_{2} . \mathrm{OZ}\right) \llbracket \mathrm{R}^{\prime} \rrbracket$ for the set of traces conforming to the CSP part.

In order to facilitate reasoning about the individual component's parts $S_{i}$.main and $S_{i}$.OZ, we show that $\mathrm{R}^{\prime}$ can be distributed over the parallel composition operator. Precisely, we show the following equivalence in the semantic domain of the CSP trace model:

$$
\begin{aligned}
& \left(S_{1} \cdot \text { main } \|_{E_{\mathrm{C}}} S_{2} \cdot \mathrm{main}\right) \llbracket \mathrm{R}^{\prime} \rrbracket \|_{E_{S}}\left(S_{1} \cdot \mathrm{OZ} \|_{E_{\mathrm{C}}} S_{2} \cdot \mathrm{OZ}\right) \llbracket \mathrm{R}^{\prime} \rrbracket={ }_{T} \\
& \left(\left(S_{1} \cdot \mathrm{main} \|_{E_{\mathrm{C}}} S_{2} \cdot \mathrm{main}\right) \|_{E_{S^{\prime}}}\left(S_{1} \cdot \mathrm{OZ} \|_{E_{\mathrm{C}}} S_{2} \cdot \mathrm{OZ}\right)\right) \llbracket \mathrm{R}^{\prime} \rrbracket .
\end{aligned}
$$

Here, the crucial aspect is that the synchronisation alphabet for the outer parallel composition changes from $E_{S}$ to $E_{S^{\prime}}$, as the right hand side now synchronises over events after the renaming took place.

We start the proof by showing a property about the composition of a general relation and its inverse: if a relation $\mathrm{Re}$ is total and injective, the composition $\mathrm{Re}^{-1} \circ \mathrm{Re}$ is the identity relation. 
Lemma 5.4.1. (Composition law for injective and total relations)

Let $\operatorname{Re} \subseteq \mathcal{A} \times \mathcal{B}$ be a relation, and let

$$
\operatorname{Re}^{-1}:=\{(b, a) \mid(a, b) \in \operatorname{Re}\}
$$

be its inverse relation. If $\mathrm{Re}$ is total and injective, then

$$
\left(\operatorname{Re}^{-1} \circ \operatorname{Re}\right)=\operatorname{ld}_{\mathcal{A}} .
$$

Proof. We recall the definitions for a relation being total and injective and the one for the composition of two relations:

- Re is total, if, and only if, $\forall a \in \mathcal{A} \exists(a, b) \in \mathcal{A} \times \mathcal{B} \bullet(a, b) \in \operatorname{Re}$.

- Re is injective, if, and only if, $\forall(a, b),\left(a^{\prime}, b^{\prime}\right) \in \operatorname{Re} \bullet b=b^{\prime} \Rightarrow a=a^{\prime}$.

- $\operatorname{Re}^{-1} \circ \operatorname{Re}=\left\{(x, y) \in \mathcal{A} \times \mathcal{A} \mid \exists z \in \mathcal{B} \bullet(x, z) \in \operatorname{Re} \wedge(z, y) \in \operatorname{Re}^{-1}\right\}$.

Based on the fact that $\operatorname{Re}$ is total, $\mathrm{Id}_{\mathcal{A}} \subseteq\left(\mathrm{Re}^{-1} \circ \mathrm{Re}\right)$ holds. Now assume some $(x, y) \in$ $\left(\operatorname{Re}^{-1} \circ \operatorname{Re}\right)$ and $x \neq y$. But then, there exists $z \in \mathcal{B}$, such that $(x, z) \in \operatorname{Re}$ and $(z, y) \in \operatorname{Re}^{-1}$. The definition of $\operatorname{Re}^{-1}$ yields $(x, z),(y, z) \in \operatorname{Re}$, contradiction to Re being injective.

For an application of this property on our renaming relation, we show that $R, R^{C}$ and $R^{0}$ are both, total and injective:

Lemma 5.4.2. (Properties of event renaming)

The following properties for $\mathrm{R}, \mathrm{R}^{\mathrm{C}}$ and $\mathrm{R}^{0}$ are satisfied:

a) $\mathrm{R}, \mathrm{R}^{\mathrm{C}}$ and $\mathrm{R}^{0}$ are total.

b) $\mathrm{R}, \mathrm{R}^{\mathrm{C}}$ and $\mathrm{R}^{0}$ are injective.

c) $\left(\mathrm{R}^{\prime} \circ \mathrm{R}\right)=\left(\mathrm{R}^{\prime} \circ \mathrm{R}^{\mathrm{C}}\right)=\left(\mathrm{R}^{\prime} \circ \mathrm{R}^{0}\right)=\mathrm{Id}_{E_{S}}$ and for any CSP process $\mathrm{Q}$ :

- $Q=Q^{\prime} \llbracket \mathrm{R} \rrbracket$ for some process $Q^{\prime}$ implies $Q \llbracket\left(\mathrm{R} \circ \mathrm{R}^{\prime}\right) \rrbracket=Q$.

- $Q=Q^{\prime} \llbracket \mathrm{R}^{\mathrm{C}} \rrbracket$ for some process $Q^{\prime}$ implies $Q \llbracket\left(\mathrm{R}^{\mathrm{C}} \circ \mathrm{R}^{\prime}\right) \rrbracket=Q$.

- $Q=Q^{\prime} \llbracket \mathrm{R}^{0} \rrbracket$ for some process $Q^{\prime}$ implies $Q \llbracket\left(\mathrm{R}^{0} \circ \mathrm{R}^{\prime}\right) \rrbracket=Q$.

\section{Proof.}

a) As the renaming relations are defined with respect to the whole set $E_{S}$, we immediately deduce this property.

b) Immediately follows by the implication

$$
\left(o p_{1} \cdot x_{1} \cdot t_{1} \cdot a_{1}=o p_{2} \cdot x_{2} \cdot t_{2} \cdot a_{2}\right) \Rightarrow\left(o p_{1} \cdot x=o p_{2} \cdot x\right)
$$

c) Based on Lemma 5.4.2, the combination of a) and b) yields

$$
\left(\mathrm{R}^{\prime} \circ \mathrm{R}\right)=\left(\mathrm{R}^{\prime} \circ \mathrm{R}^{\mathrm{C}}\right)=\left(\mathrm{R}^{\prime} \circ \mathrm{R}^{0}\right)=\mathrm{Id}_{E_{S}} .
$$

Thus, $\left(\mathrm{R} \circ \mathrm{R}^{\prime} \circ \mathrm{R}\right)=\mathrm{R},\left(\mathrm{R}^{\mathrm{C}} \circ \mathrm{R}^{\prime} \circ \mathrm{R}^{\mathrm{C}}\right)=\mathrm{R}^{\mathrm{C}}$ and $\left(\mathrm{R}^{0} \circ \mathrm{R}^{\prime} \circ \mathrm{R}^{0}\right)=\mathrm{R}^{0} . \checkmark$ 
We use these properties in the following theorem, showing the already mentioned distributivity law for the inverse renaming. The core idea for its proof is the following: both additional parameter types are either restricted by the decomposition's Object-Z part (transmission parameters) or by its CSP part (address parameters), but neither of them by both. Therefore, if a synchronisation of some op.x between $\left(S_{1}\right.$.main $\|_{E_{\mathrm{C}}} S_{2}$.main) and $\left(S_{1} . O Z \|_{E_{\mathrm{C}}} S_{2} . O Z\right)$ is possible after removing the additional parameters, there exists some op.x.t.a on which both parts can synchronise beforehand: the intersection of the newly constructed event sets of the CSP part and the Object-Z part is non-empty.

Theorem 5.4.3. (Distributivity law for inverse renaming)

The inverse renaming relation $\mathrm{R}^{\prime}$ distributes over the parallel composition $\|_{E_{S}}$, that is:

$$
\begin{aligned}
& \left.\left(S_{1} \cdot \text { main } \|_{E_{\mathrm{C}}} S_{2} \cdot \text { main }\right) \llbracket \mathrm{R}^{\prime} \rrbracket\right] \|_{E_{S}}\left(S_{1} \cdot O Z \|_{E_{\mathrm{C}}} S_{2} \cdot O Z\right) \llbracket \mathrm{R}^{\prime} \rrbracket={ }_{T} \\
& \left(\left(S_{1} \cdot \mathrm{main} \|_{E_{\mathrm{C}}} S_{2} \cdot \mathrm{main}\right) \|_{E_{S^{\prime}}}\left(S_{1} . \mathrm{OZ} \|_{E_{\mathrm{C}}} S_{2} \cdot O Z\right)\right) \llbracket \mathrm{R}^{\prime} \rrbracket .
\end{aligned}
$$

Proof. First, note that $E_{S}=\mathrm{R}^{\prime}\left(E_{S^{\prime}}\right)$ holds as $\mathrm{R}$ is total (Lemma 5.4.2, a)) and thus, $\mathrm{R}^{\prime}$ is surjective. Let $P:=S_{1}$.main $\|_{E_{\mathrm{C}}} S_{2}$.main and $Q:=S_{1} . O Z \|_{E_{\mathrm{C}}} S_{2}$.OZ. We prove the theorem by showing that $\left(P \|_{E_{S^{\prime}}} Q\right) \llbracket \mathrm{R}^{\prime} \rrbracket$ and $P \llbracket \mathrm{R}^{\prime} \rrbracket \|_{\mathrm{R}^{\prime}\left(E_{S^{\prime}}\right)} Q \llbracket \mathrm{R}^{\prime} \rrbracket$ are the initial states of a strong bisimulation [Mil89]

$$
\mathcal{R}:=\left\{(A, B) \mid A=\left(C \|_{E_{S^{\prime}}} D\right) \llbracket \mathrm{R}^{\prime} \rrbracket, B=C \llbracket \mathrm{R}^{\prime} \rrbracket \|_{\mathrm{R}^{\prime}\left(E_{S^{\prime}}\right)} D \llbracket \mathrm{R}^{\prime} \rrbracket\right\},
$$

where $C$ depicts any reachable state within the labelled transition system of $P$, and $D$ denotes any reachable state within the labelled transition system of $Q$. Based on the definition of bisimulation, we need to show two directions:

(1) If $(A, B) \in \mathcal{R}$ and $B \stackrel{e}{\rightarrow} B^{\prime}$ for $e \in\left(E_{S} \cup\{\tau\}\right)$, then there exists some $A^{\prime}$, such that $A \stackrel{e}{\rightarrow} A^{\prime}$ and $\left(A^{\prime}, B^{\prime}\right) \in \mathcal{R}$.

(2) If $(A, B) \in \mathcal{R}$ and $A \stackrel{e}{\rightarrow} A^{\prime}$ for $e \in\left(E_{S} \cup\{\tau\}\right)$, then there exists some $B^{\prime}$, such that $B \stackrel{e}{\rightarrow} B^{\prime}$ and $\left(A^{\prime}, B^{\prime}\right) \in \mathcal{R}$.

Based on the firing rules for CSP, renaming has no effect on $\tau$-transitions [Ros98]. Thus, for the $\tau$-case, both directions are immediate.

(1): Let $(A, B) \in \mathcal{R}$ and $B \stackrel{\text { op.x }}{\rightarrow} B^{\prime}$. Since $\mathrm{R}^{\prime}\left(E_{S^{\prime}}\right)=E_{S}$, both processes $C \llbracket \mathrm{R}^{\prime} \rrbracket$ and $D \llbracket \mathrm{R}^{\prime} \rrbracket$ need to synchronise on op.x. Based on the operational semantics of CSP, there exist $B_{1}^{\prime}, B_{2}^{\prime}$, such that $B^{\prime}=B_{1}^{\prime} \|_{\mathrm{R}^{\prime}\left(E_{S^{\prime}}\right)} B_{2}^{\prime}$ and

$$
C \llbracket \mathrm{R}^{\prime} \rrbracket \stackrel{o p \cdot x}{\longrightarrow} B_{1}^{\prime} \text { and } D \llbracket \mathrm{R}^{\prime} \rrbracket \stackrel{o p \cdot x}{\longrightarrow} B_{2}^{\prime} .
$$

From 5.4.2, c), we deduce $B_{1}^{\prime}=B_{1}^{\prime} \llbracket\left(\mathrm{R}^{\prime} \circ \mathrm{R}^{\mathrm{C}}\right) \rrbracket$ and $B_{2}^{\prime}=B_{2}^{\prime} \llbracket\left(\mathrm{R}^{\prime} \circ \mathrm{R}^{0}\right) \rrbracket$. Thus,

$$
C \llbracket \mathrm{R}^{\prime} \rrbracket \stackrel{o p \cdot x}{\longrightarrow} B_{1}^{\prime} \llbracket\left(\mathrm{R}^{\prime} \circ \mathrm{R}^{\mathrm{C}}\right) \rrbracket \text { and } D \llbracket \mathrm{R}^{\prime} \rrbracket \stackrel{o p \cdot x}{\longrightarrow} B_{2}^{\prime} \llbracket\left(\mathrm{R}^{\prime} \circ \mathrm{R}^{0}\right) \rrbracket .
$$

By applying $R$ and the firing rule for relational renaming from [Ros98], we get

$$
C \stackrel{\text { op.x.t.t. }}{\longrightarrow} B_{1}^{\prime} \llbracket \mathrm{R}^{\mathrm{C}} \rrbracket \text { and } D \stackrel{\text { op.x.t.t. }}{\longrightarrow} B_{2}^{\prime} \llbracket \mathrm{R}^{0} \rrbracket
$$


for all op.x.t. $t_{1} \cdot a_{1} \in \mathrm{R}^{\mathrm{C}}($ op. $x)$ and op.x.t. $t_{2} \cdot a_{2} \in \mathrm{R}^{0}($ op. $x)$. Here, we again apply Lemma 5.4.2, c), as $C \llbracket\left(\mathrm{R}^{\mathrm{C}} \circ \mathrm{R}^{\prime}\right) \rrbracket=C$ and $D \llbracket\left(\mathrm{R}^{0} \circ \mathrm{R}^{\prime}\right) \rrbracket=D$ holds. The following observation is the crucial point in this proof: the intersection of $\mathrm{R}^{\mathrm{C}}\left(\right.$ op. $x$ ) and $\mathrm{R}^{0}($ op.x) is nonempty, since the CSP part solely restricts the address parameters, whereas the Object-Z part solely restricts the transmission parameters of an operation. Precisely,

- $\mathrm{R}^{\mathrm{C}}($ op. $x)=\{$ op.x.?tr.a $\mid a$ addressing extension for $o p\}$ and

- $\mathrm{R}^{0}($ op. $x)=\{$ op.x.t?add $\mid t$ transmission parameters for $o p\}$.

Thus, there exists some op.x.t.a $\in\left(\mathrm{R}^{\mathrm{C}}(\right.$ op.x $) \cap \mathrm{R}^{0}($ op.x $\left.)\right)$ on which $C$ and $D$ can synchronise on. We deduce

$$
\left(C \|_{E_{S^{\prime}}} D\right) \stackrel{\text { op.x.t.a }}{\longrightarrow}\left(B_{1}^{\prime} \llbracket \mathrm{R}^{\mathrm{C}} \rrbracket \|_{E_{S^{\prime}}} B_{2}^{\prime} \llbracket \mathrm{R}^{0} \rrbracket\right) .
$$

Again applying the firing rule for relation renaming, we get

$$
\left(C \|_{E_{S^{\prime}}} D\right) \llbracket \mathrm{R}^{\prime} \rrbracket \stackrel{o p \cdot x}{\longrightarrow}\left(B_{1}^{\prime} \llbracket \mathrm{R}^{\mathrm{C}} \rrbracket \|_{E_{S^{\prime}}} B_{2}^{\prime} \llbracket \mathrm{R}^{0} \rrbracket\right) \llbracket \mathrm{R}^{\prime} \rrbracket,
$$

based on $\mathrm{R}^{\prime}($ op.x.t.a) = op.x. Finally,

$$
\left(A^{\prime}, B^{\prime}\right)=\left(\left(B_{1}^{\prime} \llbracket \mathrm{R}^{\mathrm{C}} \rrbracket \|_{E_{S^{\prime}}} B_{2}^{\prime} \llbracket \mathrm{R}^{0} \rrbracket\right) \llbracket \mathrm{R}^{\prime} \rrbracket,\left(B_{1}^{\prime} \llbracket\left(\mathrm{R}^{\prime} \circ \mathrm{R}^{\mathrm{C}}\right) \rrbracket \|_{\mathrm{R}^{\prime}\left(E_{S^{\prime}}\right)} B_{2}^{\prime} \llbracket\left(\mathrm{R}^{\prime} \circ \mathrm{R}^{0}\right) \rrbracket\right)\right) \in \mathcal{R} .
$$

The bisimulation diagram for this case is given next.

\begin{tabular}{|ccccc||}
\hline \hline$B=C \llbracket \mathrm{R}^{\prime} \rrbracket \|_{\mathrm{R}^{\prime}\left(E_{S^{\prime}}\right)} D \llbracket \mathrm{R}^{\prime} \rrbracket$ & $\stackrel{o p \cdot x}{\longrightarrow}$ & $B_{1}^{\prime} \llbracket\left(\mathrm{R}^{\prime} \circ \mathrm{R}^{\mathrm{C}}\right) \rrbracket \|_{\mathrm{R}^{\prime}\left(E_{S^{\prime}}\right)} B_{2}^{\prime} \llbracket\left(\mathrm{R}^{\prime} \circ \mathrm{R}^{0}\right) \rrbracket$ & $=B^{\prime}$ \\
$\mid$ & & $\vdots$ & \\
$\mathcal{R}$ & & $\mathcal{R}$ & \\
$\mid$ & & $\vdots$ & \\
$A=$ & $\left(C \|_{E_{S^{\prime}}} D\right) \llbracket \mathrm{R}^{\prime} \rrbracket$ & $\stackrel{o p . x}{\longrightarrow}$ & $\left(B_{1}^{\prime} \llbracket \mathrm{R}^{\mathrm{C}} \rrbracket \|_{E_{S^{\prime}}} B_{2}^{\prime} \llbracket \mathrm{R}^{0} \rrbracket\right) \llbracket \mathrm{R}^{\prime} \rrbracket$ & $=A^{\prime}$ \\
\hline
\end{tabular}

(2): For the second implication, assume that $(A, B) \in \mathcal{R}$ and $A \stackrel{o p . x}{\rightarrow} A^{\prime}$, that is,

$$
\left(C \|_{E_{S^{\prime}}} D\right) \llbracket \mathrm{R}^{\prime} \rrbracket \stackrel{o p \cdot x}{\rightarrow} A^{\prime} .
$$

Again, based on Lemma 5.4.2, we have the identity $\left(R \circ R^{\prime}\right)$, yielding

$$
\left(C \|_{E_{S^{\prime}}} D\right) \stackrel{\text { op.x.t.a }}{\rightarrow} A^{\prime} \llbracket \mathrm{R} \rrbracket
$$

for any op.x.t.a $\in \mathrm{R}($ op.x). Based on the operational semantics of CSP, there need to exist some $A_{1}^{\prime}$ and $A_{2}^{\prime}$, such that

$$
A^{\prime} \llbracket \mathrm{R} \rrbracket=A_{1}^{\prime} \|_{E_{S^{\prime}}} A_{2}^{\prime} .
$$

Following up, we get

$$
C \stackrel{\text { op.x.t.a }}{\rightarrow} A_{1}^{\prime} \text { and } D \stackrel{\text { op.x.t.a }}{\rightarrow} A_{2}^{\prime} \text {. }
$$


Application of $\mathrm{R}^{\prime}$ leads to

$$
C \llbracket \mathrm{R}^{\prime} \rrbracket \stackrel{o p \cdot x}{\rightarrow} A_{1}^{\prime} \llbracket \mathrm{R}^{\prime} \rrbracket \text { and } D \llbracket \mathrm{R}^{\prime} \rrbracket \stackrel{o p \cdot x}{\rightarrow} A_{2}^{\prime} \llbracket \mathrm{R}^{\prime} \rrbracket
$$

and finally,

$$
C \llbracket \mathrm{R}^{\prime} \rrbracket\left\|_{\mathrm{R}^{\prime}\left(E_{S^{\prime}}\right)} D \llbracket \mathrm{R}^{\prime} \rrbracket \stackrel{\text { op.x }}{\rightarrow} A_{1}^{\prime} \llbracket \mathrm{R}^{\prime} \rrbracket\right\|_{\mathrm{R}^{\prime}\left(E_{S^{\prime}}\right)} A_{2}^{\prime} \llbracket \mathrm{R}^{\prime} \rrbracket .
$$

This concludes the left-to-right implication, as

$$
\left(A^{\prime}, B^{\prime}\right)=\left(\left(A_{1}^{\prime} \|_{E_{S^{\prime}}} A_{2}^{\prime}\right) \llbracket \mathrm{R}^{\prime} \rrbracket, A_{1}^{\prime} \llbracket \mathrm{R}^{\prime} \rrbracket \|_{\mathrm{R}^{\prime}\left(E_{S^{\prime}}\right)} A_{2}^{\prime} \llbracket \mathrm{R}^{\prime} \rrbracket\right) \in \mathcal{R}
$$

holds.

\begin{tabular}{|ccccc||}
\hline \hline$A=$ & $\left(C \|_{E_{S^{\prime}}} D\right) \llbracket \mathrm{R}^{\prime} \rrbracket$ & $\stackrel{\text { op.x }}{\longrightarrow}$ & $\left(A_{1}^{\prime} \|_{E_{S^{\prime}}} A_{2}^{\prime}\right) \llbracket \mathrm{R}^{\prime} \rrbracket$ & $=A^{\prime}$ \\
$\mathcal{R}$ & & $\vdots$ & & \\
$\mid$ & & $\mathcal{R}$ & & \\
$B=$ & $C \llbracket \mathrm{R}^{\prime} \rrbracket \|_{\mathrm{R}^{\prime}\left(E_{S^{\prime}}\right)} D \llbracket \mathrm{R}^{\prime} \rrbracket$ & $\stackrel{o p \cdot x}{\longrightarrow}$ & $A_{1}^{\prime} \llbracket \mathrm{R}^{\prime} \rrbracket \|_{\mathrm{R}^{\prime}\left(E_{S^{\prime}}\right)} A_{2}^{\prime} \llbracket \mathrm{R}^{\prime} \rrbracket$ & $=B^{\prime}$ \\
\hline
\end{tabular}

The previous theorem showed that the renaming relation can be distributed over the parallel composition $\|_{E_{S}}$, allowing us to reason about $S_{i}$.main and $S_{i} . O Z$ and its parallel composition, without considering the renaming relation.

\subsection{CSP Laws for Parallel Composition}

The last step in the chain of proof steps is the easiest one: we need to show that within the parallel composition

$$
\left(S_{1} \text {.main } \|_{E_{\mathbf{C}}} S_{2} \text {.main }\right) \|_{E_{S^{\prime}}}\left(S_{1} . O Z \|_{E_{\mathbf{C}}} S_{2} . O Z\right),
$$

$S_{1} . \mathrm{OZ}$ and $S_{2}$.main can be redistributed, such that the resulting parallel composition constitutes the assembly of $S_{1}$ and $S_{2}$. In particular, the respective synchronisation alphabets need to be correctly adapted.

The following lemma shows a generalisation of this property for arbitrary processes with certain restrictions on their alphabets. Afterwards, we instantiate the lemma for our specific case:

Lemma 5.5.1. (Redistribution of CSP processes, alphabetised parallel) Let $P_{i}, Q_{i}$ be CSP processes and $A_{i}, B_{i}$ alphabets. Then,

$$
\left(P_{1 A_{1}} \|_{A_{2}} P_{2}\right)_{A_{1} \cup A_{2}}\left\|_{B_{1} \cup B_{2}}\left(Q_{1 B_{1}} \|_{B_{2}} Q_{2}\right)=\left(P_{1 A_{1}} \|_{B_{1}} Q_{1}\right)_{A_{1} \cup B_{1}}\right\|_{A_{2} \cup B_{2}}\left(P_{2 A_{2}} \|_{B_{2}} Q_{2}\right) .
$$


Proof. We use rules (2.4) $X \|_{Y}-$ sym

$$
P_{X}\left\|_{Y} Q=Q_{Y}\right\|_{X} P
$$

and (2.5) ${ }_{X} \|_{Y}-$ assoc

$$
\left(P_{X} \|_{Y} Q\right)_{X \cup Y}\left\|_{Z} R=P_{X}\right\|_{Y \cup Z}\left(Q_{Y} \|_{Z} R\right)
$$

from [Ros98] and incrementally deduce the equation:

$$
\begin{aligned}
& \left(P_{1 A_{1}} \|_{A_{2}} P_{2}\right)_{A_{1} \cup A_{2}} \|_{B_{1} \cup B_{2}}\left(Q_{1 B_{1}} \|_{B_{2}} Q_{2}\right) \\
& =P_{1 A_{1}} \|_{A_{2} \cup B_{1} \cup B_{2}}\left(P_{2 A_{2}} \|_{B_{1} \cup B_{2}}\left(Q_{1 B_{1}} \|_{B_{2}} Q_{2}\right)\right) \quad\left(X \|_{Y}-\text { assoc }\right) \\
& =P_{1 A_{1}} \|_{B_{1} \cup B_{2} \cup A_{2}}\left(\left(Q_{1} B_{1} \|_{B_{2}} Q_{2}\right)_{B_{1} \cup B_{2}} \|_{A_{2}} P_{2}\right) \quad\left(x \|_{Y}-\text { sym }\right) \\
& =P_{1 A_{1}} \|_{B_{1} \cup A_{2} \cup B_{2}}\left(Q_{1 B_{1}} \|_{B_{2} \cup A_{2}}\left(Q_{2 B_{2}} \|_{A_{2}} P_{2}\right)\right) \quad\left(X \|_{Y} \text { - assoc }\right) \\
& =P_{1 A_{1}} \|_{B_{1} \cup A_{2} \cup B_{2}}\left(Q_{1 B_{1}} \|_{A_{2} \cup B_{2}}\left(P_{2} A_{2} \|_{B_{2}} Q_{2}\right)\right) \quad\left(X \|_{Y}-\text { sym }\right) \\
& =\left(P_{1 A_{1}} \|_{B_{1}} Q_{1}\right)_{A_{1} \cup B_{1}} \|_{A_{2} \cup B_{2}}\left(P_{2 A_{2}} \|_{B_{2}} Q_{2}\right) \quad\left(x \|_{Y}-\text { assoc }\right)
\end{aligned}
$$

In order to use the previous lemma in our context, we have to apply it with respect to interface parallel. This can only be achieved, if all participating processes never communicate outside their respective synchronisation alphabets:

Corollary 5.5.2. (Redistribution of CSP processes, interface parallel)

Let $P_{i}, Q_{i}$ be CSP processes and $A_{i}, B_{i}$ their respective alphabets, that is, $P_{i}$ never communicates outside of $A_{i}$, and $Q_{i}$ never communicates outside of $B_{i}$, respectively. Then:

$$
\begin{aligned}
& \left(P_{1} \|_{A_{1} \cap A_{2}} P_{2}\right) \|_{\left(A_{1} \cup A_{2}\right) \cap\left(B_{1} \cup B_{2}\right)}\left(Q_{1} \|_{B_{1} \cap B_{2}} Q_{2}\right)= \\
& \left(P_{1} \|_{A_{1} \cap B_{1}} Q_{1}\right) \|_{\left(A_{1} \cup B_{1}\right) \cap\left(A_{2} \cup B_{2}\right)}\left(P_{2} \|_{A_{2} \cap B_{2}} Q_{2}\right) .
\end{aligned}
$$

Proof. Follows immediately from Lemma 5.5.1 and the fact that $P\|\|_{X \cap Y} Q=P_{X} \|_{Y} Q$ holds, if $P, Q$ never communicate outside $X$ and $Y$ ([Ros98]).

In the following section, the corollary will be instantiated by setting

- $P_{i}:=S_{i} \cdot \operatorname{main}$,

- $Q_{i}:=S_{i} \cdot O Z$,

- $A_{i}:=E_{S_{i}}$ and

- $B_{i}:=E_{S_{i}}$. 


\subsection{Proof of the Main Theorem}

Finally, we show Theorem 4.3.25 by subsuming the results of the previous sections:

Theorem 5.6.1. (Correctness of the decomposition)

Let $S$ be a specification, and let $\mathbf{C}=\left(\mathbf{C}_{1}, \mathbf{C}_{2}\right)$ be a cut, yielding a decomposition into $S_{1}$ and $S_{2}$, according to Definition 4.3.24. Then, the following holds:

$$
S={ }_{T}\left(S_{1} \|_{E_{\mathrm{C}}} S_{2}\right) \llbracket \mathrm{R}^{\prime} \rrbracket,
$$

where $\mathrm{R}^{\prime}: E_{S^{\prime}} \rightarrow E_{S}$ is defined as

$$
\mathrm{R}^{\prime}(\text { op.x.t.a) :=op.x, }
$$

with $x$ depicting the original parameter values, $t$ denoting the valuation for the possible transmission parameters and a the valuation for the possible address parameters.

\section{Proof.}

$S$

$={ }_{T} \quad$ S.main $\|_{E_{S}} S . O Z$

(Def. of $S$ )

$==_{T} \quad\left(S_{1} \cdot\right.$ main $\|_{E_{\mathrm{C}}} S_{2} \cdot$ main $) \llbracket \mathrm{R}^{\prime} \rrbracket \|_{E_{S}}\left(S_{1} . O Z \|_{E_{\mathrm{C}}} S_{2} . O Z\right) \llbracket \mathrm{R}^{\prime} \rrbracket$

(Theorem 5.2.4, Theorems 5.3.14 and 5.3.17)

$=_{T} \quad\left[\left(S_{1} \cdot\right.\right.$ main $\|_{E_{\mathbf{C}}} S_{2} \cdot$ main $\left.) \|_{E_{S^{\prime}}}\left(S_{1} \cdot O Z \|_{E_{\mathbf{C}}} S_{2} \cdot O Z\right)\right] \llbracket \mathbb{R}^{\prime} \rrbracket$

$={ }_{T} \quad\left[\left(S_{1} \cdot\right.\right.$ main $\|_{E_{S_{1}} \cap E_{S_{2}}} S_{2} \cdot$ main $\left.) \|_{E_{S^{\prime}}}\left(S_{1} \cdot O Z \|_{E_{S_{1}} \cap E_{S_{2}}} S_{2} \cdot O Z\right)\right] \llbracket \mathrm{R}^{\prime} \rrbracket$

$=_{T} \quad\left[\left(S_{1}\right.\right.$.main $\left.\|_{E_{S_{1}}} S_{1} . O Z\right) \|_{E_{S_{1}} \cap E_{S_{2}}}\left(S_{2}\right.$.main $\left.\left.\|_{E_{S_{2}}} S_{2} . O Z\right)\right] \llbracket \mathrm{R}^{\prime} \rrbracket$

$=_{T} \quad\left[\left(S_{1}\right.\right.$.main $\left.\|_{E_{S_{1}}} S_{1} . O Z\right) \|_{E_{\mathrm{C}}}\left(S_{2}\right.$.main $\left.\left.\|_{E_{S_{2}}} S_{2} . O Z\right)\right] \llbracket \mathrm{R}^{\prime} \rrbracket$

$={ }_{T} \quad\left(S_{1} \|_{E_{\mathrm{C}}} S_{2}\right) \llbracket \mathrm{R}^{\prime} \rrbracket$

(Def. of $S_{1}$ and $S_{2}$ )

Note that an application of Lemma 5.5.2 is indeed possible, as $S_{i}$.main and $S_{i}$.OZ never communicate outside of $E_{S_{i}}$.

This completes the proof of the main result of this thesis, Theorem 4.3.25. The theorem allows us to apply the assume-guarantee-based proof rules from Chapter 3: as $S$ and $S_{1} \|_{E_{\mathrm{C}}} S_{2}$ are trace equivalent modulo renaming, we can safely replace $S_{1} \| S_{2}$ by $S$ in an application of (B-AGR) and (P-AGR).

After showing correctness of our decomposition approach, the next chapter will deal with the question on how to identify reasonable decompositions, that is, correct decompositions, which most likely result in efficient compositional verification. 


\title{
Finding Reasonable Decompositions
}

\author{
Contents \\ 6.1 Decomposition Heuristics . . . . . . . . . . . . . . . . . . . 168 \\ 6.1 .1 First Heuristic: Cut Size . . . . . . . . . . . . . . . . . . . . . 169 \\ 6.1.2 Second Heuristic: Even Distribution _ . . . . . . . . . 170 \\ 6.1.3 Third Heuristic: Few Transmission ． . . . . . . . . . 170 \\ 6.1.4 Fourth Heuristic: Few Addressing . . . . . . . . . . . . . . . 172 \\ 6.2 Evaluation of Decomposition Heuristics . . . . . . . . . . 172 \\ 6.3 Candy Machine Revisited: Evaluation of Cuts . . . . . . . . . 174 \\ 6.4 Case Study: Two Phase Commit Protocol . . . . . . . . . . . . . 175

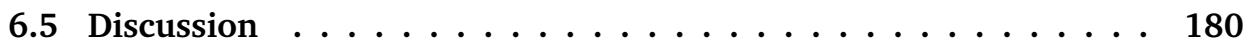 \\ 6.6 Related Work . . . . . . . . . . . . . . . . . 181
}

The overall goal of our decomposition technique is an application within the compositional verification framework, as introduced in Chapter 3. So far, we showed the correctness of our approach: the decomposition does not change the behaviour of the specification in terms of our semantic domain. This allows us to apply assume-guaranteebased proof rules with respect to the decomposed system and to infer a global result for the original system.

However, for compositional verification to have a practical impact, the technique needs to provide an advantage over monolithic, that is, non-compositional verification. Therefore, it is essential to evaluate the effectiveness of the decomposition as, in general, compositional verification does not automatically result in comparatively small time and memory consumption during model checking.

Example 6.0.2. Recall the specification of a candy machine from Figure 2.3. The set $\mathbf{C}=\{$ term $\}$ defines a valid (single) cut of the specification. A decomposition with respect to $\mathrm{C}$ is impractical, as it results in $S_{1}=_{T} S$. Yet, even though we consider compositional verification, the need to deal with the full state space of $S$ remains. ${ }^{1}$

The question is how to describe and detect reasonable decompositions. In [CAC06], the authors investigate the usefulness of assume-guarantee reasoning by evaluating all possible decompositions on five different case studies. The overall results are not very encouraging as, in terms of the size of the explored state spaces, monolithic verification often succeeds over compositional verification. This leads the authors to the following statements:

\footnotetext{
${ }^{1}$ From now on, as a valid cut uniquely defines a decomposition, we will synonymously use both terms.
} 
Deciding how to partition the subsystems into $S_{1}$ and $S_{2}$ is not easy and can have a significant impact on the time and memory needed for verification. [...] Thus, randomly selecting decompositions would likely not yield a decomposition better than monolithic verification.

[CACO6]

As a possible solution to this problem, the authors recommend to investigate heuristics to guide the software engineer towards the best possible decompositions: in this case, assume-guarantee-based verification most often outperforms non-compositional verification.

These considerations motivate the following strategy: in order to evaluate the valid decompositions which our technique generates, we define several context-specific heuristics, focusing on the underlying verification framework and the definition of the decomposition itself. These heuristics serve as the basis for a classification of all correct decompositions: those, which are unreasonable or dominated by other ones (see Section 6.2), are no longer considered - the remaining decompositions can be further compared by prioritising specific heuristics.

This chapter is organised as follows: in Section 6.1, we motivate and define our contextspecific heuristics. The following section discusses an evaluation of the results by giving a very brief introduction into the topic of multi-valued optimisation [Ehr00]. In Sections 6.3 and 6.4, respectively, we illustrate and apply the heuristics and evaluate them for the candy machine specification and a second, slightly bigger, case study. The final sections discuss the approach and related work.

\subsection{Decomposition Heuristics}

Several factors influence the effectiveness of compositional reasoning in general. In $\left[\mathrm{dRHH}^{+} 01\right]$, the authors elaborate on the question of when to use a compositional style of proof and when to use a non-compositional one. For instance, they argue that compositional verification becomes infeasible, if a system is tightly-coupled, that is, any decomposition will result in a lot of common elements and shared behaviour, or if the system comprises global invariants, which cannot be split up.

Choosing the most effective decompositions cannot be established in an automatic manner. Due to the context-specific verification frameworks, the usage of different model checkers or the structure of the specifications and verification properties, there is no universally optimal decomposition. However, one particular issue exerts the dominating influence on the efficiency of compositional verification and model checking in general: the size of the state space, which needs to be explored. Thus, according to [CAC06], we state that one decomposition is better than another one, if the number of states explored during model checking is comparatively smaller. We need to define heuristics, favouring decompositions with a relatively small state space.

The evaluation of our approach will use an implementation of the learning-based framework from Section 3.2.3 and compare it to direct model checking of the original system. We derive our heuristics from the following two requirements:

Small Interface: The size of $E_{\mathbf{C}}$ within the system $S_{1} \|_{E_{\mathrm{C}}} S_{2}$ should be small. In general, 
the smaller the interface between both components, the less shared behaviour and fewer communication between them, and the looser the components are coupled. This results in a smaller number of states, which have to be explored during model checking. In the context of AGR and according to [CS07], the smaller the assumption alphabet, the more efficient the $\mathrm{L}^{*}$ algorithm in the learning-based framework from Section 3.2.3. More precisely, the number of $\mathrm{L}^{*}$ membership queries directly depends on the assumption alphabet, which itself closely depends on the size of $E_{\mathrm{C}}$.

Equal Size of Components: The size of the components $S_{1}$ and $S_{2}$ within $S_{1} \|_{E_{\mathrm{C}}} S_{2}$ should approximately be the same. The question of how to find a good partitioning of a system is discussed in [Nam07]. The author argues that an even distribution of the number of system variables over the components leads to a more effective compositional verification. Moreover, in [GMF07], the authors state that the execution time of the $\mathrm{L}^{*}$ algorithm is exponential in the size of $S_{1}$ and $S_{2}$. If we assume $s_{1}$ to denote the size of $S_{1}$ and $s_{2}$ the size of $S_{2}, 2^{s_{1}}+2^{s_{2}}$ is minimal for $s_{1}=s_{2}$, in case that $s=s_{1}+s_{2}$ is fixed. This justifies the requirement that both components should have about the same size.

Based on these two requirements, we derive four different heuristics, with one of them related to Equal Size of Components and the remaining three based on Small Interface. These heuristics will be given as functions, mapping a specific decomposition on a certain value within the natural numbers. A comparatively better decomposition has a lower value, that is, we aim at a minimisation of the function values. For each heuristic, we start by stating the principal characteristic and give an intuitive description. Subsequently, we introduce the mathematical definition.

\subsubsection{First Heuristic: Cut Size}

The first heuristic, which we call cut size, is related to the requirement that the interface between both components should be relatively small. A small number of nodes within the cut is preferable, as the size of $E_{\mathrm{C}}$ depends on the number of corresponding operation nodes, that is,

$$
E_{\mathbf{C}}=\left\{\left|O p_{\mathbf{C}}\right|\right\}=\left\{\left|l\left[\mathbf{C}_{1}\right] \cup l\left[\mathbf{C}_{2}\right]\right|\right\}
$$

holds. This leads to the following objective for the first heuristic:

$h_{\mathrm{cs}}$ : Minimise the number of cut nodes.

The fewer nodes contained in the cut, the smaller the common elements to both specification parts and thus, the smaller the shared behaviour and the assumption alphabet. A mathematical definition for this heuristic obviously maps a cut on its number of elements. We summarise the first heuristic in Table 6.1. 


\begin{tabular}{|l|l|l|l|}
\hline Notation & Name of Heuristic & Description & Motivation \\
\hline$h_{\mathrm{CS}}$ & cut size & $\begin{array}{l}\text { Minimise number of cut } \\
\text { nodes. }\end{array}$ & Small Interface. \\
\hline \hline \multicolumn{3}{|c|}{ Mathematical Definition } \\
\hline \multicolumn{3}{|c|}{$h_{\mathrm{CS}}(\mathbf{C}):=\# \mathbf{C}$} \\
\hline
\end{tabular}

Table 6.1: Heuristic $h_{\text {cs }}$ : cut size

\subsubsection{Second Heuristic: Even Distribution}

The second heuristic, even distribution, targets the Equal Size of Components. In order to measure the size of a component, we count the number of operation nodes corresponding to $S_{1}$ and $S_{2}$, leading to the following objective:

$h_{\mathrm{ED}}$ : Minimise the difference between the number of operation nodes corresponding to $S_{1}$ and $S_{2}$.

Based on Definition 4.3.1, we get

- $\mathbf{P h}_{1} \cup \mathbf{P h}_{3} \cup \mathbf{C}_{1} \cup \mathbf{C}_{2}$ for the set of nodes according to $S_{1}$ and

- $\mathbf{P h}_{2} \cup \mathbf{C}_{1} \cup \mathbf{C}_{2}$ for the set of nodes according to $S_{2}$.

The mathematical definition for the second heuristic from Table 6.2 computes the absolute value of the difference between these sets. As the set of cut nodes is contained in both of them, it can be neglected.

\begin{tabular}{|l|l|l|l|}
\hline Notation & Name of Heuristic & Description & Motivation \\
\hline$h_{\mathrm{ED}}$ & even distribution & $\begin{array}{l}\text { Minimise size difference be- } \\
\text { tween both components. }\end{array}$ & $\begin{array}{l}\text { Equal Size of Com- } \\
\text { ponents. }\end{array}$ \\
\hline \hline \multicolumn{3}{|c|}{ Mathematical Definition } \\
\hline \multicolumn{3}{|c|}{$h_{\mathrm{ED}}(\mathbf{C}):=\left|\#\left(\mathbf{P h}_{1} \cup \mathbf{P h}_{3}\right)-\# \mathbf{P h}_{2}\right|$} \\
\hline
\end{tabular}

Table 6.2: Heuristic $h_{\mathrm{ED}}$ : even distribution

\subsubsection{Third Heuristic: Few Transmission}

The final two heuristics are again related to the requirement Small Interface. In Section 4.3.2, we introduced the concept of transmission parameters to ensure a preservation of a specification's data flow within the decomposition. These parameters are required to 
ensure the correctness of the technique. Unfortunately, they increase the set of cut events, that is, the set $E_{\mathbf{C}}$.

In order to measure the additional amount of cut events, we need to refer to the types of these parameters: simply counting the number of parameters would be too coarse. For instance, one additional parameter of type $\{1, \ldots, 10\}$ causes the size of $E_{\mathrm{C}}$ to be increased by the factor of 10 , whereas two parameters of type $\{1,2\}$ only increase it by the factor of 4 . In order to define the third heuristic $h_{\mathrm{FT}}$, we proceed as follows:

- The number of elements of $\{|o p|\}$ (see Definition 2.2.5) increases by the amount of possible parameter extensions with respect to all transmission parameters. Thus, for any cut operation op, we compute the product over the cardinality of each transmission parameter type.

- An operation can have multiple occurrences within the cut. Even though this is not reflected in the size of $E_{\mathbf{C}}$, we still need to deal with it by multiplying the previous result with the number of cut-occurrences of the operation.

- Finally, we compute the sum over the results for all cut operations.

Henceforth, $t r_{-} i$ denote transmission parameters, and $t y_{p}^{o p}$ depicts the type of the parameter $p$ of the operation op. We illustrate the weight computation for the third heuristic with an example:

Example 6.1.1. Let $\mathbf{C}=\left\{o p_{1}, o p_{2}\right\}$ be a valid cut for some specification $S$, such that $o p_{1}$ occurs once, and $\mathrm{op}_{2}$ occurs twice within S.main. Let op ${ }_{1}$ comprise two transmission parameters of types $\mathbb{B}$ and $\{1,2,3\}$. Furthermore, let op $p_{2}$ comprise one transmission parameter of type $\mathbb{P}(\mathbb{B})$. For the first operation, we get \#ty tr $\_1_{1}^{o p_{1}} * \# t_{\text {tr } \_2}^{o p_{1}}=2 * 3=6$. Moreover, $\# t_{t r-1}^{\text {op }_{2}}=2^{2}=4$. As op p $_{2}$ occurs twice within S.main, we multiply the second value by 2 , which results in the overall weight of $h_{\mathbf{F T}}(\mathbf{C})=6+8=14$ for the third heuristic.

Another question is how to deal with infinite data types. One solution could be the definition $h_{\mathrm{FT}}(\mathbf{C}):=\infty$ in case that there exists at least one operation within $\mathbf{C}$ with one transmission parameter of infinite type. However, in this case, any number of transmission parameters of infinite types would result in the same value for the given heuristic. During model checking, infinite data types need to be abstracted to some finite subset - either by the model checker or the user itself. Therefore, we follow a different approach: in our cardinal arithmetic, we assume that $\infty$ can be mapped to some bound MaxInf. Based on the actual cardinality of $\infty$ for the model checker, MaxInf can appropriately be instantiated.

Subsuming, we require:

$h_{\mathrm{FT}}$ : Minimise the amount and the type cardinality of the transmission parameters.

The third heuristic is summarised in Table 6.3. According to the previous considerations, $\# t y_{p}^{o p}=$ MaxInf is possible. 


\begin{tabular}{|l|l|l|l|}
\hline Notation & Name of Heuristic & Description & Motivation \\
\hline$h_{\mathrm{FT}}$ & few transmission & $\begin{array}{l}\text { Minimise amount and type car- } \\
\text { dinality of transmission pa- } \\
\text { rameters. }\end{array}$ & Small Interface. \\
\hline \hline \multicolumn{3}{|c|}{ Mathematical Definition } \\
\hline \multicolumn{2}{|c|}{$h_{\mathbf{F T}}(\mathbf{C}):=$ let $T_{o p}:=\left(\# l^{-1}(o p) * \# t t_{t r_{1} 1}^{o p} * \cdots * t y_{t r \_n}^{o p}\right)$ in $\sum_{o p \in l[\mathbf{C}]} T_{o p}$} \\
\hline
\end{tabular}

Table 6.3: Heuristic $h_{\mathrm{FT}}$ : few transmission

\subsubsection{Fourth Heuristic: Few Addressing}

In correspondence to transmission parameters, Section 4.3.3 introduced the concept of address parameters to preserve the control flow within the decomposition. Again, these parameters increase the size of $E_{\mathbf{C}}$.

Contrary to transmission parameters, address parameters never have an infinite type. Thus, we can precisely determine the weight for these parameters, motivating separate measurements for both parameter types. We introduce a new heuristic, which mainly corresponds to the previous one, and we set the following objective:

$h_{\mathrm{FA}}$ : Minimise the amount and the type cardinality of the address parameters.

\begin{tabular}{|l|l|l|l|}
\hline Notation & Name of Heuristic & Description & Motivation \\
\hline$h_{\mathrm{FA}}$ & few addressing & $\begin{array}{l}\text { Minimise amount and type car- } \\
\text { dinality of address parame- } \\
\text { ters. }\end{array}$ & Small Interface. \\
\hline \hline \multicolumn{3}{|c|}{ Mathematical Definition } \\
\hline \multicolumn{2}{|c|}{$h_{\mathbf{F A}}(\mathbf{C}):=$ let $A_{o p}:=\left(\# l^{-1}(o p) * \# t y_{a d \_1}^{o p} * \cdots * \# t y_{a d \_n}^{o p}\right)$ in $\sum_{o p \in l[\mathbf{C}]} A_{o p}$} \\
\hline
\end{tabular}

Table 6.4: Heuristic $h_{\mathrm{FA}}$ : few addressing

The mathematical definition for this heuristic corresponds to the one for few transmission, except that now $\# t y_{p}^{o p}=$ MaxInf is impossible. In the definition, ad_i denote address parameters.

\subsection{Evaluation of Decomposition Heuristics}

The previous section introduced several individually defined heuristics, which are possibly conflicting with each other. In order to evaluate the set of valid decompositions (or 
solutions, as we will call them in the context of this chapter), the joint application of all heuristics is required. This obviously results in a trade-off between the specific requirements for good decompositions: for instance, assigning a high priority to heuristic $h_{\text {ED }}$ will result in a set of cuts with potentially high value for heuristic $h_{\mathrm{Cs}}$. The general problem is well known as the task of multi-objective optimisation [Ehr00, Zel74].

Besides this trade-off and despite allowing the specific heuristics to be scaled and thus prioritised, some decompositions or solutions can be neglected entirely. These are the ones for which one resulting component is on the scale of the original specification: here, compositional verification needs to deal with at least the same state space as non-compositional one.

Definition 6.2.1. (Unreasonable decomposition)

Let $S$ be a CSP-OZ class specification, and let $\mathcal{C}$ denote the set of all valid cuts of $S$. We say that $\mathbf{C} \in \mathcal{C}$ is unreasonable, if, and only if,

$$
\mathbf{P h}_{1} \cup \mathbf{P h}_{3} \cup \mathbf{C}=\text { op }(N) \text { or } \mathbf{P h}_{2} \cup \mathbf{C}=\text { op }(N) .
$$

Regarding our heuristics, we immediately deduce that a decomposition is unreasonable, if, and only if, the sum over the values for the heuristics $h_{\mathrm{CS}}$ and $h_{\mathrm{ED}}$ is equal to the size of all operation nodes of the DG:

Lemma 6.2.2. (Connection between unreasonable decompositions and heuristics)

Let $S$ be a CSP-OZ class specification, and let $\mathcal{C}$ denote the set of all valid cuts of $S . \mathbf{C} \in \mathcal{C}$ is unreasonable, if, and only if,

$$
h_{\mathrm{CS}}(\mathbf{C})+h_{\mathrm{ED}}(\mathbf{C})=\# \mathrm{op}(N) .
$$

Proof. Immediate: any operation node is uniquely assigned to one of the sets $\mathbf{P h} \mathbf{h}_{1}, \mathbf{P h}_{2}$, $\mathbf{P h}_{3}$ and $\mathbf{C}$. Furthermore, $h_{\mathbf{C S}}(\mathbf{C})=\# \mathbf{C}$ and $h_{\mathbf{E D}}(\mathbf{C})=\left|\#\left(\mathbf{P h}_{1} \cup \mathbf{P h}_{3}\right)-\# \mathbf{P h}_{2}\right|$ holds.

Unreasonable decompositions will generally not be considered within our evaluation. For the further restriction of the set of valid cuts, we reason about dominated decompositions. Intuitively, they are outmatched by some other decomposition in any heuristic. In the context of multi-objective optimisation, the remaining solutions are called Pareto-optimal [Par71]. We will introduce the definition for our context, where we refer to the one from [DW04]. Let $h_{1}, \ldots, h_{4}$ denote the heuristics, as introduced in Section 6.1:

Definition 6.2.3. (Weakly dominated decomposition [DW04])

Let $S$ be a CSP-OZ class specification, and let $\mathcal{C}$ denote the set of all valid cuts of $S$. We say that $\mathbf{C} \in \mathcal{C}$ is (weakly) dominated by $\mathbf{C}^{\prime} \in \mathcal{C}$ (with respect to $\left\{h_{1}, h_{2}, h_{3}, h_{4}\right\}$ ), if, and only if,

$$
\left(\forall i \in\{1,2,3,4\} \bullet h_{i}\left(\mathbf{C}^{\prime}\right) \leq h_{i}(\mathbf{C})\right) \wedge\left(\exists i \in\{1,2,3,4\} \bullet h_{i}\left(\mathbf{C}^{\prime}\right)<h_{i}(\mathbf{C})\right) .
$$

We illustrate the definition by an example.

Example 6.2.4. Recall the candy machine specification from Section 2.2.1. Both, $\mathbf{C}=$ $\{$ switch, abort $\}$ and $\mathbf{C}^{\prime}=\{$ switch $\}$ denote valid cuts. For the evaluation of the different heuristics, we get: 


$$
\begin{array}{lll}
h_{\mathbf{C S}}(\mathbf{C})=2 & h_{\mathbf{C S}}\left(\mathbf{C}^{\prime}\right)=1 \\
h_{\mathrm{ED}}(\mathbf{C})=2 & h_{\mathbf{E D}}\left(\mathbf{C}^{\prime}\right)=1 \\
h_{\mathbf{F T}}(\mathbf{C})=\text { MaxInf } & h_{\mathbf{F T}}\left(\mathbf{C}^{\prime}\right)=\mathbf{M a x I n f} \\
h_{\mathbf{F A}}(\mathbf{C})=0 & h_{\mathbf{F A}}\left(\mathbf{C}^{\prime}\right)=0
\end{array}
$$

Here, $h_{\mathrm{FT}}(\mathbf{C})=h_{\mathrm{FT}}\left(\mathbf{C}^{\prime}\right)=$ MaxInf, due to one transmission parameter of type $\mathbb{N}$. As $h_{i}\left(\mathbf{C}^{\prime}\right) \leq h_{i}(\mathbf{C})$ for any of the four heuristics and as strictly smaller holds for the first two, \{switch, abort $\}$ is weakly dominated by $\{$ switch\}.

Independent of the scaling, a weakly dominated cut never achieves the relatively best results. In the implementation of our decomposition approach, solutions dominated by other ones will thus accordingly be marked and can be suppressed. Note that even if highly unlikely, a dominated solution might still be the most efficient one. This is due to the respective property under interest, the specific characteristics of the model checker and the general nature of a heuristic approach, which is experience-based and only points the direction.

For the remaining near-optimal solutions, no general classification is possible. Yet, an elimination of all dominated cuts results in a smaller set of possible decompositions, which can then be further interpreted, according to the priority for each heuristic.

\subsection{Candy Machine Revisited: Evaluation of Cuts}

Next, we are interested in the evaluation of the set of all valid cuts of a specification based on our heuristics. We recall the case study of a candy machine from Chapter 2 . Here, we restrict ourselves to the special case of a single cut from Definition 4.2.10 due to two reasons:

- The set of all possible general cuts is too large for an effective comparison.

- Defining two different cut sets is impractical, as the specification does not comprise any outer recursion.

Subsuming, there are 26 valid (single) cuts, which are depicted in Table 6.5. We additionally denote if the respective cut is, according to Definitions 6.2.1 and 6.2.3, non-reasonable or weakly dominated by another one.

\begin{tabular}{|l|l|l|l|}
\hline No. & Cut & Reasonable? & Non-Dominated? \\
\hline 1 & $\{$ abort, deliver, order, pay, payout, select, switch $\}$ & No & No \\
\hline 2 & $\{$ abort, deliver, order, payout, select, switch, term $\}$ & No & Yes \\
\hline 3 & $\{$ abort, deliver, order, payout, select, switch $\}$ & Yes & No \\
\hline 4 & $\{$ abort, deliver, order, payout, select, term $\}$ & No & Yes \\
\hline 5 & $\{$ abort, deliver, order, select, switch, term $\}$ & No & Yes \\
\hline 6 & $\{$ abort, deliver, order, select, switch $\}$ & Yes & No \\
\hline 7 & $\{$ abort, deliver, order, select, term $\}$ & No & Yes \\
\hline 8 & $\{$ abort, deliver, payout, term $\}$ & No & Yes \\
\hline 9 & $\{$ abort, deliver, term & No & Yes \\
\hline
\end{tabular}




\begin{tabular}{|c|c|c|c|}
\hline 10 & $\{$ abort, order, pay, payout, select, switch $\}$ & No & No \\
\hline 11 & $\{$ abort, order, payout, select, switch $\}$ & Yes & No \\
\hline 12 & $\{$ abort, order, select, switch $\}$ & Yes & Yes \\
\hline 13 & $\{$ abort, pay, payout, switch $\}$ & No & No \\
\hline 14 & $\{$ abort, payout, switch $\}$ & Yes & No \\
\hline 15 & $\{$ abort, payout, term $\}$ & No & Yes \\
\hline 16 & $\{$ abort, payout $\}$ & No & Yes \\
\hline 17 & $\{$ abort, switch $\}$ & Yes & No \\
\hline 18 & $\{$ abort, term\} & No & Yes \\
\hline 19 & $\{$ abort $\}$ & No & Yes \\
\hline 20 & $\{$ deliver, order, select, switch, term $\}$ & No & Yes \\
\hline 21 & $\{$ deliver, order, select, switch $\}$ & Yes & No \\
\hline 22 & $\{$ deliver, order, select, term $\}$ & No & Yes \\
\hline 23 & $\{$ deliver, term $\}$ & No & Yes \\
\hline 24 & $\{$ order, select, switch $\}$ & Yes & No \\
\hline 25 & $\{$ switch $\}$ & Yes & Yes \\
\hline 26 & $\{$ term $\}$ & No & Yes \\
\hline
\end{tabular}

Table 6.5: Set of valid cuts for the candy machine

Even though the set of valid cuts is rather large, only two solutions are reasonable and non-dominated. These are $\{$ abort, order, select, switch $\}$ and $\{$ switch $\}$. In Chapter 7 , we will compare both cuts.

\subsection{Case Study: Two Phase Commit Protocol}

In order to further illustrate and exemplify our decomposition technique, we introduce a second case study: a specification of the Two-Phase-Commit Protocol (TPCP) [BHG87, $\left.\mathrm{dRHH}^{+} 01\right]$. The purpose of the protocol is to guarantee consistency of $N$ local sites (or pages) of a distributed database. Instructed by a coordinator process, the protocol results in either all pages committing their transaction or all pages aborting it. The basic system structure and communication is illustrated in Figure 6.1. As the name says, the protocol works in two phases:

Phase 1 - Commit-Request: The protocol starts with the coordinator process informing all participating pages about a request to commit the current transaction. Next, all pages execute the transaction and send a vote to the coordinator, dependent on whether the local transaction succeeded (YES) or failed (NO). The coordinator collects the votes and decides to either COMMIT, in the case that all votes agree on $Y E S$, or to $A B O R T$ the transaction. Figure 6.2 illustrates the workflow of phase one for the coordinator and, for simplification, for one instance of Page.

Phase 2 - Commit: The coordinator informs all pages about the decision. All participating sites behave accordingly: an abort leads to an undo of the transaction, while a commit leads to completion. In any case, the sites output the result and send an acknowledgement to the coordinator. An illustration is given in Figure 6.3. 


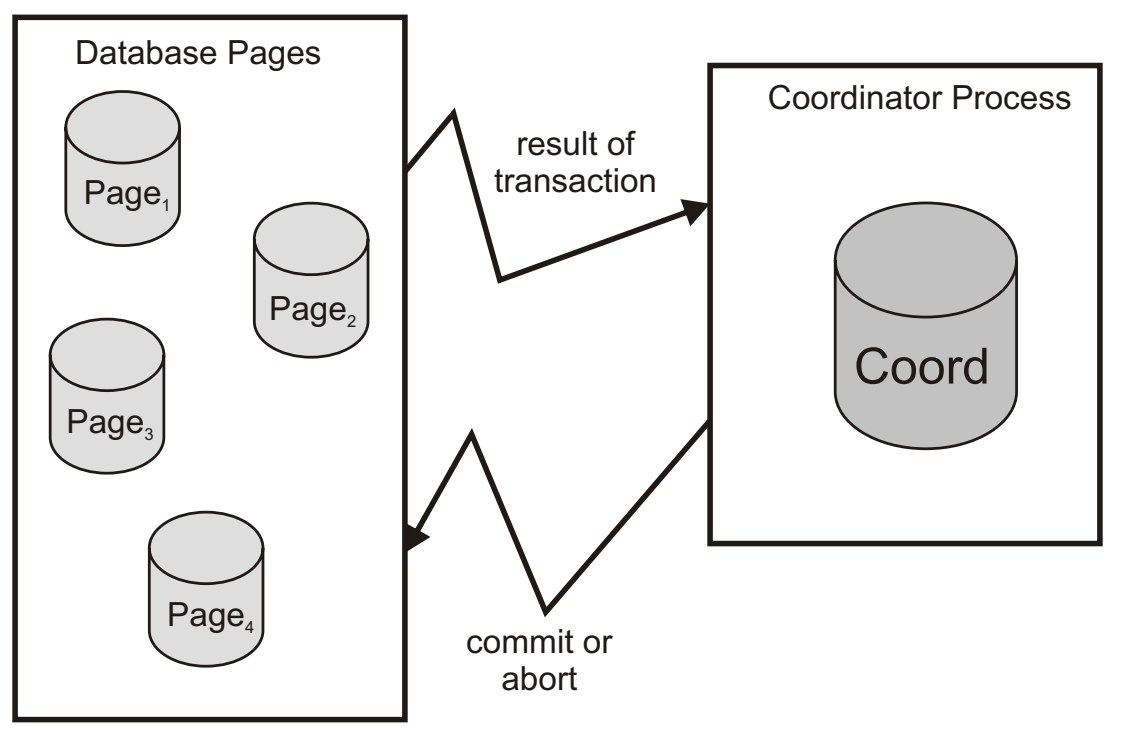

Figure 6.1: Illustration of the Two Phase Commit Protocol

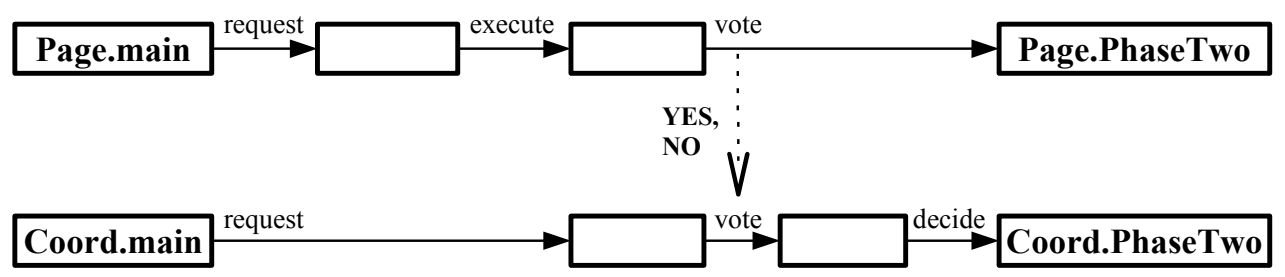

Figure 6.2: Phase one of the Two Phase Commit Protocol

Let $N$ be the number of pages participating in the protocol, and let Votes and Trans be the following two base types:

$$
\begin{aligned}
& \text { Votes }==\{Y E S, N O\} \\
& \text { Trans }==\{\text { COMMIT, ABORT }\}
\end{aligned}
$$

Here, Votes represents the possible votes of the pages, dependent on whether the transaction succeeded or not, whereas Trans describes the actual decision to either commit or abort the transaction.

The specification, as given in Figure 6.4, is the CSP-OZ class for the central coordinator. The ordering of events within the CSP part corresponds to Figures 6.2 and 6.3.

For the Object-Z part, the class' state space comprises two variables: decC of type Trans, for holding the final decision and votes of type $\mathbb{P}$ Votes, for storing the votes of the different pages. The operation vote comprises an input parameter of type Votes, and its value is 


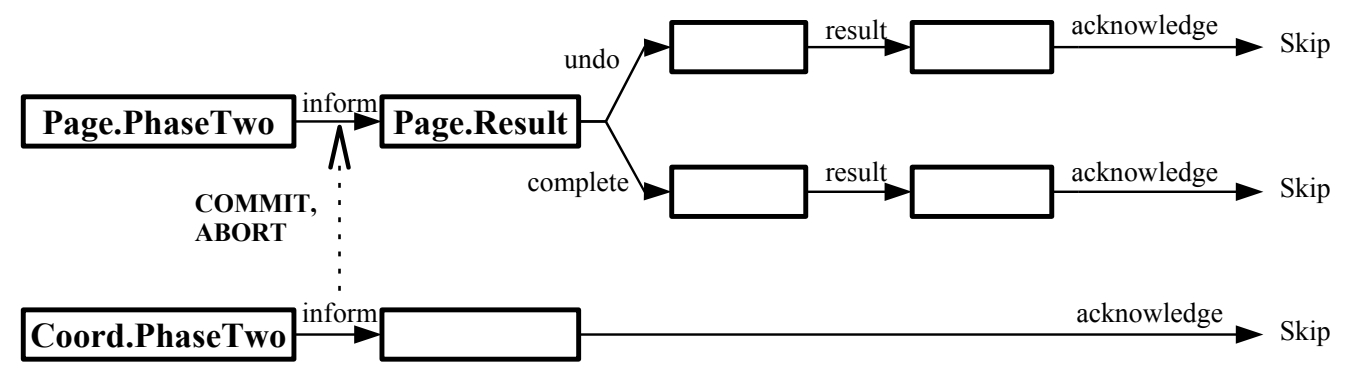

Figure 6.3: Phase two of the Two Phase Commit Protocol

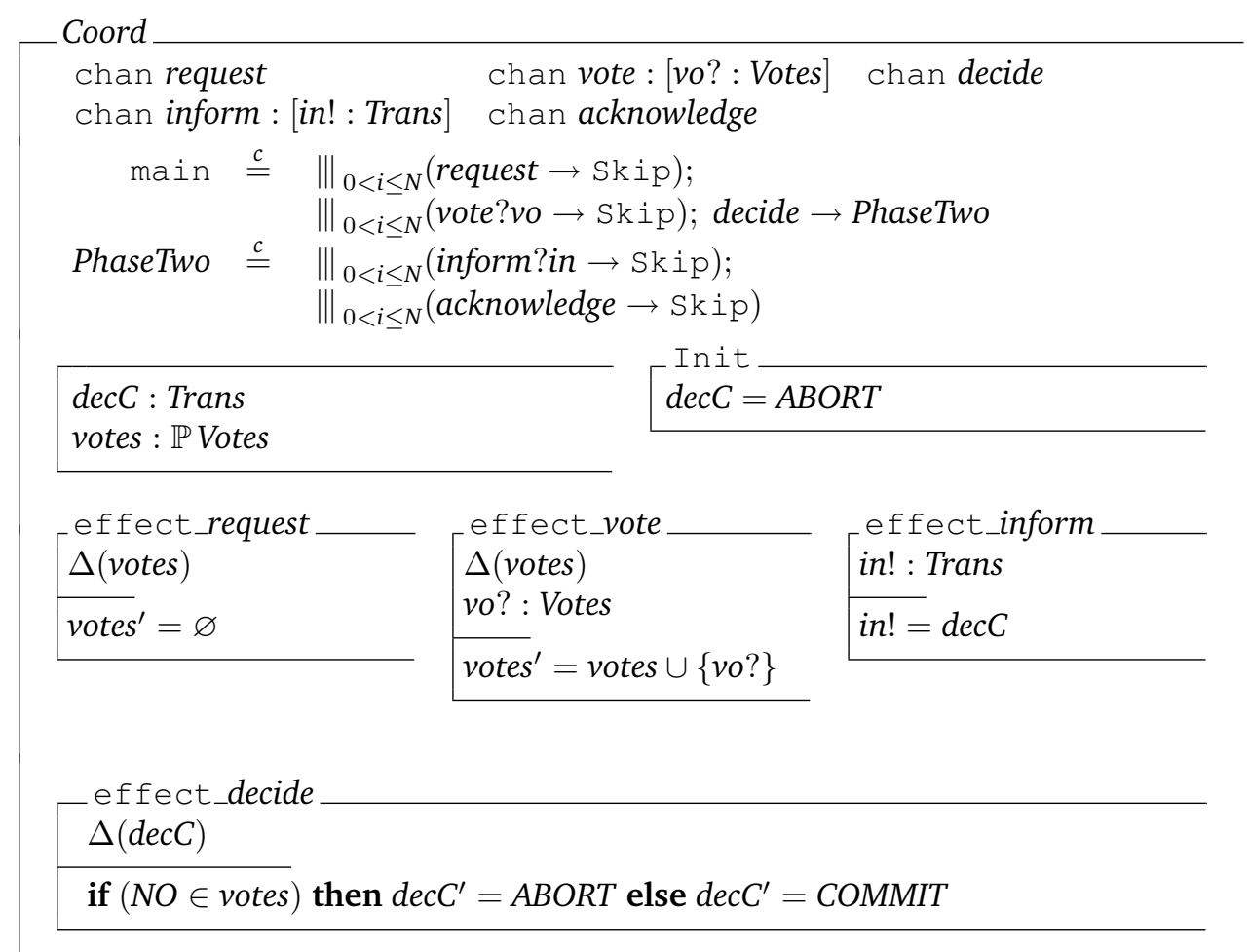

Figure 6.4: Two Phase Commit Protocol: Coord specification

added to the set votes. decide evaluates the set by assigning $\operatorname{dec} C$ to $A B O R T$ in case that at least one page votes with $N O$ and to COMMIT otherwise. Finally, inform sends the evaluation result to all participating pages by using an output parameter of type Trans.

The class Coord operates in parallel with $N$ instantiations of the class Page, as given in Figure 6.5. The state space of the Object-Z part of Page holds two variables decP of type Trans, corresponding to Coord.decC, and stable of type $\mathbb{B}$, for representing a successful (true) or unsuccessful (false) execution of the transaction. execute nonderministically 


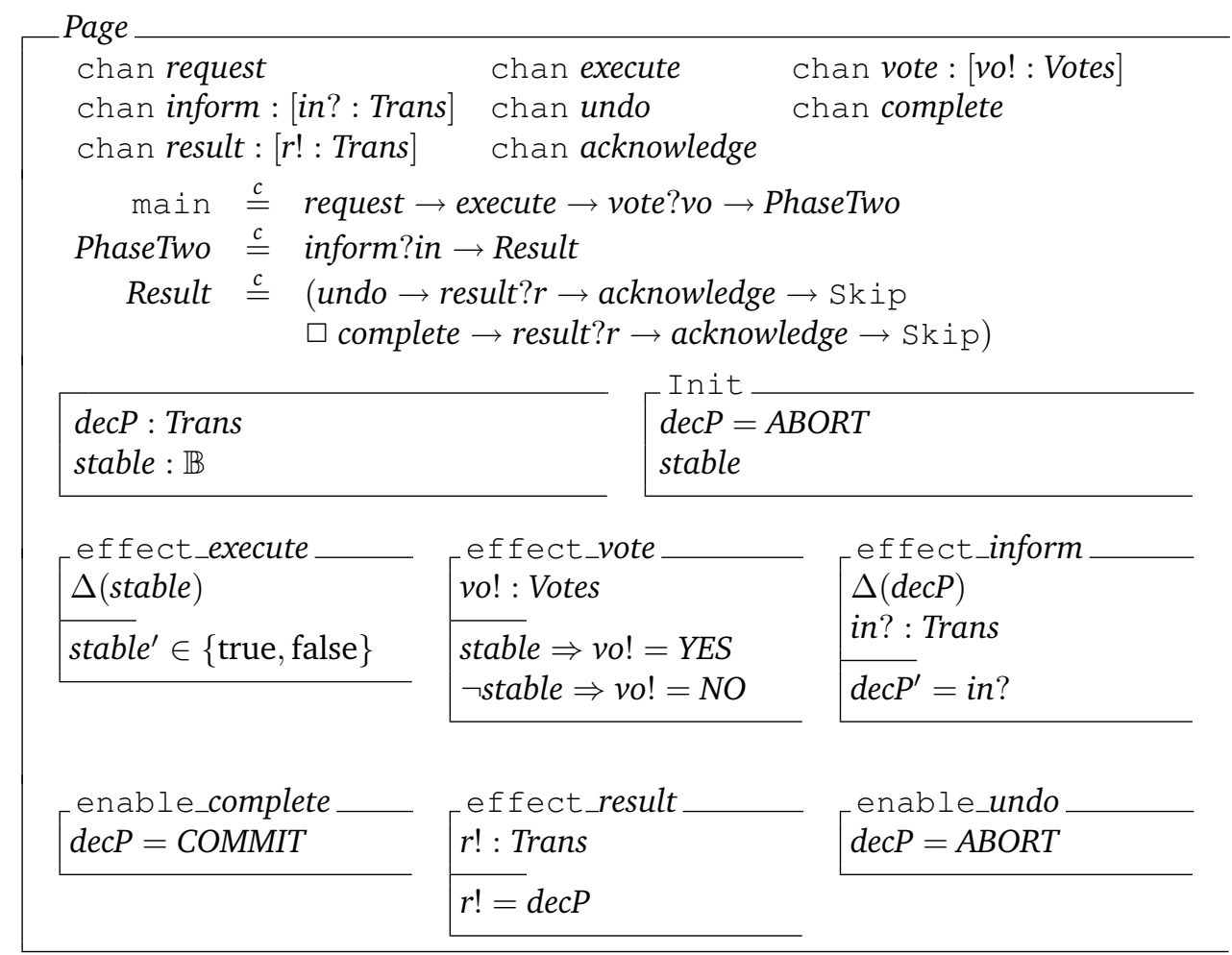

Figure 6.5: Two Phase Commit Protocol: Page specification

assigns a value to stable, dependent on which vote decides to either vote YES or NO. inform receives the decision to commit or abort the transaction, after which the specification either conducts a rollback (undo) or a permanent write (complete). Finally, the result of the transaction is communicated.

The full system is specified as

$$
\text { System }=\text { Coord } \|_{I}\left(\|_{0<i \leq N} \text { Page }\right),
$$

where $I=\{\mid$ request, vote, inform, acknowledge $\mid\}$ denotes the synchronisation alphabet for both classes.

Again, we are interested in an evaluation of the set of all valid cuts. For simplicity, we again solely deal with single cuts. Independent of the number of pages, 42 valid cuts can be identified. These are given in Table 6.6, where an operation name is abbreviated by its first four letters. Whether a certain cut is dominated by another one depends on the value of $N$. Thus, within the following table, we assume $N \geq 3$. Overall, there exist 9 reasonable and non-dominated cuts for the specification of the Two Phase Commit Protocol. 


\begin{tabular}{|c|c|c|c|}
\hline No. & Cut & Reasonable? & Non-Dominated? \\
\hline 1 & $\{$ ack, comp, deci, exec, info, resu, undo, vote $\}$ & No & No \\
\hline 2 & $\{$ ack, comp, deci, info, resu, undo, vote $\}$ & No & No \\
\hline 3 & $\{$ ack, comp, deci, info, resu, undo $\}$ & No & No \\
\hline 4 & $\{$ ack, comp, info, resu, undo $\}$ & No & No \\
\hline 5 & $\{$ ack, comp, resu, undo $\}$ & No & No \\
\hline 6 & $\{a c k$, comp, resu $\}$ & No & No \\
\hline 7 & $\{$ ack, resu, undo $\}$ & No & No \\
\hline 8 & $\{a c k, r e s u\}$ & No & Yes \\
\hline 9 & $\{a c k\}$ & No & Yes \\
\hline 10 & $\{$ comp, deci, exec, info, requ, resu, undo, vote $\}$ & No & No \\
\hline 11 & $\{$ comp, deci, exec, info, requ, undo, vote $\}$ & No & No \\
\hline 12 & $\{$ comp, deci, exec, info, requ, vote $\}$ & No & No \\
\hline 13 & $\{$ comp, deci, exec, info, resu, undo, vote $\}$ & Yes & No \\
\hline 14 & $\{$ comp, deci, exec, info, undo, vote $\}$ & Yes & No \\
\hline 15 & $\{$ comp, deci, exec, info, vote $\}$ & Yes & No \\
\hline 16 & $\{$ comp, deci, info, resu, undo, vote $\}$ & Yes & Yes \\
\hline 17 & $\{$ comp, deci, info, resu, undo $\}$ & Yes & Yes \\
\hline 18 & $\{$ comp, deci, info, undo, vote $\}$ & Yes & No \\
\hline 19 & $\{$ comp, deci, info, undo $\}$ & Yes & Yes \\
\hline 20 & $\{$ comp, deci, info, vote $\}$ & Yes & No \\
\hline 21 & $\{$ comp, deci, info $\}$ & Yes & No \\
\hline 22 & $\{$ comp, info, resu, undo $\}$ & Yes & Yes \\
\hline 23 & $\{$ comp, info, undo $\}$ & Yes & Yes \\
\hline 24 & $\{$ comp,info $\}$ & Yes & Yes \\
\hline 25 & $\{$ deci, exec, info, requ, undo, vote $\}$ & No & No \\
\hline 26 & $\{$ deci, exec, info, requ, vote $\}$ & No & No \\
\hline 27 & $\{$ deci, exec, info, undo, vote $\}$ & Yes & No \\
\hline 28 & $\{$ deci, exec, info, vote $\}$ & Yes & No \\
\hline 29 & $\{$ deci, exec, requ, vote $\}$ & No & No \\
\hline 30 & $\{$ deci, exec, vote $\}$ & Yes & No \\
\hline 31 & $\{$ deci, info, undo, vote $\}$ & Yes & No \\
\hline 32 & $\{$ deci, info, undo $\}$ & Yes & No \\
\hline 33 & $\{$ deci, info, vote $\}$ & Yes & No \\
\hline 34 & $\{$ deci, info $\}$ & Yes & No \\
\hline 35 & $\{$ deci, vote $\}$ & Yes & Yes \\
\hline 36 & $\{$ exec, requ, vote $\}$ & No & No \\
\hline 37 & $\{$ exec, requ $\}$ & No & No \\
\hline 38 & $\{$ exec, vote $\}$ & Yes & No \\
\hline 39 & $\{$ info, undo $\}$ & Yes & Yes \\
\hline 40 & $\{$ info $\}$ & Yes & Yes \\
\hline 41 & $\{r e q u\}$ & No & No \\
\hline 42 & $\{$ vote $\}$ & Yes & No \\
\hline
\end{tabular}

Table 6.6: Set of valid cuts for the TPCP 
Some of the valid cuts are unreasonable. For instance, the decomposition corresponding to \{acknowledge $\}$ results in an equal size of the first component and the original specification.

It is interesting to note that some cuts, which one would intuitively expect to result in a decomposition effective for compositional reasoning, are dominated and thus ruled out. One example is the cut $\{$ vote $\}$, which is dominated by $\{$ inform $\}$. Dependent on the number of pages $N$, we get

$$
\begin{aligned}
& h_{\mathbf{C S}}(\{\text { vote }\})=2 * N \\
& h_{\mathrm{ED}}(\{\text { vote }\})=6 * N+1 \\
& h_{\mathbf{F T}}(\{\text { vote }\})=8 * N \\
& h_{\mathbf{F A}}(\{\text { vote }\})=2 * N^{3}
\end{aligned}
$$

$$
\begin{aligned}
& h_{\mathbf{C S}}(\{\text { inform }\})=2 * N \\
& h_{\mathrm{ED}}(\{\text { inform }\})=2 * N-1 \\
& h_{\mathrm{FT}}(\{\text { inform }\})=4 * N \\
& h_{\mathbf{F A}}(\{\text { inform }\})=2 * N^{3}
\end{aligned}
$$

The values for $h_{\mathrm{FA}}$ and $h_{\mathrm{CS}}$ are identical. However, $\{$ inform $\}$ results in a distribution of the set of operation nodes closer to an even distribution than the one for $\{v o t e\}$. Additionally, $\{$ vote $\}$ requires one transmission parameter of cardinality $\# \mathbb{P}$ Votes $=4$, reflecting the variable Coord.votes, whereas \{inform $\}$ sufficiently uses one additional parameter of cardinality \#Trans $=2$, corresponding to Page.decP within the decomposition.

In contrast to the specification of a candy machine, the evaluation does not yield a small set of possible solutions. A thorough evaluation and comparison of the remaining set of reasonable and non-dominated cuts will be conducted in Chapter 7 , where we introduce our implementation framework and give the experimental results for both case studies.

\subsection{Discussion}

As the name implies, a heuristic approach, setting up context-specific rules-of-thumb, cannot be expected to precisely and completely cover all aspects of the underlying problem, neither can it generate a single optimal solution. Hence, we keep the approach as least restrictive as possible by still guiding the engineer to head into the right direction.

First, our aim for introducing the described heuristics is a classification of the set of valid cuts or decompositions of a specification. Even though the implementation of our approach focusses on the model checker FDR2, we do not define the heuristics by exploiting its specific characteristics. By doing so, we keep the approach independent of a specific model checker.

Second, in contrast to the slicing technique, as introduced in [Brü08], we do not consider the property under interest. As the alphabet of the generated assumption during learning not only depends on the set of cut events but also on the set of events occurring in the verification property, it could be reasonable to integrate the alphabet of the property as well. However, we choose not to do so, as we want to keep the decomposition approach independent of a certain verification property.

Finally, the previously introduced heuristics can be applied in any compositional verification setting - they are not limited to the learning based framework, which we consider. This is due to the fact that we try to keep the state space of the decomposition (and thus the interdepences between both components) small, which is a reasonable strategy, independent of any compositional verification framework. 
Yet, the following question remains: why do the previously defined heuristics most likely result in a set of practical solutions?

We investigated the different possibilities, causing a large state space, which needs to be explored during model checking. Here, we referred to two certain paradigms, which are generally valid for compositional verification [CAC06, $\mathrm{dRHH}^{+}$01, GL91, CGP03]: a strong connection between both components results in a high memory consumption and an increased run-time during verification. In addition, large components cause a large state space, which needs to be built up during model checking. The previous heuristics are closely related to both paradigms, as they investigate the definition of our decomposition technique and evaluate different possibilities to keep the cohesion between the components and their individual state spaces relatively small.

The different heuristics cannot be seen as equally important for any kind of specification. In addition, they conflict with each other. For instance, often, the larger the cut size for a decomposition, the smaller the size difference between both components, simply because the cut is neglected for the second heuristic.

Therefore, the actual evaluation of the set of valid decompositions must not be restricted to the specific values, given by the mathematical definitions of the heuristics. In fact, as we will see in Chapter 7, our implementation framework allows the user to prioritise certain heuristics by computing the weighted sum over all values.

However, in order to not mislead the user, several solutions can be neglected. We discussed this topic in Section 6.2: an evaluation of valid decompositions comparatively worse than other ones, that is, weakly dominated ones, is unnecessary. The same applies to unreasonable decompositions.

Summarising, the approach presented in this chapter automatically restricts the set of valid solutions as much as possible. This is done by eliminating those decompositions, which are impractical with respect to our heuristics or the generated state space size. Due to the nature of a heuristics-based approach, human intervention is still required for an evaluation of the remaining set of valid decompositions. However, this set is comparatively small in relation to the set of all valid cuts.

\subsection{Related Work}

Several works from different areas investigate heuristic approaches to cope with the state explosion problem during model checking. The work closest to ours is presented in [Nam07]. For learning-based compositional verification for models, described as symbolic transition systems (STS), the author chooses to partition a given system into several components, based on an algorithm for hypergraph partitioning [KK99]. The approach follows the general idea for an even distribution of the state variables of the STS and also aims at a minimisation of the interdependences between the components. The decomposition is performed fully automatically, not allowing the user to guide the framework to a potentially better partitioning, not complying to the static requirements. In addition, the author does not consider the control flow or a dependence analysis, and the approach does not take the alphabet of the generated assumptions into account. 
In order to cope with the state explosion problem during model checking of systems already composed of several components, in [SLU89], the authors present several alternative heuristic rules to reduce the state space of the system, focusing on the LTS semantics of a system. The work presented in [TJ02] follows a similar approach by, for instance, developing heuristics to fusion states or transitions or eliminating redundant states.

In the context of the $\mathrm{L}^{*}$ algorithm, in [GP09], the authors present a strategy for interface generation of software components. They implemented their approach for Java PathFinder (JPF) [NAS], a verification framework for Java byte code. Based on their learning framework for interface specifications, the authors also implemented assumeguarantee reasoning in JPF. JPF itself uses different search heuristics for an effective identification of possible bugs, eventually complementing compositional verification.

Further away from our approach, Dirks and Olderog [OD08] investigate the specification and the model checking of real-time systems. In their semantic domain, the first author developed an approach for heuristics-based planning and model checking [Die05]. Another heuristics-based approach, in order to more efficiently direct a model checker to potential counterexamples, is directed model checking [ESB $\left.{ }^{+} 09\right]$. Edelkamp et. al investigate directed model checking for SP IN [Hol03].

Multicriteria optimisation is an extensively researched area with a lot of different textbooks and articles giving a profound overview and insight on the topic [Ehr00, DW04, SNT85]. We concentrate on the definition of Pareto-optimality which was introduced in [Par71] and we restrict ourselves to discrete optimisation, that is, we do not consider real values within our heuristics.

This concludes the current chapter. The next chapter will introduce the implementation of our approach, including the modelling, the heuristics-based decomposition of a system and a subsequent direct or learning-based compositional verification. In addition, we evaluate the non-dominated and reasonable decompositions for both case studies and provide some significant experimental results. 


\title{
Implementation and Experimental Re- sults
}

\author{
Contents \\ 7.1 Syspect . . . . . . . . . . . . . . . . . . 184 \\ 7.1 .1 Class Diagrams . . . . . . . . . . . . . . . . 184 \\ 7.1 .2 State Machines . . . . . . . . . . . . . . 186 \\ 7.1 .3 Component Diagrams . . . . . . . . . . . . . . . 187 \\ 7.1 .4 Export to CSP-OZ . . . . . . . . . . . . . . . . 187 \\ 7.2 Decomposition Framework for Syspect … . . . . . . . . . 188 \\ 7.2.1 Decomposition Plug-In . . . . . . . . . . . . . . . . . . . . 189 \\ 7.2 .2 Mass Validation . . . . . . . . . . . . . . . . . . . . . . 191 \\ 7.2.3 Model Checking with FDR2 and the CSPLChecker . . . . . . . 192 \\ 7.2 .4 Counterexample Analysis . . . . . . . . . . . . . 196 \\ 7.2 .5 Overall Workflow . . . . . . . . . . . . . . . . . . 198 \\ 7.3 Experimental Results . . . . . . . . . . . . . . 200

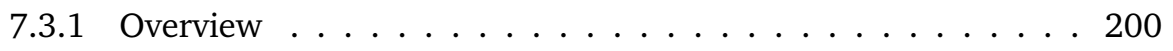 \\ 7.3.2 Verification Results for the Candy Machine . . . . . . . . . . 201 \\ 7.3.3 Verification Results for the Two Phase Commit Protocol . . . . . 204 \\ 7.3.4 Verification Results for the Number Swapper . . . . . . . 207 \\ 7.3 .5 Discussion . . . . . . . . . . . . . . . 207
}

The previous chapters introduced an approach for the decomposition of formal specifications, allowing for an application of compositional verification. Furthermore, we presented several heuristics for a classification of all valid decompositions. In order to substantiate our method and to measure its effectiveness, the technique has been implemented, and several case studies have been evaluated.

The present chapter describes the implementation framework for the theory of the previous chapters. Section 7.1 introduces Syspect [Sys06], a graphical modelling environment for CSP-OZ specifications, developed by the research group "Correct System Design" in Oldenburg. By using one of our case studies, we give a short overview on Syspect's different diagram types for modelling different aspects of a specification. The following Section 7.2 presents our context-specific extensions, realised to integrate the decomposition approach into Syspect. In the last section, the experimental results for three case studies, the candy machine from Section 2.2, the Two Phase Commit Protocol from Section 6.4 and the number swapper from Section 4.4, are given. We measure the different optimal and reasonable cuts by comparing direct model checking with FDR2 and compositional (learning-based) model checking. Finally, we discuss the results and 
draw some conclusions: some context-specific characteristics for good decompositions are pointed out, and we comment on when the application of our technique most likely results in a speed-up of model checking.

\subsection{Syspect}

The underlying platform for the implementation of our decomposition approach is the System Specification Tool (Syspect, [Sys06]). Syspect is a graphical and UML-based modelling environment for specifications, written in the integrated formalism CSP-OZ-DC [Hoe06]. By extending the language of CSP-OZ with the formalism Duration Calculus (DC) [ZH04], CSP-OZ-DC additionally allows to reason about real time aspects of a software model. Within this thesis, we do not consider DC. However, as CSP-OZ is naturally embedded into CSP-OZ-DC by simply declaring the DC-part to be empty, we can use Syspect to model CSP-OZ specifications as well.

Syspect has been developed within a student project, carried out at the research group "Correct System Design" in Oldenburg. The basis for their work is a specific UML profile for CSP-OZ, described in [MORW08]. A UML model can then be translated into a CSP-OZ specification. One focus for the definition of the UML model is the choice of a suitable subset of the UML, which is expressive enough to represent a significant part of CSP-OZ. In order to achieve this, the profile uses three different diagrams of the UML, namely

- class diagrams,

- state machines and

- component diagrams.

Next, we will shortly introduce the Syspect representation of the different diagram types by modelling the specification of the Two Phase Commit Protocol from Section 6.4. For a more detailed introduction into Syspect and the underlying UML profile, we refer to [Sys06, MORW08, Brü08].

\subsubsection{Class Diagrams}

In order to describe the static behaviour of a system specification, UML class diagrams [Obj05] can be used. Such a diagram comprises the specification's classes including their attributes: data variables (according to the state variables of the Object-Z part of a class) and methods (corresponding to the operations of the CSP-OZ class). Additionally, the definition of relationships between classes is possible: for the purpose of connecting classes and class-interfaces, different associations, such as aggregation or composition, can be used. These relationships represent the specification's composition- and synchronisation structure.

For the specification of the Two Phase Commit Protocol, the class diagram contains both classes Coord and Page. An interface ISyncCoordPage describes the set of synchronised 
operations of both classes. One additional class System is defined, representing the composition of Coord and Page, without defining additional attributes.

Figure 7.1 displays a screenshot of Syspect, showing the class diagram of the TPCP within the Syspect class diagram editor.

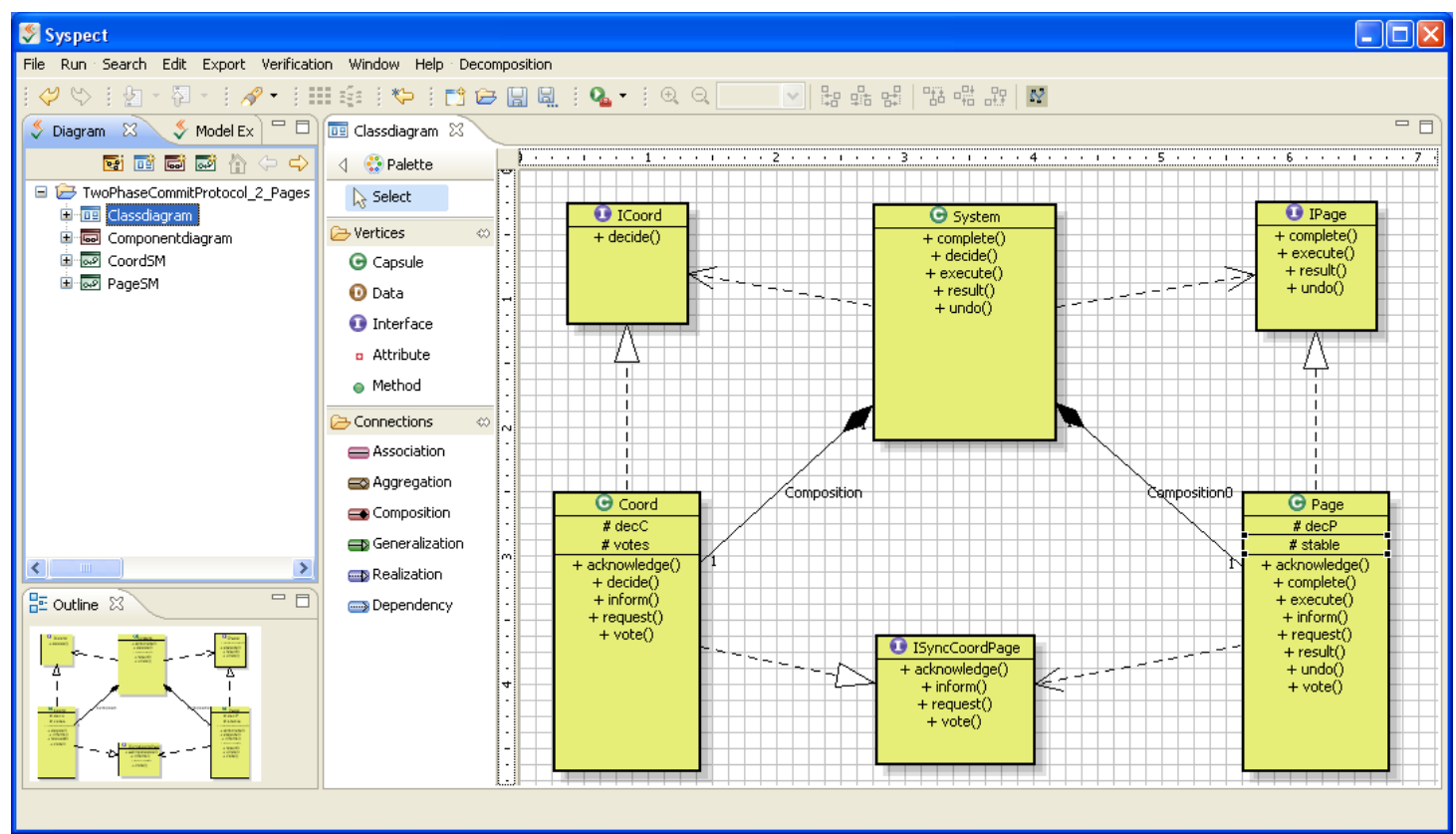

Figure 7.1: Syspect class diagram for the TPCP

Within a certain class, its set of variables and operations can be defined. The types of the variables and the behaviour of an individual operation can be described within the associated property view. In our example, both base types Votes and Trans are represented by $\mathbb{B}$, the set of boolean variables. Figure 7.2 shows the property view, associated with the operation Page.inform.

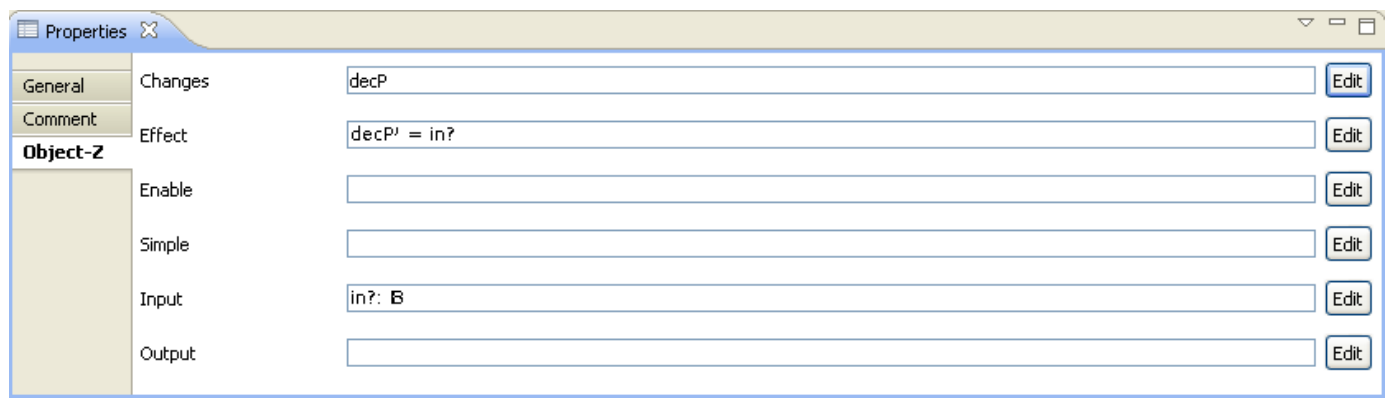

Figure 7.2: Syspect property view for the operation Page.inform 


\subsubsection{State Machines}

UML state machines are defined for representing the CSP parts of the individual classes of a CSP-OZ specification. Transitions of a state machine are labelled with an event corresponding to the associated class, or they are unlabelled for representing non-determinism. States are either

- ordinary states, representing a CSP process,

- initial states, representing the specific initial process main,

- final states, representing successful termination, that is, the process Skip, or

- complex states, containing a number of regions for modelling concurrency, that is, interleaving of several processes in terms of CSP.

In order to describe (non-deterministic- or deterministic-) choice, branching can be used.

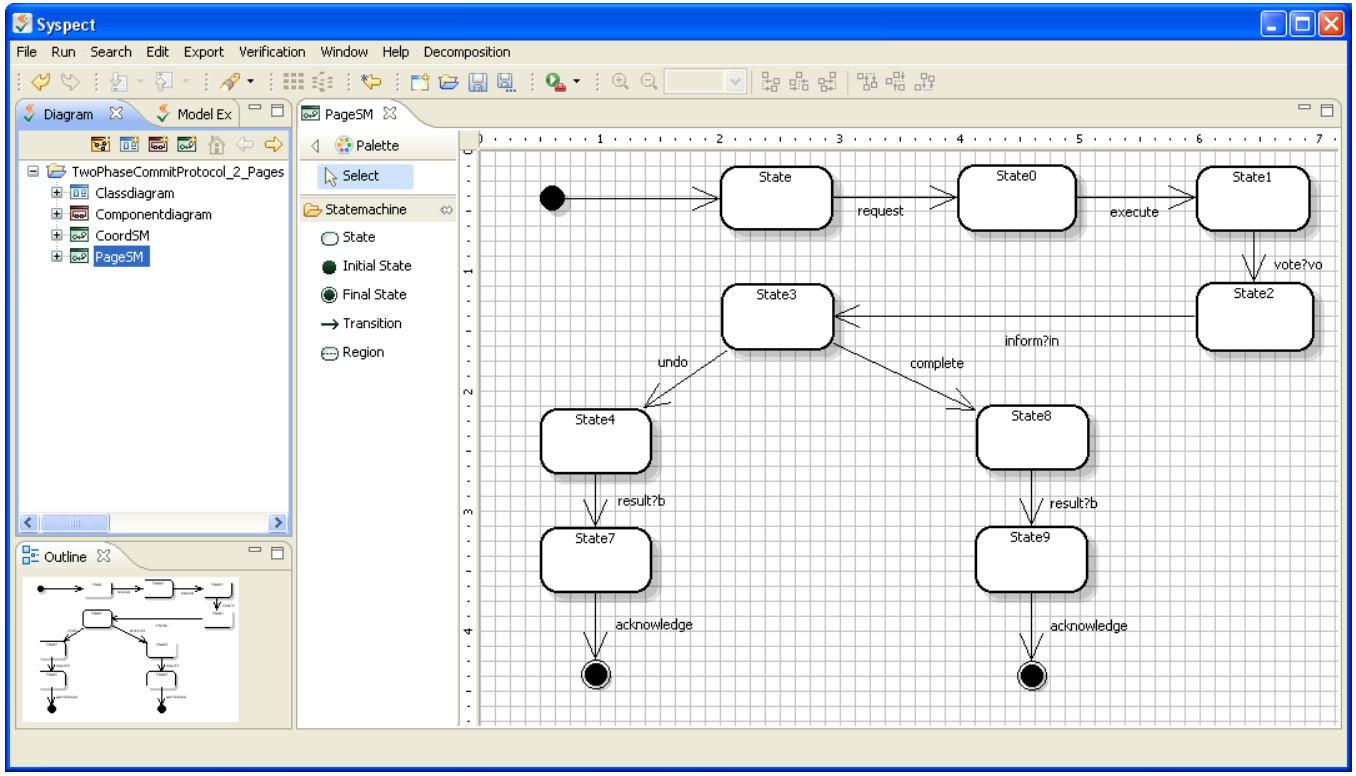

Figure 7.3: Syspect state machine for the class Page of TPCP

For the TPCP, there are two state machines, one corresponding to Page.main and one describing Coord.main. Figure 7.3 shows the state machine for Page.main. As the process Coord.main comprises interleaving of several processes, complex states are required. Here, we set $N:=2$, that is, the specification comprises two instances of class Page. Therefore, two regions are used, corresponding to the processes

$$
\|\|_{i \in\{1,2\}}(o p \rightarrow \text { Skip })
$$

for $o p \in\{$ request, vote, inform, acknowledge $\}$. The according state machine is given in Figure 7.4 . 


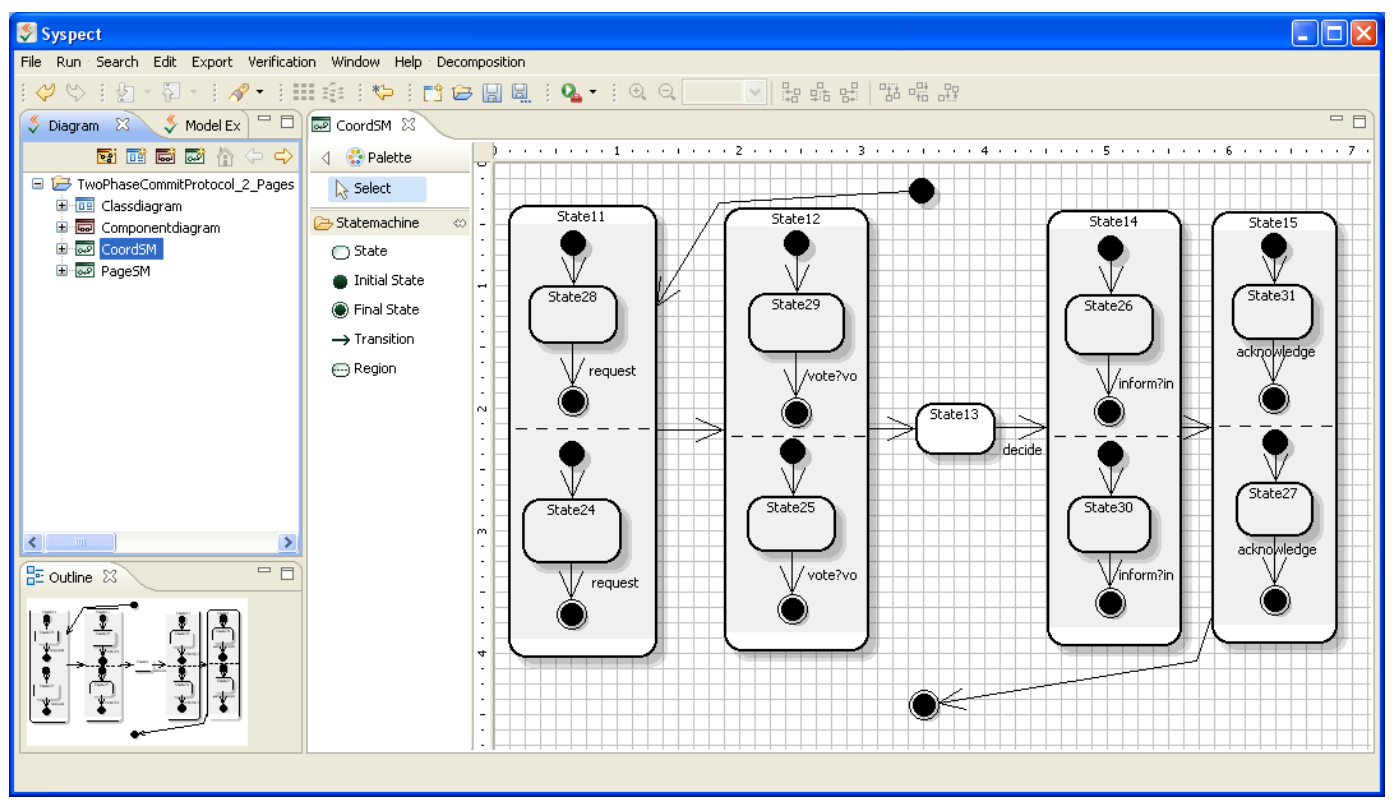

Figure 7.4: Syspect state machine for the class Coord of TPCP

\subsubsection{Component Diagrams}

The component diagram of a specification complements the class diagram and describes the composition and instantiations of its different constituents. Intuitively, it represents the overall system composition, that is,

$$
\text { System }=\text { Coord } \|_{I}\left(\|_{i \in\{1,2\}} \text { Page }\right)
$$

for the overall specification of the TPCP in the case of $N=2$. Complementary to the class diagram, the number of instances of Page is specified, along with the associations between all class instances, based on the interface connections.

Figure 7.5 shows the component diagram for the Two Phase Commit Protocol. It describes that both instances of Page synchronise with the sole instance of Coord via the interface ISyncCoordPage. Conjointly, this synchronisation yields the System-class.

\subsubsection{Export to CSP-OZ}

Syspect provides an export functionality for the translation of an UML model into a CSP-OZ representation of the model. Here, a translation into various formats can be carried out. In this thesis, we are solely concerned with the $\mathrm{BT}_{\mathrm{E}} \mathrm{X}$-export of a CSP-OZ specification: Syspect allows the generation of $\mathrm{AT}_{\mathrm{E}} \mathrm{X}$ mark-up, conforming to [ISO00] and the style file csp-oz . sty, as documented in [Fis99]. Within our verification framework, the export will be further processed and translated into the input language of the model checker FDR2. 


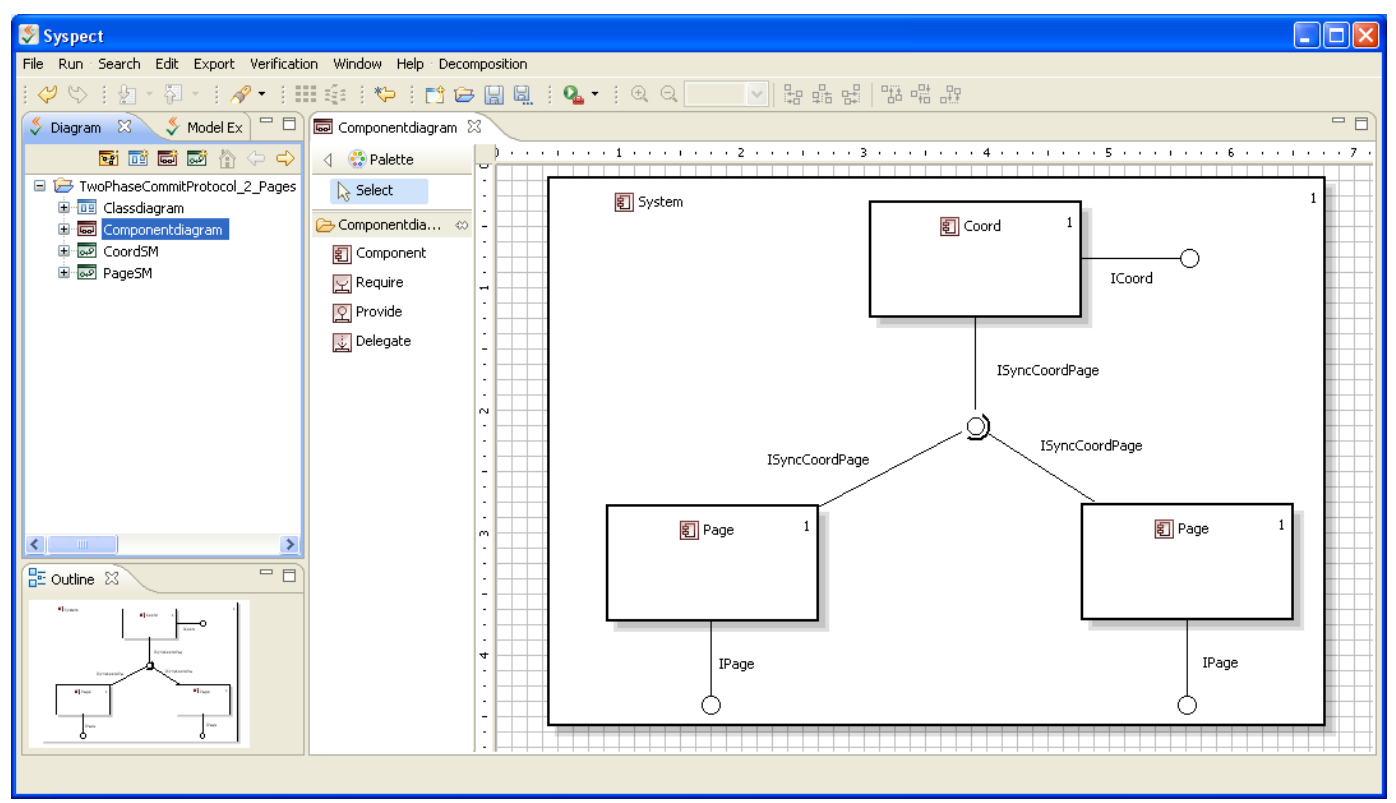

Figure 7.5: Syspect component diagram for the TPCP

\subsection{Decomposition Framework for Syspect}

After a brief introduction into Syspect, we will now describe the various additional features and extensions of Syspect, which have been developed in order to provide tool support for our decomposition technique. Namely, we present

- an implementation for the decomposition of a specification, based on the selection and validation of a (single) cut, the fragmentation of the specification's dependence graph and the subsequent decomposition of the specification itself, both according to Chapter 4 (Section 7.2.1),

- a mass validation framework to efficiently compute the set of all valid cuts, sorting out unreasonable and (weakly) dominated decompositions and scaling the remaining decompositions, based on the definitions from Chapter 6 (Section 7.2.2),

- an integration of the model checker FDR2 [For05] into Syspect, including a compiler from the Syspect export to the input language of FDR2, along with an interface to an implementation of the learning-based compositional verification framework as presented in Chapter 3 (Section 7.2.3) and

- a counterexample analysis for visualising error traces, possibly generated by FDR2, within the Syspect model (Section 7.2.4).

Figure 7.6 sketches the overall workflow. Given a Syspect specification of the software model, a user can choose

- to generate the dependence graph of a specification, manually select a cut and - in case of a valid cut - accordingly decompose the specification or 


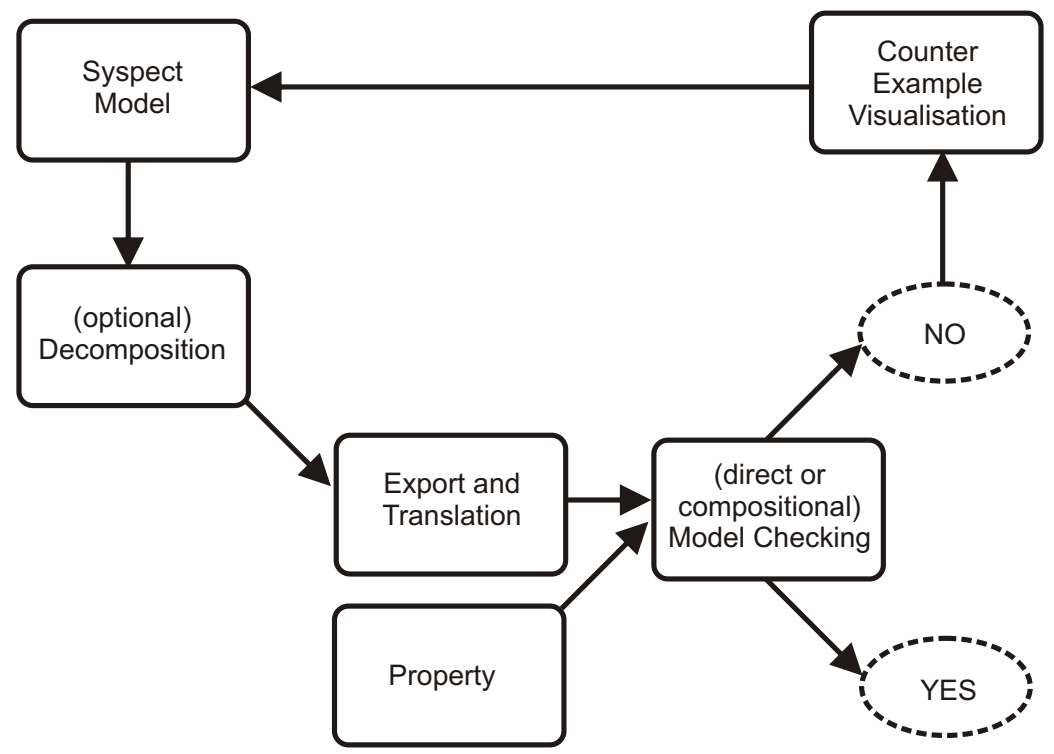

Figure 7.6: Toolchain for the verification framework

- initiate a computation of the set of all valid cuts, evaluate them with respect to our heuristics, select a specific cut and accordingly decompose the specification or

- not to decompose the specification at all.

As a next step, an export and subsequent compilation of the (decomposed) specification to the input language of the model checker FDR2 can be carried out. Afterwards, either compositional model checking or direct model checking can be applied, additionally requiring the definition of the verification property under interest. A possible counterexample is visualised within the Syspect model.

Next, we give a survey over the specific extensions of Syspect. We close this section with a more detailed description of the verification framework by using UML activity diagrams [Obj05].

\subsubsection{Decomposition Plug-In}

In Chapter 4, we defined a cut of a dependence graph, yielding a decomposition of the underlying specification. The foundation for the corresponding integration into Syspect is the decomposition plug-in, developed by Klaus Herbold as part of his diploma thesis [Her09]. The plug-in can optionally be used within Syspect, allowing the user to visualise the dependence graph of a specification and select a set of operation nodes. Afterwards, the selected cut-candidate is validated against the different correctness criteria from Section 4.2.2. To this end, the decomposition plug-in comprises the selection and validation of a single cut. In case of an invalid cut, the responsible set of violations is displayed. Otherwise, the user can proceed to decompose the specification. The decomposition is 


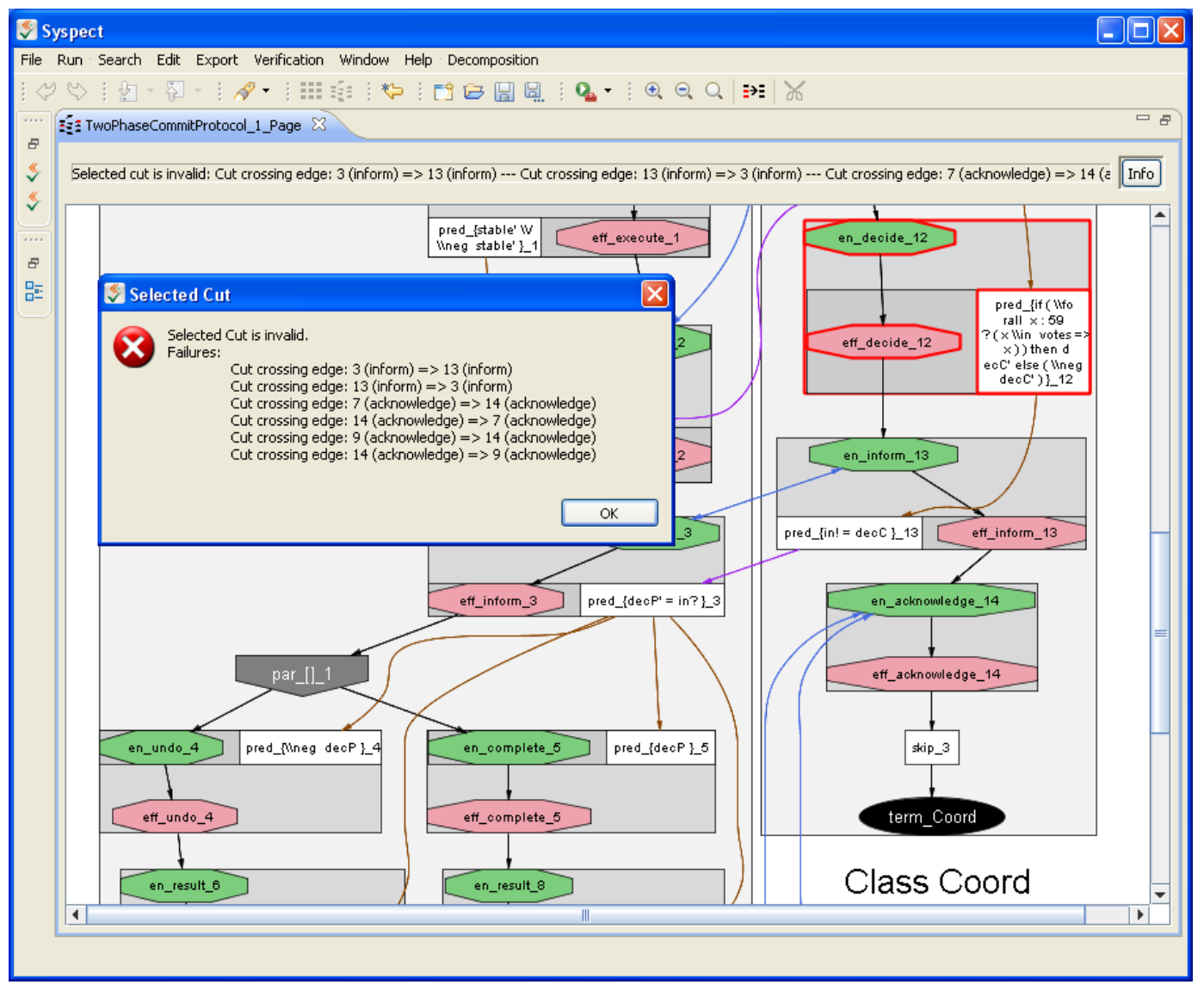

Figure 7.7: Screenshot of a selected invalid cut

carried out by applying the definitions from Section 4.3 and the addressing algorithm from Section 5.1.

Figure 7.7 shows a screenshot of an excerpt of the DG of the Two Phase Commit Protocol, as it is displayed within Syspect. For illustration purposes, we refer to the specification solely comprising one instance of the class Page. The visualisation builds up on the implementation of Brueckner's definition of the DG, which was carried out within the slicing plug-in, developed by Sven Linker [Brü08]. The graph is defined according to our modifications of the DG, as given in Section 2.3.4.

In general, a user can interact with the displayed DG and select a set of operation nodes. In the example, as displayed in the screenshot, the single node Coord.decide is selected. According to the correctness criteria, \{Coord.decide\} does not define a valid (single) cut: no nodes within the DG of Page are selected. Thus, its set of operation nodes is assigned to $\mathbf{P h}$, and several synchronisation edges, connecting nodes from the DGs of Page and Coord, violate the correctness criterion no crossing. In line with the selected cut, these violations are indicated.

In the event of the selection of a valid cut, the validity is displayed, and a further decomposition with respect to the cut can be carried out. Here, several options are 


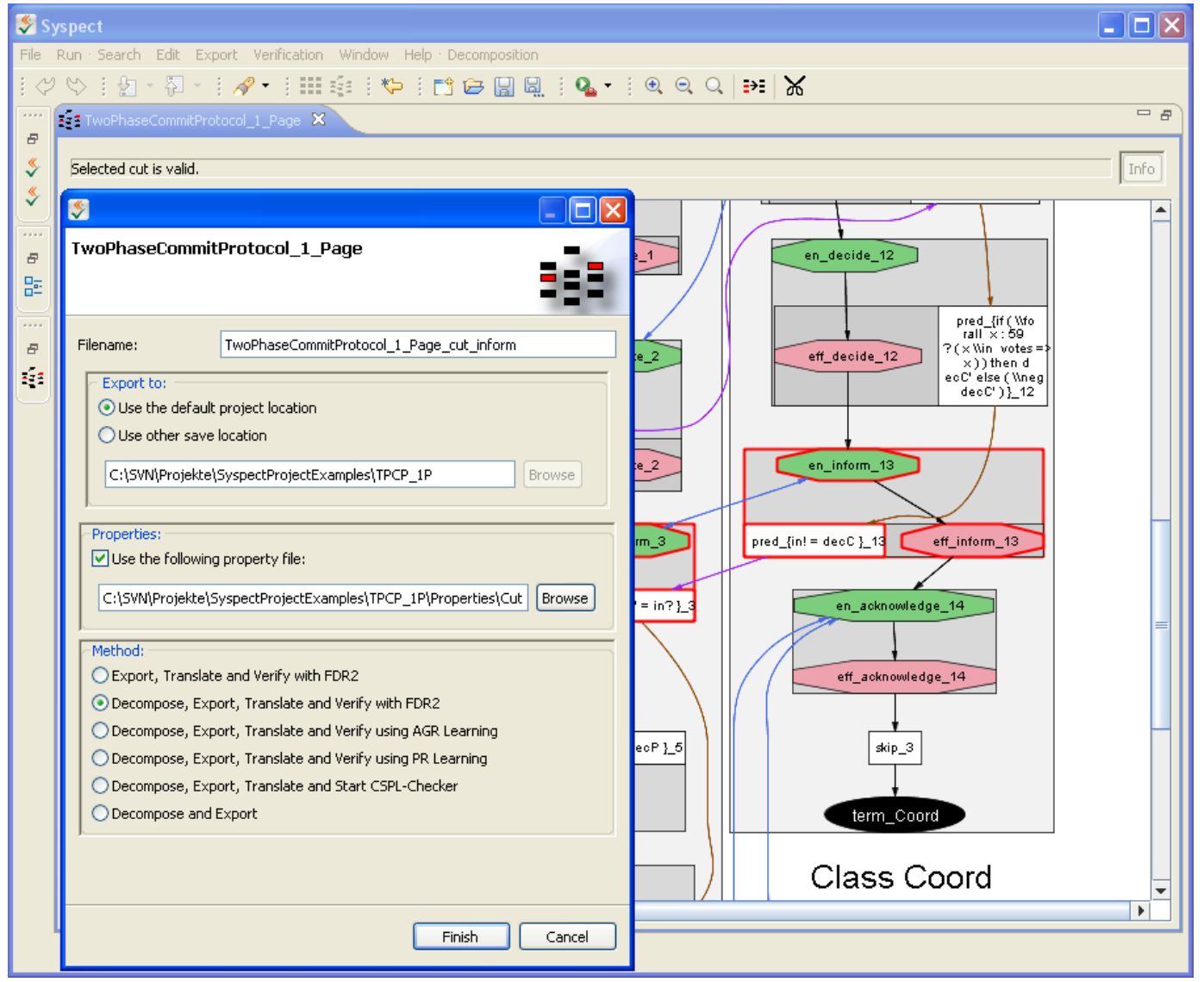

Figure 7.8: Screenshot of the decomposition options after selection of a valid cut

possible. Figure 7.8 displays the dialogue box after the selection of the correct single cut $\{$ Coord.inform, Page.inform . The various options regarding the export and model checking will be explained in Section 7.2.3. A decomposition of $S$ results in $\mathrm{AT}_{\mathrm{E}} \mathrm{X}$ markup for $S_{1} \| S_{2}$, which can then further be processed. In order to generate a valid decomposition, transmission parameters and addressing parameters are added.

Within the plug-in, several features and optimisations are implemented. For instance, according to the correctness criterion all-or-none from Section 4.2.2, either all or no nodes with the same operation name have to be contained in a cut. Thus, to facilitate a cut selection, in case that a user picks an operation node, all correspondingly named nodes are automatically selected. For more details on the implementation, we refer to [Her09].

\subsubsection{Mass Validation}

The previous section introduced the general functionality of the Syspect decomposition plug-in. A user can select and deselect operation nodes within a specification's DG, until he identified a valid cut, for which he chooses to carry out a decomposition. 
The larger the DG of a specification, the more tedious becomes a manual search for a cut. Moreover, according to Chapter 6, a valid cut must not automatically yield a decomposition suitable for an application of compositional reasoning.

In order to facilitate the choice of a valid cut and to guide the user to a decomposition most likely outmatching the original model in terms of model checking run-times, Meik Piepmeyer developed an extension of the decomposition plug-in, called the mass validation framework. Within his diploma thesis [Pie10], he mainly investigated the following question.

Given the DG of a specification, how can the set of all valid cuts efficiently be computed?

This question particularly becomes relevant if the DG comprises a large set of operation nodes: assuming $\# \mathrm{op}(N)=k$, the number of cut-candidates is $2^{k}$. Piepmeyer showed that the general problem of identifying all valid cuts is NP-complete [Coo71]. However, he developed and implemented several strategies and algorithms to efficiently validate a set of operation nodes against the various correctness criteria. One of his strategies uses a SAT solver [PBG05].

Additionally, Piepmeyer implemented the different heuristics from Chapter 6, along with the identification of all unreasonable and dominated cuts. In the latter case, one of the dominating cuts is displayed. Unreasonable cuts and dominated cuts can be removed from the set of all valid cuts, and the remaining cuts can be scaled according to the different heuristics.

Figure 7.9 shows a screenshot of the mass validation framework after choosing to compute all valid cuts for the TPCP for three instances of Page. Unreasonable cuts are marked with a minus, whereas a plus signalises reasonable cuts. In addition, optimal cuts are indicated by a small histogram. Furthermore, the amounts of valid cuts, optimal cuts and reasonable cuts are displayed. According to the results of Chapter 6, the amount of cuts which are both, optimal and reasonable, is equal to 9 .

In order to further classify the set of optimal and reasonable cuts, to this end, the weighted sum over all heuristics is used as the scaling function. In the example, in case that all heuristics are equally rated, \{inform $\}$ obtains the smallest value.

After scaling the different heuristics to identify a subjectively optimal cut, the user can proceed to decompose the specification and model check the result. For more details on the mass validation, see [Pie10].

\subsubsection{Model Checking with FDR2 and the CSPLChecker}

Following up on the selection of a valid cut and a corresponding decomposition of the model, we aim at an evaluation of direct model checking in comparison to the compositional one. The decomposition approach of this thesis is not restricted to a particular model checker. Yet, we require an existing translation from CSP-OZ to the respective input language.

In order to evaluate the effectiveness of our theory, we choose the CSP model checker FDR2 (Failure Divergence Refinement [For05]), developed by Formal Systems (Europe) Ltd. Several reasons substantiate this choice. First, FDR2 is the most commonly used 


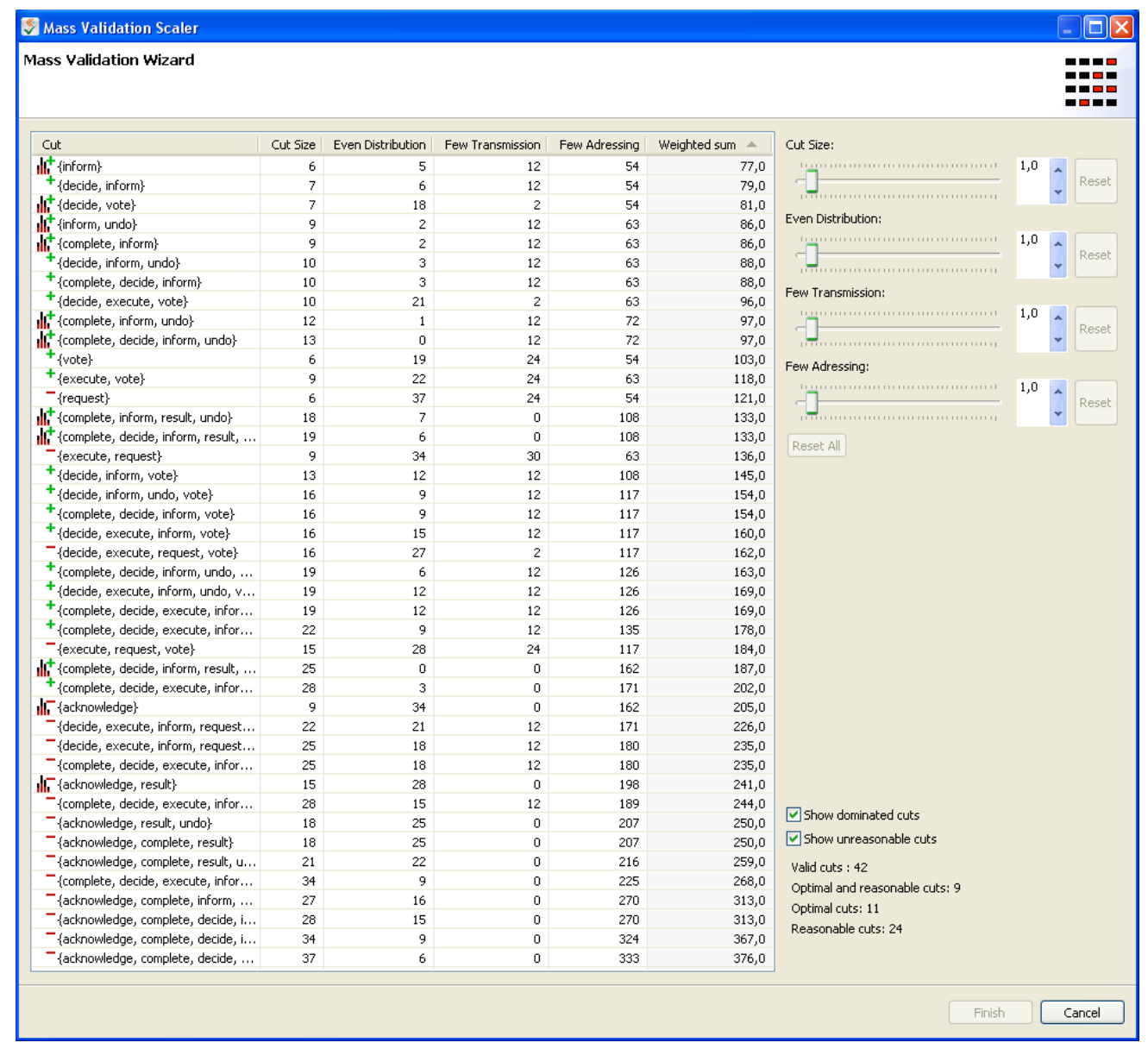

Figure 7.9: Screenshot of the mass validation framework

CSP model checker: at the time of writing his book [Ros98], Roscoe called it the chief proof and analytic tool for CSP, and this fact did not change over the recent years. Second, Wonisch [Won08] implemented the assume-guarantee-based learning framework based upon FDR2. Finally, FDR2 is well suited for our purpose, due to an already existing translation from CSP-OZ to the input language of FDR2 [FW99].

The tool inputs process specifications, written in a machine-readable dialect of CSP, called $\mathrm{CSP}_{\mathrm{M}}$. As the underlying verification concept, FDR2 uses refinement checks: given two CSP-processes $S$ and Prop, the refinement Prop $\sqsubseteq S$ can be evaluated within CSP's different semantic models. In this thesis, we are solely concerned with checking trace inclusion and do not consider any other refinement checks. For more details on FDR2, we refer to [Ros98, For05], with the latter reference comprising a full documentation of FDR2 along with the syntax of $\mathrm{CSP}_{\mathrm{M}}$.

Already, several works investigated a translation from either CSP-OZ or a related formalism to the input language of FDR2. For instance, in [MS01], the authors present a translation from CSP-Z to $\mathrm{CSP}_{\mathrm{M}}$. In her diploma thesis and simultaneously to the development of Syspect, Stamer [Sta06] investigated a translation to $\mathrm{CSP}_{\mathrm{M}}$ for models 


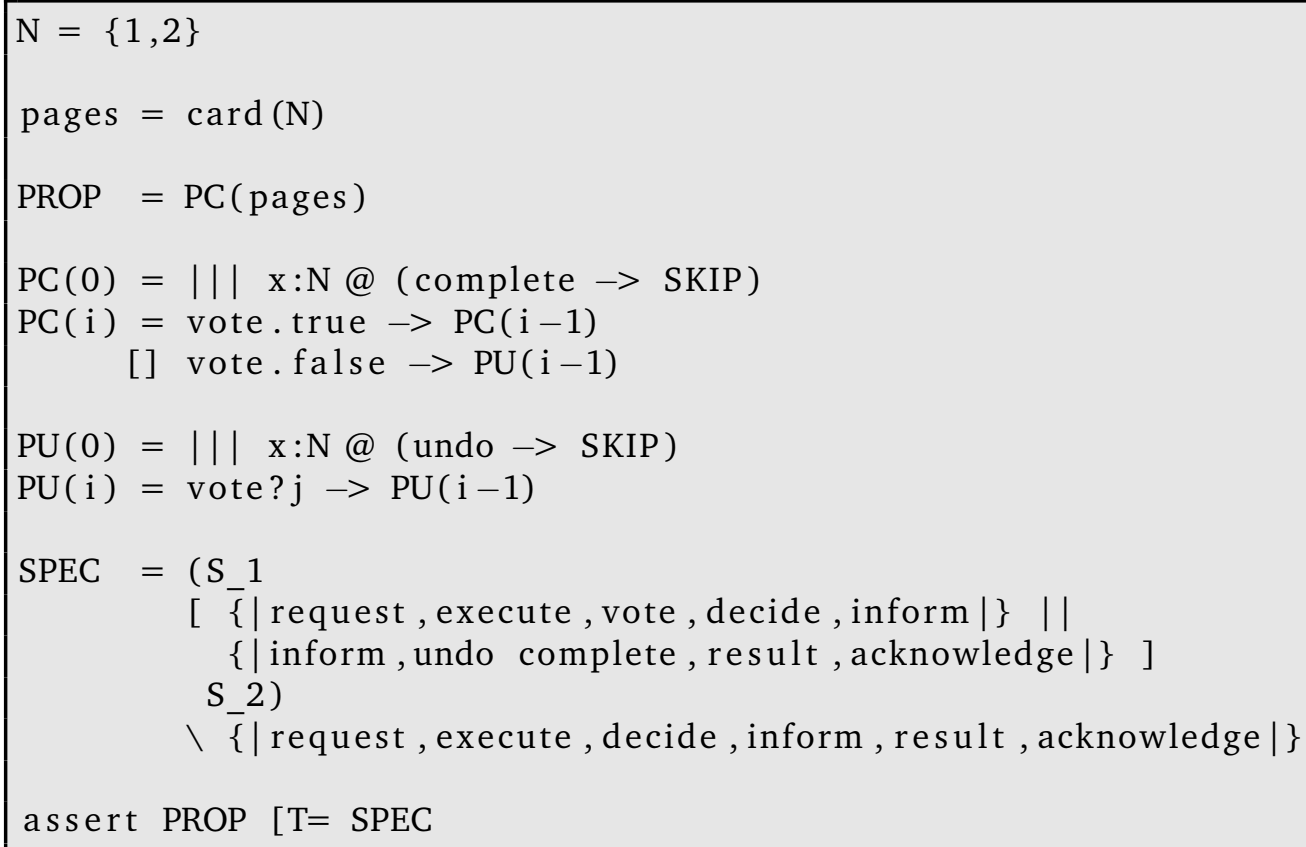

Figure 7.10: Correctness requirement for the TPCP in terms of $\mathrm{CSP}_{\mathrm{M}}$

specified in the UML profile for CSP-OZ [MORW08]. Obviously, such a translation has certain limits: it is clearly restricted by the expressiveness of the input language of the model checker. Moreover, as CSP-OZ exemplary allows to use infinite and underspecified data types, such as basic type definitions, a translation is limited to a subset of the integrated formalism.

In our context, we target the translation of the Syspect-ATEX-export to $\mathrm{CSP}_{\mathrm{M}}$. As part of his work as a student assistant, Wonisch implemented a corresponding compiler. The translation builds up on the definition from [FW99] and thus, the $\mathrm{CSP}_{Z}$ semantics, as given in Section 2.2.4. Some of the accomplishments in the compiler development include the support to translate finite sequences and various mathematical tool kit functions, along with axiomatic definitions.

Some restrictions have to be applied in order to model the different case studies within Syspect and to allow for a translation to $\mathrm{CSP}_{\mathrm{M}}$. For the case study of a candy machine, we require sequences of finite length, for which we define a corresponding constant. For specifying the Two Phase Commit Protocol, both base types are mapped to $\mathbb{B}$. For a translation of the Syspect export, a user needs to specify the maximal value for an element of $\mathbb{Z}$. By default, this value is set to 5 , meaning that $\mathbb{Z}$ is mapped onto the set $\{-5, \ldots, 5\}$ within the $\mathrm{CSP}_{\mathrm{M}}$-script (and, accordingly, $\mathbb{N}$ mapped onto $\{0, \ldots, 5\}$ ). The maximal integer is consistently used within the mass validation framework, where we implicitly set MaxInf to this specific value.

Besides the actual specification, model checking additionally requires a verification property. Currently, the user manually needs to define this property in terms of $\mathrm{CSP}_{\mathrm{M}}$. 


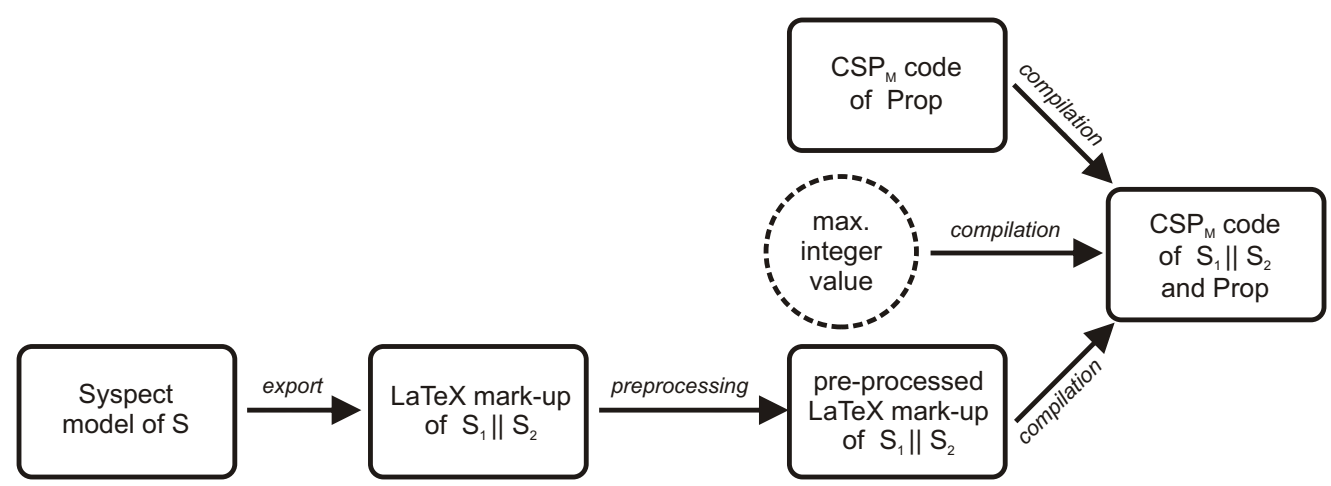

Figure 7.11: Compilation from $\mathrm{ET}_{\mathrm{E}} \mathrm{X}$ to $\mathrm{CSP}_{\mathrm{M}}$

Moreover, he needs to declare an assertion, specifying the individual trace refinement under investigation. Figure 7.10 defines a CSP process Prop, specifying a correctness requirement Prop for the TPCP. Intuitively, the property states:

"If, and only if, at least one page votes $N O$, all pages will undo the transaction. "

More technically, the process $P C(i)$ allows for $i$ votes (and thus Prop for $N$ votes, where $N=2$ ) and - if the control flow has not left the process before - an amount of $N$ subsequent events complete. As soon as one vote has the value NO, PC switches to a process $P U$. $P U(i)$ also allows for $i$ votes, independent of the parameter value, but always terminates with undos. Thus, as soon as one event vote.NO occurs, the final events of PROP will be undo.

Subsequently to the definition of the property, we define an assertion, stating that the individual specification SPEC refines the property PROP. Here, we additionally need to consider the respective decomposition we are dealing with: in the example, we evaluate the decomposition with respect to the cut $\{$ inform $\}$. Thus, SPEC needs to be accordingly defined.

Figure 7.11 illustrates the compilation framework. First, the export is preprocessed, mainly to adapt CSP-OZ-DC specific syntax according to the one for CSP-OZ. Subsequently, the $\mathrm{CSP}_{\mathrm{M}}$-code for the specification, including the one for the verification property, is generated.

Recall the different options for exporting and verifying a specification against a certain requirement, as displayed in Figure 7.8. In order to apply non-compositional model checking, the first option can be selected, yielding a direct verification of Prop $\bigsqcup_{T} S$. In this case, $S$ is not decomposed at all. In any of the following options, the specification is decomposed according to the selected cut. Here, the second option again initiates direct model checking, this time to prove or contradict Prop $\sqsubseteq_{T}\left(S_{1} \| S_{2}\right)$, whereas the last option solely exports the resulting specification to $\mathrm{CSP}_{\mathrm{M}}$.

For the remaining options, compositional verification based on the learning-based approaches from [CGP03] and [BGP03] can be carried out. As part of his bachelor's thesis, Wonisch [Won08] implemented the approach by using the CSP model checker FDR2 as the teacher. The tool is called CSPLChecker [Won] and supports an assumption 


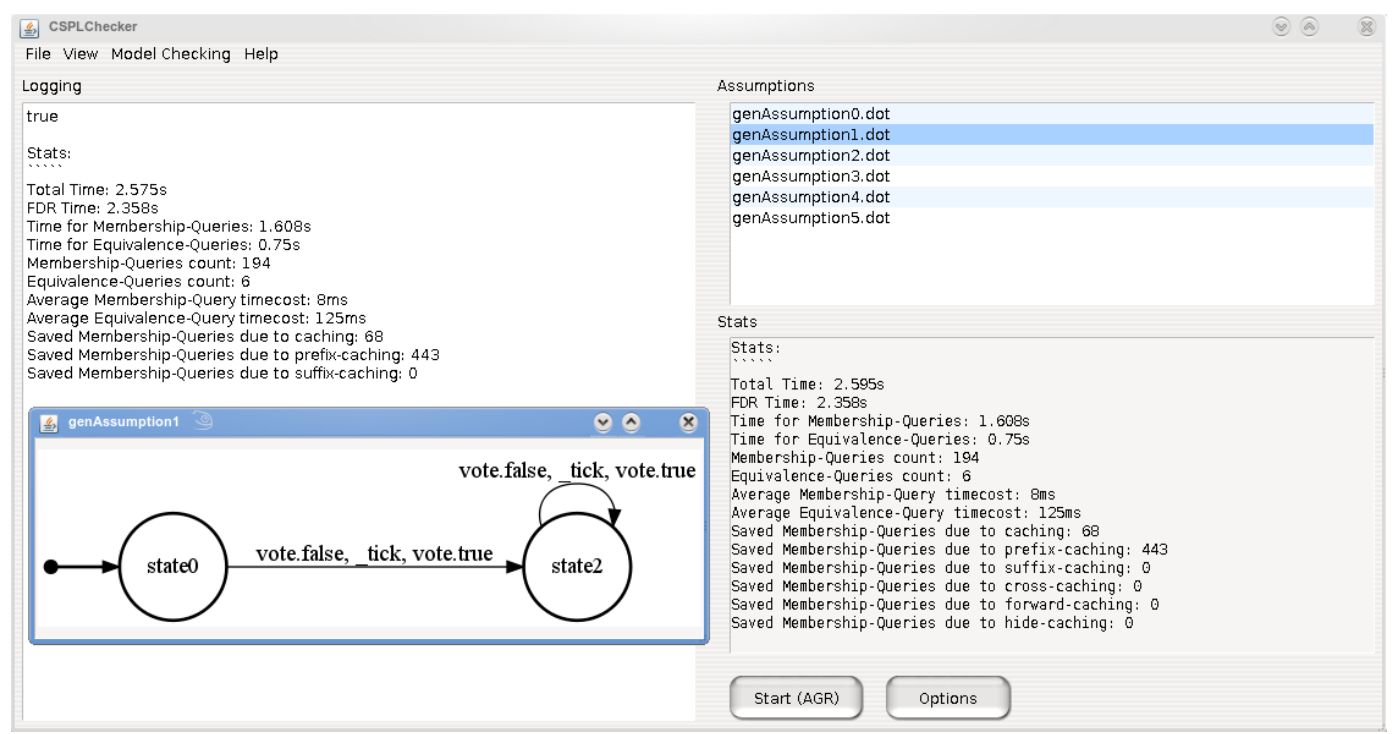

Figure 7.12: Screenshot of the CSPLChecker

generation, according to the learning frameworks for both assume-guarantee proof rules, (B-AGR) and (P-AGR) (see Section 3.3). Various optimisations as, for instance, different caching strategies, are implemented. During and after model checking, several statistics, such as the amount of membership queries or equivalence queries, can be displayed. For direct model checking with FDR2, the CSPLChecker is likewise called, forwarding the FDR2 output and showing several statistics.

The screenshot of the CSLChecker from Figure 7.12 shows the verification result for the decomposition of the TPCP. The decomposition is carried out with respect to the cut $\{$ inform $\}$, model checking refers to the property from Figure 7.11. At run-time, six intermediate assumptions are generated, the second one is displayed on the bottom left hand side. Overall, model checking takes approximately two seconds. The tool can freely be downloaded [Won], a more detailed description can be found in [Won08].

\subsubsection{Counterexample Analysis}

Independent of a direct call of FDR2 or a compositional verification, model checking a specification against a requirement possibly results in a counterexample. Such an error trace comprises a sequence of events, constituting a violation of the respective verification property. As part of the CSPLChecker output, this trace is displayed within the Syspect console. However, a purely textual representation of a counterexample is difficult to analyse. In particular, recovering the counterexample within the model can become tedious.

In order to guide the user to the specific behaviour of the model which violates the verification property, Micus [Mic10] developed an additional extension to Syspect, the countertrace plug-in. By evaluating the textual representation of a counterexample and linking it back to the specification's state machines, the error trace is visualised within 


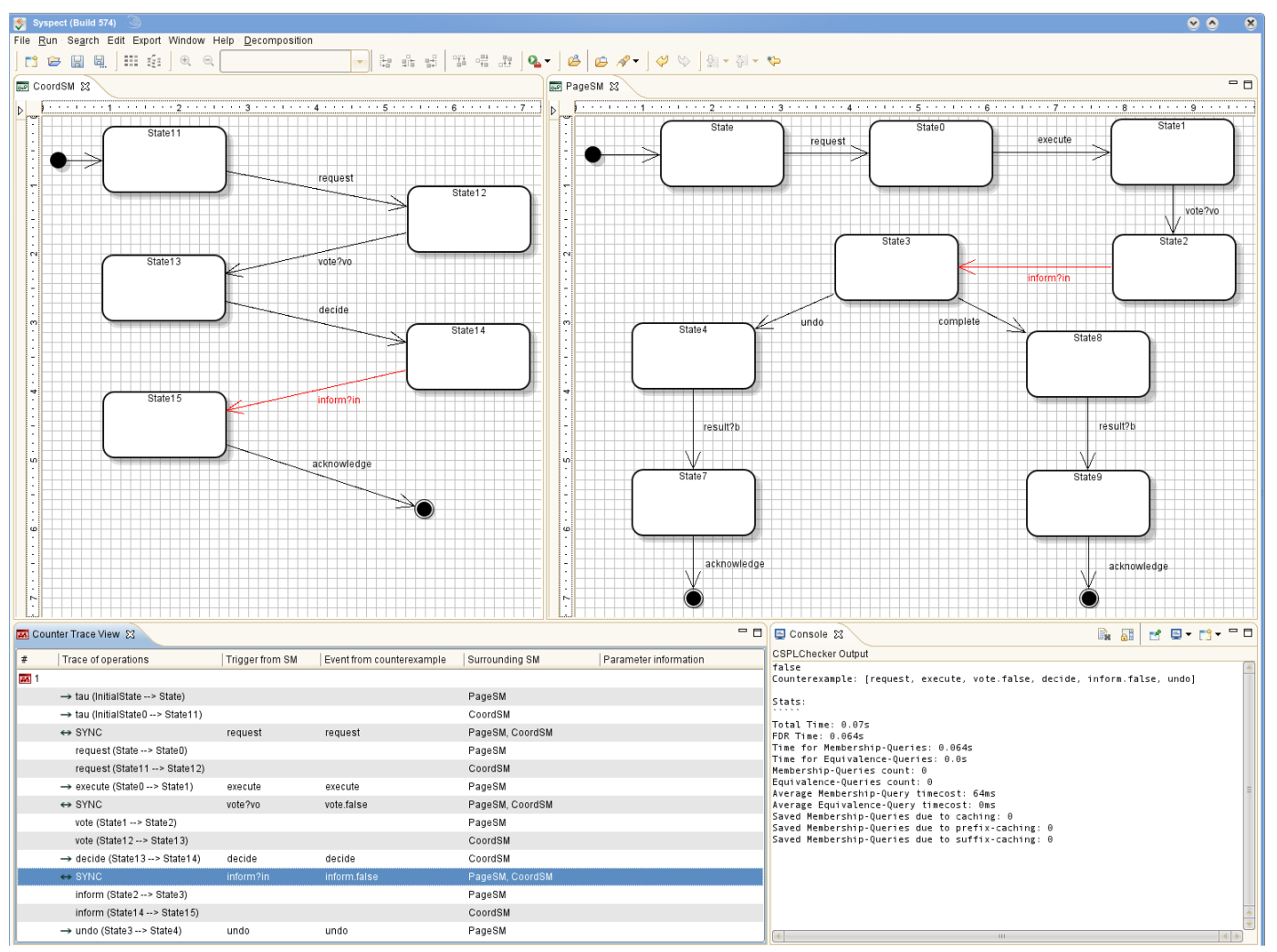

Figure 7.13: Screenshot of the counterexample visualisation

the Syspect model.

Consider a modification of the verification property from Figure 7.10: if we replace pages $=\operatorname{card}(\mathrm{N})$ by pages $=\operatorname{card}(\mathrm{N})+1$, for $N=1$, a verification will result in the following error trace: ${ }^{1}$

$$
\operatorname{tr}=\langle\text { request, execute, vote.false, decide, inform.false, undo }\rangle .
$$

This is due to the fact that Prop requires the execution of two votes before the first undo, which is clearly impossible for the TPCP with one instance of Page.

tr comprises events, solely executed by one class, along with synchronised events between the classes Coord and Page. A visualisation of $t r$ thus requires its events to be distributed over both state machines.

Figure 7.13 shows a screenshot of the visualisation of the error trace tr within both state machines. Here, the synchronised operation inform is selected, yielding the corresponding state machine triggers to be highlighted in red. Along with this, a visualisation of the complete error trace is possible.

Up to now, only the CSP part of the specification is analysed. In case there is more than one visualisation of the error trace, all of them are displayed. More details on the countertrace plug-in can be found in [Mic10].

\footnotetext{
${ }^{1}$ Recall, that Votes and Trans are mapped onto $\mathbb{B}$.
} 


\subsubsection{Overall Workflow}

After introducing the several context-specific extensions of Syspect, we concludingly assemble and summarise them. Figure 7.14 shows the decomposition framework by using an UML activity diagram [Obj05].

Given a specification $S$ and a property $P$, a user can either choose to apply direct model checking or compositional verification. In the first case, $S$ is exported to $\mathrm{ET}_{\mathrm{E}} \mathrm{X}$-mark-up (Syspect export plug-in, Section 7.1.4) and both, $S$ and $P$ are translated into a $\mathrm{CSP}_{\mathrm{M}}$-script (CSP $_{\mathrm{M}^{-}}$-export, Section 7.2.3). Here, $P$ is simply forwarded, as it is already specified in the input language of FDR2. In the latter case, $S$ is decomposed into some $S_{1} \| S_{2}$ - either by using the manual choice of a cut (Section 7.2.1) or the mass validation framework (Section 7.2.2), both realised within the Syspect decomposition plug-in. The property $P$ possibly needs to be adapted to some property $P^{\prime}$, according to the respective decomposition: $P^{\prime}$ needs to comply to the specification in terms of transmission parameters and address parameters. Along with that, a modified assertion now refers to the decomposed system. Again, a compilation of $S_{1} \| S_{2}$ and $P^{\prime}$ can be carried out, resulting in corresponding $\mathrm{CSP}_{\mathrm{M}}$-code.

Next, the actual model checking takes place. Direct, FDR2-based, model checking with respect to $S$ or $S_{1} \| S_{2}$ is generally possible. Compositional verification using the CSPLChecker (Section 7.2.3) requires the system to be composed of two components. This is clearly the case for $S_{1} \| S_{2}$ and, if $S$ itself is already assembled of two parts, for $S$ as well. In any case, if the model checking yields an error trace, the counterexample is visualised within the Syspect model (Syspect countertrace plug-in, Section 7.2.4). 


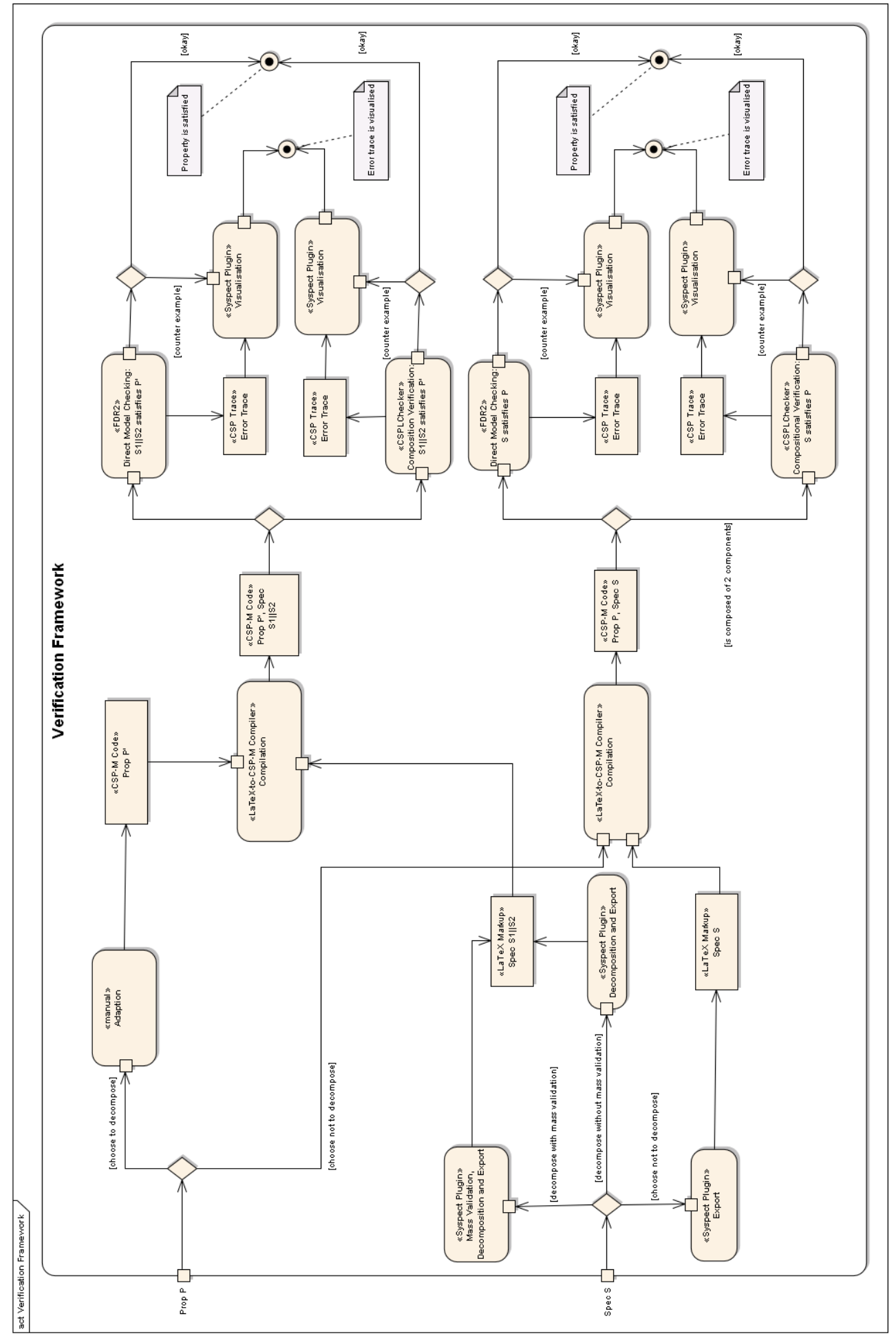

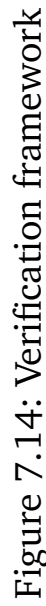




\subsection{Experimental Results}

Within this thesis, we specified several case studies, serving as an illustration of the main concepts, definitions and algorithms. The present section provides the experimental results for three specifications: the candy machine from Section 2.2, the Two Phase Commit Protocol from Section 6.4 and the number swapper from Section 4.4. In order to evaluate our approach, the examples have been specified within Syspect, decomposed and exported, after which the run-times during model checking were investigated. For all three case studies, we stepwise enlarged the size of the specification, namely, by increasing the maximal value for $\mathbb{Z}$ and, for the Two Phase Commit Protocol, the amount of participating pages. This allows us to estimate how the approach scales with an increasing size of the model.

We start this section with an overview on the technical conditions for our evaluation. Afterwards, we separately analyse the candy machine, the TPCP and the number swapper, and we draw some first conclusions. Finally, we discuss the evaluation results.

\subsubsection{Overview}

In order to accomplish an experimental evaluation of our approach, we analysed our case studies on a Dell PC, equipped with an Intel Core 2 Duo CPU, 4 GB RAM and openSUSE Linux 11.1. Besides that, we used Syspect version 1.4.0 with an integration of the various extensions, as described in Section 7.2. For model checking, we employed FDR in version 2.83 .

All case studies have been modelled within Syspect. The tool is available from its public subversion directory [Cor], the various extensions and the case studies along with the corresponding exports are freely accessible from [Res].

We provide some more background information on the conducted experimental studies.

- Up to now, the implementation of our approach within Syspect does not allow for a decomposition with respect to a general cut. Thus, for the case study of the number swapper, we manually decomposed the model, before proceeding with model checking. For the remaining case studies, we evaluated those sets of decompositions, which correspond to the set of optimal and reasonable single cuts, as given in Chapter 6.

- According to the two different proof rules, (B-AGR) and (P-AGR), we used two learning strategies, which will from now on be called basic reasoning (BR) and parallel reasoning (PR). In general, an assertion must be formed as Prop $\sqsubseteq_{T}\left(S_{1} \|\right.$ $S_{2}$ ), where $S_{1}$ denotes the first component of the decomposed system and $S_{2}$ the second.

- Some manual modifications of the export were necessary to achieve a fair comparison between the model checking results for the different systems. For instance, parameters were ordered such that the original ones are denoted first, followed by address parameters and transmission parameters. 
- Instead of comparing the sizes of the generated state spaces during model checking, we choose to compare verification run-times along with the amount of equivalence queries and membership queries during learning. This is owed to the model checker FDR2, not allowing for the computation of the size and visualisation of the overall state space, generated during model checking. In fact, it is possible to display the state space of the final generated transition system. However, in order to compare the amount of states constructed and visited during model checking, the transition systems of the intermediate processes would have to be considered as well. In our context, comparing run-times along with the amount of the different $\mathrm{L}^{*}$-queries, is a satisfactory way of an evaluation.

- In general, we specified verification properties, which turn out to be valid for the respective specification. Model checking is carried out, until either the system ran out of memory or the verification result is true.

Besides direct model checking of the original system and compositional, learning-based verification of the decomposition, we also evaluated direct model checking with respect to our generated decompositions. Therefore, our evaluation will investigate run-times for three different systems:

Original System: Given a specification $S$ and a requirement Prop, we consider direct model checking of Prop $\sqsubseteq_{T} S$.

Decomposed System, no AGR: For a valid decomposition of $S$ into $S_{1} \| S_{2}$, we investigate direct model checking of Prop $\bigsqcup_{T}\left(S_{1} \| S_{2}\right)$.

Decomposed System, AGR based on Learning: For a valid decomposition of $S$ into $S_{1} \| S_{2}$, model checking of Prop $\sqsubseteq_{T}\left(S_{1} \| S_{2}\right)$ with respect to the proof rules (B-AGR) and (P-AGR) is examined.

Section 7.3.5 will comment on the most likely reasons for the verification results. Next, we present the experimental results for the three investigated examples in detail.

\subsubsection{Verification Results for the Candy Machine}

Our experimental evaluation starts with the specification of a candy machine, as defined in Section 2.2. In Chapter 6, we already filtered all valid (single) cuts according to the criteria unreasonable (Definition 6.2.1) and dominated (Definition 6.2.3). The remaining two cuts are

- $\mathbf{C}_{1}:=\{$ switch $\}$ and

- $\mathbf{C}_{2}:=\{$ abort, order, select, switch $\}$.

Therefore, we will investigate three different systems: the undissected candy machine specification and two decompositions, according to the single cuts $\mathbf{C}_{1}$ and $\mathbf{C}_{2}$. Direct model checking is conducted for all three systems. In addition, for both decomposed systems, we consider basic reasoning and parallel reasoning. 


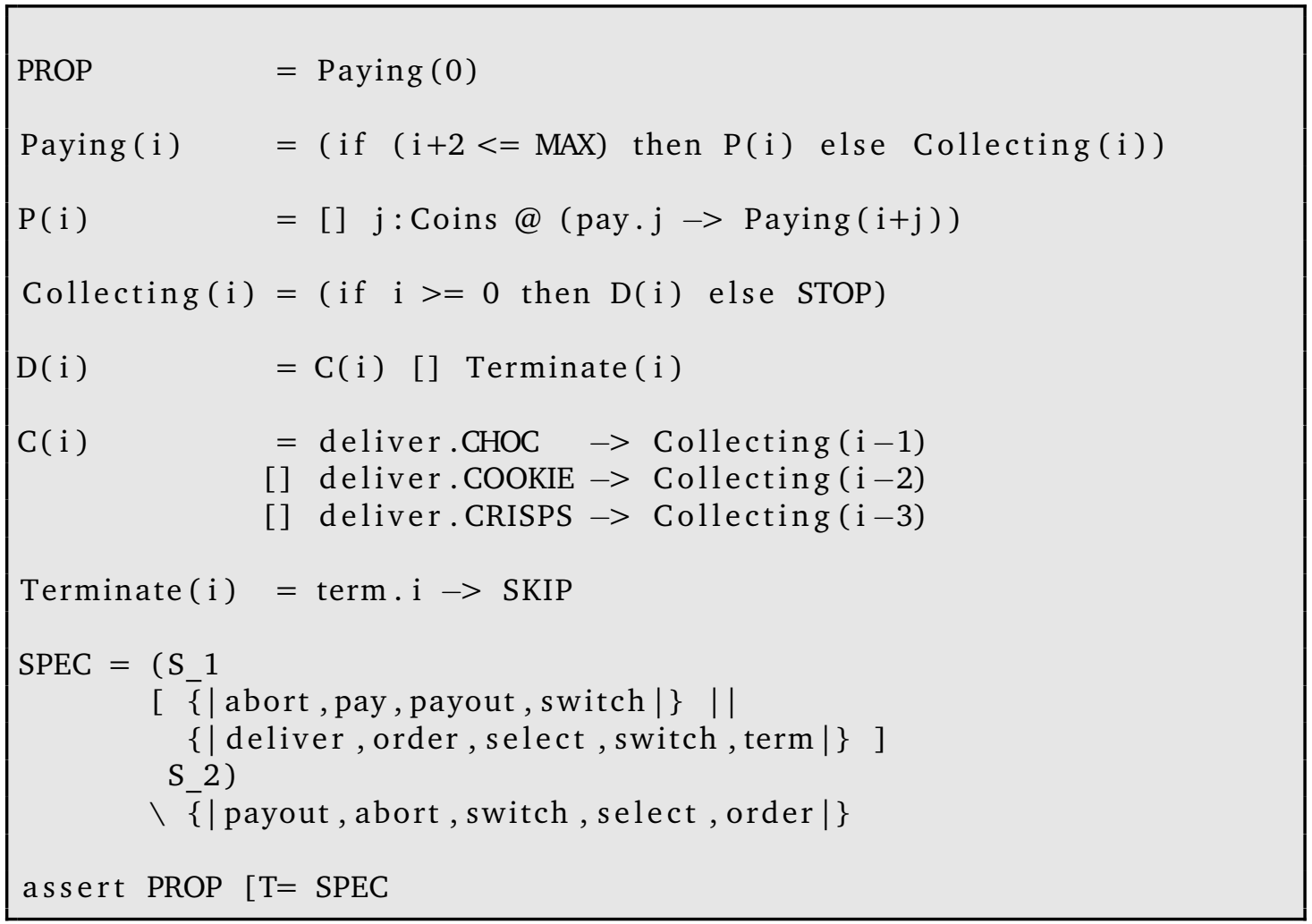

Figure 7.15: Correctness requirement for the candy machine in terms of $\operatorname{CSP}_{M}$

The verification property, which we consider, is the one defined in Figure 2.6. Rephrased in terms of $\mathrm{CSP}_{\mathrm{M}}$, the property is denoted in Figure 7.15. Here, we additionally need to to disallow the usage of integer values greater than MAX and smaller than 0 , which is specified within the property. The definition also contains an assertion for the decomposed system with respect to the cut $\{$ switch $\}$.

In the following, we scale the size of the evaluated model by increasing the maximal integer value MaxInf within the $\operatorname{CSP}_{\mathrm{M}}$-code. Precisely, MaxInf equal to $n$ means that $\mathbb{Z}$ is mapped to $\{-n, \ldots, n\}$ whereas $\mathbb{N}$ is mapped to $\{0, \ldots n\}$. The value for MaxInf determines a corresponding value for the constant Max (see Section 2.2.1).

We denote the run-times in seconds and, in case of learning, the membership queries and equivalence queries. The amount of equivalence queries is identical to the number of generated intermediate assumptions during learning.

The symbol $(*)$ indicates that the memory limit was exceeded during model checking, causing FDR2 to cancel the verification process with the message std: :bad_alloc. In addition, (-) denotes that the respective verification was not conducted, as model checking for the same system already failed for a smaller value of MaxInf. Finally, n/a denotes that compositional verification was not applicable, as the original system is not composed of two components. 


\begin{tabular}{|l|c|ccc|ccc|}
\hline \multicolumn{1}{|c|}{ Cut } & DC & \multicolumn{3}{c|}{ BR } & \multicolumn{3}{c|}{ PR } \\
& sec & sec & EQ & MQ & sec & EQ & MQ \\
\hline \hline None & $<1$ & n/a & n/a & n/a & n/a & n/a & n/a \\
\hline switch & $<1$ & $<1$ & 1 & 8 & 1 & 8 & 6 \\
\hline abort, order, select, switch $\}$ & $<1$ & $<1$ & 1 & 20 & 5 & 16 & 2000 \\
\hline
\end{tabular}

(a) Results for MaxInf $=1$

\begin{tabular}{|l|c|ccc|ccc|}
\hline \multicolumn{1}{|c|}{ Cut } & DC & \multicolumn{3}{c|}{ BR } & \multicolumn{3}{c|}{ PR } \\
& sec & sec & EQ & MQ & sec & EQ & MQ \\
\hline \hline None & 17 & $\mathrm{n} / \mathrm{a}$ & $\mathrm{n} / \mathrm{a}$ & $\mathrm{n} / \mathrm{a}$ & $\mathrm{n} / \mathrm{a}$ & $\mathrm{n} / \mathrm{a}$ & $\mathrm{n} / \mathrm{a}$ \\
\hline switch & $<1$ & 2 & 3 & 25 & 7 & 18 & 448 \\
\hline abort, order, select, switch $\}$ & 53 & 62 & 2 & 188 & 1916 & 92 & $156 \mathrm{~K}$ \\
\hline
\end{tabular}

(b) Results for MaxInf $=2$

\begin{tabular}{|l|c|ccc|ccc|}
\hline \multicolumn{1}{|c|}{ Cut } & DC & \multicolumn{3}{c|}{ BR } & \multicolumn{3}{c|}{ PR } \\
& sec & sec & EQ & MQ & sec & EQ & MQ \\
\hline \hline None & $(*)$ & n/a & n/a & n/a & n/a & n/a & n/a \\
\hline$\{$ switch & 12 & 107 & 5 & 88 & 162 & 23 & 944 \\
\hline abort, order, select, switch $\}$ & $(*)$ & $(*)$ & $(-)$ & $(-)$ & $(*)$ & $(-)$ & $(-)$ \\
\hline
\end{tabular}

(c) Results for MaxInf $=3$

\begin{tabular}{|l|c|ccc|ccc|}
\hline \multicolumn{1}{|c|}{ Cut } & DC & \multicolumn{3}{c|}{ BR } & \multicolumn{3}{c|}{ PR } \\
& sec & sec & EQ & MQ & sec & EQ & MQ \\
\hline \hline None & $(-)$ & $\mathrm{n} / \mathrm{a}$ & $\mathrm{n} / \mathrm{a}$ & $\mathrm{n} / \mathrm{a}$ & $\mathrm{n} / \mathrm{a}$ & $\mathrm{n} / \mathrm{a}$ & $\mathrm{n} / \mathrm{a}$ \\
\hline$\{$ switch & 183 & $(*)$ & $(-)$ & $(-)$ & 3044 & 25 & 1527 \\
\hline abort, order, select, switch $\}$ & $(-)$ & $(-)$ & $(-)$ & $(-)$ & $(-)$ & $(-)$ & $(-)$ \\
\hline
\end{tabular}

(d) Results for MaxInf $=4$

Table 7.1: Experimental results for the candy machine

The improvement from Section 4.3.7, allowing for a neglect of specific initial data dependences, was not yet implemented in Syspect. Therefore, the cut $\{$ switch $\}$ is not indicated as a valid cut. In order to cope with this problem, we removed the initial predicate items $=\langle\rangle$ from the model and manually re-added it within the $\mathrm{CSP}_{\mathrm{M}}$-code.

Finally, we give the experimental evaluation for the candy machine specification. Table 7.1 displays the results for MaxInf $=1$ to MaxInf $=4$. Most importantly, in case that the machine did not exceed its memory limit, we denote the amount of seconds until the verification terminated with the result true. DC indicates direct model checking, and, as already mentioned, $\mathbf{B R}$ and $\mathbf{P R}$ indicate basic reasoning and parallel reasoning. The number of equivalence queries and membership queries are given in the columns marked with EQ and MQ, respectively. For MaxInf $=5$, the two remaining evaluations for the cut \{switch\} lead to an out-of-memory exception. 
It turns out that direct model checking of the original system can only be carried out for MaxInf $\in\{1,2\}$. The same applies for the cut $\mathbf{C}_{2}=\{$ abort, order, select, switch $\}$, independent of monolithic or compositional verification. For this specific cut, run-times are even worse compared to model checking of the undissected model. The best results are achieved for the cut $\mathbf{C}_{1}=\{$ switch $\}$. Quite surprisingly, direct model checking outperforms the compositional one.

Summing up, due to the decomposition of the model, we can verify the investigated property on larger systems. Even though learning-based reasoning already outperforms monolithic verification of the original system, the best results are achieved for direct model checking of the decomposed systems according to one specific cut. This particularly shows that effective model checking for a decomposed system not automatically requires compositional, assume-guarantee-based strategies. We will further elaborate on these particular results in Section 7.3.5.

\subsubsection{Verification Results for the Two Phase Commit Protocol}

The next case study under investigation is the Two Phase Commit Protocol, specified in Section 6.4. Again, we only consider the set of optimal and reasonable cuts, as given in Table 6.6. In order to refer to the different cuts, we occasionally use the according numbers from the respective table. We verify the system against the property, given in Figure 7.10.

According to Section 6.4, there are nine optimal and reasonable cuts. As already argued, a heuristics-based approach solely points the direction, but it does not automatically determine the (set of) qualified cut(s). Instead of comparing all nine cuts, we filter the set by further analysing its elements:

- The cuts numbered as 24 and 39 result in two symmetric decompositions, which only differ in the two different operation names undo and complete. Therefore, we select one of these cuts for the evaluation, namely the one numbered as 39 , that is, \{inform, undo $\}$.

- The sole reason why the cut $\{$ complete, inform, result, undo $\}$ does not dominate the cuts numbered as 16 and 17, is the value for the heuristic even distribution. However, as the first cut is a subset of the latter two cuts, the difference appears simply due to a shift of node(s) into the cut. Clearly, this does not improve the decomposition, and we solely consider the first of these three cuts.

- The same argument applies in case we compare the cuts numbered as 23 and 19 with $\{$ inform, undo $\}$.

The remaining four (single) cuts will be evaluated. These are

- $\mathbf{C}_{1}:=\{$ inform $\}$,

- $\mathbf{C}_{2}:=\{$ vote, decide $\}$,

- $\mathbf{C}_{3}:=\{$ inform, undo $\}$ and 
- $\mathbf{C}_{4}:=\{$ complete, inform, result, undo $\}$.

Along with them, we will also consider the dominated cut $\mathbf{C}_{5}:=\{$ vote $\}$ : even though this cut seems to be a sensible one, our heuristics reject it. In order to draw some further conclusions on the plausibility of the heuristics, we exemplify an evaluation of a dominated cut on $\mathbf{C}_{5}$.

\begin{tabular}{|l|c|ccc|ccc|}
\hline \multicolumn{1}{|c|}{ Cut } & DC & \multicolumn{3}{c|}{ BR } & \multicolumn{3}{c|}{ PR } \\
& sec & sec & EQ & MQ & sec & EQ & MQ \\
\hline \hline None & $<1$ & 4 & 9 & 464 & 5 & 33 & 973 \\
\hline$\{$ inform $\}$ & $<1$ & 2 & 6 & 194 & 16 & 28 & 4050 \\
\hline$\{$ vote, decide & $<1$ & 2 & 5 & 230 & 3 & 16 & 464 \\
\hline inform, undo $\}$ & $<1$ & 76 & 11 & 5011 & 29 & 28 & 6611 \\
\hline complete, inform, result, undo $\}$ & $<1$ & 308 & 16 & $18 \mathrm{~K}$ & 2 & 5 & 416 \\
\hline \{vote & $<1$ & 4 & 3 & 226 & 7 & 13 & 976 \\
\hline
\end{tabular}

(a) Results for two pages

\begin{tabular}{|l|c|ccc|ccc|}
\hline \multicolumn{1}{|c|}{ Cut } & DC & \multicolumn{3}{c|}{ BR } & \multicolumn{3}{c|}{ PR } \\
& sec & sec & EQ & MQ & sec & EQ & MQ \\
\hline \hline None & $<1$ & 19 & 14 & 1071 & 25 & 52 & 3248 \\
\hline$\{$ inform $\}$ & 7 & 10 & 8 & 567 & 192 & 52 & $38 \mathrm{~K}$ \\
\hline$\{$ vote, decide $\}$ & $<1$ & 11 & 7 & 933 & 9 & 23 & 1875 \\
\hline$\{$ inform, undo & 7 & 1459 & 21 & $38 \mathrm{~K}$ & 567 & 61 & $97 \mathrm{~K}$ \\
\hline$\{$ complete, inform, result, undo $\}$ & 5 & 8449 & 23 & $148 \mathrm{~K}$ & 8 & 6 & 1403 \\
\hline$\{$ vote & 1 & 43 & 4 & 1218 & 40 & 19 & 4265 \\
\hline
\end{tabular}

(b) Results for three pages

\begin{tabular}{|l|c|ccc|ccc|}
\hline \multicolumn{1}{|c|}{ Cut } & DC & \multicolumn{3}{c|}{ BR } & \multicolumn{3}{c|}{ PR } \\
& sec & sec & EQ & MQ & sec & EQ & MQ \\
\hline \hline None & 17 & 319 & 18 & 1839 & 6807 & 67 & $46 \mathrm{~K}$ \\
\hline$\{$ inform $\}$ & 3657 & 42 & 10 & 1251 & 1970 & 83 & $205 \mathrm{~K}$ \\
\hline$\{$ vote, decide $\}$ & 47 & 52 & 9 & 2672 & 74 & 29 & 5040 \\
\hline inform, undo $\}$ & 3142 & $(*)$ & $(-)$ & $(-)$ & $(*)$ & $(-)$ & $(-)$ \\
\hline complete, inform, result, undo $\}$ & 1796 & $(*)$ & $(-)$ & $(-)$ & 781 & 7 & 3614 \\
\hline vote & 57 & 336 & 6 & 4721 & 161 & 20 & $10 \mathrm{~K}$ \\
\hline
\end{tabular}

(c) Results for four pages

Table 7.2: Experimental Results for the TPCP, first part

Tables 7.2 and 7.3 show the evaluation results for the TPCP, comprising two to seven pages. The table is correspondingly configured to the one for the candy machine, and it uses the same symbol to indicate an out-of-memory failure during model checking. As the specification itself is composed of two components Coord and Pages, we can apply compositional verification for the original system as well. 
The evaluation yields the following results: first of all, direct verification for the undissected system and for the decompositions according to $\mathbf{C}_{2}$ and $\mathbf{C}_{5}$ can be carried out for an amount of five pages, before the memory limit exceeded. For the remaining decompositions, direct verification is only possible for an amount of four pages.

Compositional verification results in comparatively worse run-times for two and three pages. However, the larger the model, the more effective becomes compositional reasoning and, in particular, basic reasoning. The best results are achieved for the decomposition according to $\mathrm{C}_{2}$, that is, \{vote, decide\}. Here, model checking can be carried out for up to seven pages, before the memory limit exceeds. Regarding the dominated cut $\{v o t e\}$, it is outmatched by basic reasoning with respect to the cuts $\{$ inform $\}$ and $\{$ vote, decide $\}$. Thus, even though one might intuitively assume $\{$ vote $\}$ to declare a better decomposition than $\{$ vote, decide $\}$, the heuristics prove this conjecture wrong. A more detailed discussion will be part of Section 7.3.5.

\begin{tabular}{|l|c|ccc|ccc|}
\hline \multicolumn{1}{|c|}{ Cut } & DC & \multicolumn{3}{c|}{ BR } & & PR \\
& sec & sec & EQ & MQ & sec & EQ & MQ \\
\hline \hline None & 926 & 8696 & 23 & 2926 & $(*)$ & $(-)$ & $(-)$ \\
\hline$\{$ inform $\}$ & $(*)$ & 571 & 12 & 2342 & $(*)$ & $(-)$ & $(-)$ \\
\hline$\{$ vote, decide $\}$ & 3584 & 241 & 11 & 6174 & $11 \mathrm{~K}$ & 35 & $11 \mathrm{~K}$ \\
\hline$\{$ inform, undo $\}$ & $(*)$ & $(-)$ & $(-)$ & $(-)$ & $(-)$ & $(-)$ & $(-)$ \\
\hline$\{$ complete, inform, result, undo $\}$ & $(*)$ & $(-)$ & $(-)$ & $(-)$ & $(*)$ & $(-)$ & $(-)$ \\
\hline$\{$ vote & 4220 & 1877 & 7 & $11 \mathrm{~K}$ & $10 \mathrm{~K}$ & 17 & $20 \mathrm{~K}$ \\
\hline
\end{tabular}

(a) Results for five pages

\begin{tabular}{|l|c|ccc|ccc|}
\hline \multicolumn{1}{|c|}{ Cut } & DC & \multicolumn{4}{c|}{ BR } & & PR \\
& sec & sec & EQ & MQ & sec & EQ & MQ \\
\hline \hline None & $(*)$ & $(*)$ & $(-)$ & $(-)$ & $(-)$ & $(-)$ & $(-)$ \\
\hline$\{$ inform $\}$ & $(-)$ & $(*)$ & $(-)$ & $(-)$ & $(-)$ & $(-)$ & $(-)$ \\
\hline$\{$ vote, decide $\}$ (6 pages) & $(*)$ & 1738 & 13 & $12 \mathrm{~K}$ & $(*)$ & $(-)$ & $(-)$ \\
$\{$ vote, decide (7 pages) & $(-)$ & 5846 & 15 & $22 \mathrm{~K}$ & $(-)$ & $(-)$ & $(-)$ \\
\hline$\{$ inform, undo & $(-)$ & $(-)$ & $(-)$ & $(-)$ & $(-)$ & $(-)$ & $(-)$ \\
\hline complete, inform, result, undo $\}$ & $(-)$ & $(-)$ & $(-)$ & $(-)$ & $(-)$ & $(-)$ & $(-)$ \\
\hline$\{$ vote & $(*)$ & $(*)$ & $(-)$ & $(-)$ & $(*)$ & $(-)$ & $(-)$ \\
\hline
\end{tabular}

(b) Results for six and seven pages

Table 7.3: Experimental Results for the TPCP, second part

Summing up, contrary to the evaluation results for the candy machine, direct verification of the decomposed system results in higher run-times than basic reasoning. Moreover, basic reasoning performs significantly better than parallel reasoning. The example shows that assume-guarantee-based compositional verification can indeed lead to a significant speed-up during model checking. 


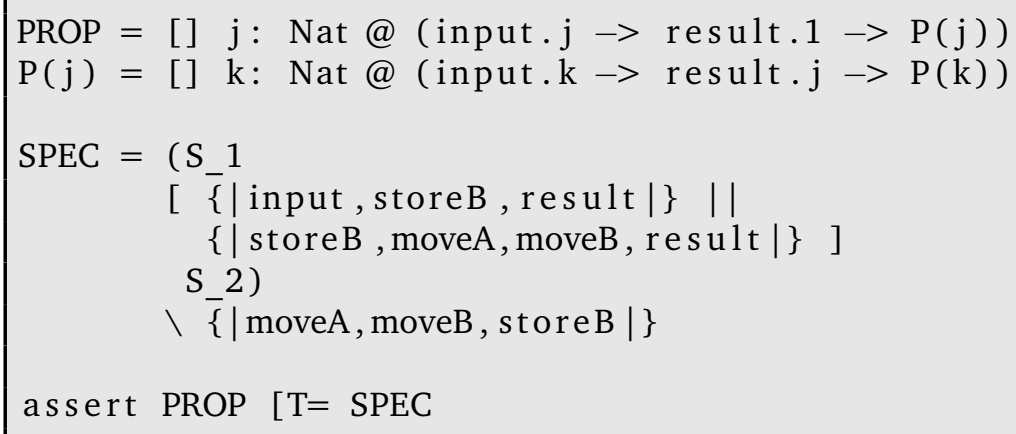

Figure 7.16: Correctness requirement for the number swapper in terms of $\operatorname{CSP}_{M}$

\subsubsection{Verification Results for the Number Swapper}

The final case study under investigation is the (extended version of the) number swapper from Section 4.4, defined in Figure 4.26. Here, due to the specific recursive structure of the CSP part, a decomposition with respect to a single cut is impossible. Moreover, based on the different data dependences, there is only one reasonable general cut, namely $\mathbf{C}=\left(\mathbf{C}_{1}, \mathbf{C}_{2}\right)$, for $\mathbf{C}_{1}=\{$ store_b $\}$ and $\mathbf{C}_{2}=\{$ result $\}$. Thus, we manually decomposed the specification according to $\mathbf{C}$, and we compared run-times for direct verification of the original system and the decomposed system with the ones for compositional verification of the decomposed system.

In order to carry out the model checking, we refer to the verification property, as given in Figure 4.29. Rephrased in terms of $\operatorname{CSP}_{\mathrm{M}}$ with an additional assertion in regard of the decomposed system, the property is specified in Figure 7.16. It states:

"The parameter value, received by input, corresponds to the output value of result in the next iteration of the protocol."

According to the experimental evaluation of the candy machine, we scale the specification by stepwise increasing the maximal integer value MaxInf. The individual results are given in Tables 7.4 and 7.5, respectively.

Compositional reasoning, particularly with respect to the proof rule (B-AGR), results in drastically worse run-times than non-compositional one. The comparison between direct verification of the original system and the decomposed one mainly yields a draw. Thus, for this case study, decomposing the specification does not yield an advantage over model checking of the undissected system.

\subsubsection{Discussion}

In this section, we evaluated three case studies, and we compared direct model checking with the compositional one with diverse results: 


\begin{tabular}{|l|c|ccc|ccc|}
\hline \multicolumn{1}{|c|}{ Cut } & DC & \multicolumn{3}{c|}{ BR } & \multicolumn{3}{c|}{ PR } \\
& sec & sec & EQ & MQ & sec & EQ & MQ \\
\hline \hline None & $<1$ & n/a & n/a & n/a & n/a & n/a & n/a \\
\hline$\{$ store_b $\},\{$ result $\}$ & $<1$ & 26 & 22 & 2740 & 3 & 32 & 591 \\
\hline
\end{tabular}

(a) Results for MaxInf $=1$

\begin{tabular}{|l|c|ccc|ccc|}
\hline \multicolumn{1}{|c|}{ Cut } & DC & \multicolumn{3}{c|}{ BR } & \multicolumn{3}{c|}{ PR } \\
& sec & sec & EQ & MQ & sec & EQ & MQ \\
\hline \hline None & $<1$ & $\mathrm{n} / \mathrm{a}$ & $\mathrm{n} / \mathrm{a}$ & $\mathrm{n} / \mathrm{a}$ & $\mathrm{n} / \mathrm{a}$ & $\mathrm{n} / \mathrm{a}$ & $\mathrm{n} / \mathrm{a}$ \\
\hline$\{$ store_b $\},\{$ result $\}$ & $<1$ & 249 & 40 & $15 \mathrm{~K}$ & 13 & 57 & 2243 \\
\hline
\end{tabular}

(b) Results for MaxInf $=2$

\begin{tabular}{|l|c|ccc|ccc|}
\hline \multicolumn{1}{|c|}{ Cut } & DC & \multicolumn{3}{c|}{ BR } & \multicolumn{3}{c|}{ PR } \\
& sec & sec & EQ & MQ & sec & EQ & MQ \\
\hline \hline None & $<1$ & n/a & n/a & n/a & n/a & n/a & n/a \\
\hline$\{$ store_b $\},\{$ result $\}$ & $<1$ & 1888 & 66 & $56 \mathrm{~K}$ & 58 & 97 & 5883 \\
\hline
\end{tabular}

(c) Results for MaxInf $=3$

\begin{tabular}{|l|c|ccc|ccc|}
\hline \multicolumn{1}{|c|}{ Cut } & DC & \multicolumn{3}{c|}{ BR } & \multicolumn{3}{c|}{ PR } \\
& sec & sec & EQ & MQ & sec & EQ & MQ \\
\hline \hline None & 1 & n/a & n/a & n/a & n/a & n/a & n/a \\
\hline$\{$ store_b $\},\{$ result $\}$ & 1 & $11 \mathrm{~K}$ & 98 & $155 \mathrm{~K}$ & 230 & 147 & $12 \mathrm{~K}$ \\
\hline
\end{tabular}

(d) Results for MaxInf $=4$

\begin{tabular}{|l|c|ccc|ccc|}
\hline \multicolumn{1}{|c|}{ Cut } & DC & \multicolumn{4}{c|}{ BR } & \multicolumn{3}{c|}{ PR } \\
& sec & sec & EQ & MQ & sec & EQ & MQ \\
\hline \hline None & 3 & n/a & n/a & n/a & n/a & n/a & n/a \\
\hline$\{$ store_b $\},\{$ result $\}$ & 3 & $62 \mathrm{~K}$ & 136 & $355 \mathrm{~K}$ & 779 & 207 & $25 \mathrm{~K}$ \\
\hline
\end{tabular}

(e) Results for MaxInf $=5$

Table 7.4: Experimental results for the (extended) number swapper, first part

1.) For the specification of a candy machine, direct model checking based on the cut $\{$ switch $\}$ outmatches learning-based verification along with direct verification of the original system.

2.) Regarding the Two Phase Commit Protocol, the learning-based method performs best, particularly for the decompositions according to the cuts $\{$ vote, decide $\}$ and $\{$ inform $\}$.

3.) The evaluation of the final case study, the number swapper, showed that a decomposition of the system does not always improve the run-times during model checking in a significant way. 


\begin{tabular}{|l|c|c|c|c|}
\hline \multicolumn{1}{|c|}{ Cut } & $\begin{array}{c}\text { DC / PR } \\
\text { MaxInf }=6\end{array}$ & $\begin{array}{c}\text { DC / PR } \\
\text { MaxInf =7 }\end{array}$ & $\begin{array}{c}\text { DC / PR } \\
\text { MaxInf =8 }\end{array}$ & $\begin{array}{c}\text { DC / PR } \\
\text { MaxInf =9 }\end{array}$ \\
\hline \hline None & 10 & 23 & 52 & 108 \\
\hline$\{$ store_b $\},\{$ result $\}$ & $10 / 2498$ & $24 / 7209$ & $52 / 19 \mathrm{~K}$ & $10 /(-)$ \\
\hline
\end{tabular}

(a) Results for MaxInf $\in\{6,7,8,9\}$

\begin{tabular}{|l|c|c|c|}
\hline \multicolumn{1}{|c|}{ Cut } & $\begin{array}{c}\text { DC } \\
\text { MaxInf }=10\end{array}$ & $\begin{array}{c}\text { DC } \\
\text { MaxInf }=11\end{array}$ & $\begin{array}{c}\text { DC } \\
\text { MaxInf }=12\end{array}$ \\
\hline \hline None & 209 & 379 & $(*)$ \\
\hline$\{$ store_b $\},\{$ result $\}$ & 207 & 372 & $(*)$ \\
\hline
\end{tabular}

(b) Results for MaxInf $\geq 10$

Table 7.5: Experimental results for the (extended) number swapper, second part

A summary of the results is given in Table 7.6. They will lead us to some context-specific conjectures, which we will discuss next. In order to draw some conclusions and develop an intuition on when decomposing a system plus applying compositional verification might pay off, we investigate the specific model checker FDR2 and the structure of the different case studies. Note that the following interpretations and considerations are mostly educated guesses and conjectures: neither can we precisely estimate the model checking procedure of FDR2, nor can we draw detailed and irrefutable conclusions from a heuristics-based technique.

\begin{tabular}{|l|ccc|}
\hline Verification Technique & \multicolumn{3}{|c|}{ Case Study } \\
& Candy Machine & TPCP & Number Swapper \\
\hline \hline Direct, original system & - & - & + \\
\hline Direct, decomposition & + & - & + \\
\hline Compositional, decomposition & $\circ$ & + & - \\
\hline
\end{tabular}

Table 7.6: Summary of the experimental results

\section{General Conclusions}

Based on the experimental results, we discuss some general observations. First, we experienced that the order of both components within the assertion is relevant for basic reasoning and - due to the nature of the symmetric proof rule - irrelevant for parallel reasoning. For basic reasoning, model checking of Prop $\bigsqcup_{T}\left(S_{1} \| S_{2}\right)$ generally performed better than the one of Prop $\sqsubseteq_{T}\left(S_{2} \| S_{1}\right)$. The previous tables thus always refer to the case of Prop $\sqsubseteq_{T}\left(S_{1} \| S_{2}\right)$.

Next, we observed that two particular criteria were most relevant for the measured 
run-times of model checking. The first one is related to the additional address- and transmission parameters: parameters of high cardinality significantly increase the runtimes. As the type of transmission parameters is arbitrary, decompositions without transmission parameters or, at least, transmission parameters of small type-cardinality should be favoured. As a second, closely related criterion, the amount of cut nodes highly influences the duration of model checking. For our case studies, we experienced that cuts with a size of more than two nodes generally lead to comparatively bad results. As both criteria determine the number of events, which have to be synchronised in the decomposition, both observations substantiate the claim from Section 6.1: the interface between both components needs to be small.

Another observation is related to the specific model checker we used for the evaluation: the behaviour of FDR2 in the context of the learning based approach is generally non-deterministic, and it is nearly impossible to draw conclusions on how the amount of membership queries and equivalence queries can be reduced [Won08]. For instance, a reordering of the specification's parameters changes the number of intermediate assumptions. However, there is no general rule which orderings should generally be favoured.

Regarding the comparison of parallel reasoning and basic reasoning, basic reasoning mostly outmatched the parallel one. This might be related to the specific case studies which we investigated: the candy machine and the Two Phase Commit Protocol can be seen as sequential systems without outer recursion, thus favouring the specific sequential structure of the rule (B-AGR). Yet, for the case study of the number swapper, even though parallel reasoning performed better that the basic one, run-times were significantly higher compared to direct model checking. This raises doubts on the usefulness of parallel reasoning in general.

Finally, we want to substantiate the claim that not only the final transition graph, generated during model checking, is relevant for the number of explored states. We illustrate this by an example.

Example 7.3.1. Let us consider the following simple CSP-OZ specification.

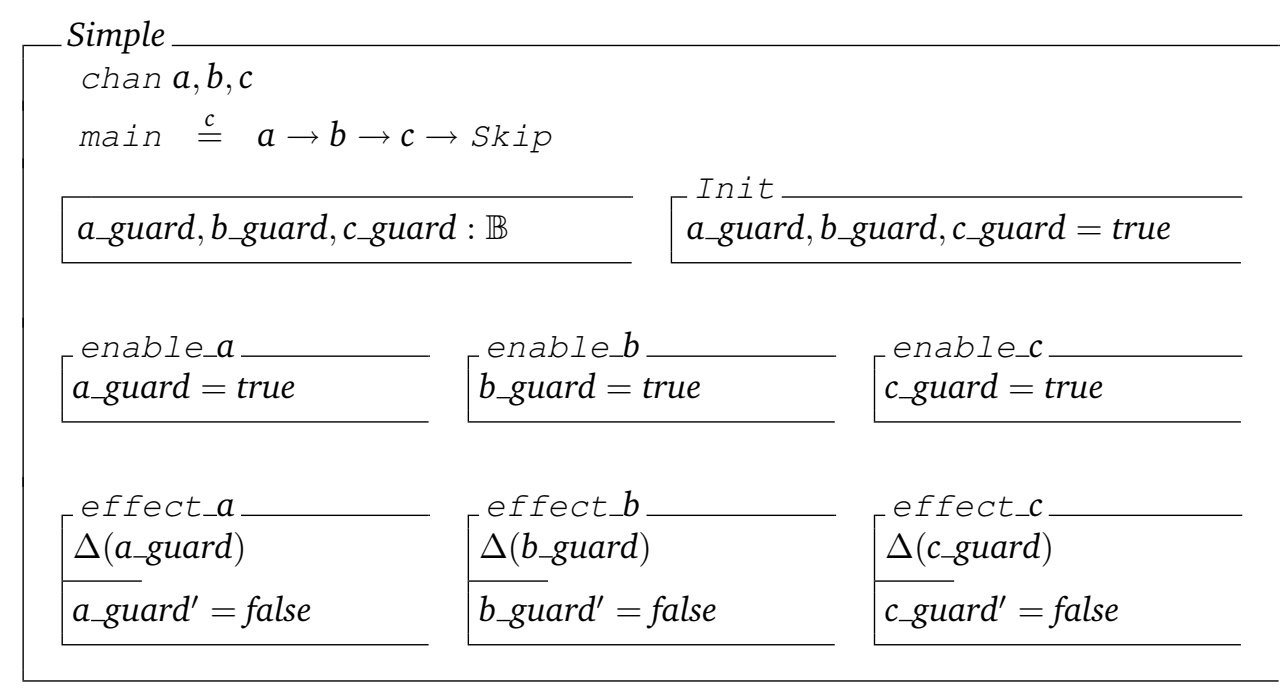


The CSP process of the class solely allows for the trace $\langle a, b, c\rangle$. For the Object-Z part, any ordering of operations is possible, as long as each operation is only called once. Thus, in order to analyse the specification, the parallel composition of the two transition systems

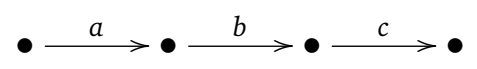

and

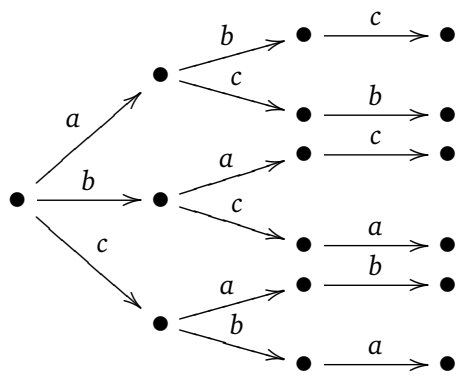

needs to be computed (without denoting the state variables of the Object-Z part). Even though the transition system of the overall process is identical to the one for the CSP part, the much bigger transition system of the Object-Z part must be computed as well before the parallel composition can be carried out.

Now assume we decompose the specification based on the valid single cut $\mathbf{C}=\{b\}$. In this case, the transition system for the first component is a parallel composition of

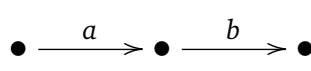

and

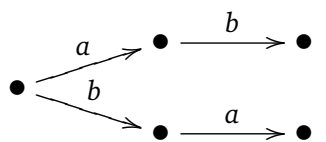

For the transition system of the second component, the event $b$ is simply replaced by the event $c$. The final transition systems for the original specification and the decomposed one are identical and according to the one of the original CSP part. However, the size of the intermediate system differs: for the original system, there are 19 states and 18 transitions, the decomposed systems needs to cope with only 16 states and 12 transitions. Thus, (direct) model checking with respect to the original model needs to explore more states than the compositional one.

The example particularly shows that direct model checking of a decomposed system can indeed outmatch direct model checking of the original specification. This can be the case if the decomposition results in smaller intermediate transition systems due to, for instance, a significant reduction of interleaving or an effective distribution of the set of state variables.

\section{Evaluation Analysis: Candy Machine}

We quote some further case-study-specific observations, and we start by analysing the results for the candy machine. The state space of the specification particularly comprises two sequences paid and items. Even though FDR2 supports the specification of sequences, generating the set over all possible sequences of finite length $n$ for some specific data type with cardinality $k$ results in $k^{n}$ elements. This is further substantiated by the fact 
that FDR2 mainly applies explicit model checking techniques and generally needs to deal with the full state space of a system.

Consider the decomposition of the specification with respect to the cut $\{$ switch $\}$, as given in Section 4.3.6. It results in a distribution of the state variables paid and items over both components - paid is assigned to CandyMachine ${ }_{1}$, and items is assigned to CandyMachine $_{2}$. Thus, the individual state spaces of the Object- $Z$ parts of the specification are significantly smaller than the state space of the original system. Hence, it is most likely that model checking with respect to $\mathbf{C}_{1}=\{$ switch $\}$ performs comparatively better than the one for the original system.

Regarding the cut $\mathbf{C}_{2}=\{$ abort, order, select, switch $\}$, the corresponding decomposition requires a transmission parameter of type seq Candies. In addition, the number of cut nodes is equal to four. Thus, model checking with respect to $\mathbf{C}_{2}$ leads to comparatively poor results.

Yet, the question remains why direct model checking outperforms the compositional one. As already mentioned, the performance of learning-based compositional reasoning depends on the number of intermediate assumptions. According to [Won08] and due to the black-box character of FDR2, it seems quite difficult to pre-estimate this number.

\section{Evaluation Analysis: Two Phase Commit Protocol}

In $\left[\mathrm{dRHH}^{+} 01\right]$, the motivation for introducing and specifying the Two Phase Commit Protocol is its particular structure, allowing for an appliance of the Communication-ClosedLayers law (CCL) [EF82]. Our way of decomposing a specification is one particular way of adopting the CCL, which leads to a transformation of a specification with a distributed or concurrent structure - such as the parallel composition of several processes - to a sequential or layered structure, consisting of several phases.

The evaluation of this specific case study shows that the structure of the TPCP facilitates an application of compositional techniques. In particular, the protocol itself consists of two phases, which are nearly independent.

Quite surprisingly, the cut yielding the minimal run-times during model checking is $\mathbf{C}=\{$ vote, decide $\}$. Figures 7.17 and 7.18 show the decomposition of the specification according to $\mathrm{C}$. In order to address specific instances of Page ${ }_{1}$ and $\mathrm{Page}_{2}$, we adopt CSP-OZ's concept of constant parameters ([Fis00]).

This specific cut reflects the loose connection between the commit-request-phase and the commit-phase: the corresponding decomposition only requires one transmission parameter of type Trans $=\{C O M M I T, A B O R T\}$ for the operation decide. This parameter represents the final decision to either commit or abort a transaction and thus, the point of intersection between both phases. As decide only occurs once within the specification, this parameter of type cardinality 2 is only used once as well. Contrary, the cut $\{$ vote $\}$ requires a transmission parameter of type $\mathbb{P}$ Votes for the operation vote. Thus, the cardinality of the type of this operation is larger than the one for the parameter of decide. Moreover and more importantly, vote occurs once in each instance of Page and $N$ times within Coord. This requires the transmission parameter to be added to all $N$ occurrences within Coord. Therefore, even though the cut $\mathbf{C}_{5}=\{$ vote $\}$ only comprises one operation schema, model 


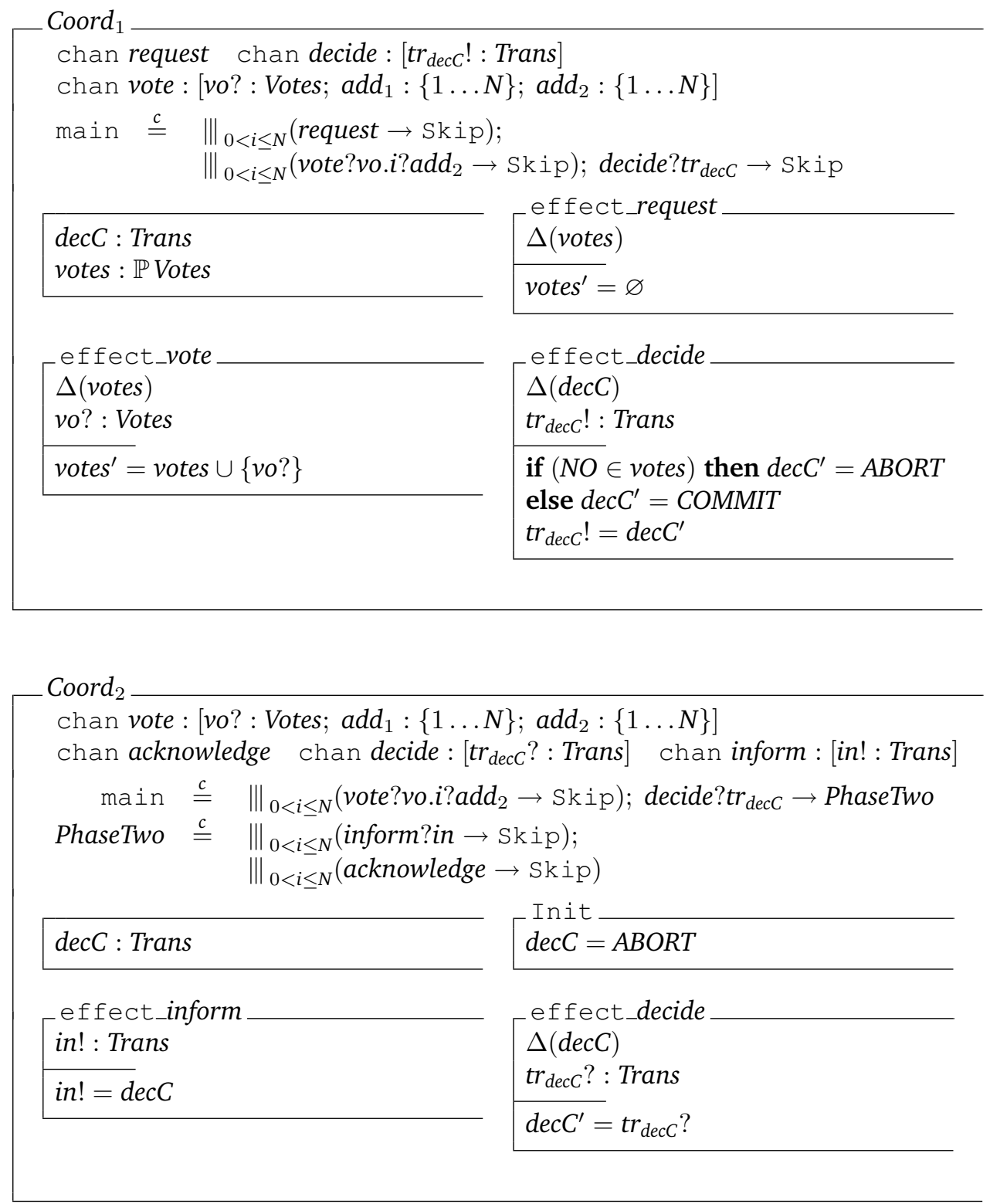

Figure 7.17: Decomposition of the TPCP: Coord specification

checking needs to cope with comparatively more events than the one of the decomposition according to $\{v o t e$, decide $\}$. Figure 7.19 illustrates the predominance of $\{v o t e$, decide $\}$.

Similarly, for the decomposition with respect to the cut $\mathbf{C}_{1}=\{$ inform $\}$, one additional transmission parameter of type Trans is required, and inform occurs multiple times within the specification. The larger the amount of pages, the more occurrences of vote and inform and thus, the more cut events for $\mathbf{C}_{1}$ and $\mathbf{C}_{5}$. This is reflected in the evaluation results: the larger the model, the better performs $\{$ vote,decide $\}$ in comparison to the 

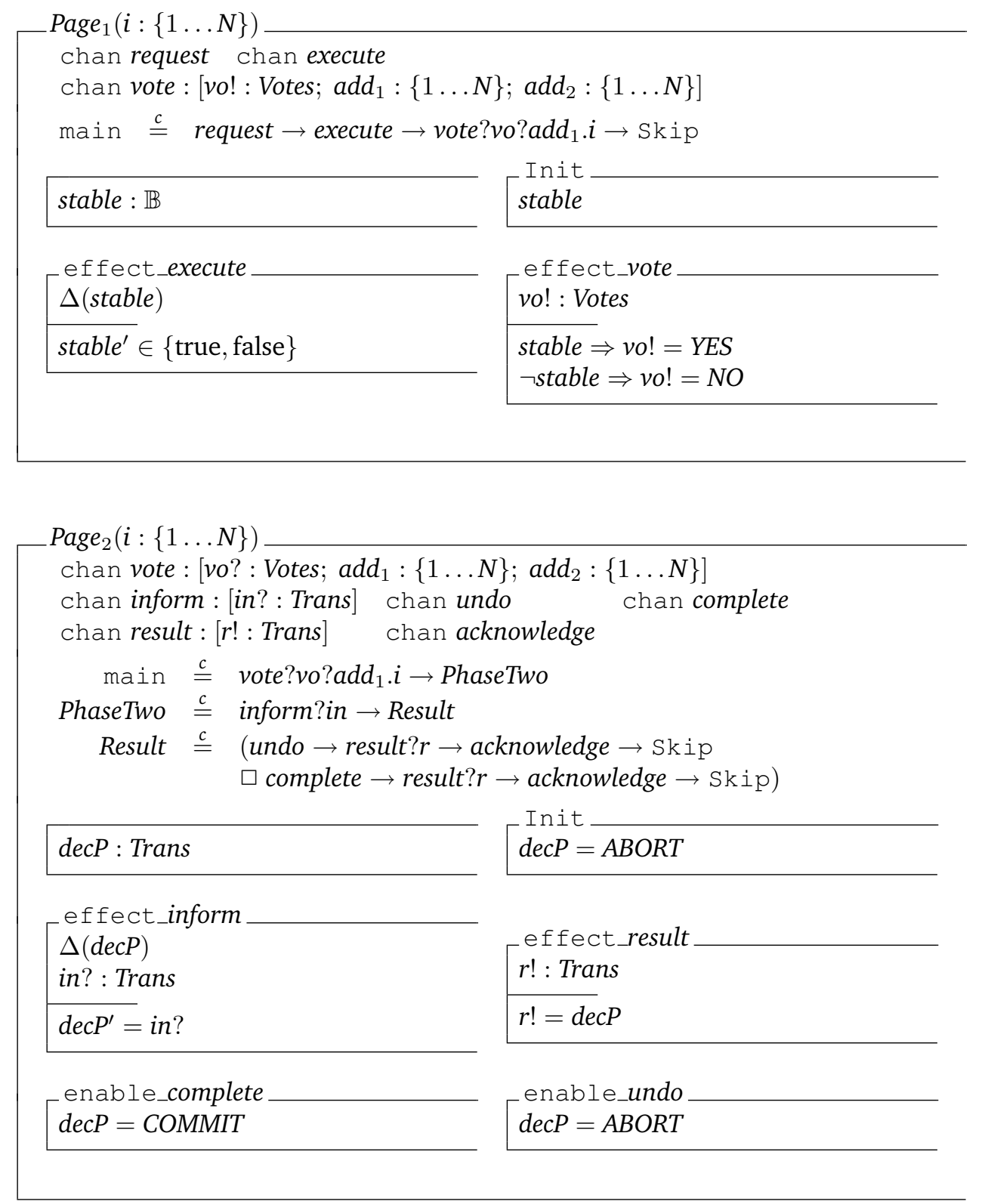

Figure 7.18: Decomposition of the TPCP: Page specification

other cuts.

Still, within the mass validation framework, the cut $\{$ inform $\}$ receives the minimal value, if we set an equal weight for all heuristics. This is due to the comparatively smaller values for the heuristics cut size and even distribution. The example substantiates to offer a possibility of scaling the different heuristics: a higher weight for few transmission will cause $\{$ vote, decide $\}$ to pass $\{$ inform $\}$ in terms of the overall value. 

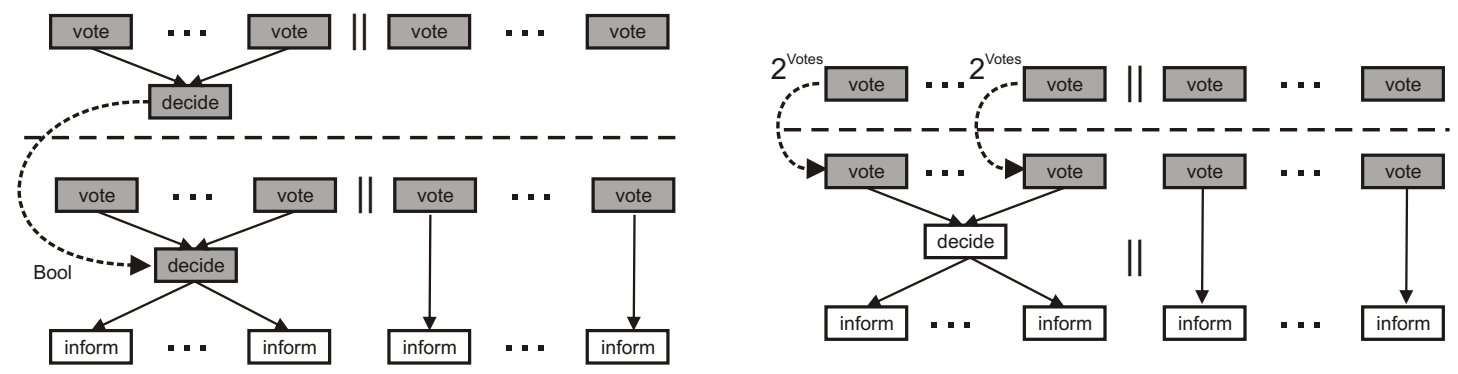

Figure 7.19: Justification for predominance of cut $\{$ vote, decide $\}$

\section{Evaluation Analysis: Number Swapper}

The results for the number swapper showed that the decomposition of a system does not generally lead to a significant improvement in regard of model checking run-times. In this specific case, the structure of the system does not allow for a decomposition beneficial for compositional verification: the CSP part of the specification itself comprises five events and only allows for a general cut. Thus, one of the components inevitably comprises four events. Moreover, store $b$ requires an additional transmission parameter, increasing the size of the interface between both components. In conclusion, learning-based model checking results in poor run-times.

Still, by decomposing the number swapper, the structure of the specification is mainly maintained. There is no advantage in the application of direct model checking of the original system over direct model checking of the decomposed system.

\section{Summary}

The evaluation results for the different case studies of this thesis highly differ. Summing up, we can conclude that there is no universal best strategy which type of verification one should choose. Still, we identified some rules-of-thumb for when to apply which strategy: in general, applying the decomposition technique can be promising, if the underlying system can be distributed in a reasonable way. This can either mean a split-up of the CSP part into two phases without a large intersection between both parts (as, for instance, the Two Phase Commit Protocol and the cut $\{$ vote, decide $\}$ ) or a reasonable distribution of its set of state variables (as, for instance, the candy machine and the cut $\{$ switch $\}$ ).

The decomposition approach will not always be beneficial. In particular, if the system is tightly coupled, a decomposition might not significantly reduce run-times during (compositional) verification. However, the technique is generally applicable and for none of the case studies did direct model checking of the original system perform best. Even though one of our case studies represents a tightly coupled system, direct model checking of its decomposition results in run-times, which are comparable to the ones for the model checking of the undissected system. 



\section{Conclusion}

\section{Contents}

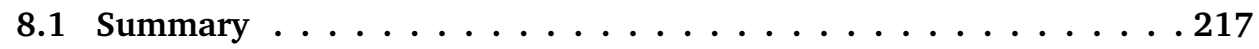

8.2 Future Work . . . . . . . . . . . . . . . . 219

The present chapter concludes this thesis with a summary. Subsequently, we discuss some topics for future research.

\subsection{Summary}

Within this thesis, we introduced a decomposition technique for software models, specified in an integrated formalism. The primary motivation for this approach arose from the major challenge of automated software verification: the state explosion problem. In order to allow model checking to scale to complex systems, appropriate measures need to be taken. Compositional verification is one possible way of dealing with the state explosion. The technique follows a "divide and conquer" approach: instead of verifying the system as a whole, the components of the system are independently verified. An appropriate combination of the verification results yields the correctness of the system. Compositional reasoning avoids the state explosion problem to a certain extent, if the overall state space of the components is comparatively smaller than the one of the original system.

After a short introduction to the topic, Chapter 2 provided background information on the modelling and the analysis of software models. First, we surveyed the field of integrated formal methods. Next, we presented the syntax and the semantics of the underlying integrated formalism of this thesis, CSP-OZ, and we exemplified it by means of a case study. Furthermore, a dependence analysis for CSP-OZ specifications was introduced. Here, we defined the specification's dependence graph, reflecting the control flow and the data flow of a software model. The dependence graph provides the basis for a further analysis and, eventually, a decomposition of a specification.

The second background chapter, Chapter 3, focussed on strategies for the automated verification of a software model and, in particular, compositional verification. We provided an overview on related and complementary techniques to cope with the state explosion problem. The assume-guarantee paradigm of compositional reasoning was introduced, along with two inference proof rules. Both proof rules are applied within our implementation framework, and they use the $L^{*}$ algorithm for an automatic detection of intermediate assumptions during model checking.

The first core chapter of this thesis, Chapter 4, described the actual decomposition of a CSP-OZ specification. The general idea for the approach is the definition of a cut of the 
specification's dependence graph. In order to ensure the validity of the decomposition, that is, the semantic equivalence of the decomposed system and the original system, a cut needs to comply with four correctness criteria. We separated the general case from the specific case of a single cut, mainly to allow a certain class of systems to be decomposed in a more effective way. Subsequently, we defined a model's decomposition with respect to a valid cut. In order to guarantee the equivalence between the original and the decomposed system, additional modifications of the resulting components had to be introduced. Mainly, these modifications required the introduction of additional parameters in order to restore the specification's original control flow and data flow.

Chapter 5 showed correctness of the decomposition technique in terms of the trace equivalence of the original and the generated model. The proof employed the operational semantics of CSP-OZ. We compositionally showed the correctness of both, the decomposition of the CSP part and the one of the Object-Z part. For the CSP part, we showed bisimilarity of the considered CSP processes, taking into account the additional address parameters within the CSP part of the decomposition. For the correctness proof of the Object-Z part, we showed trace equivalence by explicitly constructing the respective transition paths and by using transmission parameters. Finally, both individual results were used to deduce the overall correctness of the decomposition, additionally requiring several CSP-related laws for renaming and for a redistribution of CSP processes.

As the validity of a decomposition does not automatically yield a system for which model checking can effectively be carried out, Chapter 6 introduced several contextspecific heuristics to measure the quality of a decomposition. A classification of all valid cuts is carried out in two steps: first, all unreasonable and dominated cuts are sorted out. Second, the remaining cuts can be scaled, according to the heuristics.

Finally, Chapter 7 evaluated the approach on three case studies. As the underlying platform, we chose the UML-based modelling environment Syspect and the CSP model checker FDR2. In order to integrate the decomposition technique into the existing framework, several extensions to Syspect were carried out. We compared run-times for direct and compositional model checking for the original systems and the decompositions.

In summary, within this thesis, we developed a technique to effectively apply compositional verification for software models, specified in an integrated formalism. We mainly answered the following two questions:

1. How can we determine the set of all valid decompositions of a specification?

2. How can these decompositions be classified and measured regarding their effectiveness for compositional model checking?

We further implemented the approach by integrating it into an existing tool for the modelling and the analysis of software specifications. Based on the results obtained, we observed that the decomposition technique can lead to a considerable speed-up of both, compositional verification and direct verification. 


\subsection{Future Work}

The present work opened several perspectives and ideas for future research, which we will discuss next. Particularly, we detail some further extensions and some possibilities on how to combine the approach of this thesis with complementary techniques.

Target Area of Application: The decomposition technique of this thesis has been carried out with respect to the integrated formal method CSP-OZ. Yet, the approach presents the major benefit of being independent from a specific formalism: the theory of Chapter 4 used the (program) dependence graph (DG) of a specification in order to decompose a software model. DGs are a commonly-used and languageindependent way of representing a software system [HR92]. Thus, the technique can be transformed to fit to any language with an underlying dependence graph representation for its models. The general idea behind the decomposition of the model in terms of restoring the original control flow and the data flow can be used accordingly. The correctness criteria need to be adapted, according to the context specific semantic model and the equivalence requirements.

Semantic Model: As the semantics of CSP-OZ are given in terms of CSP alone [Fis00], our correctness proof referred to the semantic domain of CSP. Within the general context of learning-based model checking of safety properties, we were interested in analysing the observable behaviour of a specification. This allowed us to restrict ourselves to the semantic model of traces, that is, the sequences of communication events: the trace semantics is sufficient for showing the observable equivalence of two systems [CGP03, Weh00]. In order to analyse liveness properties as, for instance, deadlock or livelock freedom, the decomposition technique could as well be extended to the more discriminating failures-divergences model of CSP. This semantic model additionally takes the refusals of events and infinite sequences of internal actions into account. The extension would require a modification of the correctness proof and, possibly, some additional correctness criteria in order to ensure the failure-divergence equivalence of the original system and its decomposition.

Evaluation with ProB: An evaluation of the decomposition technique was carried out by using the CSP model checker FDR2. The choice was justified by several aspects, which were given in Section 7.2.3. In order to realise a more profound analysis, as a meaningful measure, the approach could be evaluated for a second model checker. Recently, ProB [Leu], an animator and model checker for the B-Method [Abr96], was extended to support $\mathrm{CSP}_{\mathrm{M}}$ as the input language [LF08]. Thus, an implementation of the learning framework from Chapter 3 for ProB and a further comparison of evaluation results for ProB and FDR2 could be advisable.

Weakening of Correctness Criteria: One correctness criterion for a valid fragmentation of the DG states that data dependences must not circumvent the set of cut operations. The criterion was justified by the fact that the influence from one specification part on the other one needs to be preserved. Our decomposition approach ensured this 
by using transmission parameters. A possible weakening of the cut definition could be a neglect of this criterion. In this case, transmission parameters would have to be used for state variables which are modified before the cut as well. In general, these considerations result in a trade-off between, on the one hand, the growing amount of valid cuts and, on the other hand, the more complex evaluation of the set of all valid cuts. Yet, we observed that a large set of additional parameter values considerably slows down model checking, raising doubts on whether this strategy is a way to success.

Recursive Learning: Previous works [GGP07, $\mathrm{PGB}^{+}$08] extended the learning framework to systems with an arbitrary number of components. Here, the specification is stepwise decomposed, using a recursive application of the learning algorithm. Wonisch already integrated the method into the CSPLChecker [WW09]. His extension allows to recursively apply both assume-guarantee-based proof rules with respect to a specific split-ratio and systems which are parallel composed of $n$ components. The theory of our thesis implicitly supports the recursive decomposition of a system, as the two resulting specification components are CSP-OZ specifications as well. In order to integrate this extension into Syspect, a re-import of the decomposed model and, in particular, a computation of the respective dependence graphs is necessary.

Arbitrary Amount of Cut Sets: According to Definition 4.2.8, a general cut refers to two cut sets, and it yields a fragmentation of the DG into two parts. Clearly, this approach might be extended to an arbitrary amount of lines of intersection, yielding a decomposition of a model into a corresponding number of components. However, within this thesis, we restricted ourselves to two cut sets: as previously explained, the approach supports a recursive decomposition, already allowing for a decomposition into an arbitrary amount of components without the need to generalise and further complicate Definition 4.2.8.

Combination with other Techniques: Another motivation for the re-import of a CSP-OZ class specification into Syspect is given by the possibility to combine two techniques, the slicing approach from [Brü08] and the decomposition method of this thesis: if the verification requirement is at hand, an obvious strategy is to first slice the original model with respect to the given requirement, re-import the slice and decompose it according to our technique. Moreover, the presented technique is generally compatible to other approaches to the state explosion problem.

Decomposition Implementation: Chapter 7 presented the implementation of the decomposition approach within the UML-based modelling tool Syspect. Here, several future extensions are possible, mainly for closing the gaps between the theory and the implementation and for facilitating the tool handling.

- Implementation of General Cut Theory: The implementation of the decomposition technique within Syspect is currently restricted to the special case of a single cut. In order to allow the tool to support the decomposition of arbitrary 
specifications, an extension of the according plug-in is required. Here, the main aspect to deal with is to allow the definition of two separate cut sets, with each of them complying to the implemented theory for one cut set.

- Implementation of Decomposition Improvement: In Section 4.3.7, we discussed an improvement of the decomposition approach in terms of reducing the set of initial data dependences. This optimisation is not yet implemented within Syspect and thus, several valid cuts are currently rejected. An implementation of this improvement, dependent on the respective specification, would lead to larger set of valid cuts.

- Modelling of Verification Properties: Currently, a manual specification of verification properties in terms of $\operatorname{CSP}_{\mathrm{M}}$ is required. As a facilitation, a modelling of the system requirements as a transition system is imaginable. Such an editor could be similar to the existing Syspect state machine editor.

- Extension of Counter Trace Plug-In: Section 7.2.4 introduced the countertrace plug-in, a Syspect extension for visualising counterexamples. Currently, the analysis only considers the CSP part of a specification. Thus, the set of detected error traces is possibly too large. An additional analysis of the Object-Z part would yield an exact counterexample analysis.

- Elimination of System Classes: As a more technical aspect, the $\mathrm{BT}_{\mathrm{E}} \mathrm{X}$-export of a Syspect specification, comprising more than one class, requires the definition of an additional class for describing the overall system composition. These classes may be replaced by a simple CSP process, as they comprise an empty Object-Z part. In order to speed-up model checking, the definition of the overall system within Syspect should be given by a CSP process instead of a CSP-OZ class. 



\title{
Glossary of Symbols
}

\author{
CSP-OZ (Section 2.2)
}

S.I

the interface definition of a specification $S$

S.Events

the global set of events of a specification $S$

S.OZ

the Object-Z part of a CSP-OZ specification $S$

S.main

the CSP part of a CSP-OZ specification $S$

\section{Object-Z part (Section 2.2)}

\begin{tabular}{|c|c|}
\hline State & the state schema of $O Z$ \\
\hline Init & the initial state schema of $O Z$ \\
\hline$O p$ & the set of operation schemas of $O Z$ \\
\hline enable_op & the precondition of the operation schema op \\
\hline effect_op & the effect of the the operation schema $o p$ \\
\hline op.delta & the delta list of the operation schema op \\
\hline op.dec & the parameter declaration part of the operation schema op \\
\hline op.pred & the predicate part of the operation schema op \\
\hline $\operatorname{In}(o p)$ & the set of possible values for the input parameters of $o p$ \\
\hline Simple(op) & the set of possible values for the simple parameters of op \\
\hline Out(op) & the set of possible values for the output parameters of $o p$ \\
\hline ref $($ op $)$ & the set of referenced variables of the operation schema op \\
\hline $\bmod (o p)$ & the set of modified variables of the operation schema $o p$ \\
\hline $\operatorname{all}(o p)$ & the union of the sets of referenced and modified variables of $o p$ \\
\hline$s \uparrow V$ & the state $s$, projected onto the set of state variables $V$ \\
\hline$M^{O Z}$ & the labelled transition system of $O Z$ \\
\hline $\operatorname{Traces}(\mathrm{OZ})$ & the set of traces of the Object- $Z$ part \\
\hline$\pi[i]$ & the $i$-th state of $\pi \in \operatorname{Traces}(\mathrm{OZ})$ \\
\hline$\pi . i$ & the $i$-th event of $\pi \in \operatorname{Traces}(\mathrm{OZ})$ \\
\hline $\operatorname{traces}(\mathrm{OZ})$ & the set of traces of $O Z$, projected onto events \\
\hline $\operatorname{races}(\mathrm{OZ}) \triangleright O p$ & the set of traces of $O Z$, projected onto operation names \\
\hline
\end{tabular}




\section{CSP part (Section 2.2)}

$\begin{array}{ll}\text { Skip } & \text { termination } \\ \text { Stop } & \text { deadlock } \\ a \rightarrow P & a \text { then } P \\ P_{1} \square P_{2} & P_{1} \text { external choice } P_{2} \\ P_{1} \sqcap P_{2} & P_{1} \text { internal choice } P_{2} \\ P_{1} \|_{A} P_{2} & P_{1} \text { parallel on } A P_{2} \\ P_{1} A_{1} \|_{A_{2}} P_{2} & P_{1} \text { parallel on } A_{1}, A_{2} P_{2} \\ P_{1} \| P_{2} & P_{1} \text { interleave } P_{2} \\ P_{1} \backslash A & P \text { hide } A \\ P \llbracket \mathrm{R} \rrbracket & P \text { renamed by R (relational renaming }) \\ \text { main } & \text { the initial CSP process of a CSP-OZ specification } \\ L C S P & \text { the set of all CSP terms } \\ M^{C S P} & \text { the labelled transition system of main } \\ \operatorname{tr}\lceil E & \text { the restriction of the trace } t r \text { on events in } E \\ \text { traces }(\text { main }) \triangleright O p & \text { the set of traces of main, projected onto operation names } \\ \operatorname{tr.i} & \text { the } i \text {-th event of the trace tr } \\ \text { initials }(P) & \text { the initial events of the process } P \\ f o o t(t r) & \text { the last event of the trace } t r \\ \left.P\right|_{E} & \text { the projection of the process } P \text { on events in } E \\ P \sqsubseteq T & Q \text { is a trace refinement of } P \\ P={ }_{T} Q & P \text { and } Q \text { are trace equivalent } \\ \alpha P & \text { the alphabet of the process } P \\ \{|C|\} & \text { the extension set for the set of channels } C \\ c_{p} & \text { a partial event for the channel } c\end{array}$




\section{Dependence Graphs (Section 2.3)}

\begin{tabular}{|c|c|}
\hline $\mathrm{DG}_{\mathrm{S}}=\left(N, \longrightarrow_{\mathrm{DG}}\right)$ & the dependence graph of a specification $S$ \\
\hline $\mathrm{CFG}_{\mathrm{S}}=(N, \longrightarrow)$ & the control flow graph of a specification $S$ \\
\hline $\mathrm{DDG}_{\mathrm{S}}=(\mathrm{op}(N),--\rightarrow)$ & the data dependence graph of a specification $S$ \\
\hline $\mathrm{op}(N)$ & the set of operation nodes of a dependence graph \\
\hline $\operatorname{cf}(N)$ & the set of CSP operator nodes of a dependence graph \\
\hline$\longrightarrow$ & a control dependence \\
\hline$\stackrel{d d}{--\rightarrow}$ & a direct data dependence \\
\hline$\stackrel{\mathrm{dd}}{--\rightarrow}(v)$ & a direct data dependence by reason of $v$ \\
\hline$\stackrel{\text { idd }}{-\rightarrow-\rightarrow}$ & an initial data dependence \\
\hline$\stackrel{s d}{\leftrightarrow--\rightarrow}$ & a synchronisation dependence \\
\hline$\stackrel{\text { sdd }}{--\rightarrow}$ & a synchronisation data dependence \\
\hline$\underset{--\rightarrow}{i f d d}$ & an interference data dependence \\
\hline$\underset{-i f d d}{i-\rightarrow}(v)$ & an interference data dependence by reason of $v$ \\
\hline $\mathrm{path}_{\mathrm{DG} / \mathrm{CFG}}$ & the paths of the DG / CFG \\
\hline $\operatorname{path}_{\mathrm{DG}} / \mathrm{CFG}\left(\mathrm{n}, \mathrm{n}^{\prime}\right)$ & the paths of the DG / CFG from $n$ to $n$ ' \\
\hline $\operatorname{succ}(n)$ & the sole successor of the node $n$ \\
\hline succ_one(n) & the first successor of the node $n$ \\
\hline succ_two(n) & the second successor of the node $n$ \\
\hline
\end{tabular}

\section{Compositional Reasoning (Sections 3.2 and 3.3)}

(B-AGR)

(P-AGR)

(C-AGR)

$\mathcal{L}(A)$

$\mathcal{L}(A)^{C}$

$\Sigma^{*}$

$\Sigma^{\omega}$ the basic assume-guarantee proof rule

the parallel assume-guarantee proof rule

the circular assume-guarantee proof rule

the language of the assumption $A$, given as a DFA

the complement of the language $\mathcal{L}(A)$

the set of finite words over $\Sigma$

the set of infinite words over $\Sigma$ 


\section{Cut of a Dependence Graph (Section 4.2)}
$\mathbf{C}=\left(\mathbf{C}_{1}, \mathbf{C}_{2}\right)$
a (general) cut of a dependence graph
$\mathbf{C}=\left(\mathbf{C}_{1}, \varnothing\right)$
a single cut of a dependence graph
$\mathrm{Ph}_{i}$
the $i$-th phase of a fragmentation of a dependence graph
$N_{1} \stackrel{\text { to }}{\longrightarrow} N_{2}$
the interval of nodes from $N_{1}$ to $N_{2}$ of a dependence graph
disjointness
the first correctness constraint on a valid cut
no crossing
the second correctness constraint on a valid cut
no reaching back
the third correctness constraint on a valid cut
all-or-none
the fourth correctness constraint on a valid cut

\section{Decomposition of a Specification (Section 4.3)}

$S_{1}$

$S_{2}$

CV

op.orig

op.add

op.tr_in

op.tr_out

$O p_{i}$

$O p_{\mathrm{C}}$

$O p^{\prime}$

$E_{S_{i}}$

$E_{S^{\prime}}$

$\mathrm{R}^{\mathrm{C}}$

$\mathrm{R}^{\prime}$

$\operatorname{InitClos}(x)$ the first component of a decomposition of $S$

the second component of a decomposition of $S$

the set of cut variables

the set of original parameters of the operation $o p$

the set of address parameters of the operation op

the set of transmission parameters of the operation $o p$, decorated with "?"

the set of transmission parameters of the operation $o p$, decorated with "!"

the set of local operation schemas of the component $S_{i}$

the set of cut operation schemas

the union $O p_{1} \cup O p_{2} \cup O p_{\mathrm{C}}$

the set of events of the component $S_{i}$

the union $E_{S_{1}} \cup E_{S_{2}}$

the (relational) event renaming for a decomposition of $S$

the inverse renaming relation

the initial closure of the state variable $x$ 


\section{Correctness Proof (Chapter 5)}

noev a special event to denote stuttering

$\overline{C V} \quad$ the shared state variables, excluding cut variables

$s \oplus t \quad$ the state $t$ overrides the state $s$

\section{Decomposition Heuristics (Section 6.1)}

$h_{\mathrm{CS}}$

$h_{\mathrm{ED}}$

$h_{\mathrm{FT}}$

$h_{\mathrm{FA}}$

\section{Predicate Logic}

$\operatorname{Free}(p)$

$p[x / a]$

$\operatorname{Atoms}(p)$

$\operatorname{vars}(p)$

\section{Miscellaneous}

$\operatorname{ld} X$ the heuristic for minimising the cut size

the heuristic for minimising the size difference

the heuristic for minimising the transmission

the heuristic for minimising the addressing

the set of free variables occurring in the predicate $p$

the predicate $p$ with all free occurrences of $x$ replaced with $a$

the set of atomic sub-predicates of the predicate $p$

the set of variables occurring in the predicate $p$

the identity on $X$, that is, the set $\{(x, x) \mid x \in X\}$ 



\section{Bibliography}

[Abr96] J.-R. Abrial. The B Book - Assigning Programs to Meanings. Cambridge University Press, 1996.

[Abr06] J.-R. Abrial. Formal methods in industry: achievements, problems, future. In ICSE '06: Proceedings of the 28th international conference on Software engineering, pages 761-768, New York, NY, USA, 2006. ACM.

[ACHH92] R. Alur, C. Courcoubetis, T. A. Henzinger, and P.-H. Ho. Hybrid automata: An algorithmic approach to the specification and verification of hybrid systems. In Hybrid Systems, volume 736 of Lecture Notes in Computer Science, pages 209-229. Springer, 1992.

[AČMN05] R. Alur, P. Černý, P. Madhusudan, and W. Nam. Synthesis of interface specifications for Java classes. ACM SIGPLAN Notices, 40(1):98-109, 2005.

[AD94] R. Alur and D. L. Dill. A theory of timed automata. Theoretical Computer Science, 126(2):183-235, 1994.

[AH06] J.-R. Abrial and S. Hallerstede. Refinement, decomposition, and instantiation of discrete models: Application to Event-B. Fundamenta Informaticae, XXI, 2006.

[All70] F. E. Allen. Control flow analysis. SIGPLAN Not., 5(7):1-19, 1970.

[AMN05] R. Alur, P. Madhusudan, and W. Nam. Symbolic compositional verification by learning assumptions. In Computer Aided Verification, 17th International Conference, CAV 2005, Edinburgh, Scotland, UK, July 6-10, 2005, Proceedings, volume 3576 of Lecture Notes in Computer Science, pages 548-562. Springer, 2005.

[Ang87] D. Angluin. Learning regular sets from queries and counterexamples. Information and Computation, 75:87-106, 1987.

$\left[\mathrm{APR}^{+} 01\right] \quad$ T. Arons, A. Pnueli, S. Ruah, J. Xu, and L. D. Zuck. Parameterized verification with automatically computed inductive assertions. In CAV'01: Proceedings of the 13th International Conference on Computer Aided Verification, pages 221-234, London, UK, 2001. Springer-Verlag.

$\left[\mathrm{BBK}^{+}\right.$04] M. Balser, S. Bäumler, A. Knapp, W. Reif, and A. Thums. Interactive verification of UML state machines. In Formal Methods and Software Engineering, 6th International Conference on Formal Engineering Methods, ICFEM 2004, Seattle, WA, USA, November 8-12, 2004, Proceedings, volume 3308 of Lecture Notes in Computer Science, pages 434-448. Springer, 2004.

[BCC98] S. Berezin, S. Campos, and E. M. Clarke. Compositional reasoning in model checking. Lecture Notes in Computer Science, 1536:81-102, 1998.

[BCCZ99] A. Biere, A. Cimatti, E. Clarke, and Y. Zhu. Symbolic model checking without BDDs. In Tools and Algorithms for the Construction and Analysis of Systems. Part of European Conferences on Theory and Practice of Software, ETAPS'99, Amsterdam, volume 1579 of LNCS, pages 193-207. Springer-Verlag, 1999. 
$\left[\mathrm{BGH}^{+}\right.$05] S. Burmester, H. Giese, M. Hirsch, D. Schilling, and M. Tichy. The FUJABA real-time tool suite: Model-driven development of safety-critical, real-time systems. In Proc. of the 27th International Conference on Software Engineering (ICSE), St. Louis, Missouri, USA, pages 670-671. ACM Press, 2005.

$\left[\mathrm{BGL}^{+}\right.$00] S. Bensalem, V. Ganesh, Y. Lakhnech, C. Muñoz, S. Owre, H. Rueß, J. Rushby, V. Rusu, H. Saïdi, N. Shankar, E. Singerman, and A. Tiwari. An overview of SAL. In LFM 2000: Fifth NASA Langley Formal Methods Workshop, pages 187-196, 2000.

[BGP03] H. Barringer, D. Giannakopoulou, and C. S. Pasareanu. Proof rules for automated compositional verification through learning. In International Workshop on Specification and Verification of Component Based Systems, Finland, 2003.

[BHG87] P. Bernstein, V. Hadzilacos, and N. Goodman. Concurrency Control and Recovery in Database Systems. Addison-Wesley, 1987.

[BJR99] G. Booch, I. Jacobson, and J. Rumbaugh. The Unified Modeling Language User Guide. Addison-Wesley, 1999.

[Bow09] J. Bowen. Formal Methods Wiki. http://formalmethods.wikia. com, 2009.

[Brü08] I. Brückner. Slicing Integrated Formal Specifications for Verification. PhD thesis, Universität Paderborn, 2008.

[Bry86] R. Bryant. Graph-Based Algorithms for Boolean Function Manipulation. IEEE Transactions on Computers, C-35(8):677-691, 1986.

[BS03] E. Börger and R. Stärk. Abstract State Machines: A Method for High-Level System Design and Analysis. Springer-Verlag, Berlin, 2003.

[But09] M. Butler. Decomposition structures for event-B. In Integrated Formal Methods, 7th International Conference, IFM 2009, Düsseldorf, Germany, February 16-19, 2009. Proceedings, volume 5423 of Lecture Notes in Computer Science, pages 20-38. Springer, 2009.

[CAC06] J. Cobleigh, G. Avrunin, and L. Clarke. Breaking up is hard to do: an investigation of decomposition for assume-guarantee reasoning. In ISSTA '06: Proceedings of the 2006 international symposium on Software testing and analysis, pages 97-108, New York, NY, USA, 2006. ACM.

[CBRZ01] E. M. Clarke, A. Biere, R. Raimi, and Y. Zhu. Bounded model checking using satisfiability solving. Formal Methods in System Design, 19(1):7-34, 2001.

[CC77] P. Cousot and R. Cousot. Abstract interpretation: A unified lattice model for static analysis of programs by construction or approximation of fixpoints. In Fourth ACM Symposium on Principles of Programming Language, Los Angeles, pages 238-252. ACM Press, New York, 1977.

[CCST05] S. Chaki, E. M. Clarke, N. Sinha, and P. Thati. Automated assume-guarantee reasoning for simulation conformance. In $C A V$, volume 3576 of Lecture Notes in Computer Science, pages 534-547. Springer, 2005.

[CES86] E. Clarke, E. Emerson, and A. Sistla. Automatic verification of finite-state concurrent systems using temporal logic specifications. ACM Transactions on Programming Languages and Systems, 8(2):244-263, 1986.

$\left[\mathrm{CGJ}^{+}\right.$03] E. Clarke, O. Grumberg, S. Jha, Y. Lu, and H. Veith. Counterexample-guided abstraction refinement for symbolic model checking. J. ACM, 50(5):752-794, 2003. 
[CGK97] S. C. Cheung, D. Giannakopoulou, and J. Kramer. Verification of liveness properties using compositional reachability analysis. In Proceedings of the Sixth European Software Engineering Conference (ESEC/FSE 97), pages 227-243. Lecture Notes in Computer Science Nr. 1013, Springer-Verlag, 1997.

[CGL94] E. Clarke, O. Grumberg, and D. Long. Model checking and abstraction. ACM Transactions on Programming Languages and Systems, 16(5):1512-1542, 1994.

[CGP99] E. Clarke, O. Grumberg, and D. Peled. Model checking. MIT Press, 1999.

[CGP03] J. Cobleigh, D. Giannakopoulou, and C. Pǎsăreanu. Learning assumptions for compositional verification. In TACAS, pages 331-346, 2003.

[CJEF96] E. M. Clarke, S. Jha, R. Enders, and T. Filkorn. Exploiting symmetry in temporal logic model checking. Formal Methods in System Design, 9(1/2):77-104, 1996.

[CMP94] E. Chang, Z. Manna, and A. Pnueli. Compositional verification of real-time systems. In Logic in Computer Science (LICS '94), pages 458-467, Los Alamitos, Ca., USA, 1994. IEEE Computer Society Press.

[Coo71] S. A. Cook. The complexity of theorem-proving procedures. In Conference record of third annual ACM symposium on theory of Computing, pages 151-158, Shaker Heights, Oh., 1971. ACM.

[Cor] Correct System Design Group. Syspect subversion repository. https://homer. informatik.uni-oldenburg.de/svn/syspect.

[CS07] S. Chaki and O. Strichman. Optimized L*-based assume-guarantee reasoning. In TACAS, volume 4424 of Lecture Notes in Computer Science, pages 276-291. Springer, 2007.

[CS08] S. Chaki and O. Strichman. Three optimizations for assume-guarantee reasoning with L*. Formal Methods in System Design, 32(3):267-284, 2008.

[CW96] E. M. Clarke and J. M. Wing. Formal methods: state of the art and future directions. ACM Computing Surveys, 28(4):626-643, 1996.

[Den74] J. B. Dennis. First version of a data flow procedure language. In Colloque sur la Programmation. Springer-Verlag, Berlin, DE, 1974.

[Die05] H. Dierks. Time, Abstraction and Heuristics - Automatic Verification and Planning of Timed Systems using Abstraction and Heuristics. Habilitation thesis, 2005.

[Dij72] E. W. Dijkstra. Notes on structured programming. In Structured Programming. Academic Press, London, 1972.

[DNS08] J. Derrick, S. North, and A. J. H. Simons. Z2SAL - building a model checker for Z. In Abstract State Machines, B and Z, First International Conference, ABZ 2008, London, UK, September 16-18, 2008. Proceedings, volume 5238 of Lecture Notes in Computer Science, pages 280-293. Springer, 2008.

[DP60] M. Davis and H. Putnam. A computing procedure for quantification theory. Journal of the ACM, 7(3):201-215, 1960.

$\left[\mathrm{dRHH}^{+}\right.$01] W. de Roever, U. Hanneman, J. Hooiman, Y. Lakhneche, M. Poel, J. Zwiers, and F. de Boer. Concurrency Verification. Cambridge University Press, Cambridge, UK, 2001. 
[DW04] O. L. De Weck. Multiobjective optimization : history and promise. 2004.

[DW07] J. Derrick and H. Wehrheim. On using data abstractions for model checking refinements. Acta Inf, 44(1):41-71, 2007.

[DWQQ01] W. Dong, J. Wang, X. Qi, and Z. Qi. Model checking UML statecharts. In APSEC, pages 363-370. IEEE Computer Society, 2001.

[EC80] E. A. Emerson and E. M. Clarke. Characterizing correctness properties of parallel programs using fixpoints. In Proceedings of the 7th International Colloquium on Automata, Languages and Programming, ICALP'80, volume 85 of LNCS, pages 169181. Springer-Verlag, Berlin-Heidelberg-New York-London-Paris-Tokyo-Hong Kong, 1980.

[EDK89] E.M. Clarke, D.E. Long, and K.L. McMillan. Compositional Model Checking. In Proceedings of Fourth Annual Symposium on Logic in Computer Science, pages 353361, Washington D.C., 1989. IEEE Computer Society Press.

[EF82] T. Elrad and N. Francez. Decomposition of distributed programs into communicationclosed layers. Science of Computer Programming, 2(3):155-173, 1982.

[Ehr00] M. Ehrgott. Multicriteria optimization. Lecture Notes in Economics and Mathematical Systems. Springer-Verlag, 2000.

[ESB ${ }^{+}$09] S. Edelkamp, V. Schuppan, D. Bošnački, A. Wijs, A. Fehnker, and H. Aljazzar. Survey on directed model checking. pages 65-89, 2009.

[Fis97] C. Fischer. CSP-OZ: A combination of Object-Z and CSP. In Formal Methods for Open Object-Based Distributed Systems (FMOODS'97), volume 2, pages 423-438. Chapman \& Hall, 1997.

[Fis99] C. Fischer. Printing CSP-OZ documents with latex; documentation for csp-oz.sty. Technical report, University of Oldenburg, 1999.

[Fis00] C. Fischer. Combination and Implementation of Processes and Data: from CSP-OZ to Java. PhD thesis, University of Oldenburg, 2000.

[FMS01] A. Farias, A. Mota, and A. Sampaio. Java translator from CSP-Z to CSPM notation. http://www.di.ufpe.br/ acf/translator/CSPZtoCSPM.html, 2001.

[For05] Formal Systems (Europe) Ltd. Failure divergence refinement: FDR2 user manual. http://www.fsel.com/documentation/fdr2/fdr2manual.pdf, 2005.

[FOW87] J. Ferrante, K. J. Ottenstein, and J. D. Warren. The program dependence graph and its use in optimization. ACM Transactions on Programming Languages and Systems, pages 319-349, 1987.

[FP78] N. Francez and A. Pnueli. A proof method for cyclic programs. Acta Informatica, 9(2), 1978.

[FW99] C. Fischer and H. Wehrheim. Model-checking CSP-OZ specifications with FDR. In IFM, pages 315-334, 1999.

[Gal04] D. Galin. Software quality assurance. Pearson Education Limited, Harlow, England, 2004.

[GGP07] M. Gheorghiu, D. Giannakopoulou, and C. S. Păsăreanu. Refining interface alphabets for compositional verification. In TACAS, pages 292-307, 2007. 
[GL91] O. Grumberg and D. E. Long. Model checking and modular verification. In CONCUR '91: Proceedings of the 2nd International Conference on Concurrency Theory, pages 250-265, London, UK, 1991. Springer-Verlag.

[GMF07] A. Gupta, K. L. McMillan, and Z. Fu. Automated assumption generation for compositional verification. In $C A V$, pages 420-432, 2007.

[God96] P. Godefroid. Partial-Order Methods for the Verification of Concurrent Systems. Springer, Berlin, 1996.

[GP08] D. Giannakopoulou and C. S. Păsăreanu. Special issue on learning techniques for compositional reasoning. Form. Methods Syst. Des., 32(3):173-174, 2008.

[GP09] D. Giannakopoulou and C. S. Pasareanu. Interface generation and compositional verification in Java PathFinder. In Fundamental Approaches to Software Engineering, 12th International Conference, FASE 2009, Held as Part of the Joint European Conferences on Theory and Practice of Software, ETAPS 2009, York, UK, March 22-29, 2009. Proceedings, volume 5503 of Lecture Notes in Computer Science, pages 94-108. Springer, 2009.

[GPB02] D. Giannakopoulou, C. S. Pǎsăreanu, and H. Barringer. Assumption generation for software component verification. In ASE '02: Proceedings of the 17th IEEE international conference on Automated software engineering, pages 3-12, Washington, DC, USA, 2002. IEEE Computer Society.

[GPY02] A. Groce, D. Peled, and M. Yannakakis. Adaptive model checking. In TACAS '02: Proceedings of the 8th International Conference on Tools and Algorithms for the Construction and Analysis of Systems, pages 357-370, London, UK, 2002. SpringerVerlag.

[Gri97] A. Griffiths. Modular reasoning in Object-Z. In Proceedings: 4th Asia-Pacific Software Engineering and International Computer Science Conference, pages 140-149. IEEE Computer Society Press, 1997.

[Gri98] A. Griffiths. A formal semantics to support modular reasoning in Object-Z. $\mathrm{PhD}$ thesis, University of Queensland, 1998.

[Har87] D. Harel. Statecharts: A visual formalism for complex systems. Science of Computer Programming, 8(3):231-274, 1987.

[Her09] K. Herbold. Konzeption \& Implementierung eines Dekompositions-Werkzeugs für kompositionelle Verifikation. Diploma's thesis, Universität Paderborn, 2009.

[HJ98] C. A. R. Hoare and H. Jifeng. Unifying Theories of Programming. Prentice Hall, London, 1998.

[Hoa78] C. Hoare. Communicating sequential processes. CACM, 21:666-677, 1978.

[Hoa85] C. Hoare. Communicating Sequential Processes. Prentice Hall, 1985.

[Hoe06] J. Hoenicke. Combination of Processes, Data, and Time. PhD thesis, University of Oldenburg, 2006.

[Hol03] G. J. Holzmann. The SPIN Model Checker. Pearson Education, 2003.

[HR92] S. Horwitz and T. Reps. The use of program dependence graphs in software engineering. In ICSE '92: Proceedings of the 14th international conference on Software engineering, pages 392-411, New York, NY, USA, 1992. ACM. 
[ISO89] ISO - International Standards Organization. Information processing systems - open systems interconnection - LOTOS - A formal description technique based on the temporal ordering of observational behaviour. Technical report, 1989. ISO/IEC 8807.

[ISO00] ISO - International Standards Organization. Information technology - Z formal specification notation - syntax, type system and semantics. Technical report, 2000. ISO/IEC 13568.

[ISO01] ISO - International Standards Organization. Information technology - enhancements to LOTOS (E-LOTOS). Technical report, 2001. ISO/IEC 15437.

[JEK ${ }^{+}$90] J.R. Burch, E.M. Clarke, K.L. McMillan, D.L. Dill, and L.J. Hwang. Symbolic Model Checking: $10^{20}$ States and Beyond. In Proceedings of the Fifth Annual IEEE Symposium on Logic in Computer Science, pages 1-33, Washington, D.C., 1990. IEEE Computer Society Press.

[Jon83] C. Jones. Specification and design of (parallel) programs. In IFIP Congress, pages 321-332, 1983.

[KK99] G. Karypis and V. Kumar. Multilevel k -way hypergraph partitioning. In DAC, pages 343-348, 1999.

[KP88] S. Katz and D. Peled. An efficient verification method for parallel and distributed programs. In Workshop on Linear Time, Branching Time and Partial Order in Logics and Models for Concurrency, number 354 in LNCS, pages 489-507, Noordwijkerhout, The Netherlands, 1988. Springer-Verlag.

[KS01] G. Kassel and G. Smith. Model checking Object-Z classes: Some experiments with FDR. In APSEC, pages 445-452. IEEE Computer Society, 2001.

[Kur94] R. P. Kurshan. Computer-aided Verification of Coordinating Processes - The AutomataTheoretic Approach. Princeton Univ. Press, 1994.

[Leu] M. Leuschel. ProB homepage. http://Www.stups.uni-duesseldorf.de/ ProB.

[LF08] M. Leuschel and M. Fontaine. Probing the depths of CSP-M: A new fdr-compliant validation tool. In Formal Methods and Software Engineering, 10th International Conference on Formal Engineering Methods, ICFEM 2008, Kitakyushu-City, Japan, October 27-31, 2008. Proceedings, volume 5256 of Lecture Notes in Computer Science, pages 278-297. Springer, 2008.

[LMC01] M. Leuschel, T. Massart, and A. Currie. How to make FDR spin - LTL model checking of CSP by refinement. In FME 2001: Formal Methods for Increasing Software Productivity, International Symposium of Formal Methods Europe, Berlin, Germany, March 12-16, 2001, Proceedings, volume 2021 of Lecture Notes in Computer Science, pages 99-118. Springer, 2001.

[LZ74] Liskov and Zilles. Programming with abstract data types. Sigplan Notices, 9, 1974.

[Mai03] P. Maier. Compositional circular assume-guarantee rules cannot be sound and complete. In FoSSaCS, pages 343-357, 2003.

[MC81] J. Misra and K. M. Chandy. Proofs of networks of processes. IEEE Trans. Softw. Eng., 7(4):417-426, 1981. 
[McM93] K. L. McMillan. Symbolic Model Checking: An Approach to the State Explosion Problem. Kluwer Academic Publishers, 1993.

[MDA] OMG model driven architecture. http://www.omg.org/mda.

[MG07] N. Moffat and M. Goldsmith. Assumption-commitment support for CSP model checking. Electron. Notes Theor. Comput. Sci., 185:121-137, 2007.

[Mic10] S. Micus. Rückführung und Visualisierung von Gegenbeispielen aus einem Model Checker. Bachelor's thesis, Universität Paderborn, 2010.

[Mil89] R. Milner. Communication and Concurrency. Prentice-Hall, 1989.

[Mil99] R. Milner. Communicating and Mobile Systems: The $\pi$ Calculus. Cambridge University Press, Cambridge, England, 1999.

[Moo90] A. P. Moore. The specification and verified decomposition of system requirements using CSP. IEEE Transactions on Software Engineering, 16:932-948, 1990.

[MORW08] M. Möller, E.-R. Olderog, H. Rasch, and H. Wehrheim. Integrating a formal method into a software engineering process with UML and Java. Formal Apsects of Computing, 20:161-204, 2008.

[MS98] A. Mota and A. Sampaio. Model-checking CSP-Z. In FASE, pages 205-220, 1998.

[MS01] A. Mota and A. Sampaio. Model-checking CSP-Z: strategy, tool support and industrial application. Sci. Comput. Program, 40(1):59-96, 2001.

[MWW08] B. Metzler, H. Wehrheim, and D. Wonisch. Decomposition for compositional verification. In Formal Methods and Software Engineering, 10th International Conference on Formal Engineering Methods, ICFEM 2008, Kitakyushu-City, Japan, October 27-31, 2008. Proceedings, volume 5256 of Lecture Notes in Computer Science, pages 105-125. Springer, 2008.

[NA06] W. Nam and R. Alur. Learning-based symbolic assume-guarantee reasoning with automatic decomposition. In ATVA, pages 170-185, 2006.

[Nam07] W. Nam. Synthesis and Compositional Verification Using Language Learning. PhD thesis, University of Pennsylvania, 2007.

[NAS] NASA Ames Research Center. Java PathFinder. http://babelfish.arc.nasa. gov/trac/jpf.

[Obj05] Object Management Group. OMG unified modeling language 2.0. http: //www . omg.com/uml, 2005.

[OD08] E.-R. Olderog and H. Dierks. Real-Time Systems: Formal Specification and Automatic Verification. Scientific and Engineering Computation Series. Cambridge University Press, New York, NY, 2008.

[OL82] S. Owicki and L. Lamport. Proving liveness properties of concurrent programs. ACM Transactions on Programming Languages and Systems, 4(3), 1982.

[OW05] E.-R. Olderog and H. Wehrheim. Specification and (property) inheritance in CSP-OZ. Science of Computer Programming, 55(1-3):227-257, 2005.

[Par71] V. Pareto. Manual of Political Economy. Kelley, New York, 1971. Originally published 1927. Translated from the Italian by A. S. Schwier, edited by A. S. Schwier and A. N. Page. 
[PBG05] M. R. Prasad, A. Biere, and A. Gupta. A survey of recent advances in SAT-based formal verification. International Journal on Software Tools for Technology Transfer (STTT), 7(2):156-173, 2005.

$\left[\mathrm{PGB}^{+}\right.$08] C. S. Pasareanu, D. Giannakopoulou, M. G. Bobaru, J. M. Cobleigh, and H. Barringer. Learning to divide and conquer: applying the $\mathrm{L}^{*}$ algorithm to automate assumeguarantee reasoning. Formal Methods in System Design, 32(3):175-205, 2008.

[Pie10] M. Piepmeyer. Effiziente Validierung und Bewertung von Modellzerlegungen. Diploma's thesis, Universität Paderborn, 2010.

[Pnu84] A. Pnueli. In transition from global to modular temporal reasoning about programs. In Logics and Model of Concurrent Systems, volume 13 of NATO ASI, pages 123-144. Springer-Verlag, Berlin, New York, 1984.

[Pnu85] A. Pnueli. Linear and branching structures in the semantics and logics of reactive systems. Lecture Notes Comp. Sci., 194:15-32, 1985.

[Rei85] W. Reisig. Petri Nets: An Introduction. Springer-Verlag, 1985.

[Res] Research Group Specification and Modelling of Software Systems. Syspect extensions subversion repository. https://svn-serv.cs.upb.de/syspect-plugins.

[Ros98] W. A. Roscoe. Theory and Practice of Concurrency. Prentice-Hall, 1998.

[RR95] T. Reps and G. Rosay. Precise interprocedural chopping. In SIGSOFT FSE, pages 41-52, 1995.

[RW94] A. Rensink and H. Wehrheim. Weak sequential composition in process algebras. In Proceedings of the Fifth International Conference on Concurrency Theory CONCUR'94, Uppsala (Sweden), pages 226-241, Berlin-Heidelberg-New York, 1994. Springer.

[Sch99] S. Schneider. Concurrent and Real Time Systems: The CSP Approach. John Wiley \& Sons, Inc., New York, NY, USA, 1999.

[Sch02] P. Schnoebelen. The complexity of temporal logic model checking. In Advances in Modal Logic, pages 393-436. King's College Publications, 2002.

[Sch05] S. Schneider. Non-blocking data refinement and traces-divergences semantics. Technical report, University of Surrey, 2005.

[Sch09] S. Schneider. Personal communication, 2009.

[SGT ${ }^{+}$03] W. Schäfer, H. Giese, M. Tichy, S. Burmester, and S. Flake. Towards the compositional verification of real-time UML designs. In ESEC/SIGSOFT FSE, pages 38-47. ACM, 2003.

[SLU89] K. K. Sabnani, A. M. Lapone, and M. U. Uyar. An algorithmic procedure for checking safety properties of protocols. IEEE Trans. Communications, 37(9):940-948, 1989.

[Smi95] G. Smith. A fully abstract semantics of classes for Object-Z. Formal Aspects of Computing, 7(3):289-313, 1995.

[Smi00] G. Smith. The Object-Z Specification Language. Kluwer Academic Publisher, 2000.

[SNT85] Y. Sawaragi, H. Nakayama, and T. Tanino. Theory of multi-objective optimization. Academic Press, Inc., Orlando, FL, 1985.

[Spi92] J. M. Spivey. The $Z$ notation: a reference manual. Prentice Hall International (UK) Ltd., Hertfordshire, UK, 1992. 
[ST02] S. Schneider and H. Treharne. Communicating B machines. In ZB '02: Proceedings of the 2nd International Conference of $B$ and $Z$ Users on Formal Specification and Development in $Z$ and B, pages 416-435, London, UK, 2002. Springer-Verlag.

[ST04] S. Schneider and H. Treharne. Verifying controlled components. In IFM, pages 87-107, 2004.

[ST05] S. Schneider and H. Treharne. CSP theorems for communicating B machines. Formal Asp. Comput., 17(4):390-422, 2005.

[Sta06] A. Stamer. Integration von CSP-OZ in die OO-Softwareentwicklung für die automatische Verifikation. Diploma's thesis, Universität Oldenburg, 2006.

[STE05] S. Schneider, H. Treharne, and N. Evans. Chunks: Component verification in CSP $\mid$ B. In IFM'2005, pages 89-108, 2005.

[SW05] G. Smith and L. Wildman. Model checking Z specifications using SAL. In ZB, pages 85-103, 2005.

[SWC02] A. Sampaio, J. Woodcock, and A. Cavalcanti. Refinement in Circus. In FME 2002: Formal Methods - Getting IT Right, volume 2391 of Lecture Notes in Computer Science, pages 451-470. Springer-Verlag, 2002.

[Sys06] Syspect. Endbericht der Projektgruppe Syspect. Technical report, Carl von Ossietzky University of Oldenburg, 2006.

[TA97] K. Taguchi and K. Araki. The state-based CCS semantics for concurrent Z specification. In ICFEM, pages 283-292, 1997.

[TJ02] J. J. P. Tsai and E. Y. T. Juan. Model and heuristic technique for efficient verification of component-based software systems. In IEEE ICCI, pages 59-68. IEEE Computer Society, 2002.

[TS99] H. Treharne and S. Schneider. Using a process algebra to control B operations. In IFM '99: Proceedings of the 1st International Conference on Integrated Formal Methods, pages 437-456, London, UK, 1999. Springer-Verlag.

[WC02] J. Woodcock and A. Cavalcanti. The semantics of Circus. In ZB 2002: Formal Specification and Development in $Z$ and B, volume 2272 of Lecture Notes in Computer Science, pages 184-203. Springer-Verlag, 2002.

[Weh00] H. Wehrheim. Data abstraction techniques in the validation of CSP-OZ specifications. Formal Aspects of Computing, 12, 2000.

[Wei81] M. Weiser. Program slicing. In Proceedings of the 5th International Conference on Software Engineering, pages 439-449. IEEE Computer Society Press, 1981.

[Wik06] Wikipedia. Retrieved from http://en.wikipedia.org/wiki/Wikipedia, 2006.

[Won] D. Wonisch. CSPLChecker homepage. http://www.cs.uni-paderborn.de/ index . php? $i d=8967 \& \mathrm{~L}=1$.

[Won08] D. Wonisch. Automatisiertes kompositionelles Model Checking von CSP Spezifikationen. Bachelor's thesis, Universität Paderborn, 2008.

[WS03] K. Winter and G. Smith. Compositional verification for Object-Z. In $Z B$, pages 280-299, 2003. 
[WW09] H. Wehrheim and D. Wonisch. Compositional CSP traces refinement checking. In Proceedings of the Eighth International Workshop on Automated Verification of Critical Systems (AVoCS 2008), volume 250, Issue 2, pages 135-151. Elsevier B.V., 2009.

[Xie96] M. Xie. Handbook of Software Reliability Engineering. McCraw Hill, New York, 1996.

[Zel74] M. Zeleny. Linear Multiobjective Programming, volume 95 of Lecture Notes in Economics and Mathematical Systems. Springer, Berlin/New York, 1974.

[ZH04] C. Zhou and M. R. Hansen. Duration Calculus: A Formal Approach to Real-Time Systems. EATCS: Monographs in Theoretical Computer Science. Springer, 2004. 


\section{List of Figures}

1.1 Decomposition of a specification $S$ into $S_{1}$ and $S_{2} \ldots \ldots \ldots \ldots$

1.2 Illustration of the overall approach of this thesis . . . . . . . . . . . 4

2.1 Structure of a CSP-OZ specification . . . . . . . . . . . . . . 12

2.2 Structure of the Object-Z part of a CSP-OZ specification . . . . . . . . . . 12

2.3 Candy machine specification . . . . . . . . . . . . . . . . . . 14

2.4 Illustration of the CSP part of the candy machine specification . . . . . . 16

2.5 Simplified grammar of CSP . . . . . . . . . . . . . . . . 20

2.6 Correctness requirement for the candy machine specification . . . . . . . . 24

2.7 Translation of a CSP-OZ specification into a CSP process . . . . . . . . . . 25

2.8 Simple CSP-OZ class specification for swapping two numbers . . . . . . . . 28

2.9 Control flow graph (CFG) for the candy machine specification . . . . . . . 31

2.10 Simple CSP-OZ class specification for a ticket machine . . . . . . . . . . 35

2.11 Data dependence graph (DDG) for the ticket machine specification . . . . 36

2.12 Extract of DDG for the candy machine specification . . . . . . . . . . 37

2.13 Dependence graph (DG) for the candy machine specification . . . . . . . . 39

3.1 Basic assume-guarantee proof rule (B-AGR) . . . . . . . . . . . . . . . 44

3.2 Parallel assume-guarantee proof rule (P-AGR) . . . . . . . . . . . . . 44

3.3 Circular assume-guarantee rule (C-AGR) . . . . . . . . . . . . . 45

3.4 Illustration of the $\mathrm{L}^{*}$ algorithm $\ldots \ldots \ldots \ldots$. . . . . . . . . 46

3.5 Illustration of the $\mathrm{L}^{*}$ based learning framework . . . . . . . . . . . 47

3.6 Rule (B-AGR) rephrased in terms of CSP trace refinement . . . . . . . . . . 49

3.7 Rule (P-AGR) rephrased in terms of CSP trace refinement . . . . . . . . 49

3.8 CSP specification of a simple elevator system . . . . . . . . . . . . 50

4.1 Overview of the cut identification and the decomposition . . . . . . . . . 57

4.2 Illustration of Definition $4.2 .1 \ldots \ldots \ldots \ldots$

4.3 Fragmentation of the DG . . . . . . . . . . . . . . . . 60

4.4 Disallowed control flow edges based on disjointness . . . . . . . . . . . . . 62

4.5 Motivation for the correctness criterion no crossing . . . . . . . . . . . 62

4.6 Disallowed data dependences based on no crossing . . . . . . . . . . 63

4.7 Motivation for the correctness criterion no reaching back . . . . . . . . 64

4.8 Disallowed edges based on no reaching back . . . . . . . . . . . . . 65

4.9 Fragmentation of the set of operation nodes in general case . . . . . . . 67

4.10 Assignment of DG edges to the subgraphs . . . . . . . . . . . . 68

4.11 Fragmentation of the set of operation nodes in the special case . . . . . . . 69

4.12 Cut of the dependence graph for the candy machine . . . . . . . . . 71

4.13 Constituents of a CSP-OZ class specification . . . . . . . . . . . 72 
4.14 Correspondence between graph nodes and specification operations . . . . 73

4.15 Simple CSP-OZ specification for increasing two natural numbers . . . . . . 80

4.16 Intermediate decomposition of Increaser . . . . . . . . . . . . . . 80

4.17 Possible data dependences targeting the cut and originating from the cut . 81

4.18 Illustration of the transmission parameters . . . . . . . . . . . . . . . 84

4.19 Decomposition of Increaser, modified according to Definition 4.3.10 . . . . 85

4.20 Synchronisation of events for external choice . . . . . . . . . . . . 87

4.21 Addressing extension for CFG branching . . . . . . . . . . . . . . . . 89

4.22 Addressing extension for nested branching . . . . . . . . . . . . 90

4.23 Illustration of Theorem 4.3.16 . . . . . . . . . . . . . . . . . . . 96

4.24 Decomposition of the candy machine, first component . . . . . . . . . 104

4.25 Decomposition of the candy machine, second component . . . . . . . . . 105

4.26 CSP-OZ specification for swapping two numbers, extended . . . . . . . . 107

4.27 Decomposition of the number swapper, first component . . . . . . . . . . 108

4.28 Decomposition of the number swapper, second component . . . . . . . . 108

4.29 Correctness requirement for Swapper . . . . . . . . . . . . . . . . . . 109

5.1 Illustration of the steps of the correctness proof . . . . . . . . . . . . . 112

5.2 Algorithm for the address extension: procedure ADDRESSMAIN . . . . . 114

5.3 Algorithm for the address extension: procedure ADDRESSCUT . . . . . . 114

5.4 Algorithm for the address extension: procedure ModifyCuT . . . . . . . 115

5.5 Algorithm for the address extension: procedure ADD . . . . . . . . . 116

5.6 Illustration of a violation of Lemma 5.2 .1 . . . . . . . . . . . . . . 120

5.7 Illustration of a violation of Lemma 5.2 .2 . . . . . . . . . . . . . . . 120

5.8 Illustration of the CSP correctness proof of binary operators . . . . . . . 121

5.9 Case differentiation for Lemma 5.2.3, parallel composition . . . . . . . . 131

5.10 Illustration of Definition 5.3.2 and Lemmas 5.3.3, 5.3.4 . . . . . . . . . . 141

5.11 Illustration of Lemma $5.3 .12 \ldots \ldots$. . . . . . . . . . . . . 151

5.12 Illustration of Lemma $5.3 .15 \ldots$. . . . . . . . . . . . . . 157

6.1 Illustration of the Two Phase Commit Protocol . . . . . . . . . . . . . . . 176

6.2 Phase one of the Two Phase Commit Protocol . . . . . . . . . . . . . 176

6.3 Phase two of the Two Phase Commit Protocol . . . . . . . . . . . . . . . 177

6.4 Two Phase Commit Protocol: Coord specification . . . . . . . . . . . . . . 177

6.5 Two Phase Commit Protocol: Page specification . . . . . . . . . . . . . 178

7.1 Syspect class diagram for the ТРСР . . . . . . . . . . . . . . . . 185

7.2 Syspect property view for the operation Page.inform . . . . . . . . . . . . 185

7.3 Syspect state machine for the class Page of TPCP . . . . . . . . . . . . . 186

7.4 Syspect state machine for the class Coord of TPCP . . . . . . . . . . . . . 187

7.5 Syspect component diagram for the TPCP . . . . . . . . . . . . . . 188

7.6 Toolchain for the verification framework . . . . . . . . . . . . . . . . 189

7.7 Screenshot of a selected invalid cut . . . . . . . . . . . . . . . . . . 190

7.8 Screenshot of the decomposition options after selection of a valid cut . . . 191 
7.9 Screenshot of the mass validation framework . . . . . . . . . . . . . 193

7.10 Correctness requirement for the TPCP in terms of $\mathrm{CSP}_{\mathrm{M}} \ldots \ldots$. . . . . . 194

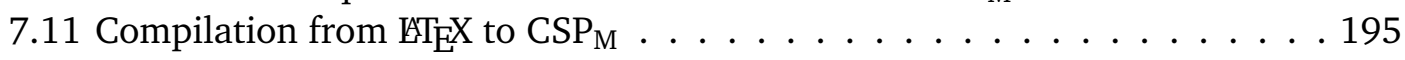

7.12 Screenshot of the CSPLChecker . . . . . . . . . . . . . . . . 196

7.13 Screenshot of the counterexample visualisation . . . . . . . . . . . 197

7.14 Verification framework . . . . . . . . . . . . . . . . . . . . . . 199

7.15 Correctness requirement for the candy machine in terms of $\operatorname{CSP}_{\mathrm{M}} \ldots$. . . 202

7.16 Correctness requirement for the number swapper in terms of $\operatorname{CSP}_{\mathrm{M}} \ldots . .207$

7.17 Decomposition of the TPCP: Coord specification . . . . . . . . . . . . 213

7.18 Decomposition of the TPCP: Page specification . . . . . . . . . . . . . 214

7.19 Justification for predominance of cut $\{$ vote, decide $\} \ldots$. . . . . . . 215 



\section{List of Tables}

1.1 Contributions of this thesis $\ldots \ldots \ldots \ldots \ldots \ldots$

2.1 Comparison between the different semantics for CSP-OZ . . . . . . . . . . 27

2.2 Table of nodes of the control flow graph . . . . . . . . . . . . . 30

2.3 Table of edges of the data dependence graph . . . . . . . . . . . 33

4.1 Comparison between the general cut and the single cut . . . . . . . . . 70

4.2 Comparison of two traces for Increaser and its components . . . . . . . . . 82

4.3 Comparison of two traces of Increaser and its components after modification 85

6.1 Heuristic $h_{\mathrm{cs}}$ : cut size . . . . . . . . . . . . . . . . . . . . . 170

6.2 Heuristic $h_{\mathrm{ED}}$ : even distribution . . . . . . . . . . . . . 170

6.3 Heuristic $h_{\mathrm{FT}}$ : few transmission . . . . . . . . . . . . . . 172

6.4 Heuristic $h_{\mathrm{FA}}$ : few addressing . . . . . . . . . . . . . . . 172

6.5 Set of valid cuts for the candy machine . . . . . . . . . . . . . . . 175

6.6 Set of valid cuts for the TPCP . . . . . . . . . . . . . . . . . 179

7.1 Experimental results for the candy machine . . . . . . . . . . . . . . 203

7.2 Experimental Results for the TPCP, first part . . . . . . . . . . . . . 205

7.3 Experimental Results for the TPCP, second part . . . . . . . . . . . 206

7.4 Experimental results for the (extended) number swapper, first part . . . . 208

7.5 Experimental results for the (extended) number swapper, second part . . . 209

7.6 Summary of the experimental results . . . . . . . . . . . . . . . . . 209 



\section{Index}

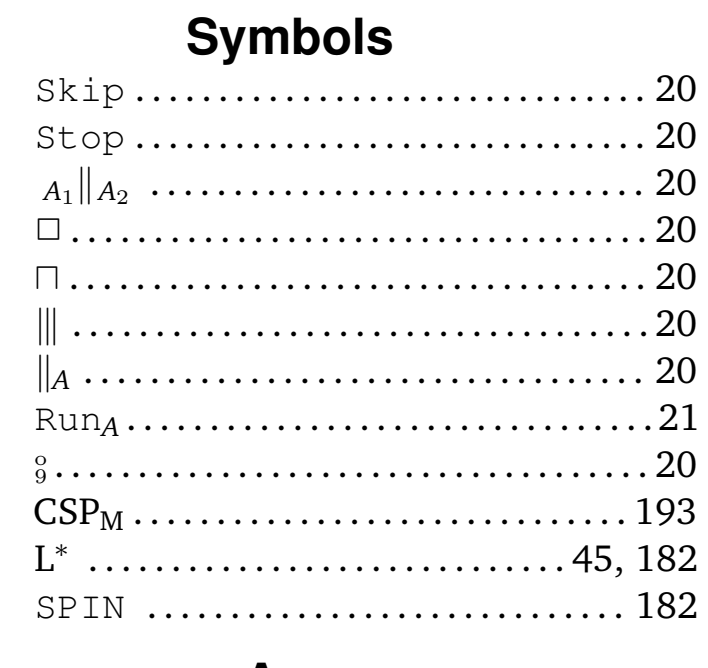

A

abstract interpretation............44

address algorithm................ 114

correctness ................. 118

termination................. 117

allowed synchronisation...........992

assume-guarantee reasoning ...... 3, 43

basic proof rule ............. 44

circular proof rule............44

parallel proof rule.............44

soundness of basic proof rule....50

soundness of parallel proof rule . 51

\section{B}

black box checking...............54

bounded model checking .......... 43

\section{C}

CCS ......................... 10

CCS-Z........................10

CFG ............ see control flow graph

Circus........................ 11

Communicating Sequential Processessee CSP

Communication Closed Layers law . 212 compositional verification.........3, 43 learning ................. 45

cone-of-influence reduction.........42

control flow analysis ............. 29

control flow graph ............... 29

fragmentation................59

completeness .............6 60

labelling of nodes ............. 32

paths ....................... 30

recursion-free.............. 141

phase..................... 59

CSP ....................11, 20

compositional verification .......53

failures-divergences model ...... 22

semantics..................22

set of CSP terms..............21

stable failures model ........... 22

CSP||B .....................10

compositional verification .......53

CSP process

alphabetised parallel..........20

channel.....................20

extension set..............21

channel type................20

choice ...................... 20

guarding of events ............ 25

hiding .................... 20

indexed choice ...............21

indexed parallel composition ....21

interface parallel.............20

interleaving $\ldots \ldots \ldots \ldots \ldots \ldots 20$

partial event ................ 22

prefix.....................20

prefix choice................ 21

process call ................. 20

projection................... 76

redistribution laws........164, 165

renaming ................... 20

traces.....................22

initials................... 23 
projection.................23 $\mathrm{CSP}_{Z} \ldots \ldots \ldots \ldots \ldots \ldots \ldots \ldots 24,194$ CSP-OZ

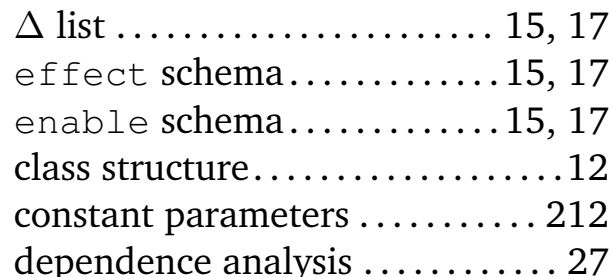

failures-divergences semantics...25 initial state schema........13, 17 input parameter...............13

interface.................... 12

operation schema ......... 12, 16

declaration part.............18

delta list.................. 18

modified variables ........... 17

predicate part............... 18

referenced variables..........17

output parameter............. 13

parameter.................. 13

semantics...................24

set of events ............... 16

simple parameter ............. 13

state ..................... 17

projection.................17

state invariants..............17

state schema .............13, 16

state variable ............... 16

initial closure.............. 106

CSP-OZ-DC ..................... 11

CSP-Z..................10, 193

CSPLChecker .............. 192, 195

cut........................66

comparison of single and general 70 correctness criteria

all-or-none................66

disjointness ..............661

no crossing ...............6 63

no reaching back............65

general ...................66 66

interval between cut sets ....... 58

single....................68

properties..................69
D

data abstraction .............. 3, 42

data dependence................. 33

direct...................... 34

direct by reason ...............34

initial.......................33

interference.................34

interference by reason..........34

synchronisation................ 34

data dependence graph.............33

decomposition .................. 100

correctness ................. 101

no distribution of initial events 120

no split of synchronisation ... 121

redistribution of CSP processes122

correctness proof ............ 166

correctness proof of CSP part .. 132

correctness proof of Object-Z part 152,157

Pareto-optimal.............. 173

unreasonable............... 173

connection to heuristics......173

weakly dominated ........... 173

decomposition components

Init schemas . ............. 77

correctness ........... 143, 144

State schemas.............. 77

conditions for correct synchronisation.....................90 90

correctness proof ........... 93

CSP parts.................. 100

cut variables ................. 83

event sets ................... 97

interfaces ................. 99

operation schemas ........... 83

renaming of channels ......... 98

renaming of events............999

distributivity law ........... 162

properties ................. 161

sets of operations ............ 75

decomposition heuristics

cut size.................... 169

even distribution..............170

few addressing................172 
few transmission............. 170 dependence graph.................37 DG ............. see dependence graph directed model checking ......... 182 Duration Calculus ................. 11

\section{E}

E-LOTOS ......................11

earlier stage .................... 140

equivalence relation ............. 106

Event-B ....................... 10

compositional verification .......53

$\mathbf{F}$

FDR2 …...........25, 53, 192

formal methods.................2, 9

formal verification ................2

\section{H}

hypergraph partitioning 181

\section{I}

identity relation............106, 123

integrated formal methods ........ 2, 9

$$
\mathbf{J}
$$

Java PathFinder

labelled transition system .......... 18

CSP part...............23

language................44

Object-Z part.................19

parallel composition .......... 26

path ................... 19

liveness property ..............48

LOTOS ........................ 10

LTS ....... see labelled transition system

\section{M}

model checking............ 3, 41, 42

model driven development (MDD) .... 1 multicriteria optimisation ..... 173, 182

\section{N}

NP completeness 192
Object-Z..................11, 16

compositional verification .......53

history semantics ........... 18

semantics................. 18

structure................... 12

operational semantics

CSP part .................. 22

CSP-OZ .................... 24

Object-Z part ............... 18

$\mathbf{P}$

partial order reduction........... 3,42 PDG ........... see dependence graph $\pi$-calculus.................... 10

program dependence graph ......... see dependence graph

$\mathbf{R}$

real-time systems .............. 182

refinement....................23

\section{$\mathbf{S}$}

safety properties ............... 22

safety property $\ldots \ldots \ldots \ldots \ldots \ldots \ldots 48$

SAL ......................... 53

SAT solving .................... 192

software quality assurance (SQA) ..... 1

software testing $\ldots \ldots \ldots \ldots \ldots \ldots \ldots 1$

software verification...............

state explosion............. 3, 41, 42

symbolic model checking ........ 3, 43

symbolic transition systems ... 110, 181

symmetry reduction ..............42

synchronisation dependence.........34

realisation of ...............91

Syspect...................... 184

class diagram ............... 184

component diagram...........187

countertrace plug-in .......... 196

decomposition framework ..... 188

decomposition plug-in ........ 189

export to $\mathrm{AT}_{\mathrm{E}} \mathrm{X}$................. 187

mass validation ............... 191

property view................ 185 
state machine............... 186

\section{$\mathbf{T}$}

timed automata .................. 10

trace equivalence.................23

trace refinement ................. 23

Two Phase Commit Protocol.........175

\section{U}

Unified Modelling Language........1, 9 activity diagram ............ 198

compositional verification ....... 53

\section{Z}

Z notation.....................11

axiomatic definition............ 12

basic type.................. 11

free type $\ldots \ldots \ldots \ldots \ldots \ldots \ldots 11$ 